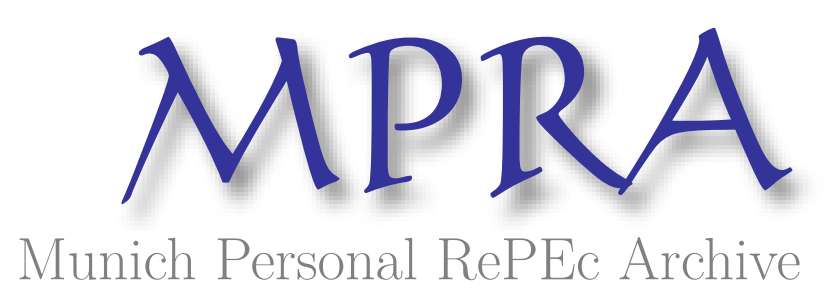

\title{
Demographic convergence and its linkage with health inequalities in India
}

Srinivas, Goli

Jawaharlal Nehru University

5 November 2014

Online at https://mpra.ub.uni-muenchen.de/79823/

MPRA Paper No. 79823, posted 22 Jun 2017 12:53 UTC 


\title{
Demographic convergence and its linkage with health inequalities in India
}

\author{
Srinivas Goli \\ Thesis submitted for the award of \\ Doctor of Philosophy \\ in \\ Population Studies
}

International Institute for Population Sciences (Deemed University)

Deonar, Mumbai-40008

2014 


\title{
Demographic convergence and its linkage with health inequalities in India
}

\author{
Srinivas Goli \\ Thesis submitted for the award of \\ Doctor of Philosophy \\ in \\ Population Studies
}

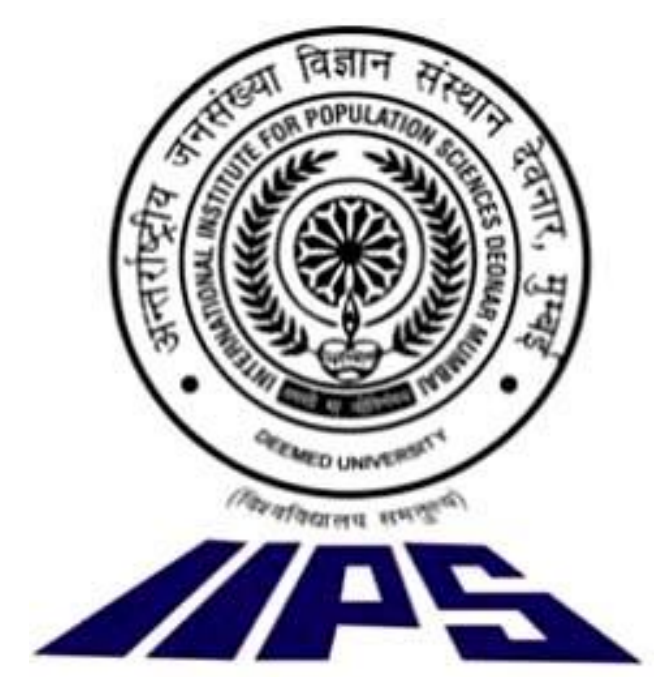

International Institute for Population Sciences

(Deemed University)

Deonar, Mumbai-40008

2014 


\section{$\underline{\text { Declaration }}$}

The work presented in this thesis entitled "Demographic convergence and its linkage with heal th inequalities in India" has been carried out entirely by me under the guidance of Prof. P. Arokiasamy, Head, Department of Development Studies, International Institute for Population Sciences, Deonar, Mumbai.

I further declare that this work is original and has not been submitted in part or full to any University/ Institute for the award of any Degree/ Diploma.

Place: Mumbai

$19^{\text {th }}$ August, 2014

(Srinivas Goli)

\section{CERTIFICATE}

I certify that the above- mentioned declaration is true to the best of my knowledge and belief.

Place: Mumbai

$19^{\text {th }}$ August, 2014

Studies
(Dr. P. Arokiasamy)

Professor \& Head

Department of Development

IIPS, Mumbai 


\section{Acknowledgement}

I express my cordial gratitude and appreciation to all who have bestowed their support, motivation, encouragement, cooperation, suggestions and blessings for the successful completion of this thesis.

I express my heartiest sense of gratitude and indebted to my research guide, Prof. P. Arokiasamy, Head Department of Development Studies, International Institute for Population Sciences (IIPS), who not only gave me insights into the topic but also helped me in generating ideas and motivating to complete this work successfully.

I owe a deep sense of gratitude to Prof. F. Ram, Director of IIPS for giving me permission to commence this thesis in the first instance to do the necessary research work and for providing me with all the required facilities.

I wish to convey my earnest gratitude to Dr. Shawn F. Dorius for his expertise suggestions and guidance on the research topic throughout my research period.

I would like to thank Prof. Jean Dreze, Prof. Christopher Wilson, Prof. Lalit Dandona, Prof. Vladimir M. Shkolnikov and Prof. Alan Lopez for their valuable suggestions and guidance.

I wish to convey my deep gratitude to all faculty members of IIPS and research staff for their cooperation and encouragement throughout my research period.

I express my sincere thanks to Mr. M. Sekhar, Assistant Registrar (Academic) for his kind cooperation and support at various point of time. I am also thankful to Mr. Somnath and Mr. Ankush at the academic section for their constant help and encouragement. 
The valuable support provided by the exquisite richness of the IIPS library deserves special acknowledgement. In this connection, the unfailing cooperation and help extended by Mr. D. D. Mestri (Library and Information officer) is worth remembering. The friendly nature and support of other library staff especially, Vasu, Ashish, Santosh, Sudhir, Rakesh and Mr. Vidhate deserve thanks.

I take this opportunity to thank my wife and friends for their friendly encouragement and moral support.

Srinivas Goli 


\section{Glossary/Acronyms}

DTM

TFP

GDP

SES

NSDP

TFR

LEB

IMR

NNMR

MMR

DMM

DMF

AID

OLS

CI

CIs

HDI

CV

MDG

SD

ST

SC

OBC

DF

SRS

RBI

NFHS

WHO

UN

UNDP

DHS

RGI

CIM

$\mathrm{CU}$

$\mathrm{Cr}$

EAG

$\beta$

$\sigma$
Demographic Transition Model

Total Factor Productivity

Gross Domestic Product

Socio-Economic Strata

Net State Domestic Product

Total Fertility Rate

Life Expectancy at Birth

Infant Mortality Rate

Neonatal Mortality Rate

Maternal Mortality Rate

Dispersion Measure of Mortality

Dispersion Measure of Fertility

Average Interstate Differences

Ordinary Least Square

Confidence Interval

Concentration Indices

Human Development Index

Coefficient of Variation

Millennium Development Goals

Standard Deviation

Scheduled Tribe

Scheduled Caste

Other Backward Caste

Degree of Freedom

Sample Registration System

Reserve Bank of India

National Family Health Survey

World Health Organization

United Nations

United Nations Development Programme

Demographic Health Surveys

Registrar General of India

Children Full Immunisation

Children Underweight

Correlation

Empowered Action Group

Beta

Sigma 


\section{Contents}

Acknowledgements

Glossary

Tables

Figures

Background

\subsection{1.}

Socioeconomic Transition and Convergence

1.1.2.

Demographic Transition and Convergence

1.1.3.

Health Transition and Convergence

1.1.4.

Health Inequalities

1.2.

Health Inequalities versus Socioeconomic, Demographic and Health Transition and Convergence

Literature Review

Socioeconomic Transition and Convergence

1.5 .

Objectives

1.6.

Hypotheses

\section{CHAPTER 2}

Data and Methodology

Data sources

2.2 .

Methods

Concepts

\section{CHAPTER 3}

3.1 .

3.2 .

3.3 .

3.4 . 
$\begin{array}{lll}\text { 3.4.2. } & \text { Population Size } & 57\end{array}$

3.4.3. TFR $\quad 60$

3.4.4. LEB 62

$\begin{array}{lll}3.5 . & \text { Discussion } & 64\end{array}$

\section{CHAPTER 4}

4.1.

4.2.

4.3 .

4.4

4.5.

4.5.1.

4.5.2.

4.5.3.

4.5.4.

4.5.5.

4.5.6.

4.5.7.

4.5.8.

4.6.

\section{CHAPTER 5}

5.1 .

5.2 .

5.3.

5.4 .

5.4.1.

5.4.2.

5.4.3.

5.4.4.

5.4.5.

5.4.6.

5.4.7.

5.4.8.

5.5.

CHAPTER 6

6.1.

6.2.

6.3 .

\section{Socioeconomic Convergence}

Introduction

Concept of Unequal Regional Progress and Approaches

Previous Evidence of Socioeconomic Progress and Convergence among Indian States

Data and Methods

Results

Descriptive Statistics

Trend Analyses

Catching-up Process

$\beta$-convergences

Conditional $\beta$-Convergence

$\sigma$-Convergence

Kernel Density Estimates and Convergence Clubs

Inequality based measure of Convergence

Discussion
67-93

94-128

Introduction

94

98

99

99

99

105

108

110

112

114

115

120

127

Discussion
129-155

131

131

132 
$\begin{array}{lll}\text { 6.3.2. } & 133\end{array}$

6.4. Results $\quad 134$

6.4.1. Fertility Trends in India and States 134

6.4.2. Closing and Catching-Up Process of Gaps in Fertility Rates

6.4.3. Convergence Estimates 140

6.4.3.1. Convergence in Average Fertility Rates: Absolute $\beta-$ Convergence Model Estimates

6.4.3.2. Conditional $\beta$-convergence model estimates 141

$\begin{array}{lll}\text { 6.4.4. } & 142 \\ 6.4 .5 & \text { Convergence Clubs }\end{array}$

$\begin{array}{lll}\text { 6.4.5. Sigma Convergence } & 148\end{array}$

6.4.6. Inequality Adjusted Convergence Measures: Convergence in Absolute and Relative Dispersion of State Level Fertility Rates

6.4.7. Nonparametric Models of Convergence 151

6.5. Discussion

\section{CHAPTER 7}

7.1.

\section{Convergence in Child Health Care Utilisation and} Outcomes

7.3.1. Trends in Averages of Child Health Care Utilisation and Child Health Outcomes

7.3.4. Convergence in Child Health Care Utilisation and Outcome Indicators: Absolute B-Convergence in Child Health Status

7.3.9. Convergence Clubs in Child Health Utilisation and Outcome Indicators

8.3.1. Time Series Association Between Trends in Averages and Inequalities in Socioeconomic, Demographic and Health 
8.3.2. Zero Order/ Partial Correlation Between Change in Averages of Socioeconomic and Demographic Indicators and SES Inequalities in Child Health

8.3.3. Pooled OLS Regression Estimates 224

8.3.4. PanelData Regression 226

8.3.5. Demographic [Fertility] Convergence and Its Linkages with Health Inequalities $\quad 229$

\section{CHAPTER 9}

9.1

9.2 .

9.2.1

9.2.2

9. 3 .

9.3.1.

9.3.2

9.3.3

9.4.

CHAPTER 10

10.1

10.2

10.3

10.4

10.5

References

Annexure

Abstract
Distance to Line of Equity and Line of Highest Achie vement: How Much Demographic Differences are Avoidable in India

Background and Rationale

Methods and Materials

235

Data

Methods

Results

Funnel plots

Line of Equity Plots

Line of Highest Achievement: Avoidable Demographic

Differences

Discussion

234-267

235

235

235

235

239

250

265

Summary and Conclusion

267-278

Summary of Findings

269

Conclusion

274

Policy Implications

275

Practical Implications

Limitations of the Study
277

278

279

309

318 


\section{List of tables}

Table no.

Title

Table 2.1

Absolute versus Relative difference and change in the total fertility rate

Table 4.1. Descriptive statistics of socioeconomic indicators in 15 major states of India.

Table 4.2.

Absolute $\beta$-convergence model estimates for per capita NSDP of the major Indian states, 1981-2010.

Table 4.3. Absolute $\beta$-convergence model estimates for poverty ratios of the major Indian states, 1974-2005.

Table 4.4. Absolute $\beta$-convergence model estimates for literacy rates of the major Indian states, 1981-2011.

Absolute $\beta$-convergence model estimates for HDI of the

Table 4.5. major Indian states, 1981-2006.

Table 4.6. Conditional $\beta$ - convergence model estimates for NSDP of the major Indian states, 1981-2010.

Table 4.7. Conditional $\beta$-convergence model estimates for poverty ratios of the major Indian states, 1974-2005.

Conditional $\beta$-convergence model estimates for Literacy

Table 4.8. Rate of the major Indian states, 1981-2010.

Conditional $\beta$-convergence model estimates for HDI of the

Table 4.9. major Indian states, 1981-2010.

Estimates of change-point analyses: significant change-points

Table 5.1. for IMR of India and selected major states, 1950-2009

Trends in concentration index for infant deaths in India and

Table 5.2. major states, 1992- 2006

Absolute $\beta$ convergence for life expectancy at birth across the

Table 5.3. major states in India, 1981-2011

Absolute $\beta$ Convergence for infant mortality rate across the

Table 5.4. major states, India, 1992-2006

Absolute $\beta$ convergence for maternal mortality ratio across the

Table 5.5. major states, India, 1992-2009

Conditional $\beta$ convergence for life expectancy at birth across

Table 5.6. the major states in India, 1981-2006

Conditional $\beta$ convergence for infant mortality rate across the

Table 5.7. major states, India, 1992-2006

Table 5.8.

Conditional $\beta$ convergence for maternal mortality ratio across the major states, India, 1992-2009

Estimates of change-point analyses: significant change-points

Table 6.1. for TFR of India and selected major states, 1950-2009

Table 6.2. Absolute $\beta$ - convergence estimates for TFR among the major Indian states, 1981-2009

Table 6.3. Conditional $\beta$-convergence estimates for TFR among the major Indian states, 1981-2009

Table 6.4. $\beta$ - convergence estimates for TFR among the socioeconomic groups of major Indian states and club of south Indian states 
1992-2006

Trends in wealth based concentration index for selected child

Table 7.1. health care utilisation and outcome indicators in India and major states, 1992- 2006

Table 7.2a Absolute $\beta$ convergence for full immunisation of children aged

Table 7.2b. 12-23 across the major states, India, 1992-2006

Table 7.2c. Absolute $\beta$ convergence for children underweight across the major states, India, 1992- 2006

Absolute $\beta$ Convergence for neonatal mortality rate across the major states, India, 1981-2001

Table 7.3a. Conditional $\beta$ convergence for full immunisation of children the major states, India, 1992-2006

Table 7.3c. $\begin{aligned} & \text { Conditional } \beta \text { convergence for ne } \\ & \text { the major states, India, 1981-2009 }\end{aligned}$

Table 8.1. Pooled OLS: inequality in children full immunisation

Table 8.2. Pooled OLS: inequality in children underweight

Table 8.3. Pooled OLS: inequality in NNMR

Results of Panel data regression analysis with the lagged

Table 8.4. effects $(1992,1998)$ of TFR on inequality in children full immunisation (2006)

Table 8.5. (1992, 1998) of TFR on inequality in children underweight (2006)

Table 8.6.

Results of Panel data regression analysis with lagged affects 


\section{List of figures}

Figure no.

Title

Figure 2.1. Conceptual framework of relationship between demographic and health transition, demographic convergence and health inequalities

Figure 2.2. Pathways of influence of Demographic transition, Convergence on Health inequalities

Figure 2.3 CUSUM charts of long-term trends of selected population and health indicators of India, 1872-2011

Figure $2.4 \quad \beta$-convergence versus Sigma Convergence

Figure 3.1 Change point analyses of trend in infant mortality rate for India, 1901-2008

Figure 3.2. Change point analyses of trend in population size for India, 1856-2011

Figure 3.3. Change point analyses of trend in total fertility rate for India, 1951-2009

Figure 3.4. Change point analyses of trend in life expectancy at birth for India, 1872-2011

Figure 4.1. Trends in selected socioeconomic indicators during 19732010

Figure 4.2. Scatter plots of change in selected socioeconomic indicator by values in the initial period of 15 major states of India

Figure 4.3. Trends in coefficient of variation (CV) in selected socioeconomic indicators of the major states in India during 1973-2005.

Figure 4.4. Kernel density estimates and distribution curves for selected socioeconomic Indicators of 15 major states of India.

Figure 4.5 Trends in population weighted AID and Gini index in selected socioeconomic indicators of the major states in India during 1981-2010.

Figure 5.1. Trends in life expectancy at birth, maternal mortality ratio, infant mortality rate in India and major states; 1981-2006

Figure 5.2. Cumulative distribution of the population of major state of India by LEB, IMR, MMR levels, 1981 to 2011

Figure 5.3. Trends in DMM and Gini Index measures for LEB of major Indian states,1980-2006

Figure 5.4. Trends in DMM and Gini Index measures for IMR of major Indian states,1981-2008

Figure 5.5. Trends of DMM and Gini Index for MMR of major Indian states, 1997-2009

Figure 5.6. Change in LEB, IMR and MMR during 1981-2011 for major states of India by LEB, IMR and MMR levels in initial period, 1981

Figure 5.7. Sigma convergence in average LEB across the major states in India, 1992-2006

Figure 5.8. Sigma convergence in averages of IMR across the major 
states in India, 1992-2006

Figure 5.9. Sigma convergence in averages of MMR across the major states in India, 1997-2009

Figure 5.10. Absolute convergence rate (in percentage) in LEB across the major states in India, 1980-2006

Figure 5.11. Absolute convergence rate (in percentage) in IMR across the major states in India, 1981-2008

Figure 5.12. Absolute Convergence rate of MMR for major Indian states, 1997-2009

Figure 5.13. Relative convergence rate (in percentage) for overall health inequality in LEB across the major states in India, 1980-2006

Figure 5.14. Relative convergence rate (in percentage) for overall health inequality in IMR across the major states in India, 1981-2008

Figure 5.15. Relative convergence rate (in percentage) overall inequality in MMR across the major states in India, 1997-2009

Figure 5.16. Sigma convergence in socioeconomic inequality (concentration indices) in IMR indicators across the major states in India; 1992-2006

Figure 5.17. Trends in convergence clubs in LEB, 1981-2011 121

Figure 5.18. Trends in convergence clubs in IMR, 1981-2011 121

Figure 5.19. Trends in convergence clubs in MMR, 1997-2009 122

Figure 5.20. Changing patterns of infant mortality clubs among socioeconomic groups of major states of India, 1992-2006

Figure 5.21. Trends in Kernel density plots for LEB, IMR and MMR

Figure 6. 1. Conceptual framework: Fertility transition and convergence model

Figure 6.2. Trends in mean TFR for major states of India, 1981-2009

Figure 6.3. Change in TFR during 1981-2009 for major states of India by TFR levels in initial period, 1981

Figure 6.4a. Cumulative distribution of the population of major state of India by TFR levels, 1981 to 2009

Figure $6.4 \mathrm{~b}$. Cumulative distribution of the population of major state of India by TFR levels in rural areas, 1981 to 2009

Figure 6.4c. Cumulative distribution of the population of major state of India by TFR levels in rural areas, 1981 to 2009

Figure 6.5. Changing patterns of fertility clubs among major states of India, 1951-2009

Figure 6.6. Changing patterns of fertility clubs among socioeconomic groups of major states of India, 1992-2006

Figure 6.7. Sigma convergences for unweighted TFR across the major states of India, 1981-2009

Figure 6.8. Trends in dispersion measure and Gini index of the TFR distribution among the major Indian states, 1981-2009

Figure 6.9. Trends in the rate of convergence in absolute dispersion of TFR among the major states of India, 1981-2009

Figure 6.10. Trends in the rate of convergence in relative dispersion of TFR among the major states of India, 1981-2009

Figure 6.11. Kernel density estimates of Total Fertility Rate distribution in 
India and major states, 1981-2011

Figure 6.12 Kernel density estimates of Total Fertility Rate distribution in rural areas of India and major states, 1981-2011

Figure 6.13. Kernel density estimates of Total Fertility Rate distribution in urban areas of India and major states, 1981-2011

Figure 7.1. Trends in children full immunisation coverage, children underweight, NNMR in India and major states, 1992-2006

Figure 7.2a. Trends of AID and Gini Index of child health care and outcome indicators for major Indian states, 1981-2009

Figure 7.2b. Trends of DMM and Gini Index of child health care and outcome indicators for major Indian states by place of residence 1981-2009

Figure 7.3a. Change in children full immunisation, children underweight, NNMR during 1992-2006 for major states of India by children full immunisation, children underweight, NNMR levels during initial period, 1992-93

Figure 7.3b. Cumulative distribution of the population of major state of India by children full immunisation, children underweight, NNMR levels, 1992 to 2009

Figure 7.4. Sigma convergence in averages of child health indicators across the major states, India; 1992-2006

Figure.7.5a. Trends of percentage of absolute convergence in Children immunisation, Children underweight, NNMR among major Indian states, 1981-2009

Figure 7.5b. Convergence in measure in relative inequalities based on the Gini estimates among the major states of India, 1992-2009

Figure 7.5c. Trends of percentage of absolute inequality convergence in Children full immunisation, Children underweight, NNMR among major Indian states by Rural-Urban, 1981-2009

Figure 7.5d. Trends of percentage of relative inequalities convergence in Children full immunisation, Children underweight and NNMR among major Indian states by Rural-Urban, 19812009

Figure 7.6a. Sigma convergence in socioeconomic inequality (concentration indices) in child health indicators across the major states of India; 1992-2006

Figure 7.6b. Sigma convergence in socioeconomic inequality (concentration indices) in NNMR indicators across the major states of India; 1992-2006

Figure 7.7a Changing patterns of children immunisation clubs among the major states of India, 1992-2006

Figure 7.7b Changing patterns of children underweight clubs among the major states of India, 1992-2006

Figure 7.7c Changing patterns of neonatal mortality clubs among major states of India, 1981-2009

Figure 7.7d Changing patterns of children immunisation clubs among social groups in major states of India, 1992-2006 
Figure 7.7e Changing patterns of Children Underweight clubs among social groups in major states of India, 1992-2006

Figure.7.7f. Changing patterns of neonatal mortality clubs among social groups in major states of India, 1981-2009

Figure 7.8. Trends in Kernel density plots for children full immunisation (CIM), children underweight (CU) and neonatal mortality rate (NNMR)

Figure 8.1. Panayotov's framework showing average health status and health inequalities relationship

Figure 8.2. Times series association between growth in averages and inequalities in socio economic indicators, 1981-2010

Figure 8.3. Times series association between growth in averages and inequalities in Fertility indicators, 1981-2010

Figure 8.4. Times series association between growth in averages and inequalities in LEB, 1981-2006

Figure 8.5. Times series association between growth in averages and inequalities in IMR, 1981-2010

Figure 8.6. Times series association between trends in averages and inequalities in NNMR, 1981-2010

Figure 8.7. Times series association between trends in averages and inequalities in children full immunisation, 1992-2006

Figure 8.8. Times series association between trends in averages and inequalities in children underweight, 1992-2006

Figure 8.9. Zero order correlation (pooled cross-sectional association) between change in averages of per capita NSDP, Poverty ratios, Literacy rates, TFR and change in inequalities in child health indicators, 1992-2006

Figure 8.10. Partial correlation (pooled cross-sectional) analyses showing three dimensional linkages of fertility with child health inequalities by adjusting to child health averages, 1992-2006

Figure 8.11. Demographic convergence and its linkages with child health inequalities

Figure 9.1. Funnel charts: Measuring of dispersal of states from line of the national average in terms of child health care and outcome indicators

Figure 9.2. Line of equity plots: Measuring the dispersion of states from the line of equity in terms of TFR by place of residence and socioeconomic status

Figure 9.3. Line of equity plots: Measuring the dispersion of states from the line of equity in terms of Mortality indicators by place of residence and socioeconomic status

Figure 9.4. Line of equity Plots: Measuring the dispersion of states from the line of equity in terms of child care and outcome indicators by place of residence and socioeconomic status

Figure 9.5. Plots of line of highest achievement: Measuring the dispersion of states from the line of highest achievement in terms of TFR by socioeconomic groups, 2011 
Figure 9.6. Plots of line of highest achievement: Measuring the dispersion of states from the line of highest achievement in terms of mortality indicators by socioeconomic groups

Figure 9.7. Plots of line of highest achievement: Measuring the dispersion of states from the line of highest achievement in terms of full immunisation among children by socioeconomic groups

Figure 9.8. Plots of line of highest achievement: Measuring the dispersion of states from the line of highest achievement in terms of underweight among children by socioeconomic groups

Figure 9.9. Plots of line of highest achievement: Measuring the dispersion of states from the line of highest achievement in terms of NNMR by socioeconomic groups 


\section{CHAPTER 1}

\section{Introduction}

\subsection{Background}

The contemporary theory of population change revolves around the concept of the classical demographic transition model enunciated by Notestein (1945). Demographic Transition Model (DTM) is defined as a shift from a regime of negligible population growth characterized by high birth and high death rates to one of equally little growth based on low birth and low death rates (Notestein, 1945; Davis, 1951; Davis, 1963; Dyson, 2004; Dyson, 2010; Casterline, 2011). Though, from a theoretical perspective, demographic transition, health transition, nutritional transition and epidemiological transition overlap, it is difficult to affirm which one precedes the other (Omran, 1971, 1998; Karar, 2009), but demographers believe that the DTM provides a scientific framework for studying many aspects of development. One school of thought argues that, together with industrialization a demographic modernization took place (Coale, 1987). The second school of thought argues that, the demographic transition has largely been responsible for the process of urbanization and indeed the larger process of development $-\mathrm{a}$ fact that deserves much greater recognition ${ }^{1}$ (Dyson, 2010). Since 1950, demographic transition has occupied center stage in demographic analyses and, therefore, the progress in demographic transition need to be understood and interpreted correctly. However, a perfect portrayal of demographic transition is required to investigate the demographic changes and its linkage with social, economic, health and political structures of nations.

Theoretically, following homogeneous pre-transition phase of demographic transition, progressive transition generates multidimensional geographic and socioeconomic heterogeneity, until the reappearance of a homogeneous post-transition phase. The process through which heterogeneity of transition will become homogeneous over time is called 'convergence' (Balabdaoui et al., 2001). The idea of convergence is largely debated in the

\footnotetext{
${ }^{1}$ Other classical demographic literature provides evidences for two way causal linkages among demographic transition and urbanization that urbanization effected by, and effects the demographic transition.
} 
economics literature where, it stems from the neoclassical model of growth ${ }^{2}$. Convergence is largely used as a framework for studying income inequality transition (Firebaugh, 2003). The demographic transition process is also described to go through the process of equilibrium and disequilibrium in terms of fertility and mortality convergence, divergence and re-convergence across the geographical units and socioeconomic stratum (Mc Michael et al., 2004, Moser et al., 2005; Dorius, 2008, 2010). The concept of convergence lies at the heart of demographic transition theory. However, as Oeppen (1999) puts it, 'despite this, until 1990, there seems to have been no attempt to address convergence in a formal way in population policy context'.

Post-1990 observed a growing interest in convergence methodologies in demography (Watkin, 1990; Herbertsson et al., 2000; Wilson, 2001; Coleman, 2002; Dorius, 2008, 2010). Watkin (1990) showed that, during the $19^{\text {th }}$ and $20^{\text {th }}$ centuries, there has been a tendency of greater demographic homogeneity within nations than in the past. Rising and converging life expectancy is established by a growing body of empirical research (Easterlin, 2000; Bourguignon and Morrison, 2002; Gosling and Firebaugh, 2004; Cole and Niemeyer, 2003, Niemeyer, 2004; Becker et al., 2005). Wilson (2001) provided a valuable quantitative assessment of the extent to which rising life expectancy and the decline in fertility, led him to describe as a 'global demographic convergence'. Taking the population of the world as a whole, the United Nations Population Division estimates that life expectancy at birth rose from 46.5 years in the early 1950s to 70 years by the late 2012. During the same period, fertility fell from a global average of 5 children per woman to 2.4 over the same time scale (United Nations, 2012; WHO, 2012). In wide social terms, there are several evidences of convergence in Europe. Vallin and Mesle perhaps best summarized global transitions in their discussion of health where, "each major improvement in matter of health is likely to first lead to a divergence in mortality since most favoured segments of the population benefit most from the improvement. When the rest of the population access the benefit of the improvement (through improved social conditions, behavioural changes, health policies, etc.) a phase of convergence begins and can lead to homogenisation until a new major advance occurs. The entire health transition

\footnotetext{
${ }^{2}$ A growth model where the capital-output ratio, 'V', was precisely the adjusting variable that would lead a system back to its steady-state growth path, i.e. that ' $\mathrm{V}$ ' would move to bring $\mathrm{S} / \mathrm{V}$ into equality with the natural rate of growth (n). In simple terms neoclassical growth theory is mostly that of the equilibrium of a competitive economy through time.
} 
process is identified into successive stages, each including a specific divergenceconvergence sub-process" (Vallin and Mesle 2004:14). However, evidences from empirical analyses of the convergence model across developing countries are virtually non-existent.

Demographic convergence is an emerging phenomenon in India. Comparatively south Indian states are approaching convergence in fertility and mortality while many of the northern Indian states still have high and divergent fertility rates. However, on an average both demographic and health trends have been positive in recent decades in India and heading towards convergence from both geographic and socioeconomic contexts. Over the period, the progress in fertility decline, life expectancy, and average health status in India is remarkable (Rele, 1987; Guilmoto, 1992; Ram and Ram, 2009; RGI, 2007, 2012). There are some assumptions about current and near future demographic scenario which looks to be a period of continued convergence. In the current phase of demographic transition, India's demographic trends are transitioning from third to the fourth stage of the demographic transition (Visaria, 2004b). The country is also amidst the second phase of epidemiological transition (Visaria, 2004a). But this decline is not uniform across the states and social stratum (Rele, 1987; Guilmoto, 1992; IIPS and Macro Internationals, 1992-2006; James and Nair, 2005; Kulkarni, 2011). More importantly, the combined effect of differential decline in fertility and mortality and better survivorship is affecting the level and patterns of health inequalities across all age groups in developing countries (Wagstaff, 2003; Maclanahan, 2004). Thus, understanding the demographic transition and convergence allows us to make comparatively firm statements about where the country is heading. However, recent research in Indian demography largely ignored the connection between demographic progress towards convergence and its linkage with health transition and health inequalities.

\subsubsection{Socioeconomic transition and Convergence}

The concept of 'socioeconomic transition' refers to change in socioeconomic conditions of people with the progress in technology and production over the time. More specifically, it is the transformation of societies from a pre-dominantly agricultural to industrial societies (Kalediene and Petrauskiene, 2004). The socioeconomic transition is often measured by change in living standards and income levels. However, the definition of socioeconomic 
transition has been widening over the period as progress in societies or development has been measured on a variety of scales. Socioeconomic progress or development is measuring on multi-dimensional scales such as income, education, living conditions, gender equality, health and political stability (Sen, 1973, 1983).

The concept of convergence was introduced into the analysis of transition economies about two decades ago, but has gained more significance during mid-1990s (Barro and Sala-I-Martin, 1991, 1992; Sirioppulos and Asteriou, 1997). In the context of economic growth, convergence is said to occur in a cross section of economies, if there is a negative relationship between the growth rate of income and the initial level of income (Barro, 1991; Sala-I-Martin, 1994, 1996a, 1996b; Barro and Sala-I-Martin, 1995). In other words, convergence occurs, in a cross-section of economies if poor economies tend to grow faster than wealthy ones. Similarly, Baumol (1994) defines 'convergence is the process of diminishing in the degree of economic inequality among the countries'.

Convergence is a concept that has gained popularity among economists, not only because of the importance of the issue about poor countries catching up with rich ones, but also because this analysis can serve as a way to verify the validity of different growth models. The convergence debate is also vital as it is concerned with the gaps in living standards between countries, i.e. whether these gaps are narrowing or rather widening across countries and over time. Moreover, despite the persisting disputes among economists on the determinants of long-run growth, the convergence debate has also enormous policy implications for policy makers both in the developed and developing countries (Pritchett, 1996; Workie, 2008). Convergence process can be analysed for a range of aspects. Real convergence describes the convergence of income levels, nominal convergence reflects the convergence of price levels, and institutional convergence implies harmonization of legislation. In addition, one can also speak about the convergence of social stratification and social outcomes such as literacy, fertility and mortality, and so on (Quah, 1996, 1996a, 1996b; Varblane and Vahter, 2005).

During the period from 1956 until the mid-1980s, the leading theory was clearly the Solow-Swan exogenous growth model (Solow, 1956). The majority of the recent empirical research on economic growth begins from the 'Slow growth framework' (Pfaffermayr, 2009; Workie, 2008). The Solow model is closed economy framework 
(Barro, 1991; Caselli et al., 1996; Galor, 1996; Sutcliffe, 2005), where the output (Y) is a function of input variable, such as labour $(\mathrm{L})$ and Capital $(\mathrm{K})$. This can formally be written as:

$$
Y=F(K, L)
$$

There are three basic assumptions that are linked to this model:

1. The production function in eq. (1) assumes positive and marginal products with respect to each input variable.

$$
\frac{\partial F}{\partial K}>0, \quad \frac{\partial F}{\partial L}>0 ; \frac{\partial^{2} F}{K^{2}} \prec 0 ; \frac{\partial^{2} F}{\partial L^{2}} \prec 0
$$

2. The production function exhibits constant returns to scale, indicating a proportionate increase in output as the results of changes in all input variables. This can formally be written as:

$$
F(\lambda K, L)=\lambda \cdot F(K, L), \text { for all } \lambda>0
$$

3. The third assumption is referred to as the so called 'Inada conditions'.

$$
\begin{aligned}
& \lim _{k \rightarrow 0} F_{K}=\lim _{L \rightarrow 0} F_{L}=\infty \\
& \lim _{k \rightarrow \infty} F_{K}=\lim _{L \rightarrow \infty} F_{L}=0
\end{aligned}
$$

The Inada conditions expressed in eq. (1.3) state that while production in the absence of input variables is impossible, their excess abundance also makes their marginal product diminished over time. The assumption of constant returns to scale in eq. (1.2) is also consistent with a balanced growth path along which capital and effective labour grow at the same time.

Based on this Solow growth model Barro and Sal-I-Martin (1991, 1995) and Sala-I-Martin (1996), elaborate the convergence debate more broadly. They used Beta $(\beta)$-convergence and Sigma -convergence concepts. Convergence in terms of both growth rate and income level is called $\beta$-convergence. $\beta$-convergence is typically tested by regressing the growth measured as gross domestic product (GDP) per capita or purchasing power parity (PPP) on the initial relative level across a cross-section of states (regions). The name of this type of convergence is derived from the coefficient of the initial income variable in these regressions $(\beta)$ and is supposed to be negative if the hypothesis holds (Barro, 1991; Sala-IMartin, 1994, 1996a, 1996b; Barro and Sala-I-Martin, 1995). Sala-I-Martin (1996) points out that the lack of convergence means that the degree of cross-country income inequality not only fails to disappear, rather tends to increase over time (Sigma-divergence). The 
economies (states) which are predicted to be richer a few decades from now are the same countries (states) that are rich today.

In the mid-1980s, a new branch of economic growth theory, endogenous growth theory, was proposed (Romer, 1986). According to the new growth theory the creation of the newly enlarged market allows better utilisation of the economies of scale effect and this will have a long lasting positive effect on growth. Instead of the diminishing returns to investment stipulated by the Solow model, in the new growth model the knowledge "spillovers" produce increasing returns to scale to capital accumulation (Romer, 1986; Lucas, 1988; Grossman and Helpman, 1991). According to the new theory of endogenous growth, there may not be convergence in every context, especially, when the low income countries continue to suffer from the lack of adequate social capability which can be a serious barrier to it (Grossman and Helpman, 1991). In addition, for example, Lucas (1988) showed that under the conditions where human capital with increasing returns is the main driving force of economic growth, the possibility of a brain drain acting as a vehicle of cross-country growth divergence. Other authors insisted that research and development and human capital creation, being the most important engines of growth, would also cause growing inequality between countries and, instead of convergence on the global scene, divergence could start to dominate as poor countries have much less resources to invest in these areas (Romer, 1986).

In order to understand the current trends in the dispute over convergence, it is very helpful to use the classification proposed by Islam (2003). The whole discussion may be described in the form of the following seven dichotomies: 1) Convergence within an economy vs. convergence across economies; 2) Convergence in terms of growth rate vs. convergence in terms of income level; 3) $\beta$-convergence vs. $\sigma$ convergence; 4) Unconditional (absolute) convergence vs. conditional convergence; 5) Global convergence vs. local or clubconvergence; 6) Income-convergence vs. total factor productivity (TFP) -convergence; 7) Deterministic convergence vs. Stochastic convergence.

Although the Indian states have long shared common political institutions and national economic policies, the wide diversity in geographic, demographic, socio-cultural and economic features is also readily apparent. States have experienced different pace of economic growth with some states showing fast progress and others languishing behind, 
although the national growth has been reasonably doing well for the past two decades. Also, the per capita incomes in the states of India are quite diverse (Dholakia, 1994; Ghosh et al., 1998; Dreze and Khera, 2012). The states that lag far behind the others in literacy rates are Uttar Pradesh, Bihar, Madhya Pradesh, and Rajasthan and these states are also in a disadvantageous position in terms of poverty ratios and demographic indicators (Cashin and Sahay, 1996; Marjit and Mitra, 1996; Bandopadhyay, 2004). However, much of the earlier literature in Indian context heavily focused on GDP per capita convergence while there is not much evidence of convergence of other socioeconomic indicators (Cashin and Sahay, 1996; Marjit and Mitra, 1996; Ghosh et al., 1998; Dholakia, 1994; Trivedi, 2002).

\subsubsection{Demographic transition and Convergence}

Demographic transition stands for "the transition from high birth and death rates to low birth and death rates as a country progresses from a pre-industrial to an industrialized economic system" (Notestein, 1945). The theory is based on an interpretation of demographic history developed in 1929 by the American demographer Warren Thompson. Thompson observed changes in birth and death rates in industrialized societies over the previous 200 years. In countries that are now developed, demographic transition began in the $18^{\text {th }}$ century and continues today. In less developed countries, this demographic transition started later. While most developed countries are in third or fourth stage of the model and the majority of developing countries have reached third stage. The major exceptions are some poor countries, mainly in sub-Saharan Africa and some Middle Eastern countries (Caldwell, 1976; Campbell, 1991; Kirk, 1996, Montgomery, 1999; Dyson, 2010).

Montgomery (1999) described the demographic transition in four stages, however, recently the stage five also recognized. Stage one belongs to pre-industrial society where death rates and birth rates are high and more or less in balance. Thus, the population growth was typically very slow (Notestein, 1945; Montgomery, 1999; Dyson, 2010). In stage two, the death rates drop rapidly due to improvements in food supply and sanitation, access to technology, basic healthcare, and education which increases life spans and reduce disease. However, without a corresponding fall in birth rates this produces an imbalance. Generally, the countries in this stage experience a large increase in population. 
In stage three, birth rates fall due to access to contraception, increases in wages, urbanization, a reduction in subsistence agriculture, an increase in the economic and educational status of women, a reduction in the value of children's work, an increase in parental investment in the education of children and other social changes, population growth begins to decrease. However, it is important to note that the birth rate decline is caused also by a transition in values, not just because of the availability of contraceptives. During stage four, there are both low birth rates and low death rates. Birth rates may drop to well below replacement level, which has happened in countries like Germany, Italy and Japan, leading to a shrinking population, a threat to many industries that rely on population growth. It creates an economic burden on the shrinking working population and growing aging population. This stage also witnesses growing burden of noncommunicable diseases in a population (Campbell, 1991; Kirk, 1996; Lee, 2003; Montgomery, 2009; Bloom, 2010).

Though, above mentioned model is an idealized picture of population change in European countries but the model is a generalization that applies to world countries as a group and may not accurately describe all individual cases. The extent to which it applies to lessdeveloped societies today remains to be seen. For example, many countries such as China, Brazil and Thailand have passed through the demographic transition model very quickly due to faster social and economic change, political factors and diffusion of fertility limiting behaviour. Some countries, particularly African countries, appear to be stalled in the second stage due to stagnant development and the effect of life style diseases (Weeks, et al., 2004; Dyson, 2010).

"Different timing of the demographic transition has segmented countries into different regimes and their simultaneous progress makes the concept of convergence meaningless if neglected" (Wilson, 2001). The pathways of the process of demographic convergence lie in the demographic transition. In its original form, the demographic transition theory had a strong homeostatic perspective, directly and indirectly arguing for a demographic steady state, or what Easterlin (2000) referred to as an "imminent stationary state". Thus, the concept of convergence, or declining inequality, is central to the demographic transition theory and yet, formal empirical treatment of cross-national convergence is in short supply (Wilson, 2001). 
During mid-1990s, the notion of demographic convergence has generated considerable curiosity around the question of assessing convergence by standard and innovative methods in varying developmental context. And while patterns of convergence and divergence in life expectancy (Bourguignon and Morrisson, 2002; Goesling and Firebaugh, 2004; Neumayer, 2004; Ram, 2006), and to a lesser extent, infant and child mortality (Neumayer, 2004; Deaton, 2007), have received recent attention, we know far less about level and change in the variance of within country estimates of fertility (Arokiasamy and Goli, 2012). Few studies have focused either exclusively or explicitly on international or global variance in fertility rates (Wilson, 2001, 2011; Dorius, 2008, 2010).

Though Dyson et al., (2004) and other researchers (Rele, 1987; Guilmoto, 1992; IIPS and Macro Internationals, 1992-2006; Visaria, 2004a, 2004b; James and Nair, 2005; Alagarajan and Kulkarni, 2005; Srinivasan et al., 2006; Kulkarni, 2011) have presented a detailed discussion on demographic trends and their relevance to policy shifts for India but there is no study in India that focused on empirics of demographic convergence and explained demographic transition in terms of their steady state differentials and inequalities in progress across the states, rural-urban and socioeconomic groups.

\subsubsection{Health transition and Convergence}

The term 'health transition' is a two and half decade old. It was coined at a summit of demographers, medical scientists and social scientists held in 1985 at Bellagio, Italy (Caldwell and Santow, 1989). Since then considerable literature as come-up on the precise meaning of the term, yet, there is no agreement among researchers about what actually it means (Cleland, 1990; Palloni, 1990; Van de Walle, 1990; Caldwell, 1994; Omran, 1998). Van de Walle (1990) suggested that transition of infant mortality from high levels to low levels could be an important part of health transition. Palloni (1990) focused on mortality decline only but he conceded that, the term 'health transition' has implications for health also. Cleland (1990) said 'it is not a matter of formal definition but rather its emphasis on social, cultural and behavioural determinants of health' but the health transition is a much broader concept than epidemiological transition. Explaining that the term 'health transition' is broader than the two terms, 'mortality transition' and 'epidemiological transition', Caldwell (1990) wrote: "health transition embraces changes in levels of sickness as well as mortality". Omran, (1998) said that the term' health transition' is derived from earlier 
terms like demographic transition, fertility transition, mortality transition and epidemiological transition. Demographic transition, health transition and epidemiological transition overlap and it is difficult to affirm which one precedes the others.

However, the health transition is not uniform all over the world. While some countries are achieving greater progress in overall health, but many developing countries, such as Asia and African countries lagging behind. The focus of developed countries shifted from communicable to non-communicable diseases while developing countries still facing the dual burden of (communicable and non-communicable) diseases. Some developing countries are also experiencing resurgence of communicable disease such as Malaria and Dengue and other environmental relative diseases. Few studies also suggested such resurgence led to a recent setback in convergence of life expectancy at birth across the countries worldwide. This is also mainly attributable to unequal progress of public health outcomes across the world countries (Neumayer, 2004; McMichael et al., 2004; Becker et al., 2005; Moser et al., 2005; Bloom and Canning, 2007; Dorius, 2008, 2010; Clark, 2011; Gächter and Engelbert, 2011). However, there are no studies across the world or in India, which focused on transition in maternal and child health care and outcome indicators. Transition in health status of vulnerable population group such as children is a major focus in the world as well as in India. Many of the child health indicators considered to be sensitive indicator of human wellbeing. Reduction in children underweight and improvement in children full immunisation thereby reduction in early childhood mortality become the thrust of national health policies. Though, India is experiencing transition in child health indicators but there are no studies which focused on convergence/divergence of such progress.

\subsubsection{Health inequalities}

The study of health inequalities considered as the measurement of differences in health outcomes and health care utilisation across different populations (WHO, 2012). Health inequalities between and within countries is a matter of growing concern internationally (Marmot, 2005). This may include differences in the "presence of disease, health outcomes, or access to health care" across gender, place of residence and socio-economic groups. The term "disparities" generally refers to differences of which the writer disapproves (Goldberg et al., 2004). Health inequality is not only an infringement of 
equity: the avoidable mortality and morbidity of lower socioeconomic groups also impedes productivity and threatens to undermine economic growth and prosperity (WHO, 2001).

Equitable distribution of health care is a principle subscribed in many countries, often explicitly in legislation or official policy documents (van Doorslaer et al., 1993). "Egalitarian equity goals distinguish between horizontal equity- equal treatment of equals - and vertical equity - appropriate unequal treatment of unequal' ( $\mathrm{O}$ ' Donnell et al., 2008). In healthcare, most attention, both in policy and research, has been given to the horizontal equity principle, defined as equal treatment for equal medical need, irrespective of other characteristics such as income, race, place of residence, etc (Van Doorslaer et al., 2000; Wagstaff and van Doorslaer, 2000; Wagstaff et al., 1991). In practice, it is not possible to examine the extent to which the horizontal equity principle is violated without simultaneously specifying a vertical equity norm. Researchers have usually assumed, implicitly or explicitly, that, on average, vertical equity is satisfied. That is, the observed differentials in utilisation of health care across individuals in different states. For example, one seeks to establish whether there is a differential utilisation of health care by income after standardising for differences in the need for health care in relation to income. In empirical analyses, expected utilisation, given characteristics such as age, gender and measures of health status, is used as a proxy for "need" (O'Donnell et al., 2008). Other important dimensions of health inequalities are 'relative and absolute inequalities'. Relative inequality is about ratios and absolute inequality is about differences. Absolute inequality may change without change in relative inequalities (Ravallion, 2003). A generalized theory of inequality transitions or life cycle of inequality transition has applied to a broad range of domains: change in the shape and composition of between-nations or global inequality, within national inequalities and social inequalities (Whitehead et al., 2004).

In India, while gender and place of residence play significant factors in explaining healthcare inequality in India, socioeconomic status is the greatest determining factor in an individual's level of access to health care (Subramanian et al., 2006, 2009; Goli et al., 2013). Not surprisingly, individuals of lower socioeconomic status in India have lower levels of overall health and less access to adequate health care (Pradhan and Arokiasamy, 2010; Arokiasamy and Pradhan, 2011; Arokiasamy et al., 2012; Jain, Goli and 
Arokiasamy, 2012; Arokiasamy and Goli, 2012; Goli et al., 2013). However, an important quest in health inequality research in India is what relationship does health inequalities pose with average health status? Do health inequalities swim against the tide that health inequalities are increasing with increases in average health status? However, there is no research in India which theoretically and empirically addresses these research questions.

\subsubsection{Health inequalities versus Socioeconomic, Demographic, Health and Transition and Convergence}

Though, socioeconomic status and health outcomes, health inequalities relationship are well established (Wagstaff and Doorslaer, 1991; Kakwani et al., 1997; Gwatkin, 2000; Kawachi et al., 2002; Wagstaff, 2002a, 2002b; Mormot, 2002; Van Doorslaer and Gerdtham, 2003; Wagstaff et al., 2003; Mackenbach, 2003; Van Doorslaer et al., 2004; Subramanian and Kawachi, 2004; Hosseinpoor et al., 2006; Subramanian et al., 2006, 2009; Harper and Lynch, 2007; Van de Poel et al., 2008; Subramanian, 2008; Speybroeck et al., 2009; Konda et al., 2009; Po and Subramanian, 2010; Pradhan and Arokisamy, 2010; Marmot, 2005, 2010; Po and Subramanian, 2011; Arokiasamy et al., 2012; Suzuki et al., 2012), but there are not many studies which have examined the relationship between the demographic and health transition with transition health inequalities. In particular, the question 'Does socioeconomic, demographic and health progress is leading to greater uniformity in average health status or health inequalities are swimming against the tide' is not given much attention. The way of quantifying the tradeoff between health inequalities and socioeconomic status is tricky task given that increased health inequality associated with rising per capita incomes is a bad thing and increased average health levels associated with rising incomes are a good thing (Deaton and Paxson, 2001). Some previous studies attribute the association between "health and income inequality" to structural changes undergoing hand-in-hand with economic growth, coupled with a tendency for the better-off to assimilate new technology, ahead of the poor (Wagstaff and Doorslaer, 1991, 2004; Dorius, 2010). These important research questions are not examined in the previous studies in India.

\subsection{Literature review}

\subsubsection{Socioeconomic transition and Convergence}

Economic literature foster that economists have always been concerned with variations in income and living standards over time and across space. Baumol (1986) has been the first 
growth economist to examine the convergence across 16 industrialized countries (18701979) using Madison's 1982 data. However, Romer (1986), Sala-I-Martin (1996) and Quah (1996) criticizes the traditional empirical analysis of growth and convergence for overemphasizing physical capital and de-emphasizing endogenous technological progress and externalities that are main determinants of growth and convergence. Later a number of studies addressed the question about the role of integration in the process of long-term growth and further economic convergence between countries and states/provisions in countries (Barro, 1991; Barro and Sala-I-Martin, 1995; Sala-I-Martin, 1996; Barro and Sala-I-Martin, 2002). Until now, studies identified multiple definitions of convergence, reviewed different concepts of convergence and used various research methodologies. Furthermore, the links between the theory of growth, economic integration and convergence are well established in empirical research, particularly in terms of economic indicators i.e. per capita income. The empirical analyses are carried out using descriptive statistical methods and econometric models (Barro and Sala-I-Martin, 1995).

In the early stage unfortunately, the empirical analyses often failed to support $\beta$ convergence hypothesis. For example, World Bank economists discovered that the growth rates of GDP per capita, show little correlation with the starting levels of GDP per capita in a sample of over 80 countries for the period 1965-89 (Barbone, 1996). Similar results were obtained also by other authors working with different data sets (Workie, 1998). Unsuccessful attempts to show absolute $\beta$-convergence stem from the most important assumption of the neoclassical growth model. In general, the neoclassical model assumes that countries are more similar in all other ways than their per capita physical and human capital. In reality, there is great variation between countries with regard to factors relevant for growth. This also implies that each country/state may have its own steady level of growth (Quah, 1996). The theory opposite to absolute $\beta$-convergence accepts the idea that the steady-state of countries could be different and consequently the regression model should contain other variables than the initial income level. This allows an estimation of the impact of different factors through a conditional $\beta$-convergence model (Quah, 1996a; Galor, 1996; Workie, 1998; Haider, 2010). However, in recent times there is ample empirical evidence confirming the "Solow view of regional convergence" (Barro, 1991; Barro, Sala-I-Martin, 1991; Sala-I-Martin, 1996; Barro and Sala-I-Martin, 2002; Rey and Montouri, 1999; Dorius, 2008). In particular, this literature provides evidence on spatial correlation in income growth rate, $\beta$-convergence (typically found in Solow's growth 
model) and emphasizes the importance of spatial knowledge "spillovers" that diminish with time.

Literature also discussed the concept of club-convergence that the convergence hypothesis may hold for countries with relatively similar starting positions with similar economic, political and social environments. This idea was realised in convergence theory as the concept of "club-convergence". According to this concept, countries with relatively similar conditions tend to converge, meaning, during the process of growth the income levels of countries will become more equal and the variation between their per capita GDP levels will gradually lessen (Young et al., 2004; Dorius, 2008). Countries with very different conditions will not converge on their own, but if certain economic policy instruments should allow for eliminating the differences in their conditions, then the countries may turn out to converge. However, according to the Solow growth model, changes in economic policy will have only a temporary effect on economic activity, that is, they cannot drive long-term growth and convergence (Sala-I-Martin, 1996).

The large empirical literature on regional income convergence under spatial externalities uses two workhorse models (Solow and Endogenous models) to motivate the estimated econometric specifications. However, this study also discussed the third model, in which the growth process follows Verdoorn's law. This model relates growth in GDP per capita output growth and emphasizes the increasing return to scale. In both cases economic growth is associated with convergence and spatial knowledge spillovers. Under Verdoorn's law the growth rate of income per capita as a proxy of labour productivity is found to be positively related to output growth. Fingleton (2001) argues that the Verdoorn's model provides a more realistic description of the regional growth process. It is compatible with both endogenous growth models and models of economic geography that allows for agglomeration of economic activity. Evidence on Verdoorn model comes from McCombie (1998), Fingleton (2001) and Fingleton and Lopez-Bazo (2006). Further, Islam (2003) and Abreu et al. (2005) provide comprehensive surveys on this issue.

However, based on our extensive literature, I have classified convergence approaches under four broad headings. 
Mechanism of economic convergence

\begin{tabular}{|c|c|c|c|c|}
\hline & $\begin{array}{c}\text { Standard } \\
\text { neoclassical growth } \\
\text { model }\end{array}$ & $\begin{array}{l}\text { New growth theory } \\
\text { or Endogenous } \\
\text { growth model }\end{array}$ & $\begin{array}{l}\text { New geography, } \\
\text { literature }\end{array}$ & Verdoorn's model \\
\hline Authors & $\begin{array}{l}\text { Barro and Sala-I- } \\
\text { Martin (1991) }\end{array}$ & $\begin{array}{l}\text { Romer (1986) } \\
\text { Lucas (1988) }\end{array}$ & $\begin{array}{c}\text { Krugman (1991) } \\
\text { Puga (1996), } \\
\text { Ottaviano and Puga } \\
\text { (1997) }\end{array}$ & $\begin{array}{l}\text { McCombie (1998), } \\
\text { Fingleton (2001), } \\
\text { Fingleton and } \\
\text { Lopez-Bazo (2006) }\end{array}$ \\
\hline $\begin{array}{l}\text { Sources of } \\
\text { Convergence }\end{array}$ & $\begin{array}{l}\text { Exogenously given } \\
\text { technical progress }\end{array}$ & $\begin{array}{l}\text { Research and } \\
\text { Development, } \\
\text { Expenditures in } \\
\text { human capital }\end{array}$ & $\begin{array}{l}\text { Imitation of } \\
\text { technology, trade } \\
\text { and FDI as } \\
\text { channels for } \\
\text { technology } \\
\text { spillovers }\end{array}$ & $\begin{array}{c}\text { Convergence with } \\
\text { spatial knowledge } \\
\text { spillovers }\end{array}$ \\
\hline
\end{tabular}

The issues of regional economic growth and inequality have attracted considerable attention among researchers, planners and policy makers in India. Since independence, the Indian government has been concerned about how to strengthen national unity and promote economic growth with regional equality (Pal and Gosh, 2006; Jha, 2006; Nair, 2004; Chandrashekar and Gosh, 2003). Although growth performance has improved substantially in the post-reform period compared to the pre-reform, there has been a wide variation in growth performance across the states (Kurian, 2007). Previous literature on the evidence of $\sigma$-convergence and absolute $\beta$-convergence shows that the states have diverged in per capita income, particularly after the implementation of large-scale economic reforms (Marjit et al., 1998). The evidence of conditional $\beta$-convergence indicates that interstate variations in the steady-state levels of per capita income are due to interstate variations in human capital, production structures, and physical, social and economic infrastructure. The states following different steady-state paths are organised into two clubs - one club consists of the five states (viz., Gujarat, Karnataka, Kerala, Rajasthan and Tamil Nadu) that are converging to, and the other club consists of the remaining ten states (viz., Andhra Pradesh, Assam, Bihar, Haryana, Madhya Pradesh, Maharashtra, Orissa, Punjab, Uttar Pradesh, and West Bengal) that are diverging from the national average steady-state path. The results of earlier studies suggest that the policy measures improving human capital and physical, social and economic infrastructure can have a significant effect on long-run growth potential of the states. Targeting public investment in human capital and infrastructure for the states with lower steady-state levels could improve overall growth performance and reduce the regional imbalance. The ongoing economic reforms that seem to have led to an increase in the growth rate as well as 
regional inequality, therefore, need appropriate planning and policies for reducing regional disparities in development in the country (Nagaraj et al., 1997; Rao et al., 1999; Aiyar, 2001; Trivedi, 2002). Overall, the literature on the empiricism of socioeconomic convergence in India is plenty but there are no studies which investigated socioeconomic convergence by using non-parametric models and population weighted inequality based convergence models.

\subsubsection{Demographic transition and Convergence}

Given convergent socioeconomic trends, demographic convergence is assumed to follow (Coleman, 2002). The last 50 years have seen major demographic changes that have affected all regions and countries. As a result of changes in fertility and mortality of the global population has increased from 2.5 to 6 billion. Demographers over the past quarter century have assessed a worldwide convergence of nations towards low fertility and mortality, with resultant higher life expectancy. They foster that cross-national inequality trends in life expectancy and infant mortality have followed different trajectories over the past half century with an increase in per capita income of the world countries (Dorius, 2008).

The studies in terms of Life expectancy averages have demonstrated long-term convergence since the mid-twentieth century, while infant mortality rates have continuously diverged during same time period (Preston, 1970; Wilson, 2001; Becker et al., 2005; Bourguignon and Morrison, 2002; Easterlin, 2000, Gosling and Firebaugh, 2004; Neumayer, 2004; McMichael et al., 2004; Lanzieri, 2010). Drawing on data for a panel of more than 150 countries, Wilson (2001) found that differences in life expectancy across countries declined sharply from 1950 to 2000, a trend he attributed to the global "demographic convergence" that occurs as longevity increases and mortality rates decline. In contrast, based on data from a panel of 186 countries, Neumayer (2004) found that inequality in the international distribution of life expectancy increased in the 1990s, after falling in the 1980s (Firebaugh and Goesling, 2004; Mazumdar, 2003; Neumayer, 2003). Neumayer predicted that the trend of rising international health inequality would persist through the early twenty-first century but this hypothesis has not been tested with more recent international data. In fact, in several regions of the world, the rate of progress in life expectancy has declined over the past 1-2 decades. This trend suggests that the 
expectation that emerged in 1960s and 1970s of world gains and convergence in population health status is not guaranteed by a general deterministic process ${ }^{3}$. Crossnational inequality trends in life expectancy and infant mortality have followed different trajectories over the past half century. Life expectancy averages have demonstrated longterm convergence since the mid-twentieth century, while infant mortality rates have continuously diverged during this same time period (Mc Michael et al., 2004).

Studies that have assessed fertility also foster different views, Hosseini and Haghshenas (2009) identified fertility convergence of Muslim countries with world fertility level. Over the period of 1952-1995, Italian regional fertility converged at a rate greater than 2 per cent with an increase in per capita income (Franklin, 2004). However, according to the diffusion school perspective, diffusion of fertility decline progresses faster than socioeconomic development (Montgomery and Casterline, 1996; Lesthaege and Vanderhoeft, 2001). A growing volume of recent literature recognizes that the fertility reduction among uneducated women in India is a complementary phenomenon to the early phase of fertility decline among educated women (McNay et al., 2003; Arokiasamy, 2009).

On the other hand, Casterline (2001) modelled the pace of fertility in less developed countries from 1950-2050 and found a significant level of inter-country and intra-regional variation in the pace of fertility decline. In a recent study, Dorius (2008) argued that evidences are pointing to divergence rather than convergence. He focused on relative rather than absolute, inter-country differences in fertility intensities to measure the variation in inequality. He noted how common economic distinction between poor and rich countries is becoming of less importance for demography, and that the demographic convergence can be seen as one element of the socio-demographic change which seems to have taken place more rapidly than the economic development. However, most of the earlier studies in terms of empirical attempts of quantification of the volume of

\footnotetext{
${ }^{3}$ The newly arising combinations of social, economic, and political conditions, and altered relations with the microbiological world, cause reversals in death rates. That is, gains in longevity could be less smooth and less certain than earlier notions of global convergence suggested. reflecting deleterious socio-economic and political conditions, often with unequal access to health care (Whitehead et al. 2001); increase in various infectious diseases, especially to se associated with poverty, such as tuberculosis and HIV/AIDS; the growing toll-especially in poorer states-of injury, impaired mental health, and death from violence (WHO 2002), conflict, and war; and the health risks consequent on large scale environmental changes caused by human pressures (McMichael 2001).
} 
demographic convergence were focused on global context or between groups of countries (e.g. Oeppen, 1999; Wilson, 2001, 2011; Coleman, 2002; Neumayer, 2004; Dorius, 2008, 2010; Montegomery, 2009; Bloom, 2010; Lanzieri, 2010).

Very few studies, for instance O’Connell (1981) Evans (1986) Alagarajan and Kulkarni (1998) Bongaarts (2003) Franklin (2002), Alagarajan (2003), James and Nair (2005) and Alagarajan and Kulkarni (2008) presented evidence that fertility rates conceal considerable differences in reproductive behaviour among socioeconomic groups within countries. Further, quantitative assessments of convergence models within and between developing countries are rare. Within countries, fertility is usually higher in less developed region (states) than developed region, higher in rural than urban areas, higher among uneducated women than their better-educated counterparts, and higher in households with low incomes than their higher income counterparts (Merrick, 2001). A situation of this kind is ideal for testing convergence models to assess the progress of within country convergence in fertility rates across the states and socioeconomic groups.

Over the period, on an average, the progress in fertility decline in India is remarkable (Rele, 1987; Registrar General of India, 1971-2007, 2009; James and Goswami, 2012). The total fertility rate in India fell from an average of 5.8 children per woman (1951) to 2.6 children per woman (2009). India's national demographic trends are currently transitioning from third to fourth stage of the demographic transition (Visaria, 2004b). However, the progress in fertility transition is not uniform across all the states and socioeconomic groups of India. Total Fertility Rates (TFR) in India are declining with divergent destinies across states, rural-urban and socioeconomic groups (Guilmoto and Rajan, 2001; Visaria, 2004b; James and Nair, 2005; Kulkarni and Alagarajan, 2005; Alagarajan and Kulkarni, 2008; James and Goswami, 2012). Comparatively, the south Indian states, urban areas, and higher socioeconomic groups are approaching low fertility rates with some categories such as the upper wealth and education quintiles of low fertility states reaching lowest-low fertility rate (TFR of 1.3), while many of the north Indian states, rural areas, and disadvantageous socioeconomic groups still have higher fertility rates (Registrar General of India 1971-2007, 2009; International Institute for Population Sciences [IIPS] and Macro Internationals 1992-2006; James and Nair, 2005; James and Goswami, 2012). 


\subsubsection{Health transition and Convergence}

Throughout the world, health trends in the $20^{\text {th }}$ century were generally positive and average life expectancy doubled. Death rates continue to decline in many countries, including all developed countries. For the world as a whole life expectancy at birth has increased from 46.5 years in 1950-1955 to 65.0 years in 1995-2000, although the rate of increase has slowed over the past 25 years. Life expectancy at birth for the world is 68 years in 2009 (United Nations, 2010, Wilson, 2011). However, there are different views on cross-national progress in health over the period. Wilson (2001) claims globally, life expectancy has been in a long uptrend and in the last half of the twentieth century has been characterized as an era of cross-national health convergence (Wilson, 2001), as well as an era featuring rapid economic growth in a number of developing nations (Firebaugh, 2003). However, the emerging picture of variable mortality trends and regional setbacks indicates that future health gains are not guaranteed by any general deterministic process of convergence. Rather, there is an increased heterogeneity between countries (Neumayer, 2003). The recent report of WHO (2011) also reveals that the improvement in health gains is not uniform across the countries and still there exists vast differentials in the pace of mortality decline. The life expectancy at birth in the developed countries is more than 70 years while the developing countries are still in the way to achieve this in the future. The important reason for setbacks in life expectancy in developing countries is poor health care in childhood. Poor coverage of children vaccination, nutritional deficiency and early neonatal mortality are costing poor life expectancy in these countries (Moser et al., 2005).

In case of India there has been a significant increase in the life expectancy at birth. It has increased from 23.7 years in 1881 to 62.6 years in 2002-06 among males and from 25.6 years to 64.2 years for females (RGI, 2007; Visaria, 2004b; Bhat, 2008; Saikia et al., 2011). This is majorly attributable to increases in survival chances at early ages which mainly depend on improvement of child health care services. Though, there are reports and studies (RGI, 2007; IIPS and Macro-Internationals, 1992-2006; Singh et al., 2011; Kumar and Mohanty, 201; Pathak and Singh, 2011; Goli and Arokiasamy, 2013) which reported progress in reduction of child mortality, nutrition status and children basic vaccinations in India in recent past. However, there is dearth of studies which empirically assessed the convergence or divergence in observed progress in child health status across the states and social groups. 


\subsubsection{Health inequalities}

A large body of research in economics, sociology and development has focused on national income inequality trends across the states and more recently, the empirical tools of measuring income inequality have been extended to the study of other types of inequality such as health (Firebaugh, 2004). Health inequalities have recently started to receive a good deal of attention in the developing world. They are recognized to be a major problem in all countries studied (Subramanian et al, 2006, 2009; Harper and Lynch, 2007; Van de Poel et al., 2008; Subramanian, 2008; Speybroeck et al., 2009; Konda et al., 2009; Po and Subramanian, 2010). The evidence from trends in health inequalities in both the developing and developed world supports the notion that health inequalities rise with rising per capita incomes (Wagstaff and Doorslaer, 1991; Kakwani et al., 1997; Gwatkin, 2000; Kawachi et al., 2002; Wagstaff, 2002a, 2002b; Mormot, 2002; Wagstaff et al., 2003; Mackenbach, 2003; van Doorslaer et al., 2004; Subramanian and Kawachi, 2004; Hosseinpoor et al., 2006; Pradhan and Arokisamy, 2010; Marmot, 2005, 2010; van Bon-Martern et al., 2012; Arokiasamy et al., 2012; Suzuki et al; 2012). However, countries differ in their definitions of inequalities in health and their assessment of the scale of the problem. In high-income countries, poorer individuals consume more health care resources as a result of their lower health status and so greater need for health care. Obviously, such inequality in health care use cannot be interpreted as inequity. In low-income countries, lack of health resources and purchasing power among the poor typically mean that their utilisation of health care is less than that of the better-off despite their greater need (Gwatkin et al, 2003; O’Donnell et al., 2008).

Nevertheless, inequalities in health are most commonly presented as the difference in health status between socioeconomic groups but inequalities in health are also described by geographic location, employment status, gender and ethnic groups (Marmot, 2010). Thus, the root causes of inequalities in health are the complex interaction between personal, social, economic and environmental factors (Marmot and Wilkinson, 1999). Mackenbach points out that the most fundamental approach to reducing such inequalities in health is to address directly inequalities in education, occupation and income (Mackenbach and Bakker, 2002). Action is required at many levels: international, national and regional, city and local community, and at individual, family and other social group level (Marmot, 2010). 
Studies in measuring size and trends in health inequalities in India in terms of various health indicators are plenty (e.g. Joe et al., 2009; Pradhan and Arokiasamy, 2010; Saikia et al., 2011; Pathak and Singh, 2011; Kumar and Mohanty, 2011; Subramanian et al., 2007, 2009; Po and Subramanian, 2011; Subramanyam and Subramanyam, 2011; Arokiasamy et al., 2012). However, there are no studies which have assessed convergence in relative and absolute distribution of health status across the population sub-groups. Further, there are no studies which have addressed health inequalities in terms of 'health averages versus health inequalities' and 'overall versus socioeconomic health inequality' with a comprehensive framework of showing an association between them.

\subsubsection{Health inequalities versus Socioeconomic, Demographic, Health and Transition and Convergence}

Economic growth has often been a powerful force for improved health status, although it also depended on countering the accompanying adverse health effects at institutional and individual levels (Deaton, 2011). Previous empirical research has established a strong connection between international income, literacy and health status, such that richer countries enjoy better health (Preston, 1975; Deaton, 2003). Moreover, many theories of development also predict a strong connection between socioeconomic status and inequality in health, reasoning that gains in population health often stem from the technological advances and improved literacy with national income growth (e.g., Ram, 1982, 1992; Pritchett and Summers, 1996; Easterlin, 2000). In the countries of rapid economic growth (e.g., India, China, and Indonesia), the mortality is declining and survival of children is increasing (WHO, 2002).

However, the linkage between health inequalities and socioeconomic status, particularly economic status received two views. One view is there is a negative association between increases in income is associated with decrease in health inequalities. For example, a study by Becker and colleagues (2005) have assessed the recent trend in international health inequality by estimating the economic value of recent gains in longevity and using these figures to adjust estimates of national income growth. The study found a sharp drop in international inequality using this health-adjusted income data, with much of the drop reflecting declining inequality in health. Analysing data for a comparable period, Kenny 
(2006) found similar results for several other key measures of population health, including infant mortality and malnutrition rates, as well as average caloric intake.

The second view is that health inequalities are positively associated with socioeconomic and demographic status. In general, the ongoing demographic and epidemiological transition in developed countries has resulted in a more skewed distribution of health and has affected the health inequalities (Wagstaff, 2000; Segall et al., 2000). In spite of convergence in demographic indicators such fertility rates and life expectancy at birth, the evidences from the trends in child health inequalities in both developing and developed world support the notion that health inequalities rise with rising per capita incomes and average demographic and health status. This is largely due to the health care seeking pattern of poor in transitional economies (Segall et al., 2000; Whitehead et al., 2001; Wagstaff, 2002). Mclanahan (2004) argues that the trends associated with second demographic transition are following two trajectories to greater disparities in children's resources. Rich and poor are benefiting alike with returns of fertility and mortality decline. A growing body of research also reports evidences of divergence in health disparities by socioeconomic status in later life (Ross and Wu, 1996; Brown and Prus, 2004; Diprete and Eirich, 2006; Wilson et al., 2007). However, optimistic thinkers like Neumayer (2004) suggests that the current divergence health trends with progress in socioeconomic status and fertility decline will re-convergence, possibly as early as 2015 .

\subsection{Need for the study}

First and foremost, the need of this study arises out of the fact that the discourse of demographic convergence is ever receiving a greater attention in other parts of the world, whereas in India, the discourse on demographic convergence is barely given any attention. Demographic convergence, low fertility and their implications are typically discussed in the context of Western Europe and other Northern countries of the world. However, over the last two decades, these trends have begun to be replaced by the high fertility and mortality in developing country populations too. As such, demographic convergence is a world-wide phenomenon, but developed and developing countries are likely to experience the effects of these trends differently. Though, the rapid fall in fertility carries concerns over distribution of resources, both in developed and developing countries, the 
ramifications are much more severe in developing countries because they are compounded by the challenges of socioeconomic development and inequality.

In India, during the past 50 years, there has been a gradual improvement in life expectancy at birth. There has been a general decrease in mortality in India leading to significant gains in life expectancy, both at the country and state level over the last three decades (Dyson et al., 2004; Goli and Arokiasamy, 2013). Similarly, there is also an accelerating decline in fertility (James and Nair, 2005; Arokiasamy et al., 2013). However, an important issue emerging out of the national progress in demographic outcomes is, whether progress across the states is converging or not? However, there are virtually no studies in Indian context precisely answering this question.

Second, though the progress in health outcomes in India appears to be positive across all the states and socioeconomic groups, yet convergence in such progress yet to be examined. The population and health in India are improving with divergent pattern across states, rural-urban and socioeconomic groups. Therefore, the future challenges for health policies at this junction are to reduce inequality, not just to lengthen the life. There are studies on health inequality, but such studies have not moved beyond quantifying health inequalities to its socioeconomic determinants. Until now, we do not have a logical and theoretical framework to assess, monitor and review the health inequalities alongside the demographic and health progress. Although India is experiencing significant socioeconomic, demographic and health transition, there are no evidence about socioeconomic, demographic and health convergence. To our knowledge, there is no major study that has attempted to assess the patterns, volume, and speed of demographic convergence and its linkage with health inequalities in India. With ambiguity in extant literature in India, we cannot predict with confidence whether SES differentials in health, especially in child health will diminish or grow with the convergence in fertility decline. Thus, more empirical evidences on the convergence pattern of SES differential in demographic status, especially in a different social context, can help us to evaluate the merit and applicability of the convergence or divergence measures in population parameters. Therefore, there is a need to look far beyond customary demographic statistics to measure and monitor the progress in demographic imbalance between different states, rural-urban and socioeconomic groups. 
Thirdly, demographic convergence and rising health inequalities and causal relationships are critical to understand the future direction and progress in population and health. This necessitates positioning of the convergence framework in terms of demographic indicators and its linkage with health inequalities. Thus, this study aims to examine the health inequalities in the context of ongoing demographic change and demographic convergence. This also attempts to address the speculative ideas prevailing over the hypotheses that "demographic change and demographic convergence reflects convergence in health status too" or does health inequalities are swimming against the tide of economic growth and demographic progress until post-transition homogeneity appears? The assessment of how different geographic units are moving and converging demographically is important for the projection and prediction of future population and health. Studying demographic convergence can further inform our understanding of socioeconomic, demographic and health progress in terms of equity, since demographics are affected by causality dilemma of the socioeconomic status.

Finally, there is a need for a generalized framework for the study of 'life cycle of inequality transition'. An important weakness in the current demographic literature in India is the lack of a generalized and unifying framework that is relevant to study the demographic inequality similar to other types of inequality, such as income, education and health. The current demographic scenario, in conjunction with increasing demands for accountability, requires intensified efforts to innovate and bridge the gap between diverse disciplinary theories to ensure that demographic methods and tools to take advantage of the other social science and provide valid, reliable and comparable measurements for wider implementation. What has been missing from the Indian health calendar is a crosscutting population and health measurement tools that unites the different disciplines that have something to contribute to an enhanced capacity of Indian population and health measurement and evaluation. Therefore, the important rationale of this study is to provide the cutting-edge summary measures in terms of convergence tools to assess and monitor the socioeconomic, demographic and health progress, and inequalities in India by using available data. Convergence measures act as a catalyst for population health measurement and evaluation that bridge the gaps in policy formulation, monitoring, reviews and remedies. Therefore, this study attempts to fill critical gaps, said above. 


\subsection{Research questions}

The following critical research questions are addressed in this study.

1. What are the critical change-points in demographic trends in India?

2. Are the trends in socioeconomic, demographic and health indicators across the states in India heading to converge?

3. Do the improvements in average SES, demographic and health status reduce inequalities in the same indicators?

4. Do the improvements in the average SES, demographic and health status reduce health inequalities?

5. Has demographic convergence reduced inequalities in child health status?

6. What is the magnitude of a gap that needs to be reduced to bring Indian states to the line of equity and to the line of highest achievement in demographic and health status?

\subsection{Objectives}

The major objectives of the study are

1. To assess the process of demographic transition in India using change-point analyses

2. To measure socioeconomic convergence across the major states of India

3. To measure fertility convergence across major states, rural-urban and socioeconomic stratum

4. To measure mortality convergence across the major states, rural-urban and socioeconomic stratum

5. To assess the convergence in child health status across the major states, rural-urban and socioeconomic stratum

6. To examine the linkage of demographic transition, convergence and inequalities in child health status

7. To understand the prospects of convergence to the line of equity and the line of highest achievement in India and major states

\subsection{Hypotheses}

Following null hypotheses are formulated to address in this study

1. There are no critical changes in demographic trends in India

2. There is no convergence in socioeconomic progress

3. There is no convergence in demographic (fertility and mortality) progress 
4. There is no convergence in child health progress

5. There is no relation between improvements in average socioeconomic, demographic and children's health status and inequalities

6. There is no relation between demographic convergence and inequalities in children's health status

7. There are no avoidable demographic and health disparities in India 


\section{CHAPTER 2}

\section{Data and Methodology}

This chapter provides the data sources and describes the methods of analyses used in the study.

\subsection{Data sources}

In this study, I used data from several secondary sources. These include Sample Registration System (SRS) (RGI, 1971-2007) and three rounds of National Family Health Survey (NFHS) (IIPS and Macro Internationals, 1992-2006) to assess the demographic trends and convergence, and their linkage with health inequalities in India and major states. Since the early 1970s, India's SRS has been the reliable source of fertility and mortality estimates for the country. SRS (1971-2007) data on Total Fertility Rate (TFR), Life Expectancy at Birth (LEB), Maternal Mortality Rate (MMR) and Infant Mortality Rate (IMR) are used as indicators to assess the demographic trends and convergence. Neonatal Mortality Rate (NNMR) from SRS is used as the indicators of child health status across the major states and rural-urban. The three rounds of India's NFHS are widely used source of information for estimating fertility, mortality and child health trends. TFR, IMR, NNMR, Full immunisation of child and Child underweight are the indicators considered from data of three NFHS rounds. This study also used population totals and literacy rates from the Census of India (1971-2001). Compiled data (1971-2005) on socioeconomic indicators of India from official statistics from Reserve Bank of India (RBI) and Planning commission are also used in this study to examine the socioeconomic trends and convergence. The historical, Life Expectancy at Birth (LEB), Total Fertility Rate (TFR) and Infant Mortality Rate (IMR) data in pre-1971 for India was constructed based on indirect estimates from various sources (Chandrasekhar, 1972; Rele, 1987, Guilmoto and Rajan, 2001; Mitra, 2005). Pre-1951 population totals are taken from Mukherjee, (1969). However, the fusion of data from multiple sources is important for convergence analyses, pooled OLS and panel data regression analyses.

\subsection{Methods}

This study used a unified framework of theory and measures from not only demography but also from economics, geography and sociology. Thus, the framework of this study is 
mainly multidisciplinary, but remains within the conventional margins of demography. The methodology of the present study is described in the following sections: concepts, indicators, conceptual framework and statistical models used in the assessment of the socioeconomic, demographic and child health trends, convergence and their linkage with child health inequalities.

\subsubsection{Concepts:}

a) Demographic transition: 'Demographic transition is the shift from a regime of negligible population growth characterised by high birth and death rates to one of equally little growth based on low birth and low death rates, during which there is a rapid increase in numbers due to the demographic gap, the lag of fertility decline behind the mortality decline' (Notestein, 1945).

b) [Demographic] Convergence: In the economic literature, it is possible to find several definitions of convergence (see, Barro, 1992; Barro and Sala-I-Martin, 1992, 1995). The first and widely used concepts describe the convergence of a group of geographic units (states, regions, countries, etc.) as the reduction of dispersion of a given indicator over the time (e.g. the TFR). Operational definition of demographic convergence for this study is defined as 'Closing of gap between states, rural-urban and socioeconomic groups over the time, more specifically convergence of indicators to the same point can be hypothesised as a process of demographic convergence'.

c) Inequality: Inequality is the uneven distribution of status, goods or services across a population and a measure of relative disproportionality (Sen, 1998; Deaton, 2001; Deaton, 2003; Firebaugh, 2003). Further, the magnitude and direction of inequality also important concepts to relate to inequality (see also Dorius, 2010).

d) Absolute and Relative inequality: The distinction between absolute and relative change in an indicator is necessary for understanding change in inequality because the two stand to affect interstate or group inequality trends. In the context of inequality trend analysis, this is sometimes also referred to as level versus proportional change. When considering changes in the level of inequality, it is possible that the absolute gap between states with the highest and lowest values might be increasing, while inequality is simultaneously decreasing (Deaton, 2003; Deaton, 2007). Consider the example, in Table 2.1, where the 
absolute decline in fertility was larger for state ' $A$ ' and therefore, the fertility gap between states A and state B decreased. Now consider the relative change, where, the rates of change for states A and B were 17 and 25 percent, respectively. Since the rate of change in fertility was greater for state $\mathrm{B}$, the relative difference between the two states, measured using the ratio of state A's fertility to State B's fertility increased, even though, the absolute difference between the two states decreased. An absolute decline in fertility gaps is neither a necessary nor a sufficient condition of declining inequality in fertility. The more uneven distribution of $Y$ across the states (i.e. the greater the variance), the greater is the magnitude of interstate inequality. For between-state inequality to be reduced, the relative decline among lagging states must be greater than the relative decline among leading states. For this reason, studies of inequality are typically interested in the absolute difference between individuals or groups only in so far as it affects the relative difference.

Table 2.1. Absolute versus Relative difference and change in the total fertility rate

\begin{tabular}{lcccc}
\hline & 1950 & 2000 & Absolute $\Delta$ & Relative $\Delta$ \\
\hline Country A & 6.0 & 5.0 & 1.0 & $17 \%$ \\
Country B & 2.0 & 1.5 & 0.5 & $25 \%$ \\
Absolute Difference (Fertility Gap) & 4.0 & 3.5 &. &. \\
Relative Difference (Fertility Ratio) & 3 & 3.33 &. &. \\
\hline
\end{tabular}

Notes: The absolute difference between countries A and B is measured as the fertility gap (Country A TFR - Country B TFR), while the relative difference is measured using the fertility ratio (Country A TFR/Country B TFR). Similarly, absolute change is measured as the simple difference between 1950 and 2000 TFR, while relative change is measured as the percentage change from 1950 to 2000. Inequality (relative difference) may be rising even though the absolute gap between countries is declining. This condition of opposing trends occurs when the relative fertility decline of low fertility countries is greater than the relative decline of high fertility countries.

Source: Dorius (2010)

e) Life cycle of inequality: 'Life cycle of inequality' is defined as a consistent pattern of change in inequality which follows an inverted U-shaped curve. It moves from equilibrium to disequilibrium and returning to equilibrium (Firebaugh, 2003; Dorius, 2010).

\subsubsection{Assumptions of convergence, demographic convergence}

The assumption of convergence follows from a number of considerations: first, the socioeconomic characteristics, ways of life and demographic characteristics will become similar across the countries and states over the period (e.g. Nerlove, 1973). It is expected that the end-product of demographic transition would be stabilization around convergent low rates of birth and death, which would generate zero or negative rates of natural 
increase (Coward, 1986). Thus, the assumptions of economic convergence ${ }^{4}$ well applicable in case of demographic indicators (TFR and LEB) which have logical limits if not absolute limits of their metric scale of upper bound. If this is so, then the end of demographic history may be towards the same demographic 'point omega', convergence.

\subsubsection{Conceptual framework}

Human ecology-evolutionary theory (Crenshaw et al., 200; Lenski, 2005), with its focus on an historical and homeostatic perspective in demography (Wilson 1999; Wilson and Airey 1999) offers a promising framework for demographic transition and convergence analyses. In its original form, demographic transition theory has a strong homeostatic perspective, directly and indirectly arguing for demographic steady state or what Easterlin (2000:23) referred to as an "imminent stationary state". The pathways of demographic convergence lie in the demographic transition process and regimes ${ }^{5}$. This study assumes that different timeline of the socioeconomic and demographic transition has segmented the Indian states into different stages of transition or regimes ${ }^{6}$ (form of a variance roughly approximates a beta distribution). A similar process can be observed with health transition. The conceptual framework presented here (Figure 2.1) illustrates the pathways of the process of socioeconomic, demographic and health transition, convergence and inequalities. The framework present simultaneous process of socioeconomic, demographic and health transition because, in a theoretical perspective, socioeconomic, demographic, health, nutritional and epidemiological transitions overlaps and it is difficult to affirm which one precedes the other (Pressat, 1985; Omran, 1998; Karar, 2009).

\footnotetext{
${ }^{4}$ Diminishing returns to capital-labour ratio is a major assumption in Solow's growth model. An economy with lower capital-labour ratio exhibits a higher marginal product of capital and thus, grows faster compared to a similar economy with a higher capital-labour ratio. The differences across the countries will tend to fade over the time, with per capita income and its growth rate gradually converging until reaching an identical long-run equilibrium level for both the states, respectively.

5 Demographic regimes was introduced by Landry (1934) but has never gained the prominence of the demographic transition. According to dictionary of demography, the definition of demographic regime is "the particular combination of interrelated demographic characteristics that pertains in a given population". For example, a situation in which fertility and mortality are in balance and where social arrangements maintain this balance is sometimes referred to as a homeostatic regime" (Pressat, 1985:196).

${ }^{6}$ Many scholars have argued that the trends as sociated with demographic transition are fueled by a common factor, such as modernization, technology and development which effects rich-poor populations differently (Lesthaeghe, 1995; Mason and Jesen, 1995; Mclanahan, 2004).
} 
On the other hand, failure to incorporate diffusion into the study of inequality transition is now challenged in recent times. Economic determinism in demography is challenged by the claim that in modern societies, demographic behaviour is influenced more by ideational factors than material ones (van de kaa, 1987, 1994; Lesthaeghe, 1995). Therefore, a rough generalization is, the engine of first demographic transition is socioeconomic development, medical, technological innovations and reduction in mortality. The engine of the second demographic transition is fertility which mainly driven by diffusion (van de kaa, 2004). However, all the transitions pass through three stages 1) pre-transition: homogeneity regime ${ }^{7}$ 2) progressive transition: heterogeneity regime 3) post-transition: homogeneity regime. Specifically, looking at hypothesis of this study, I presume that during progressive transition period, different states, rural-urban and socioeconomic groups improve at different pace leading to divergent trends until the reappearance of post transition homogeneity. The process from progressive transition or heterogeneity regime to post-transition or homogeneity regime is called 'convergence'. The pathways of this process are two: 1) "Spillover" and "Diffusion" effects from developed to developing societies 2) Policy intervention can lift the developing societies to the level of developed societies in terms of socioeconomic, demographic and health conditions.

However, the story will not end with the end of demographic transition. Although the spillover and diffusion process of convergence story has merit, we believe there are reasons to be concerned about the changes. Particularly, how children are faring under demographic transition and convergence? Does the forces driving the demographic transition are leading to two different trajectories for children's health status? On other hand, it has been reported that many developed countries have experienced a sharp rise in income inequalities with progress in economic growth rate. In a capitalistic mode growth process, often few social groups and states become top earners and captured a larger share of the overall income gains, while for others income has raised only a little. Interestingly, in spite of fall in absolute poverty, economic inequalities are going up (Ortiz and Cummins, 2013). Similarly, the progress of demographic and health transition in developing countries has resulted in a more skewed distribution of health, which

7 The terms homeostatic regime is denote the existence of a system of relationships between the fertility, mortality and nuptiality characteristics of a community and its socioeconomic circumstances so that any movement away from an initial position of equilibrium tends to provoke changes elsewhere in the system which restore the original state" (Pressat, 1985:97). 
considerably influenced the between and within group health inequalities (Ram, 1998; Wagstaff, 2000; Gwatkin, 2000; Mackenbach and Stronk, 2002; Victoria et al., 2003; Goesling and Glenn, 2004; Becker et al., 2005; Hosseinpoor et al., 2006). Earlier evidences from the trends in health inequalities-in both developing and developed worlds supports the notion that health inequalities rise with rising per capita incomes and average health status (Wagstaff, 2002).

Further, the notion of lower fertility leading to improved maternal and child health has long been an important rationale for national and international support for family planning and $\mathrm{MCH}$ programs in low-income countries. This notion is based, largely on the belief that, as fertility declines, it systematically alters patterns of family-building-birth spacing, and mother's age at childbirth in a ways that are beneficial to the mother's and child's health (LeGrand and Phillips, 1996; Arokiasamy et al., 2013). However, studies also reported that decline in fertility led to widening social-class disparities in children's resources (Maclanahan, 2004). Yet, the mechanisms giving rise to inequalities are still imperfectly understood. Thus, this study conceptualised a framework to study the causal relationship between fertility decline and child health inequalities.

The figure 2.2 shows the relationship between fertility and mortality decline and health inequalities. The framework proposed in this study overrides the earlier assumptions that, improvement in socioeconomic, demographic and health averages will reduce child health inequalities in normal conditions. Here, the normal condition, we mean, 1) There is no base difference in purchasing power, knowledge, adoption and diffusion; 2) there is no geographic, social group and individual disparity in the provision of socioeconomic benefits, family welfare and health care services, 3) there is no geographic, social group and individual disparity in utilisation of social, family welfare and health care services. However, this could be a utopian expectation, if one says the relationship between fertility decline and improvement in child health is straight forward. In reality, this relationship is much complex. The societies divided on the grounds of social and economic status, and this division further perpetuated in differential purchasing power and knowledge which further contributed to the unequal utilisation of social and family welfare benefits and health care services, there by outcomes. As a consequence, the demographic transition is widening social-class disparities in children's resources (Haveman and Wolff, 1995, 2004; 
Maclanahan 2004). Children who were born to mothers from the most-advantaged backgrounds are making substantial gains in resources and relative to their counterparts.

Based on this framework, I propose two theoretical postulations for this study: 1) socioeconomic, demographic and child health progress in averages doesn't necessarily bring equal change in inequalities in same indicators and thereby inequalities in child health status and 2) The reduction in fertility not only contribute to increase in average health status but also influence the inequalities in child health care and child health outcomes. The demographic [fertility] convergence not necessarily brings convergence in health inequalities among children. Thus, this study addresses an important research question that 'does improvements in fertility, mortality and average health status increased child health inequalities'? 
Figure 2.1. Conceptual framework of relationship between Demographic and Health transition, Demographic convergence and Health Inequalities

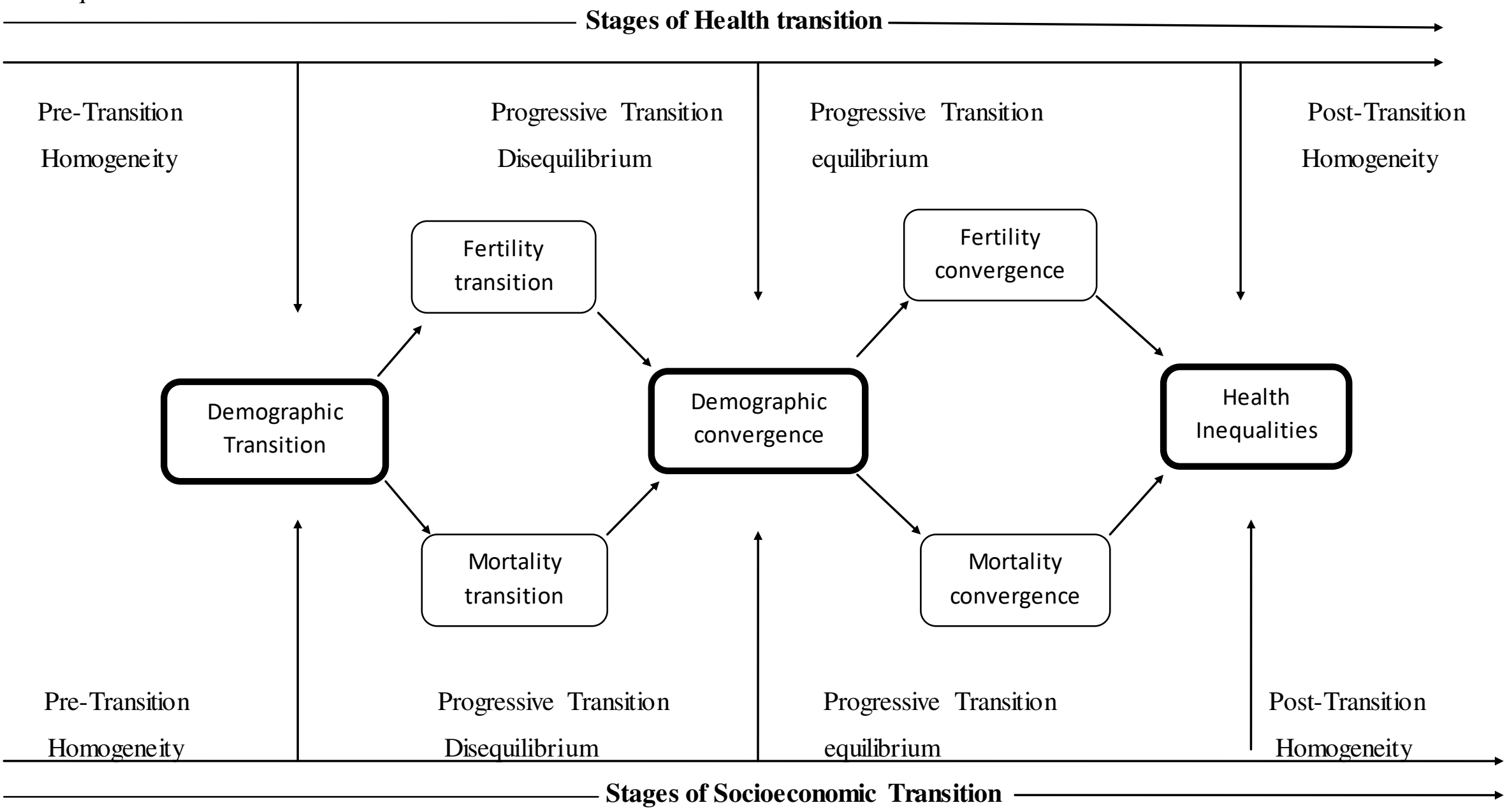

Note: This framework is a synthesized from ideas presented in works of Pressat, 1985; Barro, 1991; Wagstaff et al. 1999, 2002; Lesthaeghe, 1995; Wilson 2001, 2013; Van de kaa, 2004; Maclanahan, 2004; McMichael et al., 2004; Moser et al., 2004; Dorius, 2008, 2010 
Figure 2.2. Pathways of influence of Demographic transition, Convergence on Health inequalities
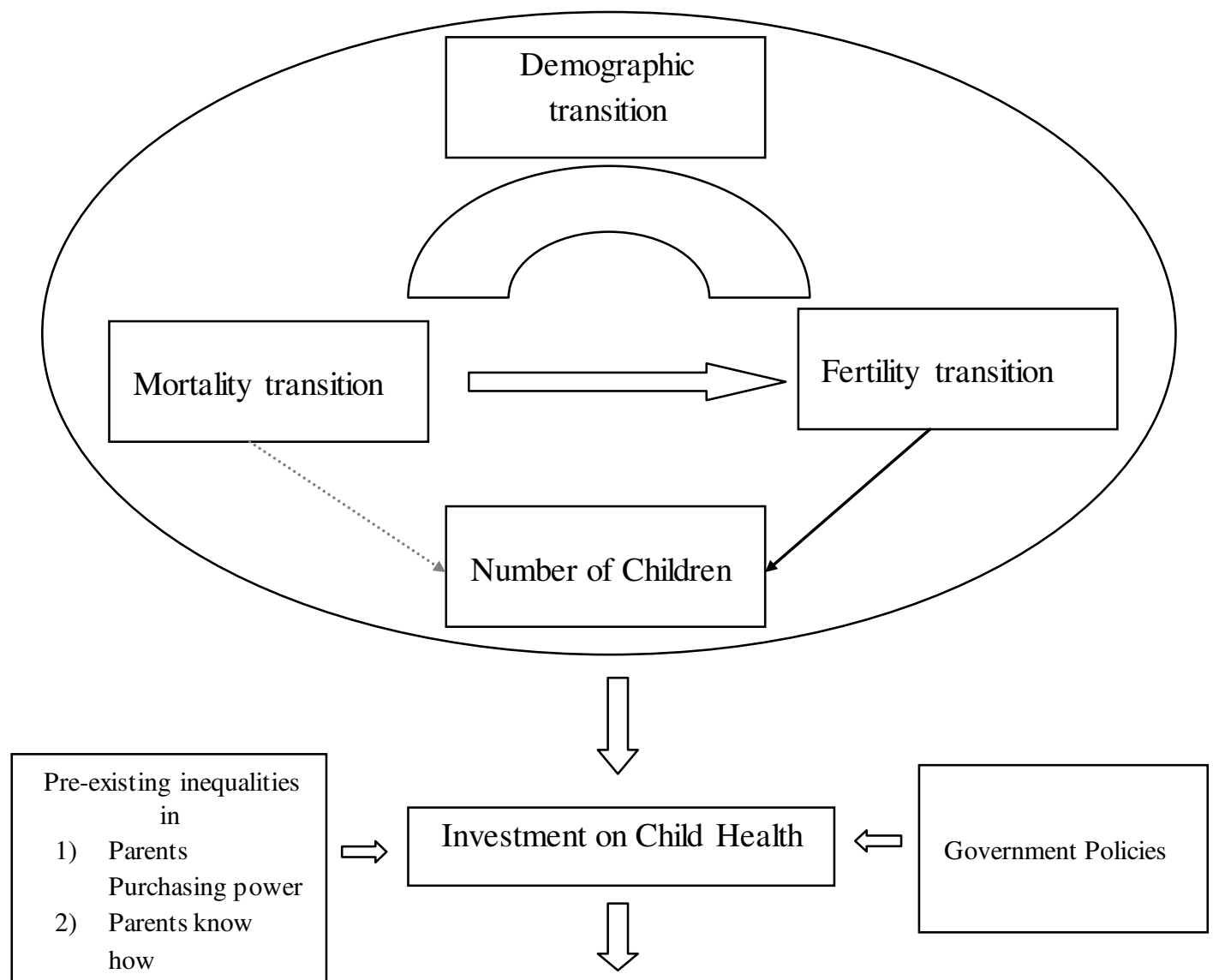

Health care utilisation<smiles>[CH]1C=C1</smiles>

Health inequalities

This relationship borne out the fact that the decline in fertility and mortality is led to increase in investments on quality of health services in which rich people are investing more than poor. Further, this led to differential health outcomes that the rich - poor are receiving the returns of fertility and mortality decline differently. Fewer number of children mean the growing concern about child quality thereby increased investment in education and public health care of children where, rich spend much higher compared to poor which result into health inequalities (Figure 2.2). 


\subsubsection{Indicators}

The study focused on following vital indicators for assessment of socioeconomic, health and demographic trends, convergence and health inequalities:

1. Socioeconomic indicators: per capita Net State Domestic Product (NSDP), Poverty ratio, Literacy rate and Human Development Index (HDI)

2. Fertility trends and Convergence: Total Fertility Rate (TFR)

3. Mortality trends and Convergence: Life Expectancy at Birth (LEB), Maternal Mortality Rate (MMR) and Infant Mortality Rate (IMR)

4. Child health trends, Convergence and inequalities: Neonatal Mortality Rate [NNMR] (Outcome indicators), Child underweight and Child immunisation coverage (Process indicators)

5. Population size is used to estimate the population weighted estimates

\subsubsection{Statistical models}

Assessment of demographic trends

Demographic trends in terms of key demographic indicators were plotted against time scales with simple line graphs. The different regimes of demographic progress were identified based on CuSum charts and 'change-point analyses'. CuSum charts and 'change-point analyses' provides comparative information that can be useful in analysis of trend data to find potential trend shifts in several series and insights about convergence points vis-à-vis population policy shifts in the plots. A general usage of word 'change-point' indicates 'the time at which a change began to occur' (Taylor, 2011). However, a critical change-point is the point where a major shift in the trend is recognised. There are several approaches to performing a trend analyses and 'changepoint analyses' (Page, 1955; Chow, 1960; Page, 1957; Hinkley, 1971; Hinkley and Schechtman, 1987; Pettitt, 1980; Box and Luceño, 1997; Sibanda and Sibanda, 2007; Fujisaki et al. 2008; Taylor, 2000a; Taylor, 2011). However, this study used the method proposed by Taylor for performing the 'change-point analyses' which generally use a combination of 'Cumulative Sum' (CUSUM) Charts and bootstrapping to detect changes. "The outliers in any population data create additional distinction in the data, making it more complicated to detect a change. The 'change-point analyses' technique is more robust to such outliers. Change-point analyses involve following procedures which explained for in case of IMR (Figure 2.3). 
Let $\mathrm{IMR}_{1}, \mathrm{IMR}_{2} \ldots, \mathrm{IMR}_{43}$ represent the 43 data points. From this, the cumulative sums $\mathrm{S}_{0}, \mathrm{~S}_{1} \ldots, \mathrm{S}_{43}$ are calculated. The procedure for estimating the CUSUM proposed by Taylor (2000a) is given below:

- The first step in estimating CUSUM is to estimate the average

$\bar{X}=\frac{I M R_{1}+I M R_{2}+\ldots I M R_{43}}{43}$

- In the second step one should begin the CUSUM at zero by setting $S_{0}=0$. Then estimate the other CUSUM by adding the difference between the current value and the average to the previous sum, i.e. $S_{1}=S_{1-I}+X_{1}-\bar{X}$ for $i=1,2 \ldots, 43$.

Figure 2.3. CUSUM Charts of long-term trends of selected population and health indicators of India, 1872-2011

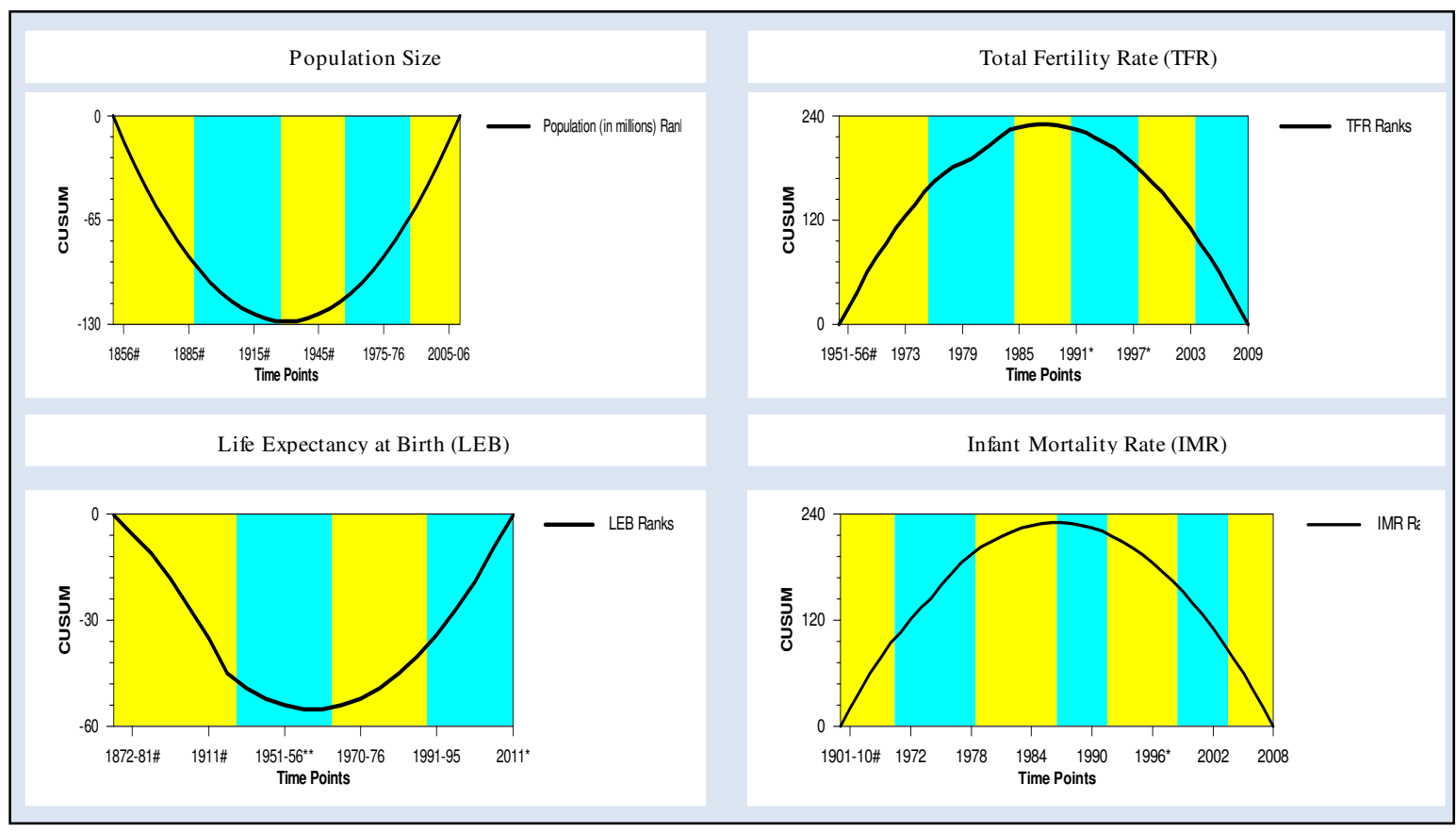

Here, the CUSUMs are not the CUSUMs of the values instead they are the CUSUMs of differences between the values and the average. "These differences sum up to zero, so the CUSUM always ends at zero (S43=0)" (Taylor, 2000a). However, the difficulty with CUSUM charts is that they require exceptional skill to understand correctly. Further, one cannot affirm that these changes took place? For this, the confidence levels of each change-point and significant changes were estimated to understand the apparent change by undertaking a bootstrap analysis. Before undertaking the bootstrap analysis, 
an estimator of the amount of the change is required (Taylor, 2000a). According to Taylor (2000a) one option, which works well in any case of the distribution and regardless of multiple changes, is $S_{\text {diff }}$ defined as:

$$
\begin{aligned}
& S_{\text {diff }}=\mathrm{S}_{\max }-S_{\min } \text { where } \\
& \mathrm{S}_{\max }=\max _{i=0, \ldots .43} S_{i} \\
& \mathrm{~S}_{\min }=\min _{i=0, \ldots .43} s_{i}
\end{aligned}
$$

Following this, bootstrap analysis can be performed when the estimator of the amount of the change has been fixed (Hinkley and Schechtman, 1987; Taylor, 2000a; Karkal, 1985). According to Taylor (2000a) a single bootstrap is performed through the following steps:

- A bootstrap sample of 43 units can be generated by randomly reordering the original 43 values and denoted: ${ }^{I M R_{1}^{0}},{ }^{I M R_{2}^{0}} \ldots . . . .{ }^{M} R_{43}^{0}$.

- Once the bootstrap sample is generated, based on this bootstrap sample, the bootstrap CUSUM is calculated and denoted by $S_{0}^{0}, S_{1}^{0}, \ldots S_{43}^{0}$.

- In the next step, the difference of the bootstrap CUSUM is calculated by taking maximum, minimum, denoted by $\mathrm{S}_{\text {mix }}, \mathrm{S}_{\text {min }}, \mathrm{S}_{\text {diff }}$.

- And the last step involves determining whether the bootstrap difference $S_{\text {diff }}^{0}$ less than or more than the original difference.

A bootstrap analysis consists of performing a large number of bootstrap and counting the number of bootstraps for which $S_{\text {diff }}^{0}<S_{\text {diff }}$. Let ' $N$ ' is the number of bootstrap samples performed and let ' $\mathrm{X}$ ' be the number of bootstraps for which $\mathrm{S}_{\text {diff }}^{0}<\mathrm{S}_{\text {diff }}$. Hence, the confidence level $=100 * \mathrm{X} / \mathrm{N}$. Typically $90 \%$ or $95 \%$ confidence is required to determine that a significant change has occurred. Out of 1,000 bootstraps, 995 had $S_{\text {diff }}^{0}<S_{\text {diff }}$. This gives a confidence level $100 * 995 / 1000=99.5 \%$. This indicates strong evidence that a change did, in fact, occur (Taylor, 2000a).

Assessment socioeconomic, demographic and health convergence measures

Global literatures on economic, demographic and health convergence were used models ranging from simple graphical tools to sophisticated econometric models: catching-up 
plots, absolute $\beta$-convergence, $\sigma$ - convergence, conditional $\beta$-convergence, nonparametric methods of convergence. In particularly, there is no harmonization among scholars on process and measures of convergence. O' Connell (1981) used graphical tools. Wilson (2001) used simple graphical methods. Dorius (2008) used three indices: population-weighted $\sigma$ - and $\beta$-convergence and inequality measures. Herbertsson et al. (2000) focused on the conditional $\beta$-convergence model. Tomka (2002) takes a different perspective, analyzing the demographic convergence. $\mathrm{He}$ proposes indices based on standardized differences from the averages. Franklin $(2002,2003)$, used $\sigma$ convergence, Kotzamanis and Duquenne (2006) and Bloom et al. (2010) used Nonparametric tools. A fact which is quite evident from the global literature is that there is no agreement on a single standard of measuring demographic convergence among the global scholars. Therefore, this study used all important convergence models to draw the conclusion on the convergence process in India. Further, this study classified all these convergence models in four heads and explained in detail in the following sections:

\section{Absolute $\beta$-convergence measure}

Absolute $\beta$-convergence measure: this type of convergence measure is used, where the gap between rich and poor shrinks specially due to higher progress in poorer social strata and states. $\beta$-convergence in a cross-section of states is the negative relationship between the growth rate of an indicator and its initial levels. It is estimated by a regression model proposed by Barro and Sala-I-Martin (1992). This model is represented in the form of the following equation:

$\operatorname{In}\left[\frac{Y_{i, t+k}}{Y_{i, t}}\right]=\alpha+\beta \cdot \ln \left(y_{i . t}\right)+\varepsilon_{i t}$

Where $\operatorname{In}\left[\frac{Y_{i, t+k}}{Y_{i, t}}\right]$ is the mean annualized growth rate of the variable $\mathrm{y}$ in the state $i$ in the period $(\mathrm{t}, \mathrm{t}+\mathrm{k}), y_{i . t}$ is the value in the initial time $\mathrm{t}$ and $\varepsilon_{i t}$ are the corresponding residuals.

Further, the speed of convergence is computed as: $s=-[\ln (1+T \beta) / T]$. Where $s=$ speed of convergence and $T \beta$ is the $\beta$-convergence in $T$ time period. 


\section{Conditional $\beta$-convergence}

When the analysis is done at national level, it will not be reasonable to assume that all states will share the same socioeconomic conditions. However, it is recognized that each state may be converging towards its own steady state across SES strata. This is referred to as conditional $\beta$-convergence and it may be detected with the inclusion of an additional set of variables in the Barro and Sala-I-Martin regression model, which account for varying socioeconomic conditions (such as percentage of illiterates and poverty ratios of the states). In the present study, I estimate conditional $\beta$-convergence by adding percent illiterate and poverty ratios of the states as additional covariates in the original $\beta$-convergence measure thus, the equation of this model can be written as

$\operatorname{In}\left[\frac{Y_{i, t+k}}{Y_{i, t}}\right]=\alpha+\beta \cdot \ln \left(y_{1, i . t} y_{2, i . t}\right)+\varepsilon_{i t}$

Where $\operatorname{In}\left[\frac{Y_{i, t+k}}{Y_{i, t}}\right]=$ is the mean annualized growth rate of the demographic variable $\mathrm{y}$ in the state $i$ in the period $(\mathrm{t}, \mathrm{t}+\mathrm{T}), Y_{i . t}$ is the value in the initial time $\mathrm{t}$ and $\varepsilon_{i t}$ are the corresponding residuals. Similarly $y_{1}$ is the percentage illiterate in state $i$ in the period $(\mathrm{t}, \mathrm{t}+\mathrm{T})$ and $y_{2}$ is percentage of poverty ratio in the state $i$ period $(\mathrm{t}, \mathrm{t}+\mathrm{T})$.

\section{Sigma-convergence measure}

In the mid 1990s, there is a heated debate regarding the relationship between $\beta$ convergence and Sigma convergences (apparently first introduced by Sala-I-Martin, 1996). The central point of controversy was the presumptions that $\beta$-convergence be a necessary prerequisite for sigma convergence. However, later it was acknowledged that $\beta$-convergence is a necessary but not a sufficient condition for sigma convergence to take place. This is because of either overtaking or divergence. This is clear from the figure 2.4. The first panel indicates the absence of both $\beta$-convergence and sigma convergence across the states, which implies that the states are diverging in terms of gap in LEB and this gap, is increasing over time. In the second panel, it is possible to notice that there is a decline in the gap of LEB between states and this was accompanied by a decline in the dispersion of LEB across the states and over the time which shows both $\beta$-convergence and sigma convergence. The last panel seems to suggest overtaking or polarization (Quah, 1996) which shows only $\beta$-convergence but not the sigma convergence. 
Sigma convergence postulates that convergence is taking place when the dispersion of demographic indicators decreases. Friedmann (1992) and Quah (1996) suggest that as the time passes, we expect the gap between states in terms of demographic status become smaller. Extending this logic to the case of Indian states, we expect the difference between variable ' $\mathrm{Y}$ ' across states to eventually shrink.

Figure $2.4 \beta$-convergence versus Sigma Convergence
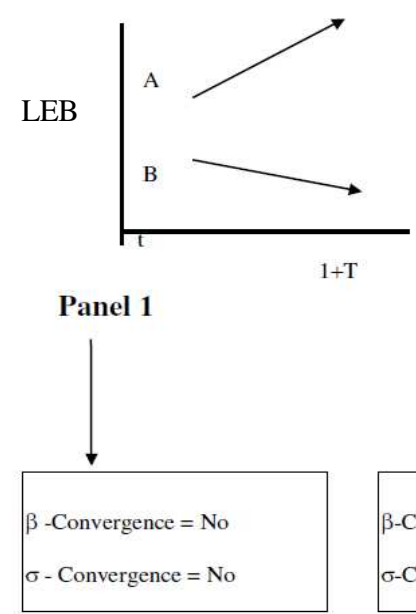
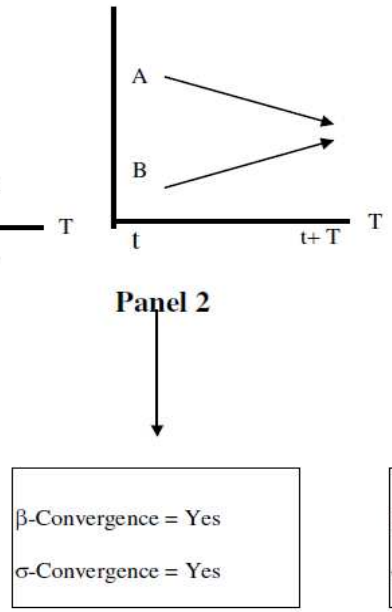

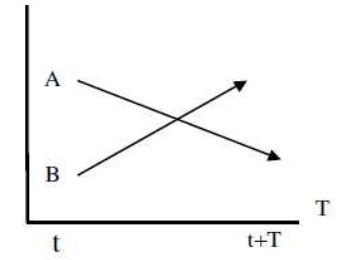

Panel 3

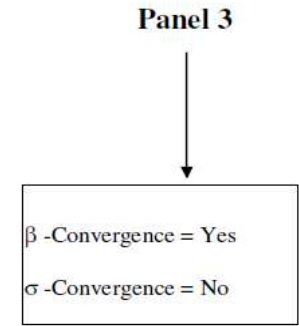

Based on the nature of the indicators (socioeconomic and demographic indicators), the earlier studies were used two methods for estimating sigma convergence: Standard Deviation (SD) and Coefficient of Variation (CV). I have also followed the same procedure. While SD shows the absolute sigma convergence and CV indicates relative sigma convergence. The absolute sigma convergence model is estimated as

$\sigma_{t}>\sigma_{t+T}$

Where $\sigma_{t}$ is the standard deviation (or assimilated measure) of the indicator at the time

$t$. If the parameter $\sigma_{t+T}$ is reduces, it implies evidences of convergence.

The relative $\sigma$-convergence is derived by using $\mathrm{CV}$. Coefficient of variation is estimated as

$C V_{t}>C V_{t+T}=\sigma_{t+T} / \mu$

Where $C V_{t}$ is the coefficient of variation of the indicator at the time $t$. If the parameter $C V_{t+T}$ is reduces, it implies evidences of convergence. 


\section{Convergence clubs}

Quah (1996) argues $\beta$-convergence is interested only in comparison of mean growth across countries, but not in the distribution of the indicator, and that cross-section regressions can represent only average behaviour, not the behaviour of the entire distribution. From Quah's hypothesis, two key results emerged: first, the coalition (convergence clubs) forms endogenously. The model delivers prediction on coalition membership across the entire cross-section of states, and secondly, different convergence dynamics are generated depending on the initial distribution of characteristics across the states. These dynamics characterized as polarization or convergence clubs. Demographic convergence may not be strong across all the states but may be strong across the regions. For example, in India, it may be possible that the southern region is faster converging than northern region. Similarly, it can also be expected that urban areas are faster converging than rural areas. In such situation, the introduction of regional, rural, and urban dummy variables in conditional $\beta$ convergence models gives a picture of convergence within the regions and within urban or rural, these portions are called convergence clubs. Estimation of the model is similar to conditional $\beta$-convergence by additional regional dummy. However, this study also used graphical plots and non-parametric models for identifying convergence clubs and short-term divergent paths of the transition.

\section{Non-parametric models of convergence clubs}

Though, parametric convergence metrics are useful to examine the convergence process across states that have been criticized for using unreal assumptions such as the normal distribution. Among non-parametric convergence metrics Kernel density estimates are widely used method. Kernel density estimates allow a closer look at changes in the distribution in relative terms. They allow data to be modelled without presuming that the data follow a normal distribution and identify the short-term divergent paths, which may occur in long convergence process (Quah, 1993a). Further, kernel density estimator is the best suitable test for identifying the number of clusters in the distribution of an indicator. The advantage of kernel density estimators is that the clusters are not prejudiced by geographical regions (which prone to huge heterogeneity within the region) rather they are emerging from the distribution of the indicator. The Nonparametric methods capture short-time divergent paths which occur along with the convergence process (Raileanu, 2011). This study used the Epanechnikov kernel which 
is an optimal in a minimum variance sense (Epanechnikov, 1969). However, for some indicators, I have also used Gaussian kernel. With reference to bandwidth, this study used adaptive bandwidth kernel density estimation. If the bandwidth is not held fixed, but is varied depending upon the location of either the estimate or the samples, this produces a particularly powerful method which makes estimates more robust. A general form of kernel densities is estimated by using the following equation:

$\hat{f}(x)=\frac{1}{h n} \sum_{i=1}^{n} k\left(\frac{x-X_{i}}{h}\right)$

Where, $\hat{f}(x)$ is the density estimation of the variable $\mathrm{x}, \mathrm{n}$ is the number of observations, ' $h$ ' is the bandwidth (smoothing parameter) and $\mathrm{K}$ (.) is the smooth and symmetric kernel function integrated to unity.

\section{Assessment of demographic and health inequalities}

Previous studies that examined inequality adjusted convergence adopted various metrics such as Theil index, Gini coefficient and Gussian density function (Franklin 2002; Dorius 2008; Bloom 2011). However, in view of the widespread state and socioeconomic disparities in India, this study used measures which give more robust test of convergence and account for 'absolute' and 'relative' distributions of the indicator. To assess the overall absolute inequality, this study used Dispersion Measure of Mortality (DMM). With slight modification, this model was also applied for estimating dispersion in fertility and health status. The DMM measures the degree of dispersion that exists at any point of time in the mortality experiences of states in a country. It is calculated as the average absolute inter-state mortality difference, weighted by population size, between each and every pair of the country. Change in the DMM over time indicates whether mortality is becoming more or less similar across the country, thus, the decrease indicates convergence and increase indicates divergence. The DMM for life expectancy at birth is measured in years of life and the DMM for infant mortality is measured in infant deaths per thousand live births. It is obtained by the formula given below (Shkolnikov et al., 2003; Moser et al., 2005).

$\mathrm{DMM}=\frac{1}{2\left(\mathrm{~W}_{\mathrm{Z}}\right)^{2}} \sum_{\mathrm{i}} \sum_{\mathrm{J}}\left(\left|\mathrm{M}_{\mathrm{i}}-\mathrm{M}_{\mathrm{j}}\right| * \mathrm{~W}_{\mathrm{I}} * \mathrm{~W}_{\mathrm{J}}\right)$

Where, $i, j$ are states, and $1 \leq i, j \leq 15$

$\mathrm{Z}$ is equal to 1 
$\mathrm{M}$ is the mortality rate

$\mathrm{W}$ is the weighting, and $\sum_{\mathrm{i}} \mathrm{W}_{\mathrm{i}}=\sum_{\mathrm{j}} \mathrm{W}_{\mathrm{j}}=\mathrm{W}_{\mathrm{z}}$

When applied to life expectancy at birth, $\mathrm{M}=$ life expectancy at birth of the state,

$\mathrm{W}_{\mathrm{z}}=1$ and $\mathrm{W}_{\mathrm{I}}$ represents the relative population size of the state $i$.

To assess the overall relative inequality, this study used the Gini concentration index. The estimation of Gini in case of Life Expectancy at Birth (LEB) is equal to DMM divided by the average life expectancy of the states (Shkolnikov et al., 2003).

$\mathrm{G}=\frac{\mathrm{DMM}}{\overline{\mathrm{e}_{0}^{0}}}$, where $\overline{\mathrm{e}_{0}^{0}}=\left[\sum_{\mathrm{i}} \mathrm{Pi}_{0}^{\mathrm{i}}\right]$

Where $\mathrm{G}=$ Gini index value, $\mathrm{DMM}=$ Dispersion Measure of Mortality, $\overline{\mathrm{e}_{0}^{0}}$ is average life expectancy at birth adjusted by the population proportion of the states $i_{\ldots} . . i_{n}$.

Subsequently, this model is slightly adjusted to apply on fertility to estimate Dispersion Measure of Fertility (DMF). The DMF is calculated as the average absolute interstate fertility difference, weighted by the size of the population between each and every pair of states. Change in the DMF over time indicates, whether, fertility rates are becoming more or less similar across the states. A decrease in DMF indicates convergence and an increase indicates divergence.

$\mathrm{DMF}=\frac{1}{2\left(\mathrm{~W}_{\mathrm{Z}}\right)^{2}} \sum_{\mathrm{i}} \sum_{\mathrm{J}}\left(\left|\mathrm{TFR}_{\mathrm{i}}-\mathrm{TFR}_{\mathrm{j}}\right| * \mathrm{~W}_{\mathrm{I}} * \mathrm{~W}_{\mathrm{J}}\right)$

Where, $i, j$ are states, and $1 \leq i, j \leq 15$

$\mathrm{z}$ is the India

TFR is the total fertility rate

$\mathrm{W}$ is the weighting, and $\sum_{\mathrm{i}} \mathrm{W}_{\mathrm{i}}=\sum_{\mathrm{j}} \mathrm{W}_{\mathrm{j}}=\mathrm{W}_{\mathrm{z}}$

$\mathrm{W}_{\mathrm{z}}=1$ and $\mathrm{W}_{i}$ represents the relative population size of the state $i$.

To assess the relative inequality, the Gini concentration index is used. In case of estimation of relative inequality of total fertility rate, $\mathrm{G}$ is equal to DMF divided by the average TFR. 
$\mathrm{G}=\frac{\mathrm{DMF}}{\overline{\mathrm{TFR}}}$, where $\overline{\mathrm{TFR}}=\left[\sum_{\mathrm{i}}\right.$ Pi TFRi $]$

Where $\mathrm{G}=$ Gini index value, $\mathrm{DMF}=$ Dispersion Measure of Fertility, $\overline{\mathrm{TFR}}$ is average total fertility rate adjusted by the population proportion of the states $i_{\text {.... }} i_{n}$.

Further, for estimating absolute overall inequalities in socioeconomic and child health indicator, this study used Average Interstate Differences (AID) to indicate the absolute dispersion in the 'variable' across the states. The AID is estimated by using the following equation proposed by Shkolnikov et al. (2011).

$$
A I D=\frac{1}{2(u)^{2}} \sum_{x} \sum_{y} d_{x} d_{y}|\bar{x}-\bar{y}|
$$

Where, AID is Average Interstate Differences, $u$ is the mean of socioeconomic variable, $d x$ is the population proportion of states $x, d y$ is the population proportion of states $y$, $\overline{\boldsymbol{x}}-\overline{\boldsymbol{y}}$ is the difference in socioeconomic variables of states $x$ and $y$.

To assess the socioeconomic inequality in health, this study used concentration index proposed by Wagstaff et al. (1991). The concentration index is computed as twice the (weighted) covariance of the health variables, and a person's relative rank in terms of economic status, divided by the 'variable' mean. This can be written as

$C=\frac{2}{\mu} \operatorname{cov}_{\mathrm{w}}\left(\mathrm{y}_{\mathrm{i}}, \mathrm{R}_{\mathrm{i}}\right)$

Where $y_{i}$ and $R_{i}$ are, respectively, the health status of the $i^{\text {th }}$ individual and the $R_{i}$ fractional rank of the $i^{\text {th }}$ individual (for weighted data), in terms of the index of household economic status. $\mu$ is the (weighted) mean of the health variable of the sample and $\operatorname{cov}_{w}$ denotes the weighted covariance.

Assessment of inequality measure based convergence in demographic and health indicators

Convergence in demographic and health inequalities was estimated based on change in inequalities estimated by using the DMM, AID and Gini index. The convergence in 
overall absolute and relative inequalities was calculated in terms of percentage reduction in DMM or AID and Gini index, respectively.

Assessment of demographic and health convergence and its linkage with health inequalities

Times series and cross-sectional scatter plots, correlation and regression estimates are used to link the demographic progress with health inequalities.

Zero order and Partial correlation

Zero order correlation is a general correlation in which there is no control variable. On the other hand, partial correlation represents the correlation between the criterion and a predictor after common variance with other predictors has been removed from both the criterion and the predictor of interest. That is, after removing variance that the criterion and the predictor have in common with other predictors, the partial expresses the correlation between the residualised predictor and the residualised criterion. This can be expressed as

$$
r_{y x z}=\frac{r_{y x}-r_{y z} * r_{x z}}{\sqrt{\left(1-r^{2}{ }_{x z}\right)\left(1-r^{2}{ }_{y z}\right)}}
$$

Where $r_{y x . z}=$ the (first-order) partial correlation of $\mathrm{y}$ and $\mathrm{x}$ controlling for $\mathrm{z}$ Zero order correlation can written as $r_{y x}=$ the bivariate (zero-order) correlation of $\mathrm{y}$ and $\mathrm{x}$ $r_{y z}=$ the bivariate (zero-order) correlation of $\mathrm{y}$ and $\mathrm{z}$ $r_{x z}=$ the bivariate (zero-order) correlation of $\mathrm{x}$ and $\mathrm{z}$

The formula suggests that the zero order correlation is the function of the bivariate correlations among the three variables. It can also define as correlation between two variables which does not include a control variable.

\section{Pooled Ordinary Least Square (OLS) Regression Model}

The simplest estimator for panel data is pooled OLS. The pooled OLS regression assesses the association between panels of inequalities in child health indicators, demographic and socioeconomic factors. As usual OLS, this model treats all 
observations as equivalent. In this case the error term captures "everything". The pooled OLS specification may be written as

$\mathrm{Y}_{\mathrm{it}}=\mathrm{X}_{\mathrm{it}} \beta_{1}+u_{\mathrm{it}}$

Where $\mathrm{Y}_{\mathrm{it}}$ is the observation on the dependent variable for cross-sectional unit $i$ in a period $\mathrm{t}, \mathrm{X}_{\mathrm{it}}$ is a $1 \mathrm{x} \mathrm{k}$ vector of independent variables observed for unit $i$ in period $\mathrm{t}, \beta$ is a $\mathrm{K} \times 1$ vector of parameters, and $u_{\mathrm{it}}$ is an error or disturbance term specific to unit $i$ in the period $t$. But pooled regression ignores time and space dimensions. Therefore, I have also carried out panel data regression.

Panel data regression: Fixed effects

Panel data are repeated pooled cross-sections over time that, in essence, there will be a space as well as time dimensions. Panel data regression analysis is used to estimate the effect of demographic transition and rising average health on health inequalities. In panel data analysis, the term fixed effects indicate that the intercept may differ across individuals, but it does not vary over time, that is, it is time-invariant. If I assume fixed effects, I impose time independent effects for each entity that are possibly correlated with the repressor. Moreover, before selecting a suitable panel regression model that fixed or random effect model, I used 'Hausman test'. This test confirmed that error terms are not correlated then decided to apply only fixed effect model. The 'fixed effect model' allows for heterogeneity among individuals by allowing each one to have its own intercept value. Fixed effect model removes the effect of those time-invariant characteristics of the predictor variables so I can assess the predictors' net effect. Fixed effects panel regression is represented by the following equation

$Y_{i t}=\propto_{i}+\beta_{1} X_{i t}+\mu_{i t} \quad i=1 \ldots . . N ; t=1 \ldots \ldots \ldots . T$

Where

$-\propto_{i} \quad(\mathrm{i}=1 \ldots . . \mathrm{n})$ is the unknown intercept for each entity (n entity-specific intercepts).

- $Y_{i t}$ is the dependent variable where $\mathrm{i}=$ entity and $\mathrm{t}=$ time.

- $X_{i t}$ represents one independent variable,

- $\beta_{1}$ is the coefficient for that independent variable,

- $\mu_{i t}$ is the error term 


\section{Measure of distance to line of equity and line of highest achievement}

To make need assessment of future prospects of health equity across the states and socioeconomic groups, I have used three methods 1) Funnel plots 2) Distance to line of equity plots and 3) Distance to line of highest achievement plot. These analyses are inspired by the social welfare function proposed by Sen (1973), and holds that a development process in which society attempts to equitably supply of basic opportunities, ensuring that as many individual as possible to have access to those basic opportunities, with a target of Universalism. It requires distributing available basic opportunities increasingly towards the most disadvantaged groups.

\section{Funnel charts}

'Funnel plots' are also graphical aid for showing the distribution of an indicator across population subgroups, in which an estimate of an underlying quantity is plotted against an interpretable measure of its 'Control limits'. Funnel plots are flexible and attractively simple. Funnel plots are adapted to mapping through the use of Confidence intervals (Upper and Lower limits). In the mathematical form this can be written as

$$
\frac{P_{i}-\bar{p}}{\sqrt{\frac{\bar{P}(1-\bar{p})}{n i}}}
$$

Where $\bar{p}$ the national average, $P_{i}$ is the $i^{\text {th }}$ states value, and $n_{i}$ is the $i^{\text {th }}$ exposed population. The confidence limits correspond to the 95\% upper and 95\% lower control limits in the funnel plot.

\section{Distance to line of equity plot}

Distance to line of equity plot shows the dispersal of states from the line of equity in terms of selected demographic indicators. Based on this plot, one can identify which of the states are located at a greater distance from the line of equity. The measures of distance to line of equity have a hypothetical reference from the same population. Here, I measured how two contrasting groups poorest and richest tend to be distributed for a given indicator from a line of equity. This plot shows whether, the indicator is skewed towards poorest or richest groups. This method is only a graphical tool and the line of equity often based on a hypothetical series taken from the same distribution of the indicator from the same population (Goli, 2012). 
Distance to line of highest achievement plot

Plot of distance to line of highest achievement shows the gap between the location of states and the line of equity in terms of selected demographic indicators. Based on this plot one can, not only identify which states located at a greater distance from the line of highest achievement, but also quantify the gap that need to be reduced for the convergence in the line of highest achievement. The distance from the line of highest achievement was estimated based on a reference population. The reference group is one which has the best progress and it is taken not only from the same population, but also from any population in the state or country or even at a global level. It is the best achievement made in a given indicator by any population. By this measure, one can estimate and show the avoidable level of a given indicator for states or socioeconomic groups.

\subsubsection{Organization of thesis}

The study is organized into ten chapters

Chapter 1: This chapter includes introduction, review of literature, need for the study and objectives of the study.

Chapter 2: This chapter describes the sources of data and methodology used in the study.

Chapter 3: This chapter assesses demographic transition in India: evolutionary interpretation of long-term trends using change-point analysis.

Chapter 4: This chapter assesses the socioeconomic convergence.

Chapter 5: This chapter examines mortality convergence.

Chapter 6: This chapter measures the fertility convergence.

Chapter 7: This chapter measures the convergence in child health care utilisation and outcomes.

Chapter 8: This chapter assesses the linkage of demographic convergence with child health inequalities in India.

Chapter 9: This chapter measures the distance to line of equity and line of highest achievement: how much demographic differences can be avoidable in India.

Chapter 10: This chapter provides the summary, conclusion, policy and practical implications, and limitation of the study. 


\section{CHAPTER 3}

\section{Demographic transition in India: Evolutionary interpretation of long-term trends using Change-point analysis}

\subsection{Introduction}

The understanding of demographic transition was once called a fundamental problem of human socio-biology (Vinning, 1986, p167). As I mentioned in the earlier part of this study that the modern suppositions of population change revolve around the idea of the classical demographic transition model articulated by Notestein (1945). He noted that 'all the societies, at one time or other, move from a near-equilibrium condition of high mortality and high fertility towards a presumed low-fertility and low-mortality equilibrium' (Notestein, 1945). This largely acceptable characterization of demographic transition consists of five components: mortality decline, natural increase in population size, fertility decline, urbanisation and population aging. Although, this conception is only a rough generalization, it has such wide applicability that it has become a central axis of conceptualisation about population trends in world (Notestein, 1945; Davis, 1951; Davis, 1963; Sinding, 2001; Dyson, 2004; Caldwell, 2004; Dyson 2010; Casterline, 2011).

However, some of the Notestein contemporaries found transformation in portrayal of demographic theory. For example, Caldwell (2004: 297) stated:

"Demographic transition theory has been largely transformed over the last half-century from grand theory to short-term theory, often endowed with such immediacy as to so limit our vision of the future that even population policy makers are made difficult. Demographic theorists lost their nerve as the globalization of decline in mortality and fertility proceeded much more rapidly than they anticipated".

Taking his stand forward, I say that a perfect portrayal of demographic transition is required to investigate the impact of demographic changes and its linkages to social, economic and health status of the nations. Numerous studies have assessed demographic transition in terms of fertility, mortality and health trends and transition at the global scale (Dyson 2010; Van and Knodel, 1980; Bloom and Williamson, 1998; Wilson and Airey, 1999; Casterline, 2001; Wilson, 2001; Wilson, 2011; Bongaarts, 
2003; Weeks et al., 2004; Caldwell, 2004; Moser et al., 2005; Doepke, 2005; Dorius, 2008; Dorius and Firebaugh, 2010; Angeles, 2010). A growing number of studies in India had also assessed the trends and transition in population health indicators (Dyson, 2005; Ghosh, 1956; Rele, 1982; Rele, 1987; Dyson, 1989; Bhat, 1989; Bhat, 1997; Bhat et al., 1984; James, 1995, James and Nair, 2005; Guilmoto and Rajan, 2001; Visaria and Visaria, 1994; Visaria and Visaria, 2003; Kulkarni and Alagarajan, 2005; Visaria, 2004b; Kulkarni, 2011; Visaria, 2011). However, all these studies have used basic trend line plots, control charts or descriptive tables with annual or decadal changes to determine transition points in population and health indicators. Historical studies that estimated on set of fertility [demographic] transition believed that it starts when there is at least $10 \%$ decline in fertility which begins an irreversible trend downwards said to be "completed when replacement level fertility levels are achieved" (Bongaarts and Watkins, 1996; Ross et al. 2005; United Nations, 2003; Bongaarts, 2008). This approach cannot be used here because historical data are lacking and the level of pre-transitional fertility cannot be determined accurately. To our knowledge and to date, there are no studies across the globe or in India that has attempted an evolutionary interpretation of long-term trends in population and health indicators through more sophisticated tools and techniques, which talk about origin of sustainable demographic change and have properties to estimate, and determine critical changepoints and multiple changes, and affirm its statistical significance.

This study adopted an innovative procedure is termed as 'change-point analyses' to study, the long-term trends in population and health indicators in India and states. The main distinguishing factor between 'change-point analyses' and simple trend line plots or charts is that, simple trend line plots are in general better at identifying isolated uneven points and major changes whereas a 'change-point analyses' can also detect modest changes that are frequently unobserved by control charts. Moreover, the simple trend line plots or charts cannot quantify the change with its statistical significance including confidence levels. Estimation of confidence levels of a change is essential to validate and determine the robustness of a change that appears in graphical plots and charts. While examining the historical data, particularly when data sets are large 'change-point analyses' is favoured, as it is scientifically robust than the control charts in terms of identifying the pattern of trend. The other key advantage of a 'change-point analyses' is that it controls the change-wise error rate. Therefore, each change 
identified is possibly the truest. Simple trend line plots or 'control charts' or descriptive tables of annual or decadal change do not control the point wise error rate and, therefore, may provide an inaccurate assessment of demographic trends. When analyses are performed in large numbers of data points, several points can go beyond the set limits even when no change may have occurred. The main advantage of 'change point analyses' is:

"it is simple to apply and construe, especially for large data sets and when multiple changes may have occurred" (Taylor, 2011).

Through 'change-point analyses' of the population and health indicators, this study addressed five questions: 1) Did change occur at all? 2) Did more than one change occur? 3) When did the changes occur? 4) With what confidence level, did the change occur? and 5) What demographic expositions emerge from the observed change-points? By assessing these five questions in Indian context, this study attempts to identify the onset of critical changes and multiple change points in various demographic indicators over a long period of demographic transition. Implicitly, this chapter is an effort to integrate demographic change with population and health policy shifts and other historical events. Thus, assesses the temporal dimension of the demographic progress. With the determination of critical change-points and their time points, this study innovate to more accurately interpret the accounting factors of progress in demographic and health transitions. To establish the causal relationship, this study compares historical trends and pattern of fertility, mortality and population size by relating the trend to time trajectories of population policies and development strategies as drivers of change.

\subsection{Data source}

Though, in the chapter 2, I did mention about the data source of the study, but this particular chapter needs little more discussion on the data source because analysing long-term data involves issues like 'availability' and 'quality of data' in the countries like India where official mechanism to maintain such data has evolved very late (Kulkarni, 2011). This chapter attempted to investigate long-term (around 150 years) demographic trend data for various reasons. The long term trends observed better than short intervals of time because of the defectiveness of the available statistical material, where specific annual totals and rates in births and deaths do not have much significance. While examining and establishing trends in health and demographic 
transition, one hundred and fifty years constitute a convenient and significant stretch of time for identifying a pattern and progress in pre and progressive transition and prediction of post transition regimes of the population. This has been proved adequate in case of Western Europe, where the demographic cycle began around 1750 and lasted until the depression - a period of more than 200 years (Davis, 1963; Chandrasekhar, 1972). However, the major impediment of historical analyses of demographic trends in India is the absence of good quality of data as the vital registration system is far from functioning well (Bhat, 1989). It is well known that in India, the coverage of vital registration system is far from being complete to provide reliable estimates of population and health indicators for the period until 1970 (Kulkarni, 2011). This chapter, therefore, uses myriad sources of data: Census data, indirect estimates, survey based and other official estimates to assess the long-run trends of population size, fertility and mortality indicators. The detailed description of different data sources used in this study is presented below.

Mortality and Fertility indicators: The historical Total Fertility Rate (TFR) and Infant Mortality Rate (IMR) data in pre-1971 India is constructed based on indirect estimates of various sources: 1) Data on TFR and Life Expectancy at Birth (LEB) during 19511971 are based on Rele estimates (Rele, 1987). The Rele estimates are considered to be robust compared to other estimates of fertility and mortality prior to the Sample Registration System (SRS) estimates. 2) The LEB data for the pre-1951 period was obtained from estimates provided in Mitra (2005). Mitra provided LEB estimates based on Registrar General of India (RGI) and Census during 1872-1941. The estimates of total LEB are averages of male-female LEB (Office of Registrar General of India, 1971-2005). However, we don't have reliable TFR estimates for the pre-1951 period. The recent data on LEB, fertility and mortality indicators are taken from the SRS, available on an annual basis (Census of India, 1871-2001). 3) Pre-1971 IMR estimates were adopted from Chandrasekhar (1972).

Population size: The data on population size prior to 1955 were based on estimates of Mukherjee (1969). I have chosen Mukherjee estimates (1969) over Davis (1951), Ghosh (1956) and Mahalanobis and Bhattacharya (1976) etc. because his estimates of population totals from, 1856 to 1951 followed a uniform method, which are comparable over time. However, the recent statistics on population size obtain from Reserve Bank 
of India (RBI) (1955-2011). Mukherjee (1969) and RBI (1955-2011) both gives population totals for five year intervals based on linear interpolation between Censuses (Census of India, 1871-2001). Therefore, both these estimates are comparable over a long-term period. However, the population totals for the year 2011 is obtained from provisional Census results of 2011 (Census of India, 2011).

\subsection{Change-point analyses}

As mentioned above, I have used 'change-point analysis' technique to assess the critical and multiple change-points over the long-term population and health trends of India. Though, a detailed discussion on the procedure of plotting and estimation of changepoints has been discussed in the chapter 2. Here, I discussed few important issues which must be considered while interpreting the results of change-point analyses. A general usage of word 'change-point' indicates 'the time at which a change began to occur'. However, a critical change-point is the point, where a major shift in the trend is recognized. The change-points highlighted in this study are critical change-points. A critical change will not appear immediately after a growth or decline, rather an accumulative growth or decline will results into a critical change for a given indicator. However, the time period for a change resulting into a critical change depends on sensitivity nature of the indicators to its external factors. For example, in comparison to population size, a critical change in child mortality rates and for some extent the fertility rates yield in lesser time. For each change, 'change-point analyzer' provides detailed information including volume of change, confidence levels and change-wise error rate during the trend period. 'The procedure of performing a 'Change-Point analyses' is very flexible, as it can be carried out on all the types of time series data as well as attribute data, that data from non-normal distributions, ill-behaved and complaint data, and data with outliers' (Taylor, 2011). However, the change-point analyses yield better results on long period data because on long period data, we can observe the pattern and time shifts more clearly.

\subsection{Results}

The results of this chapter demonstrate trends and patterns in key population and health indicators of India: 1) IMR 2) Population size 3) TFR and 4) LEB. These four demographic indicators represent four critical components of demographic transition in 
their sequential order: mortality decline, increased rate of natural increase, fertility decline and increasing life expectancy or population aging (Dyson, 2010). The results of 'change-point analyses' are also presented in accordance with the sequence of these four key components of the demographic transition process.

\subsubsection{IMR}

IMR is the most sensitive and widely used summary measure to understand the overall progress in mortality decline and improvement in population health. Numerous demographic and public health research studies have overwhelmingly concluded that the decline in IMR is majorly the consequence of the complementary progress in modern health care and socioeconomic advancement. As indicated by Davis in the Change Response model, it is the first component of demographic transition (Davis, 1951; Davis, 1963). In the recent refreshing exposition of demographic transition, Dyson (2010) believed:

"Mortality decline is the crucial catalyst. Once it occurs, the other four components necessarily follow, and most often sequentially because one component generates the next. Mortality decline leads to an increased rate of natural increase, which produces the conditions that cause fertility decline, which in turn leads to increase in life expectancy and population aging”.

Over the last more than half a century, India experienced a phenomenal decline in IMR. Nevertheless, India's current level of IMR (54/1000 by 2008) is seen to be relatively higher compared to many developed and developing countries of the world. By analyses of historical trends of mortality, Chandrasekhar (1972), Bhat et al (1984) and Dyson (2004) have documented the phenomenal decline of infant mortality rate from 1921 — which accelerated sharply after 1947. Until the late 1970s, the IMR showed a declining trend but with considerable discrepancies, followed by a continuous decline in post-1970 - which led to the steady increase in natural growth rate and acceleration in the population size.

However, virtually all previous studies that assessed trends in IMR have used simple trend line plots. As trend lines are methodologically imperfect tools, they have been not useful to detect and quantify the critical change-points in long-term trends of infant 
mortality transition. Figure 3.1 presents a plot of IMR trend and change-point estimates during 1901-2008. During the past, more than 100 years, results indicate five critical change-points mostly in the last 30 years $(1978,1984,1990,1996$, and 2002) for IMR trends in India. All the five change-points showed much higher confidence levels (CI $>95 \%)$. The rate of decline in infant mortality rate was more remarkable during 1911-78. During this period, infant mortality rate dropped by 157 per 1000 live births. This pronounced change in IMR is majorly attributed to progress against communicable diseases such as dysentery, tetanus, polio, leprosy and absence of major famines in the post independence period (Dyson, 2004; Chandrasekhar, 1972; Karkal, 1985; Efron and Tibshirani, 1993).

Figure 3.1. Change point analyses of trend in infant mortality rate for India, 1901-2008

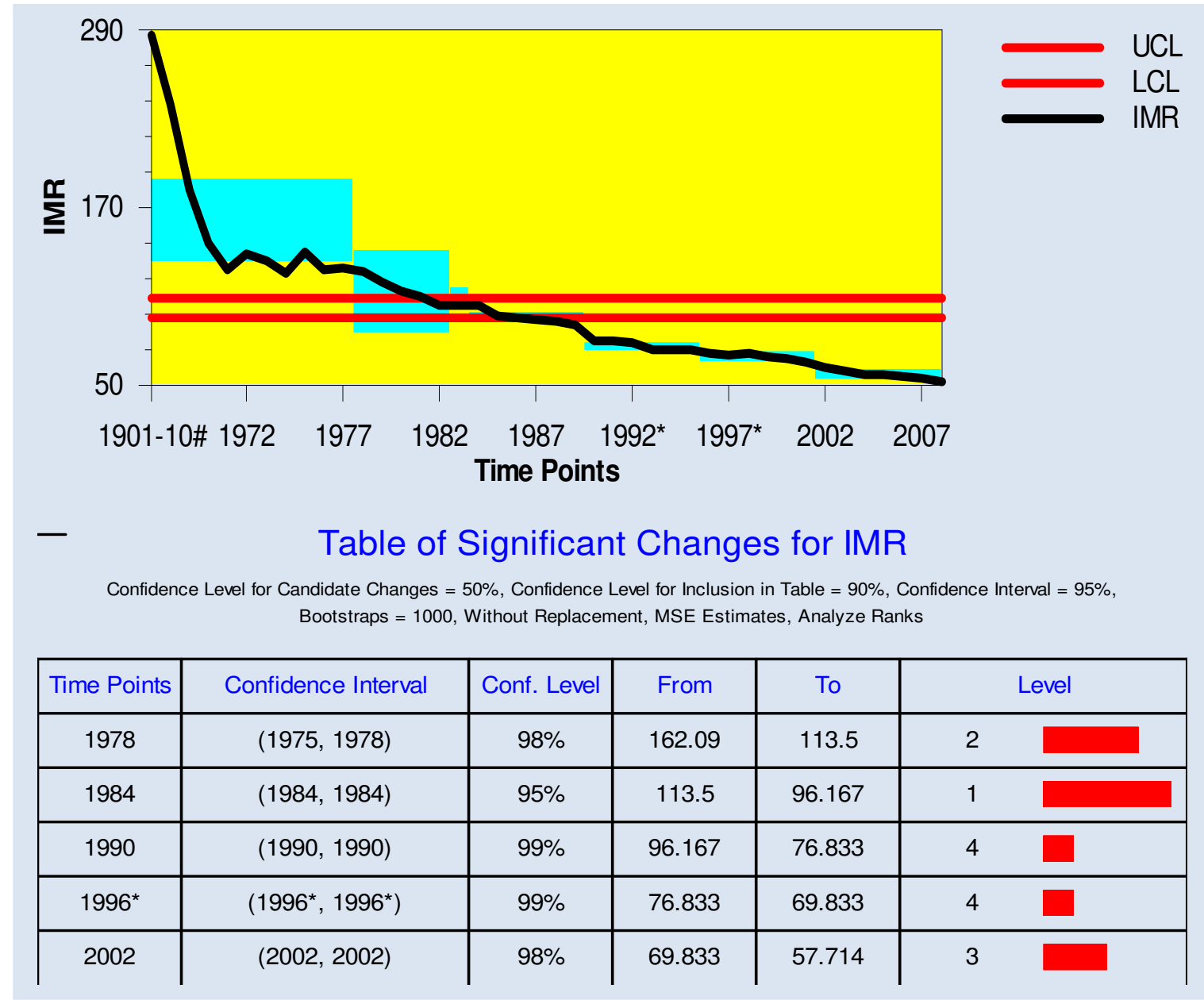

Note: 1. UCL- Upper side of Confidence Level; LCL- Lower side of Confidence Level

2. Level- Confidence Level

Source: 1. " Rele, 1987

2. Office of Registrar General of India, 1971-2008

3. * Excludes Jammu and Kashmir due to non-receipt of returns. 
Further, a careful observation of trends in short-term intervals indicates that there are ups and downs in the progress until 1977. Primarily due to this reason, the first critical change-point was appeared in 1978. However, more sustained critical changes in IMR that occurred in the year 1990, 1996 and 2002 were closely attributable to progress in socioeconomic conditions and steady improvement through wide-ranging mother and child health services. Commensurately, the government spending on maternal and child health programmes has also increased manifold during this period (Dyson, 2004; Chandrasekhar, 1972; Efron and Tibshirani, 1993; Dasgupta, 2005).

\subsubsection{Population size}

India is currently the second most populous and not too far to overtake China soon to become the most populous country in the world. Of the four principal components that this study addressed in assessing the long-term trends, the eventual but a critical component of the demographic transition following mortality change is the natural increase in population size. Persistent decline in infant mortality rate in the early stages of high fertility accelerates the natural increase in population size. To find critical change-points in the trends of population size of India, this study performed the change-point analyses of historical trends in population size. The population size estimates of India mainly come from decadal census counts of the population. The history of the Census began in 1800 when England had begun its Census, but the population of dependencies was not known at that time. In its continuation based on this methodology, a census was conducted in some towns and provinces of British India. Though, the first census of India began in 1871 but the modern census started in 1881. Since then, the Census of India has provided uninterrupted counts of population for every ten years (Chandrasekhar, 1972; Bhat et al., 1984; Bhat, 1989; Office of Registrar General of India, 1971-2005; Dyson, 2010). In trend analysis, however, the ten year period is rather a larger interval to assess the change-points in its entirety. It is possible that there may be changes within the ten year duration. Hence, to get a fairly good picture of change-points, I have used population size estimates of five year interval based on Census population counts (RBI (1955-2011). Similarly, Mukherjee (1969) provided population size estimates for five year intervals for pre-independence India. 
The trend assessment of population size during the 1856-2011, through 'Change-Point Analyzer' show that, in a period of 155 years, India experienced critical changes in its population size at four time points: 1890, 1930, 1960-61, 1990-91 (figure 3.2). All the four change points, however, conveniently fall in census years. The change point estimates were statistically significant with above 95 per cent confidence levels, but estimates in the years, 1890 and 1930 showed the greater confidence level (CI=100\%) compared to 1960-61 (CI=99\%) and 1990-61 (CI=96\%). A greater volume of change in population size (432 million) has been observed in 1990-91, followed by a change of 254 million in 1960-61. This is obvious as last two change-points were observed in the phase of India's rapid population growth (1951-1991) whereas 1890 represented the stagnant phase of population growth (prior to 1921) and 1930 was the phase of steady increase in population growth during 1921-1951 (Ghosh, 1956; Dyson, 1989; Dyson, 2004).

Figure 3.2. Change point analyses of trend in population size for India, 1856-2011

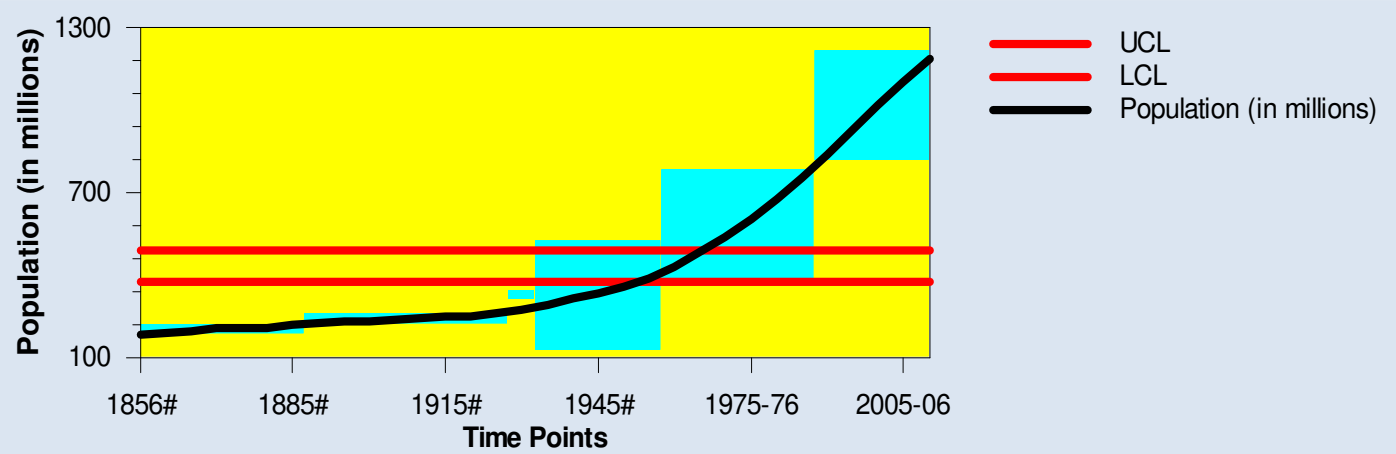

- Table of Significant Changes for Population (in millions)

Confidence Level for Candidate Changes $=50 \%$, Confidence Level for Inclusion in Table $=90 \%$, Confidence Interval $=95 \%$, Bootstraps $=1000$, Without Replacement, MSE Estimates, Analyze Ranks

\begin{tabular}{|c|c|c|c|c|c|}
\hline Time Points & Confidence Interval & Conf. Level & From & To & \multicolumn{2}{|c|}{ Level } \\
\hline $1890 \#$ & $(1890 \#, 1890 \#)$ & $100 \%$ & 204.14 & 244.62 & 2 \\
\hline $1930 \#$ & $(1925 \#, 1930 \#)$ & $100 \%$ & 244.62 & 329.67 & 3 \\
\hline $1960-61$ & $(1960-61,1960-61)$ & $99 \%$ & 329.67 & 583.5 & 3 \\
\hline $1990-91$ & $(1985-86,1990-91)$ & $96 \%$ & 583.5 & 1015.6 & 3
\end{tabular}

Note: 1. UCL- Upper side of Confidence Level; LCL- Lower side of Confidence Level

2. Level- Confidence Level

3. \# Estimates based on Mukherjee (1969)

Available literature (Davis, 1951; Ghosh, 1956; Mukherjee, 1969; Mahalanobis and Bhattacharya, 1976) advances a variety of plausible explanations that may be 
attributable to these four time points as a critical change point in population growth trends. The year, 1890 as a critical change-point in population size is likely due to changes in the procedures of estimation of population of India. India underwent first modern Census in 1881 for counting population size. The improved counting of the population in 1881 provided a fair chance of having a critical change in population size of India compared to its earlier estimates. Secondly, it is again obvious to notice that 1930 as a critical change point in the trends of population size in India as there was an important event which set as a crossroads for the preceding period of 1921. The year 1921 was called the year of the 'Great Divide' because it distinguished the earlier period of chequered population growth from the period of moderately increasing growth. Thirty years prior to 1921, the varying fortunes of mortality levels were responsible for variation in the growth rates of the population. The decade 1901-11 witnessed several local famines and Plague, Influenza and Malaria epidemic which caused the death of an estimated 7 percent of the total population of India (Dyson, 1989; Office of Registrar General of India, 1971-2005; Mukherjee, 1969). Consequently, the growth of the Indian population was negative during the period of 1901-1911. However, in the succeeding decade (1921-31), following recovery from famines and epidemics, India experienced 28 million or more than one and a half times increase in population. The 1921 sowed the seeds of future phenomenal and accelerating growth that was witnessed during 1921-31. An accumulative growth in India's population size was resulted in a critical change-point in 1930. The rapid growth of population in the post-1950 following a steady mortality decline was might be responsible for a critical-change point in population size during 1960-61. Several researchers affirm that this growth was majorly driven by fall of death rate (Dyson, 1989; Bhat and Rajan, 1997). The estimates of the average birth and death rates in India show that while death rates have sharply declined in each successive decade up to 1951, birth rates have virtually remained unchanged up to 1961 (Mahalanobis and Bhattacharya, 1976; Bhat, 1989; Office of Registrar General of India, 1971-2005). This period represented the inevitably long demographic lag period between the onset of mortality decline in 1920 and the onset of fertility decline in the 1960s. However, during the decade of 1981-91, the population of India increased by 24 percent and the average annual exponential growth rate peaked 2.14. This has led to a phenomenal change in population size during 1990-91 rendering this as one of the critical change- 
point in the history of India's population growth. The considerable decline in mortality may be attributed to increase in the population size in this period.

\subsubsection{TFR}

While the onset of infant mortality began post-1921, but the onset of fertility decline occurred in 1965. After a lag of 45 years, fertility in India has declined and the pace of decline has accelerated from 1980s and consequently the country is currently passing through the third stage of fertility transition. Given the scale and diversity of India's population and the provision of voluntary choice in family size norm, a decline from around six births per women in 1970 to less than half that level within a span of 30-40 years is a significant achievement (Preston, 1980; Rele, 1987; Bhat, 1989; Guilmoto and Rajan, 2001; Visaria and Visaria, 1994; Kulkarni and Alagarajan, 2005). However, changes in fertility across the time scale are not uniform. The decline in fertility has been highly sensitive to mortality scenarios, population-health policies and programme shifts (Preston, 1980; Rele, 1987; Visaria and Visaria, 1994; Kulkarni and Alagarajan, 2005). In general, the previous literature indicates that the year 1965-70 is determined as the period of onset of fertility decline in India. However, none of the above studies have tried to determine multiple critical changes that marked the long-term fertility trends of India based on more sophisticated tools of trend assessment. In this study, I have not only assessed critical change-points in long-term fertility trends, but also presented plausible reasons for such changes during that particular time point. Figure 3.3 presents 'Change-point analysis' trend line plot and estimates of critical changepoints for fertility rates during 1951-2009. The assessment of the trend line plot and estimate of change-points indicated that India experienced five critical change-points (1976, 1985, 1991, 1998 and 2008) during the past half a century of fertility trend. The first critical change in Indian fertility trend was observed in 1976; during this year, fertility declined by almost 20 percent from a TFR of 5.4 to 4.5 per women with a confidence level of 100 percent. The year 1976 clearly marked a turning point in India's fertility trends because fertility remained more or less stable (or even to have increased) during the initial period until 1961-66. The estimate for the period, 1966-71 marked the true beginning of fertility decline. The onset of India's fertility decline in 1961-66 also appears to coincide with a major change in the Indian family planning programme from a clinic-based approach to the extension approach in the late 1963. 
Additionally, the establishment of a full-fledged department of Family Planning in the Ministry of Health and Family Planning in 1966 was deemed to be an important step to pursue predetermined goals of fertility decline.

Figure 3.3. Change point analyses of trend in total fertility rate for India, 1951-2009

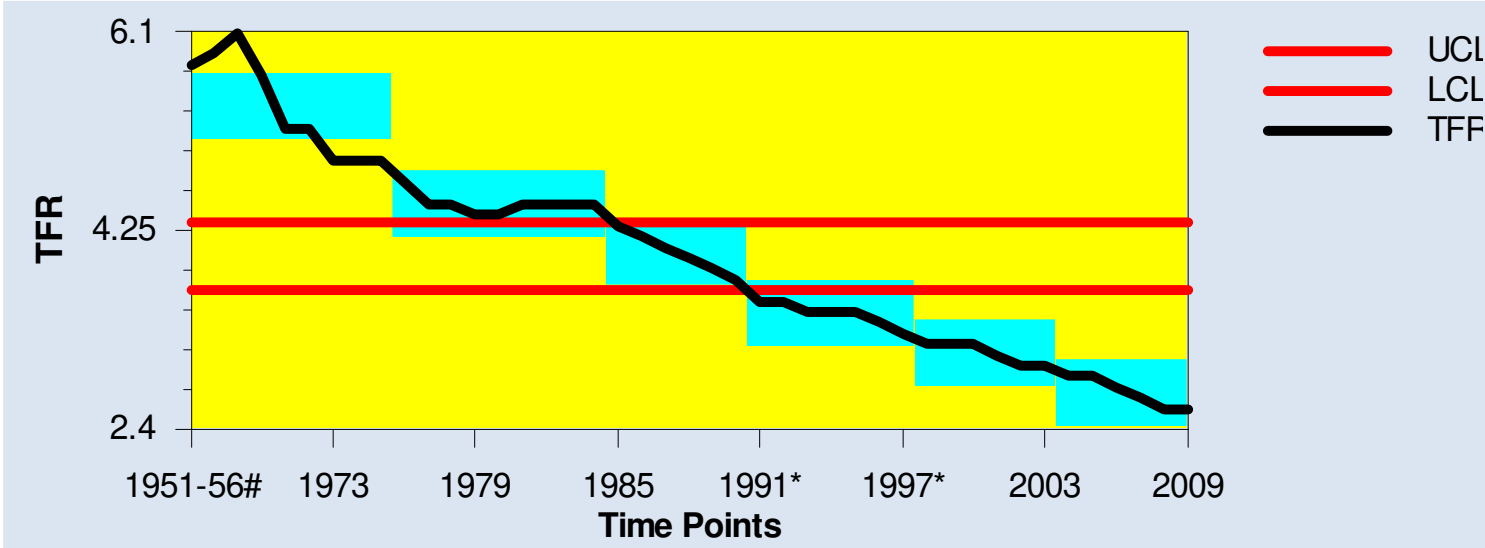

Table of Significant Changes for TFR
Confidence Level for Candidate Changes = 50\%, Confidence Level for Inclusion in Table = 90\%, Confidence Interval = $95 \%$,
Bootstraps = 1000, Without Replacement, MSE Estimates, Analyze Ranks
\begin{tabular}{|c|c|c|c|c|c}
\hline Time Points & Confidence Interval & Conf. Level & From & To & Level \\
\hline 1976 & $(1976,1976)$ & $100 \%$ & 5.4 & 4.5 & 2 \\
\hline 1985 & $(1983,1985)$ & $100 \%$ & 4.5 & 4.05 & 3 \\
\hline $1991^{*}$ & $\left(1991^{*}, 1991^{*}\right)$ & $99 \%$ & 4.05 & 3.4857 & 3 \\
\hline 1998 & $\left(1997^{*}, 1998\right)$ & $97 \%$ & 3.4857 & 3.1167 & 2 \\
\hline 2004 & $(2004,2004)$ & $99 \%$ & 3.1167 & 2.75 & 3 \\
\hline
\end{tabular}

Note: 1. UCL- Upper side of Confidence Level; LCL- Lower side of Confidence Level

2. Level- Confidence Level

3. * Excludes Jammu and Kashmir due to non-receipt of returns.

Source: 1. "Rele, 1987

2. Office of Registrar General of India, 1971-2005

The establishment of the department of family planning was accompanied by a substantial increase in total expenditure for India's family planning programme. The objective was to make family planning programme services widely and easily accessible and over time a variety of incentives were introduced and offered to acceptors of family planning programme as part of the strategy to voluntarily promote family planning and fertility control. Consequently, fertility decline accelerated with an estimated TFR of 5.78 in 1966-71, 5.37 in 1971-76, and 4.65 in 1976-81. A decline of about one child per woman during the decade of 1971-81, or to refer to the midpoints of 
this period, approximately 1975-76, the year 1976 represents the first critical changepoint of fertility trend in India.

The most notable change-point in fertility trends in terms of greater volume of decline in TFR were observed in 1991. At this point of time, India experienced a decline of TFR from 4.05 to 3.48, which was the highest volume of TFR decline among all the observed change-points. The greatest decline of TFR in 1991 is closely associated with the shift in family planning and target oriented family welfare goals during the late 1970s and 1980s. However, the volume of TFR declined in other two observed changepoints (1998 and 2004) was also substantial. Change-point estimates in figure 3.3 showed greater statistical confidence levels for all the five change-points. However, the decline in TFR during the post-1990s and an observed critical change point in 1998 are more likely to be the result of a change in socioeconomic status in the post-economic reform period. The recent change-point, in 2004 can be stated as the result of sizeable changes in fertility rates of traditionally high fertility states such as the Empowered Action Group (EAG) states on account of more focused and intense fertility reduction policy drive in these states and widespread diffusion of small family norms and contraception use among uneducated women. Studies have demonstrated that since the 1990s and through the period of 2000s, India's fertility decline has been driven by major fertility decline among the illiterate and poor women through widespread use of female sterilization (James and Nair, 2005). Second, complementary to this, since the year 2001, the government of India has made much bigger budget allocations to improve population and health indicator of EAG states, which majorly contributed to the decline in fertility rates in these states. Overall, the pro-women and child health programmes and the population stabilization policy drive have helped in accelerating the pace of fertility decline and which are cloning forces for convergence in national fertility levels (Preston, 1980; Visaria and Visaria, 1994; Kulkarni and Alagarajan, 2005).

\subsubsection{LEB}

LEB is the most widely used aggregate mortality measures in public health research (Srinivasan et al., 2007). In the Indian context, many recent studies on mortality in general and LEB in particular focused on the recent trends (post-1970s) are typically 
based on sample registration system data. Declining infant mortality rate has been considered as a direct consequence on the improvement in overall survival times of a given population (Bhat et al., 1984; Srinivasan et al., 2007). However, all such studies used simple trend line plots and charts as tools for analyses. With the exception of studies that dealt with analysis of mortality patterns and their determinants, no recent study examined the long-term historical trends in LEB. Regardless of the discrepancies in annual rates of LEB, however, in the long-run, on an average, LEB level in India has more than doubled since pre-Independence days (Bhat et al., 1984; Rele, 1987; Bhat, 1989; Office of Registrar General of India, 1971-2005). In search of historical trends in LEB, I have reconstructed the historical change-points in LEB and quantified the magnitude of change-points based on robust methodological tools.

Figure 3.4. Change point analyses of trend in life expectancy at birth for India, 18722011

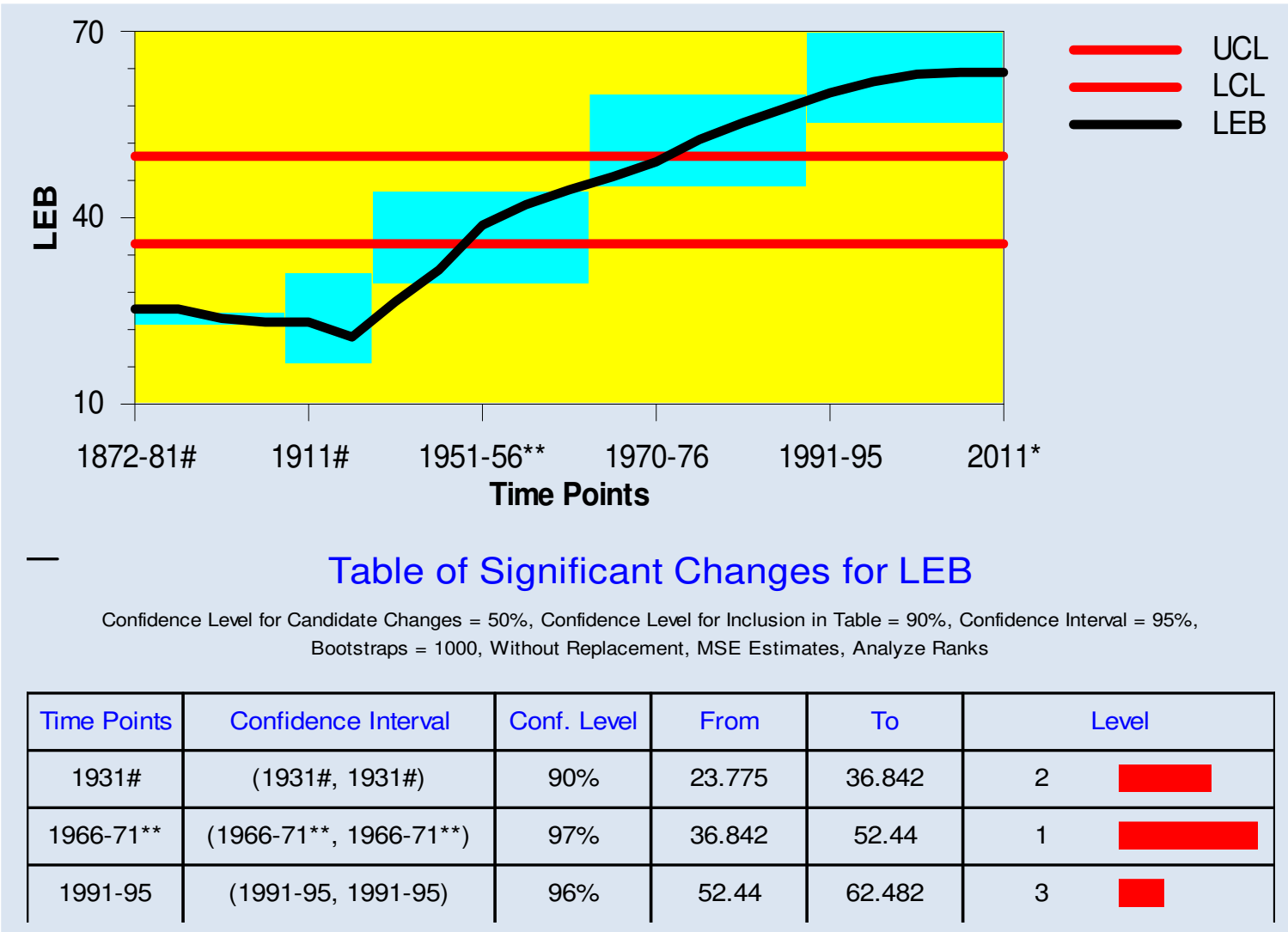

Note: 1. UCL- Upper side of Confidence Level; LCL- Lower side of Confidence Level 2. Level- Confidence Level

Source: 1. " Mitra, 2005 documented LEB estimates based on Registrar General of India and Census during 1872-1941. The estimates presented in the table are male female averages 2. ${ }^{* *}$ Rele, 1987

3. Office of Registrar General of India, 1970-2006

4. *UNDP, 2011 
Figure 3.4 shows change-point analyses for LEB trends during 1871-2011. The results unravel three critical change-points $(1931,1966-71,1991-95)$ for LEB over the period of 140 years. All the three change-points show high confidence levels (CI>90\%). However, the long-term trend in India revealed a greater change in LEB during 196671 with greater confidence level $(\mathrm{CI}>97 \%)$. The patterns of critical change-point in LEB are seen closely tied to epidemics and famines, medical and mortality scenarios and major changes in socioeconomic conditions in India. The critical change in LEB in 1931 was mainly due to the spectacular progress in food supplies and emerging chronic food shortage, health care provision to control epidemics and communicable diseases after dreadful famines during 1901-11. Thereafter, it was followed by a recovery period. The graphical presentation of trend line in figure 3.4 also shows that the rate of decline in LEB in the post-1931 period slowed down after 1950, but regained progress since 1960 .

The progress following slowdown is consistent with the swift reduction in infant mortality rate and further reductions in adult mortality rates in the post independence period. The accelerated increase in LEB in the post-1960 marked a critical change-point in 1966-71. This is the period where India have experienced sustained decline in death rates that lead to pronounced increases in the LEB. However, the major changes in LEB in 1991-95 are attributed to fall in IMR, improvement in socioeconomic and health status which further lengthened the life span in India. The death rate in India declined significantly from 27 per thousand in 1941-50 to 11 per 1000 population in 1986-91. During this period, India improved in terms of maternal and child health which may be greatly contributed to increase in the LEB.

\subsection{Discussion}

Although, demographic transition is quite a familiar idea during the past half century period, it was regarded more an abstraction than a description of the true trajectory of population and health indicators because demographic statistics hardly go back to the start of the cycle and there is no certainty that when the end of it will be achieved in rural areas of developing India. According to the current demographic scenario, it is tough to predict the conclusion stage of demographic transition in India. However, over the past half century, the progress in fertility and mortality decline in India is 
remarkable (Rele, 1987; Bhat, 1989; Visaria, 2004b; Office of Registrar General of India, 1971-2005; McNay et al., 2003; Visaria, 2011). Several past studies, which examined the process of demographic transition in India recognized very few major shifts and concluded that overall demographic trends in India are transitioning from third to the fourth stage of demographic transition (Visaria and Visaria, 1994; James, 1995; Bhat and Rajan, 1997; Dyson, 2004; Guilmoto and Rajan, 2001; Visaria and Visaria, 2003; Visaria, 2004b; Kulkarni and Alagarajan, 2005; Kulkarni, 2011; Visaria, 2011).

In view of considerable analytical limitations of such previous studies, this study advanced the 'change-point analyses' as a new methodological tool for the assessment of progress and changes in population and health indicators for the larger goal of understanding the true trajectories of population and health transition in India. The findings of this study foster that the trajectory of long-term demographic trends in India, in four key demographic indicators, resulted in several major shifts. 'Changepoint analyses' for IMR, Population size, TFR and LEB revealed multiple critical changes over a long-run period of demographic history in India. The shifts in IMR and TFR trends are closely associated with strategic shifts in the approach of the family welfare programme in India. However, the critical change-points observed in preindependence period for Population size and LEB are majorly attributed to fluctuations in these indicators that emerged out of famines, several communicable diseases and epidemics. A sustainable and the continued demographic transition was initiated during the mid - 1960s. Therefore, the causal linkages among four major components of demographic transition were interpreted only for the trends observed in post-1965.

In the Indian context, this study fosters that transition in fertility is initially followed by infant mortality. As evident from our trend line graphs, the infant mortality steeply declined ever since 1921 but fertility transition was initiated in post-1965. The first critical change-point in fertility emerged two years before the infant mortality is basically attributed to 1) Though, the volume of infant mortality decline during a larger interval (1921-1977) was high, but ups and downs in the progress in short-intervals delayed the emergence of first critical change-point in infant mortality until 1978. 2) On the other hand, targeted and forced family planning programme during this period facilitated advantage for a sustained decline in TFR. However, in the later periods, the 
other critical change-points in infant mortality appeared earlier than critical changepoints in fertility. Again, this is due to a sustained decline in infant mortality in post1978 and change in approach of family planning programme in a later phase of 1975. Overall, the improvement in socioeconomic conditions, family planning and maternal and child health services are the driving force of the decline in child mortality and fertility after mid-1970s. The trends in population size showed that most critical change-point in 1990-91. It took hundreds of years to reach first 500 million populations, however, the second 500 million populations were added to the total population size (1186 million) in just 40 years (from 1971 to 2011). A critical change in LEB during 1991-95 is clearly attributable to decline in mortality rates during 19701990 with improved health care provisions and socioeconomic conditions.

Taken as a whole, this chapter advanced a number of critical insights on demographic transitional change-points connecting it to contextual policy shifts in Indian context. The evolutionary construction and interpretation of long-run demographic trends and the vital demographic change-points in this study have certainly enhanced our understanding of trends of population and health indicators vis-a-vis population and health policy shifts. Critical change-points in population and health indicators in India are associated with the evolution of structural changes in population and health policy framework. The results have not only helped in reassessing the historical past and the current demographic transition trajectory, but also advanced a new statistical tool for assessing the demographic trends which are necessary for robust monitoring of the progress in population and health trends. There are no specific limitations with a change-point analyzer tool. However, it will provide the best results with data of a long period of time. Nevertheless, this study has a limitation in terms of data used: 1) in India, we do not have a long term data from a single source of official statistics. Therefore, i am compelled to use multiple data sources for this study. 2) The demographic estimates before the 1950s were mostly based on indirect estimates. 


\section{CHAPTER 4 \\ Socioeconomic Convergence}

\subsection{Introduction}

In a Global context, the latest Human Development Report (2013) present that over the last decade, all countries have accelerated their achievements in non-income dimensions of human development: the education and health dimensions as measured in the Human Development Index (HDI) - indicated convergence. As faster progress was recorded in lower HDI countries during this period, although progress was uneven within and between regions. Looking specifically at countries which lifted their HDI value substantially between 1990 and 2012 for non-income dimensions of human development, it is a remarkable achievement.

In India, pronounced economic disparities among states received substantial attention of researchers and policy makers during the post independence period, (e.g. Mishra, 1969; Rao, 1984; Dholakia, 1985; Pradhan, 1988; Choudary, 1992, 1993; Dholakia, 1994; Ghosh et al., 1998; Kurian, 2000; Ahluwalia, 2002; Trivedi, 2002; Dholakia and Ravindra, 2003; Bhattacharya et al., 2004; Jha, 2004; Dreze' and Khera, 2012). Since 1951, balanced regional development has remained one of the objectives of the planning process in India (Kurian, 2007). This is evident from the emphasis given to regional disparities in successive five year plans after independence. Many attempts have been made to address the India's regional imbalances through institutions like the Planning Commission and Finance Commission. For example, in case of financial transfers from the centre to the states, the poorer states have been given priority over the richer states. Direct investment by the centre in the development of public infrastructure in poorer states is another effort towards balance in development (Das, 1993; Ghosh et al., 1998; Kurian, 2007). There is no doubt in the fact that despite these efforts, substantial regional inequalities in socioeconomic development have persisted (Dreze' and Khera, 2012).

With the introduction of economic reforms in the early 1990s, Indian economy witnessed a major shift in economic policies (Sarkar, 1994, 1995). Subsequently, in the last two decades, even though India has experienced high economic growth rates, the 
economic growth has not been uniform across the states. Some studies show that, in the post reform period, India has experienced a sharp increase in regional inequalities (Nair, 2004; Pal and Ghosh, 2006; Jha, 2004). For instance, Pal and Ghosh (2006) in 2002-2003, pointed out that per capita Net State Domestic Product (NSDP) of the richest state, Punjab, was about 4.7 times higher than that of Bihar, the poorest state. This ratio had increased from 4.2 in 1993-1994 to 4.7 in 2002-2003. These regional inequalities are limited not only to the domain of economic indicators, but have also been reflected in the uneven human development among the states during this period. The recent Indian Human Development Report-2011 has shown that the ranking of the states in terms of HDI has not changed much between 1999-2000 and 2007-08. The report points out that, the well-performing states like Kerala, Delhi, Himachal Pradesh, Goa, and Punjab have occupied the first five ranks in both 1999-2000 and 2007-08. On the other hand, Bihar, Jharkhand, Madhya Pradesh, Uttar Pradesh, Odisha, Rajasthan, and Chhattisgarh appear at the bottom of the list in both these years (Institute of Applied Manpower Research, 2011). Moreover, Kurian (2007) warns that if the existing trend in differential rates of socioeconomic development continues, then it will not only accentuate, but also will have serious adverse implications for the Indian economy, society and polity.

\subsection{Concept of unequal regional progress and approaches}

Outside demography, several large-scale theories predict socioeconomic convergence. Marxist analyses hold convergence to be more or less historically inevitable as nations develop along defined tracks only temporarily diverted by the contradictions of capitalism from the uniform certainties of the future workers' state (Fukuyama, 1992). Thus, socioeconomic space is often contentious and debatable (Hall, 1992; Parr, 1999). How do regions grow? Why do some regions/states grow more rapidly than others? Why are disparities in levels of economic-social welfare across regions/states so persistent? These critical questions have attracted the attention of a diverse group of social scientists (see for e.g. Perroux, 1950; Friedmann, 1956, 1964; Kuznets, 1955; Myrdal, 1957; Hirschman, 1958; Williamson, 1965; Hoover, 1971; Lipton, 1977; Krugman, 1979, 1991a, 1991b; Barro, 1995; Quah, 1993; Barro and Sala-I-Martin, 1995; Sala-I-Martin, 1996). Previous studies suggest that unequal regional progress emerge from the concentration of income in the hands of a few who prefer to direct 
them in already developed areas or near growth poles (Perroux, 1950; Kuznets, 1955; Myrdal, 1957; Friedmann, 1956; Hirschman, 1958; Romer, 1986). The growth-pole strategy implies the channelling of investment to a limited number of locations to boost economic activity and which in turn leads to increased economic growth within and outside these regions (Perroux, 1950; Parr, 1999).

Following Kuznets (1955), various scholars argue that regional inequalities follow inverted U-shaped curve (Fujita et al., 1999; Lucas, 2000; Ottaviano and Theses, 2004). This implies that in the course of economic development, initially regional disparities increase and in the later period, these disparities will narrow down owing to urbanization and industrialization (Kuznets, 1955). Similarly, a number of scholars have emphasized on resource distribution in explaining the cause of regional disparities. The resources across the regions are not uniformly distributed. The propensities of different regions of a nation often differ because the regions are naturally endowed with different resources and usually have different historical, sociological and political backgrounds (Perroux, 1950; Hoover, 1971; Hall, 1992). Moreover, as a nation develops socio-economically, the different regions of the nation may or may not share the benefits of this socioeconomic development equally (Williamson, 1965). Several authors have built on this idea for regional analysis of economic growth and suggested that inequalities first rise as developed areas progress much faster compared to their counterparts. During the transition period, developed areas more benefit from external economies, political power, and labour mobility (Friedmann, 1956, 1964; Myrdal, 1957; Hirschman, 1958; Williamson, 1965; Hoover, 1971; Barro, 1991).

However, later this growth approach has been widely criticized by other scholars such as Darwent (1969), Lipton (1977), Sen (1983), Das (1993), Parr (1999), Kurian (2000). This group claims that the socioeconomic disparities across the regions are by and large, an outcome of the working of the socioeconomic and political system and its processes. They are also influenced by central and state socioeconomic policies and political systems. Hence, it is a matter of great interest to examine the manner in which inter-regional differences in the levels of socioeconomic development undergo change during the process of national economic development: whether such progress across sub-national level is converging or diverging is a pertinent research question. With this 
theoretical perspective in the background, this chapter examines the process of socioeconomic progress across the major states of India. In the first stage, this chapter reviewed the progress in socioeconomic status assessed by previous studies in India. In the second stage, it adopted an empirical approach to re-examine the socioeconomic progress by using convergence hypothesis and econometric convergence models.

\subsection{Previous evidence of socioeconomic progress and convergence among Indian states}

Though, there is no empirical evidence of demographic convergence in the past, but a number of attempts have been made to examine the regional imbalances in development in India. Most of these studies have tested convergence in economic growth across Indian states, covering different time periods, following Barro and SalaI-Martin (1992, 1995). This section briefly reviews these studies.

Dholakia (1994) analyzed the convergence of per capita Net State Domestic Product (PCNSDP) growth rates across 20 Indian states during the period 1960-61 to 1989-90. He observed the tendencies of convergence in long-term PCNSDP growth rates across the states. Cashin and Sahay (1996) also examined the convergence of per capita income for 20 states covering the period 1961-91. They found that there has been absolute $\beta$-Convergence of per capita income across the states during this period. On the contrary, Marjit et al. (1998) investigated the convergence in 'properly measured real PCNSDP' for the period 1960-61 to 1994-95 across 26 Indian states. They argued that Indian states diverged in economic growth during 1960-61 to 1994-95.

Nagraj et al. (1998) showed the existence of conditional convergence among 14 states during 1970-1994, but they ruled out the existence of any absolute convergence. They explained interstate variations in steady states in terms of variations in different factors like physical, economic and social infrastructure. Trivedi (2002) also reached a similar conclusion as Nagaraj et al (1997) and Aiyar (2001) by testing the convergence hypothesis of levels and growth rates of per capita income among 16 states during 1960-1992. He asserted that no evidence is found to support absolute $\beta$-Convergence in the growth rates, but confirmed that there is clear and robust evidence of conditional convergence. Rao et al. (1999) based on their analysis of interstate differences in 
growth rates during 1965-1995 among 14 major states conclude that interstate disparities are increasing rather than converging. These interstate disparities in income are attributed to the differential capacity of the states in attracting private investment and further it is pointed out that the allocation of private investment is determined by the availability of better infrastructure in a state (Rao et al. 1999).

Particularly after the introduction of liberalization policies in the 1990s, different studies have sought to understand the impact of economic reforms on regional inequality and convergence among Indian states. Ahluwalia (2002), using a population weighted Gini coefficient framework, analysed interstate inequalities during the 1990s. He found that inequality in real per capita regional output increased from 0.175 in 1991-1992 to 0.233 in 1998-1999 among 14 major states. He argued that the variation in the private investment across the states is responsible for cross-sectional differences in states' growth rates. Bhattacharya and Sakthivel (2004) analyzed interstate income disparities before and after the reform period. They showed that interstate income disparities almost remained unchanged during 1980s, but sharply increased during the 1990s. Kar and Sakthivel (2007) argued that regional inequality remained stable without much increase during the 1980 s due to a fall in inequality within the industrial and the service sectors during this period. Further, rise in regional inequality in the post-1990s is attributed to a sharp rise in the disparity in the industrial and service sectors' progress across the states. Another study by Ghosh (2011) also confirmed an increase in the interstate inequality of per capita income in the post-reform period since 1991 .

The review of the above studies suggests that the study of regional inequalities has attracted considerable interest from social scientists in India. However, most of these studies have emphasised convergence or divergence of per capita NSDP across the sates by assuming per capita income as a measure of standard of living of the people. Per capita income may not be a true indicator of socioeconomic well-being (Sen, 1998). Sen (1998) observed that economic variables like per capita income can be considered as an important indicator of development, but they may not be adequate to capture the standard of living or quality of life of the people. Therefore, I believe that, a more meaningful way of testing the convergence in the regional imbalances is the analyses of 
convergence in terms of standard of living by using multiple socioeconomic indicators across states over time.

Though, substantial empirical literature has dealt with interstate disparities in India, only few studies have examined the regional disparities based on multiple socioeconomic indicators across the states. Dholakia (2003) examined the trends in regional disparity in India in the average per capita NSDP and human development during 1977-80 to 1997-2000. He found that while per capita NSDP inequality does not show any significant decline, but there was a decline in inequality in the human development index among 20 major states. Ghosh (2006) examined the convergence of 15 major Indian states of human development during 1981-2001. He found that evidence for regional convergence in human development exists, despite considerable divergence in per capita income among the states. Also, the Indian Human Development Report-2011 maintains that during 1999-2000 to 2007-8 the increase in HDI in the states that are among the poorest has been much faster than the national average, and hence there is a convergence taking place between states in terms of HDI (Institute of Applied Manpower Research, 2011). However, the Indian Human Development Report-2011 does not test the convergence hypothesis for HDI among Indian states.

Findings from some of the recent studies, which have examined interstate regional disparities in social indicators, cast doubts over convergence in levels of human development across the states as pointed out by Dholakia (2003), Ghosh (2006) and IHDR (2011). Drèze and Khera (2012), based on their study on child and human development deprivation at district level, observed that still substantial gap exists in HDI of states like Bihar, Madhya Pradesh, Rajasthan, Uttar Pradesh and Odisha and the rest of the states in India. In this context, this chapter tries to complement the existing studies in understanding regional inequalities and convergence not only when measured in terms of per capita NSDP, but also in terms of a broader range of socioeconomic indicators by evaluating the performance of Indian states in socioeconomic indicators during the period of 1971-2010. 


\subsection{Data and Methods}

This chapter assesses more recent trends rather than long-term trends in socioeconomic progress vis-à-vis convergence or divergence among the states, as this period represents the most critical phase of India's socioeconomic progress. Many previous studies have demonstrated economic reforms as a critical change-point in the analyses of trends in socioeconomic conditions of India. Therefore, this chapter examines data beginning a decade before the economic reforms of the latest available statistics on various socioeconomic indicators from multiple data sources. Though, a general description of the data has been presented in Chapter 2: Methodology, a detailed description of the data used especially in this chapter is described here. Net State Domestic Per Capita Income (NSDP) for major states was taken from Reserve Bank of India (RBI, 19812010). Literacy rates were compiled from Census of India, 1981-2011 (Office of Registrar General, India 1981- 2011). The Poverty ratios were based on Planning Commission estimates of poverty, 1973-2006 (Government of India, 1973-2006). Human Development Index (HDI) estimates for major states of India during 1981-2001 were taken from Planning Commission (Government of India, 1981-2001). However, the latest HDI statistics for the states are obtained from UNDP India estimates (UNDP India, 2011). Gini index estimates of per capita consumption expenditure for major states were taken from Planning Commission reports (Government of India 1973-2006. Data on Total Fertility Rate (TFR) Infant Mortality rate (IMR) and Life Expectancy at Birth (LEB) were taken from the sample registration system (Office of Registrar General, India, 1981-2006).

This chapter used analyses of trends and catching-up process in the first stage. In the second stage, for determining convergence process in socioeconomic indicators, this chapter not only used parametric methods, but also employs nonparametric methods to overcome the assumption of linearity, normal distribution in the growth regression. This chapter also takes into account absolute and relative distributions. The convergence analyses of this study comprise three parts: 1) examination of convergence processes using standard parametric econometric models of convergence such as $\beta$ convergence and Sigma $(\sigma)$ Convergence, 2) testing non-parametric econometric models such as Kernel Density estimates, and 3) assessment of inequality adjusted convergence. For further details, refer to methodology chapter. 


\subsection{Results}

\subsubsection{Descriptive statistics}

Table 4.1 presents the descriptive statistics of socioeconomic indicators selected for this study. Sample sizes (observations) of all selected socioeconomic indicators are 15. The results show multiple increases in mean per capita net state domestic product during 1981-2010. However, in the same period, the standard deviation and range of income distribution across the 15 major states has also increased multiple times. The mean poverty ratio of the 15 states has declined by half in a period of 31 years. Minimum poverty ratios across the states were also declined, but the decline in the maximum poverty ratio was not impressive. Moreover, the standard deviation and range of poverty ratios across the states has increased. The average literacy rates of the states have increased considerably. The standard deviation and range of literacy rates across the states has decreased during 1981-2011. However, the average Gini index values in consumption expenditure increased over the given period. Further, the standard deviation and range in Gini index values across the states have also increased considerably. In case of TFR and IMR, both average and standard deviation across the states have decreased during 1981-2009. The average life expectancy at birth increased, standard deviation and range across the states have decreased during 1981-2006. The trends in average HDI of the 15 major states show a remarkable increase and standard deviation and range show a considerable decline during 1981-2006. Overall, along with the considerable progress in the average NSDP, poverty and life expectancy, the standard deviations and the Gini Index values have also increased.

Table 4.1. Descriptive statistics of socioeconomic indicators in 15 major states of India.

\begin{tabular}{|c|c|c|c|c|c|c|}
\hline Variable & Observations & Mean & $\begin{array}{c}\text { Standard } \\
\text { Deviatio } \\
n\end{array}$ & Minimum & Maximum & Range \\
\hline Per capita NSDP_1981 & 15 & 1631.4 & 507.18 & 917 & 2674 & 1757 \\
\hline Per capita NSDP_1991 & 15 & 2204.86 & 793.61 & 1197 & 3730 & 2533 \\
\hline Per capita NSDP_2001 & 15 & 16183.07 & 5670.07 & 6554 & 25986 & 19432 \\
\hline Per capita NSDP_2010 & 15 & 34539.93 & 14777.34 & 11799 & 57458 & 45659 \\
\hline Poverty ratios_1974 & & 51.23 & 9.1904 & 28.1 & 61.5 & 33.4 \\
\hline Poverty ratios_1984 & 15 & 40.75 & 11.68 & 18.5 & 58.4 & 39.9 \\
\hline Poverty ratios_1994 & 15 & 32.07 & 9.69 & 11.7 & 46.4 & 34.7 \\
\hline Poverty ratios_2000 & 15 & 24.02 & 12.57 & 6.16 & 47.15 & 40.99 \\
\hline Poverty ratios_2005 & 15 & 24.75 & 10.81 & 8.1 & 45.6 & 37.5 \\
\hline Literacy rates_1981 & 15 & 43.57 & 12.64 & 28.37 & 78.85 & 50.48 \\
\hline Literacy rates_1991 & 15 & 54.38 & 13.15 & 38.48 & 89.81 & 51.33 \\
\hline
\end{tabular}




\begin{tabular}{lcccccc}
\hline Literacy rates_2001 & 15 & 67.03 & 9.68 & 47.53 & 90.92 & 43.39 \\
Literacy rates_2011 & 15 & 75.19 & 7.42 & 63.82 & 93.91 & 30.09 \\
Gini index_1983 & 15 & .3106 & .0312 & .25 & .36 & 0.11 \\
Gini inde_1994 & 15 & .3000 & .03722 & .22 & .38 & 0.16 \\
Gini index_2000 & 15 & .3206 & .0413 & .23 & .39 & 0.16 \\
Gini index_2006 & 15 & .3360 & .04610 & .24 & .39 & 0.15 \\
TFR_1981 & & & & & & \\
TFR_1991 & 15 & 4.34 & .8683 & 2.8 & 5.8 & 3 \\
TFR_2001 & 15 & 3.46 & .9146 & 1.8 & 5.1 & 3.3 \\
TFR_2009 & 15 & 2.94 & .8666 & 1.8 & 4.5 & 2.7 \\
& 15 & 2.48 & .7398 & 1.7 & 3.9 & 2.2 \\
IMR_1981 & & & & & & \\
IMR_1991 & 15 & 100.66 & 29.58 & 37 & 150 & 113 \\
IMR_2001 & 15 & 74.06 & 25.83 & 16 & 124 & 108 \\
IMR_2009 & 15 & 62.26 & 20.10 & 11 & 91 & 80 \\
& 15 & 49.466 & 16.67 & 12 & 70 & 58 \\
LEB_1981 & & & & & & \\
LEB_1991 & 15 & 57.13 & 4.92 & 50 & 68 & 18 \\
LEB_2001 & 15 & 61.46 & 4.68 & 55 & 73 & 18 \\
LEB_2006 & 15 & 63.93 & 4.30 & 58 & 74 & 16 \\
HDI_1981 & 15 & 64.06 & 4.18 & 58 & 74 & 16 \\
HDI_1991 & & & & & & \\
HDI_2001 & 15 & .3226 & .0709 & .24 & .5 & 0.26 \\
HDI_2006 & 15 & .4033 & .0762 & .31 & .59 & 0.28 \\
\hline NOE & 15 & .4633 & .0756 & .37 & .64 & 0.27 \\
\hline
\end{tabular}

Note: NSDP-State Net Domestic Product; TFR- Total Fertility Rate; IMR-Infant Mortality Rate; LEBLife Expectancy at Birth; HDI- Human Development Index

\subsubsection{Trend analyses}

The assessment of trends in net state domestic product reveals that until 1990-91, the NSDP among the states are more or less same. However, there was a huge divergent trend in post-1991 period. States like Maharashtra, Haryana, Punjab, Gujarat and Tamil Nadu could achieve greater progress while states like Bihar, Uttar Pradesh, Madhya Pradesh and Orissa achieved very less progress. Thus, over the period 1981-2010, the gap between states in terms of NSDP has increased enormously. Though, the decline of poverty ratios across the states was universal during 1974-2005, but the interstate gap in-terms of absolute levels of poverty ratios remain same. However, a tremendous progress has been achieved in terms of literacy rates. The trend line plots show a clear evidence of closing the literacy gap across the states. Even among the states like Uttar Pradesh, Bihar and Madhya Pradesh, the progress was remarkable.

The trends in Gini index show an increase in gap across the major states. This index was calculated based on National Sample Survey Consumption expenditure data. The majority of the states showed an increase in Gini index values. The less developed 
states show lower levels of Gini value compared to developed counterparts. The state of Maharashtra showed highest inequality in consumption expenditure. Human Development Index, a composite measure of overall development also showed tremendous improvement over the period for all the major states. However, a comparison of the interstate gap between trend plots showed that though the gap was closing, but at a very slow pace (Figure 4.1).

Figure 4.1. Trends in selected socioeconomic indicators during 1971-2011

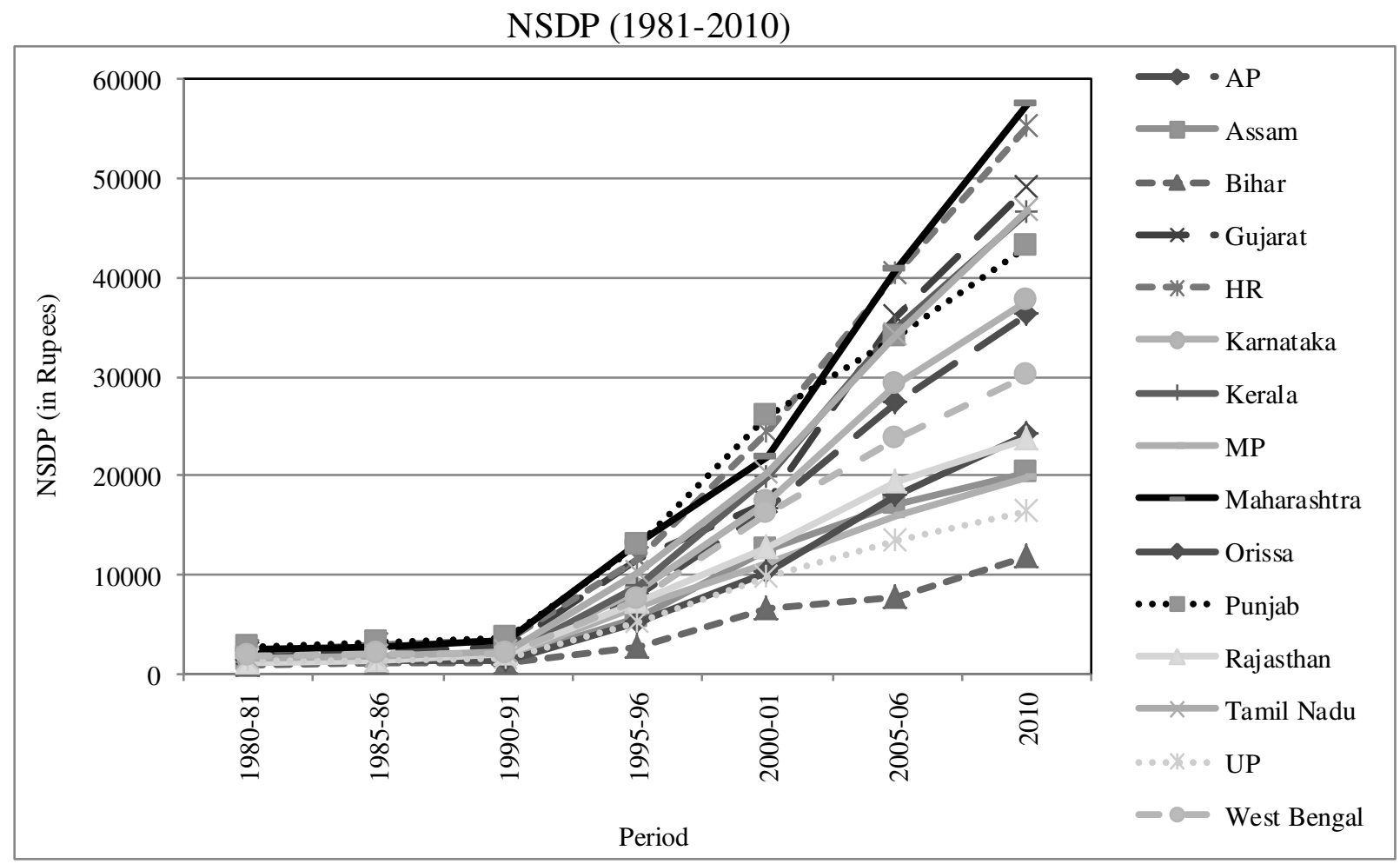


Poverty ratios 1974-2005

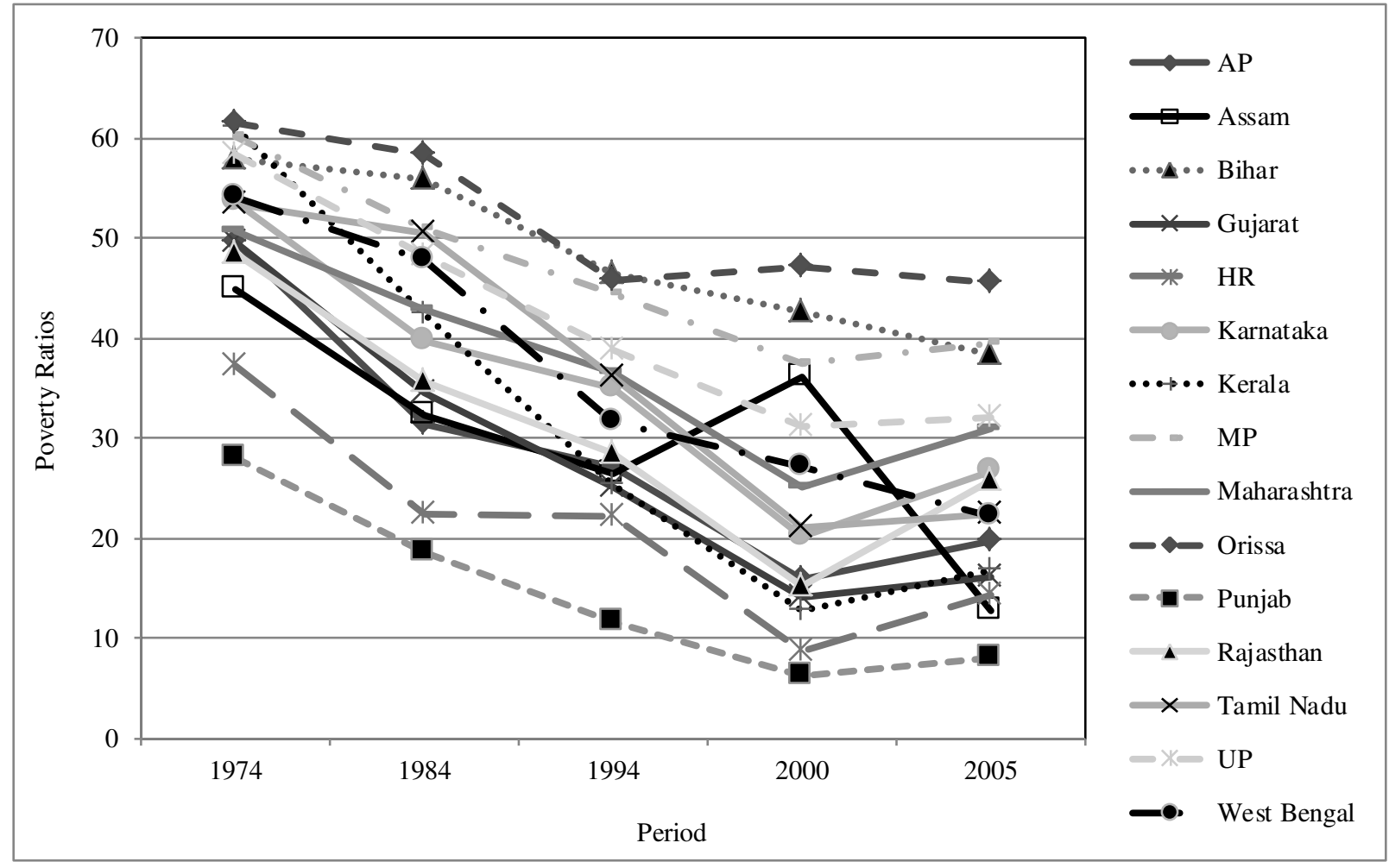

Literacy rates (1971-2011)

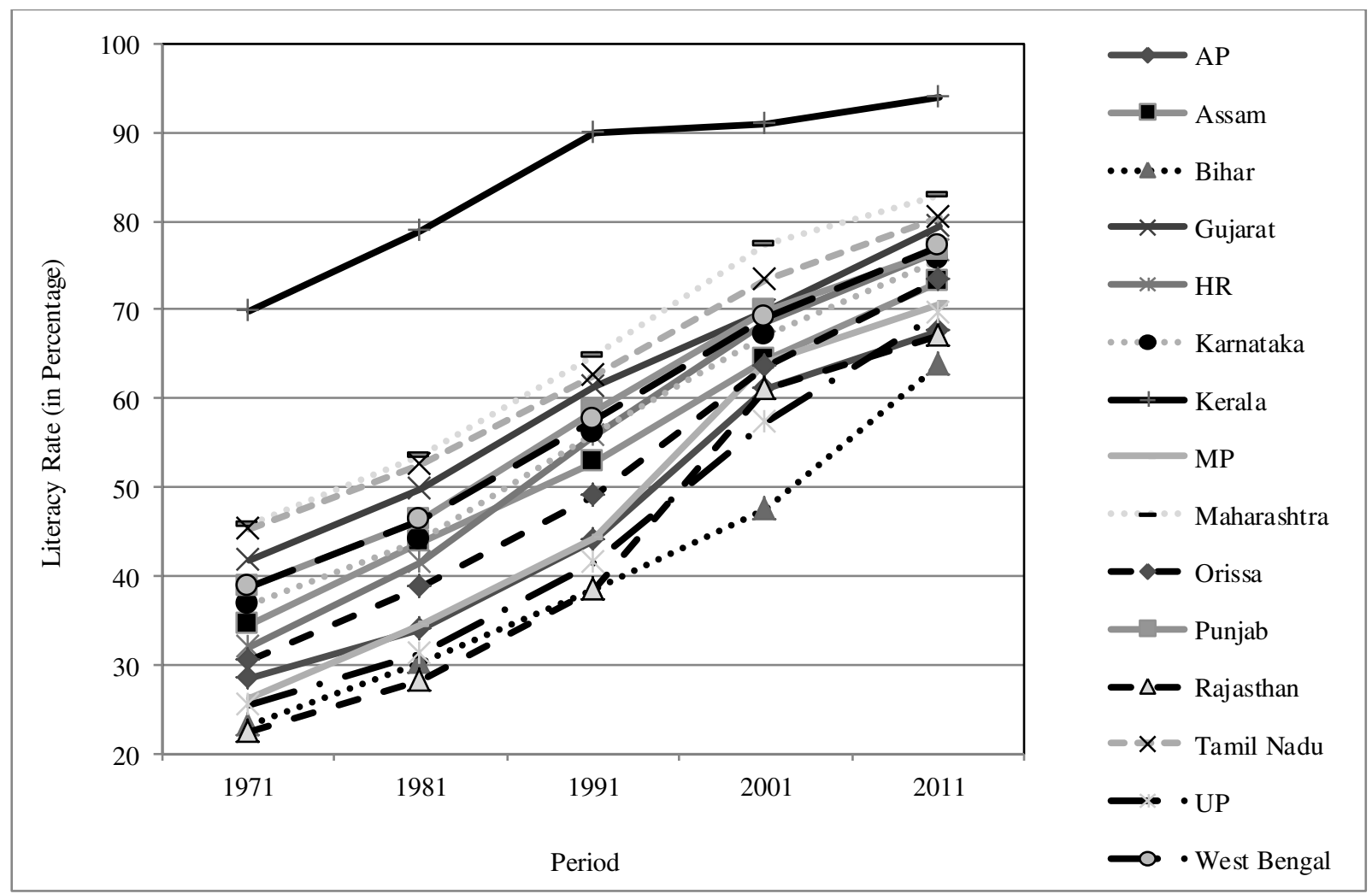


Gini index (1981-2005)

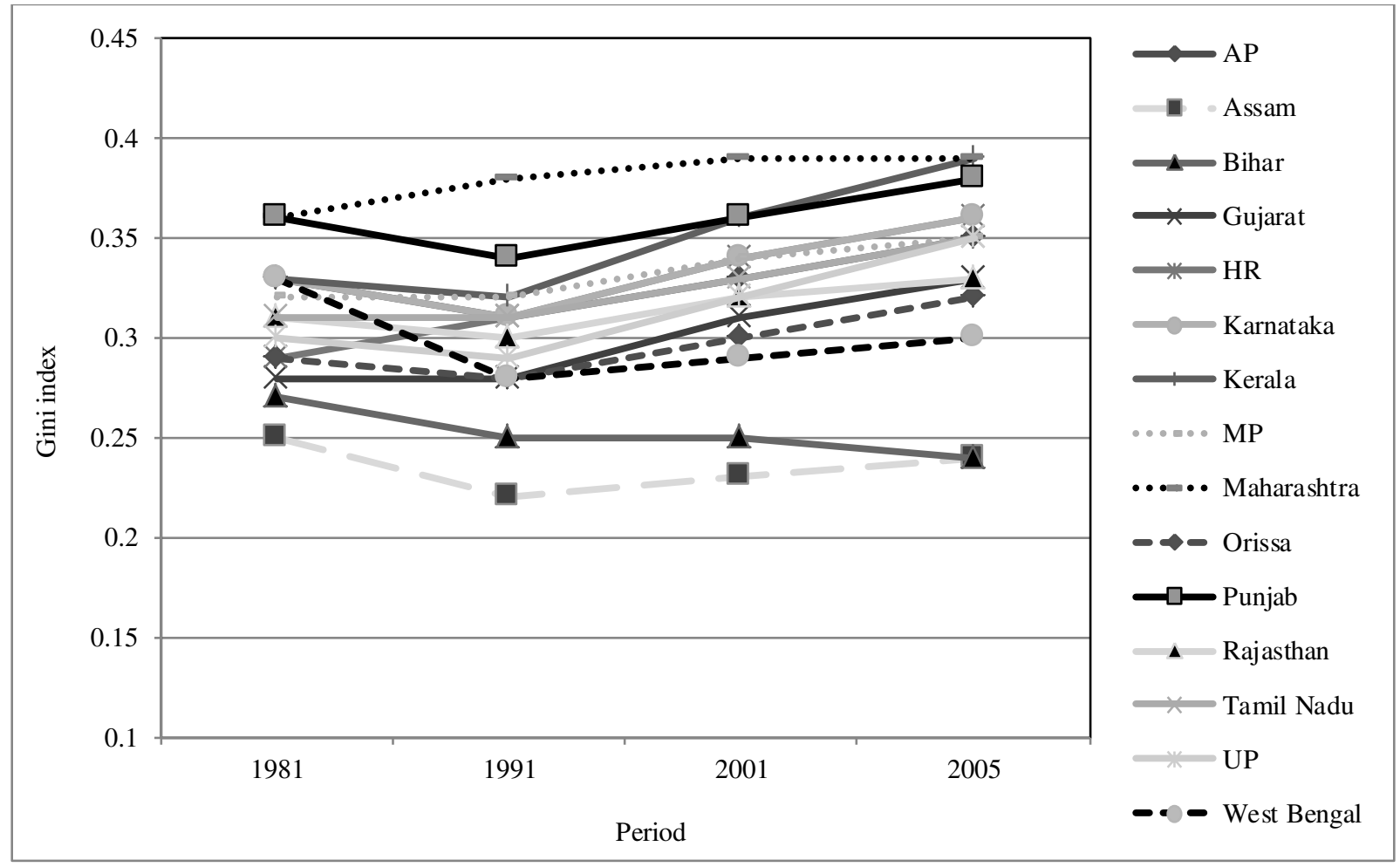

HDI (1981-2006)

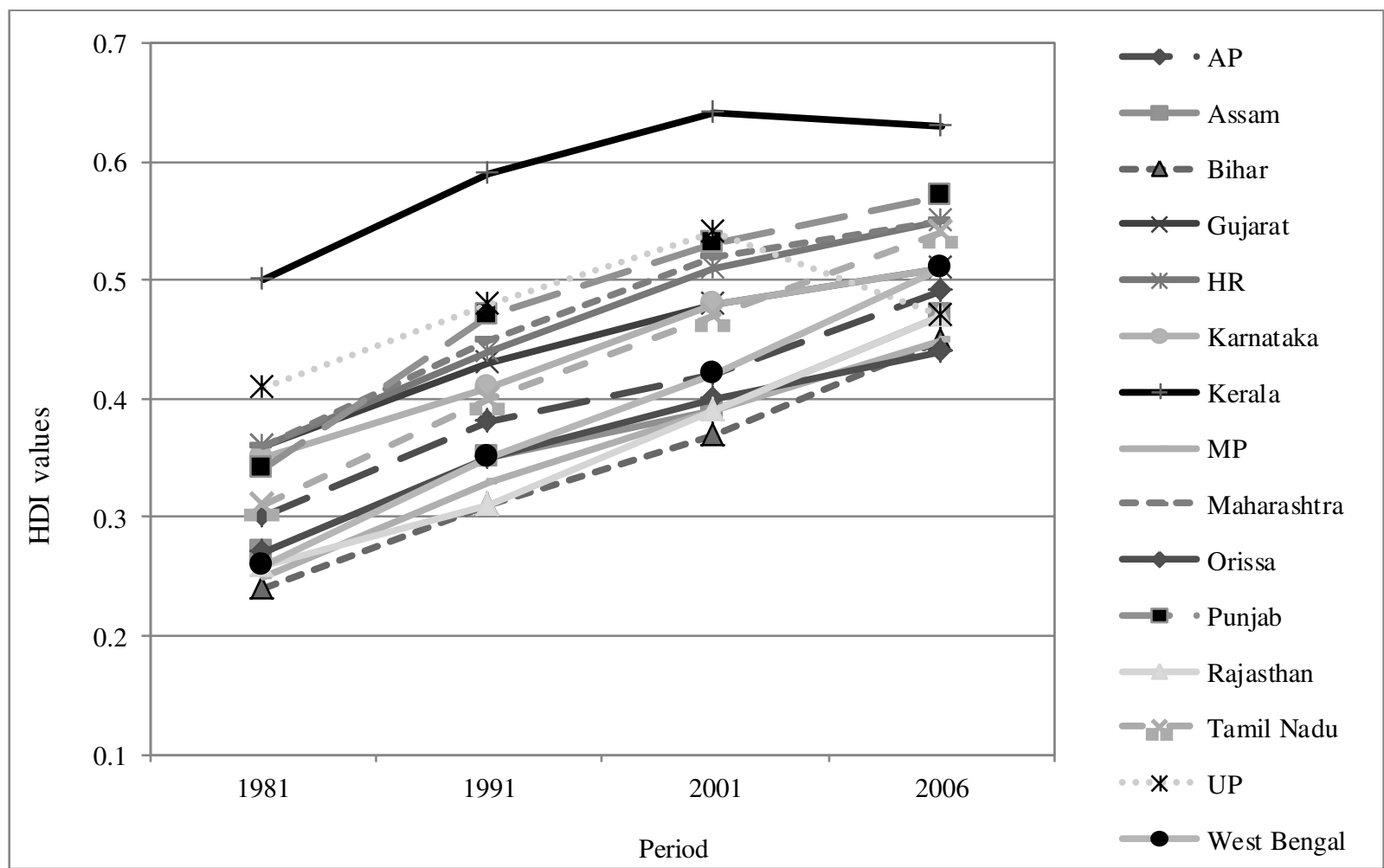




\subsubsection{Catching-up process}

In the Solow's growth framework or the neo-classical growth model, the catching-up mechanism is necessary for convergence. The catching-up process was identified by plotting a scatter diagram for change in an indicator in two points of time against values in the initial period. According to the Solow growth framework, the advanced states experience less change with better values in the initial period. In contrast the laggard states experience a greater change in poorer values in the initial period. In this chapter, I have examined the catching-up process in socioeconomic indicators for the 15 major states of India.

Figure 4.2. Scatter plots of change in selected socioeconomic indicator by values in the initial period of 15 major states of India.
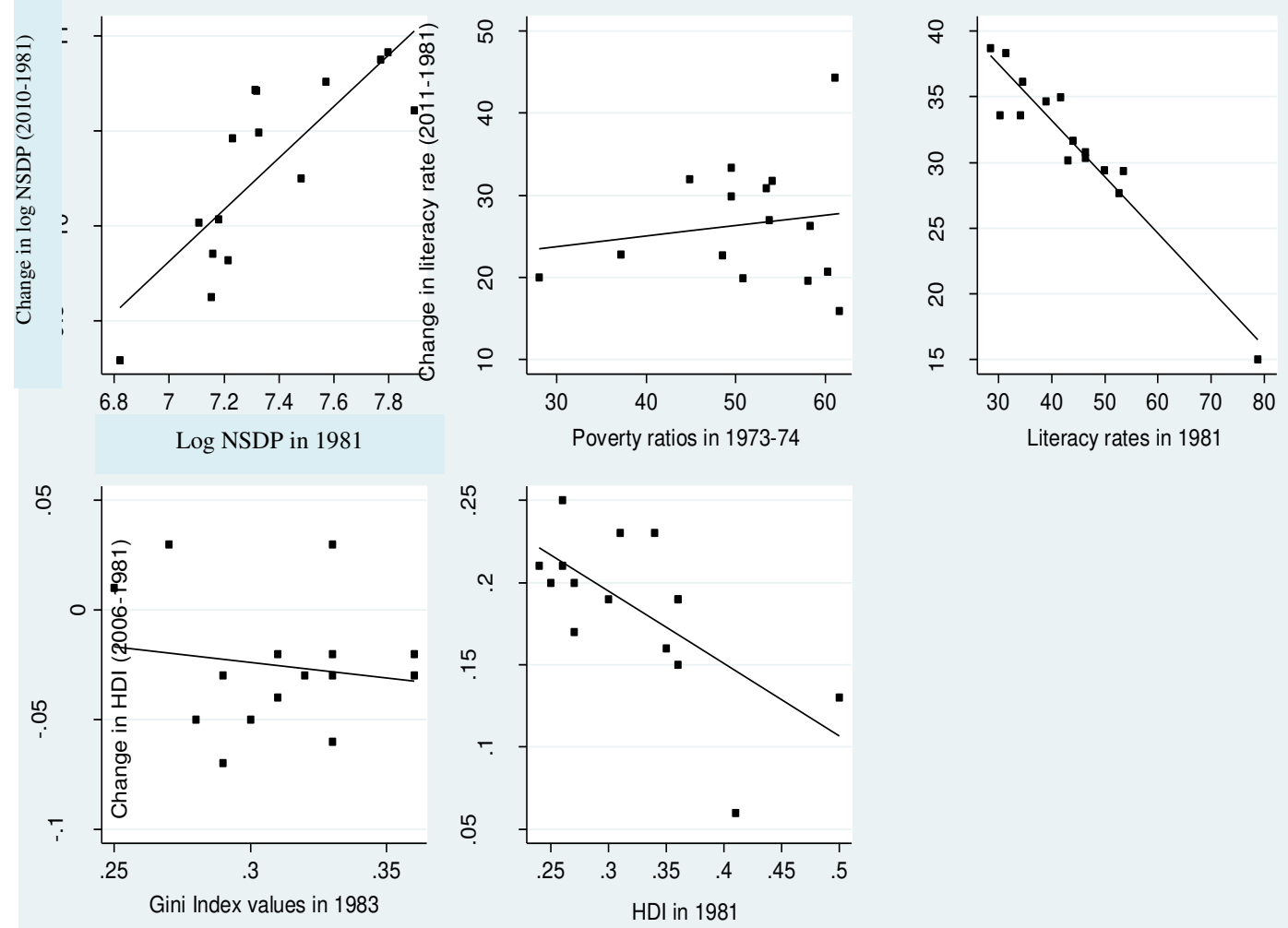

Figure 4.2 presents the scatter plots of change in selected socioeconomic indicators against the mean values in the initial period for 15 major states of India. In case of per capita NSDP, the results showed a positive association between change and initial values of per capita NSDP. This indicates that the catching-up process was not yet evident from the laggard states in case of the per capita NSDP. The economically advanced states (e.g. Punjab, Haryana, Maharashtra, Gujarat and Karnataka) in India 
have continued to experience higher growth rate in per capita NSDP compared to the laggard states (e.g. Bihar, Uttar Pradesh, Odisha, Rajasthan and Madhya Pradesh). A similar pattern was also evident for other two economic indicators: poverty ratios and Gini index. A change in both these indicators was positively associated with values in the initial period which implies that the reduction in poverty and economic inequality (in terms of Gini index) in some states was much greater in comparison to other states. The catching-up process in terms of progress in reduction of poverty and economic inequality from laggard states of India was not evident as yet.

However, the results in case of literacy rate indicate a contrasting picture. The states (e.g. Rajasthan, Bihar, Uttar Pradesh, Andhra Pradesh, Madhya Pradesh and Odisha) with low literacy rates in the initial period are experiencing greater change in literacy rates compared to their counterparts (e.g. Kerala, Tamil Nadu, Karnataka, Gujarat, Maharashtra) with higher literacy rates in the initial period. This is an indication of the catching-up process in terms of progress in literacy rates in India by laggard states to leading states. Similarly, there was also clear evidence of the catching-up process in a composite measure of human development. The results revealed a greater progress in human development index in laggard states (e.g. Bihar, Uttar Pradesh, Madhya Pradesh Rajasthan, and Odisha) compared to advanced states (e.g. Kerala, Maharashtra, Gujarat, Punjab, Haryana, Tamil Nadu, Karnataka) during 1981 to 2006.

\subsection{4. $\beta$-convergences}

Though, the catching-up process provides clues about convergence, the real convergence mechanism can be identified only by appropriate convergence models. Therefore, this chapter used $\beta$-convergence models to test the convergence hypothesis for the progress of socioeconomic indicators.

\section{Absolute $\beta$-convergence}

Table 4.2 presents the results of absolute $\beta$-convergence model for log per capita net state domestic product among the major states during 1981-2010. Though, the results revealed that during the period of 30 years, the per capita NSDP showed absolute $\beta$ Convergence ( $\beta=-0.0138$ ) across the 15 major states, but the estimate was statistically not significant and adjusted $\mathrm{R}^{2}$ was very low. Moreover, the results of piece-wise 
convergence models (estimated for sub-periods in a long period) were evidently a mixed pattern. During the initial period, 1981-91, the results showed a clear divergence phase $(\beta=.07587)$ in the NSDP. In contrast, in 1991-01, the earlier phase of divergence was replaced with convergence $(\beta=-.25090)$. Moreover, the volume of convergence was high. For the recent period, convergence phase in the previous period was replaced by divergence $(\beta=.12591 ; p<0.032)$ and the estimate was statistically significant.

Table 4.2. Absolute $\beta$-convergence model estimates for per capita NSDP of the major Indian states, 1981-2010.

\begin{tabular}{lccccc}
\hline Period & $\beta$ coefficient & $\mathrm{P}$ value & Adjusted $\mathrm{R}^{2}$ & $\mathrm{n}$ & $\mathrm{df}$ \\
\hline $1981-2010$ & -.0138 & 0.877 & 0.0749 & 15 & 14 \\
$1981-1991$ & .07587 & 0.576 & 0.0503 & 15 & 14 \\
$1991-2001$ & -.25090 & 0.120 & 0.1123 & 15 & 14 \\
$2001-2010$ & .12591 & .322 & 0.0041 & 15 & 14 \\
Constant $^{\mathrm{a}}$ & 1.278 & 0.070 & & & \\
\hline
\end{tabular}

Note- $\mathrm{n}=\mathrm{sample}, \mathrm{df}=$ degree of freedom, $\mathrm{a}=$ constant value refers to a convergence model of period, 1981-2010.

Table 4.3. Absolute $\beta$-convergence model estimates for Poverty Ratios of the major Indian states, 1974-2005.

\begin{tabular}{lccccc}
\hline Period & $\beta$ coefficient & $\mathrm{P}$ value & Adjusted R $^{2}$ & $\mathrm{n}$ & $\mathrm{df}$ \\
\hline $1974-2010$ & .06291 & 0.036 & 0.2424 & 15 & 14 \\
$1974-1984$ & .10874 & 0.008 & 0.3809 & 15 & 14 \\
$1984-1994$ & -.00388 & 0.908 & 0.0758 & 15 & 14 \\
$1994-2005$ & .12538 & 0.019 & 0.3058 & 15 & 14 \\
Constant $^{\mathrm{a}}$ & -5.82597 & 0.001 & & &
\end{tabular}

Note- $\mathrm{n}=\mathrm{sample}, \mathrm{df}=$ degree of freedom, $\mathrm{a}=$ constant value refers to the convergence model of period, 1974-2005.

Absolute $\beta$-Convergence model estimates for poverty ratios for 15 major states during 1974-2010 are presented in table 4.3. The results revealed that in the long run, the reduction of poverty ratios across the 15 major states were diverging $(\beta=0.06291$, $\mathrm{p}<0.036)$. However, the results of convergence model estimates for sub-periods during 1974-2010 again indicated a mixed pattern. The reduction of poverty rates across the states during 1974-84 showed divergent progress $(\beta=0.10874 ; \mathrm{p}<0.008)$, but followed by a convergence phase during 1984-94 $(\beta=-0.00388 ; \mathrm{p}<0.908)$ and again divergence ( $\beta=0.12538)$ during 1994-2005. Though, the results showed progress of convergence 
in the reduction of poverty ratios during 1984-94, but the model was not statistically significant and adjusted $\mathrm{R}^{2}$ value showed the poor goodness of fit. However, the absolute $\beta$-convergence estimates for the entire period, 1974-2005 and other subperiods, 1974-84 and 1994-2005 showed statistically significant divergence in the reduction of poverty across 15 major states.

Table 4.4. Absolute $\beta$-convergence model estimates for literacy rates of the major Indian states, 1981-2011.

\begin{tabular}{|c|c|c|c|c|c|}
\hline Period & $\begin{array}{c}\text { LR } \\
\beta \text { coefficien }\end{array}$ & $\mathrm{P}$ value & Adjusted $\mathrm{R}^{2}$ & $\mathrm{n}$ & $\mathrm{df}$ \\
\hline $1981-2011$ & -.04468 & 0.000 & 0.9408 & 15 & 14 \\
\hline 1981-1991 & -.03158 & 0.000 & 0.7334 & 15 & 14 \\
\hline $1991-2001$ & -.07056 & 0.000 & 0.7079 & 15 & 14 \\
\hline $2001-2011$ & -.05189 & 0.000 & 0.6777 & 15 & 14 \\
\hline Constant $^{\mathrm{a}}$ & 3.8670 & 0.000 & & & \\
\hline
\end{tabular}

Note- $\mathrm{n}=$ sample, $\mathrm{df}=$ degree of freedom, $\mathrm{a}=$ constant value refers to a convergence model of period, 1981-2011.

Table 4.5. Absolute $\beta$-convergence model estimates for human development index values of the major Indian states, 1981-2006.

\begin{tabular}{lccccc}
\hline Period & $\beta$ coefficient & P value & Adjusted R & $\mathrm{n}$ & $\mathrm{df}$ \\
\cline { 1 - 3 } $1981-2006$ & -6.3717 & 0.000 & 0.7627 & 15 & 14 \\
$1981-1991$ & -4.4534 & 0.020 & 0.3020 & 15 & 14 \\
$1991-2001$ & -3.3580 & 0.006 & 0.4042 & 15 & 14 \\
$2001-2006$ & -9.5074 & 0.000 & 0.5951 & 15 & 14 \\
Constant $^{\mathrm{a}}$ & 3.6714 & 0.000 & & &
\end{tabular}

Note- $\mathrm{n}=$ sample, $\mathrm{df}=$ degree of freedom $\mathrm{a}=$ constant value refers to the convergence model of period, 1981-2010.

Table 4.4 presents absolute $\beta$-convergence model estimates for literacy rates, for major states of India. The results suggest a strong convergence phase $(\beta=-0.04468, \mathrm{p}<0.00)$ in literacy rates during 1981-2011. The results of piecewise convergence model estimates showed that the estimates for all the sub periods support convergence. However, the convergence process was stronger in recent two decades $(\beta=-.07056$, $\mathrm{p}<0.00$ during 1991-2001 and $\beta=-.05189, \mathrm{p}<0.00$ during 2001-2011) compared to the earlier initial period, 1981-1991 $(\beta=-.07056, \mathrm{p}<0.00)$. The absolute $\beta$-convergence model estimates for literacy rates showed high goodness fit with the adjusted $R^{2}$ value greater than 0.90 . The absolute $\beta$-convergence model estimates for human development 
index values of the major states of India are presented in table 4.5. The results for the entire period, 1981-2006 $(\beta=-6.3717, \mathrm{p}<0.00)$ and for all the sub periods indicated a strong absolute $\beta$-Convergence in human development across the major states of India. However, the volume of convergence was greater in the recent period, 2001-06 ( $\beta=$ 9.5074, $\mathrm{p}<0.00)$ compared to the initial period, 1981-1991 $(\beta=-4.4534, \mathrm{p}<0.00)$. The $\beta$-Convergence model estimates for human development index values were statistically significant and goodness fit for the model was also high.

\subsubsection{Conditional $\beta$-convergence}

Table 4.6 presents the conditional $\beta$-convergence model estimates for log per capita NSDP of the major Indian states. The conditional $\beta$-convergence model estimates for per capita NSDP was estimated after controlling state's social indicators such as literacy rates, total fertility rates and life expectancy at birth of the initial period, 1981. The results showed statistically significant convergence $(\beta=-1559, \mathrm{p}<0.000)$ in the per capita NSDP of 15 major states of India during 1981-2010. The conditional $\beta$ convergence model estimates for poverty ratios of the major Indian states were presented in table 4.7. Commensurate with the absolute $\beta$-Convergence model estimates, the conditional $\beta$-Convergence model estimates showed progress in the reduction of poverty ratios for majority of the states of India $(\beta=0.1132, p<0.002)$ during the period, 1974-2005 and the model was statistically significant. The adjusted $\mathrm{R}^{2}$ value showed that the goodness fit of the model was also high.

Table 4.6. Conditional $\beta$ - convergence model estimates for NSDP of the major Indian states, 1981-2010.

\begin{tabular}{lccccc}
\hline Factors & $\beta$ coefficient & P value & Adjusted R $^{2}$ & $\mathrm{n}$ & $\mathrm{df}$ \\
\hline Log NSDP_1981 & -.1559 & 0.090 & & & \\
Literacy Rate_1981 & -.0013 & 0.660 & .4143 & 15 & 14 \\
TFR_1981 & -.0745 & 0.094 & & & \\
LEB_1981 & .0078 & 0.325 & & & \\
Constant & 2.2584 & 0.004 & & & \\
\hline
\end{tabular}

Note- $\mathrm{n}=$ sample, $\mathrm{df}=$ degree of freedom

Table 4.8 presents the conditional $\beta$-convergence model estimates for literacy rates of major Indian states. The model was conditioned for the initial levels of log NSDP, poverty ratios and TFR. Similar to absolute $\beta$-convergence model estimates for literacy rates of major states of India during 1981-2011, the conditional $\beta$-convergence model 
estimates showed that progress in literacy among 15 major Indian states have led to convergence $(\beta=-.0368, \mathrm{p}<0.000)$. The model was statistically significant and goodness of fit of the model was very high. Further, the conditional $\beta$-convergence model estimates for the human development index values of major Indian states are shown in table 9. Also, the model was conditioned for the initial situation of the states in terms of inequality, poverty and fertility rates of the states. The results revealed a strong indication of convergence $(\beta=-7.3343, \mathrm{p}<0.000)$ in terms of progress in human development across the major states of India.

Table 4.7. Conditional $\beta$-convergence model estimates for poverty ratios of the major Indian states, 1974-2005.

\begin{tabular}{lccccc}
\hline Period & $\beta$ coefficient & P value & Adjusted R & $\mathrm{n}$ & $\mathrm{df}$ \\
\hline Poverty Ratios_1974 & .1132511 & 0.002 & & & \\
Log NSDP_1981 & 2.137415 & 0.040 & & \\
TFR_1981 & -.0862611 & 0.800 & 0.6796 & 15 & 14 \\
Literacy Rate_1981 & -.0767969 & 0.012 & & & \\
Constant & -20.40596 & 0.022 & & \\
\hline
\end{tabular}

Note: $\mathrm{n}=$ sample, $\mathrm{df}=$ degree of freedom

Table 4.8. Conditional $\beta$-convergence model estimates for Literacy Rate of the major Indian states, 1981-2010.

\begin{tabular}{lccccc}
\hline Period & $\beta$ coefficient & P value & ${\text { Adjusted } \mathrm{R}^{2}}$ & $\mathrm{n}$ & $\mathrm{df}$ \\
\hline Literacy Rate_1981 & -.0368 & 0.000 & & & \\
Log NSDP_1981 & -.0515 & 0.797 & & & \\
Poverty Ratios_1974 & .0030 & 0.605 & .9502 & 15 & 14 \\
TFR_1981 & .1320 & 0.095 & & \\
Constant & 3.1727 & 0.079 & & \\
\hline
\end{tabular}

Table 4.9. Conditional $\beta$ - convergence model estimates for HDI of the major Indian states, 1981-2010.

\begin{tabular}{lccccc}
\hline Period & $\beta$ coefficient & P value & Adjusted R $^{2}$ & $\mathrm{n}$ & $\mathrm{df}$ \\
\hline HDI_1981 & -7.3343 & 0.000 & & & \\
Gini Index_1981 & 1.5779 & 0.461 & & & \\
Poverty Ratios_1974 & -.0069 & 0.292 & .8291 & 15 & 14 \\
TFR_1981 & -.1476 & 0.084 & & \\
Constant & 4.4896 & 0.001 & & \\
\hline
\end{tabular}

Note- $\mathrm{n}=$ sample, $\mathrm{df}=$ degree of freedom 


\subsection{6. $\sigma$-Convergence}

The $\sigma$-Convergence was measured by $\mathrm{CV}$ and is presented in figure 4.3. The estimates of trends in CV for per capita NSDP showed increasing dispersion over the period, 1981-2010. Hence, indicated a divergence phase in per capita NSDP across the major states of India. A more careful examination of the trends revealed that the divergence has accelerated during the recent period, 2001-2010. The estimate of trends of CV for the poverty ratios indicated a divergent trend in reduction of poverty ratios across the states until 2000, but was replaced by convergence trend during the post-2000 period. The trends of CV for literacy rates showed a clear decline over the period, 1981-2011. Hence, it supports the hypothesis of $\sigma$-convergence. The speed of convergence was highest during 1991-2001, but it slowdown in the post-2000 period. Similarly, the trends in HDI showed a continued decline in CV over the period, 1981-2006 and supported the hypothesis of convergence. Moreover, the speed of convergence was increased in the recent period, 2001-2006 in comparison with the earlier period of 1981-1991 (Figure 4.3).

Figure 4.3. Trends in coefficient of variation (CV) in selected socioeconomic indicators of the major states in India during 1973-2011.

\section{Per capita NSDP}

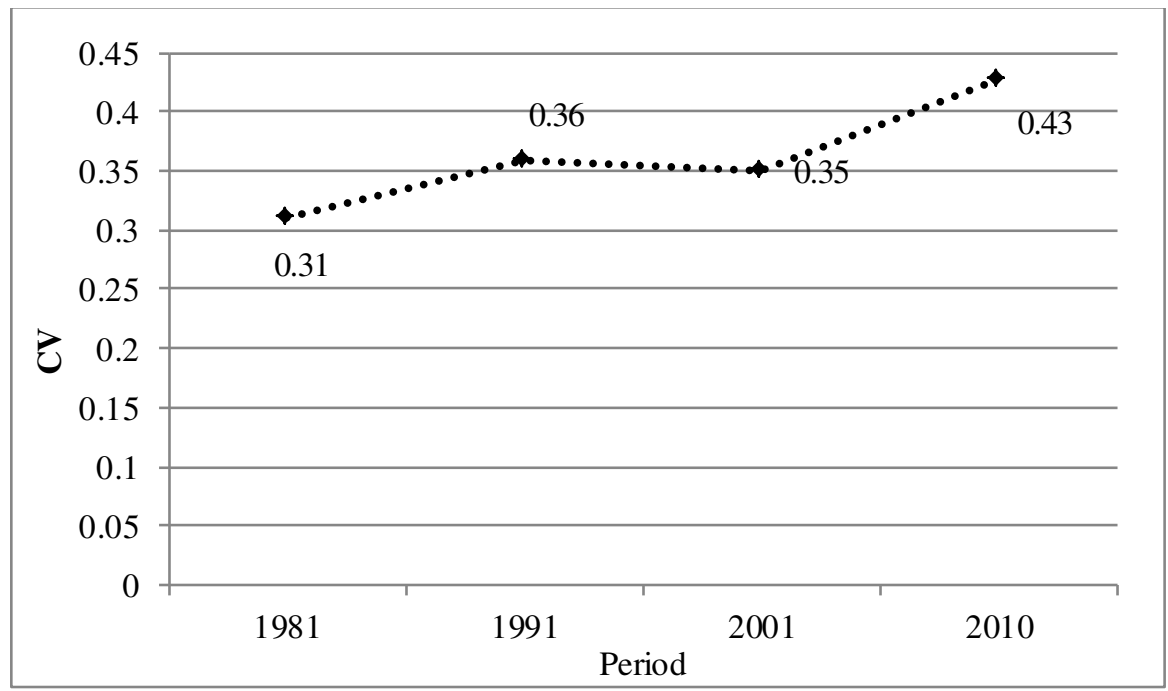


Poverty ratios

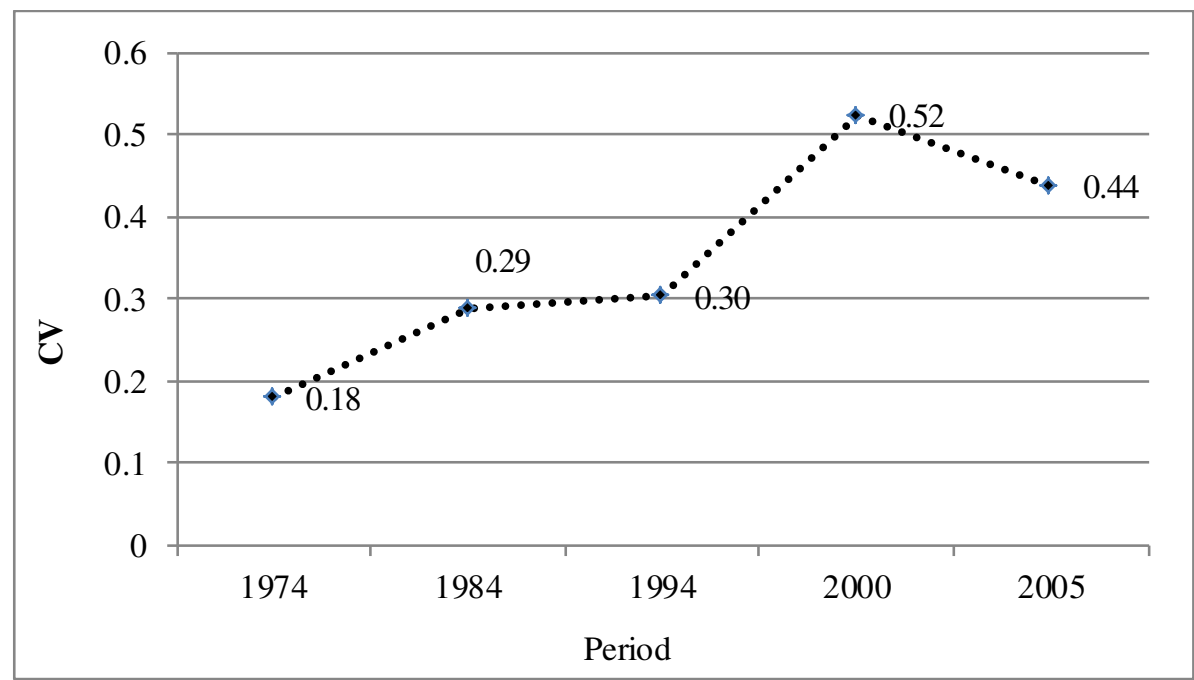

Literacy rates

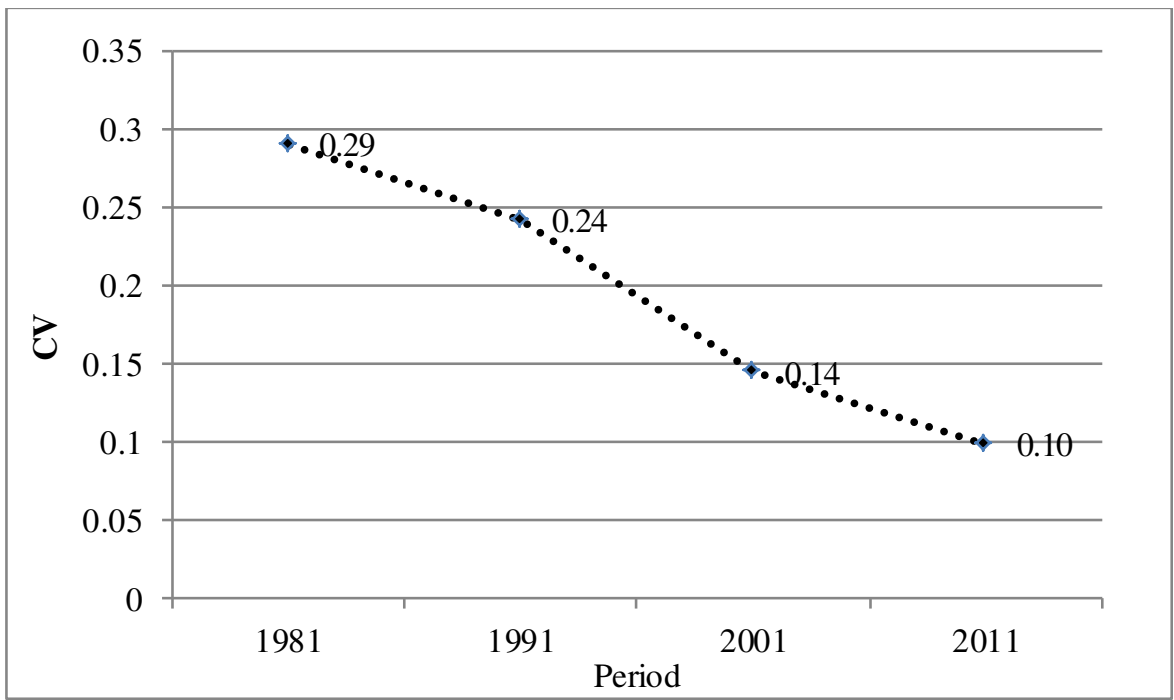

HDI values

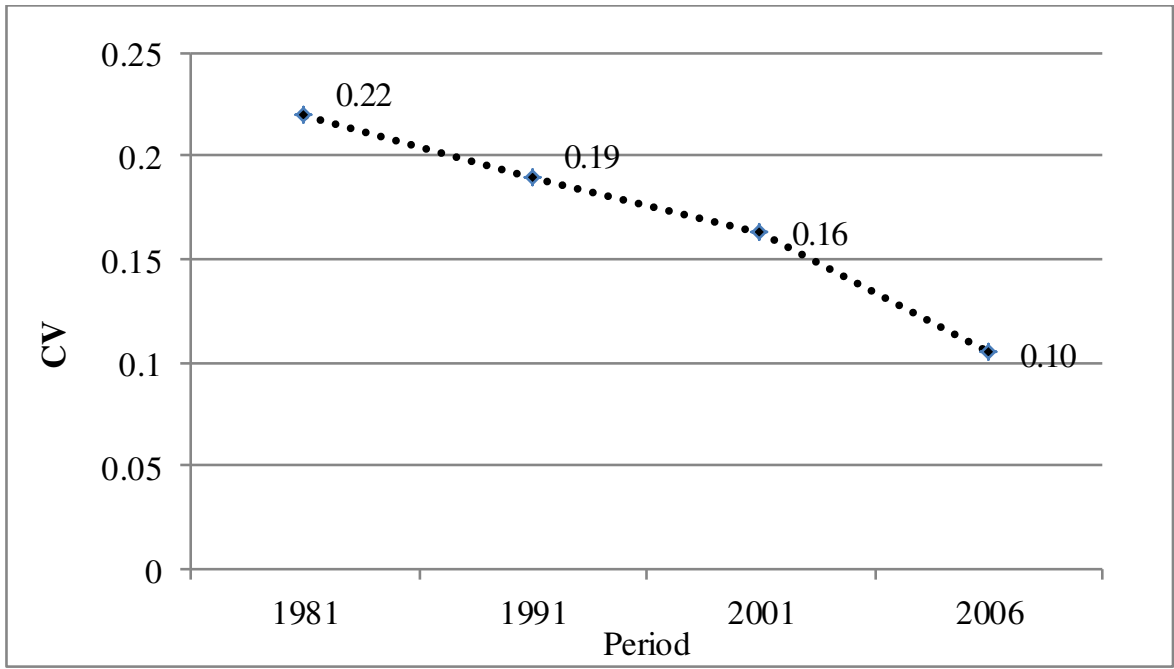




\subsubsection{Kernel density estimates}

Figure 4.4 presents the result of Gaussian Kernel density estimates. The first row shows the Kernel density plots for the per capita log NSDP during 1981-2010. For the year 1981 and 1991, the kernel density distribution plots were showed bimodality in peaks that indicates presence of convergence clubs. During these two time points, the second and smaller peaks were on the left side, indicating that most of the states fall under lower income states. For the year 2001, the second peak was clearly not evident, but in the year 2010, the kernel distribution plots clearly showed bimodality in the distribution of per capita log NSDP. In both 2001 and 2010, the major peak shifted to the right side showing maximum number of states incline to the higher income side. Thus, in case of per capita NSDP, the Kernel density estimates revealed evidence to support a divergence in progress across but convergence across the different clubs of states during 1981-2010. These clubs were formed based on income levels, such as groups of high and low income states.

The Kernel density estimates in case of poverty ratios across the major states also revealed bimodality for the year, 1974, 1984 and 1994, but were unimodal for the year 2005. Though, there was no secondary peak in the year 2005, but the Kernel plots were wider, thus, not showed a clear evidence of convergence in progress with respect to poverty ratios. The Kernel density distribution plots for literacy rates indicated the bimodal distribution for all the four periods, 1981, 1991, 2001 and 2011. However, the second peak for all the years was very small, the majority of the states fall under higher and narrow peak thus, showed evidence for convergence in literacy rates over the period. The Kernel density distribution plots for Gini indices indicated that over the period, the distribution of the Gini index across the states have become more dissimilar. During 1983, the Gini index distribution shows a unimodal, but it was slightly unsmoothed in 1994. However, during 2001 and 2005, the distribution of Gini was clearly showed a bimodal distribution. Thus, there was evidence for divergence in the progress of the reduction in inequality of economic status across the major Indian states. The Kernel estimates in case of human development index indicate a bimodal distribution for all the years from 1981-2006. Though, the size of secondary peak was very small in 2006, the distribution was wider. Kernel plots do not showed a clear 
pattern of convergence of progress in human development index in 15 major states of India.

Figure 4.4. Kernel density estimates and distribution curves for selected socioeconomic Indicators of 15 major states of India.

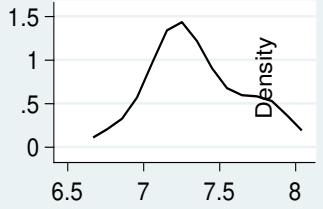

Log per capita NSDP in 1981

$\mathrm{k}=$ gaussian, $\mathrm{h}=0.1542$

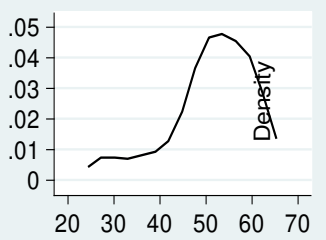

Poverty ratios in 1974

$\mathrm{k}=$ gaussian, $\mathrm{h}=3.8040$

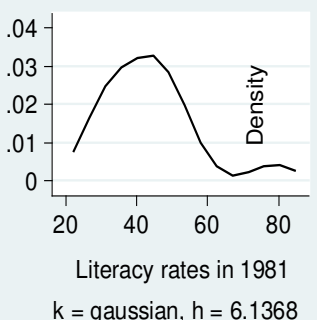

$\mathrm{k}=$ gaussian, $\mathrm{h}=6.1368$
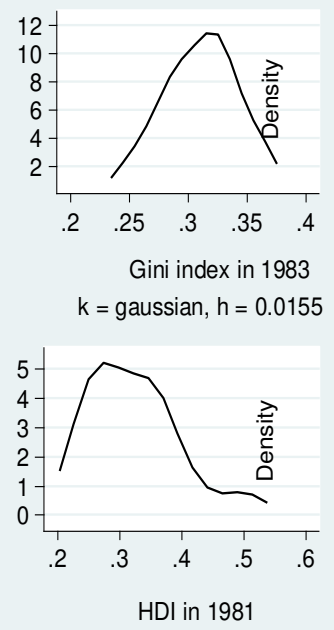

$\mathrm{k}=$ gaussian, $\mathrm{h}=0.0372$

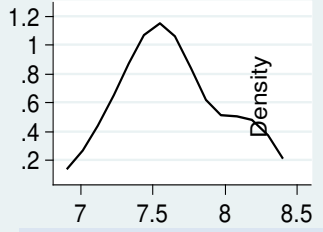

Log per capita NSDP in 1991

$\mathrm{k}=$ gaussian, $\mathrm{h}=0.1783$

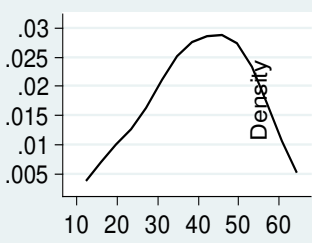

Poverty ratios in 1984

$k=$ gaussian, $h=6.1163$
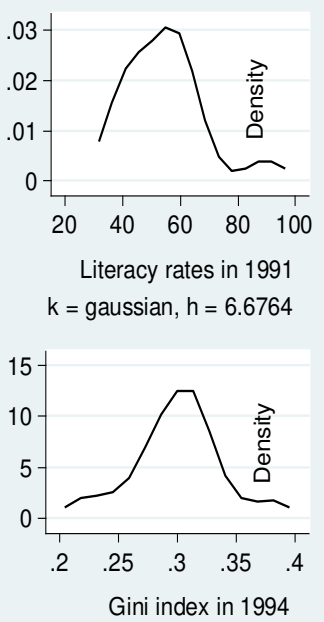

$\mathrm{k}=$ gaussian, $\mathrm{h}=0.0155$

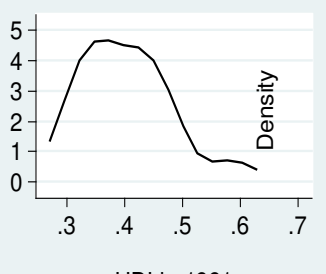

HDI in 1991

$\mathrm{k}=$ gaussian, $\mathrm{h}=0.0388$
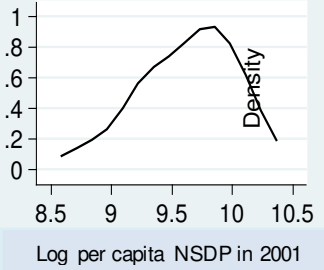

$\mathrm{k}=$ gaussian, $\mathrm{h}=0.2016$

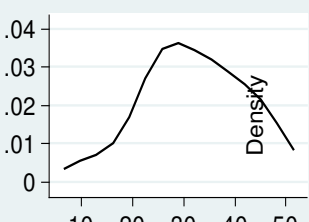

$\begin{array}{lllll}10 & 20 & 30 & 40 & 50\end{array}$

Poverty ratios in 1994

$k$ = gaussian, $h=5.0775$

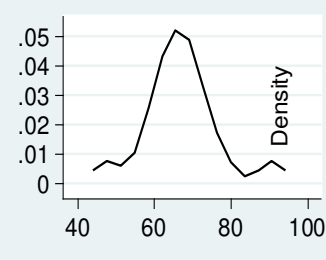

Literacy rates in 2001

$\mathrm{k}=$ qaussian, $\mathrm{h}=3.4430$

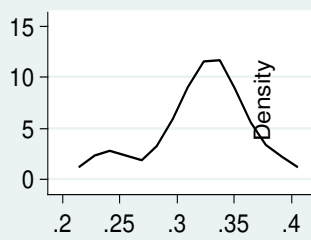

Gini index in 2001

$\mathrm{k}=$ gaussian, $\mathrm{h}=0.0155$

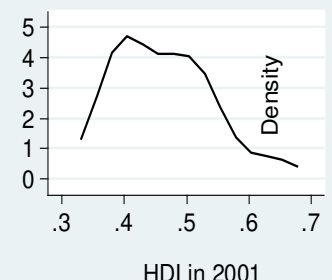

$\mathrm{k}=$ gaussian, $\mathrm{h}=0.0396$

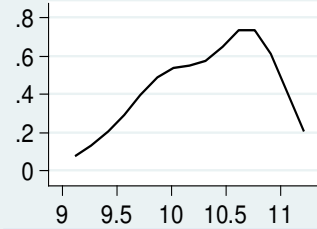

Log per capita NSDP in 2010

$\mathrm{k}=$ gaussian, $\mathrm{h}=0.2537$
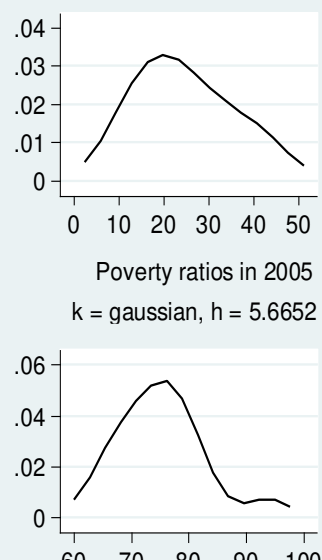

Literacy rates in 2011

$\mathrm{k}=$ gaussian, $\mathrm{h}=3.7225$
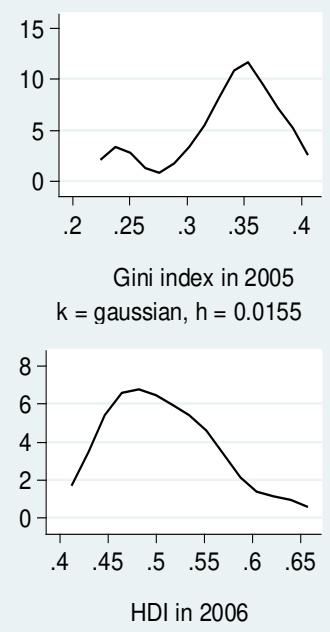

$\mathrm{k}=$ gaussian, $\mathrm{h}=0.0277$

\subsubsection{Inequality based measure of convergence}

The estimates of convergence in socioeconomic indicators by using AID and Gini index are presented in figure 4.5. The results in case of per capita NSDP showed 
increasing dispersion in both absolute and relative distribution of income. Thus, the results of inequality based measure of convergence measure for per capita NSDP support a divergence hypothesis in terms of its progress during 1981-2010. The absolute inequalities in poverty ratios across the states decreased during 1981-91, then increased sharply during 1991-2001 and declined again in post-2001 period. The trends in relative inequalities in terms of the Gini index of poverty ratios showed an increasing trend until 2000, but in decreasing phase in the post-2000 period. The trends of absolute interstate difference in literacy rates across the states indicated a rising trend during 1981-91, but showed a declining trend thereafter. However, the trends in the Gini index of literacy rates across the state showed a continuous decline, but the decline was sharper during 1991-2001. The estimates of AID for HDI of major Indian states during 1981-2010 showed declining trends for the entire period, but the decline was sharper in the recent period, 2001-10. The Gini index estimates for HDI of major states indicate a rising trend until-2000, but a sharply declined in the period after 2000 .

Figure 4.5. Trends in Population weighted AID and Gini index in selected socioeconomic indicators of the major states in India during 1981-2010.

Per capita NSDP

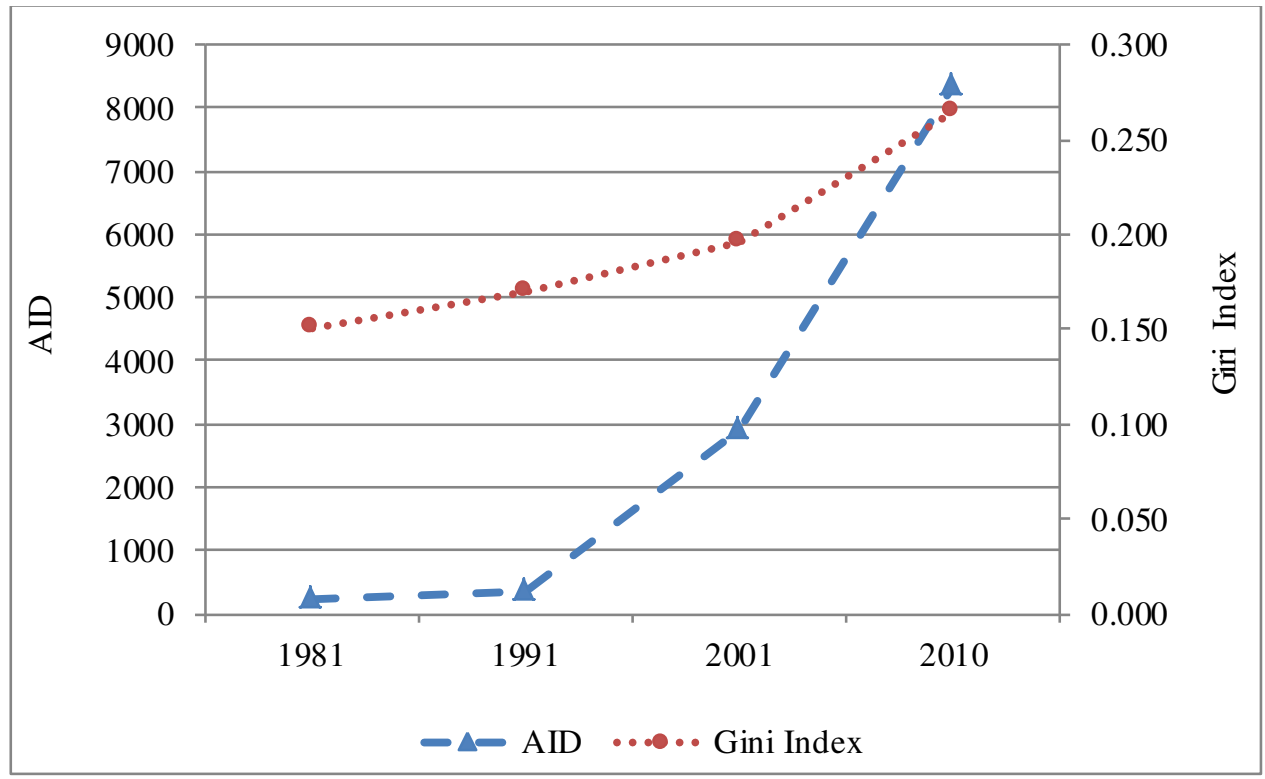


Poverty ratios

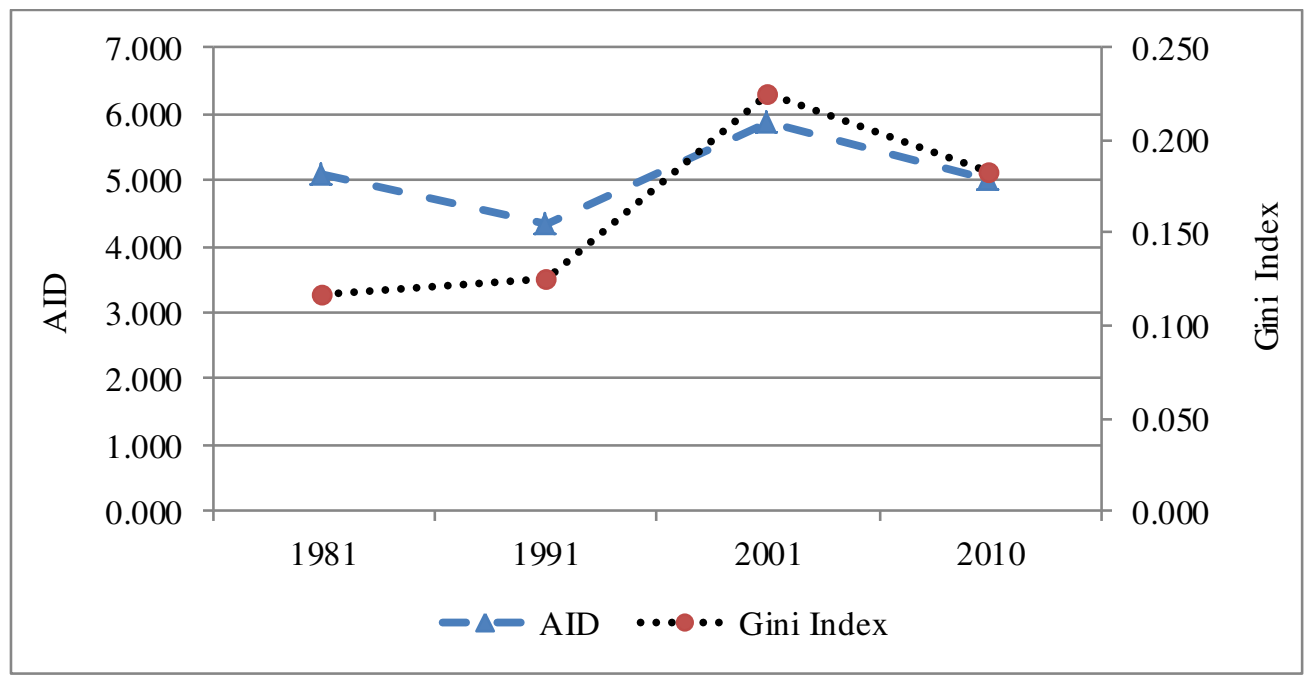

Literacy rates

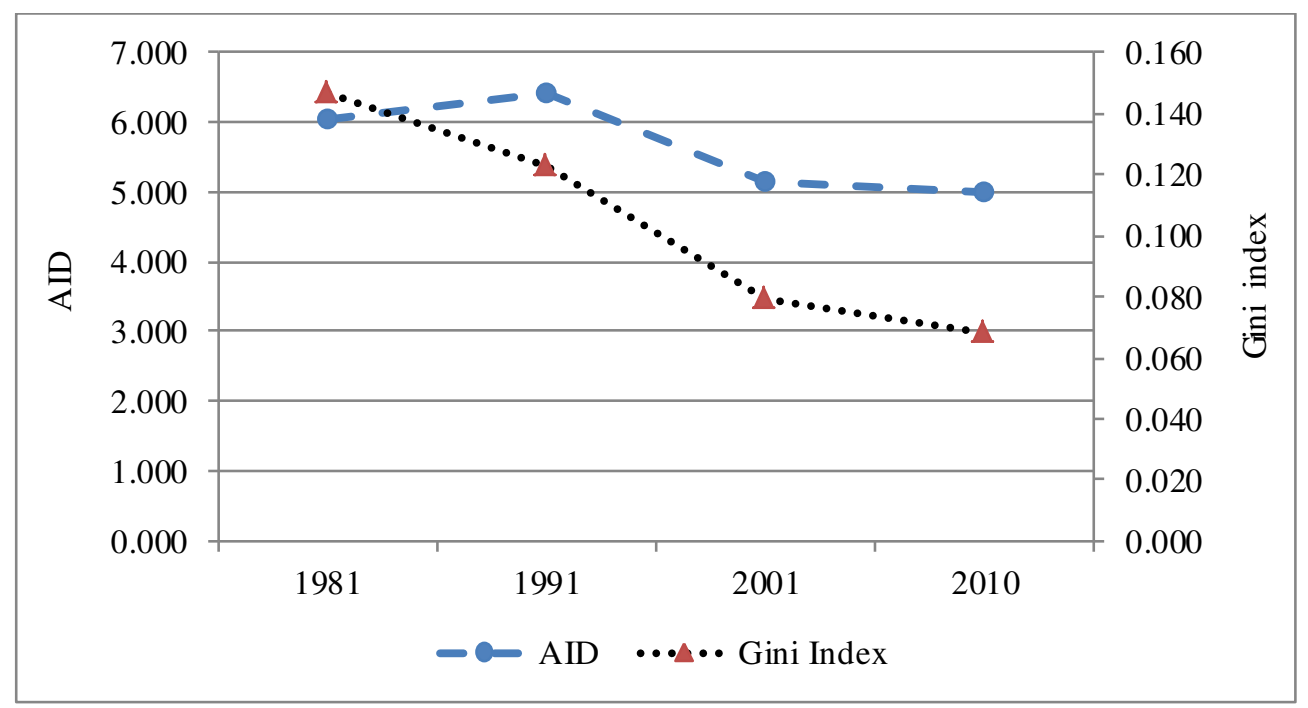

HDI

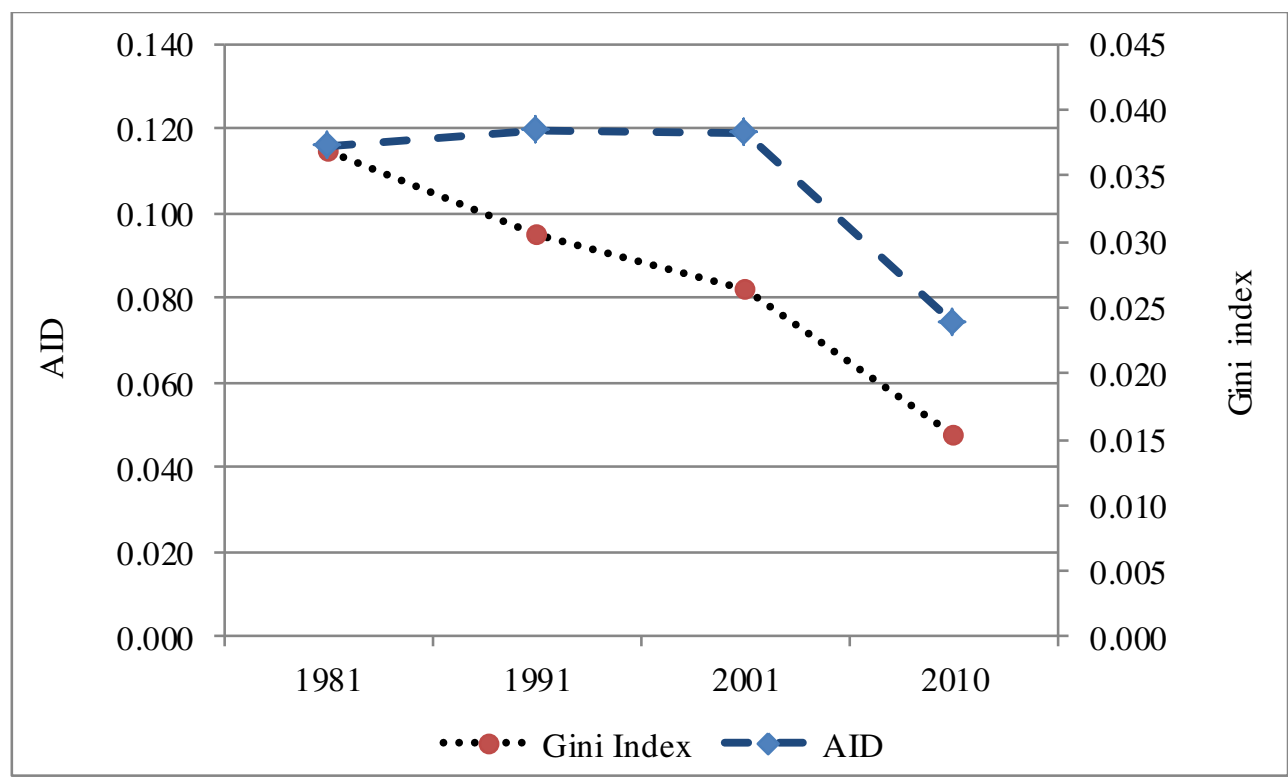




\subsection{Discussion}

This chapter tested the convergence hypothesis to determine the progress in socioeconomic indicators across 15 major states of India by using both parametric and non-parametric convergence metrics. The various convergence metrics used in this chapter give a number of interesting findings about progress in socioeconomic indicators. First, the scatter plots suggested that the laggard states are catching-up with the advanced states only in social indicators like literacy rates and HDI. However, in case of economic indicators (per capita NSDP, poverty ratios and Gini index), the advanced states showed much higher levels of improvement compared to the laggard states. Thus, no catching-up process was evident for economic indicators.

Second, the $\beta$-convergence estimates give more insights on the volume of convergence of the selected indicators. The absolute $\beta$-convergence estimates for the entire period indicated convergence in the literacy rates and HDI, but the divergence in per capita NSDP and poverty ratios across the major states. However, the estimates for per capita NSDP were not statistically significant. Further, convergence estimates for shorter intervals revealed statistically significant divergence in per capita NSDP and poverty ratios for the recent period, but convergence in literacy rates and HDI. The conditional $\beta$-convergence estimates indicated statistically significant and greater volume of convergence than absolute $\beta$-convergence for per capita NSDP and HDI, but the lesser volume of convergence in case of literacy rates. In case of poverty ratios, both absolute and conditional $\beta$-convergence measure indicated a divergence in economic progress. Another parametric convergence measure that is the $\sigma$-convergence or inequality based convergence measure revealed that in the long run, there was divergence in all economic indicators (per capita NSDP and poverty ratios) but clear convergence was seen in social and human development indicators (literacy rates and HDI).

Third, the non-parametric measure in the form of Gaussian Kernel density plots supported divergence hypothesis for per capita NSDP. However, there was a clear emergence of convergence clubs in case of per capita NSDP for all the years and the major peaks was shifted to higher values in the recent two decades. For poverty ratios, the major peaks were shifted to lower mean values as the poverty ratio has dropped down in many states, but there was still no clear evidence of convergence. In case of 
Gini index, the Kernel density plots showed a divergent progress and shifting of peak to higher values. In contrast to parametric convergence measures, Kernel density plots, in case of HDI, supported divergence hypothesis. Overall, parametric, non-parametric and inequality based convergence metrics supported the hypothesis of convergence for progress in literacy rate, but strong divergence in economic indicators like per capita NSDP and poverty ratios during 1981-2010.

Taken as a whole, the pattern of economic progress in the Indian states supported divergence rather than the absolute $\beta$-convergence hypothesis especially in post-reform period. This suggests that the economic reform in India in the post 1991 economic reform phase has certainly affected the pattern and volume of economic progress unequally in the major states. In case of both the economic measures: per capita NSDP and poverty ratios, the results clearly indicated that during a long period, there is statistically significant conditional $\beta$-convergence but there is no absolute $\beta$ convergence. These findings are also in tune with earlier studies (Bhattacharya and Sakthivel, 2004; Kar and Sakthivel, 2007 and Ghosh, 2011). Additionally, through Kernel density plots, this study rules out the existence of absolute convergence, but reveals the presence of convergence clubs and that the trends have shifted in a major fashion in post 1991.

Fifth, earlier studies (Dholakia, 2003; Ghosh, 2006) that focussed on social indicators which used only parametric convergence measure have supported convergence which is well established in our analysis. All the selected convergence measure in case of literacy rates supports the convergence hypothesis, except Kernel density plots which shows that though many states cluster together, still there are a few laggards which formed a small secondary peak. In case of the HDI, parametric measure support existences of convergence, but non-parametric measure support divergence and existence of convergence clubs. However, non-parametric measures in case of both social indicators revealed the existence of convergence clubs rather than clear convergence. Thus, this study suggests that use of non-parametric convergence measures is important to obtain insights on the overall progress as well as short-term divergent paths. 
Lastly, though, initially the regional economic disparities may result from uneven resource-endowments, but the persistence of regional imbalances in the long-run can be mainly attributed to the failure of our planning process. The convergence/divergence patterns, Kernel density distribution and inequality trends in selected socioeconomic indicators clearly indicate that the socioeconomic disparities across the regions are, by and large, an outcome of the working of the socioeconomic and political system and its processes rather than disparities in natural endowments. The increasing divergence during the post-reform period in economic indicator is a clear evidence of the influence of economic reforms on regional disparities. However, diffusion of education, health behaviour and special focus on demographically disadvantageous states contributed to improved literacy rates and life expectancy, and thus to the improvement in human development of laggard states. Unlike in the sphere of social and health policies, the laggard states had no special support in economic inputs as most of the economic decisions are guided by profit making motives of the investors. Thus, in the context of increasing competition among states for attracting private investment, the laggard states are in a more disadvantageous position compared to leading states and this can further accentuate the gap between the leading and the laggard states in terms of economic indicators. Special policy efforts to promote more investment are required in laggard states and to bridge the economic gaps between the leading and laggard states. 


\section{CHAPTER 5 \\ Mortality transition and Convergence}

\subsection{Introduction}

The near universal decline in death rates that was registered in 1960s led demographers to label the term "Mortality Transition" to refer to the passage from high and fluctuating mortality to low and stable mortality. Transition in mortality reflects improvements in the quality of life through improvements in health. Thus, a lot of attention has been given to it in the recent times (United Nations, 1973; Bloom and Canning, 2007; Clark, 2010; Goesling and Firebaugh, 2004; Mayer-Foulkes, 2003; McMichael et al., 2004; Moser et al., 2005; Neumayer, 2004). There are at least three stages of mortality transition based on the experience of mortality decline in the developed countries. The first stage comprises of lowering peaks of mortality. In the context of developed countries, this was achieved primarily through improved food supply and overall living standards and to a lesser degree through medical progress, sanitation and organised public health activities (Namboodiri, 1996). The second stage of mortality transition is characterised by a sustained decline in mortality, primarily as a result of public health measures, universal availability of safe drinking water and sanitation facilities, food hygiene, etc. (McKeown, 1976). Finally, the third stage of mortality transition comprised of a shift in the primary causes of mortality from infectious and parasitic diseases to degenerative diseases and diseases of human origin (Omaran, 1977).

The widely used indicators for assessment of mortality transition are three: Life Expectancy at Birth (LEB), Maternal Mortality Rate (MMR) and Infant Mortality Rate (IMR). Among these, LEB reflects the overall mortality level of a population. It is the most widely used indicator for analysing mortality transition. It gives the number of years a new born is expected to survive, on an average, given the prevailing age schedule of mortality (Pollard, 1982). The relationship between mortality transition and improvements in expectation of life at birth is essentially reciprocal, but the exact connection is not straight forward (Vaupal and Romo, 2003). In 2011, life expectancy at birth globally was 70 years (United Nations 1999). The increase of life expectancy across the world is not uniform. The difference ranging from 60 years in low-income 
countries to 80 years in high-income countries, giving a ratio of 1.3 between the two income groups. McMichael et al. (2005) focus on the most recent century and draw attention to three groups of countries: those that have experienced rapid improvement in their life expectancy, those that have experienced relative stagnation in their life expectancy, and those that have experienced an erosion of life expectancy. This trichotomy leads McMichael and his colleagues (2005) to argue against a deterministic process of global gains and convergence in population health. This argument is not withstanding with the argument of many of the classical analyses of the 1970s of longterm mortality trends maintained that, more significant gains in longevity in countries with low mortality is unlikely, but the death rates in countries with high mortality would fall, resulting in a worldwide convergence in mortality. In demographic perspective, the studies have shown that, during the $19^{\text {th }}$ and $20^{\text {th }}$ centuries, there has been a tendency of greater demographic homogeneity across the world countries (Omran, 1971; Preston, 1976; Watkin, 1990; Easterlin 2000, Bourguignon and Morrison 2002; Gosling and Firebaugh 2004; Cole and Niemeyer 2003, Niemeyer 2004; Becker, Philipson, and Soares, 2005; Wilson, 2001; Dorius, 2008). There are also studies which showed empirical evidence based on their valuable quantitative assessment for converging life expectancy (Watkin, 1990; Wilson, 2001, Dorius, 2008). However, later, Bloom and Canning (2009) reject the hypothesis that these changes reflect a simple convergence process. Their analyses instead, suggest two way process of improvement in LEB across the countries: one group experiencing continued improvement in life expectancy resulting in a jump from one cluster to the other but the second group of countries almost stagnant and remained with their previous clusters.

While LEB is a widely accepted summary measure of overall mortality, MMR and IMR are the sensitive indicators which explain the mortality situation of a country. Further, these two are also important because maternal and child mortality are two of eight goals for development adopted by 189 countries in the Millennium Declaration in 2000. These two issues have prompted the global health analysts and driven increased levels of development aid, policy attention and research work (Goodburn and Campbell, 2001; Child Mortality Coordination Group, 2006; Starrs, 2006; Hill et al., 2007; Murray et al., 2007; Countdown Coverage Writing Group on behalf of the Countdown to 2015 Core Group 2008; Gregson et al. 2009; Hogan et al., 2010; Rajaratnam et al., 2010). The estimates of MMRs during 1990 to 2008 for many 
countries reveals, for the first time, a substantial decline of 30-45 percent in the number of annual maternal deaths worldwide (Hogan et al., 2010; Rajaratnam et al., 2010; Yadamsuren, 2010; Lozano et al., 2011). However, a recent United Nations report (2010) on MDGs evaluation suggests "though progress has been made in terms of IMR and MMR, it is uneven. Moreover, without a considerable push forward, many of MDG targets are likely to be missed out in most regions. Old and new challenges threaten to further slow progress in some areas or even undo the successes achieved so far". WHO et al. (2012) also reported "nearly 80 percent of all maternal deaths in 2008 crop up in just 21 countries, and half of all maternal deaths are occurring in six countries: India, Nigeria, Pakistan, Afghanistan, Ethiopia and the Democratic Republic of the Congo". "A comparison of maternal mortality rates in Canada and Afghanistan reveal that Canada has one of the lowest in the world, with 7 deaths per 100,000 live births in 2008, whereas the Afghanistan's maternal mortality rate was 1575 deaths per 100,000 live births in 2008" ( Hogan et al., 2010). The rate of change in the maternal mortality ratio varies widely across countries, "from an annualised decline of more than 8 percent in the Maldives to an increase of 5.5 percent in Zimbabwe during 1990 to 2008. Moreover, only 31 developing countries in the world are likely to meet the MDG 4 goal in case of child mortality indicators" due to unequal progress in child mortality indicators across world countries (Rajaratnam et al., 2010).

Indian experience of mortality transition also foster similar pattern. According to the Sample Registration System reports, the expectation of life at birth in India increased from 50 years during 1970-75 to 63 years during 2001-05 (Office of Registrar General of India, 2007). The average life of an Indian is increased by about 13 years in a period of 30 years. The increase in the average life of a male (12 years) was, however, slower than that of a female (15 years). Similarly, an increase in the expectation of life at birth had been more rapid in rural (14 years) as compared to urban areas (10 years) (Navaneetham, 1993; Chaurasia, 2006). Further, LEB levels varied across states. Transition has been fast by international standards in Kerala, Tamil Nadu and Himachal Pradesh and very slow in Bihar, Madhya Pradesh and Uttar Pradesh etc. In recent years, considerable inter-state disparity continues to persist (Namboodiri, 1996; Goli and Arokiasamy, 2013). 
The situation of IMR also more or less similar in spite of tremendous progress achieved over the period, the latest SRS report reveals that IMR for India as a whole is 44 per 1000 live births. However, a comparison of IMR among the major states reveals that it varies from 12 per 1000 live births in Kerala to 59 per 1000 live births in Madhya Pradesh. The six states of India have IMR more than 50 per 1000 live births. Kerala and Tamil Nadu are the only states having IMR of less 30 per 1000 live births (RGI, 2009).

A comparison of the estimates of MMR of the year 2007 with the year 1997-98 shows that, over a period of 10 years, India could avert 186 maternal deaths per 100,000 live births. Similarly, Assam and Kerala could avert 178 and 69 maternal deaths per 100,000 live births, respectively (RGI, 1997-2003, 2011). The findings of a study by Mukhopadhyay (2012) foster that the decline in MMR is not uniform across the states, even though the overall MMR in India is steadily declining. According to the latest sample registration system report (RGI, 2013), the MMR in India is 212 per 100,000 live births, with the highest rate in the state of Assam (390 per 100,000 live births) and the lowest in the state of Kerala (81 per 100,000 live births).

As discussed above, studies in global context have suggested that the global progress in mortality indicators does not warrant convergence across the countries with the progress in mortality rates (Neumayer, 2004, McMichael et al., 2004, Becker et al., 2005; Moser et al., 2005, Dorius, 2008, 2010; Bloom and Canning, 2009; Goli and Arokiasamy, 2013 etc.). Few studies also suggested recent setbacks in convergence of life expectancy at birth across the countries worldwide (Neumayer 2004; McMichael et al, 2004; Becker et al., 2005; Moser et al. 2005; Bloom and Canning, 2009, Dorius, 2008, 2010; Clark, 2011; Gächter and Engelbert, 2011). The expectation that emerged in the 1960s and 1970s of the world gains in LEB does not guarantee convergence in population health status by a general deterministic process (McMichael et al., 2004). The studies suggest that such a situation arises due to three main reasons: first, the differences in health care seeking pattern by the poor and rich in transitional economies. Second, the newly arising combinations of social, economic and political conditions, and altered relations with the microbiological world and exposure to it (poor expose and more compared to the rich), which causes a reversal in death rates. That is, gains in longevity could be less smooth and less certain than what earlier notions of global convergence had suggested (Moser et al., 2004). This reflects deleterious 
socioeconomic and political conditions, often with unequal access to health care (Whitehead et al., 2001). Moreover, the burden of injury, impaired and mental health problems and deaths from violence, conflict and war are on the rise. There is also a rise in health risks a consequent to large scale environmental change caused by population pressure (WHO, 2002; Neumayer, 2004). Third, the health disparities in later life increase with the increasing proportion of the older population and the socioeconomic disparities (Perlman, 2008).

On the other hand, the optimistic view suggests that the current mortality trends towards divergence will rebound with convergence, possibly as soon as 2015 (Neumayer, 2004). Wilson (2011) believing on his simple graphical presentation of trends re-stressed the argument of global mortality convergence. However, I strongly feel the debate of convergence in health is yet to be fully explored from both theoretical and methodological perspectives. Given the importance of this question, recently few research studies have attempted to address the issue, but all of them focused typically on worldwide mortality (LEB and IMR) trends or in the developed world context (e.g. Bloom and Canning, 2007; Montero-Granados et al., 2007; Taylor, 2009; Dorius, 2010; Clark, 2011; Gächter and Theurl, 2011; Wilson, 2011).

In the developing countries, despite considerable progress in mortality reduction, there is hardly any attempt for testing convergence hypothesis for mortality transition, especially at the sub-national or local level. Moreover, till now, there are no studies in India, which attempted a comprehensive assessment of mortality transition by states and socioeconomic groups with improved models of transition and convergence analyses. Testing a convergence hypothesis for mortality transition reveal whether, the reported progress in the averages of the LEB, maternal and child mortality indicators in country is, contributed from few developed states or all the states. With this perspective, this chapter attempts to analyse the dynamics and anomalies in mortality transition in India during in a 40 year period, between 1971 through 2011.

\subsection{Data source}

In this chapter, this study used sample registration system (RGI, 1981-2011) data on life expectancy at birth, maternal mortality ratio and infant mortality rate to assess 
mortality transition and convergence. The maternal mortality ratio estimates for the year 2011 also released recently by RGI (RGI, 2013), but this study not included them as a part of our analyses. Their inclusion might bring more interesting estimates of convergence in maternal mortality ratios, but it is too late to change the entire analyses. However, these estimates will be considered in future studies. This chapter also used data on population totals and literacy rates from the Census of India (RGI and Census Commissioner, 1971-2011), and poverty ratio estimates from Planning Commission (Government of India 1973-2006). For detailed description of data source, see chapter 2 on data and methodology.

\subsection{Methods}

The first stage of analyses consisted of trend analyses of mortality measure in terms of LEB, MMR and IMR. Graphical tools and change-point analyses are carried to assess mortality transition. Second, convergences in average mortality rates for all selected indicators were measured by both absolute and conditional $\beta$-convergence measure. Third, Dispersion Measure of Mortality (DMM) and Gini indices were used to assess the convergence in absolute and relative inequalities, respectively. The percentage reduction in DMM and Gini indices are used to show the overall absolute and relative convergence in mortality progress.

\subsection{Results}

\subsubsection{Trends in averages of mortality rates}

Mortality trends in India and its states were measured in terms of progress in key mortality indicators such as LEB, MMR and IMR. Trend assessments from figure 5.1 showed that LEB in India and selected major states has improved over the last 25 years. However, the growth in LEB was slower during the recent decade (2001-2006) as compared to $1980 \mathrm{~s}$ and 1990s. Therefore, since 2001, the trend lines of LEB are plateauing for India and the selected states. However, the trend lines for IMR display a different scenario, indicated consistent declines in IMR during 1981 to 2008 for India and selected states. The gap between the states for IMR narrowed substantially, which was greater during pre-2001 compared to post-2001. However, a substantial gap exists between the demographically advanced states like Kerala and demographically lagging states like Bihar, Madhya Pradesh and Uttar Pradesh, both in terms of LEB and IMR. 
Trends in MMR showed that, over the period, the decline in MMR was universal and the gap between high and low mortality states was declined. However, the existing gap is still high: only two states, Kerala and Tamil Nadu show MMR of less than 100 maternal deaths per 100,000 live births during 2007-09. During the same period, four states showed more than 300 maternal deaths per 100000 live births. The south-north divide was clearly evident in terms of MMR. Additionally, mortality transition gap between EAG and non-EAG states also clearly evident.

Figure 5.1. Trends in life expectancy at birth, maternal mortality ratio, infant mortality rate in India and major states; 1981-2009

LEB

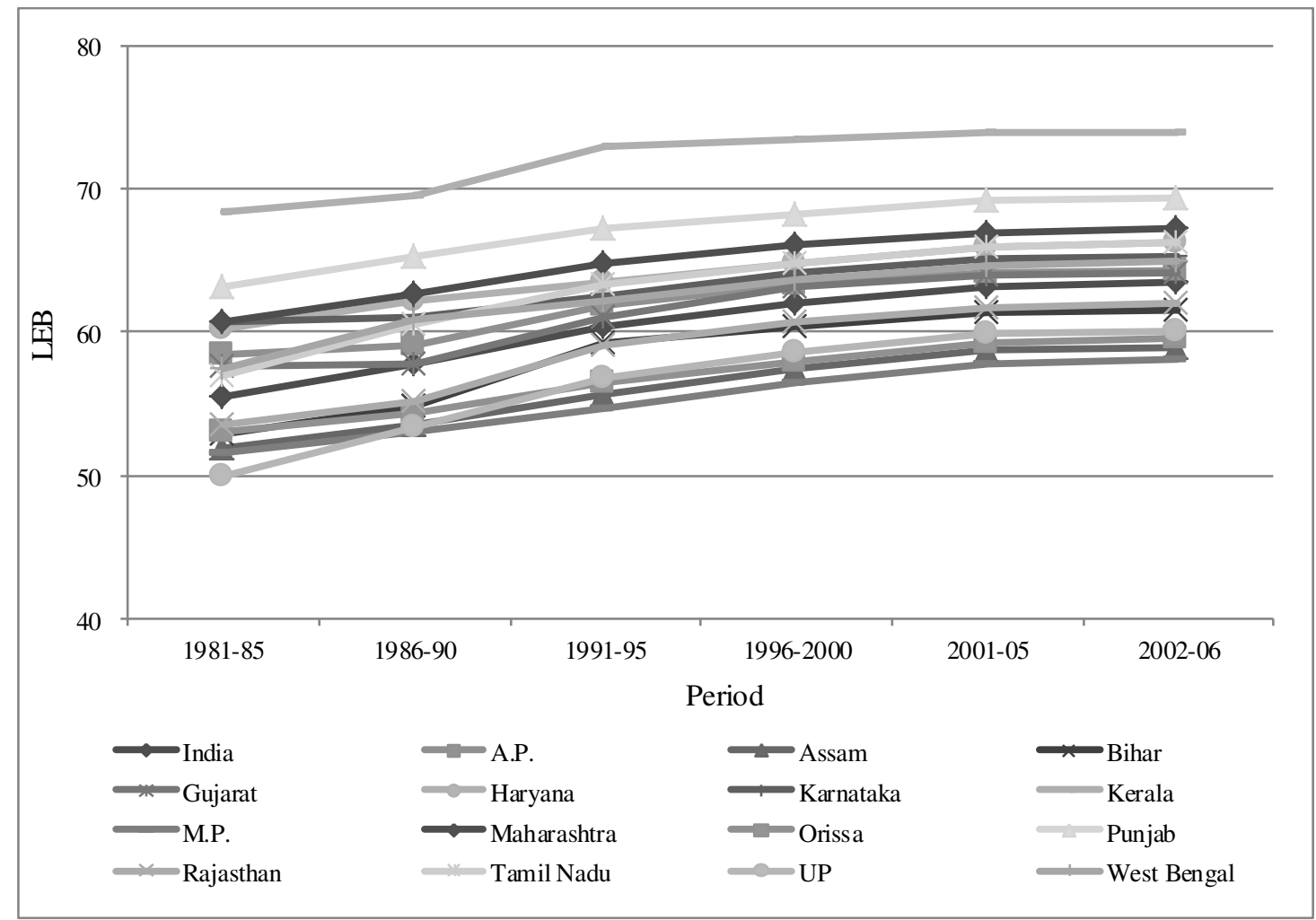


MMR

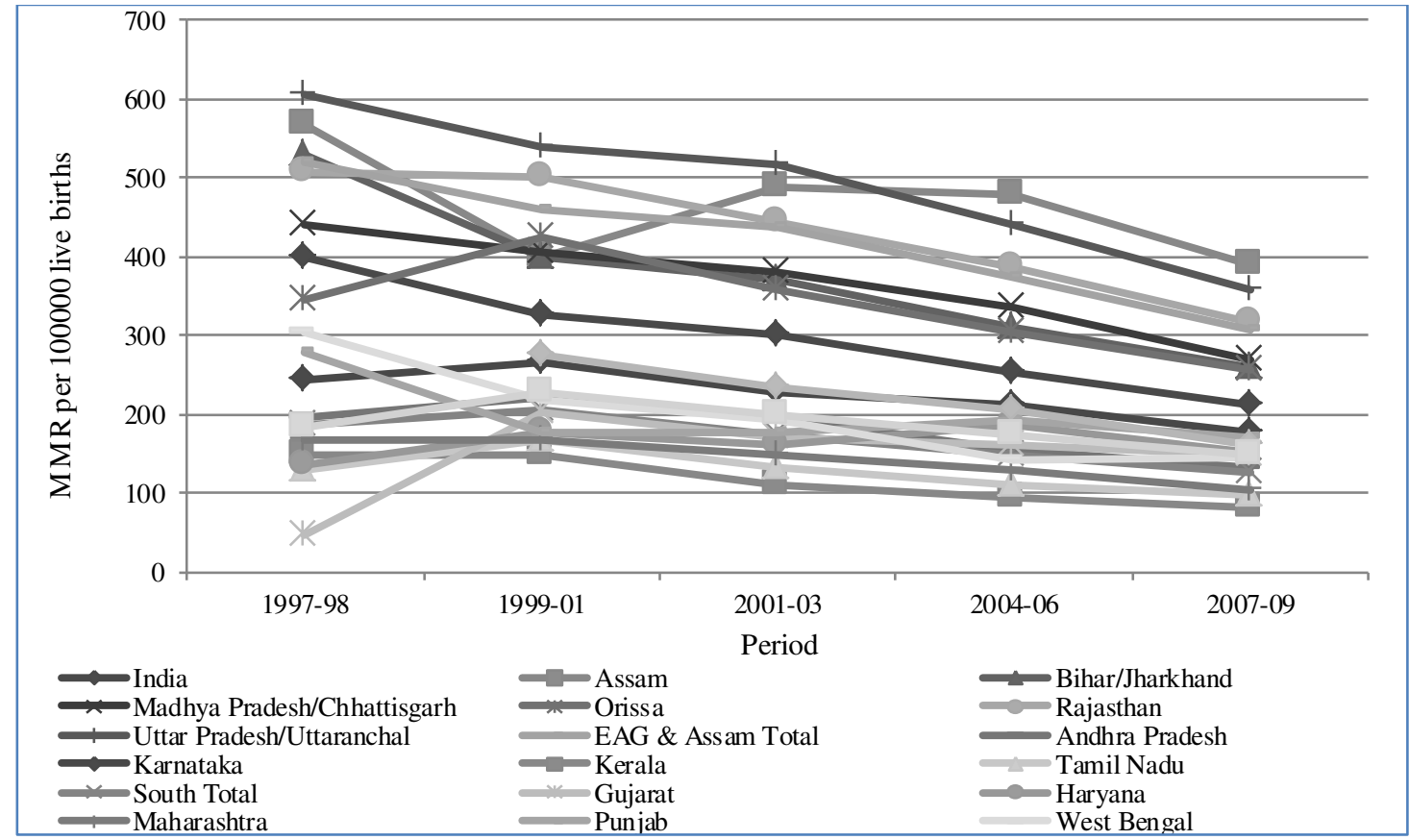

IMR

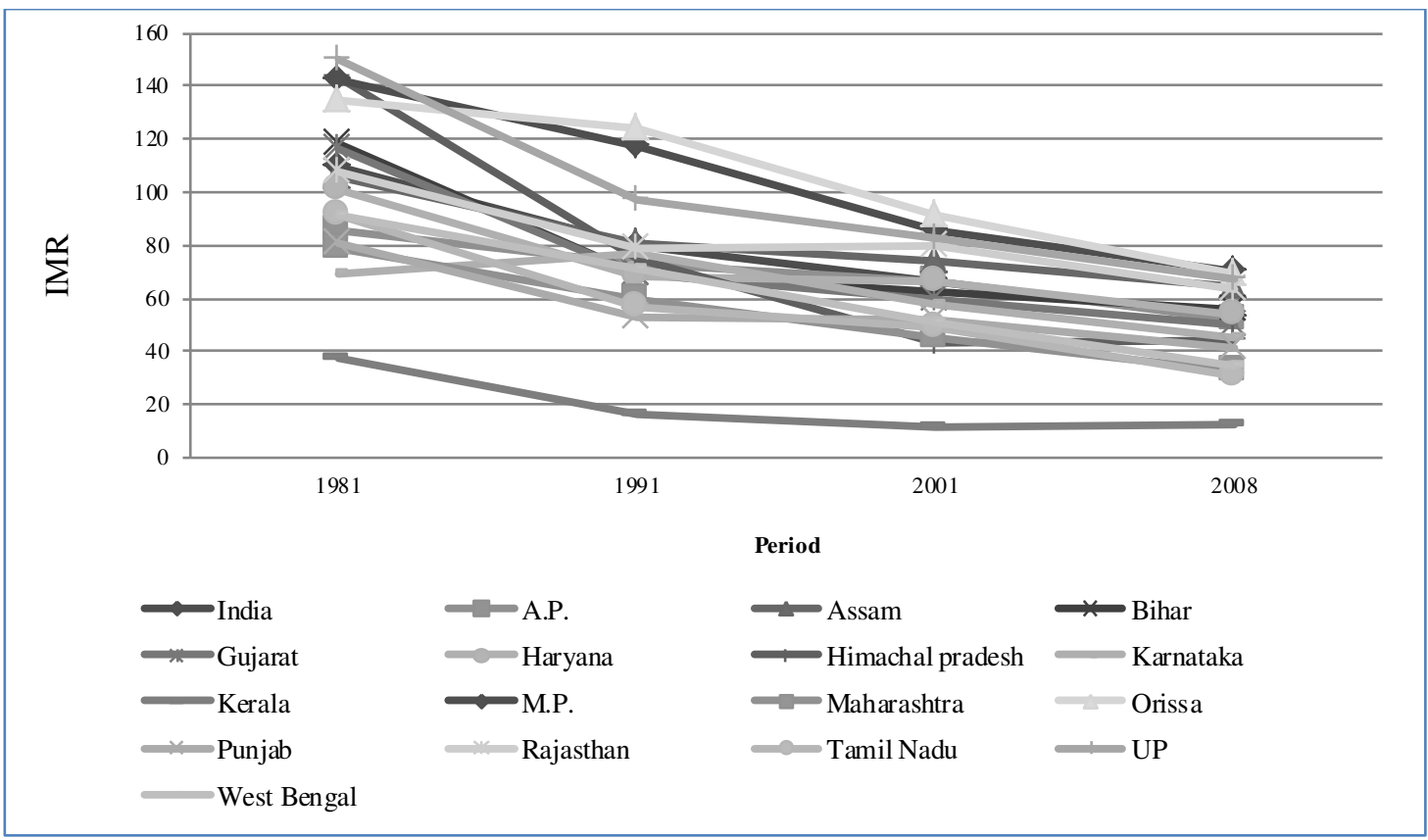

Cumulative distribution of the population of major state of India by LEB, IMR, MMR levels, 1981 to 2008 are presented in figure 5.2. In case of LEB, the results revealed that over the period the greater proportion of population shifting from lower LEB levels to higher LEB. In 1981, half of the population of 15 major states of India were with LEB below 55 years. However, there was an increase of 10 years for average life expectancy for half of the population in 15 major states of India during 1981-2006 that 
means 50 percent of the population living with 65 years and above life expectancy at birth by 2006. In case of IMR, the results showed more progress compared to LEB that over the period, majority of the population of 15 states were shifted to lower mortality rates. While in 1981, 50 percent of population of 15 major states have infant mortality rates above 100 per 1000 live birth but by 2011 half of the population of these states have just below 40 infant deaths for 1000 live births. This considered being remarkable achievement, though current levels are still high compared other developed countries. The results in case of MMR also showed a remarkable improvement. However, in these particular analyses, the assessment for MMR was carried out for only recent decade due to lack of reliable data for previous decades. The results revealed that during 2001, half of the population of 15 major states have MMR of above 250 maternal deaths per 100000 live births. In the duration of one decade, half of the population of these states have 150 maternal deaths per 100000 live births. Though, this is a sizeable achievement, but current levels of MMR in some states are still very high. Further, India as a whole and many of its states are also likely to miss the achievement of MDG goal 5 by 2015 .

Figure 5.2. Cumulative distribution of the population of major state of India by LEB, IMR, MMR levels, 1981 to 2011

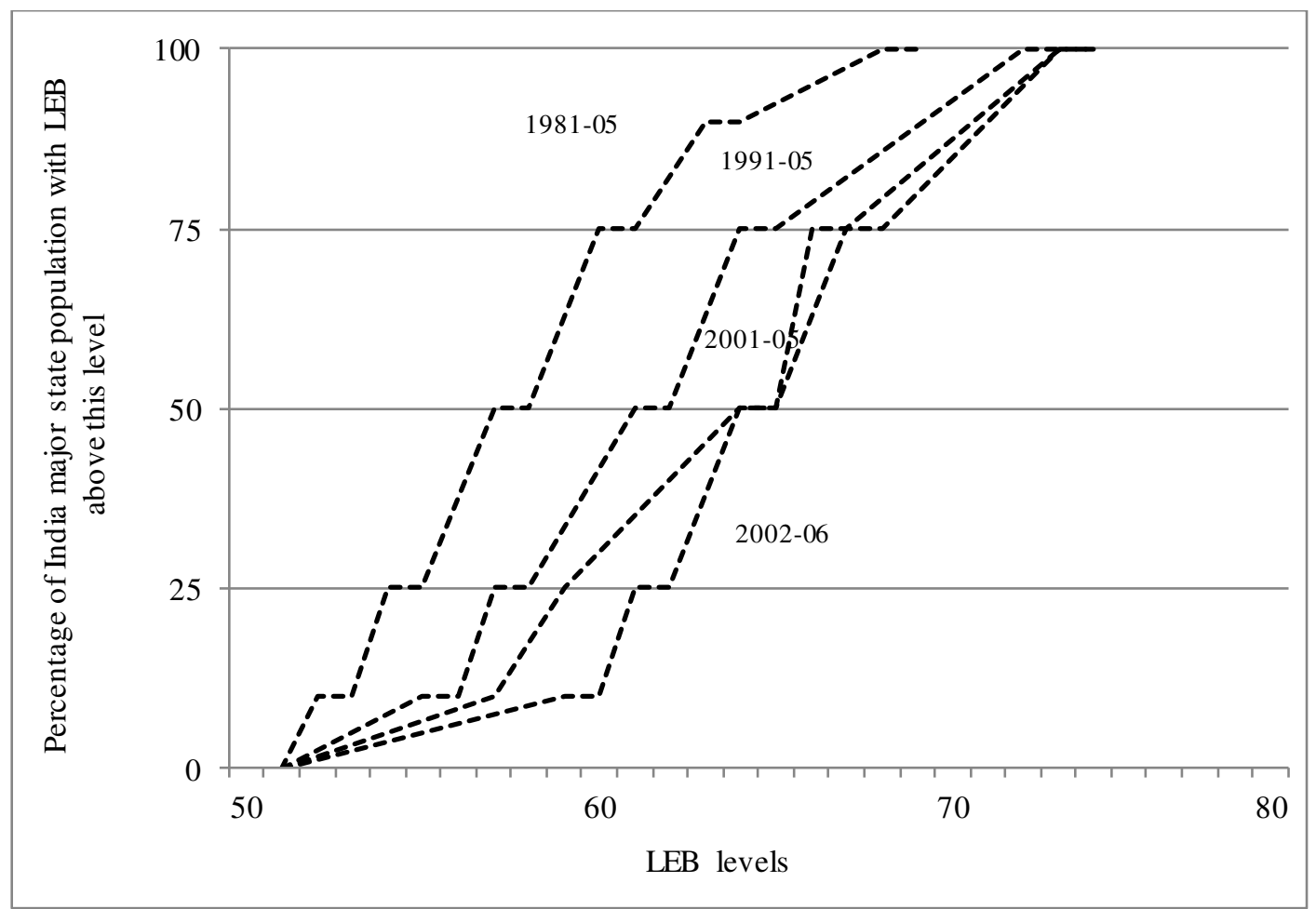



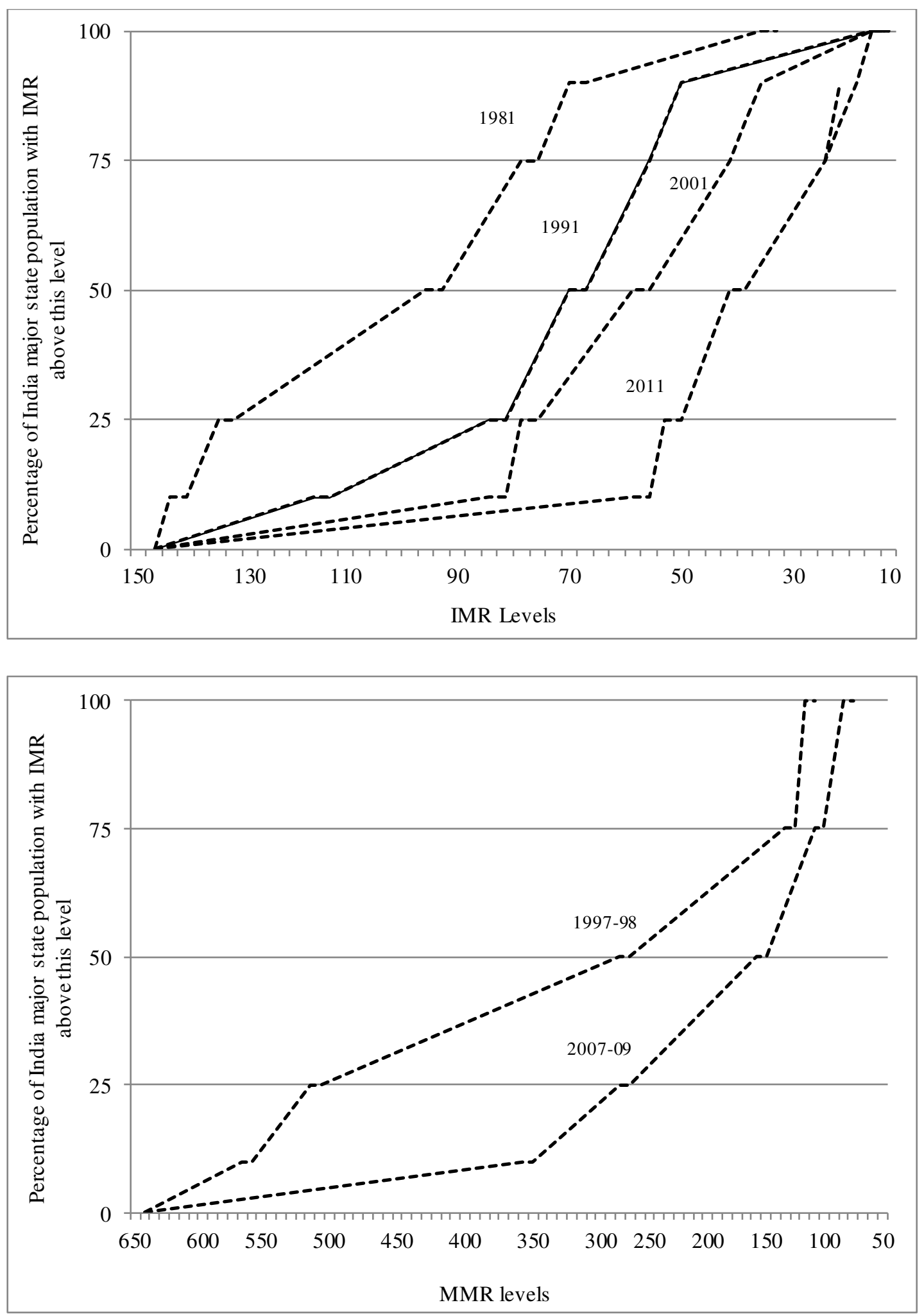

Change-point analysis was also considered to assess the trends in IMR for 15 major states during 1971-2009. In case of IMR, the results showed a huge state level variation in critical change-points. States like Haryana, Kerala, Rajasthan and Tamil Nadu experienced critical change-points in the pre-1980s or in the 1980. Out of these three 
states, Haryana experienced critical change-point in 1972 not because of decrease in IMR, but due to increase of IMR. However, other four states showed change-points due to decrease in IMR in the pre-1980s. Further, majority of the states experienced their first critical change-points in early eighties or in mid eighties. Though, some of the EAG states which experienced their first critical change-points in pre-1980s, but their second critical change-points were very late that as late as in 1990s. However, Kerala and Tamil Nadu are the two states which were also experienced their second critical change-point during early 1980s. Overall, majority of the states experienced their second critical change-point very late. The state of Bihar experienced its first critical change-point in 1991, second in 1999 and third in 2007, which is very late transition with reference to southern states of India (Table 5.1).

Table 5.1. Estimates of change-point analyses: significant change-points for IMR of India and selected major states, 1950-2009

\begin{tabular}{|c|c|c|c|c|c|}
\hline \multirow{2}{*}{ India/ states } & \multirow{2}{*}{ Change points } & \multicolumn{2}{|c|}{ Volume of change } & \multirow{2}{*}{$\begin{array}{c}\text { Confidence } \\
\text { Interval }\end{array}$} & \multirow{2}{*}{$\begin{array}{c}\text { Confidence } \\
\text { level }(\%)\end{array}$} \\
\hline & & From & To & & \\
\hline \multirow{5}{*}{ India } & 1978 & 162.09 & 113.5 & $(1975,1978)$ & 98 \\
\hline & 1984 & 113.5 & 96.16 & $(1984,1984)$ & 95 \\
\hline & 1990 & 96.16 & 76.83 & $(1990,1990)$ & 99 \\
\hline & 1996 & 76.83 & 69.83 & $(1996,1996)$ & 99 \\
\hline & 2002 & 69.83 & 57.74 & $(2002,2002)$ & 98 \\
\hline \multirow{4}{*}{ Andhra Pradesh } & 1982 & 109.9 & 77.82 & $(1982,1982)$ & 100 \\
\hline & 1993 & 77.82 & 65.22 & $(1992,1993)$ & 100 \\
\hline & 2002 & 65.22 & 58.6 & $(2001,2002)$ & 98 \\
\hline & 2007 & 58.6 & 48.8 & $(2007,2007)$ & 96 \\
\hline \multirow{2}{*}{ Assam } & 1996 & 85.22 & 74.42 & $(1995,1996)$ & 95 \\
\hline & 2003 & 74.42 & 63.56 & $(2003,2004)$ & 100 \\
\hline \multirow{3}{*}{ Bihar } & 1991 & 99.5 & 70.12 & $(1990,1991)$ & 100 \\
\hline & 1999 & 70.12 & 61.25 & $(1999,1999)$ & 100 \\
\hline & 2007 & 61.25 & 51.6 & $(2005,2007)$ & 97 \\
\hline \multirow{5}{*}{ Gujarat } & 1980 & 136.11 & 108.14 & $(1978,1981)$ & 97 \\
\hline & 1987 & 108.14 & 80.16 & $(1986,1987)$ & 95 \\
\hline & 1993 & 80.17 & 62 & $(1993,1995)$ & 99 \\
\hline & 2001 & 62 & 56.17 & $(1998,2001)$ & 100 \\
\hline & 2007 & 56.17 & 47 & $(2007,2007)$ & 98 \\
\hline \multirow{5}{*}{ Hary ana } & 1972 & 72 & 102.85 & $(1972,1975)$ & 97 \\
\hline & 1985 & 102.85 & 85.8 & $(1982,1985)$ & 95 \\
\hline & 1990 & 85.8 & 69.1 & $(1990,1992)$ & 96 \\
\hline & 2000 & 69.1 & 61.71 & $(1998,2000)$ & 100 \\
\hline & 2007 & 61.7 & 50.4 & $(2006,2007)$ & 95 \\
\hline \multirow{3}{*}{ Karnataka } & 1980 & 87 & 72.38 & $(1980,1981)$ & 100 \\
\hline & 1993 & 72.38 & 58.8 & $(1992,1993)$ & 100 \\
\hline & 2003 & 58.8 & 45 & $(2003,2003)$ & 100 \\
\hline \multirow{3}{*}{ Kerala } & 1977 & 56.5 & 41.8 & $(1977,1977)$ & 95 \\
\hline & 1982 & 41.8 & 25.18 & $(1982,1985)$ & 97 \\
\hline & 1993 & 25.18 & 13.10 & $(1993,1995)$ & 100 \\
\hline \multirow{5}{*}{ Madhy a Pradesh } & 1982 & 143.64 & 123 & $(1980,1982)$ & 100 \\
\hline & 1989 & 123 & 111 & $(1987,1989)$ & 96 \\
\hline & 1994 & 111 & 96 & $(1994,1994)$ & 96 \\
\hline & 2000 & 96 & 82.67 & $(2000,2000)$ & 99 \\
\hline & 2006 & 82.67 & 67.33 & $(2006,2006)$ & 98 \\
\hline \multirow{2}{*}{ Maharashtra } & 1980 & 95.66 & 71.55 & $(1980,1980)$ & 100 \\
\hline & 1989 & 71.55 & 56.57 & $(1988,1989)$ & 99 \\
\hline
\end{tabular}




\begin{tabular}{|c|c|c|c|c|c|}
\hline & 1996 & 56.57 & 48 & $(1995,1996)$ & 98 \\
\hline & 2001 & 48 & 40.8 & $(2001,2001)$ & 96 \\
\hline & 2006 & 40.8 & 31 & $(2006,2006)$ & 96 \\
\hline \multirow{4}{*}{ Orissa } & 1986 & 137.13 & 121.86 & $(1983,1986)$ & 100 \\
\hline & 1993 & 121.86 & 100.43 & $(1993,1993)$ & 100 \\
\hline & 2000 & 100.43 & 84.66 & $(2000,2000)$ & 99 \\
\hline & 2006 & 84.66 & 66 & $(2006,2006)$ & 96 \\
\hline \multirow{3}{*}{ Punjab } & 1982 & 102.09 & 67.66 & $(1981,1982)$ & 100 \\
\hline & 1991 & 67.66 & 52.91 & $(1991,1992)$ & 100 \\
\hline & 2003 & 52.91 & 40.88 & $(2003.2003)$ & 100 \\
\hline \multirow{4}{*}{ Rajasthan } & 1979 & 138.86 & 106 & $(1979,1980)$ & 100 \\
\hline & 1990 & 106 & 83.9 & $(1990,1990)$ & 100 \\
\hline & 2000 & 83.9 & 73.42 & $(1998,2000)$ & 100 \\
\hline & 2007 & 73.42 & 58.8 & $(2005,2007)$ & 96 \\
\hline \multirow{6}{*}{ Tamil Nadu } & 1977 & 111.67 & 94.71 & $(1977,1977)$ & 99 \\
\hline & 1984 & 94.71 & 76.16 & $(1983,1984)$ & 98 \\
\hline & 1990 & 76.16 & 57.8 & $(1989,1990)$ & 98 \\
\hline & 1995 & 57.8 & 52.66 & $(1995,1995)$ & 96 \\
\hline & 2001 & 52.66 & 41.83 & $(2001,2001)$ & 100 \\
\hline & 2007 & 41.83 & 28 & $(2007,2007)$ & 98 \\
\hline \multirow{5}{*}{ Uttar Pradesh } & 1979 & 179.75 & 154.83 & $(1978,1979)$ & 100 \\
\hline & 1985 & 154.83 & 117.12 & $(1985,1986)$ & 98 \\
\hline & 1993 & 117.12 & 87.16 & $(1992,1993)$ & 100 \\
\hline & 1999 & 87.16 & 78.71 & $(1999,1999)$ & 99 \\
\hline & 2006 & 78.71 & 64.66 & $(2005,2006)$ & 99 \\
\hline \multirow{4}{*}{ West Bengal } & 1985 & 85.75 & 70.12 & $(1985,1987)$ & 94 \\
\hline & 1993 & 70.12 & 56.14 & $(1992,1993)$ & 100 \\
\hline & 2000 & 56.14 & 47.4 & $(1999,2000)$ & 100 \\
\hline & 2005 & 47.4 & 34.85 & $(2005,2005)$ & 97 \\
\hline
\end{tabular}

Note: Estimates are based on thousand bootstraps without replacement, CUSUM estimates and analyses of IMR ranks. The confidence level for IMR changes is $50 \%$, and the confidence level for Inclusion in the table is $90 \%$.

\subsubsection{Trends in overall and socioeconomic inequalities in mortality}

In this chapter, this study measured the convergence for two types of mortality inequalities: first, overall mortality inequality and second, socioeconomic inequality in infant mortality rates. Within the domain of mortality inequality, this study measured absolute and relative inequalities in mortality by using a Dispersion Measure of Mortality (DMM) and Gini index, respectively. DMM is based on estimated absolute interstate population mortality status, weighted by their population sizes. Gini coefficient shows the relative interstate inequality in mortality. Decrease or increase in DMM shows changes in absolute inequality in mortality among the population of selected states. Similarly, change in the Gini coefficient indicates the change in relative inequality of mortality (Shkolnikov et al., 2003; Saikia, 2011). 
Figure 5.3. Trends in DMM and Gini Index measures for LEB, India, 1980-2006

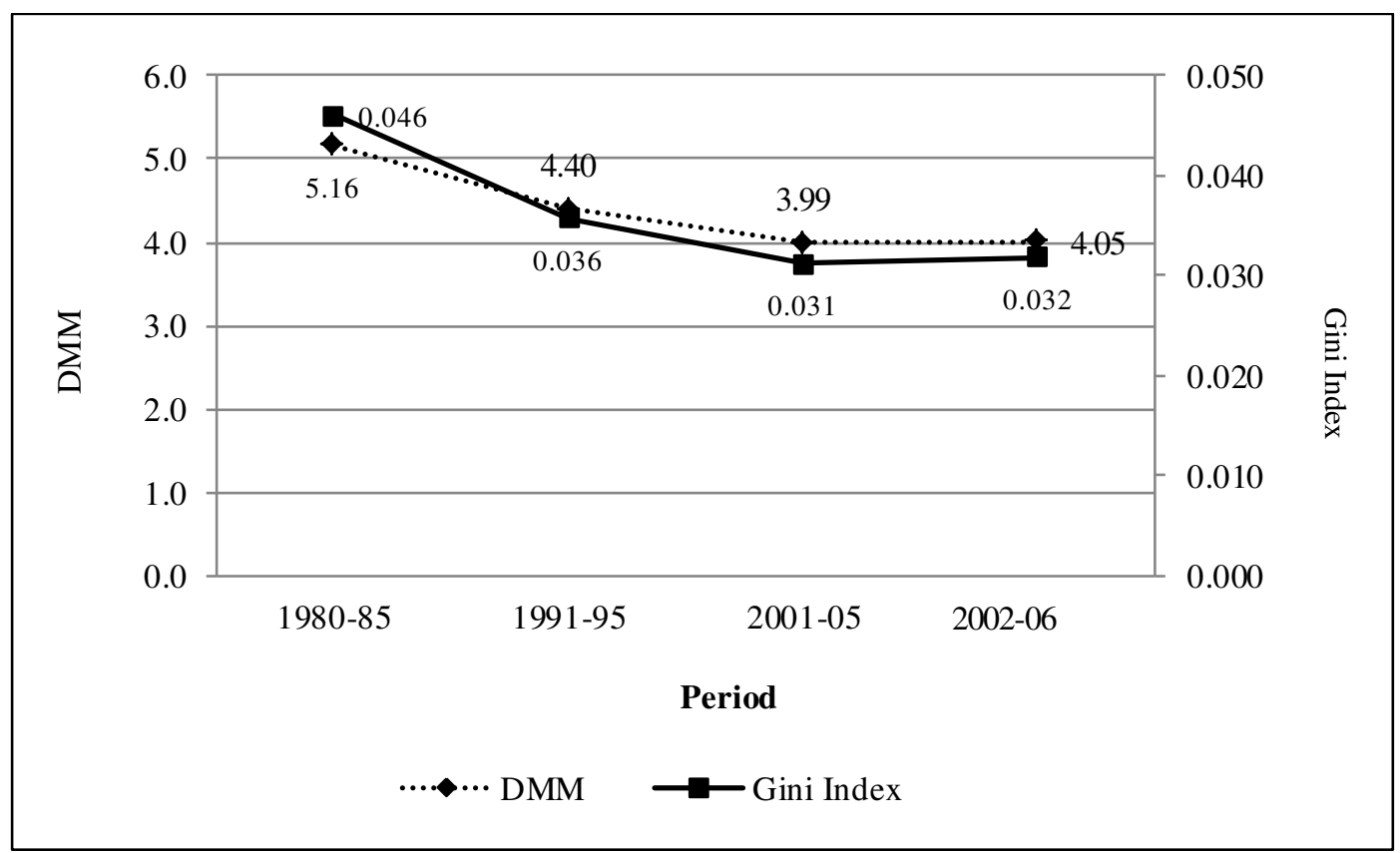

Note: 1) States: $n=15$

Figure 5.3 presents the estimates of DMM and Gini indices of LEB among major Indian states during 1981-2006. Results revealed that DMM decreased until 2001-2005, but signaled a rising trend thereafter. Similar trends were also observed for Gini index of LEB. The estimates of both indices thus, confirm that the decrease in both absolute and overall relative inequalities in LEB for earlier periods (1980s and 1990s) across Indian states was not sustained for recent time periods (2001-2006), as inequality in LEB indicated a rising trend since 2001. Unlike the trends in LEB, DMM and Gini coefficient trends in IMR indicated a more distinct pattern. DMM for IMR declined steadily during 1981-2008, but conversely, the Gini index values increased substantially. Such pattern of results suggests that though, absolute inequality in IMR was on the decline, relative inequality in IMR continues to rise (Figure 5.4). Figure 5.5 presents the results of dispersion measure of mortality for MMR. The results revealed that the absolute differences for MMR among the 15 major states declined by 100 points over the period of two decades. However, the relative inequalities declined during 1997-2003, but showed a slightly increasing trend in the recent period 20032009. 
Figure 5.4. Trends in DMM and Gini index measures for IMR, India, 1981-2008

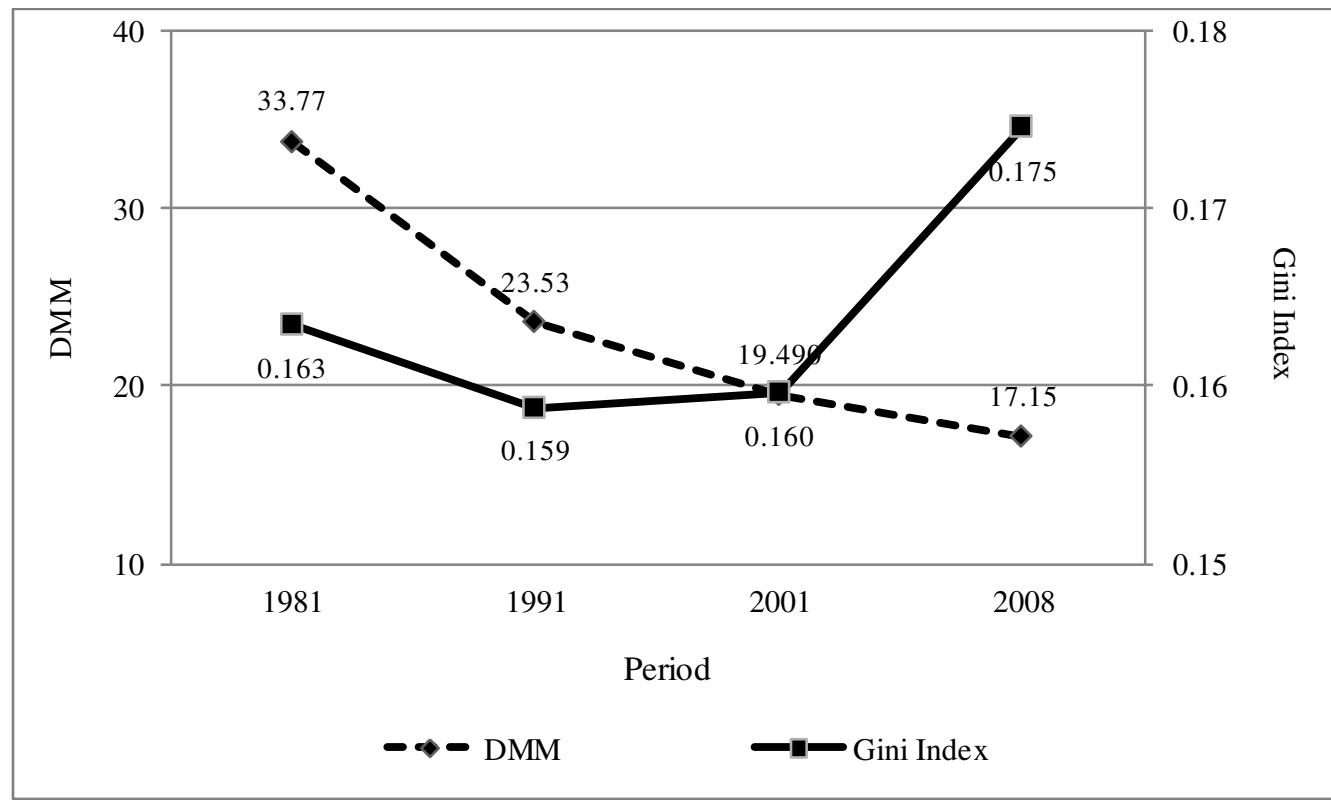

Note: States: $\mathrm{n}=15$

Figure 5.5. Trends of DMM and Gini Index for MMR of Major Indian states, 19972009

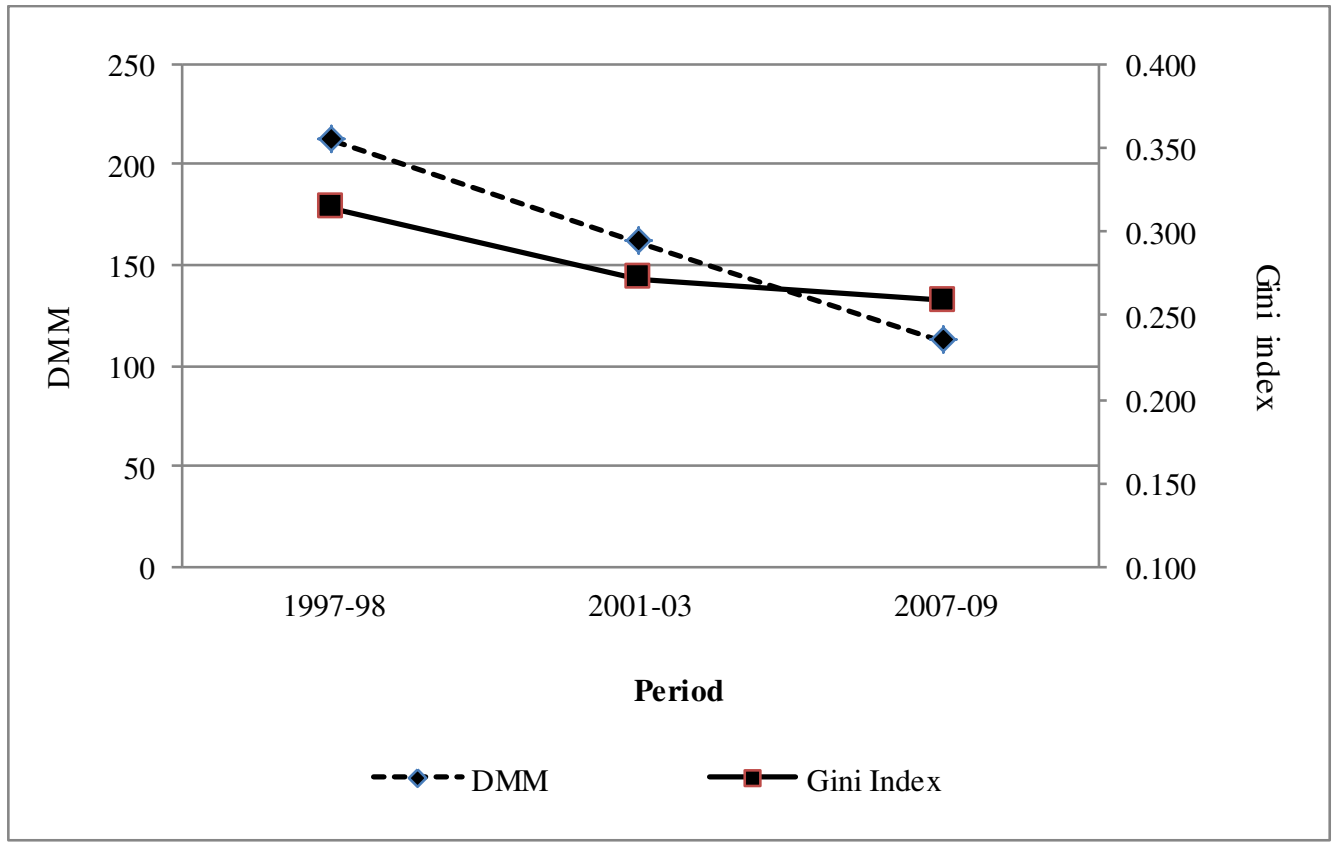

Though, LEB estimates by social groups is not available in India, the socioeconomic inequality in mortality was measured in terms of IMR among socioeconomic groups of selected major states of India (Table 5.2). The trends in socioeconomic inequality in IMR reveal a number of appealing findings. First, complementing the results of DMM, the trend assessment of CIs for IMR showed a declining trend for 11 out of the 16 
selected states of India. However, it must be noted that four of the five states, that experienced an increase in IMR were economically and demographically advanced states. Overall, the trend assessments of absolute, relative and socioeconomic inequalities evidently indicated that inequalities in IMR declined over time. However, inequalities increased for recent periods for LEB. It is important to distinguish from the results that steady improvements in the average mortality rates over time may not necessarily guarantee a reduction in inequalities in mortality rates. Therefore, in the following sections, this chapter assessed convergence for the averages of LEB and IMR and inequalities in LEB and IMR, separately.

Table 5.2. Trends in concentration index for infant deaths in India and major states, 1992- 2006

\begin{tabular}{lcccc}
\hline India/Major States & \multicolumn{4}{c}{ CIs of Infant deaths } \\
\cline { 2 - 5 } & -0.176 & I & III & III-I \\
\hline India & -0.1038 & -0.1816 & -0.1616 & 0.0144 \\
Andhra Pradesh & -0.1463 & -0.0992 & -0.1696 & -0.0658 \\
Assam & -0.1131 & -0.1386 & -0.139 & 0.0073 \\
Bihar & -0.2237 & -0.1823 & -0.1835 & 0.0225 \\
Gujarat & -0.1651 & -0.1857 & -0.0957 & 0.0402 \\
Haryana & -0.1545 & -0.0564 & -0.0722 & 0.0694 \\
Himachal Pradesh & -0.1463 & -0.1527 & -0.155 & -0.0087 \\
Karnataka & -0.2707 & -0.1154 & -0.2207 & 0.05 \\
Kerala & -0.0888 & -0.1396 & -0.1204 & -0.0316 \\
Madhya Pradesh & -0.2121 & -0.1594 & -0.1709 & 0.0412 \\
Maharashtra & -0.1345 & -0.1612 & -0.1286 & 0.0059 \\
Orissa & -0.1439 & -0.1721 & -0.1581 & -0.0142 \\
Punjab & -0.029 & -0.1571 & -0.0841 & -0.0551 \\
Rajasthan & -0.139 & -0.0126 & -0.1045 & 0.0345 \\
Tamil Nadu & -0.1326 & -0.1294 & -0.0931 & 0.0395 \\
Uttar Pradesh & -0.1083 & -0.1898 & -0.1504 & -0.0421 \\
West Bengal & Note: National Family Health Survey- I (1992-1993). National Family Health Survey- II (1998-1999). \\
\multicolumn{1}{c}{ National Family Health Survey- III (2005-2006). } & & & \\
\multicolumn{4}{c}{}
\end{tabular}

\subsubsection{Catching-up process in mortality indicators}

The catching process was examined by plotting the change in LEB, IMR and MMR during 1981-2011 among major states of India by LEB, IMR and MMR levels in initial period, 1981. The results revealed that except for LEB which showed no clear trend of catching-up process, the IMR and MMR showed clear trends of catching-up process. In case of LEB, some states with higher LEB levels also experienced greater changes along with states with lower LEB, thus, it do not showed a clear catching-up process. However, in case of both IMR and MMR the states with higher IMR and MMR showed 
greater progress than lower IMR and MMR, thus, there is a clear evidence of higher IMR and MMR states catching-up with lower IMR and MMR states (Figure 5.6).

Figure 5.6. Change in LEB, MMR, IMR during 1981-2010 for major states of India by LEB, MMR, IMR levels in initial period, 1981

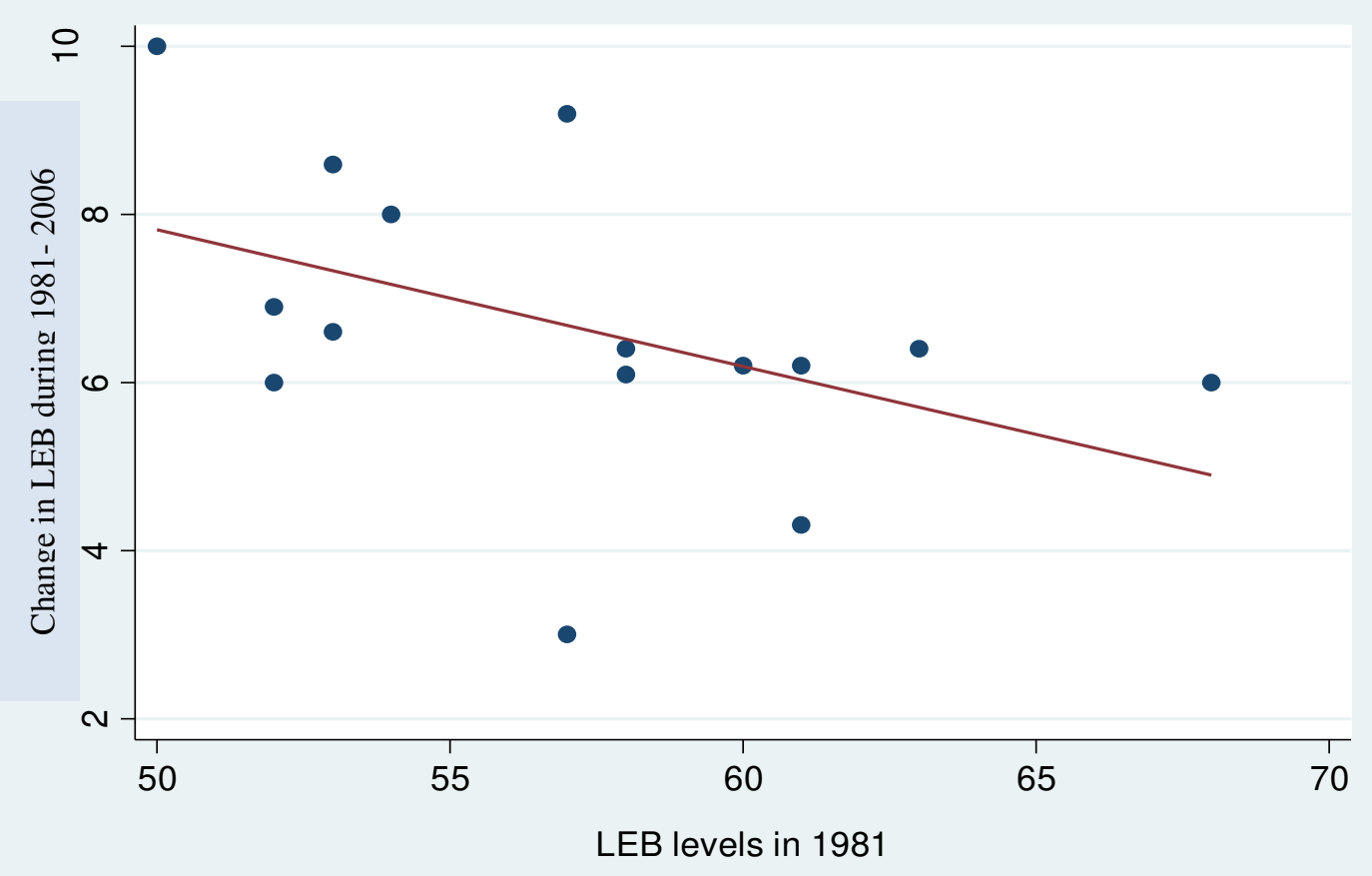

- Change in LEB during 1981- $2006 \quad$ Fitted values

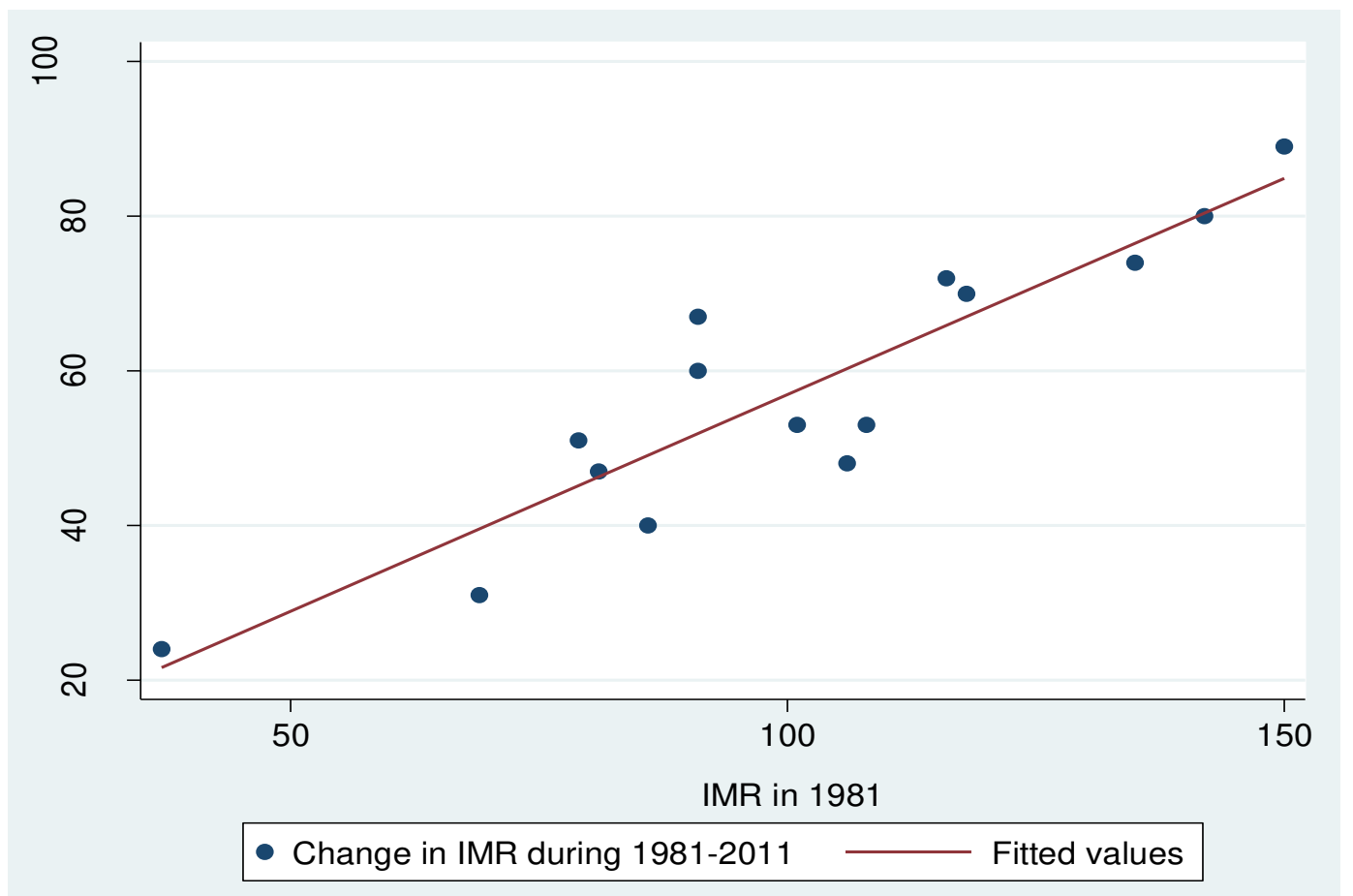




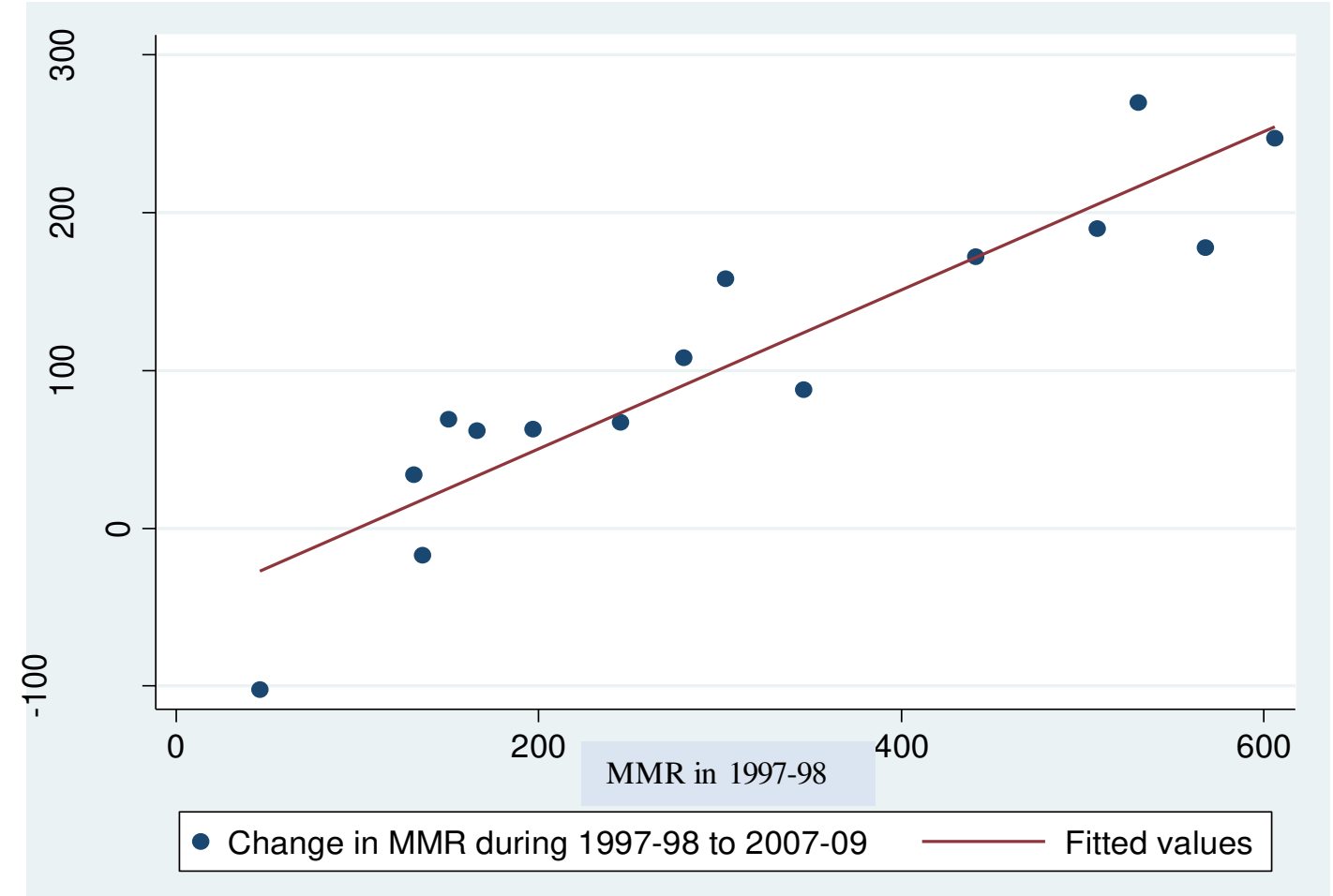

\subsubsection{Convergence in averages of mortality rates: Absolute $\beta$-convergence}

In this section, this chapter assessed the convergence in averages of mortality rates. Convergence in mortality rates was measured based on LEB, IMR and MMR. To measure the convergence in LEB and to explore the possibility of contra underlying trends during the twenty-one year period, piecewise convergence models were estimated. Piecewise convergence estimates indicated convergence process in the smaller intervals.

Table 5.3. Absolute $\beta$ convergence for life expectancy at birth across the major states in India, 1981-2006

\begin{tabular}{lcccc}
\hline Period & $\beta$ coefficient & P value & ${\text { Adjusted } \mathrm{R}^{2}}$ & $\begin{array}{c}\text { Speed of convergence } \\
\text { (\% per annum) }\end{array}$ \\
\hline 1981-85 to 2002-06 & -.015722 & 0.007 & 0.40 & 2.13 \\
$1981-85$ to $1991-95$ & -.027543 & 0.047 & 0.21 & 3.22 \\
$1991-95$ to 2001-05 & -.019977 & 0.000 & 0.85 & 2.23 \\
2001-05 to 2002-06 & -.006587 & 0.763 & 0.069 & 0.67 \\
\hline States: $\mathrm{n}=15$, df 14 & & &
\end{tabular}

The results of absolute $\beta$-convergence estimates showed statistically significant evidence of convergence in LEB during the period, 1981-2006 at the rate of 2.1\% per annum. However, when convergence coefficient was disaggregated for three smaller 
intervals, the volume and speed of convergence $(\beta=-.027543, S=3.2 \%$ per annum, $\mathrm{p}<0.047$ ) was higher during 1985-95 compared to later periods, 1995-2006 (Table 5.3).

Convergence in child mortality rate was measured based on infant mortality rate. Analogous to the process adopted for estimating convergence in LEB, the $\beta$ convergence models for IMR was estimated in two stage analyses: the first stage analyses dealt with $\beta$-convergence between the beginning and end period under observation and, the second stage analyses dealt with piece-wise analyses: $\beta$ convergence for the sub-periods. Akin to LEB, absolute convergence estimates for IMR presented in table 5.4 also revealed that the $\beta$ value for IMR during 1992-2005 was statistically significant $(\beta=-.06527, \mathrm{p}<0.007)$ indicating convergence in IMR. However, Piece-wise regression models for disaggregated periods showed that the convergence was much greater during 1992-1998 ( $\beta=-.14834, \mathrm{p}<0.002)$ than 1999$2005(\beta=-.02565, \mathrm{p}<0.427)$.

Table 5.4. Absolute $\beta$ Convergence for infant mortality rate across the major states, India, 1992-2006

\begin{tabular}{lcccc}
\hline Period & $\beta$ coefficient & $\mathrm{P}$ value & ${\text { Adjusted } \mathrm{R}^{2}}$ & $\begin{array}{c}\text { Speed of } \\
\text { convergence } \\
\text { (\% per annum) }\end{array}$ \\
\hline $1992-93$ to $2005-06$ & -.06527 & 0.007 & 0.24 & 6.7 \\
$1992-93$ to 1998-99 & -.14834 & 0.002 & 0.31 & 16.1 \\
$1998-99$ to 2005-06 & -.02565 & 0.427 & 0.14 & 2.6 \\
\hline
\end{tabular}

States: $\mathrm{n}=24$, df 23

The results presented in table 5.5 show an absolute convergence in MMR $(\beta=$ $.0003776, \mathrm{p}<.0060)$ for the period, 2001-03 to 2007-09. A comparative assessment of the convergence estimates for two mortality indicators during 1992-2005 revealed that the speed of $\operatorname{IMR}(S=7 \%$ per annum) is greater than the LEB $(S=2.13 \%$ per annum). The convergence was more for the recent period for children full immunisation, whereas convergence was greater in the earlier period for children underweight and IMR.

Table 5.5. Absolute $\beta$ convergence for maternal mortality ratio across the major states, India, 1997-2009

\begin{tabular}{|c|c|c|c|}
\hline \multirow[t]{2}{*}{ Period } & $\beta$ coefficient & \multirow{2}{*}{$\begin{array}{c}\text { Adjusted } \\
\mathrm{R}^{2}\end{array}$} & \multirow{2}{*}{$\begin{array}{c}\text { Speed of convergence } \\
\text { for infant mortality rate } \\
(\% \text { per annum })\end{array}$} \\
\hline & Maternal mortality ratio & & \\
\hline $1997-98$ to $2007-09$ & $-.0003776 \quad(.0060)$ & 0.71 & $0.04 \%$ \\
\hline
\end{tabular}

Note: 1 . States: $\mathrm{n}=15$, df 14; 2 . () P value 


\subsubsection{Conditional $\beta$-convergence in overall and child health status}

Although, the absolute convergence model presented evidential support for unconditional convergence, it is not reasonable to assume that all the states do share same socioeconomic conditions. Therefore, to account for socioeconomic variability of the states, conditional $\beta$-convergence was estimated by including two more explanatory variables: proportion of illiterate population and proportion of population in poor wealth quintile in the regression model. The conditional $\beta$-convergence estimates for life expectancy at birth also showed evidence of convergence as indicated by negative $\beta$ coefficient $(\beta=-.03197, \mathrm{p}<0.016)$ during 1981-2006 (Table 5.6). The estimates for shorter intervals within the larger intervals also support convergence hypothesis for all the three periods under consideration. Consistent with the pattern observed for absolute $\beta$-convergence model estimates, piecewise conditional $\beta$-convergence model estimates also showed greater volume and speed of convergence and statistical significance $(\beta=$ $.06573, S=10 \%$ per annum, $\mathrm{p}<0.050)$ during 1985-1995 than recent period, 20052006, $(\beta=-.01062, S=2.5 \%)$. Overall, results imply that both absolute and conditional $\beta$-convergence estimates in terms of negative coefficient point to sizeable evidence of Indian states becoming similar in terms of the levels of LEB during 1981-2006. However, conditional $\beta$-convergence estimates revealed greater volume and speed of convergence compared to unconditional $\beta$-convergence estimates.

Table 5.6. Conditional $\beta$ convergence for life expectancy at birth across the major states in India, 1981-2006

\begin{tabular}{|c|c|c|c|c|c|}
\hline \multirow[t]{2}{*}{ Period } & \multicolumn{3}{|c|}{$\beta$ coefficient } & \multirow{2}{*}{$\begin{array}{c}\text { Adjusted } \\
\mathrm{R}^{2}\end{array}$} & \multirow{2}{*}{$\begin{array}{c}\text { Speed of } \\
\text { convergence for } \\
\text { LEB } \\
(\% \text { per annum })\end{array}$} \\
\hline & LEB & $\begin{array}{l}\text { proportion of } \\
\text { illiterate } \\
\text { population }\end{array}$ & $\begin{array}{l}\text { Poverty } \\
\text { ratio }\end{array}$ & & \\
\hline $\begin{array}{l}1981-85 \text { to } 2002- \\
06\end{array}$ & $\begin{array}{l}-.03197 \\
(0.016)\end{array}$ & $\begin{array}{l}.00585 \\
(0.179)\end{array}$ & $\begin{array}{l}-.00458 \\
(0.121)\end{array}$ & 0.44 & 3.99 \\
\hline $\begin{array}{l}1981-85 \text { to } 1991- \\
95\end{array}$ & $\begin{array}{l}-.06573 \\
(0.050)\end{array}$ & $\begin{array}{l}.01558 \\
(0.180)\end{array}$ & $\begin{array}{l}-.00770 \\
(0.312)\end{array}$ & 0.21 & 10.71 \\
\hline $\begin{array}{l}1991-95 \text { to } 2005- \\
05\end{array}$ & $\begin{array}{l}-.02780 \\
(0.000)\end{array}$ & $\begin{array}{l}.00252 \\
(0.060)\end{array}$ & $\begin{array}{r}-.00168 \\
(0.062)\end{array}$ & 0.89 & 3.26 \\
\hline $\begin{array}{l}2001-05 \text { to } 2002- \\
06\end{array}$ & $\begin{array}{l}-.01062 \\
(0.711)\end{array}$ & $\begin{array}{r}-.00030 \\
(0.976)\end{array}$ & $\begin{array}{l}-.00340 \\
(0.638)\end{array}$ & 0.23 & 2.52 \\
\hline
\end{tabular}

Note: 1 . States: $\mathrm{n}=15$, df 14

2. () P value

Commensurate with absolute $\beta$-convergence estimates, the conditional $\beta$-convergence estimates for IMR indicated negative $\beta$ values for all three periods, thereby indicating 
convergence in IMR over time. Similar to absolute $\beta$-convergence estimates of IMR, conditional $\beta$-convergence estimates also suggests that the volume of convergence $(\beta=.-28982, \mathrm{p}<0.000)$ was higher during 1992-1998 than $(\beta=-.12649, \mathrm{p}<0.023) 1998$ 2006. Moreover, the estimates of speed of convergence and goodness of fit measured by adjusted $\mathrm{R}^{2}$ also indicated similar pattern (Table 5.7 ).

Table 5.7. Conditional $\beta$ convergence for infant mortality rate across the major states, India, 1992-2006

\begin{tabular}{|c|c|c|c|c|c|}
\hline \multirow[t]{2}{*}{ Period } & \multicolumn{3}{|c|}{$\beta$ coefficient } & \multirow{2}{*}{$\begin{array}{c}\text { Adjusted } \\
\mathrm{R}^{2}\end{array}$} & \multirow{2}{*}{$\begin{array}{c}\text { Speed of } \\
\text { convergence for } \\
\text { infant mortality } \\
\text { rate } \\
\text { (\% per annum) }\end{array}$} \\
\hline & $\begin{array}{c}\text { Infant } \\
\text { mortality rate }\end{array}$ & $\begin{array}{l}\text { Proportion of } \\
\text { illiterate } \\
\text { population }\end{array}$ & $\begin{array}{l}\text { Poverty } \\
\text { ratio }\end{array}$ & & \\
\hline $1992-93$ to & -.15031 & .07497 & .06381 & 0.43 & 16.3 \\
\hline $2005-06$ & (0.000) & (0.048) & $(0.068)$ & & \\
\hline $1992-93$ to & -.28982 & .12818 & .10244 & 0.27 & 34.2 \\
\hline 1998-99 & $(0.000)$ & (0.092) & $(0.144)$ & & \\
\hline $1998-99$ to & -.12649 & .07100 & .05162 & 0.23 & 13.5 \\
\hline $2005-06$ & $(0.023)$ & $(0.156)$ & $(0.226)$ & & \\
\hline
\end{tabular}

Note: 1 . States: $\mathrm{n}=24$, df 23

2. () P value

Unlike the absolute $\beta$ convergence estimates for MMR which showed evidence for convergence, the results of conditional $\beta$-convergence estimates show evidence for divergence $(\beta=.0029621, \quad \mathrm{P}<.01660)$. However, a comparison of absolute and conditional $\beta$-convergence estimates showed that after controlling for state level proportion of illiterate population and poverty ratios, the volume and speed of convergence indicated greater conditional $\beta$-convergence than unconditional $\beta$ convergence for IMR and LEB indicators.

Table 5.8. Conditional $\beta$ convergence for maternal mortality ratio across the major states, India, 1992-2009

\begin{tabular}{|c|c|c|c|c|c|}
\hline \multirow[t]{2}{*}{ Period } & \multicolumn{3}{|c|}{$\beta$ coefficient } & \multirow{2}{*}{$\begin{array}{l}\text { Adjusted } \\
\mathrm{R}^{2}\end{array}$} & \multirow{2}{*}{$\begin{array}{c}\text { Speed of } \\
\text { convergence } \\
\text { for infant } \\
\text { mortality rate } \\
\text { (\% per annum) }\end{array}$} \\
\hline & $\begin{array}{c}\text { Maternal } \\
\text { mortality } \\
\text { ratio }\end{array}$ & $\begin{array}{l}\text { Proportion of } \\
\text { illiterate } \\
\text { population }\end{array}$ & $\begin{array}{l}\text { Poverty } \\
\text { ratio }\end{array}$ & & \\
\hline $\begin{array}{l}1997-98 \text { to } \\
2007-09\end{array}$ & $\begin{array}{l}.0029621 \\
(.00692)\end{array}$ & $-.128029(.01660)$ & $\begin{array}{c}-.2413254 \\
(.000)\end{array}$ & 0.4165 & \\
\hline
\end{tabular}

Note: 1 . States: $\mathrm{n}=15$, df 14

2. () P value 


\subsubsection{Sigma convergence in mortality rates}

As it has been noted in the methodology chapter (Chapter 2) that $\beta$-convergence is necessary, but not a sufficient condition for Sigma convergence. In other words, a country experiencing $\beta$-convergence may not be experiencing a sigma convergence. Therefore, it is necessary to test for sigma convergence alongside the $\beta$-convergence. This chapter examined sigma convergence based on change in standard deviations of health status indicators over the time for the major states of India (Figure 5.7). The results for sigma convergence in LEB indicated a clear convergence in average LEB for Indian states that the standard deviation in LEB declined from 5.05 during 1981-86 to 4.33 in 2001-05. However, increase in standard deviation of $(S D=4.33$ in 2001-05 to $S D=4.41$ in 2006) LEB for the recent period indicated the emerging divergence trend.

Figure 5.7. Sigma convergence in average LEB across the major states, India; 1981-

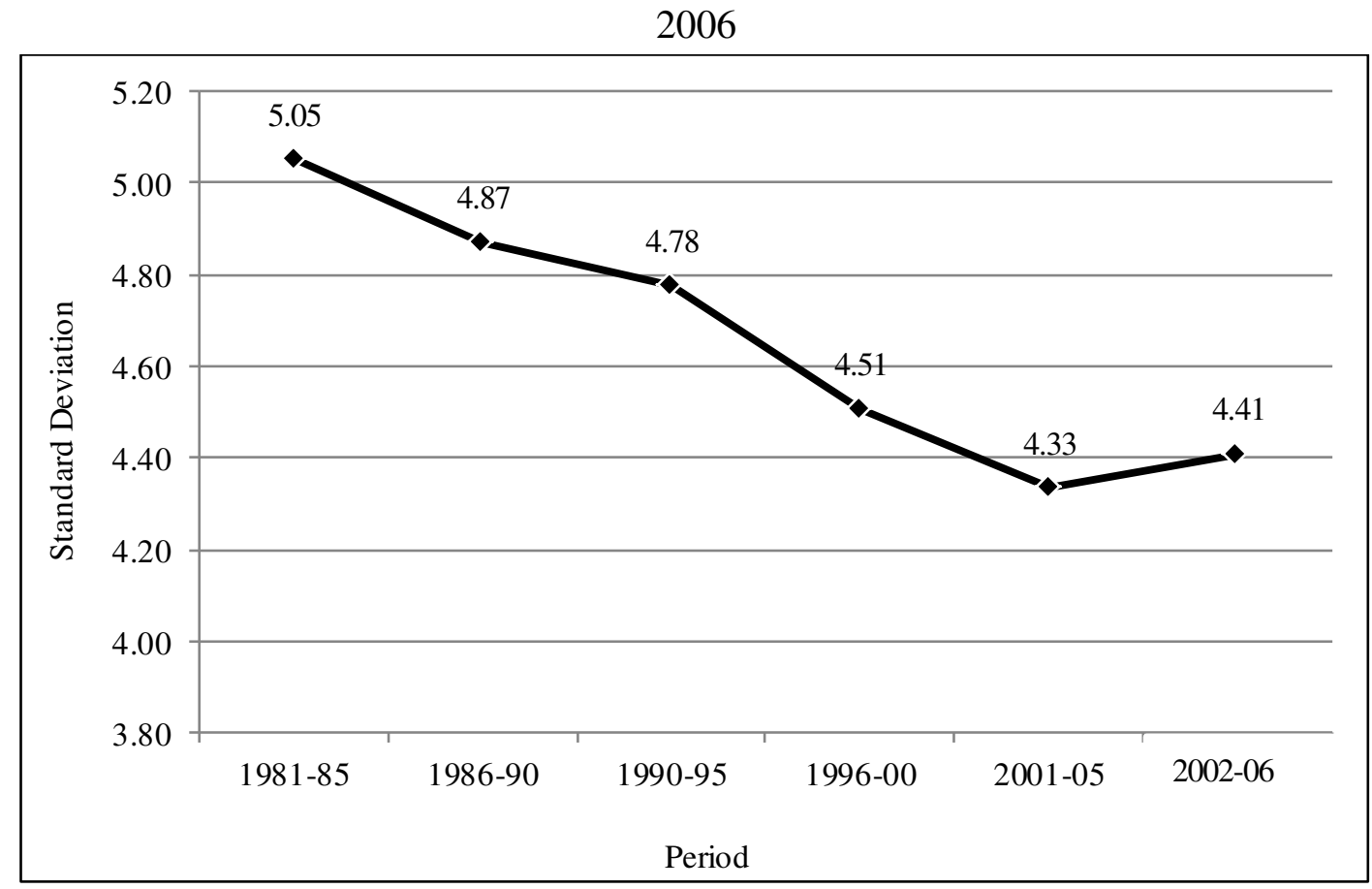

Figure 5.8 show the results of Sigma convergence in averages of IMR. In contrast to Sigma convergence results of LEB, the Sigma convergence results for IMR indicated a convergent trend over the period that the dispersion in IMR declined from 25/1000 in 1992-93 to 16/1000 live births in 2005-06. Sigma convergence results in case of MMR show a continued decline in dispersion of MMR across the states of India. Standard deviation of MMR across the states in 1997-98 was 180 maternal deaths per 100,000 live births, which increased to 97 maternal deaths per 100,000 live births (Figure 5.9). 
Figure 5.8. Sigma convergence in averages of IMR across the major states, India; 19922006

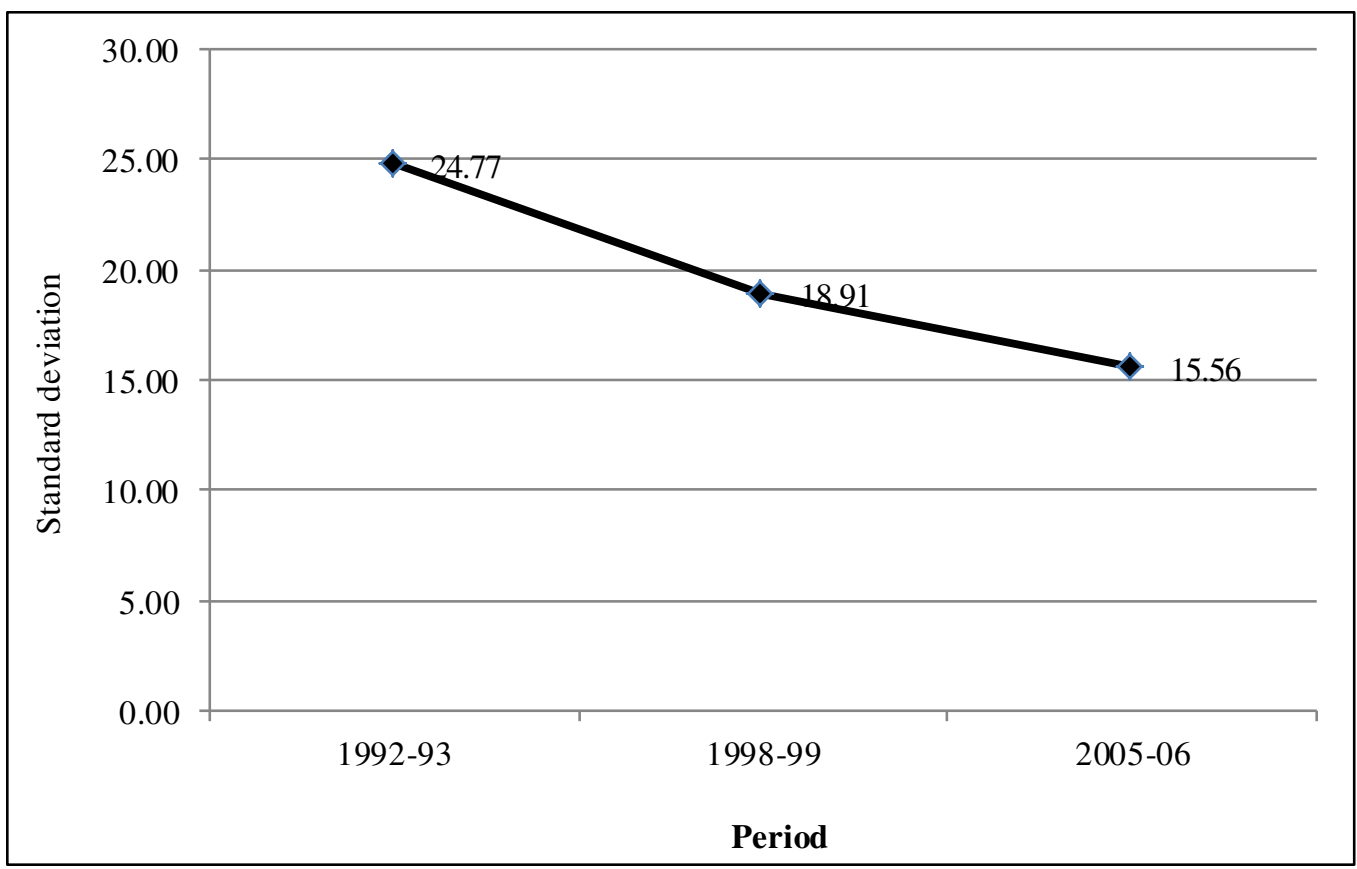

Figure 5.9. Sigma convergence in averages of MMR across the major states, India; 1997-2009

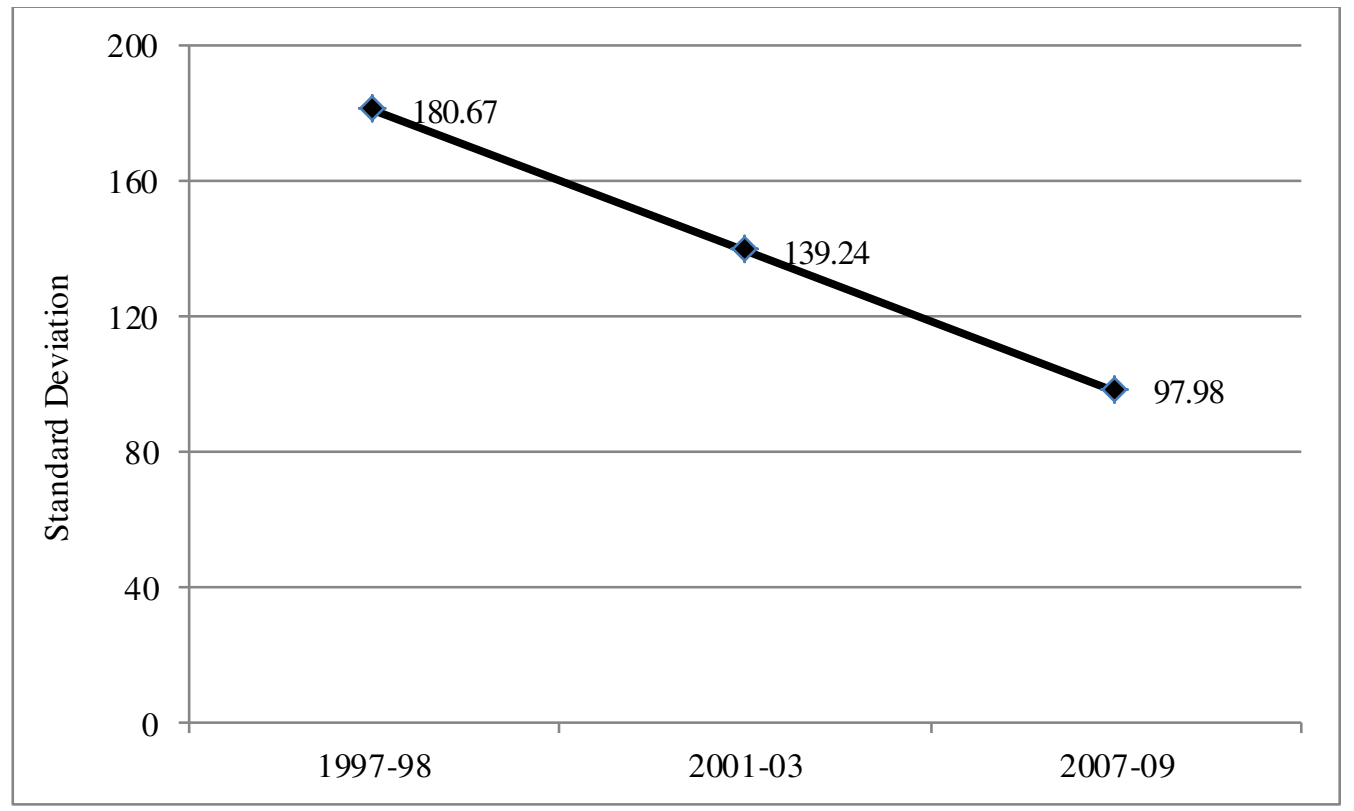

\subsubsection{Convergence in absolute and relative inequalities in mortality rates}

This section assesses the convergence in mortality across the states through inequality measures. The rate of convergence in overall mortality inequality was measured based on percentage reduction in dispersion of LEB, MMR and IMR. To deal with equity and efficiency, this chapter used two types of measures of overall inequalities in mortality: 
first, DMM was used to measure the absolute distribution of mortality and the calculation of this measure was based on an interval scale. Second, the relative distribution of mortality was measured with Gini coefficient and the calculation of Gini was based on a ratio scale. Therefore, convergence in absolute and relative inequalities in mortality was measured based on percentage reduction in DMM and Gini: a positive percentage value indicate convergence and a negative value implies divergence. However, the convergence in socioeconomic inequality in IMR was estimated using the sigma convergence measure.

Figure 5.10 presents that the percentage reduction in absolute inequality, measured by DMM declined for LEB and IMR over the period. However, the decline in DMM was greater (15\%) during the period 1980-1990 in LEB across Indian states, but turns negative (-2.4\%) during 2001-05 to 2002-06. Hence, convergence trend was replaced with divergence trend in LEB for the recent periods. In contrast to LEB, the results of convergence in absolute inequality for IMR revealed a steady progress towards convergence across the states over last three decades. However, the volume of convergence in overall absolute inequality in IMR has slowdown during the recent decade: the volume of convergence in overall inequality during 1981-1991 was 31\% compared with only 17\% during 1991-2001 and 12\% during 2001-2008 (figure 5.11).

Figure 5.10. Absolute convergence rate (in percentage) in LEB across the major states, India, 1980-2006

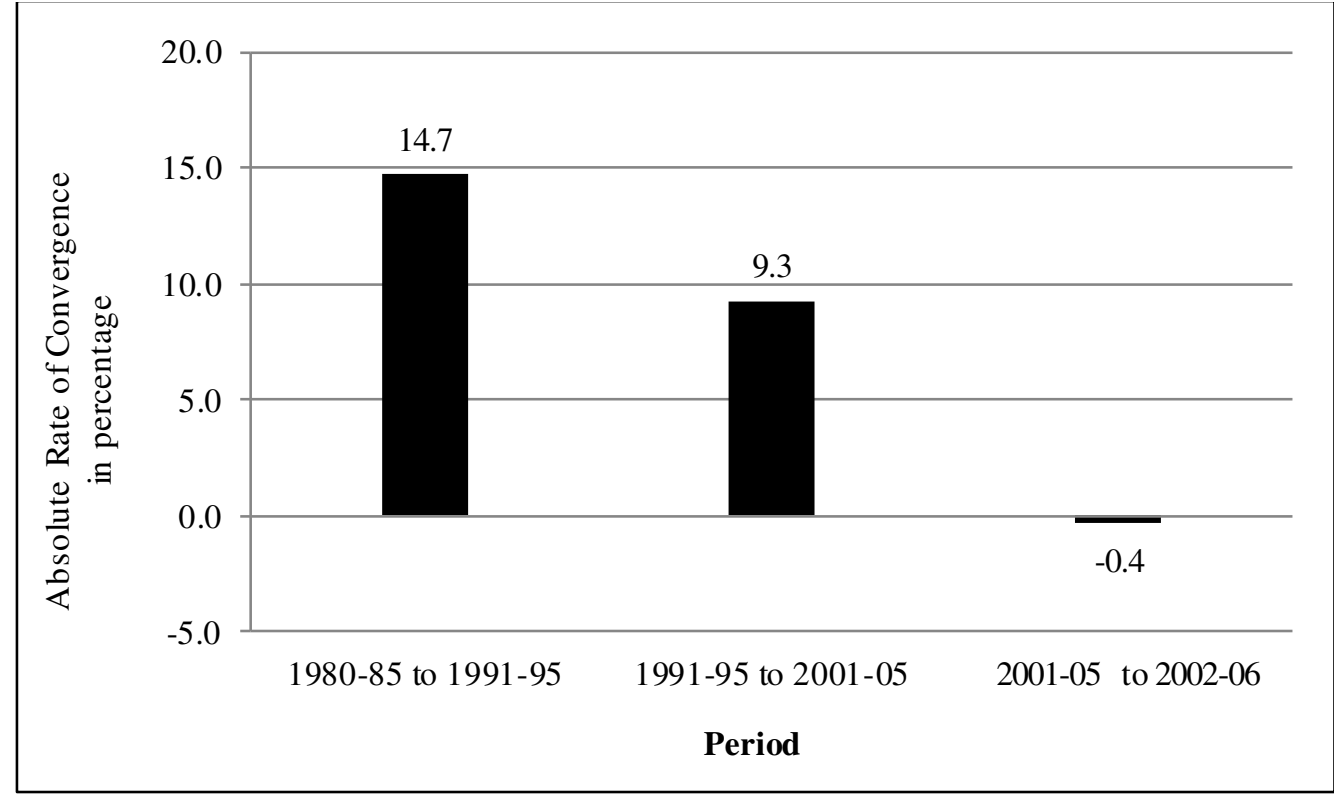

Note: Absolute convergence rate is calculated based on DMM estimates of LEB during 1980 to 2006 
Figure 5.11. Absolute convergence rate (in percentage) in IMR across the major states, India, 1981-2008

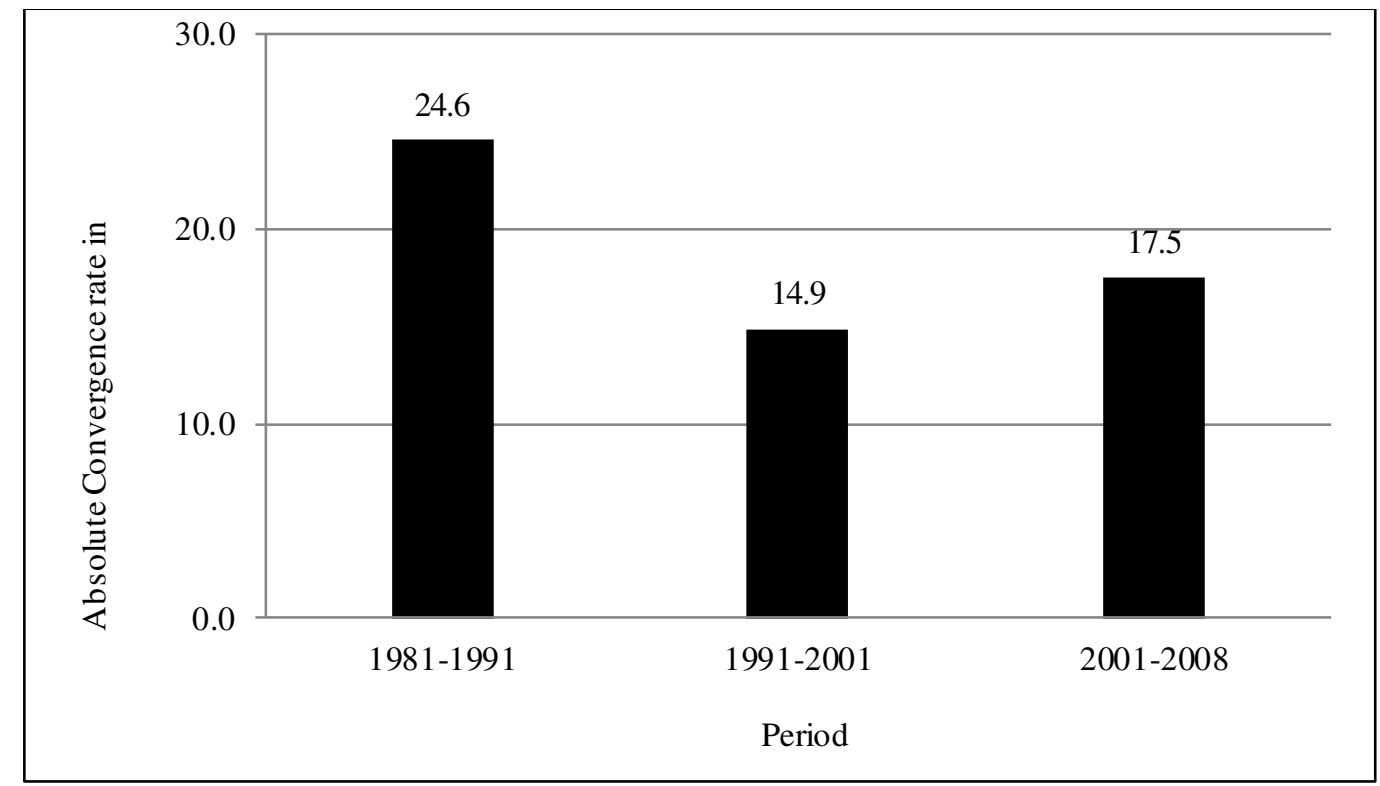

Note: Absolute convergence rate is calculated based on DMM estimates of IMR during 1981 to 2008.

Estimates of absolute convergence for inequalities in MMR showed a continued convergence trend. The absolute convergence in terms of reduction in DMM of MMR was $23.7 \%$ during $1997-2001$ rose to $30.5 \%$ during 2001-2009. Comparative assessment between LEB, MMR and IMR indicated convergence in overall inequality for both LEB and IMR was greater during the 1980s and 1990s followed by a slowdown in the recent period. In terms of overall inequality in LEB, divergence trend replaced with convergence for the recent period. However, throughout the period, IMR experienced convergence in absolute inequality across the states, but with a slowdown in speed of convergence for the recent period. In contrast, MMR showed continued convergence with an increasing speed of convergence (Figure 5.10, 5.11, 5.12).

Convergence in overall relative inequality in mortality was measured by Gini coefficient based on reduction in percentage of Gini for LEB, MMR and IMR. Compared with convergence estimates in absolute inequality, estimates of relative convergence showed greater divergence in IMR than in LEB: the rate of percentage reduction in Gini coefficient was negative for all three periods for IMR, whereas, the rate of reduction in relative inequality in LEB was negative only for the recent period. However, there was a steady decline in the percentage reduction of relative inequality for LEB. In case of MMR, the results showed decline in relative inequalities across the states, but such decline was greater during 1997-2001 (13\%) compared to 2001-07 
(5\%). In sum, the relative convergence in overall inequality in mortality showed evidence of divergence or reduction in level of convergence than continued convergence, particularly for a period since 2001 (Figure 5.13, 5.14, 5.15). The sigma convergence model was used to estimate the convergence in socioeconomic inequality (CIs) of IMR. Results revealed continued convergent trends for IMR during 1992-2006 and such convergence is increasing over the period.

Figure 5.12. Absolute convergence rate of MMR for major Indian States, 1997-2009

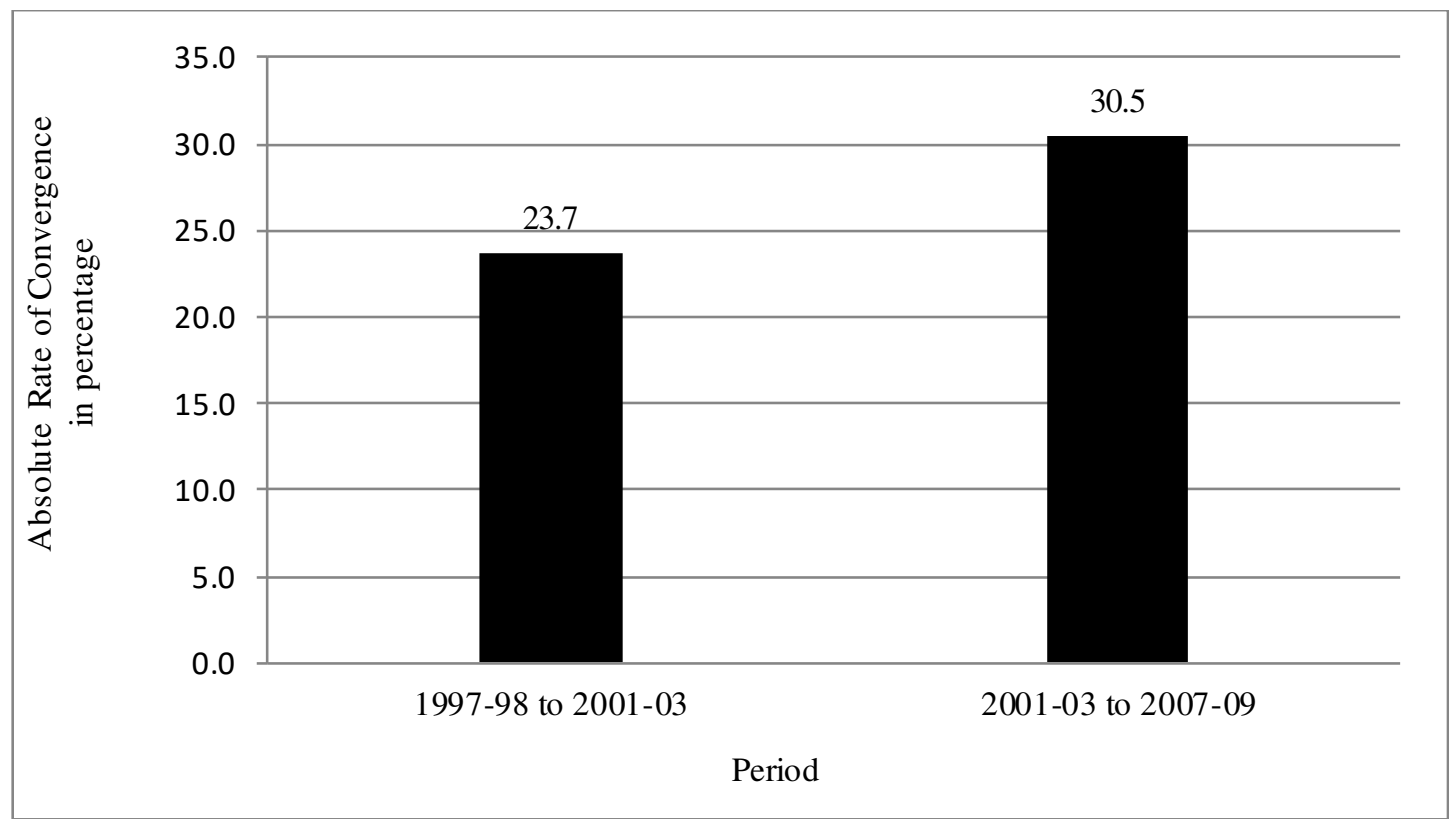

Figure 5.13. Relative convergence rate (in percentage) for overall health inequality in LEB across the major states, India, 1980-2006

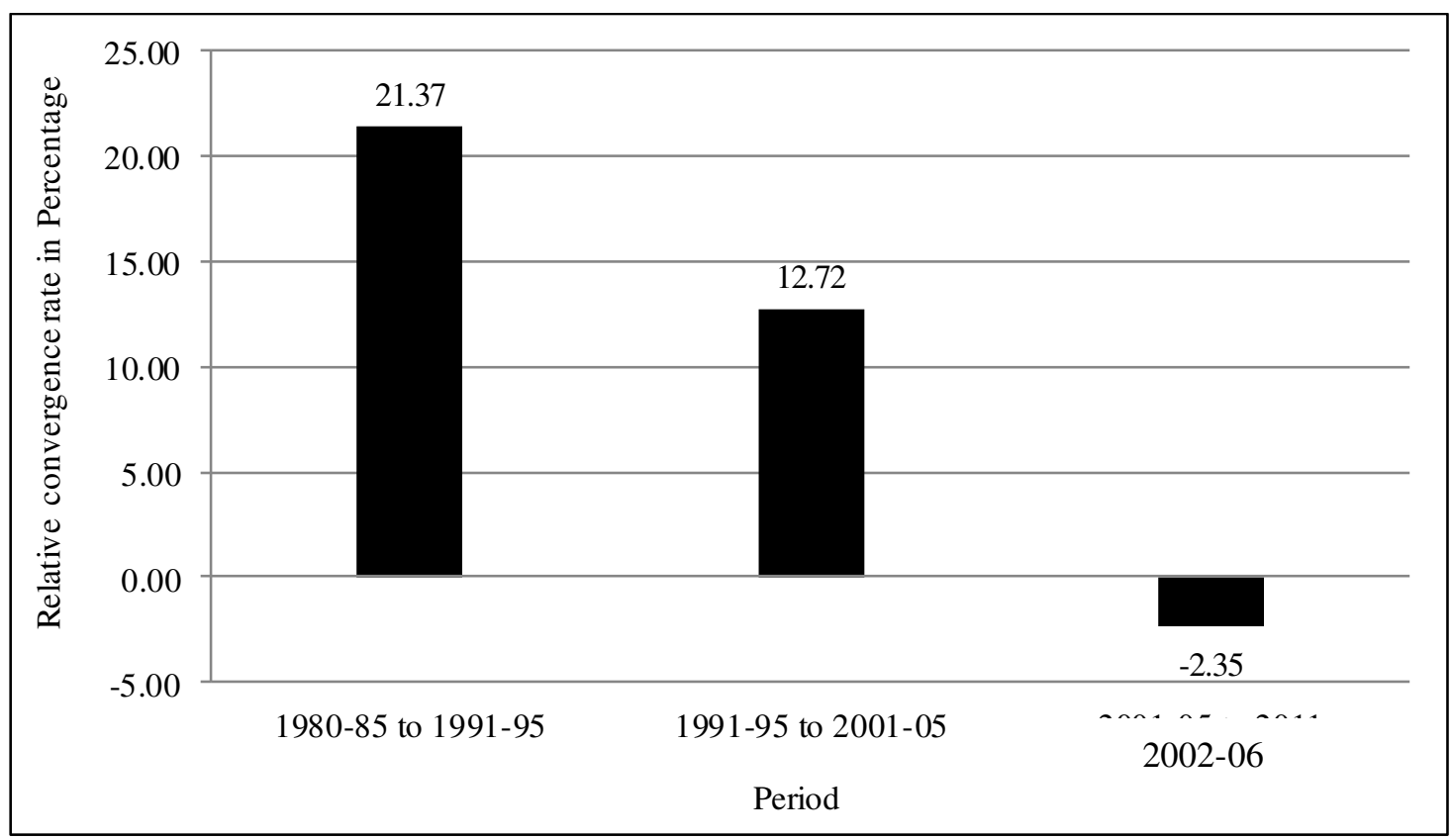

Note: Relative convergence rate is calculated based on Gini estimates of LEB during 1980 to 2006. 
Figure 5.14. Relative convergence rate (in percentage) for overall health inequality in IMR across the major states in India, 1981-2008

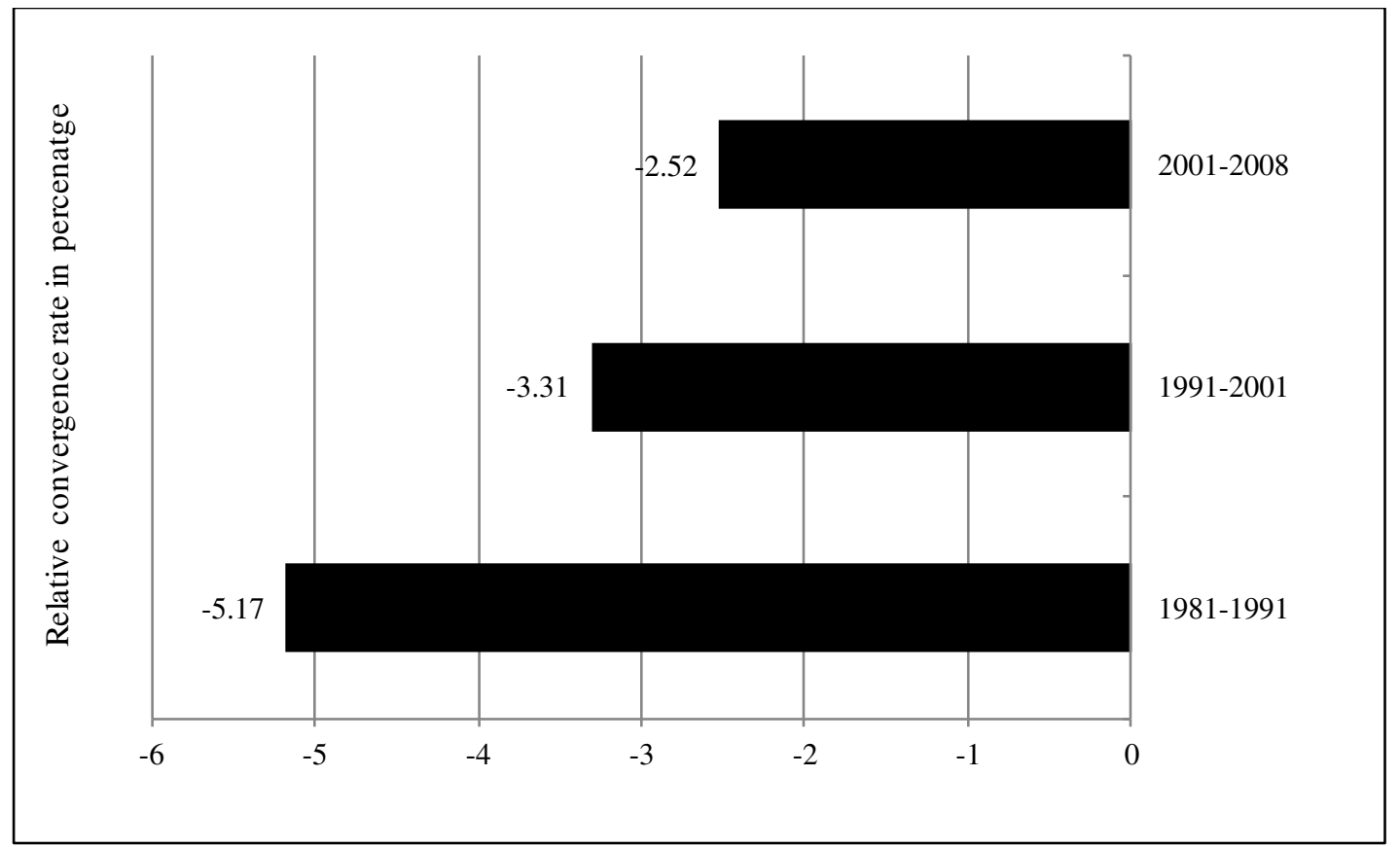

Note: Relative convergence rate is calculated based on Gini estimates of IMR during 1981 to 2008.

Figure 5.15. Relative convergence rate (in percentage) overall inequality in MMR across the major states in India, 1997-2009

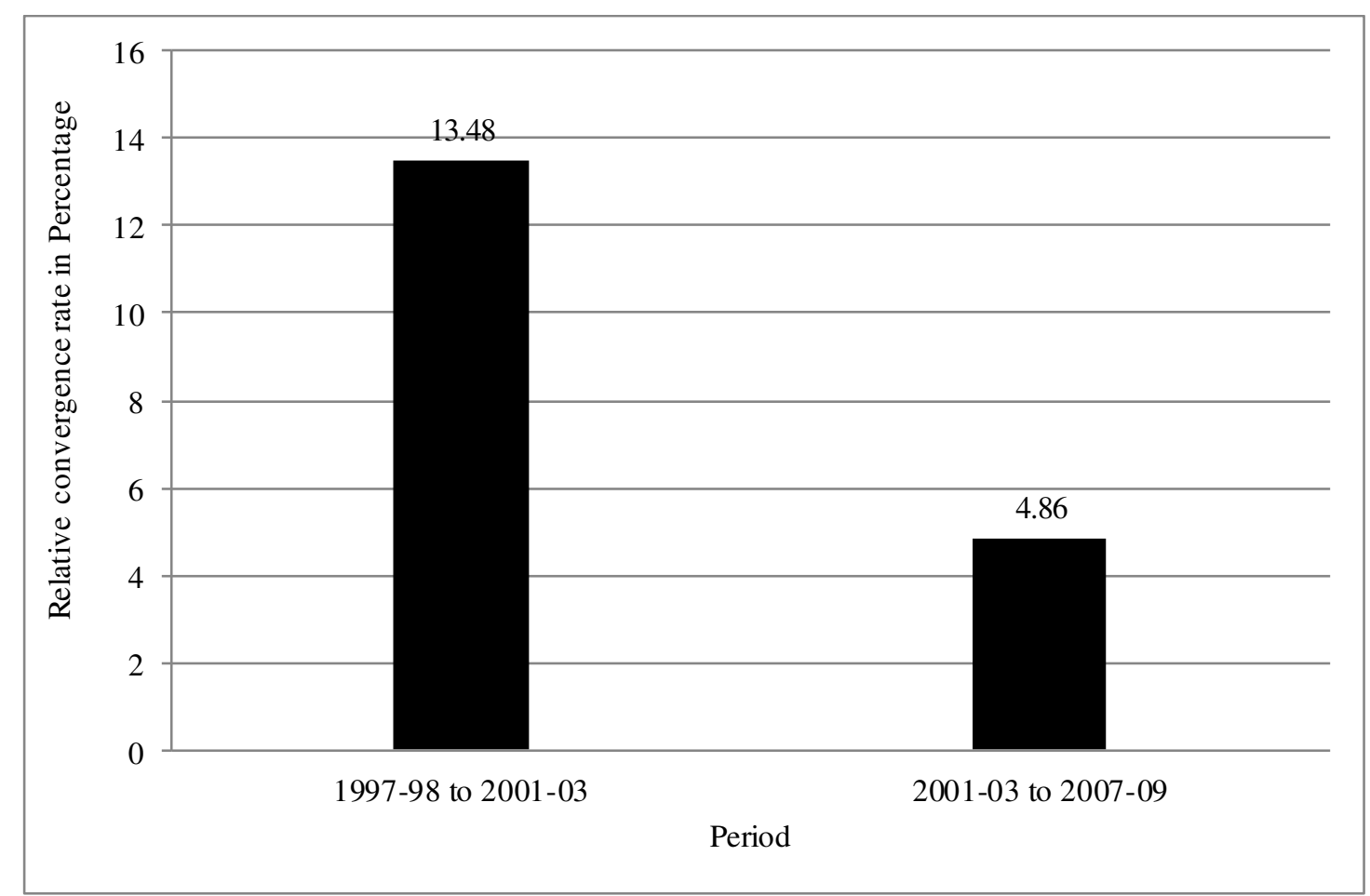

Note: Relative convergence rate is calculated based on Gini estimates of MMR during 1997- 2009. 
Figure 5.16. Sigma convergence in socioeconomic inequality (concentration indices) in IMR indicators across the major states in India; 1992-2006

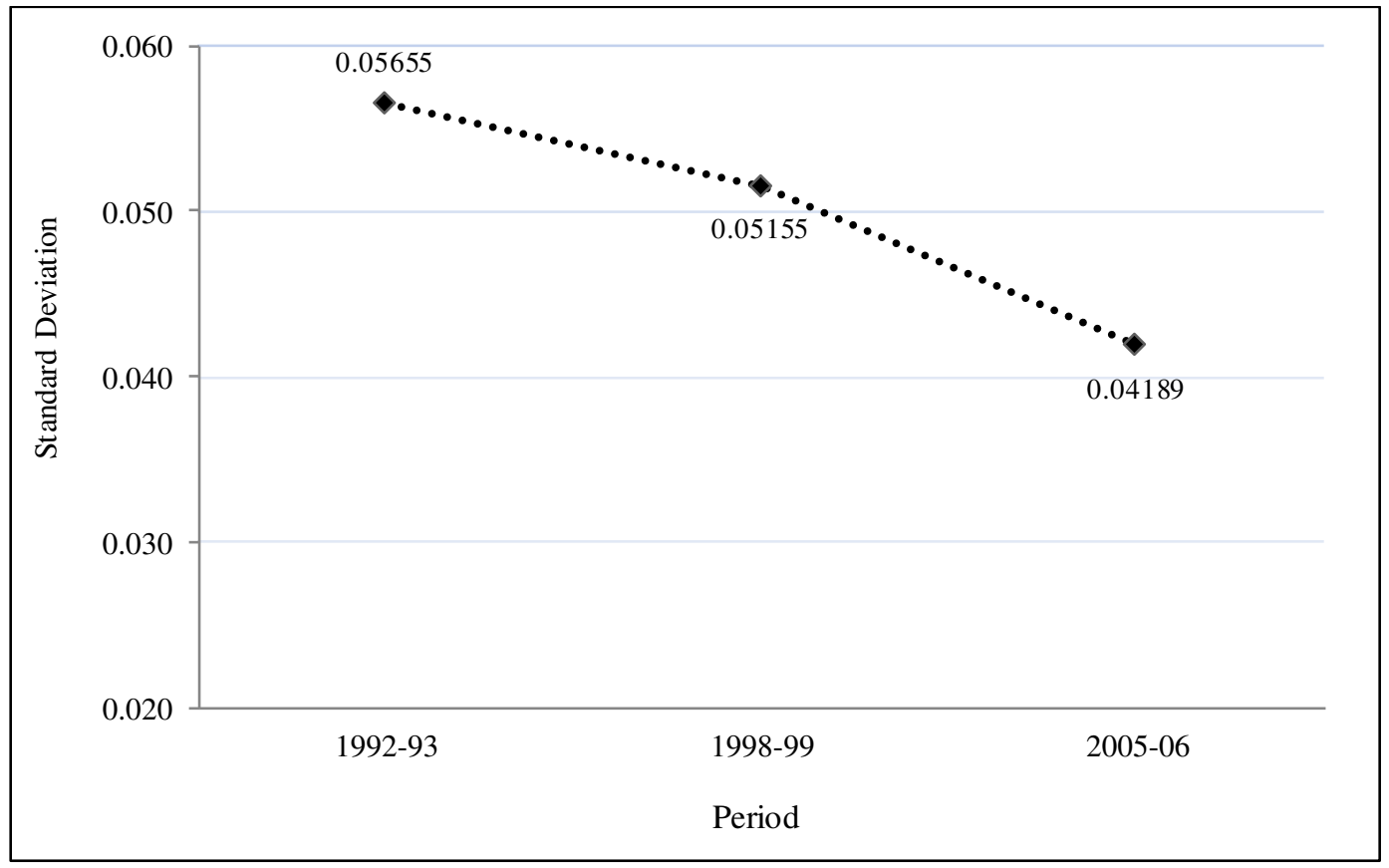

Source: NFHS 1-3

\subsubsection{Convergence clubs}

Figure 5.17 shows the assessment of convergence clubs by using a graphical plot. In case of LEB, the clubs were comprised of three groups: less than or equal to 50 years, 51-60 years, 61-70 years and more than 70 years. In 1981, Uttar Pradesh was the only state with LEB less than 50 years. At this point, majority of the states fall in between LEB 51-60 years and no state with LEB more than 70 years. However, by 2006 the Uttar Pradesh joined the club of LEB ranging between 51-60 years. Only Kerala has LEB above 70 years and majority of the states were concentrated in the club of LEB levels ranging between 61-70 years.

Convergence clubs in terms of trends in IMR levels of states during 1981-2011 is presented in Figure 5.18. The results showed that in 1981, states like Assam, Bihar, Gujarat, Haryana, Madhya Pradesh, Orissa, Rajasthan and Uttar Pradesh were in the club of IMR above 100. Kerala was the only state in the clubs-III with IMR range of 25-49. However, there was no state with IMR less than 25. By the year 2011, there is no state with IMR above 100. Greater number of states became part of the club with IMR range of 25-49. However, the three states of Kerala and Tamil Nadu in the leading club with IMR of less than 25. 
Figure 5.17. Trends in Convergence clubs in LEB, 1981-2006

\begin{tabular}{|c|c|c|c|c|c|}
\hline Clubs & LEB range & 1981-05 & 1991-05 & 2001-05 & 2002-06 \\
\hline है & $\begin{array}{l}\text { Less than } \\
\text { or equal to } \\
50\end{array}$ & Uttar Pradesh & & & \\
\hline E & $51-60$ & $\begin{array}{l}\text { Andhra Pradesh, } \\
\text { Assam, Bihar, } \\
\text { Gujarat, Haryana, } \\
\text { Madhya Pradesh, } \\
\text { Orissa, Rajasthan, } \\
\text { Tamil Nadu, West } \\
\text { Bengal }\end{array}$ & $\begin{array}{l}\text { Assam, Bihar, } \\
\text { Madhya Pradesh, } \\
\text { Orissa, } \\
\text { Rajasthan, Uttar } \\
\text { Pradesh }\end{array}$ & $\begin{array}{c}\text { Assam, Madhya } \\
\text { Pradesh, Orissa, } \\
\text { Uttar Pradesh }\end{array}$ & $\begin{array}{l}\text { Assam, Madhya } \\
\text { Pradesh, Orissa, } \\
\text { Uttar Pradesh, } \\
\text { West Bengal }\end{array}$ \\
\hline $\begin{array}{l}\text { 目 } \\
\text { 五 } \\
\text { 己 }\end{array}$ & $61-70$ & $\begin{array}{c}\text { Karnataka, Kerala, } \\
\text { Maharashtra, } \\
\text { Punjab }\end{array}$ & $\begin{array}{c}\text { Andhra Pradesh, } \\
\text { Gujarat, Haryana, } \\
\text { Karnataka, } \\
\text { Maharashtra, } \\
\text { Punjab, Tamil } \\
\text { Nadu, West } \\
\text { Bengal }\end{array}$ & $\begin{array}{c}\text { Andhra Pradesh, } \\
\text { Bihar, Gujarat, } \\
\text { Haryana, } \\
\text { Karnataka, } \\
\text { Maharashtra, } \\
\text { Punjab, } \\
\text { Rajasthan, Tamil } \\
\text { Nadu, West } \\
\text { Bengal }\end{array}$ & $\begin{array}{l}\text { Andhra Pradesh, } \\
\text { Bihar, Gujarat, } \\
\text { Haryana, } \\
\text { Karnataka, } \\
\text { Maharashtra, } \\
\text { Punjab, } \\
\text { Rajasthan, Tamil } \\
\text { Nadu }\end{array}$ \\
\hline 五 Z & $\begin{array}{c}\text { More than } \\
70\end{array}$ & & Kerala & Kerala & Kerala \\
\hline
\end{tabular}

Source: RGI (1981-2011)

Figure 5.18. Trends in Convergence clubs in IMR, 1981-2011

\begin{tabular}{|c|c|c|c|c|c|}
\hline Period & IMR range & 1981 & 1991 & 2001 & 2011 \\
\hline 官 & $\begin{array}{l}\text { More than } \\
100\end{array}$ & $\begin{array}{l}\text { Assam, Bihar, } \\
\text { Gujarat, Haryana, } \\
\text { Madhya Pradesh, } \\
\text { Orissa, Rajasthan, } \\
\text { Uttar Pradesh }\end{array}$ & $\begin{array}{c}\text { Madhya Pradesh, } \\
\text { Orissa }\end{array}$ & & \\
\hline 春 & $50-100$ & $\begin{array}{c}\text { Andhra Pradesh, } \\
\text { Karnataka, } \\
\text { Maharashtra, Punjab, } \\
\text { Tamil Nadu, West } \\
\text { Bengal }\end{array}$ & $\begin{array}{c}\text { Andhra Pradesh, } \\
\text { Assam, Bihar, } \\
\text { Gujarat, Haryana, } \\
\text { Karnataka, } \\
\text { Maharashtra, Punjab, } \\
\text { Rajasthan, Tamil } \\
\text { Nadu, Uttar Pradesh, } \\
\text { West Bengal }\end{array}$ & $\begin{array}{l}\text { Andhra Pradesh, } \\
\text { Assam, Bihar, } \\
\text { Gujarat, Haryana, } \\
\text { Karnataka, } \\
\text { Madhya Pradesh, } \\
\text { Orissa, Punjab, } \\
\text { Rajasthan, Uttar } \\
\text { Pradesh, West } \\
\text { Bengal }\end{array}$ & $\begin{array}{l}\text { Assam, Madhya } \\
\text { Pradesh, Orissa, } \\
\text { Rajasthan, Uttar } \\
\text { Pradesh }\end{array}$ \\
\hline $\begin{array}{l}\text { 貝 } \\
\text { 完 }\end{array}$ & $25-49$ & Kerala & & $\begin{array}{l}\text { Maharashtra, } \\
\text { Tamil Nadu }\end{array}$ & $\begin{array}{c}\text { Andhra Pradesh, } \\
\text { Bihar, Gujarat, } \\
\text { Haryana, Karnataka, } \\
\text { Maharashtra, Punjab, } \\
\text { West Bengal }\end{array}$ \\
\hline 官 Z & $\begin{array}{l}\text { Less than } \\
\quad 25\end{array}$ & & Kerala & Kerala & Kerala, Tamil Nadu \\
\hline
\end{tabular}

Source: RGI (1981-2011) 
Figure 5.19. Trends in Convergence clubs in MMR, 1997-2009

\begin{tabular}{|c|c|c|c|c|}
\hline Period & $\begin{array}{l}\text { MMR } \\
\text { range }\end{array}$ & $1997-98$ & 2001-03 & 2007-09 \\
\hline 官 & $\begin{array}{l}\text { More than } \\
300\end{array}$ & $\begin{array}{l}\text { Assam, Bihar, Madhya } \\
\text { Pradesh, Orissa, Rajasthan, } \\
\text { Uttar Pradesh, West } \\
\text { Bengal }\end{array}$ & $\begin{array}{c}\text { Assam, Bihar, Madhya } \\
\text { Pradesh, Orissa, } \\
\text { Rajasthan, Uttar Pradesh }\end{array}$ & $\begin{array}{c}\text { Assam, Rajasthan, Uttar } \\
\text { Pradesh }\end{array}$ \\
\hline 是 & $200-300$ & Karnataka, Punjab & Karnataka & $\begin{array}{c}\text { Bihar, Madhya Pradesh, } \\
\text { Orissa }\end{array}$ \\
\hline$\frac{\Xi}{\Xi}$ & $100-200$ & $\begin{array}{c}\text { Andhra Pradesh, } \\
\text { Maharashtra, Haryana, } \\
\text { Tamil Nadu, Gujarat, } \\
\text { Kerala }\end{array}$ & $\begin{array}{c}\text { Andhra Pradesh, } \\
\text { Maharashtra, Haryana, } \\
\text { Tamil Nadu, Kerala, } \\
\text { Gujarat }\end{array}$ & $\begin{array}{c}\text { Andhra Pradesh, } \\
\text { Maharashtra, } \\
\text { Karnataka, Punjab, } \\
\text { Haryana, Gujarat, West } \\
\text { Bengal }\end{array}$ \\
\hline 茂 & $\begin{array}{l}\text { Less than } \\
100\end{array}$ & & & Tamil Nadu, Kerala \\
\hline
\end{tabular}

Source: RGI (1997-2009)

Figure 5.19 presents convergence club trends in MMR during 1997-2009. During 199798, seven states were in the club of MMR more than 300. The majority of the states in the club-III with MMR range of 100-200 and no state in the club of MMR range less than 100. In the 2007-09, the majority of the states were in the clubs of MMR with the range of 100-200. Moreover, Tamil Nadu and Kerala also entered into the club of MMR less than 100. However, the states like Assam, Rajasthan and Uttar Pradesh were remained in the club of MMR more than 300 .

Trends in convergence clubs in mortality rates in socioeconomic groups were also assessed for IMR. However, due to non availability of LEB and MMR estimates by socioeconomic groups, the trends in convergence clubs by socioeconomic group were not assessed for these indicators. The results presented in figure 5.19 shows intriguing findings. In 1992-93, ten socioeconomic groups [Assam (Muslim); Bihar (SC; Muslim); M.P. (SC, ST); UP (SC, ST, Others; Hindu); WB (ST)] were in the club of IMR above 100. However, majority of the states were in the club of IMR range 50-100. The only Hindus in Kerala were in the club of IMR less than 25. By 2005-06, only poorest in Gujarat state came under the category of IMR with more than 100. Majority of the states again located in the club of IMR ranges 50-100. However, the number of socioeconomic groups with IMR less than 25 were rose to 24 [Assam (Richest); 
Karnataka (Richest); Kerala (OBC, Others; Hindu, Muslim, Others; Richer, Richest), MP (Muslim; Richest); Maharashtra (SC, ST, OBC]. Overall, the convergence clubs assessment by socioeconomic groups suggests huge variation in mortality transition across the socioeconomic groups and on set of mortality transition across the socioeconomic groups. 
Figure 5.20. Changing patterns of infant mortality clubs among socioeconomic groups of major states of India, 1992-2006

\begin{tabular}{|c|c|c|c|c|}
\hline Period & IMR range & 1992-93 & 2000-01 & 2005-06 \\
\hline 㝘 & $\begin{array}{c}\text { More than } \\
100\end{array}$ & $\begin{array}{l}\text { Assam (Muslim); Bihar (SC; Muslim); M.P. } \\
\text { (SC, ST); UP ( SC, ST, Others; Hindu); WB ( } \\
\text { ST) }\end{array}$ & $\begin{array}{l}\text { AP (ST); Haryana (Poorer); M.P. (SC, ST; } \\
\text { Poorer); Orissa (Poorest); Rajasthan (Poorer); } \\
\text { UP (SC, OBC; Poorer) }\end{array}$ & Gujarat (Poorest) \\
\hline 콜 & $50-100$ & $\begin{array}{l}\text { A.P. ( SC, ST, Others; Hindu); Assam ( SC, } \\
\text { Others; Hindu); Bihar (ST, Others; Hindu); } \\
\text { Gujarat (SC, ST, Others; Hindu); Haryana (SC, } \\
\text { Others; Hindu, Muslim, Others); Karnataka } \\
\text { (SC, ST, Others; Hindu, Muslim); MP (Others } \\
\text { caste; Hindu, Muslim); Maharashtra (SC, ST, } \\
\text { Others; Hindu, Others); Orissa (SC, ST); } \\
\text { Punjab (SC; Hindu); Rajasthan (SC, ST, } \\
\text { Others; Hindu); T.N. ( SC, Others); UP } \\
\text { (Muslims, Others); WB (SC, Others; Hindu, } \\
\text { Muslims) }\end{array}$ & $\begin{array}{l}\text { A.P. (SC, OBC; Hindu; Poorer, Middle); Assam } \\
\text { (ST, Others; Hindu, Muslim; Poorer, Middle); } \\
\text { Bihar (SC, ST, OBC, Others; Hindu, Muslim; } \\
\text { Poorer, Middle); Gujarat (SC, ST, OBC, Others; } \\
\text { Hindu; Poorer, Middle); Haryana (SC, OBC, } \\
\text { Others; Hindu, Muslim; Middle); Karnataka } \\
\text { (SC, ST, OBC, Others; Hindu; Poorer, Middle); } \\
\text { MP (OBC, Others; Hindu, Muslim; Middle, } \\
\text { Richer); Maharashtra (SC, ST, OBC; Hindu, } \\
\text { Others; Poorest); Orissa (SC, ST, OBC, Others; } \\
\text { Middle); Punjab (SC, OBC; Hindu, Others; } \\
\text { Richer); Rajasthan (SC, ST, OBC, Others; } \\
\text { Hindu, Muslim; Middle, Richer); T.N. (OBC; } \\
\text { Hindu, Muslim; Poorer);UP (ST, Others; Muslim; } \\
\text { Middle, Richer); WB (SC, ST; Muslim; Poorer) }\end{array}$ & $\begin{array}{l}\text { A.P. (SC, ST, OBC, Others; Hindu, Muslim; } \\
\text { Poorest, Poorer, Middle); Assam (SC, ST, } \\
\text { OBC, Others; Hindu, Muslim; Poorest, } \\
\text { Poorer, Middle, Richer); Bihar (SC, OBC, } \\
\text { Others; Hindu, Muslim; Poorest, Poorer, } \\
\text { Middle, Richer, Richest); Gujarat (SC,ST, } \\
\text { OBC; Hindu; Poorer, Middle, Richer); } \\
\text { Haryana ( SC, OBC; Muslim; Poorer, } \\
\text { Richer); Karnataka (SC, OBC; Hindu; } \\
\text { Poorest, Poorer, Middle); MP (SC, ST, OBC, } \\
\text { Others; Poorest, Middle); Maharashtra } \\
\text { (Poorest, Middle); Orissa (SC, ST, OBC, } \\
\text { Others; Poorest, Poorer, Richer); Punjab } \\
\text { (Richer); Rajasthan (SC, ST, OBC, Others; } \\
\text { Hindu, Muslim; Poorest, Poorer, Middle, } \\
\text { Richer); TN (Poorest, Poorer); UP (SC, OBC, } \\
\text { Others; Hindu, Muslim; Poorest, Poorer, } \\
\text { Middle, Richer, Richest); WB (Other Caste; } \\
\text { Muslim; Poorest, Poorer, Middle, Richer) }\end{array}$ \\
\hline
\end{tabular}




\begin{tabular}{|c|c|c|c|c|}
\hline $\begin{array}{l}\text { 코 } \\
\text { हิ }\end{array}$ & $25-49$ & $\begin{array}{l}\text { A.P. (Muslim); Kerala (Muslim, Others); } \\
\text { Maharashtra (Muslim); Punjab (Others Caste; } \\
\text { Others Religion); Rajasthan (Muslim) }\end{array}$ & $\begin{array}{l}\text { A.P. (Others Caste; Muslim; Richer); Assam } \\
\text { (SC, OBC; Richer); Bihar (Richer); Gujarat } \\
\text { (Muslim; Richer); Haryana(Religion others; } \\
\text { Richer) Karnataka (Richer); Kerala (Poorer); } \\
\text { Maharashtra (Others Caste; Muslim; Middle, } \\
\text { Richer); Orissa (Richer); Punjab (Others Caste; } \\
\text { Poorer); T.N. (SC; Middle, Richer); WB (Others } \\
\text { Caste; Middle) }\end{array}$ & $\begin{array}{l}\text { A.P. (Richer, Richest); Gujarat (Others } \\
\text { Caste; Muslim; Richest); Haryana(Others } \\
\text { Caste; Hindu; Middle, Richest); Karnataka } \\
\text { (ST, Others; Muslim; Richer, Middle); } \\
\text { Kerala (Middle); MP (Hindu; Poorest, } \\
\text { Poorer, Middle, Richer); Maharashtra } \\
\text { (Poorer, Richer, Richest); Orissa (Richest); } \\
\text { Punjab (SC, Others; Hindu, Others; Middle; } \\
\text { Richest); Rajasthan (Richest); T.N. (SC, } \\
\text { OBC; Hindu; Middle); WB (SC; Hindu) }\end{array}$ \\
\hline $\begin{array}{l}\overline{1} \\
\text { 音 }\end{array}$ & Less than 25 & Kerala (Hindu) & $\begin{array}{l}\text { Kerala (OBC, Others; Hindu, Muslim, Others; } \\
\text { Middle, Richer); WB (Richer) }\end{array}$ & $\begin{array}{l}\text { Assam (Richest); Karnataka (Richest); } \\
\text { Kerala (OBC, Others; Hindu, Muslim, } \\
\text { Others; Richer, Richest) , MP (Muslim; } \\
\text { Richest); Maharashtra (SC, ST, OBC, } \\
\text { Others; Hindu, Muslim, Others); T.N. (Others } \\
\text { Religion; Richer, Richest); WB (Richest) }\end{array}$ \\
\hline
\end{tabular}

Source: Three rounds of NFHS (1-3) 
Figure 5.21. Trends in Kernel density plots for LEB, IMR and MMR
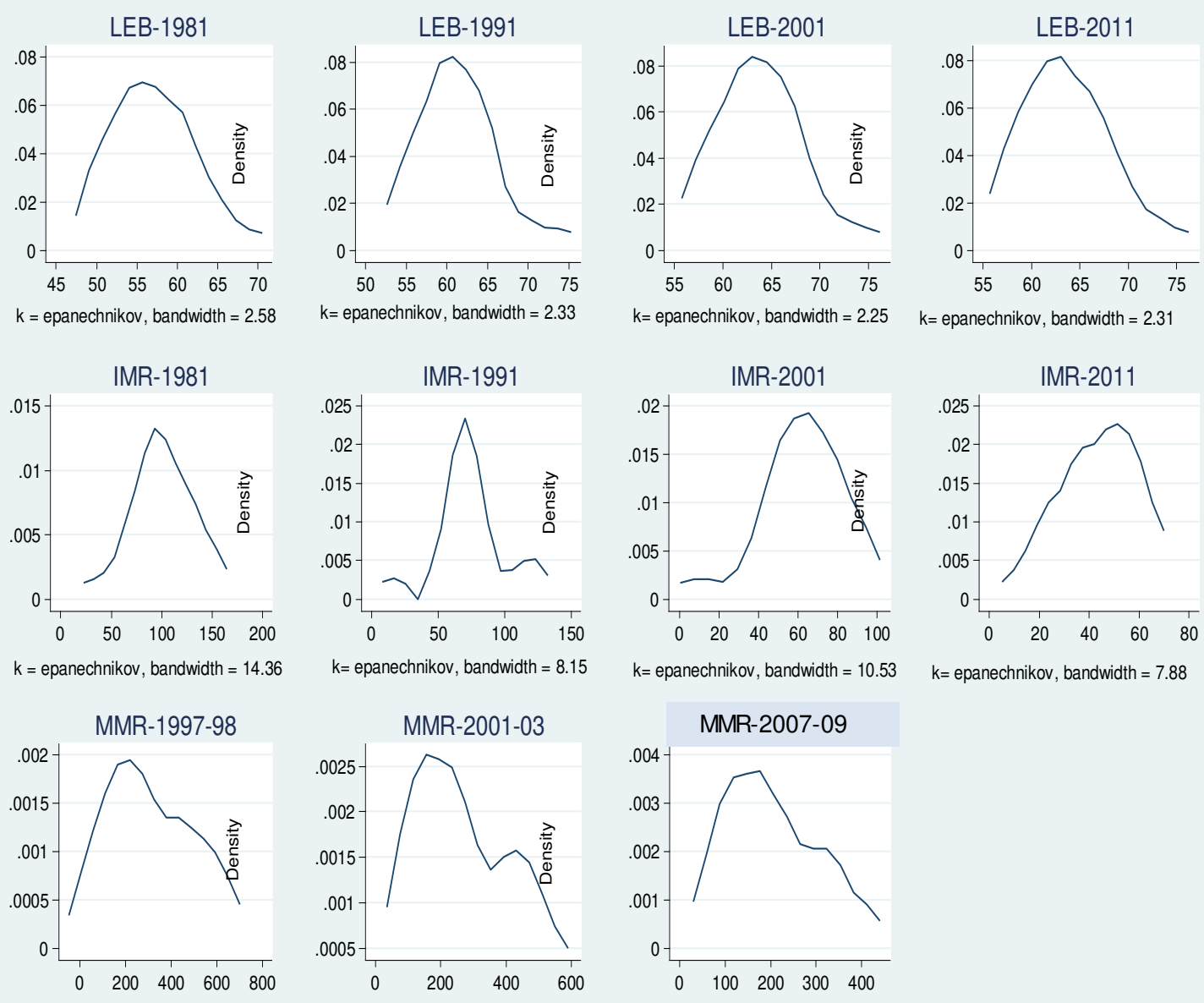

$\mathrm{k}=$ epanechnikov, bandwidth $=10.53$

$\mathrm{k}=$ epanechnikov, bandwidth $=7.88$

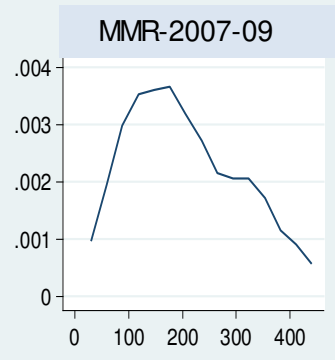

$\mathrm{k}=$ epanechnikov, bandwidth $=94.6$

$\mathrm{k}=$ epanechnikov, bandwidth $=72.91 \quad \mathrm{k}=$ epanechnikov, bandwidth $=51.3$

Note: $\mathrm{k}=$ kernel

Figure 5.21 presents trends in kernel density plots for LEB, IMR and MMR. The kernel plots in case of LEB reveal that in 1981 there was only a single peak in the distribution of LEB across the major states. However, the peak became narrow in 1991, but thereafter, it became wider again and the states with higher life expectancy started showing emergence of a separate convergence club. In LEB, there is no clear evidence of emergence of second club yet. In case of IMR, though there was no secondary peak in 1981, but there was clear evidence of multiple peaks in 1991, 2001 and 2011. The kernel distribution of MMR across the states also showed clear evidence of two peaks. One peak located at higher MMR levels range from 350 to 600 and second major peak located at MMR ranges from 90 to 200. Among the three indicators considered for the analyses, MMR showed more clear evidence of convergence clubs. 


\subsection{Discussion}

This chapter used both standard and inequality based convergence metrics to test the hypothesis of convergence in averages, absolute and relative distribution of mortality rates in India focusing on 15 major states and socioeconomic groups for three key mortality indicators. The results of trend analyses for mortality averages and inequalities of selected indicators suggested varying patterns. The gap across the states in average mortality in terms of IMR and MMR is closing. However, the gap remains the same for LEB and showed a setback in convergence trend in the recent period, 1998-2006. The trends in DMM and Gini coefficient suggested a decline in both absolute and relative dispersion initially, but indicated a reversal for the recent period, 2001-2008. The trends in socioeconomic inequalities in IMR suggested an increase in inequality for 6 out of 15 states.

A range of convergence metrics tested for averages of mortality and inequalities in this study revealed varying patterns of convergence by type of measure and time periods. The results indicated convergence in averages of mortality indicators, but with decreasing volume and speed of convergence for the recent period. The convergence in averages was principally driven by greater progress in laggard states and resultant catching-up process. However, the results present the evidence of some recent setback in convergence in absolute and relative inequalities in LEB, socioeconomic and relative inequalities in IMR. From the late 1990s, the results suggested progress in converging trend in mortality inequalities was being replaced by diverging trend. These anomalies may arise because of many reasons, but here, I point out few important reasons. First, the divergence in LEB for recent periods is more likely to be the result of unequal rate of progress in reduction of adult mortality across the states. Second, India has adapted globalisation, liberalisation and privatisation policies since 1991, and different states of India benefited differently from these policies. Such differences may have led to increase in the relative income inequality across states and socioeconomic groups in post 1990 s and consequently widened the gaps in both child and adult mortality. Third, explanations can also drawn from previous studies, for instance, Wagstaff (2002) provided alternative reasons for such situation: 'increasing per capita income leads to absolute decline in mortality averages, but increase in relative dispersion of mortality across the different population groups of the developing countries' as current experience 
of India suggests. This is mainly due to unequal rate of progress across the population sub groups. Fourth, the difference in both absolute and relative convergence process across the indicators can be attributed to nature of the indicators and factors affecting on them.

Moreover, the data analysed for IMR and MMR belongs to more recent compared to $\mathrm{LEB}$, therefore, the convergence observed in averages of IMR and MMR can also attributed to this. At the same time, one could expect a re-convergence of states in terms of LEB in the last 7-8 years, if the latest data of LEB is available in the near future. Taken as a whole, from these results, it can be said that improving state average mortality status may not always result in convergence in relative mortality situation of populations. India is a country of huge geographic, socioeconomic and cultural diversity. Therefore, state specific policies play a critical role in explaining the intrastate disparities in mortality. 


\section{CHAPTER 6 \\ Fertility transition and Convergence}

\subsection{Introduction}

Given convergent socioeconomic and mortality trends, fertility convergence is assumed to follow. Since 1960s, many developing countries have experienced rapid fertility decline. Fertility fell from a global average of 5 children per woman to 2.7 over the same time scale. As I discussed in earlier chapters that in wide social terms, there are several evidences of convergence in Europe. However, evidences from empirical analyses of convergence model across the developing countries are very less. By 2000, many of these countries have reached the replacement level fertility of 2.1 births per woman, and countries that are still in transition are widely expected to continue to decline until fertility drops to or even below replacement level (Wilson, 2001, 2011; Rutstein, 2002; Bongaarts, 2006; Dorius, 2008, 2010; Dyson, 2010). However, within countries, fertility is usually higher in less developed region (states) than developed region, higher in rural than urban areas, higher among uneducated women than their better-educated counterparts, and higher in households with low incomes than their higher income counterparts (Merrick 2001; Rutstein, 2002; Wilson, 2001, 2011; Doris, 2008 ; 2010). A situation of this kind is ideal for testing convergence models to assess the progress of within country convergence in fertility rates across the states and socioeconomic groups.

In India, it is usually considered that fertility was high in three to four decades back. However, a major problem with Indian fertility studies is the lack of historically reliable estimates of fertility as the vital registration system had not functioned well in pre1970s (Kulkarni, 2011). The indirect estimates by census actuaries and demographers show that at the beginning of the twentieth century, the Crude Birth Rate (CBR) was in the range of 45-50 per thousand populations (Davis, 1951). Estimates by the RGI showed some fall in the 1940s. Later, Bhat (1998) using the variable-r method estimated CBR and it was just above 45 and the Total Fertility Rate (TFR) around 6 up to the 1950s (Kulkarni, 2011). Applications of the modified Rele method by Rele (1987) and Ram and Ram (2009) also showed the TFR to be around 6.3 children per woman in pre1950s. During the 1960 s, especially the late 1960 s, some fall in fertility was evident. 
Fairly reliable direct estimates of fertility from the Sample Registration System are available after 1970 (Mitra, 2005; Kulkarni, 2011). Since 1970s, on an average, the progress in fertility decline in India is remarkable (Rele 1987; Registrar General of India 1971-2007, 2009). The total fertility rate for India fell from an average of 5.8 children per woman (1951) to 2.6 children per woman (2009). India's demographic trends are currently transitioning from third to fourth stage of the demographic transition (Visaria 2004b). Ten Indian states (namely, Andhra Pradesh, Delhi, Himachal Pradesh, Jammu and Kashmir, Karnataka, Kerala, Maharashtra, Punjab, Tamil Nadu, West Bengal) accounting 43 per cent of India's total population have reached replacement level fertility of 2.1 in 2010 .

However, the progress in fertility transition is not uniform across all the states and socioeconomic groups of India. In some states, the transition began in the 1960s and in other states in the 1970s. The pace also differed spatially. Thus, TFR in India are declining with divergent destinies across states, rural-urban and socioeconomic groups. The southern Indian states, urban areas, and higher socioeconomic groups are approaching low fertility rates with some categories such as the upper wealth and education quintiles of low fertility states reaching lowest-low fertility rate (TFR of 1.3). The northern Indian states, rural areas, and disadvantageous socioeconomic groups still have higher fertility rates (Guilmoto and Rajan 2001; Visaria 2004b; James and Nair 2005; Kulkarni and Alagarajan 2005; Alagarajan and Kulkarni 2008; Arokiasamy and Goli, 2012; Goli, 2013).

Even during the pre-transition period, the southern states had lower fertility (TFR below 6) than the northern states. In particular, the TFR in Tamil Nadu was around 5 for some time, even before 1950 (Rele 1987; Guilmoto, 1992). Thus, comparatively, south Indian states, urban areas, and higher socioeconomic groups are approaching low fertility rates with some categories such as the upper wealth and education quintiles of low fertility states reaching lowest-low fertility rate (TFR of 1.3), while many of the north Indian states, rural areas, and disadvantageous socioeconomic groups still have higher fertility rates (Registrar General of India 1971-2007, 2009; International Institute for Population Sciences [IIPS] and Macro Internationals 1992-2006). However, there are some assumptions about current and near future fertility scenario, which looks to be a period of continued 'convergence'. The states with higher fertility rates were achieved 
considerable progress in recent past and catching-up towards the low fertility rates. Nevertheless, there has been no formal attempt to estimate the volume and speed of fertility convergence across the Indian states and socioeconomic groups.

Being the second largest populous country with vast geographical variations and faster pace of fertility transition, India fosters an ideal condition for testing convergence hypothesis. Accordingly, the purpose of this chapter is to test fertility convergence hypothesis across sub national geographic units (the states), and socioeconomic spectrum of India.

\subsection{Data source}

This chapter utilized secondary data from the following sources: Sample Registration System (SRS) and three rounds of National Family Health Survey (NFHS) to assess the fertility trends and the progress in fertility convergence across the major states and socioeconomic groups (Registrar General of India 1971-2007, 2009; IIPS and Macro International 1992-2006). Since the early 1970s, India's Sample Registration System has been the most reliable source of fertility estimates for the country and the states (Registrar General of India 1971-2007, 2009). However, the SRS does not provide comprehensive trend data on fertility by different social groups. Therefore, I have used the three rounds of India's National Family Health Survey to assess the trends and convergence in fertility across the states and socioeconomic spectrum of India. NFHS is the equivalent to worldwide Demographic Health Surveys (DHS) in terms of survey design and questionnaire. The NFHS surveys are widely used source of information for estimating fertility and demographic indicators for India and the states (for more information visit www.nfhsindia.org; IIPS and Macro Internationals 1992-2006). Additional data used in this chapter include: fertility rates for the before 1971 from Rele (1987) estimates, the population totals and literacy rates from the Census of India (Registrar General of India and Census Commissioner 1971-2011) and, poverty ratio estimates from Planning Commission (Government of India 1973-2006).

\subsection{Methods}

The methods of analyses of this study involve 1) defining and conceptualising the process of demographic convergence in general and fertility convergence in particular, 
and b) adopted a range of measures and models to assess the progress of fertility convergence in India.

\subsubsection{Defining and conceptualising fertility convergence}

Fertility convergence can be defined as the reduction of their dispersion of a given fertility indicator such as TFR of a group of geographical units (states, regions, countries, etc.) over time. As we discussed above that in India, fertility rates fell substantially in the recent decades, but with considerable unevenness. As a result, the Indian states and rural-urban areas are at different stages of fertility transition. Closing of gap between the states, rural-urban and socioeconomic strata over the time, more specifically fall in fertility levels to a same point can be hypothesized as a process of fertility convergence. Therefore, this is a process in which the states and social groups tend to become homogeneous in terms of fertility can be termed as fertility convergence.

As discussed in the chapter 1 that the pathways of the process of demographic convergence lie in demographic transition. Moreover, the demographic transition process is also described to go through the processes of equilibrium and disequilibrium via fertility convergence, divergence, and re-convergence across the geographical units and socioeconomic spectrum. This study assumes that varying timelines of the socioeconomic and fertility transition has segmented the Indian states into different stages or regimes of fertility transition. I have designed a conceptual framework that illustrates the mechanism of interaction between socio-economic, health and fertility transition, resultant fertility convergence (see figure 6.1). 
Figure 6.1. Conceptual framework: Fertility transition and convergence model

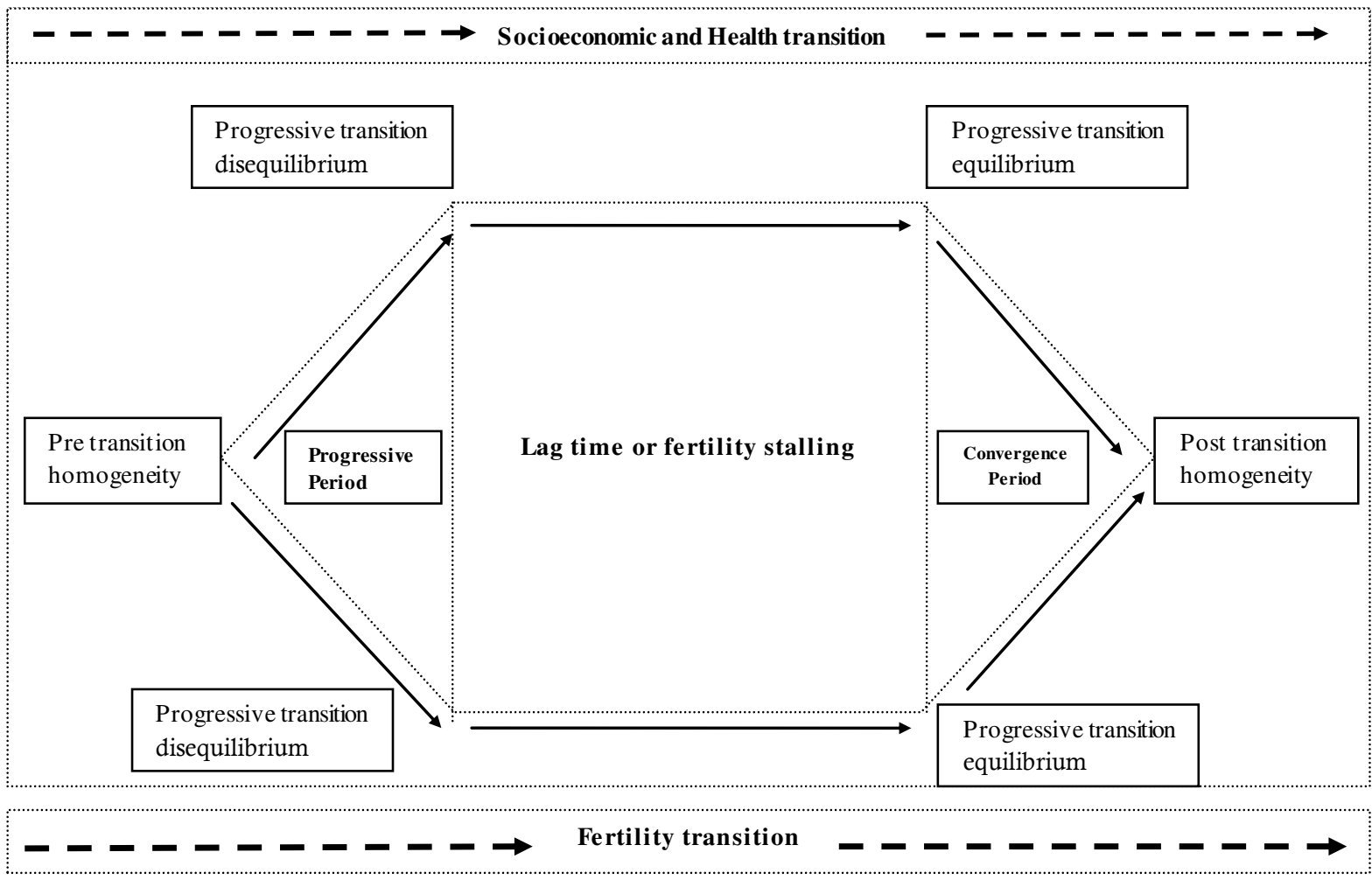

This framework envisages that during the progressive transition period, the states and different socioeconomic groups improve at varying pace leading to divergent trends until reappearance of post transition homogeneity or equilibrium. The shifting process of progressive disequilibrium into equilibrium process is termed 'convergence', and the time duration is called convergence period. During the progressive equilibrium phase, the demographically weaker states experience greater decline in fertility rates and come closer to states with lower fertility rate, thus indicating the progress towards convergence process.

\subsubsection{Measures of fertility convergence}

Previous studies on convergence in fertility rates on global scale adopted a variety of measures. However, a large number of studies used trend line plots and classical economic convergence measures such as absolute convergence, conditional $\beta$ convergence and Sigma convergence measures. In view of diverse stages of fertility transition in India, in this study I have used both standard measures of convergence (based on averages) and inequality based measures and nonparametric convergence models, which account for relative and socioeconomic distribution of fertility with 
adjustment for population sizes of the states. As I discussed earlier in chapter 2, the methods adopted in this study have been previously adopted for the study of convergence at global scale: between countries convergence, convergence across European regions and sub-national convergence in developed countries such as Italy and France (e.g. Herbertsson et al. 2000; Wilson 2001; Casterline 2001; Coleman 2002; Reher 2004, 2007; Moser et al. 2005; Dorius 2008, 2010; Lanzieri 2010; Lee and Reher 2011).

\subsection{Results}

The results of this chapter are presented in three sections: Section one presents the results of the progress in fertility transition in India and the states, encompassing the assessment of differentials in steady state: Change-point analyses. Section two examines the closing of gaps in fertility rates across the states or the catching-up process of higher fertility states with lower fertility states and polarisation of cumulative population distribution to lower fertility rates. Section three deals with a) quantification of the volume and speed of convergence in mean fertility rates (convergence in averages) across the major states and socioeconomic groups and; b) quantification of the volume and speed of convergence in absolute and relative distribution of fertility rates (convergence in inequalities) across the states and socioeconomic spectrum.

\subsubsection{Fertility trends in India and states}

The concept of demographic convergence lies at the heart of demographic transition theory (Oeppen 1999). In accordance with the conceptual framework unveiled in figure 6.1, the fertility transition process during the progressive stage indicates divergent paths with some states experiencing faster pace of decline in fertility rates e.g. Kerala, Tamil Nadu, Himachal Pradesh and Andhra Pradesh. However, more proactive fertility control programme in the demographically lagging states is likely to stimulate an acceleration of fertility decline leading to a catching-up process in low fertility states.

Many previous studies that examined the fertility transition process used trend line plots of fertility rates. However, simple trend line plots do not provide critical Change-points in the transition process and the volume of change in fertility rates. Therefore, in this chapter, I have adopted a more recently embraced innovative method of analysis namely 
'Change-point analyses' (Taylor 2011). The assessment of variations in fertility transition in terms of critical Change-points across the states helps in understanding the differentials in steady state conditions across the states. My earlier discussion on 'Change-Point analyses tool' in chapter 2 and 3 reveals that 'the advantage of 'Changepoint analyses' are: first, the Change-point analyses statistically estimate the critical Change-points and the volume of change in fertility. Second, the Change-points more effectively characterise the changes in fertility with statistical significance or confidence intervals. Third, it is a powerful tool to determine the changes in fertility more accurately and controls the overall error rate and robust to outliers. Lastly, the Changepoint analyses are capable of detecting subtle changes in fertility missed by simple trend line plots" (Taylor, 2011).

Table 6.1. Estimates of change-point analyses: significant change-points for TFR of India and selected major states, 1950-2009

\begin{tabular}{|c|c|c|c|c|c|}
\hline \multirow{2}{*}{ India/ states } & \multirow{2}{*}{ Change points } & \multicolumn{2}{|c|}{ Volume of change } & \multirow{2}{*}{ Confidence interval } & \multirow{2}{*}{$\begin{array}{c}\text { Confidence } \\
\text { level }(\%)\end{array}$} \\
\hline & & From & To & & \\
\hline \multirow{5}{*}{ India } & 1976 & 5.4 & 4.5 & $(1976,1976)$ & 100 \\
\hline & 1985 & 4.5 & 4.05 & $(1984,1985)$ & 95 \\
\hline & 1991 & 4.05 & 3.5167 & $(1991,1991)$ & 99 \\
\hline & 1997 & 3.5167 & 3.1429 & $(1997,1997)$ & 99 \\
\hline & 2004 & 3.1429 & 2.75 & $(2004,2004)$ & 99 \\
\hline \multirow{4}{*}{ Andhra Pradesh } & 1979 & 4.45 & 3.8556 & $(1979,1979)$ & 100 \\
\hline & 1988 & 3.8556 & 2.925 & $(1987,1988)$ & 100 \\
\hline & 1996 & 2.925 & 2.35 & $(1996,1996)$ & 100 \\
\hline & 2004 & 2.35 & 1.95 & $(2002,2004)$ & 98 \\
\hline \multirow{4}{*}{ Assam } & 1986 & 4.4 & 3.61 & $(1984,1986)$ & 100 \\
\hline & 1996 & 3.61 & 3.18 & $(1993,1996)$ & 96 \\
\hline & 2001 & 3.18 & 2.94 & $(2001,2001)$ & 97 \\
\hline & 2006 & 2.94 & 2.65 & $(2006,2006)$ & 93 \\
\hline \multirow{3}{*}{ Bihar } & 1985 & 5.675 & 5.2 & $(1985,1985)$ & 93 \\
\hline & 1991 & 5.2 & 4.4818 & $(1991,1993)$ & 99 \\
\hline & 2002 & 4.4818 & 4.125 & $(2002,2002)$ & 100 \\
\hline \multirow{4}{*}{ Gujarat } & 1981 & 5.07 & 3.84 & $(1981,1981)$ & 100 \\
\hline & 1991 & 3.84 & 3.0889 & $(1991,1991)$ & 100 \\
\hline & 2000 & 3.0889 & 2.8333 & $(2000,2000)$ & 100 \\
\hline & 2006 & 2.8333 & 2.575 & $(2006,2006)$ & 95 \\
\hline \multirow{5}{*}{ Hary ana } & 1977 & 6.15 & 4.9625 & $(1997,1979)$ & 98 \\
\hline & 1985 & 4.9625 & 4.38 & $(1983,1985)$ & 98 \\
\hline & 1990 & 4.38 & 3.7833 & $(1990,1990)$ & 96 \\
\hline & 1996 & 3.7833 & 3.2571 & $(1996,1996)$ & 97 \\
\hline & 2003 & 3.2571 & 2.7286 & $(2003,2003)$ & 100 \\
\hline \multirow{4}{*}{ Karnataka } & 1979 & 3.99 & 3.6125 & $(1977,1981)$ & 95 \\
\hline & 1987 & 3.6125 & 3.125 & $(1987,1987)$ & 100 \\
\hline & 1995 & 3.125 & 2.4875 & $(1995,1995)$ & 100 \\
\hline & 2003 & 2.4875 & 2.1429 & $(2003,2003)$ & 100 \\
\hline \multirow{5}{*}{ Kerala } & 1977 & 3.6833 & 2.8875 & $(1977,1977)$ & 99 \\
\hline & 1984 & 2.8875 & 2.1714 & $(1984,1984)$ & 100 \\
\hline & 1991 & 2.1714 & 1.725 & $(1991,1991)$ & 92 \\
\hline & 1995 & 1.725 & 1.811 & $(1994,1995)$ & 96 \\
\hline & 2004 & 1.811 & 1.7 & $(2004,2004)$ & 100 \\
\hline \multirow{3}{*}{ Madhy a Pradesh } & 1978 & 5.6714 & 5.2429 & $(1977,1978)$ & 98 \\
\hline & 1985 & 5.2429 & 4.7333 & $(1984,1985)$ & 100 \\
\hline & 1991 & 4.7333 & 4.32 & $(1990,1991)$ & 98 \\
\hline
\end{tabular}




\begin{tabular}{lccccc} 
& 1996 & 4.32 & 3.9667 & $(1996,1996)$ & 97 \\
& 2002 & 3.9667 & 3.55 & $(2002,2002)$ & 96 \\
\multirow{3}{*}{ Maharashtra } & 1977 & 4.2333 & 3.61 & $(1977,1979)$ & 99 \\
& 1987 & 3.61 & 3.32 & $(1983,1987)$ & 98 \\
& 1992 & 3.32 & 2.8286 & $(1992,1992)$ & 98 \\
\multirow{3}{*}{ Orissa } & 1999 & 2.8286 & 2.4 & $(1997,1999)$ & 100 \\
& 2004 & 2.4 & 2.0667 & $(2004,2004)$ & 97 \\
\hline & 1977 & 4.6833 & 4.2625 & $(1977,1979)$ & 99 \\
\multirow{3}{*}{ Punjab } & 1985 & 4.2625 & 3.82 & $(1982,1986)$ & 98 \\
& 1990 & 3.82 & 3.1778 & $(1990,1992)$ & 98 \\
& 1999 & 3.1778 & 2.5727 & $(1999,2000)$ & 100 \\
\hline
\end{tabular}

Note: Estimates are based on thousand bootstraps without replacement, CUSUM estimates and analyses of TFR ranks. The confidence level for TFR changes is $50 \%$, and the confidence level for Inclusion in the table is $90 \%$.

Table 6.1 provides the results of Change-point analyses for TFR in India and selected major states. The estimates of significant changes for India as a whole indicates that a period of more than half century (58 years) India has experienced the critical changes in fertility at five points: 1976, 1985, 1991, 1997 and 2004. The largest change in TFR (from 5.4 to 4.5) was observed in 1976 and the magnitude of change was statistically significant at perfect $100 \%$ level of confidence. Further, in 1991, India has experienced a greater change in TFR (from 4.50 to 3.51 ) at $99 \%$ confidence level.

The estimates of significant changes for states showed varying patterns across the states. However, majorly three types of patterns have been noticed: the first group comprises the south Indian states of Kerala, Andhra Pradesh, and Tamil Nadu which experienced the critical change in fertility transition in late 1970s or early 1980s. The second group of states is Punjab, Karnataka, West Bengal, Haryana, Assam, and Orissa which experienced critical change in fertility transition from early 1980s to late 1990s. And, the last group is the states of Madhya Pradesh, Rajasthan, Uttar Pradesh and Bihar that experienced critical change in fertility in the post 1990. The critical Change-point estimates of fertility for the states were statistically significant with greater confidence levels (Table 6.1).

\subsubsection{Closing and catching-up process of gaps in fertility rates across the states}

The estimates of critical Change-points across the states indicated varying patterns of steady state in fertility transition for different states. The progress of fertility decline in 
some states was faster during the 1970s and 1980s. However, in the post 1990s phase, many of the demographically lagging states of India are catching-up with the demographically advanced states. This catching-up process resulting in fertility homogenisation is more apparent from the results presented in this section. The catching-up process has been examined with three types of measures: 1) simple trend line plots of average TFR of states; 2) trend plots of the association between change in TFR of entire period under observation on initial level of TFR among major states of India; 3) and cumulative distribution of population of the major Indian states by their total fertility rates during 1981-2009.

Figure 6. 2. Trends in mean TFR for major states of India, 1981-2009

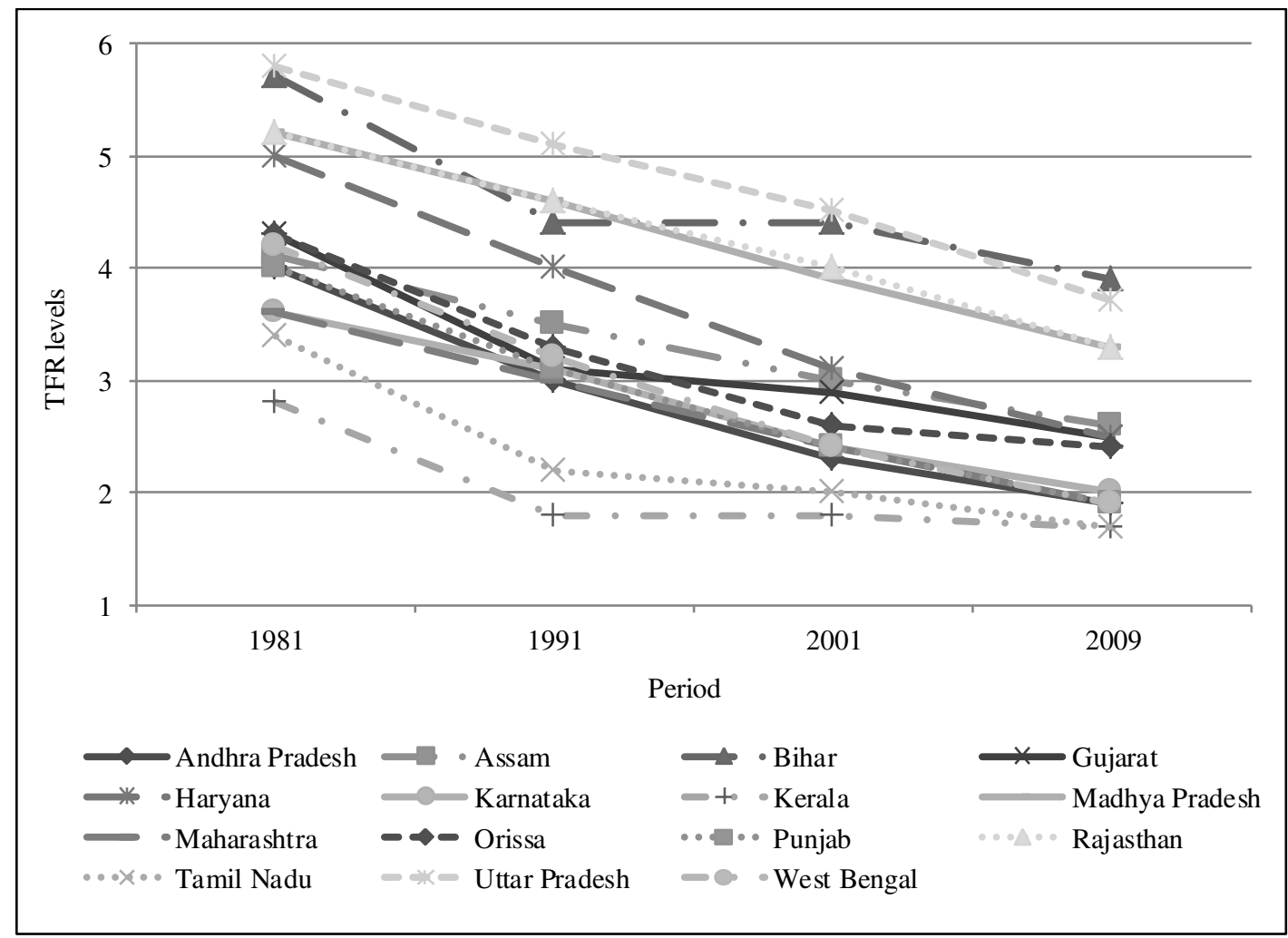

Figure 6.2 presents the simple trend line plots of fertility rates for the major states of India. The figure shows that until 1991, the fertility rates across the major states were diverged. This fertility divergence phase mainly occurred due to greater relative decline in fertility in the demographically advanced south Indian states compared with their north Indian counterparts. However, in the post 1990s period, the demographically advanced states experienced stabilisation in fertility rates between TFR of 2 to 1.8. On other hand, the demographically lagging states experienced greater pace of decline in 
TFR, thus, closing the overall gap in average TFR levels across the states. Figure 6.3 presents the complementing results for the catching up process. The relative change in TFR during entire period under observation by base year (1981) TFR levels indicate that those states which had greater initial TFR level (e.g. more than 4 and 5) have experienced greater change in TFR vis-à-vis those states which had lower levels of initial TFR have experienced lesser change in TFR during 1981-2009. Such a pattern demonstrates the evidence of catching-up process in TFR across the 15 major states of India.

Figure 6.3. Change in TFR during 1981-2009 for major states of India by TFR levels in initial period, 1981

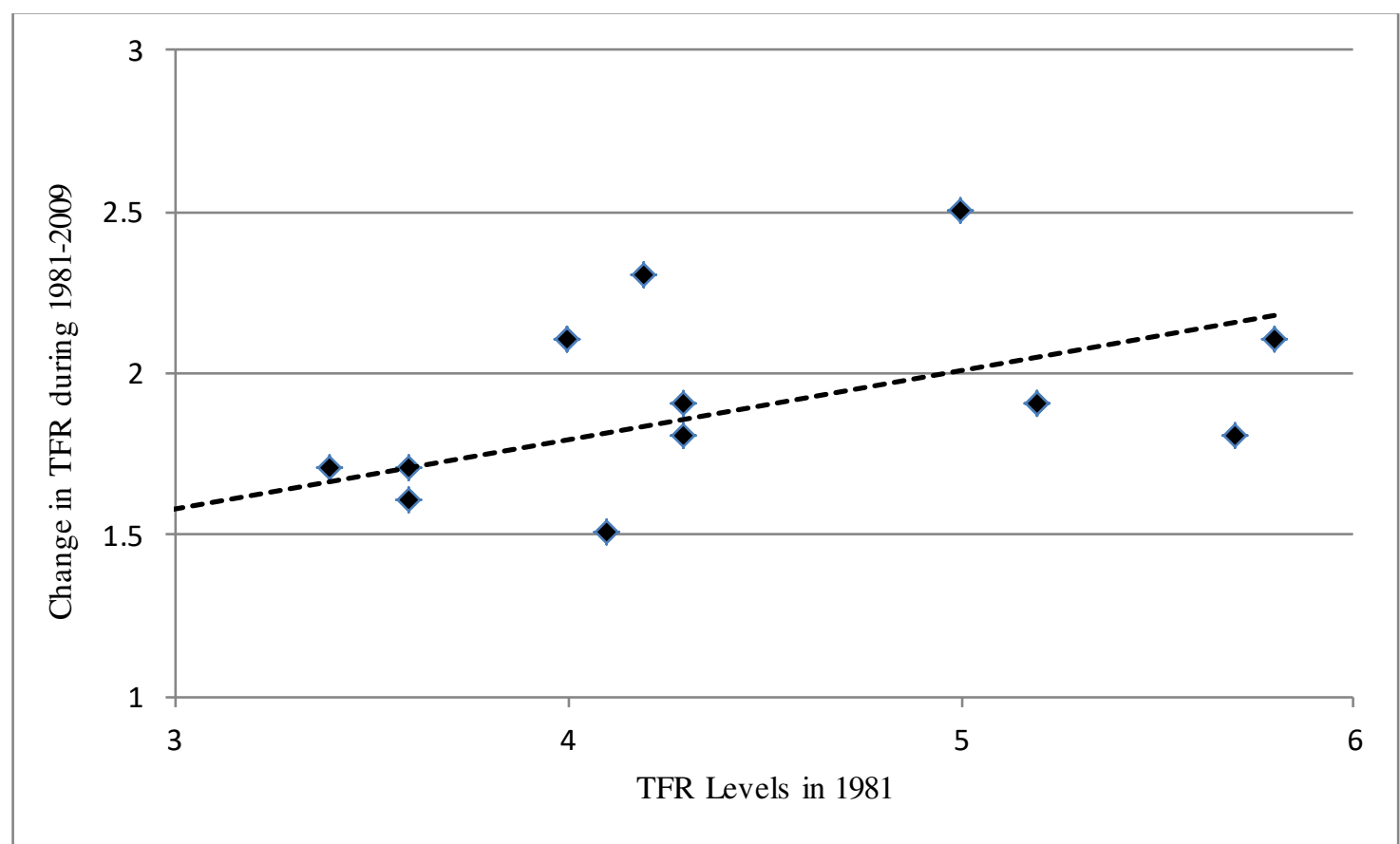

Furthermore, for greater empirical authentication of the catching-up process, Figure 6.4a presents the cumulative distribution of population of the major states of India by their total fertility levels during 1981-2009. The results indicated that over the period, a greater proportion of population is cumulating at lower fertility levels. The pace of fertility change was more significant in the post 1990s period as cumulative population curve line of 2001 and 2009 were observed greatly inclined towards lower levels of fertility. However, such process was more evident in urban areas than rural areas (Figure 6.4b and 6.4c). The three types of TFR trend line plots presented in this section indicated an emerging convergence phase in fertility rates across the Indian states and thus, portray an ideal condition for testing the convergence hypothesis. However, there 
is a need to quantify the volume and speed of convergence for better understanding of the progress and priority setting in policy interventions. Therefore, in the following section, I present the results of the volume and speed of convergence of TFR across the Indian states and socioeconomic strata.

Figure 6.4a. Cumulative distribution of the population of major state of India by TFR levels, 1981 to 2009

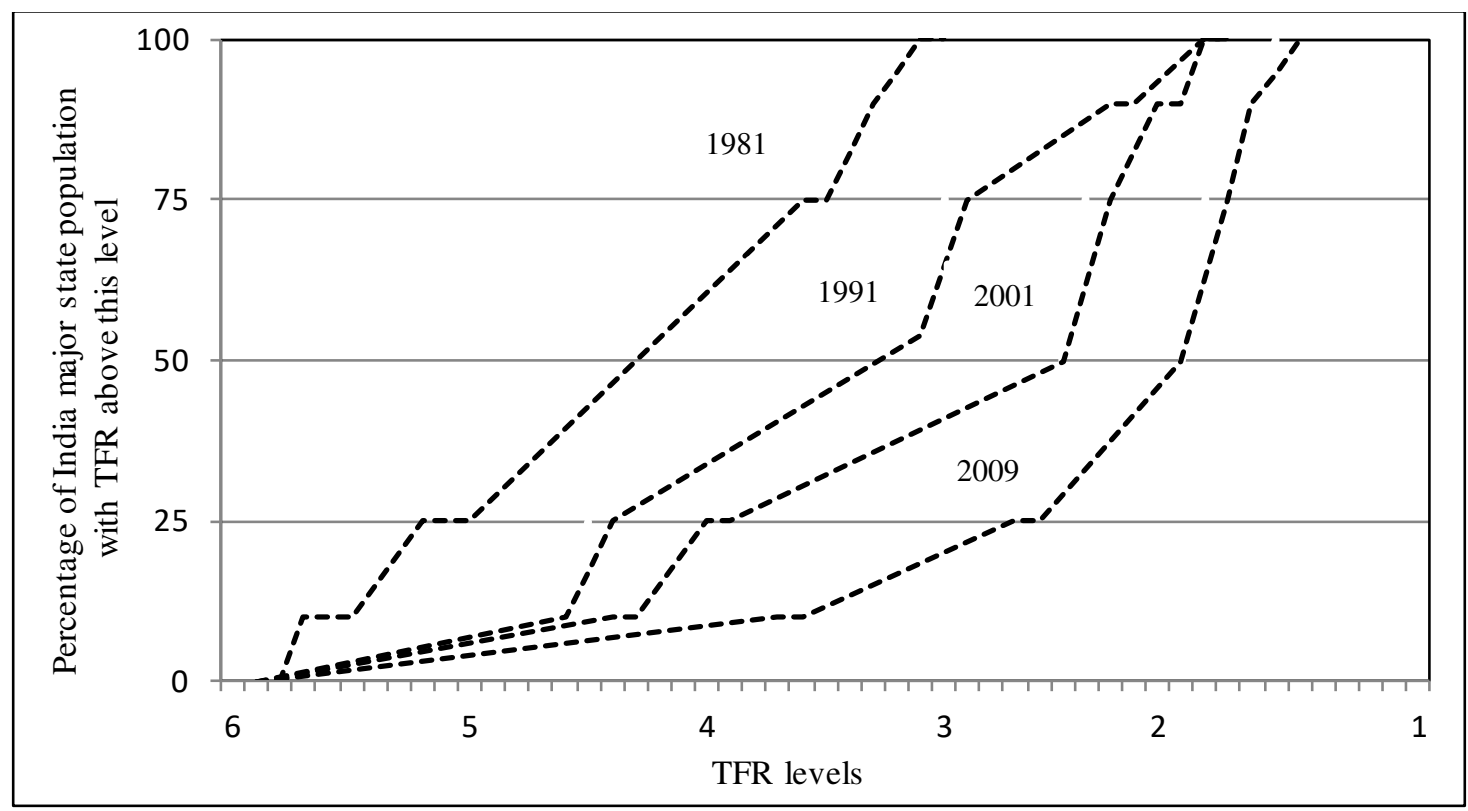

Figure $6.4 \mathrm{~b}$. Cumulative distribution of the population of major state of India by TFR levels in rural areas, 1981 to 2009

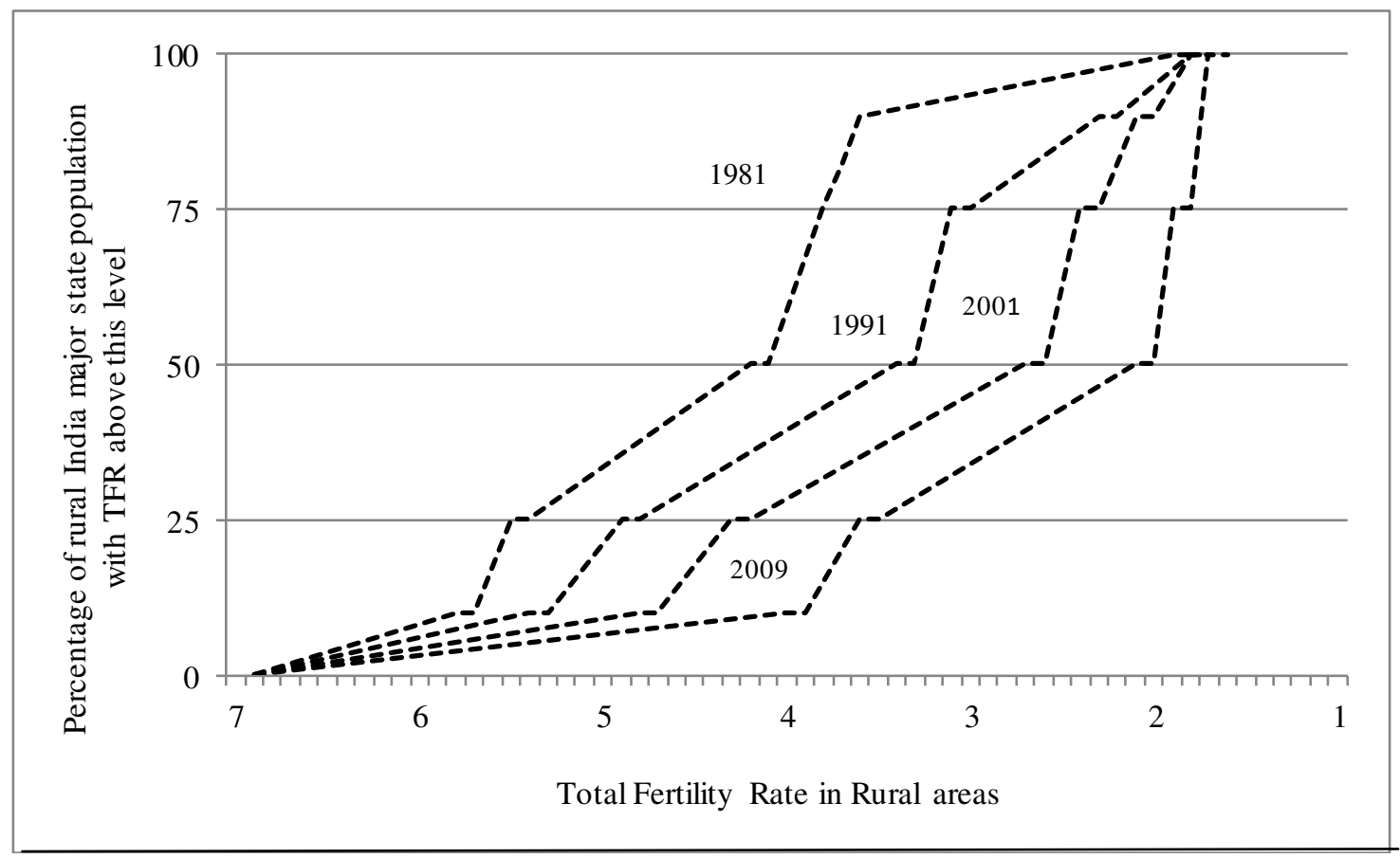


Figure 6.4c. Cumulative distribution of the population of major state of India by TFR levels in urban areas, 1981 to 2009

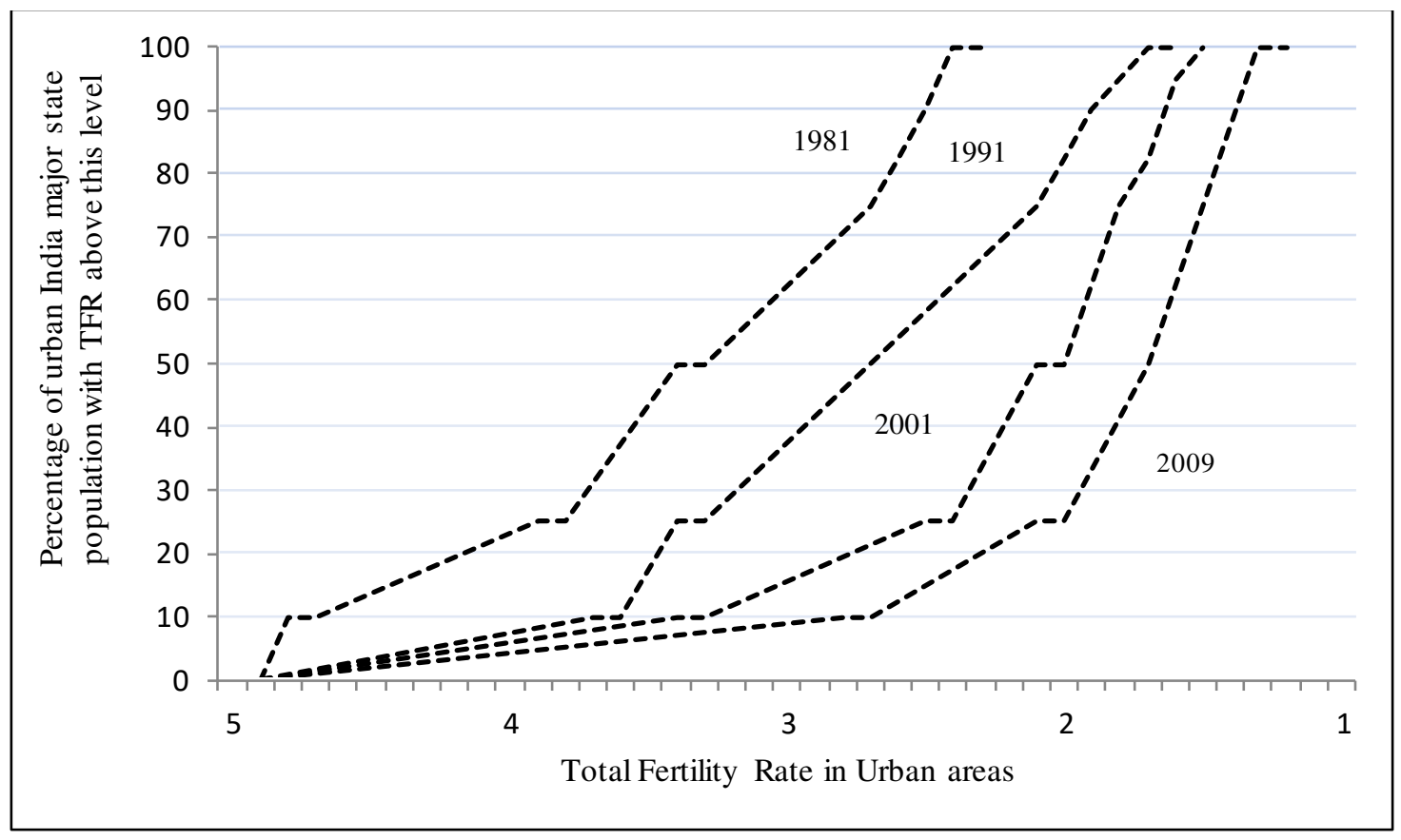

\subsubsection{Convergence estimates}

The process of convergence in fertility rates across the states and socioeconomic spectrum were examined with two categories of measures: 1) Convergence in average fertility rates was examined using absolute and conditional $\beta$-convergence and Sigma convergence and; 2) Inequality based convergence in fertility rates was assessed using absolute and relative inequality based convergence models.

\subsubsection{Convergence in average fertility rates: Absolute $\beta$ convergence model estimates}

In this section, I present the estimates of absolute $\beta$-convergence for TFR of major states during 1981-2009. The results in Table 6.2 indicated that overall fertility rates have diverged across the states during 1981-2009. However, time disaggregated piecewise convergence model estimates for small intervals indicated evidence of fertility divergence only during the earlier period of 1981-1991. In contrast, estimates for the recent periods of 1991-2001 and 2001-2009 showed evidence of convergence in fertility rates across the states $(\beta=-01157, p<0.969$ and $\beta=-.04577, p<0.834$, respectively). The volume and speed $(S)$ of convergence was greater for the most recent period of 2001-2009 ( $S=5 \%$ per annum) compared with 1991-2001 ( $S=1 \%$ per annum). 
Table 6.2. Absolute $\beta$-convergence estimates for TFR among the major Indian states, 1981-2009

\begin{tabular}{lcccccc}
\hline Period & $\begin{array}{c}\beta \\
\text { coefficient }\end{array}$ & P value & $\begin{array}{c}\text { Adjusted } \\
\mathrm{R}^{2}\end{array}$ & $\begin{array}{c}\text { Speed of } \\
\text { convergence }\end{array}$ & $\mathrm{n}$ & $\mathrm{df}$ \\
\hline $1981-2009$ & .24351 & 0.079 & 0.25 & -0.0734 & 15 & 14 \\
$1981-1991$ & .70624 & 0.020 & 0.30 & -0.2087 & 15 & 14 \\
$1991-2001$ & -.01157 & 0.969 & 0.076 & 0.0122 & 15 & 14 \\
$2001-2009$ & -.04577 & 0.834 & 0.073 & 0.0569 & 15 & 14 \\
\hline
\end{tabular}

Note- $\mathrm{n}=$ sample, $\mathrm{df}=$ degree of freedom

\subsubsection{Conditional $\beta$-convergence model estimates}

When estimating convergence rates for the states at the national level, it is not ideal to assume that all the states have the same socioeconomic steady state. Therefore, to account for and disentangle the effects of socioeconomic factors on fertility rates, I have estimated conditional $\beta$-convergence model by incorporating the control variables. Conditional $\beta$-convergence gives the convergence rate, if fertility would not have been influenced by other factors. This study estimated the conditional $\beta$-convergence by adding two critical socioeconomic covariates namely a) the percentage of literate population and $b$ ) the poverty ratio to Barro regression model.

Table 6.3. Conditional $\beta$-convergence estimates for TFR among the major Indian states, 1981-2009

\begin{tabular}{|c|c|c|c|c|c|c|c|c|}
\hline \multirow[t]{2}{*}{ Period } & \multicolumn{4}{|c|}{$\beta$ coefficient } & \multirow{2}{*}{$\begin{array}{c}\text { Adjusted } \\
\mathrm{R}^{2}\end{array}$} & \multirow{2}{*}{$\begin{array}{c}\text { Speed of } \\
\text { convergenc } \\
\mathrm{e}\end{array}$} & \multirow[t]{2}{*}{$\mathrm{n}$} & \multirow[t]{2}{*}{$\mathrm{df}$} \\
\hline & TFR & $\begin{array}{l}\text { Proportion } \\
\text { of illiterate } \\
\text { population }\end{array}$ & $\begin{array}{c}\text { Poverty } \\
\text { ratio }\end{array}$ & $\begin{array}{l}\text { TFR of club } \\
\text { of south India } \\
\text { states }\end{array}$ & & & & \\
\hline 1981- & .39946 & .01281 & .01104 & -.43388 & 0.24 & -0.1066 & 15 & 14 \\
\hline 2009 & $(0.053)$ & $(0.291)$ & $(0.079)$ & $(0.400)$ & & & & \\
\hline $\begin{array}{l}1981- \\
1991\end{array}$ & $\begin{array}{l}.32093 \\
(0.452)\end{array}$ & $\begin{array}{l}-.03201 \\
(0.240)\end{array}$ & $\begin{array}{l}-.00075 \\
(0.966)\end{array}$ & $\begin{array}{l}3.4064 \\
(0.227)\end{array}$ & 0.27 & -0.1437 & 15 & 14 \\
\hline $\begin{array}{l}1991- \\
2001\end{array}$ & $\begin{array}{l}.35333 \\
(0.498)\end{array}$ & $\begin{array}{l}.04297 \\
(0.245)\end{array}$ & $\begin{array}{l}.03223 \\
(0.233)\end{array}$ & $\begin{array}{r}-1.9237 \\
(0.002)\end{array}$ & 0.025 & -0.1511 & 15 & 14 \\
\hline $\begin{array}{l}2001- \\
2009\end{array}$ & $\begin{array}{l}.02125 \\
(0.933)\end{array}$ & $\begin{array}{l}.02402 \\
(0.308)\end{array}$ & $\begin{array}{l}.02538 \\
(0.092)\end{array}$ & $\begin{array}{l}-1.7325 \\
(0.134)\end{array}$ & 0.08 & -0.0196 & 15 & 14 \\
\hline
\end{tabular}

Note- $\mathrm{n}=$ sample, $\mathrm{df}=$ degree of freedom

Table 6.3 presents the results of conditional $\beta$-convergence estimates. Similar to absolute $\beta$-convergence estimates, conditional $\beta$-convergence estimates also showed the evidence of divergence in fertility rates across states in India during 1981-2009. Unlike, piecewise convergence estimates of absolute $\beta$-convergence model that showed divergence in the initial period and convergence in the recent period, the piecewise 
convergence estimates of conditional $\beta$-convergence model showed evidence of fertility divergence for all the three periods (1981-1991, 1991-2001, and 2001-2009). However, the rate of divergence declined for the recent period (i.e. 14\% in 1981-91 declined to just $1 \%$ in 2001-09). Overall, the conditional $\beta$-convergence model estimates showed the evidence of convergence in terms of absolute $\beta$-convergence estimates for the recent period disappeared after disentangling of the variation in literacy and poverty ratios across the states. This indicates the strong connection between fertility convergence and socioeconomic steady state conditions of the states.

$\beta$-convergence in total fertility rates by socioeconomic spectrum of major Indian states: $\beta$-convergence estimates for total fertility rates by the socioeconomic groups (Scheduled caste, Scheduled tribe, Other backward caste, Other caste, Hindus, Muslims, Other religion groups, Poorest, Poorer, Middle, Richer and Richest economic groups $[n=12]$ ) across the 15 states $\left(15^{*} 12=180\right.$ cases) indicated evidence of divergence $(\beta=.02566$, $\mathrm{p}<0.870, S=2 \%$ per annum) for entire period under observation (1992-2006). However, assessments of convergence for shorter interval indicated that fertility rates of socioeconomic groups across the states converged during initial phase of 1992-99 $(\beta=$ $.82391, \mathrm{p}<0.004, \mathrm{~S}=29 \%$ per annum), but diverged $(\beta=-.23388, \mathrm{p}<0.334, \mathrm{~S}=13 \%$ per annum) during the recent phase of 1999-2006. Overall, $\beta$-convergence estimates for socioeconomic groups across the states suggest that evidence of convergence in earlier phase was being replaced with divergence in fertility rates in the later phase.

\subsubsection{Convergence clubs}

The fertility rates may not converge as strongly and consistently across all the states, but may converge more strongly in a subset of states of a particular region. In India, it may be possible that the demographically advanced south Indian states (club) are converging faster and earlier than the north India states. Therefore, to examine such possibilities, I have incorporated south Indian regional dummy variable in the conditional $\beta$ convergence model to estimate the fertility convergence among south Indian states. Table 6.3 provides the results of fertility convergence estimates among south Indian states. The negative $\beta$ coefficient $(\beta=-.43388, \mathrm{p}<0.400)$ for south Indian states demonstrates evidence of fertility convergence during the long-term phase of 19812009. However, in the south Indian states too fertility diverged during the early phase of 
1981-91 $(\beta=3.4064, \mathrm{p}<0.227)$, but converged strongly during the last two decades. Moreover, the speed of convergence was much more pronounced during 1991-2001 ( $\beta=$ -1.9237) compared to 2001-2009 $(\beta=-0.0196)$.

Table 6.4. $\beta$-convergence estimates for TFR among the socioeconomic groups of major Indian states and club of south Indian states 1992-2006

\begin{tabular}{|c|c|c|c|c|c|c|}
\hline \multirow[t]{2}{*}{ Period } & \multicolumn{2}{|c|}{$\beta$ coefficient } & \multirow{2}{*}{$\begin{array}{l}\text { Adjusted } \\
\mathrm{R}^{2}\end{array}$} & \multirow{2}{*}{$\begin{array}{c}\text { Speed of } \\
\text { convergence }\end{array}$} & \multirow[t]{2}{*}{$\mathrm{n}$} & \multirow[t]{2}{*}{ df } \\
\hline & TFR & $\begin{array}{c}\text { TFR of club of } \\
\text { South India states }\end{array}$ & & & & \\
\hline $1992-93$ to & .02566 & -1.7956 & 0.072 & -0.0221 & 138 & 137 \\
\hline $2005-06$ & $(0.870)$ & $(0.000)$ & & & & \\
\hline $1992-93$ to & -.82391 & -4.8771 & 0.044 & 0.2970 & 161 & 160 \\
\hline 1998-99 & (0.004) & (0.000) & & & & \\
\hline $1998-99$ to & .23388 & -4.5978 & 0.004 & -0.1385 & 150 & 149 \\
\hline 2005-06 & $(0.334)$ & $(0.000)$ & & & & \\
\hline
\end{tabular}

Note- $\mathrm{n}=\mathrm{sample}, \mathrm{df}=$ degree of freedom

Table 6.4 also presents the convergence estimates for the club of south Indian states across socioeconomic spectrum using the three rounds of NFHS data. Compared to the major states, the conditional $\beta$-convergence estimates for the socioeconomic crosssections of south Indian states suggested evidence of strong fertility convergence ( $\beta=$ $1.7956, \mathrm{p}<0.000)$ for the entire period of 1992-2006. However, short-term disaggregated convergence estimates also revealed convergence in fertility rates across the socioeconomic stratum, but the volume of convergence was greater during the earlier period of 1992-99 $(\beta=-4.8771, \mathrm{p}<0.000)$ compared to the recent period of $1999-2006$ $(\beta=-4.5978, p<0.000)$. Overall, the results indicate that fertility rates did not necessarily converge strongly for all major states of India, but converged more strongly for the club of south Indian states.

In this chapter, the changing patterns of fertility clubs among major states of India are assessed. Further, low fertility and lowest-low fertility clubs are also identified. Figure 6.5 documents the shifting pattern of major states from high fertility to high, medium and low and below replacement level fertility states. During 1951-66, majority of the states were located in the high fertility club (Phase I), but Tamil Nadu is the only state which was in phase II during this period. In the successive decades, other south Indian states including Maharashtra joined club II. In the year 1991, Kerala first entered into the below replacement level fertility club. Later, in the year, 2001 Tamil Nadu also joined the Kerala. However, in 2009, 7 states out of 15 major states entered into below 
replacement level club. This number may further go up if we count small states like Himachal Pradesh, Goa, North eastern states and other Union Territories. This number is also more according to latest report of office of registrar general of India (2010).

Figure 6.5. Changing Patterns of Fertility Clubs among Major States of India, 19512009

\begin{tabular}{|c|c|c|c|c|c|c|c|}
\hline Period & $\begin{array}{l}\text { TFR } \\
\text { range }\end{array}$ & 1951-66 & 1971 & 1981 & 1991 & 2001 & 2009 \\
\hline \multirow{2}{*}{ 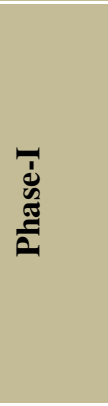 } & $\begin{array}{l}\text { More } \\
\text { than } 6\end{array}$ & $\begin{array}{c}\text { Gujarat, } \\
\text { Haryana, } \\
\text { M.P., Oris sa, } \\
\text { Punjab, U.P., } \\
\text { Rajasthan }\end{array}$ & Haryana, U.P. & & & & \\
\hline & $5.1-6$ & $\begin{array}{c}\text { A.P., } \\
\text { Karnataka, } \\
\text { Maharashtra, } \\
\text { Kerala }\end{array}$ & $\begin{array}{c}\text { Gujarat, } \\
\text { Assam, M.P., } \\
\text { Orissa, Punjab, } \\
\text { Rajasthan }\end{array}$ & $\begin{array}{l}\text { Bihar, M.P., } \\
\text { Rajasthan, } \\
\text { Uttar Pradesh }\end{array}$ & U.P. & & \\
\hline \multirow{2}{*}{ 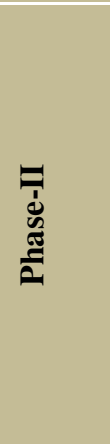 } & $4.1-5$ & Tamil Nadu & $\begin{array}{c}\text { A.P., } \\
\text { Karnataka, } \\
\text { Maharashtra, } \\
\text { Orissa, Kerala }\end{array}$ & $\begin{array}{l}\text { Assam, } \\
\text { Gujarat, } \\
\text { Orissa, West } \\
\text { Bengal }\end{array}$ & $\begin{array}{l}\text { Bihar, M.P., } \\
\text { Rajasthan, }\end{array}$ & $\begin{array}{l}\text { U.P. } \\
\text { Bihar }\end{array}$ & \\
\hline & $3.1-4$ & & Tamil Nadu & $\begin{array}{c}\text { A.P., } \\
\text { Karnataka, } \\
\text { Maharashtra, } \\
\text { Punjab, } \\
\text { Tamil Nadu }\end{array}$ & $\begin{array}{c}\text { Assam, } \\
\text { Gujarat, } \\
\text { Karnataka, } \\
\text { Orissa, } \\
\text { Punjab, West } \\
\text { Bengal }\end{array}$ & $\begin{array}{l}\text { Haryana, } \\
\text { M.P., } \\
\text { Rajasthan }\end{array}$ & $\begin{array}{c}\text { U.P. } \\
\text { Bihar, } \\
\text { Rajasthan }\end{array}$ \\
\hline 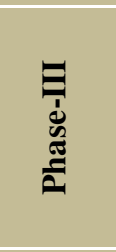 & $2.1-3$ & & & Kerala & $\begin{array}{c}\text { A.P., } \\
\text { Maharashtra } \\
\text { Tamil Nadu }\end{array}$ & $\begin{array}{l}\text { Assam, A.P., } \\
\text { Gujarat, } \\
\text { Karnataka, } \\
\text { Orissa, } \\
\text { Maharashtra, } \\
\text { West Bengal }\end{array}$ & $\begin{array}{l}\text { Assam, } \\
\text { Gujarat, } \\
\text { Haryana, } \\
\text { Orissa, }\end{array}$ \\
\hline 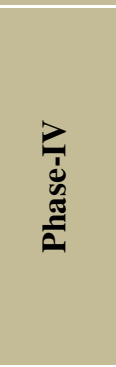 & $\begin{array}{l}\text { Less } \\
\text { than or } \\
\text { equal to } \\
2\end{array}$ & & & & Kerala & $\begin{array}{c}\text { Tamil Nadu, } \\
\text { Kerala }\end{array}$ & $\begin{array}{l}\text { A.P., } \\
\text { Karnatak } \\
\text { a, } \\
\text { Maharash } \\
\text { tra, Tamil } \\
\text { Nadu, } \\
\text { Kerala, } \\
\text { West } \\
\text { Bengal }\end{array}$ \\
\hline
\end{tabular}

Source: Rele (1951-66); Office of Registrar General of India (1971-2009)

Note: A.P.-Andhra Pradesh; M.P. Madhya Pradesh, U.P. - Uttar Pradesh.

Low and lowest-low fertility is an emerging phenomenon in India. However, yet, no empirical evidence has been documented. The lack of availability of latest unit level data on fertility is the main hindrance for this. This chapter also tried to document some evidence on changing pattern of low and lowest-low fertility clubs based on three rounds of national family health survey. The results presented in the figure 6.6 revealed 
a number of intriguing findings on less know phenomena of lowest-low fertility in India. In the year 1992-93, only eight socioeconomic groups such as A.P. (Others); Karnataka (Others); Kerala (Hindu, Others; Richest, SC, ST); Maharashtra (Richer) are identified in low fertility club. However, during this period, none of the socioeconomic groups reached to the lowest-low fertility group. By 1998-99, the number has increased to 28: A.P. (Others); Assam (OBC; Hindu, Others; Richest); Gujarat (Others); Karnataka (OBC; Hindu; Poorer, Middle, Richer, Richest); Kerala (SC, OBC, Others; Hindu, Others; Poorest, Poorer, Middle, Richer); Punjab (Others; Richest); T.N. (Others); WB (OBC; Others; Richer, Richest). Even in 1998-99, none of the socioeconomic groups in India had reached to the lowest-low fertility group. However, the more interesting findings are observed in the year, 2005-06. The number of socioeconomic groups in the club of low fertility has increased to 46: A.P. (SC, OBC, Others; Hindu, Muslim, Others; Poorer, Middle, Richer, Richest); Assam (OBC; Hindu; Richer); Gujarat (Others; Richest); Karnataka (OBC, Others; Middle); Kerala ( OBC; Hindu, SC; Middle, Richer, Richest); MP (Richest); Maharashtra (OBC; Hindu, Others; Richest); Orissa (Others; Middle, Richer, Richest); Punjab (Others; Richest); T.N. ( OBC; Hindu, Others; Poorer, Middle, Richer, Richest); UP (Others); WB ( Hindu; Richer). Three socioeconomic groups are also found with lowest-low fertility: Assam (Richest); Kerala (SC), WB (Richest). It can be expected that this number would have been much more if we would have information on fertility by socioeconomic groups for the year, 2012-13. 
Figure 6.6. Changing patterns of fertility clubs among socio-economic groups of major states of India, 1992-2006

\begin{tabular}{|c|c|c|c|c|}
\hline $\begin{array}{c}\text { Fertility } \\
\text { Clubs }\end{array}$ & $\begin{array}{c}\text { TFR } \\
\text { range }\end{array}$ & $1992-93$ & 1998-99 & $2005-06$ \\
\hline 吾 & $\begin{array}{c}\text { More } \\
\text { than } 3\end{array}$ & $\begin{array}{l}\text { A.P. (ST, Poorest), Assam (ST, Others; } \\
\text { Muslim, Others; Poorest, Poorer, Middle, } \\
\text { Richer); Bihar (SC, ST, OBC, Others; Hindu, } \\
\text { Muslim, Others; Poorest, Poorer, Middle, } \\
\text { Richer, Richest); Gujarat (ST; Muslim; } \\
\text { Poorest, Poorer), Haryana (SC, Others; } \\
\text { Hindu, Muslim, Others; Poorest, Poorer, } \\
\text { Middle, Richer, Richest); Karnataka (SC, } \\
\text { Muslim, Poorest); Kerala (Poorer, Poorest); } \\
\text { M.P. (SC, ST, Others; Hindu, Muslim; } \\
\text { Poorest Poorer, Middle, Richer, Richest); } \\
\text { Maharashtra (SC, ST; Muslim; Poorest); } \\
\text { Orissa (SC; Muslim, Others; Poorest); } \\
\text { Punjab (SC; Muslim; Others; Poorest, Poorer, } \\
\text { Middle, Richer); Rajasthan ( SC, ST; Others; } \\
\text { Hindu, Muslim, Others, Poorest, Poorer, } \\
\text { Middle, Richer, Richest); UP ( SC, ST, OBC, } \\
\text { Others; Hindu, Muslim, Others, Poorest, } \\
\text { Poorer, Middle, Richer, Richest); WB (SC, } \\
\text { ST; Muslim; Poorest, Poorer) }\end{array}$ & $\begin{array}{l}\text { Assam (Muslim; Poorest); Bihar ( SC, OBC, Others; } \\
\text { Hindu, Muslim; Poorest, Poorer, Middle); Gujarat (SC; } \\
\text { Muslim; Poorest); Haryana (SC, OBC; Muslim; Poorest, } \\
\text { Poorer, Richer); M.P. (SC, ST, OBC; Hindu, Muslim; } \\
\text { Poorest, Poorer, Middle); Maharashtra (Muslim); Orissa } \\
\text { (Muslim); Punjab (Muslim); Rajasthan (SC, ST, OBC, } \\
\text { Other; Hindu, Muslim; Poorest, Poorer, Middle, Richer); } \\
\text { UP (SC, ST, OBC, Others; Hindu, Muslim; Poorest, Poorer, } \\
\text { Middle, Richer, Richest); WB (Muslim) }\end{array}$ & $\begin{array}{l}\text { Assam (Muslim; Poorest); Bihar (SC, ST, OBC, } \\
\text { Others; Hindu, Muslim, Others; Poorest, Poorer, Middle, } \\
\text { Richer); Gujarat (Poorer); Haryana (Poorer, Middle); } \\
\text { M.P. (SC, ST, OBC; Hindu, Muslim; Poorest, Poorer, } \\
\text { Middle); Orissa (ST); Rajasthan (SC, ST, OBC; } \\
\text { Hindu, Muslim; Poorest, Poorer, Middle); UP (SC, ST, } \\
\text { OBC, Others; Hindu, Muslim; Poorest, Poorer, Middle, } \\
\text { Richer); WB (Muslim; Poorest) }\end{array}$ \\
\hline
\end{tabular}




\begin{tabular}{|c|c|c|c|c|}
\hline 苞 & 2.1-3 & $\begin{array}{l}\text { A.P. ( SC, Others; Hindu, Muslim; Poorer, } \\
\text { Middle, Richer, Richest); Assam ( SC; } \\
\text { Hindu; Richest); Gujarat (SC, Others; Hindu; } \\
\text { Middle, Richer, Richest); Karnataka (ST, } \\
\text { Others; Hindu; Poorer, Middle, Richer, } \\
\text { Richest); Kerala (Others; Muslim; Poorest, } \\
\text { Richer); MP (Others); Maharashtra (Others; } \\
\text { Hindu, Others; Poorer, Middle, Richest); } \\
\text { Orissa (ST, Others; Hindu; Poorer, Middle, } \\
\text { Richer, Richest); Punjab (Others; Hindu; } \\
\text { Richest); T.N. ( SC, Others; Hindu, Muslim, } \\
\text { Others, Poorest, Poorer, Middle, Richer, } \\
\text { Richest); WB (Others; Hindu, Others; Middle, } \\
\text { Richer, Richest) }\end{array}$ & $\begin{array}{l}\text { A.P. (SC, ST, OBC; Hindu, Muslim, Others; Poorest, } \\
\text { Poorer, Middle, Richer, Richest); Assam (SC, ST, Others; } \\
\text { Poorer, Middle, Richer); Bihar (ST; Others; Richer, } \\
\text { Richest); Gujarat (ST, OBC, Others; Hindu; Poorer, } \\
\text { Middle, Richer, Richest); Haryana (Others; Hindu, Others; } \\
\text { Middle); Karnataka (SC, ST, Others; Muslim, Others; } \\
\text { Poorest); Kerala (Muslim; Richest); MP (Others; Others; } \\
\text { Richer, Richest); Maharashtra (SC, ST, OBC, Others; } \\
\text { Hindu, Others; Poorest, Poorer, Middle, Richer, Richest); } \\
\text { Orissa (SC, ST, OBC, Others; Hindu, Others; Poorest, } \\
\text { Poorer, Middle, Richer, Richest); Punjab (SC, OBC; Hindu, } \\
\text { Others; Poorest, Poorer, Middle, Richer); Rajasthan } \\
\text { (Others; Richest); T.N. ( SC, ST, OBC; Hindu, Muslim, } \\
\text { Others; Poorest, Poorer, Middle, Richer, Richest);UP } \\
\text { (Others); WB (SC, ST, Others; Hindu; Poorest, Poorer, } \\
\text { Middle) }\end{array}$ & $\begin{array}{l}\text { A.P. (Poorest); Assam ( SC, ST, Others; Poorer, } \\
\text { Middle); Bihar (Richest); Gujarat (SC,ST, OBC; } \\
\text { Hindu, Muslim; Middle, Richer); Haryana ( SC, ST, } \\
\text { Others; Hindu; Richer, Richest); Karnataka (SC, ST; } \\
\text { Hindu, Muslim; Poorest, Poorer, Richer, Richest); } \\
\text { Kerala ( Others; Muslim, Others); MP ( Others; } \\
\text { Richer); Maharashtra ( SC, ST, Others; Muslim; } \\
\text { Poorest, Poorer, Middle, Richer); Orissa (SC, OBC } \\
\text { Hindu; Poorest, Poorer); Punjab (SC; Hindu; Middle, } \\
\text { Richer); Rajasthan (Other; Richer, Richest); TN (SC; } \\
\text { Poorest); UP (Richest); WB (SC, Other; Poorer, Middle) }\end{array}$ \\
\hline 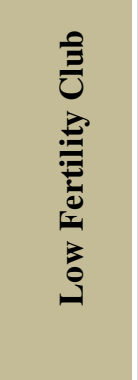 & $1.4-2$ & $\begin{array}{l}\text { A.P. (Others); Karnataka (Others); Kerala } \\
\text { (Hindu, Others; Richest, SC, ST); } \\
\text { Maharashtra (Richer) }\end{array}$ & $\begin{array}{l}\text { A.P. (Others); Assam (OBC; Hindu, Others; Richest); } \\
\text { Gujarat (Others); Karnataka (OBC; Hindu; Poorer, Middle, } \\
\text { Richer, Richest); Kerala (SC, OBC, Others; Hindu, Others; } \\
\text { Poorest, Poorer, Middle, Richer); Punjab (Others; Richest); } \\
\text { T.N. (Others); WB (OBC; Others; Richer, Richest) }\end{array}$ & $\begin{array}{l}\text { A.P. (SC, OBC, Others; Hindu, Muslim, Others; Poorer, } \\
\text { Middle, Richer, Richest); Assam (OBC; Hindu; Richer); } \\
\text { Gujarat (Others; Richest); Karnataka (OBC, Others; } \\
\text { Middle); Kerala ( OBC; Hindu; Middle, Richer, } \\
\text { Richest); MP (Richest); Maharashtra (OBC; Hindu, } \\
\text { Others; Richest); Orissa (Others; Middle, Richer, } \\
\text { Richest); Punjab (Others; Richest); T.N. (OBC; } \\
\text { Hindu, Others; Poorer, Middle, Richer, Richest); UP } \\
\text { (Others); WB ( Hindu; Richer) }\end{array}$ \\
\hline 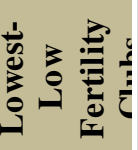 & $\begin{array}{l}\text { Less } \\
\text { than } 1.4\end{array}$ & & & Assam (Richest); Kerala (SC),WB (Richest) \\
\hline
\end{tabular}

Source: Three rounds of NFHS (1-3) and Author's estimation based on NFHS data. 


\subsubsection{Sigma convergence}

As we mentioned in the previous chapters that $\beta$-convergence is a necessary, but not a sufficient condition for Sigma convergence. Alternatively, Sigma convergence is sufficient, but not a necessary condition for $\beta$-convergence (Young et al. 2004). Therefore, in order to test for both these conditions, I have estimated Sigma convergence alongside $\beta$ convergence and it was estimated based on reduction in standard deviations in TFR of the major Indian states. Commensurate with absolute $\beta$ convergence estimates, Sigma convergence estimates of TFR across the states also indicate divergence in fertility rate during the initial period of 1981-91 and convergence in fertility in the post-1991 period. However, the speed of convergence was much greater for recent period than earlier period (Figure 6.7).

Figure 6. 7. Sigma convergences estimates of TFR across the major states of India, 1981-2009

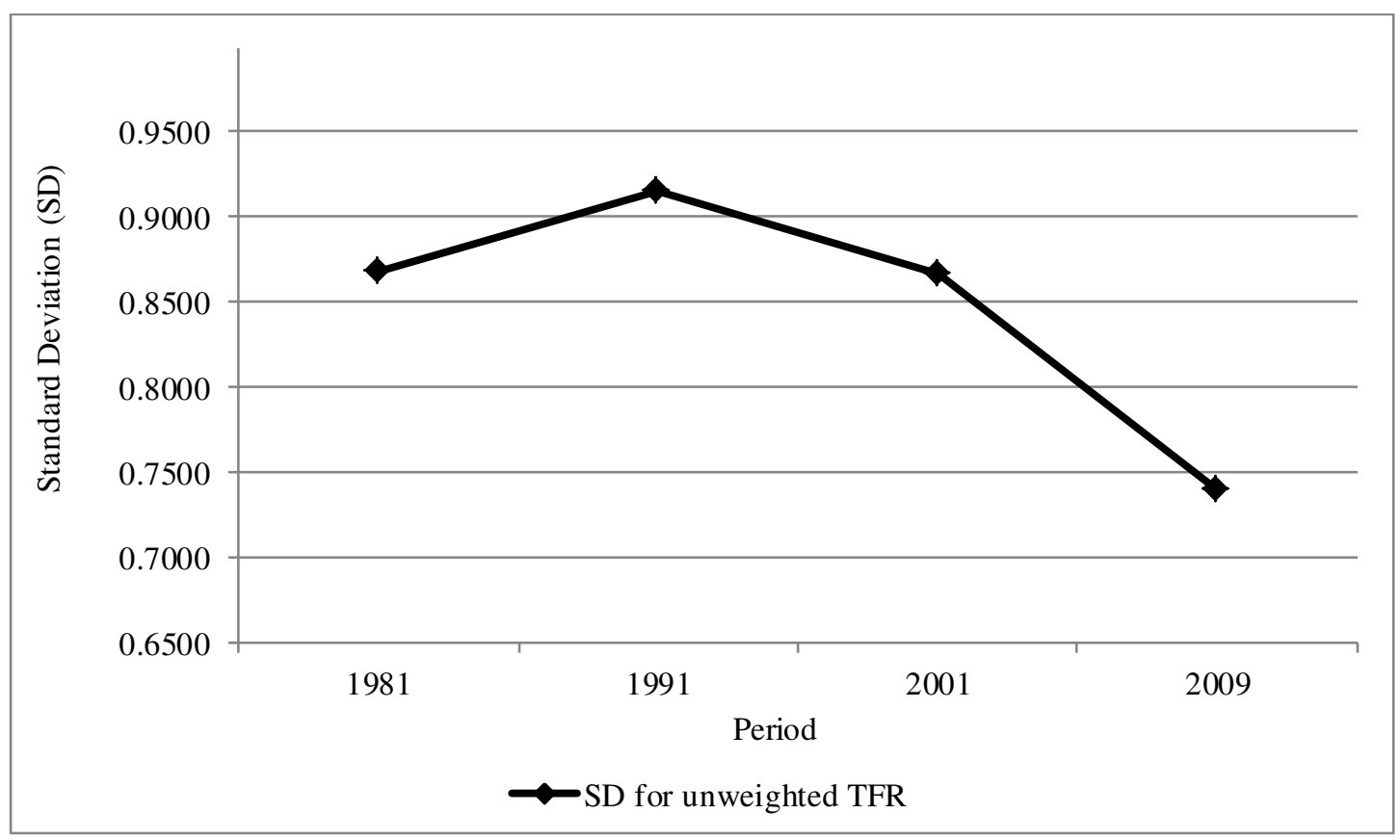

\subsubsection{Inequality adjusted convergence measures: Convergence in absolute and relative dispersion of state level fertility rates}

Decline in the gap in mean fertility rates of the states do not mean fertility across the states in India is converging. As conventionally defined in studies dealing with between-country differences, convergence occurs when relative difference between the states declines (Dorius 2008). Therefore, to overcome the shortcomings of convergence 
measures based on averages alone, I have used cutting-edge inequality based measures to assess the progress of convergence in both absolute and relative dispersion of fertility rates of the states by weighting with the proportion of population of the respective states. The inequality based convergence measures are estimated in terms of percentage reduction in absolute (DMF) and relative (Gini index) convergence measures.

Figure 6.8. Trends in dispersion measure and Gini index of the TFR distribution among the major Indian states, 1981-2009

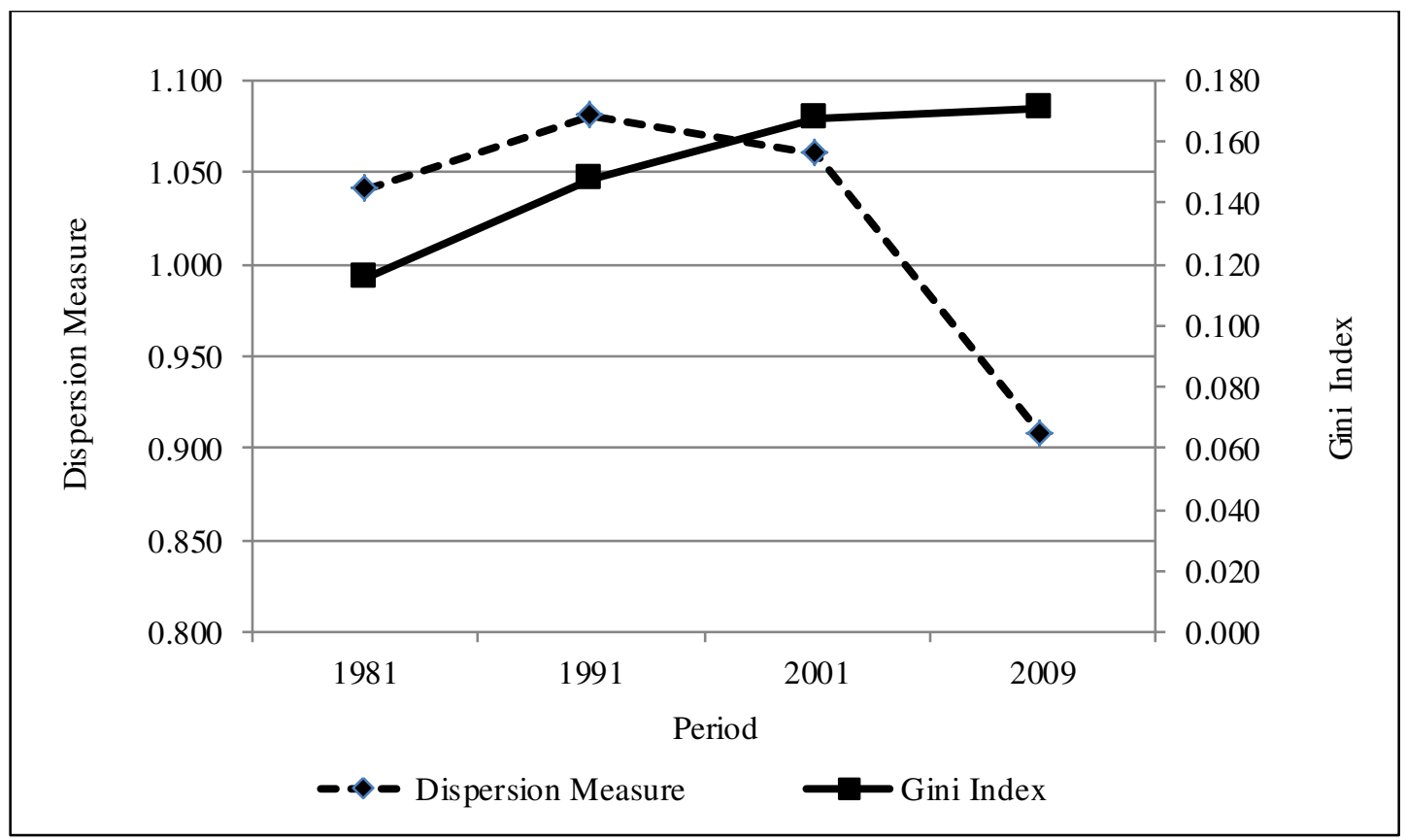

Figure 6.8 displays the trends in 'between state' inequalities in TFR in terms of DMF and Gini index. DMF values indicate absolute dispersion and Gini index values indicate relative dispersion in fertility rates. The results revealed that over the long-term period of 1981-2009), the DMF values have declined substantially, but this decline was greater for the recent period of 2001-2009. In contrast, Gini Index values indicated rising inequality trends during the long-term duration of 1981-2009. This is an indication that absolute inter-state inequalities have been decreasing, but relative inequalities have been rising (see figure 6.1: conceptual framework for understanding underlying mechanism). This is possible during the progressive stage of demographic transition, where increasing averages can also increase the relative inequalities. In the context of studying health inequalities, Wagstaff (2002) referred to this phenomenon as 'inequalities are swimming against the tide in developing countries'. 
Figure 6.9 show the results of absolute convergence i.e. the percentage reduction in absolute dispersion of TFR between the states during the long-term period of 19812009. The results indicate divergence in fertility rates for the initial period of 1981-91, followed by a strong phase of convergence in fertility for the later periods of 1991-2001 and 2001-09.

Figure 6.9. Trends in the rate of convergence in absolute dispersion of TFR among the major states of India, 1981-2009

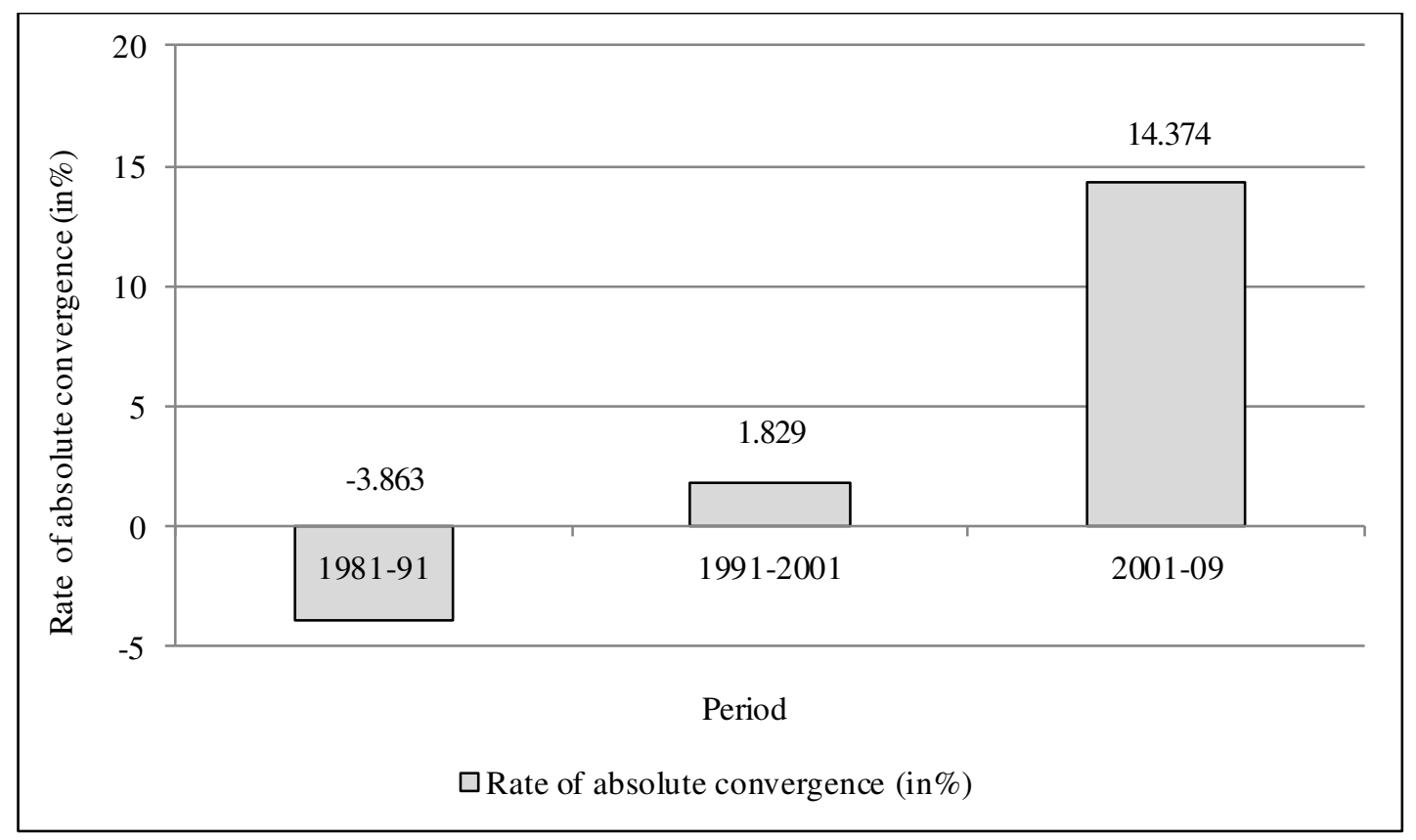

Moreover, the volume of convergence (14\% reduction in DMF) was very high during 2001-2009. In contrast, the relative convergence estimates displayed in figure 6.10 indicate evidence of divergence in fertility rates for the entire period of 1981-2009), however, divergence is greater ( $28 \%$ increase in Gini index) during the initial period of 1981-91 compared to (2\% increase in Gini index) the recent period of 2001-09. Overall, the results of inequality adjusted convergence measures suggest evidence of convergence in absolute dispersion of total fertility rate, but relative convergence suggests a divergence trend (Figure 6.9 and 6.10). However, the promising trend is that the substantial decreases in volume of divergence in terms of relative dispersion of total fertility rate indicate the prospect of near future convergence in relative dispersion of fertility. 
Figure 6.10. Trends in the rate of convergence in relative dispersion of TFR among the major states of India, 1981-2009

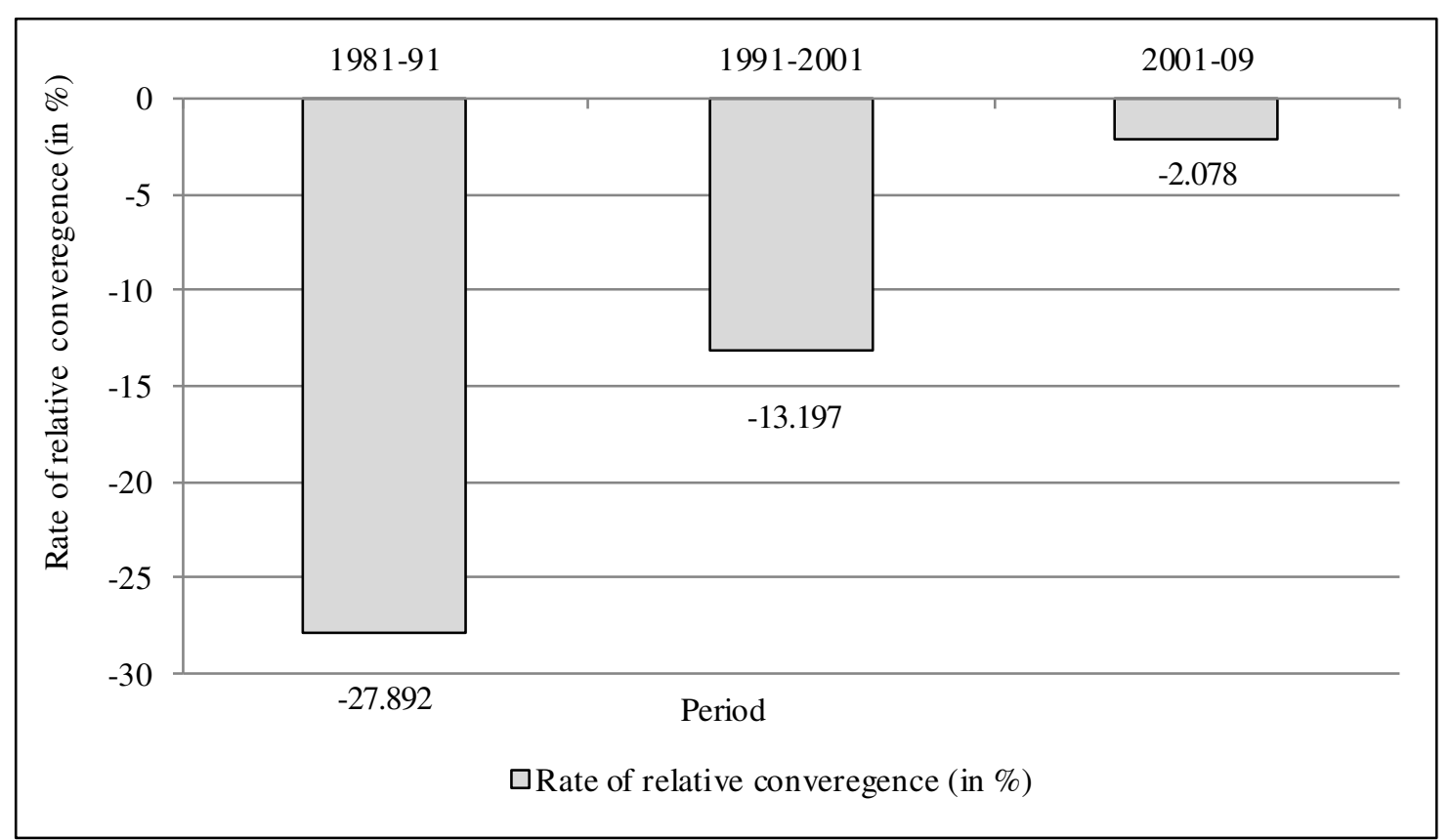

\subsubsection{Nonparametric models of Convergence}

The assessment of convergence process through nonparametric models reveals much better understanding of convergence than the above models. Kernel distribution plots clearly evident that in 1981, there is no evidence of existence of any convergence clubs. The distribution was more or less with a single peak. However, with greater pace of fertility transition from the year, 1991, emergence of a secondary peak was evident and such secondary peaks were more clearly apparent from the year 2001 and 2011. Such process clearly manifests that though, fertility transition is universal in India, but still there are unequal rate of progress across the states. The states with TFR below the national average are forming a different convergent clubs and rest of the states is forming other secondary peaks. A separate assessment of such process for rural and urban areas separately reveals that the process is more pronounced in case of rural than urban areas. Since 1991, the emergence of multiple peaks is observed in rural areas and clearly apparent in the years 2001 and 2011. However, in urban areas, the presence of secondary peaks is clearly evident only in the 2011 (Figure 6.11, 6.12, 6.13). 
Figure 6.11. Kernel density estimates of Total Fertility Rate distribution in India and major states, 1981-2011

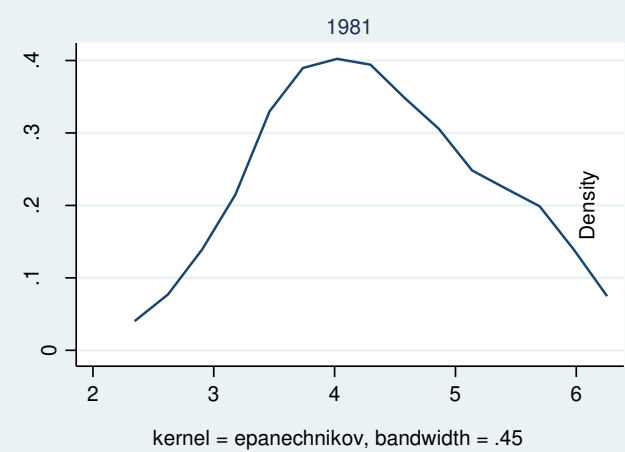

2001

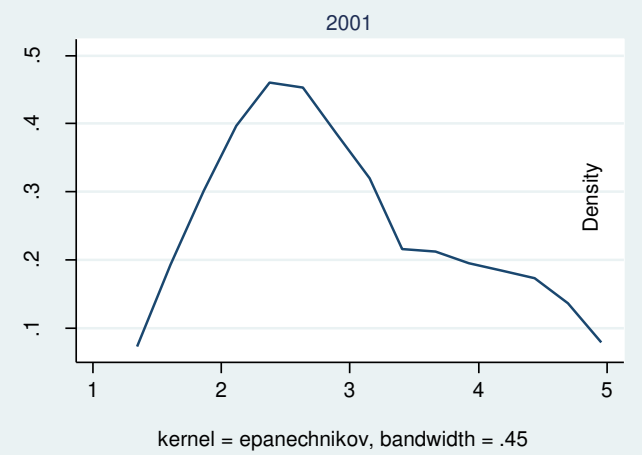

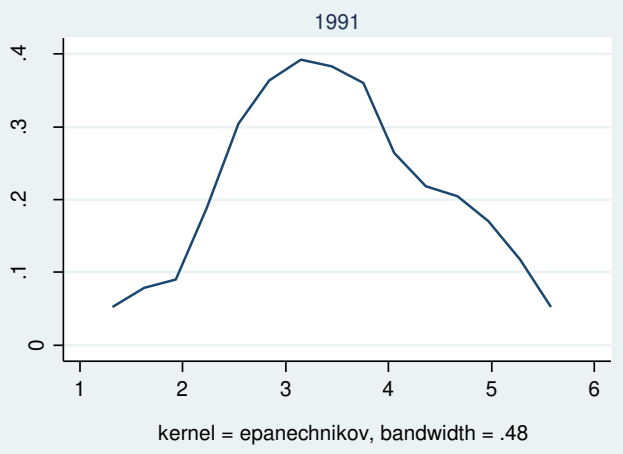

2011

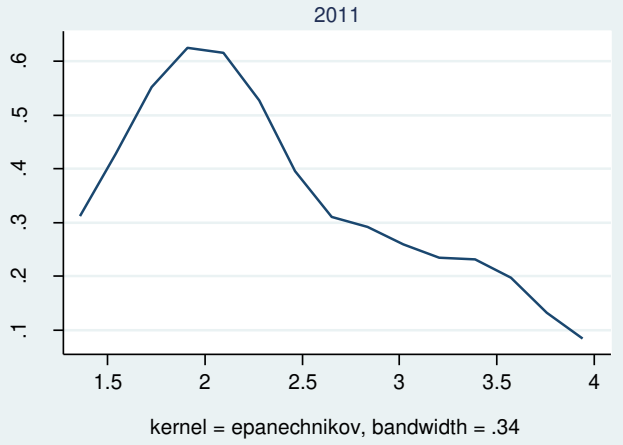

Figure 6.12. Kernel density estimates of Total Fertility Rate distribution in Rural areas of India and major states, 1981-2011

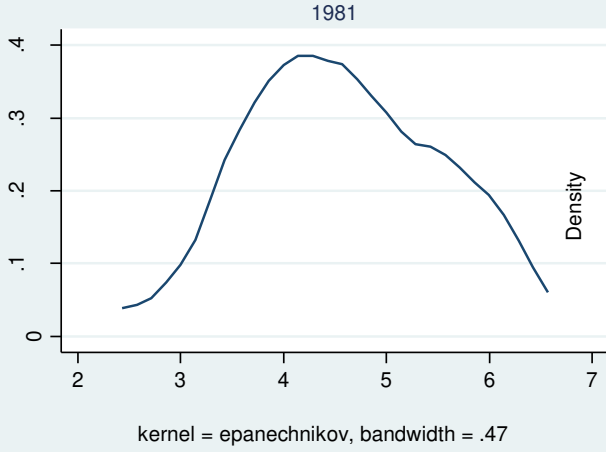

2001

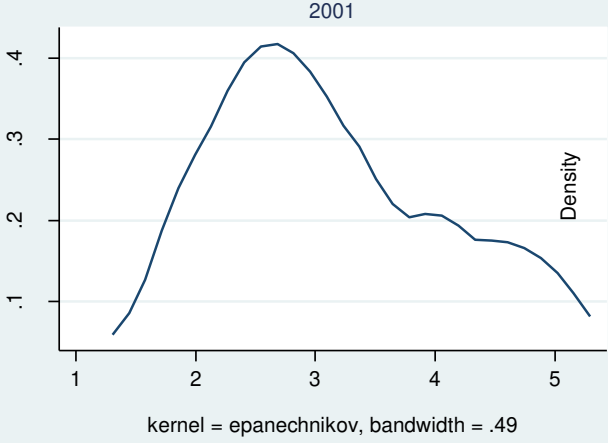

1991

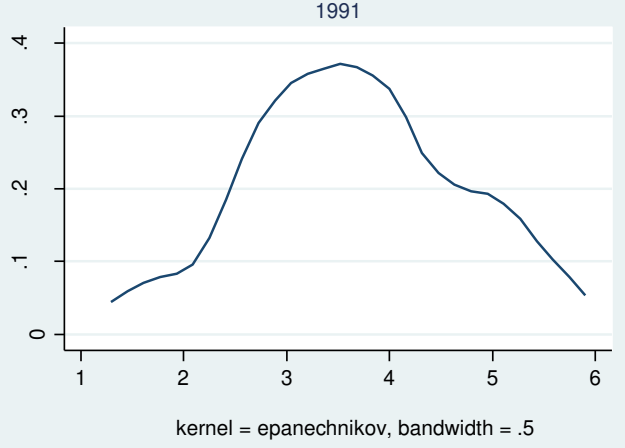

2011

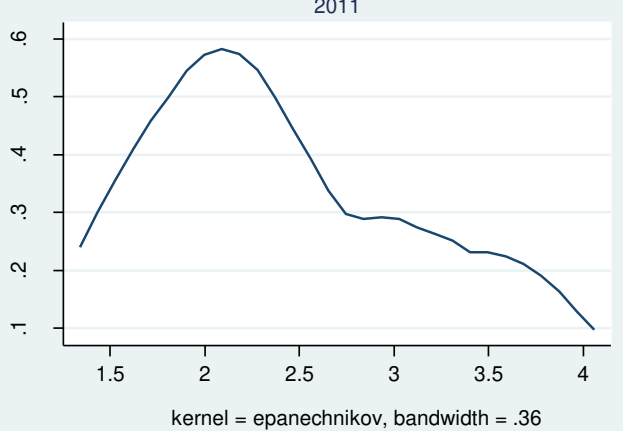


Figure 6.13. Kernel density estimates of Total Fertility Rate distribution in urban areas of India and major states, 1981-2011

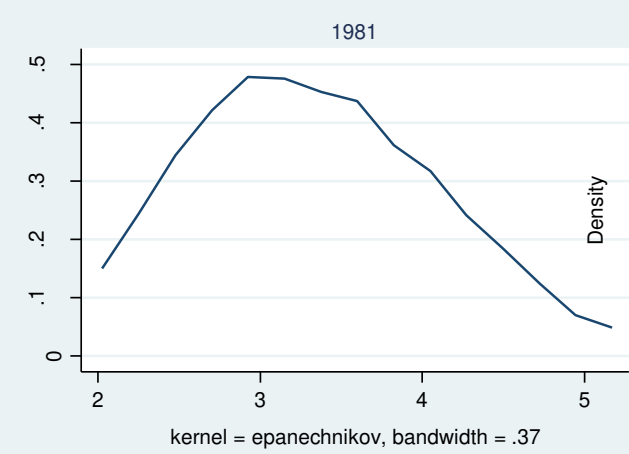

kernel $=$ epanechnikov, bandwidth $=.37$

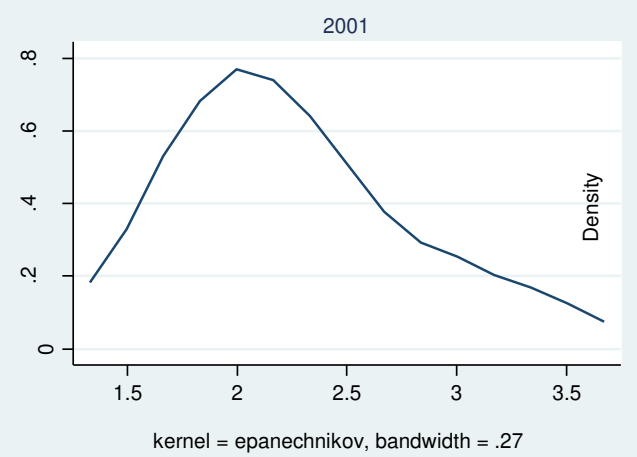

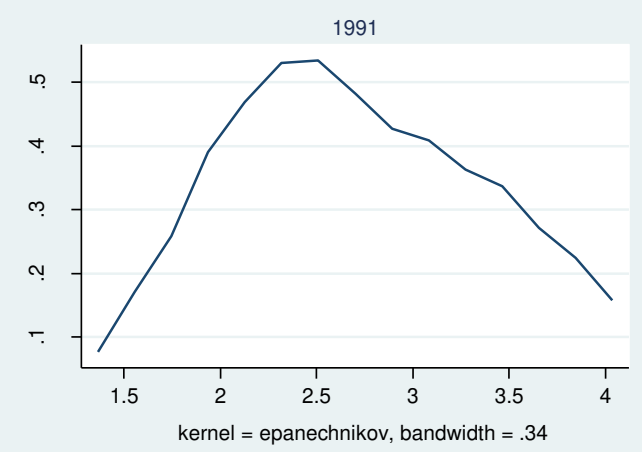

2011

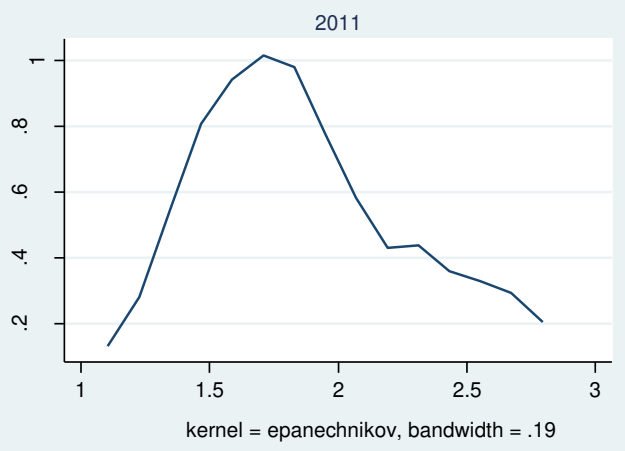

\subsection{Discussion}

This chapter is a comprehensive assessment of fertility convergence hypotheses in India over the geographic units (states) and socioeconomic spectrum. I have tested with both conventional and contemporary metrics of convergence for total fertility rates for Indian states and socioeconomic strata. From a theoretical context, this chapter makes a critical contribution to advancing knowledge on patterns of the progress in fertility transition. The key theoretical and analytical contributions of this chapter are as follow:

- An innovative framework has been conceptualised to understand the integrated process of fertility transition and fertility convergence, alongside socioeconomic and health transition. This framework serves as an important tool to describe the critical stages and pathways of fertility convergence across the Indian states.

- The fertility transition plots and Change-points, analyses indicate a varying pattern of fertility transition across the major Indian states. Critical changepoints in fertility rates across the states indicate a substantially varying pattern. While few of the states have experienced major fertility changes as early as in 
the 1970s, other states have experienced significant changes in fertility as late as the 2000s. This process has created a condition of differentials of steady state and catching-up process where, the states with higher fertility rates experienced a greater fall in fertility rates thus, catching-up with the states of lower fertility rates.

- The $\beta$-convergence model estimates suggested that due to pronounced fertility divergence during the early phase of 1981-1991, the estimates for an overall period (1981-2009) showed fertility divergence. The initial phase of divergence in TFR among the states was mainly because of the relatively greater decline in TFR of the demographically advanced (mostly comprising south Indian states) states compared with the demographically lagging states (mostly comprising north Indian states). However, due to greater fertility decline in the states with higher fertility rates in post 1990 s period, the divergence in fertility was replaced with convergence in fertility. Sigma convergence estimates revealed the similar pattern: initially showed divergence then catching-up with the fertility convergence process.

- The results of inequality based convergence measures indicated the convergence in absolute and relative dispersion in fertility rates across the 15 major states with varying time trends. The convergence estimates for absolute dispersion of fertility rates were consistent with absolute $\beta$-convergence estimates: divergence in the pre-1990 period was replaced with convergence in fertility in the post1990 period. However, the convergence estimates in the relative dispersion of fertility rates showed persistent divergence, but with a substantial decline in the volume of divergence for the recent period. This suggests that convergence in the absolute dispersion of fertility rates may not always give a guarantee of relative convergence. Because, the initiation of these two processes may not occur at the same point. This pattern implies that convergence in average fertility rates and absolute dispersion of between state fertility rates are necessary, but not a sufficient condition for relative convergence. Therefore, the convergence estimates should follow both absolute and relative distributions of any population parameters such as fertility. 
- The test of non-parametric model of convergence showed the evidence for emergence of convergence clubs with acceleration in fertility transition for the recent periods (post-1990s) rather convergence as whole.

Overall, this chapter has advanced considerable knowledge in measuring the progress of fertility transition in the Indian states using a range of convergence models both in terms of efficacy and equity across the states and socioeconomic spectrum. The assessment of India and state level fertility transition demonstrates evidence of transformation from progressive transition disequilibrium to progressive transition equilibrium phase. Moreover, based on the results of both standard and innovative metrics of convergence, this study concludes that the earlier phase of divergence in fertility rates across the 15 major states of India was being replaced by emerging convergence in fertility rates for the recent period, but the progress is not resulted into absolute convergence yet. The trends and patterns suggest the strong prospects of continued fertility convergence among the Indian states if the club of higher fertility states will further move to catch the club of lower fertility states. 


\section{CHAPTER 7}

\section{Convergence in Child health care utilisation and Outcomes}

\subsection{Introduction}

Child health is arguably the heart of the MDGs (Lawn et al., 2006). In the past two decades, newborn and child health subjects were engrossing, debatable, and fast moving as people paying greater attention to these subjects, the data are evolving. Further, these issues have driven increased levels of development aid, policy attention, and research work and, on a positive note, the child health is improving and child deaths are falling (Starrs, 2006, Hill et al., 2007, Murray et al., 2007, Gregson et al., 2009, Hogan et al., 2010, Rajaratnam et al. 2010; Ehiri, 2010). However, a recent United Nations report (2010) on MDGs evaluation suggests 'though progress has been made, it is uneven that only 31 developing countries in the world are likely to meet the MDG 4 goal' (reduction in childhood mortality by two-third). Hogan and Colleagues (2010) and Rajaratnam et al. (2010) reported that under-5 deaths have become increasingly clustered in certain regions. About one in every three deaths of children under-5 occurs in South Asia, and about one in two occurs in sub-Saharan Africa whereas, less than $1 \%$ of deaths of children under- 5 occur in high-income countries.

Akin to global scenario, the child health subject has also received greater attention of a growing number of studies in India (e.g. DasGupta and Das, 2000; Kumar, 2007; Navaneetham et al., 2008; Subramanyam and Subramanyam, 2011; Nath, 2011; Pradhan and Arokiasamy, 2010; Kumar and Mohanty, 2011; Goli et al., 2013; Goli and Arokiasamy, 2013; Ram et al., 2013). There has been a consistent childhood mortality decline in India, especially the Neonatal Mortality Rate (NNMR) which comprises a larger share of childhood mortality. However, the progress is uneven across the states and socioeconomic groups. Only six states of India are likely to achieve the NNMR target of MDG-4 (reduction by two-third): Tamil Nadu and Kerala in the South, Maharashtra in the West, West Bengal in the East, and Punjab and Himachal Pradesh in the North. Latest estimates in 2010 showed that the variation in NNMR as high as, 64 deaths per 1000 live births in Madhya Pradesh to 13 deaths per 1000 live births in Kerala (RGI, 2011). A district level assessment by Ram and Colleague (2013) suggest, just over a third of all districts are on track to reach MDG4 by 2015. 
Children full immunisation and Children underweight are considered being important process indicators in the evaluation of child health status. Though, on an average, progress in full immunisation coverage of children is positive, but it varies largely across the states: 23 percent in Uttar Pradesh compared to 81 percent in Tamil Nadu. Also, the differentials in children immunisation coverage are continued to be large by economic and social status of the households. In 2005-06, the full immunisation of children aged 12-23 months belonging to the lowest wealth quintile was 24 percent compared to 71 percent among the richest quintile (IIPS and Macro International 2007; Kumar and Mohanty, 2011; Goli et al., 2013). The prevalence of child malnutrition in India, on an average, had undergone a noticeable change in some states despite the stagnation at the national level. However, studies also suggest growing interstate differentials with respect to child malnutrition in India. The differences in the decline of malnutrition is ranged between 6 percent in the 'poorest' and 27 percent in the 'richest' category of the wealthiest quintile during 1992-2006 (Subramanian and Smith, 2006; Prathak and Singh, 2011; Arokiasamy et al., 2013; Goli et al., 2013).

Though, earlier studies have presented an ideal assessment of progress in terms of child health indicators and reported not only a remarkable progress at the national level, but also considerable variation among the states. These reported anomalies in progress of child health care utilisation developed a curiosity to test the convergence hypothesis across the states in India in terms of child health care utilisation and outcome indicators. Moreover, there is not a single study in India which have tested quantitatively convergence hypothesis to see whether such progress is reflected in convergence or divergence-if convergence, at what speed the gap is closing in terms of absolute convergence in child health care utilisation and outcome indicators for the states and socioeconomic groups of India. It is important to study, the extent to which improvements in national average health status over the last three decades have been accompanied by convergence in average health status and its influence on health inequalities across the states and socioeconomic groups. Hence, the fundamental research question of the chapter is to assess whether the progress in child health care utilisation and outcome indicators in the recent decades is reflected in convergence or divergence across the states and socioeconomic groups of India. 


\subsection{Data and Methods}

This chapter used data from the SRS (1981-2009) and three rounds of the National Family Health Survey (NFHS, 1992-2006) to assess the convergence in child health care utilisation and outcome indicators for the states of India. Akin to fertility and mortality chapters, in this chapter too, we used three types of convergence metrics: graphical plots, absolute and conditional $\beta$ convergence, sigma convergence, kernel density plots and inequality based convergence analyses. A detailed discussion on these three models of convergence is given in methodology chapter.

\subsection{Results}

\subsubsection{Trends in averages of child health care utilisation and child health outcomes}

Trends in averages of child health care utilisation status are measured in terms of progress in key indicators such as children full immunisation, children underweight and NNMR. Figure 7.1 shows the trend assessment for children full immunisation coverage. The results showed two different set of pattern of trends across the states: the first set relates to the demographically advanced states with higher levels of full immunisation coverage with an increasing trend until 1998-1999, followed by a declining trend for the later period. The second category relates to demographically lagging states, which presents a contrasting situation, as they showed a downward trend until 1998-99, followed by a substantial increase. This suggests that those demographically backward states with higher growth of full immunisation coverage rates are heading towards the levels achieved by the demographically advanced states.

However, the trends in children underweight showed dissimilar progress. All the states experienced a decline in children underweight during 1992-99, however, from 19982006 indicate a mixed pattern: the trends in the percentage of children underweight declined in the majority of the states, but increased in a smaller number of states. In case of NNMR, the results showed that U.P with largest and Kerala with lowest NNMR showed a huge gap between the states in 1981. By 2009, the overall NNMR has decreased in all the states and moving towards the lower mortality levels, but the gap is not closing at the same speed. In general, trends in child health indicators showed though, all the states are progressing, but the state level gaps are not closing much instead they remains more or less same for two out of four indicators. 
Figure 7.1. Trends in children full immunisation coverage, children underweight, NNMR in India and major states, 1981-2009

Children full immunisation

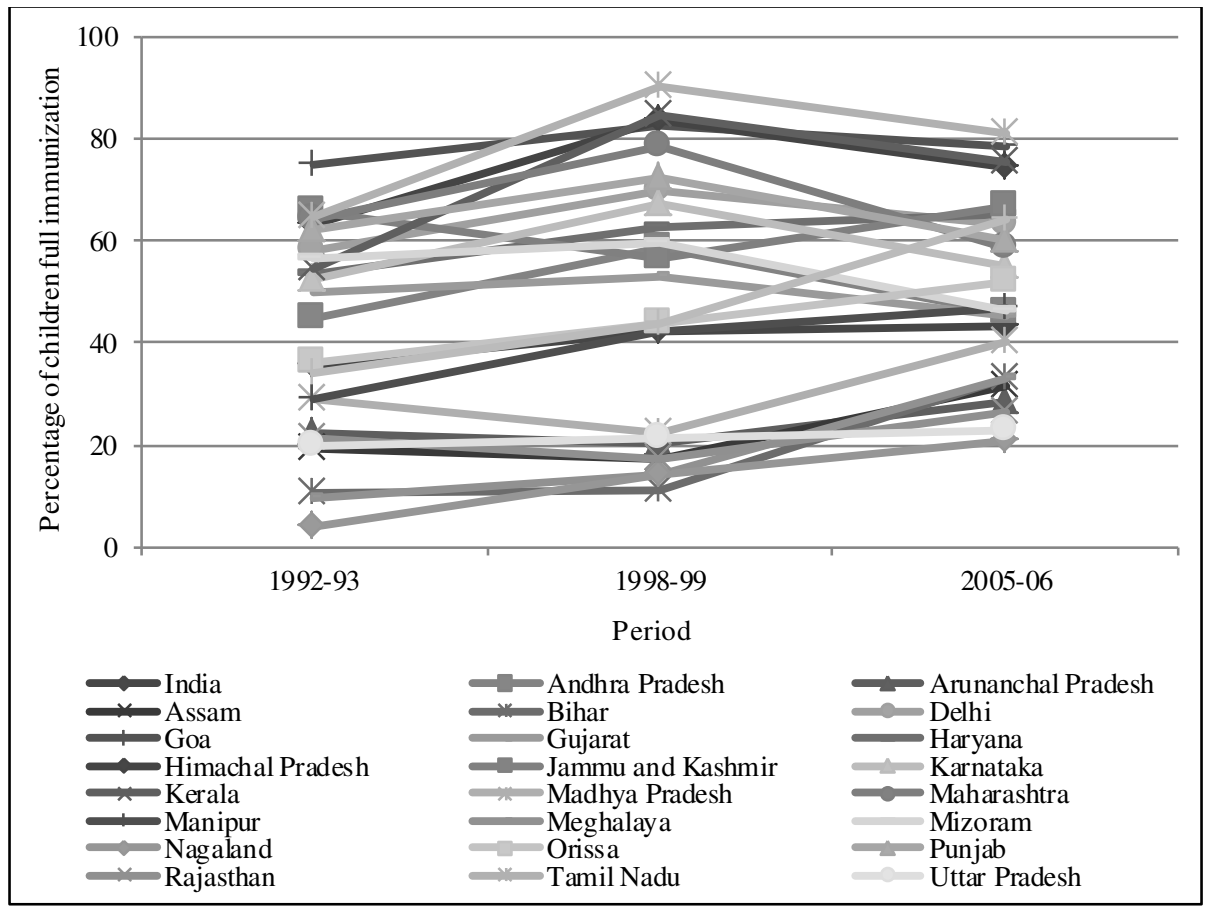

Children underweight

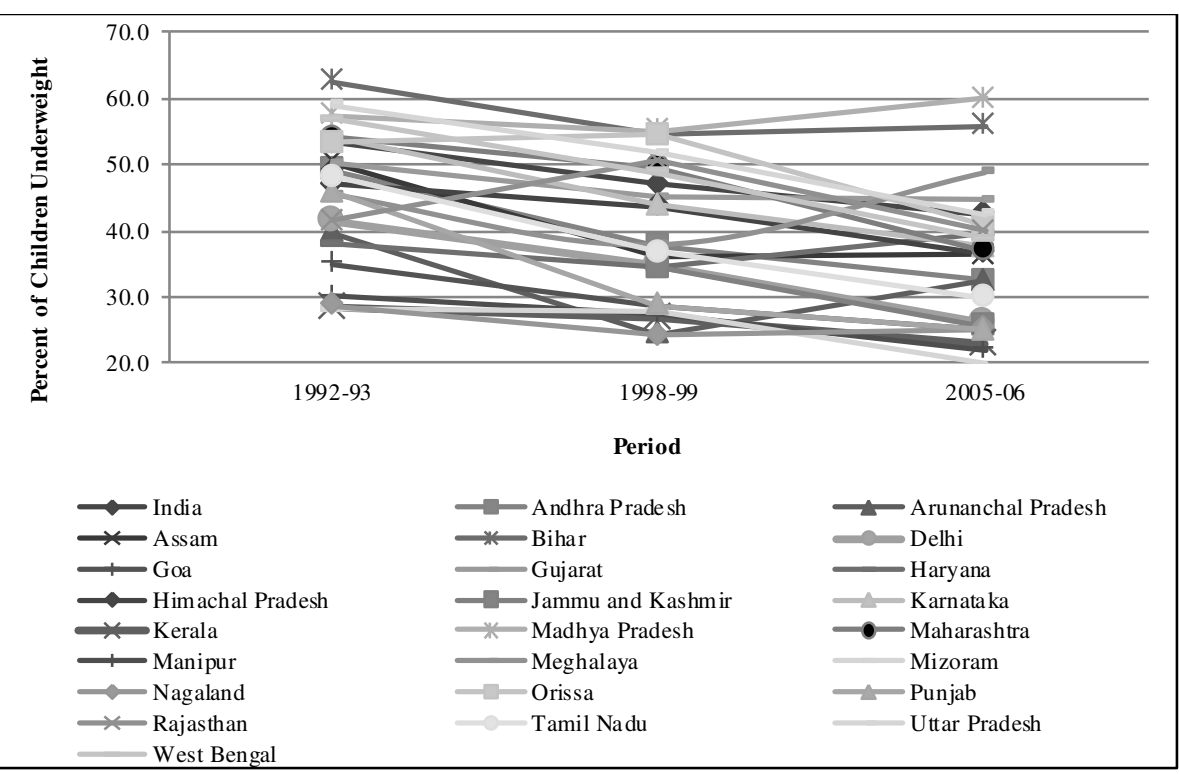


NNMR

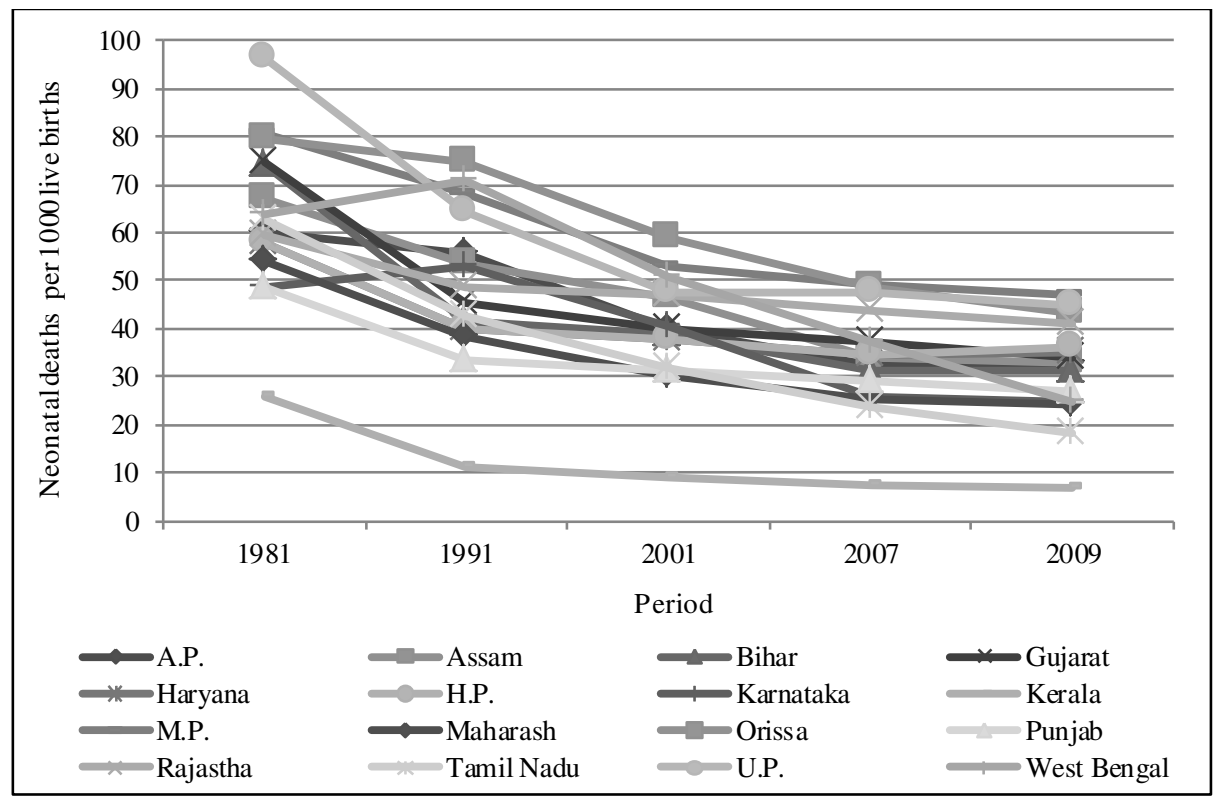

\subsubsection{Trends in overall and socioeconomic inequalities in health}

This chapter measured the convergence in two types of child health inequalities: first, overall health inequality and second, socioeconomic inequality in health. Within the overall health inequality, this study measured overall absolute health inequality and overall relative health inequality by using Average Inter-Individual Difference (AID), Dispersion Measure of Mortality (DMM) and Gini index, respectively. DMM is based on estimated absolute interstate population health status, weighted by their population sizes. Gini coefficient shows the relative interstate inequality in health. Decrease or increase in DMM shows changes in absolute inequality in health among the population of selected states. Similarly, change in the Gini coefficient indicates the relative change in inequality in health (Shkolnikov et al., 2003; Saikia, 2011). Further, socioeconomic inequalities in child health were measured in terms of Concentration Indices (CIs).

The results presented in Figure 7.2a show that the absolute inequalities in terms of AID for children full immunisation was increased (10 percent to 14 percent) during 1992-99, but decreased (14 percent to 10 percent) in 1999-2006. However, the 10 percent average inter-individual difference in children full immunisation in 2005-06 is still showing very high inequality in full immunisation coverage across the states. A similar pattern is also evident in case of relative inequalities in terms of the Gini index of child's full immunisation, which, first increased, then decreased in the recent period. 
The Gini index of 0.22 still shows a high relative inequality across the states in terms of children full immunisation convergence in India.

Figure 7.2a. Trends of AID and Gini index of child health care and outcome indicators for major Indian states, 1981-2009

Children full immunisation

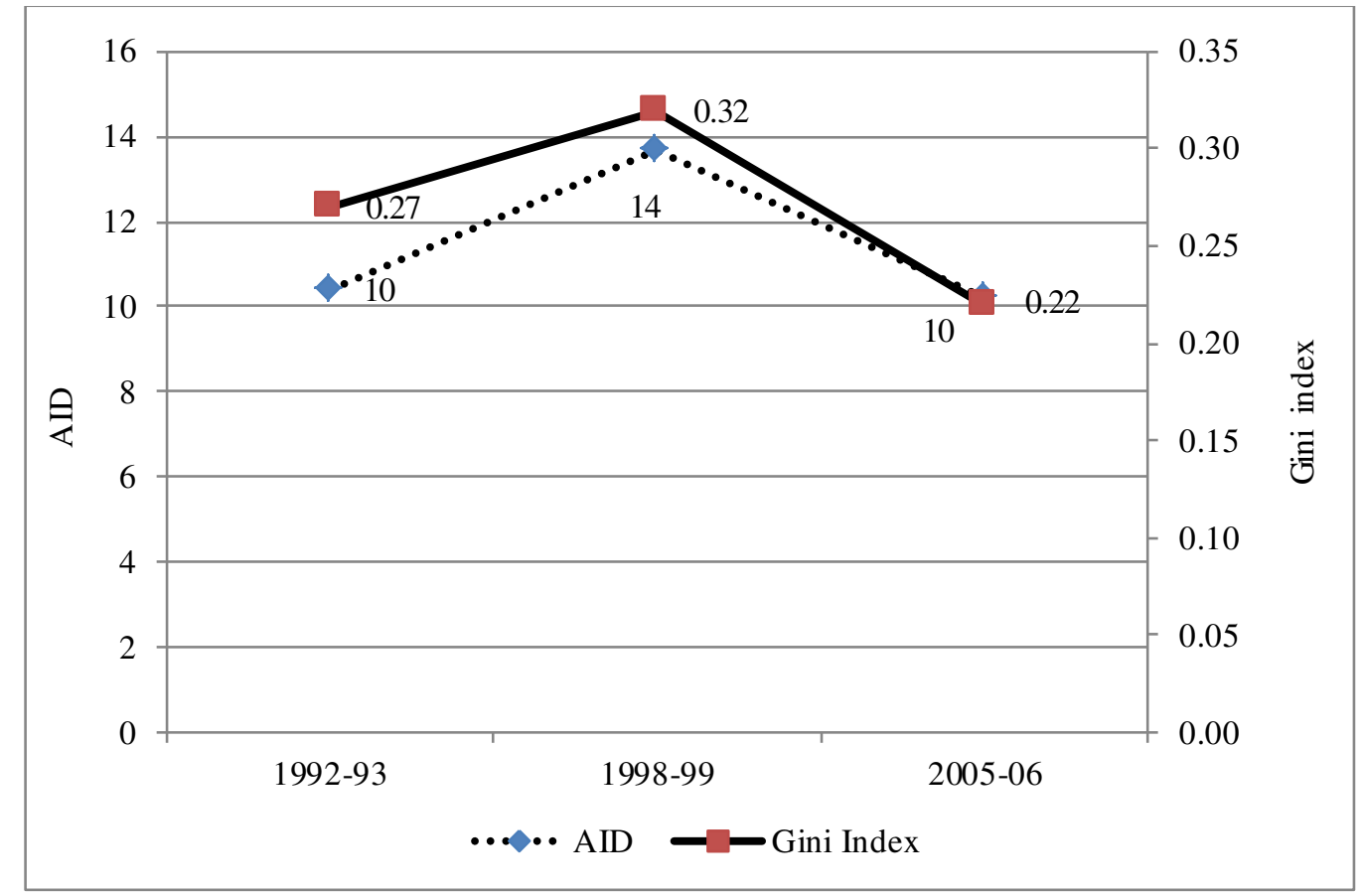

Children underweight

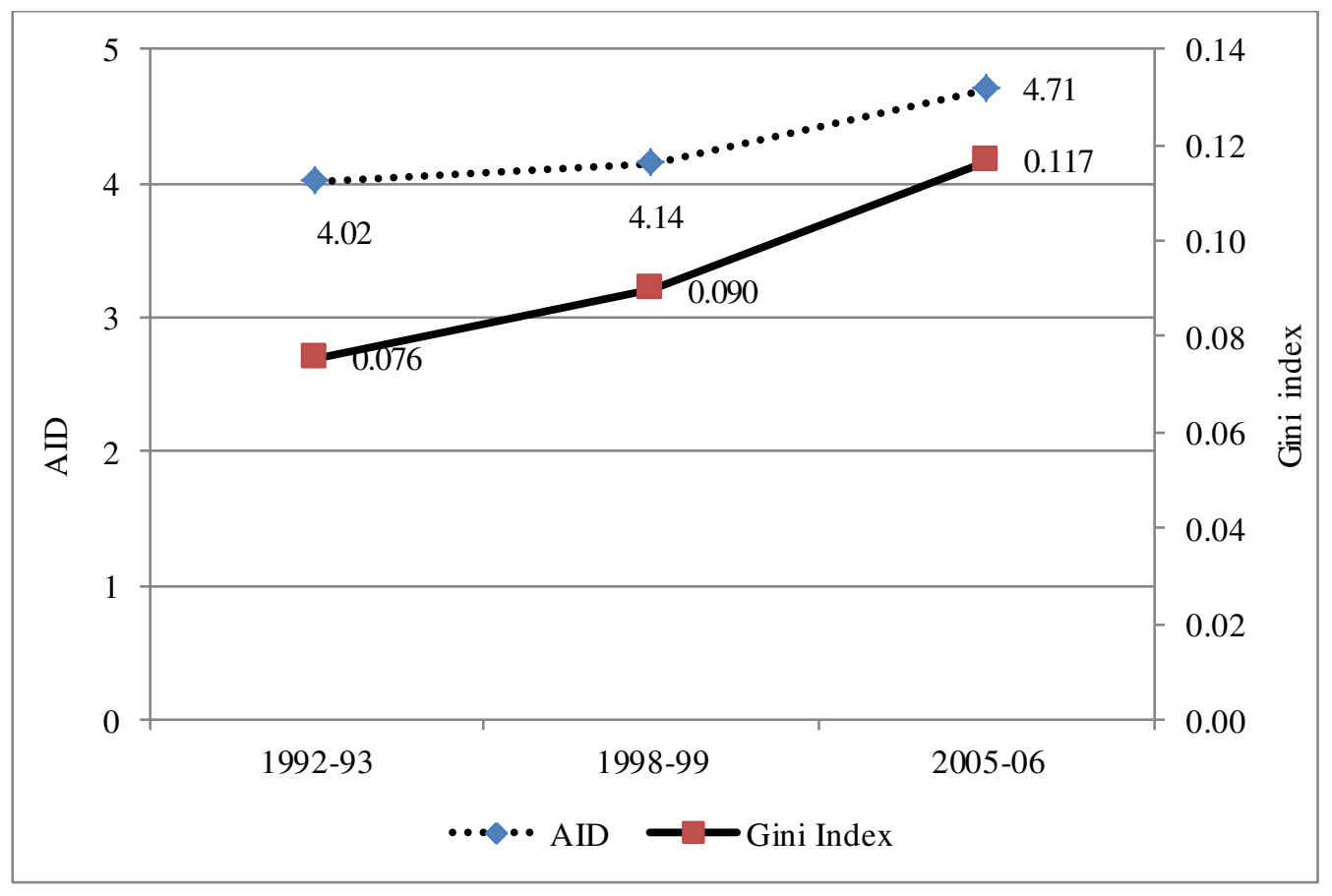


NNMR

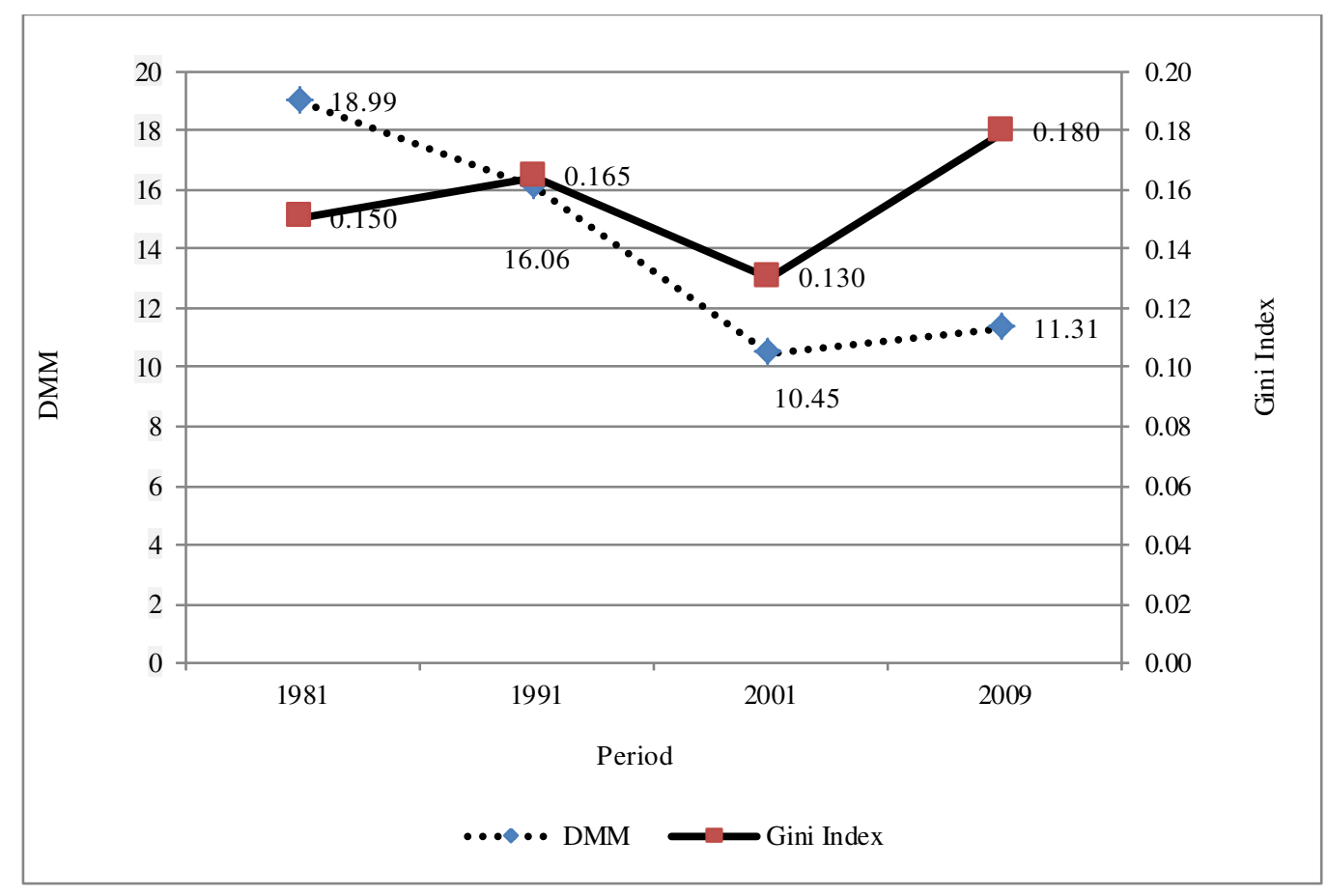

However, in case of children underweight, results showed evidence of increasing trend in both absolute and relative inequalities. However, the increase in relative inequalities is much higher compared to absolute inequalities. The absolute inequalities in terms of AID have increased from 4.02 percent to 4.71 percent where, the relative inequalities were increased from a Gini index of 0.076 to 0.11 . The results in cases of neonatal mortality present, a different picture of trends. The both absolute and relative inequalities in neonatal mortality rate were decreased until 2001, but thereafter they have increased. The increase in relative inequalities was much sharper compared to absolute inequalities that the relative inequalities increased by five points in terms of Gini index, but absolute inequalities have increased by only one percent in terms of average inter-individual differences. In general, the comparison of these three indicators revealed that in two out of three indicators, both absolute and relative inequalities are increasing. Though, in case of children full immunisation, these inequalities are declining in the recent period, but level of inequalities is much higher than children underweight and NNMR. 
Figure 7.2b. Trends of DMM and Gini index of child health care and outcome indicators for major Indian states by place of residence, 1981-2009

Children full immunisation, Rural

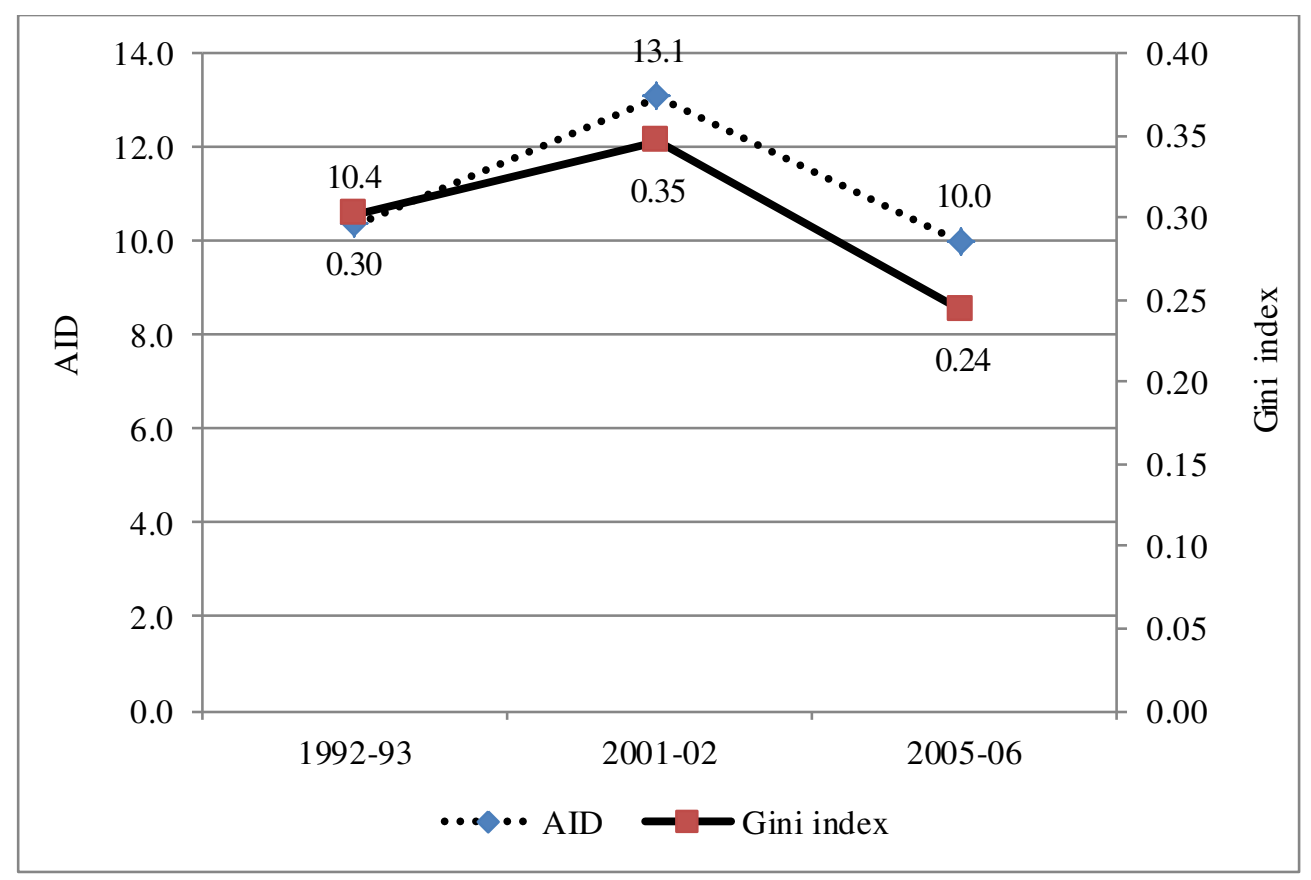

Children full immunisation, Urban

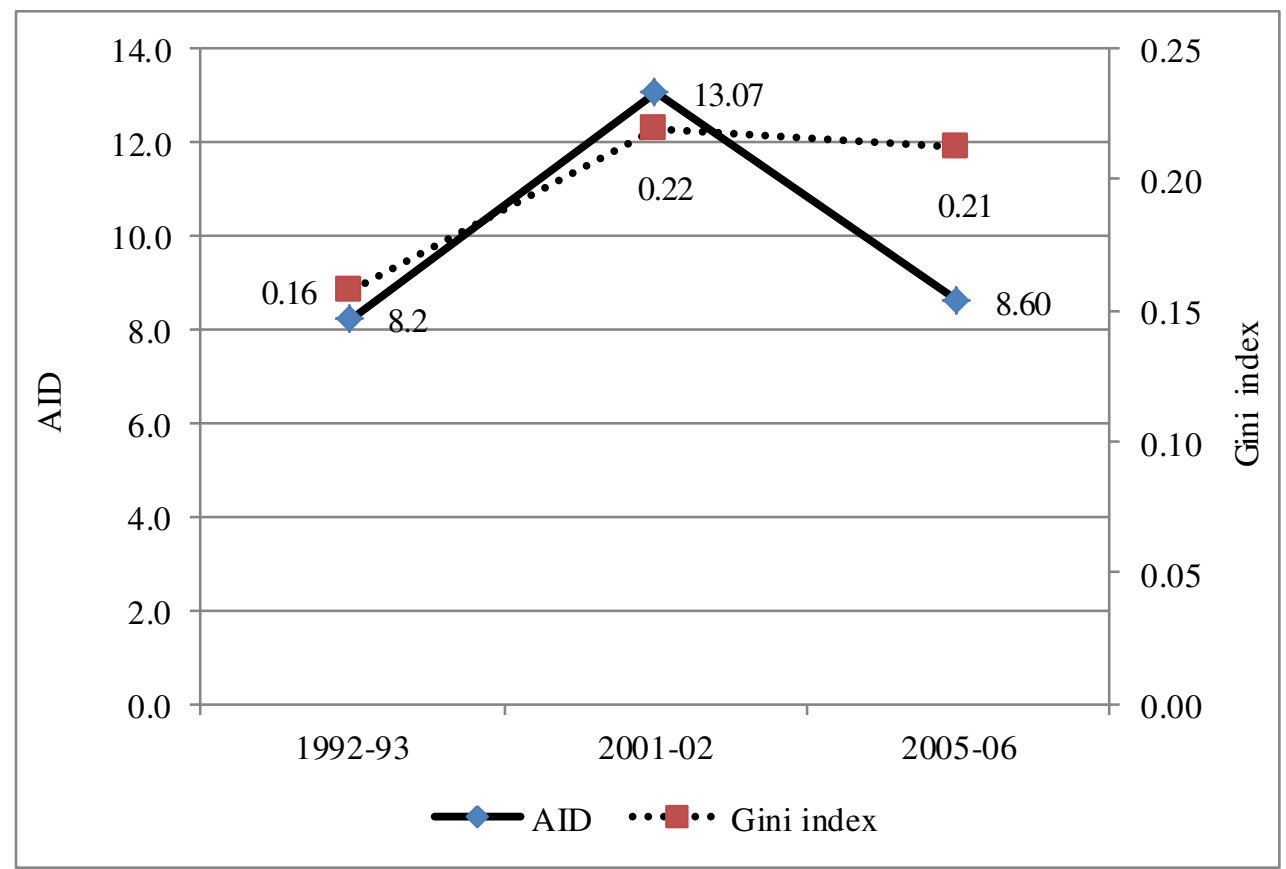


Children underweight, Rural

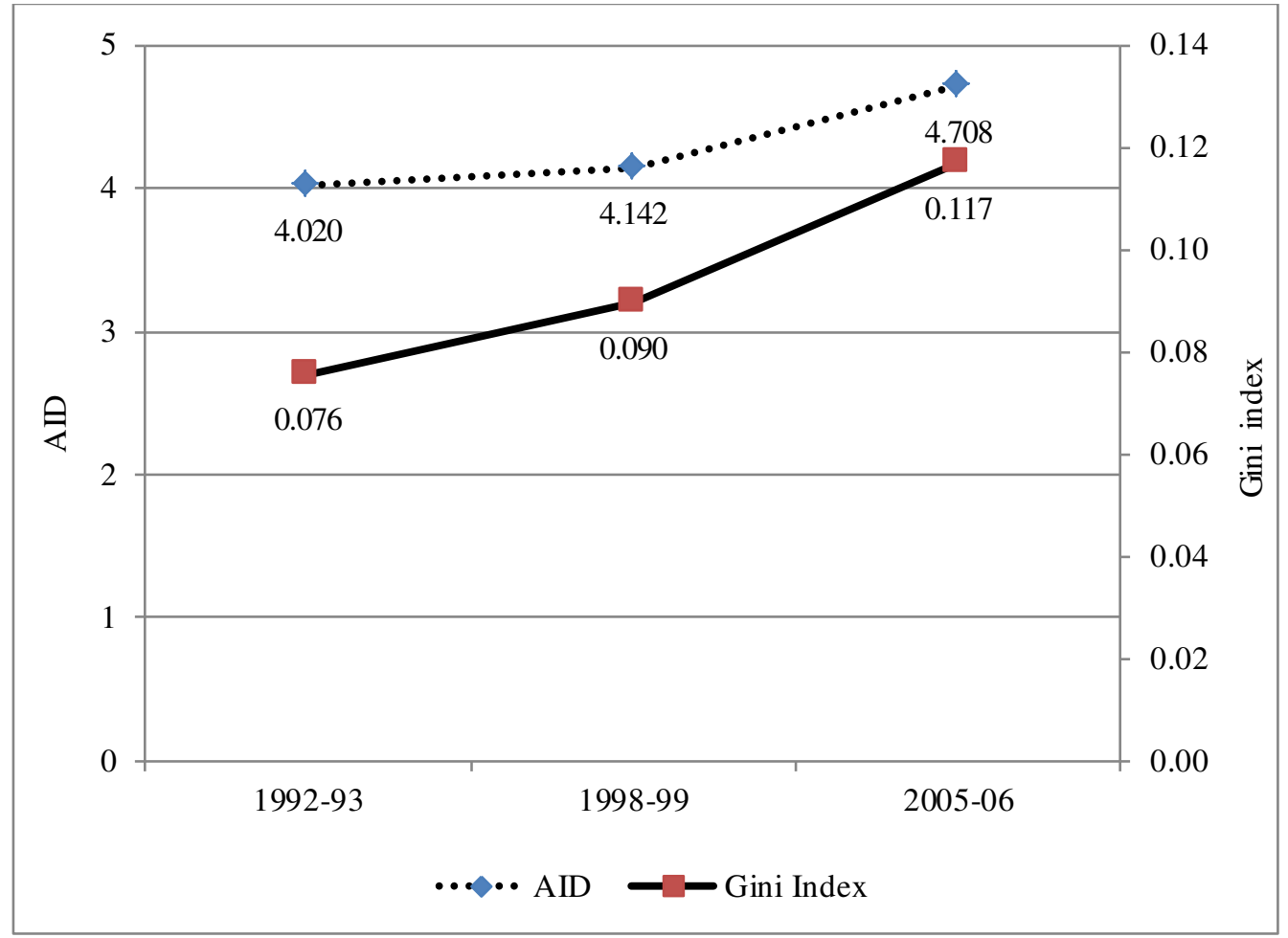

Children underweight, Urban

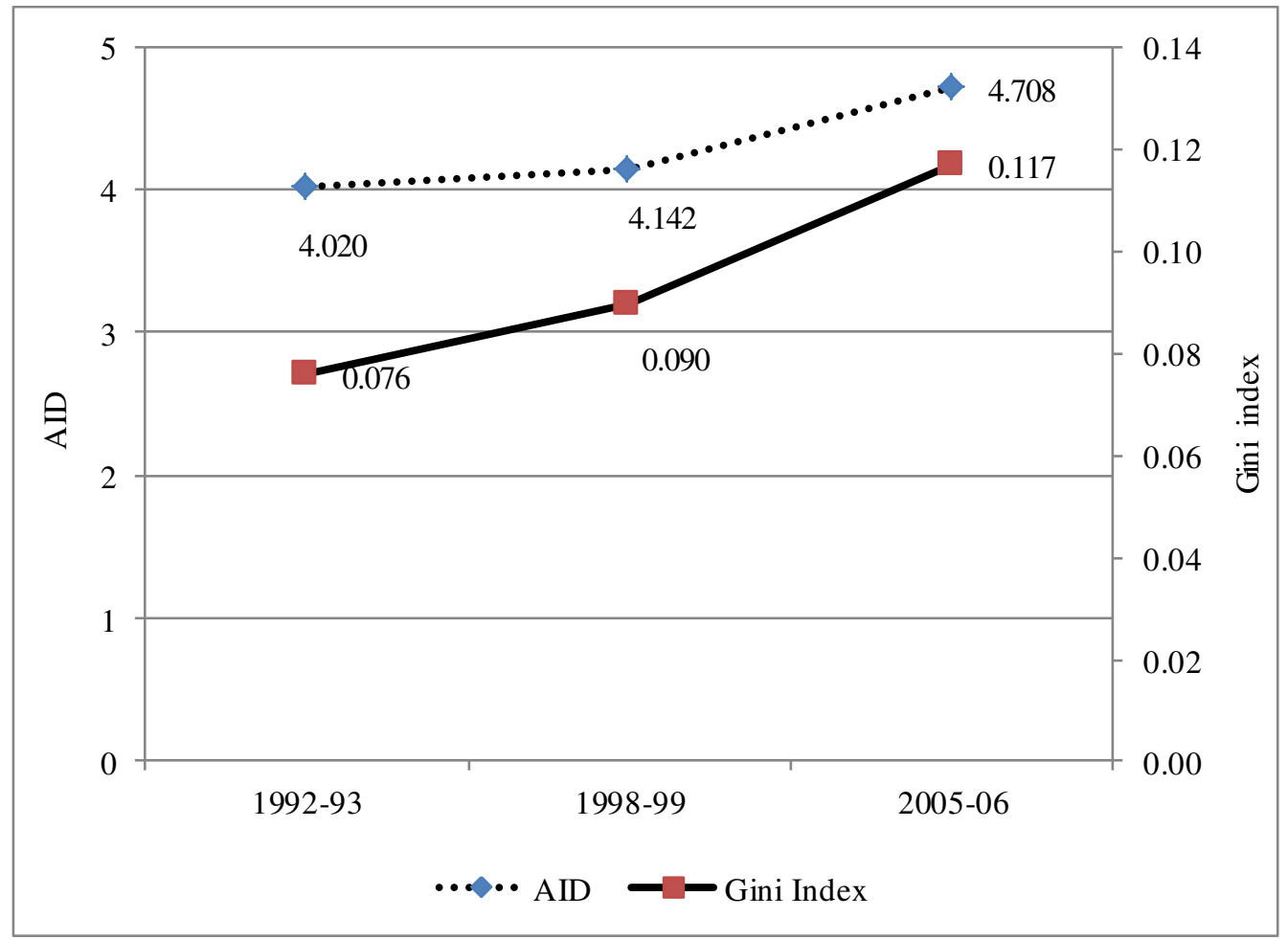


NNMR, Rural

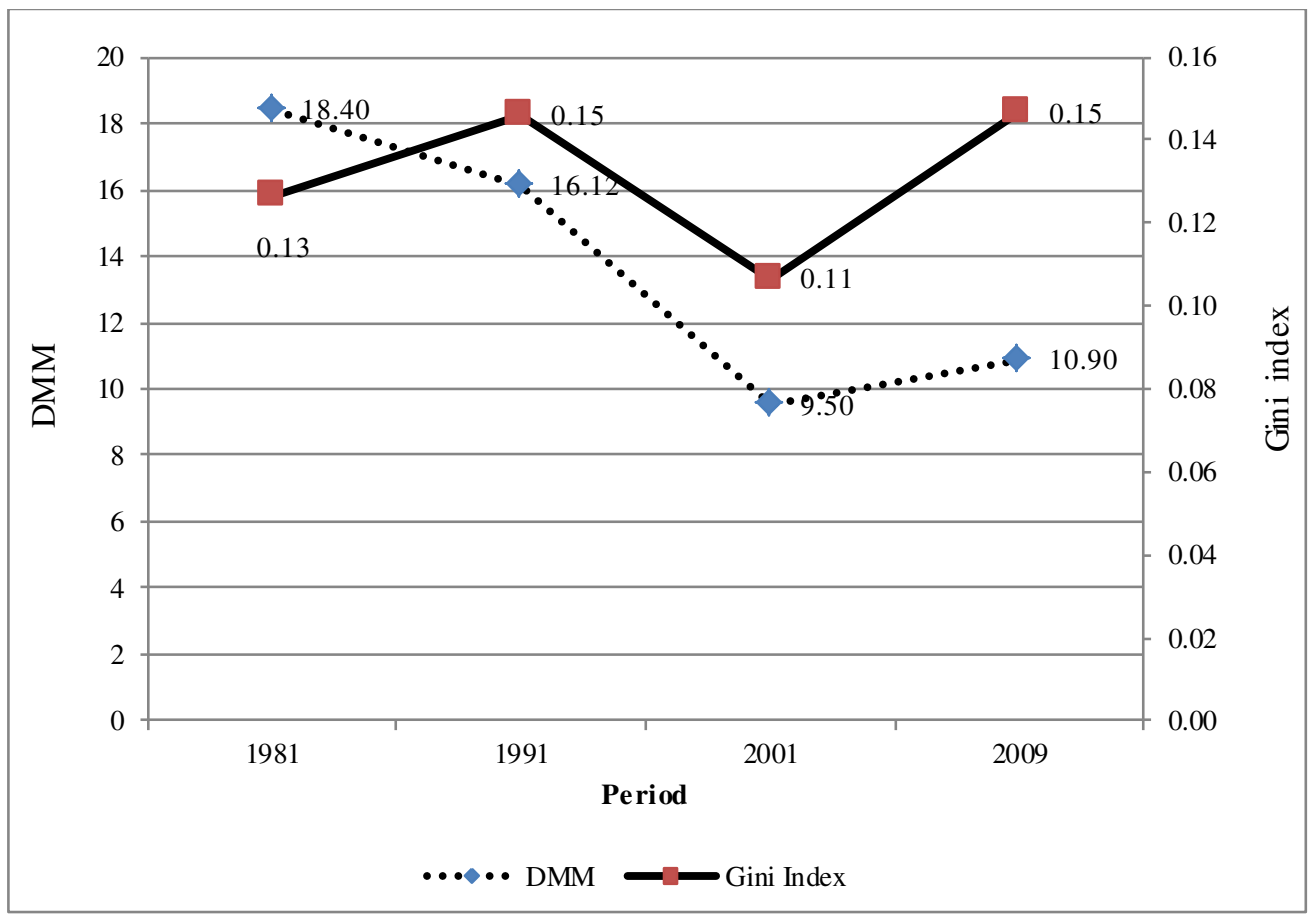

NNMR, Urban

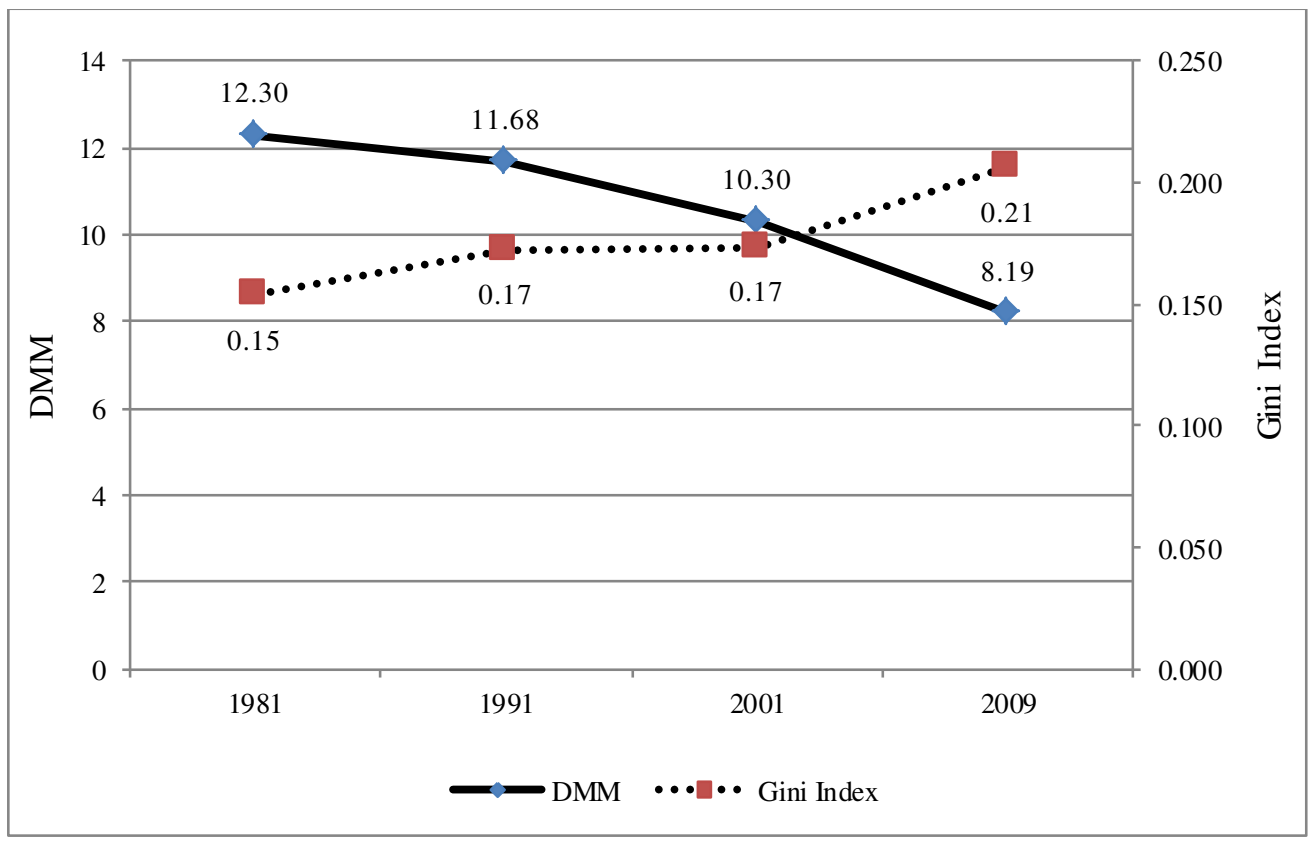

Figure $7.2 \mathrm{~b}$ presents the trends in absolute and relative inequalities in child health indicators by rural and urban areas. The results in case of rural areas of the states revealed that, absolute and relative inequalities in children full immunisation were increased in 1992-99 but decreased during 1999-2006. Similar results are also evident in case of urban areas, but the decline in relative inequalities during 1999-2006 was less 
in urban areas compared to rural areas. The trends in absolute and relative inequalities in children underweight across the major states showed that both have increased during 1992-2006, but the increase in relative inequalities was much sharper than absolute inequalities in both rural and urban areas. The results in case of NNMR in rural areas indicated decrease in both absolute and relative inequalities until-2001 and earlier decrease was replaced with the increasing trend in post-2001. However, in urban areas, the absolute inequalities are continuously decreasing, but relative inequalities are continuously rising during 1981-2009.

Socioeconomic inequality in health is measured in terms of CI for selected major states of India (table 7.1). The trends in socioeconomic inequality in health revealed a number of appealing findings. First, complementing the results of DMM, the trend assessment of CIs for NNMR showed a declining trend for 11 out of the 16 selected states of India. However, it must be noted that four out of the five states, that experienced an increase in NNMR, were economically and demographically advanced states. Second, the socioeconomic inequality in full immunisation of children showed an increase for India and states during 1992-1993 to 2005-06. The increase was greater for most of the socially, economically and demographically developed states than for their less developed counterparts. Nevertheless, the socioeconomically and demographically weaker states such as Bihar and Madhya Pradesh indicated a rise in health inequality. Third, socioeconomic inequality in children underweight increased in eleven states, but showed a reduction in four states. However, for India as a whole, socioeconomic inequality in children underweight has increased. In contrast to the trends in full immunisation of children, socioeconomic inequality for children underweight was higher among the socioeconomically and demographically weaker states. Overall, the trend assessments of absolute, relative and socioeconomic inequalities evidently indicated that inequalities in NNMR declined over time. However, inequalities increased in recent periods for all the other three indicators of health: children underweight, full immunisation of children and children underweight. It is important to distinguish from the results that steady improvements in average health status over time may not necessarily guarantee a reduction in health inequalities. Therefore, in the following sections, I have assessed the convergence in average health status and health inequalities separately. 
Table 7.1. Trends in wealth based concentration index for selected child health care utilisation and outcome indicators in India and major states, 1992- 2006

\begin{tabular}{|c|c|c|c|c|c|c|c|c|c|c|c|c|}
\hline \multirow{3}{*}{$\begin{array}{l}\text { India/Major } \\
\text { States }\end{array}$} & \multirow{2}{*}{\multicolumn{4}{|c|}{ Child immunisation coverage }} & \multicolumn{4}{|c|}{ Children underweight } & \multicolumn{4}{|c|}{ Neonatal Mortality } \\
\hline & & & & & \multicolumn{8}{|c|}{ National Family Health Survey (NFHS) } \\
\hline & $\mathrm{I}$ & II & III & III-I & I & II & III & III-I & I & II & III & III-I \\
\hline India & .1091 & .0938 & .1596 & 0.0505 & .1337 & 0.1715 & 0.1585 & 0.0248 & 0.15313 & 0.12548 & 0.16148 & 0.00835 \\
\hline $\begin{array}{l}\text { Andhra } \\
\text { Pradesh }\end{array}$ & 0685 & .0362 & .0959 & .0274 & 1213 & 0.1462 & 0.156 & 0.0347 & 4515 & .13027 & -0.18229 & 0.13714 \\
\hline Assa & .0747 & .0353 & .1132 & .0385 & .1517 & -0.0967 & -0.1328 & 0.0189 & -0.1 & -0.06637 & -0.13496 & -0.02708 \\
\hline Bihar & .035 & -0.0304 & -0.1334 & -0.0984 & -0.049 & -0.0672 & -0.0958 & -0.0468 & -0.0872 & -0.12217 & 6532 & .02188 \\
\hline Gujar & 1116 & -0.0829 & -0.1561 & -0.0445 & -0.1566 & -0.1843 & -0.1374 & 0.0192 & -0.17469 & -0.15209 & -0.15756 & .01713 \\
\hline Hary & .1211 & -0.0829 & -0.3318 & -0.2107 & -0.1825 & -0.1672 & -0.1306 & 0.0519 & -0.1623 & -0.14116 & -0.06470 & 0.09760 \\
\hline $\begin{array}{l}\text { Hima } \\
\text { Prad }\end{array}$ & .1329 & -0.1088 & -0.1501 & -0.0172 & -0.1365 & -0.1757 & -0.1316 & 0.0049 & -0.07712 & -0.03069 & -0.15828 & -0.08116 \\
\hline & & & 83 & -0.0579 & 78 & & & & & & & \\
\hline $\mathrm{Ke}$ & 15 & 551 & 56 & -0.2106 & 146 & -0 & 042 & 0.0104 & 099 & -0.2326 & 928 & 171 \\
\hline $\begin{array}{l}\text { Madhya } \\
\text { Pradesh }\end{array}$ & 685 & -0.059 & -0.1786 & -0.1101 & 663 & -0 & 802 & -0.0139 & -0.05556 & 2 & 7 & 1 \\
\hline Mahs & 0901 & -0.0509 & -0.1789 & -0.0888 & .1378 & -0.1692 & -0.1726 & -0.0 & & & 56 & 57 \\
\hline Oris & 0768 & -0.074 & -0.1318 & -0.055 & -0.1174 & -0.1163 & -0.1823 & -0.0649 & & & 8363 & 392 \\
\hline & & -0.1 & -0.2 & -0.0909 & & & & -0 . & & 21 & 08 & \\
\hline $\mathrm{Ra}$ & & -0. & -0.0894 & -0.0356 & 139 & -0 & & -0.1 & & & & \\
\hline Tamil Nac & -0.1176 & -0.0389 & -0.0415 & 0.0761 & -0.1412 & -0 & -0.1795 & -0.0383 & 33 & 288 & 08 & 325 \\
\hline $\begin{array}{l}\text { Uttar } \\
\text { Pradesh }\end{array}$ & -0.057 & -0.0362 & -0.0756 & -0.0186 & -0.0618 & -0.1994 & -0.1154 & -0.0536 & 598 & -0.06457 & -0.10150 & 0.03448 \\
\hline West Bengal & -0.0539 & -0.0905 & -0.1265 & -0.0726 & -0.1175 & -0.1045 & -0.1652 & -0.0477 & -0.12222 & -0.24417 & -0.08291 & 0.03931 \\
\hline
\end{tabular}
National Family Health Survey- III (2005-2006). 


\subsubsection{Catching-up process in child health care utilisation and outcome}

The assessment of catching-up process for children full immunisation is not showing a strong pattern of 'laggard states catching-up the leader states'. The figure 7.3a clearly showed a mixed pattern in catching-up process as some of the laggard states are still experiencing a very low rate of progress compared to some of the leading states with higher levels of achieved progress and some laggard states with low level of achieved progress experiencing the highest rate of progress. A similar pattern is also evident in case of children underweight. Though, the majority of the states with greater underweight levels are experiencing a greater reduction in underweight, but at the same time few states have not yet pick-up the progress and experiencing the lower rate of progress. Thus, the scatter pattern evident in figure 7.3a for children underweight is still, not clearly indicating the catching-up mechanism in terms of progress in children underweight. Comparatively, the scatter pattern for NNMR is showing catching-up process in terms of progress in NNMR during 1981-2009. In case of NNMR accept one or two states, all other states with a greater level of NNMR are also experiencing a greater rate of decline in NNMR thus, showing a clear evidence of catching-up process.

Figure 7.3a. Change in children full immunisation, children underweight, NNMR during 1992-2006 for major states of India by children full immunisation, children underweight, NNMR levels during initial period, 1981, 1992-93

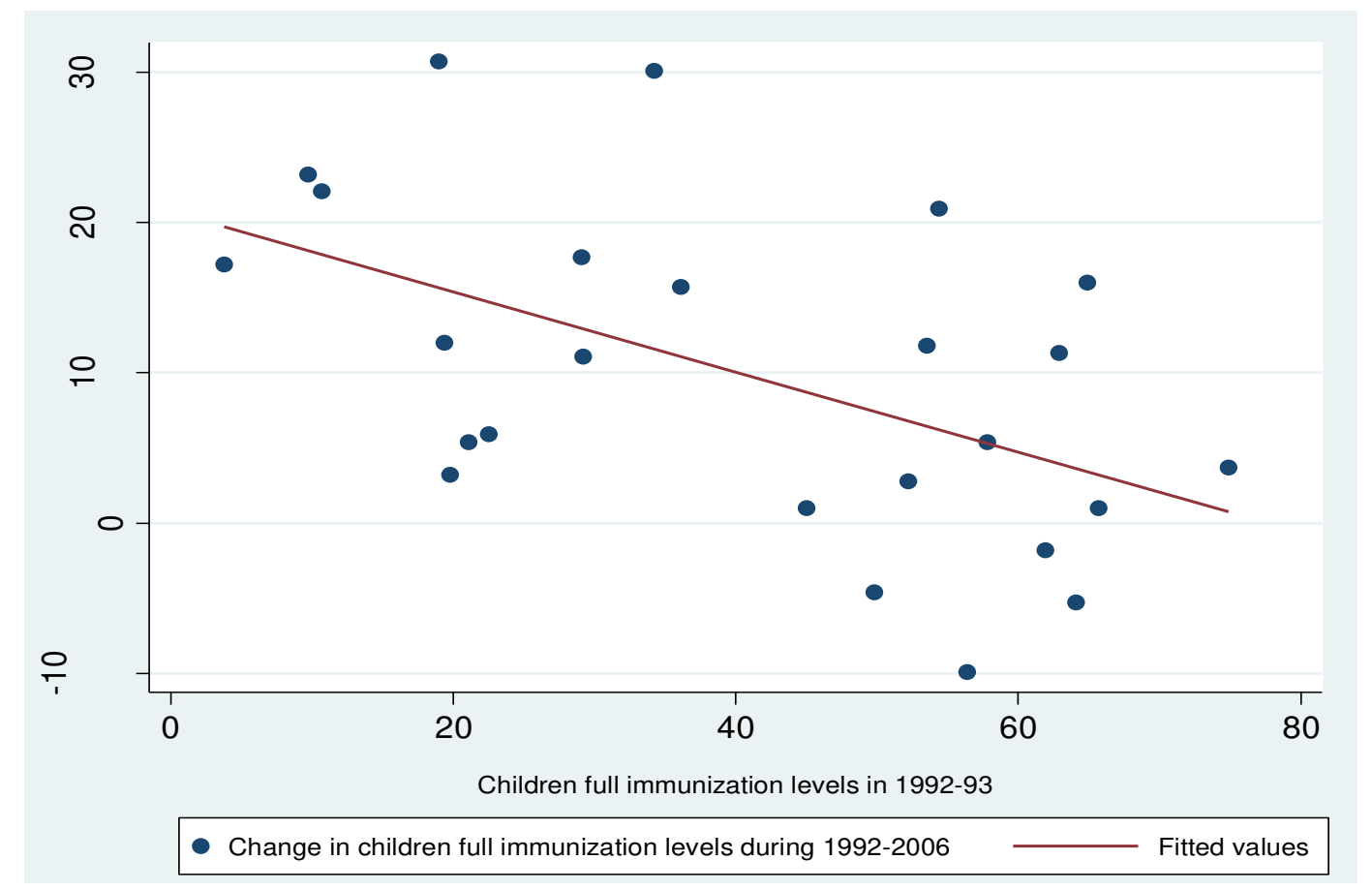



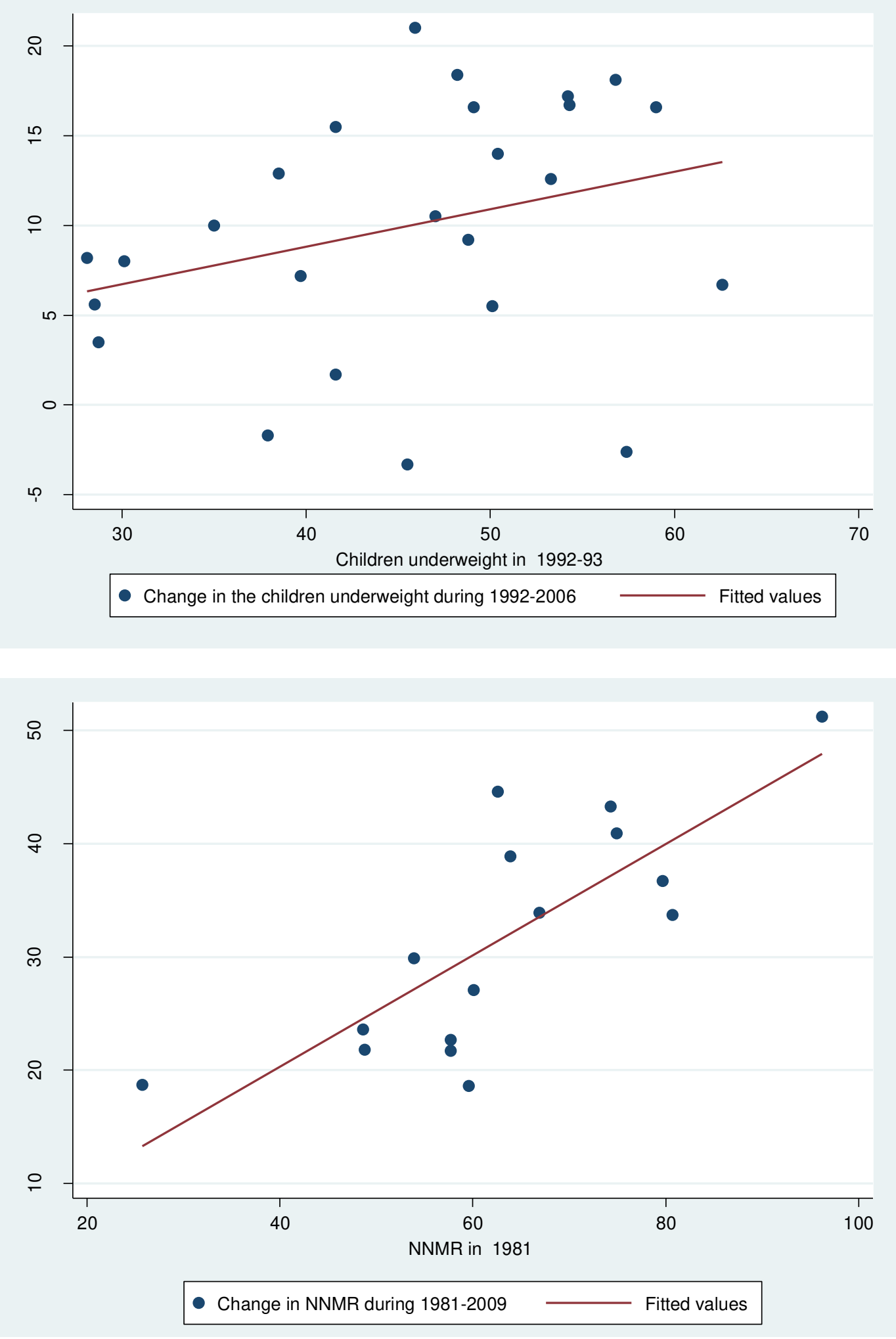
Figure $7.3 \mathrm{~b}$ presents the cumulative distribution of the population of major state of India by their initial levels of children full immunisation, children underweight, NNMR, 1992 to 2009. The plot for children full immunisation indicates a trend shift in population of the 15 major states towards higher levels of children full immunisation. However, the shift was much positive during 1992 to 1999 in comparison with 1999 to 2006. During, 1999 to 2006, many of the states with lower immunisation levels have experienced negative progress, thus, the trend shift in cumulative distribution of population is also negative. In case of children underweight, the trend shift in cumulative distribution of population towards the lower children underweight was less during 1992 to 1999. During 1999-2006, the shifts of the cumulative distribution of population was greater but, at the same time population in few laggard states have experienced the negative trend shift due to undo progress achieved in the earlier period, 1992-99. For NNMR, the results show clear trends of the cumulative distribution of population from higher NNMR to lower NNMR. However, the progress was much greater in 1981-91. In the later periods, 1991-2001 and 2001-09, though trends shift was positive, but the rate of progress was smaller the than rate of progress during 1981-91.

Figure 7.3b. Cumulative distribution of the population of major state of India by children full immunisation, children underweight, NNMR levels, 1992 to 2009

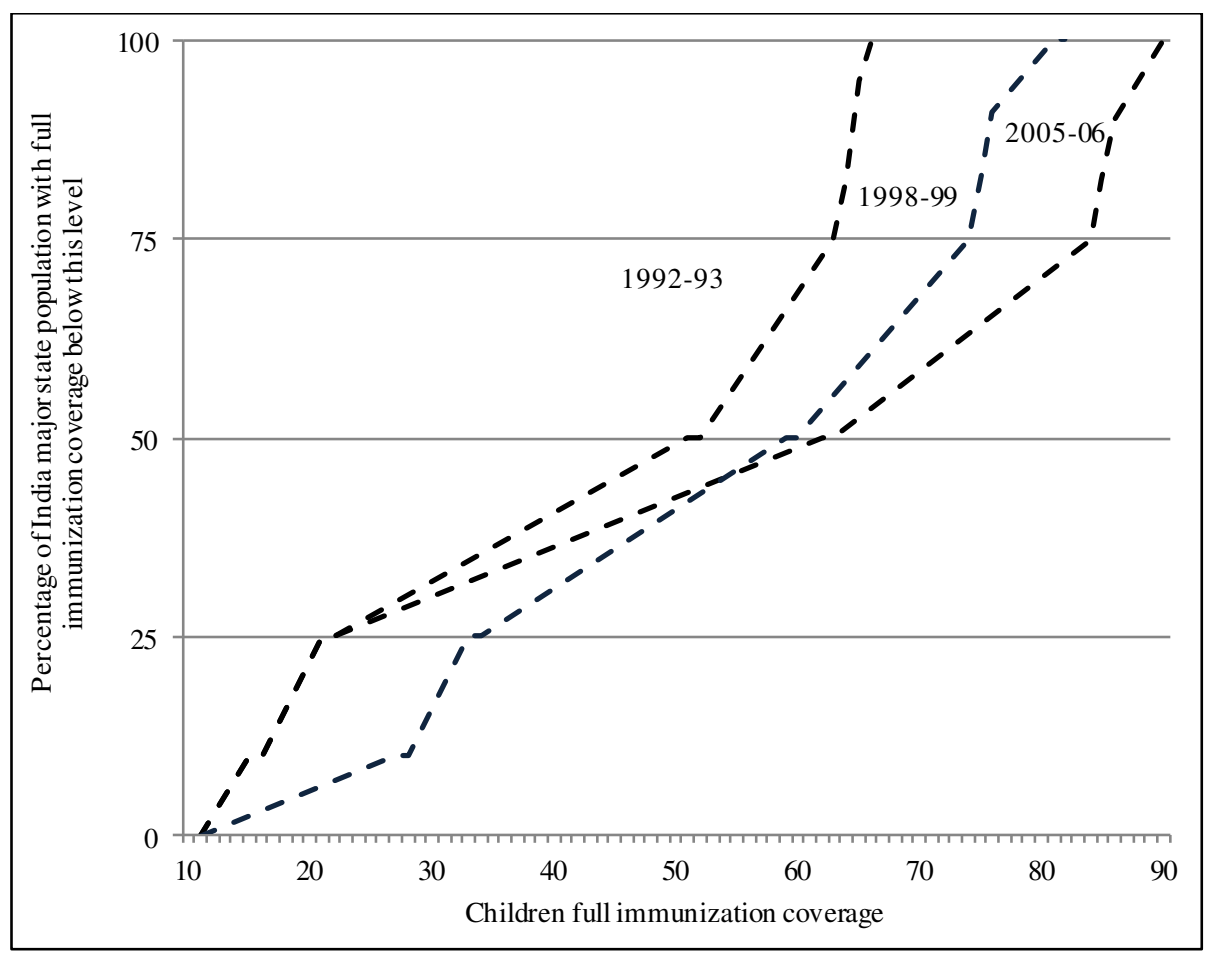



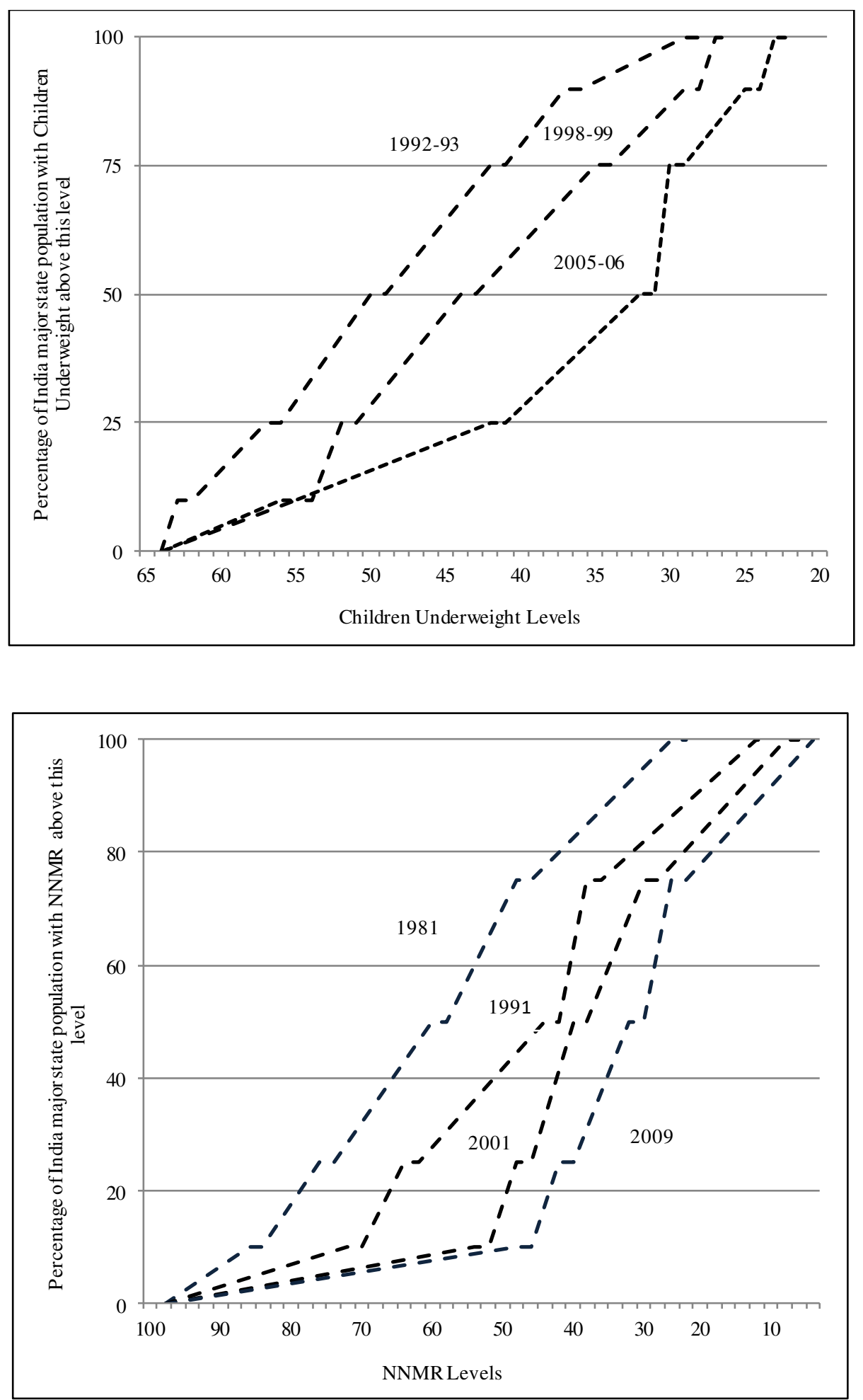


\subsubsection{Convergence in child health care utilisation and outcome indicators: Absolute $\beta$-convergence in child health status}

In this section, I have assessed the convergence in averages of child health care utilisation and outcome indicators. Convergence in child health status was measured based on three key child health indicators: full immunisation coverage of children, children underweight and NNMR. Convergence in averages of child health status has been estimated by using both absolute and conditional $\beta$-convergence measures. Further, $\beta$-convergence models for child health indicators were estimated in two stage analyses: the first stage analyses dealt with $\beta$-convergence between the beginning and end periods under observation and, the second stage analyses dealt with $\beta$-convergence for the sub-periods.

The results of absolute $\beta$-convergence estimates of coverage in child full immunisation were provided in table 7.2a. The results showed negative $\beta$ coefficients for all the three selected periods implying that there was convergence among the states in terms of full immunisation coverage. However, the volume and speed of convergence was greater during $1999-2006$ ( $S=18 \%$ per annum) compared to $1992-1998$ ( $S=5 \%$ per annum). Commensurate with the results of full immunisation coverage, the results of absolute $\beta$ convergence model estimates for children underweight showed the negative $\beta$ coefficients during 1992-2005 and for the sub-periods. Further, the volume and speed of convergence in children underweight were also greater for recent periods compared to earlier periods. However, for children underweight, the model was statistically not significant and adjusted $\mathrm{R}^{2}$ showed very poor goodness of fit (Table 7.2b). The absolute convergence estimates for NNMR presented in table $7.2 \mathrm{c}$ also revealed that the $\beta$ value during 1992-2005 was statistically significant $(\beta=-.06527, \mathrm{p}<0.007)$ indicating convergence in progress of NNMR. However, piecewise regression models for disaggregated periods showed that the convergence was much greater during 1992-1998 ( $\beta=-$. 14834, $\mathrm{p}<0.002)$ than 1999-2005 $(\beta=-.02565, \mathrm{p}<0.427)$.

A comparative assessment of convergence estimates for the three child health indicators during 1992-2005 revealed that the speed of convergence in underweight $(S=0.2 \%$ per annum) was smaller compared to full immunisation coverage $(S=14 \%)$ and $\operatorname{NNMR}(S=$ $7 \%$ per annum). The convergence was more for the recent period for children full immunisation, whereas, convergence was greater in the earlier period for children underweight and NNMR. 
Table 7.2a. Absolute $\beta$ convergence for full immunisation of children aged 12-23 across the major states, India, 1992-2006

\begin{tabular}{lcccc}
\hline Period & $\begin{array}{c}\beta \\
\text { coefficient }\end{array}$ & P value & $\begin{array}{c}\text { Adjusted } \\
\mathrm{R}^{2}\end{array}$ & $\begin{array}{c}\text { Speed of convergence } \\
\text { (\% per annum) }\end{array}$ \\
\hline 1992-93 to 2005-06 & -.12960 & 0.000 & 0.57 & 13.9 \\
1992-93 to 1998-99 & -.05292 & 0.329 & 0.002 & 5.4 \\
$1998-99$ to 2005-06 & -.16931 & 0.000 & 0.67 & 18.5 \\
\hline
\end{tabular}

States: $n=24$, df 23

Table 7.2b. Absolute $\beta$ convergence for children underweight across the major states, India, 1992- 2006

\begin{tabular}{lcccc}
\hline Period & $\begin{array}{c}\beta \\
\text { coefficient }\end{array}$ & P value & $\begin{array}{c}\text { Adjusted } \\
\mathrm{R}^{2}\end{array}$ & $\begin{array}{c}\text { Speed of convergence } \\
\text { (\% per annum) }\end{array}$ \\
\hline 1992-93 to 2005-06 & -.00186 & 0.947 & 0.04 & 0.2 \\
1992-93 to 1998-99 & -.01629 & 0.746 & 0.03 & 1.6 \\
1998-99 to 2005-06 & -.03609 & 0.479 & 0.02 & 3.7 \\
\hline
\end{tabular}

States: $\mathrm{n}=24$, df 23

Table 7.2c. Absolute $\beta$ Convergence for neonatal mortality rate across the major states, India, 1981-2009

\begin{tabular}{lcccc}
\hline Period & $\beta$ coefficient & P value & Adjusted $\mathrm{R}^{2}$ & $\begin{array}{c}\text { Speed of } \\
\text { convergence } \\
(\% \text { per annum })\end{array}$ \\
\hline $1981-2009$ & .01538 & 0.312 & 0.00 & 6.7 \\
$1981-1991$ & .03290 & 0.412 & -0.01 & 16.1 \\
$1991-2001$ & -.02792 & 0.113 & 0.10 & 2.6 \\
$2001-2009$ & -.01471 & 0.767 & -0.06 & \\
\hline
\end{tabular}

States: $\mathrm{n}=24$, df 23

\subsubsection{Conditional $\beta$-convergence in child health status}

Although, the absolute convergence model presented the evidential support for unconditional convergence, it is not reasonable to assume that all the states do share same socioeconomic conditions. Therefore, to account for socioeconomic variability of the states, conditional $\beta$-convergence was estimated by including two more explanatory variables: proportion of illiterate population and proportion of population in poor wealth quintile in the regression model. The absolute $\beta$-convergence estimates, the conditional $\beta$ 
estimates for children full immunisation rates also showed negative $\beta$ coefficients $(\beta=-$ $\left..15848, \mathrm{p}<0.000, \mathrm{R}^{2}=0.68\right)$ in the entire period, 1992-2006, thereby, supporting the convergence hypothesis. However, the piecewise conditional $\beta$-convergence model estimates for child full immunisation coverage showed greater volume, speed of convergence and goodness of fit $\left(\beta=-.18017, \mathrm{p}<0.001, R^{2}=0.58\right)$ during 1992-1998 than $\left(\beta=-.15952, \mathrm{p}<0.000, R^{2}=0.70\right) 1998-2005$ (Table 7.3a). The conditional $\beta$-convergence results for children underweight during 1992-2006 indicated a convergence, but the adjusted $\mathrm{R}^{2}$ indicated the poor goodness of fit for the model. Nevertheless, the conditional $\beta$ estimates for children underweight showed the greater volume and speed of convergence $(\beta=-.15835, \mathrm{p}<0.026, S=17 \%$ per annum) for the recent period than $(\beta=-.06652$, $\mathrm{p}<0.282, S=06 \%$ per annum) previous period (Table 7.3b). The conditional $\beta-$ convergence estimates for NNMR indicated that $\beta$-values for all three periods were negative, thereby, indicating convergence in NNMR over time. Similar to absolute $\beta$ convergence estimates of NNMR, conditional $\beta$-convergence estimates also suggests that the volume of convergence $(\beta=.-28982, \mathrm{p}<0.000)$ was higher during 1992-1998 than $(\beta=$ $.12649, \mathrm{p}<0.023$ ) 1998-2006. Moreover, the estimates of the speed of convergence and goodness of fit measured by the adjusted $\mathrm{R}^{2}$ also indicated the similar pattern (Table 7.3c).

However, a comparison of absolute and conditional $\beta$-convergence estimates showed that after controlling for the state level proportion of illiterate population and poverty ratios, the volume and speed of convergence indicated a greater conditional $\beta$-convergence than unconditional $\beta$-convergence. Moreover, comparison of pattern of convergence during 1992-2006 for different child health indicators revealed that child full immunisation showed a greater volume of convergence in comparison to other two indicators. However, piecewise regression estimates showed greater convergence in the recent period (19982006) for child full immunisation compared to the greater volume of convergence was during 1992-1998 for NNMR and underweight. 
Table 7.3a. Conditional $\beta$-convergence for full immunisation of children in age 12-23 across the major states, India, 1992-2006

\begin{tabular}{|c|c|c|c|c|c|}
\hline \multirow[t]{2}{*}{ Period } & \multicolumn{3}{|c|}{$\beta$ coefficient } & \multirow{2}{*}{$\begin{array}{c}\text { Adjusted } \\
\mathrm{R}^{2}\end{array}$} & \multirow{2}{*}{$\begin{array}{c}\text { Speed of } \\
\text { convergence for } \\
\text { child Immunisation } \\
\text { (\% per annum) }\end{array}$} \\
\hline & $\begin{array}{l}\text { Child full } \\
\text { immunisation }\end{array}$ & $\begin{array}{c}\text { Proportion of } \\
\text { illiterate } \\
\text { population }\end{array}$ & $\begin{array}{l}\text { Poverty } \\
\text { ratio }\end{array}$ & & \\
\hline $1992-93$ to & -.15848 & -.07353 & -.000093 & 0.68 & 17.3 \\
\hline $2005-06$ & $(0.000)$ & $(0.011)$ & (0.998) & & \\
\hline $1992-93$ to & -.18017 & -.02343 & .00959 & 0.58 & 19.9 \\
\hline 1998-99 & $(0.001)$ & $(0.636)$ & $(0.864)$ & & \\
\hline $1998-99$ to & -.15952 & -.03008 & -.04595 & 0.70 & 17.4 \\
\hline $2005-06$ & $(0.000)$ & $(0.553)$ & $(0.340)$ & & \\
\hline
\end{tabular}

Note: 1. States: $\mathrm{n}=24$, df 23; 2 . () P value

Table 7.3b. Conditional $\beta$-convergence for children underweight across the major states, India, 1992-2006

\begin{tabular}{|c|c|c|c|c|c|}
\hline \multirow[t]{2}{*}{ Period } & \multicolumn{3}{|c|}{$\beta$ coefficient } & \multirow{2}{*}{$\begin{array}{c}\text { Adjusted } \\
\mathrm{R}^{2}\end{array}$} & \multirow{2}{*}{$\begin{array}{c}\text { Speed of } \\
\text { convergence for } \\
\text { child immunisation } \\
\text { (\% per annum) }\end{array}$} \\
\hline & $\begin{array}{c}\text { Children } \\
\text { underweight }\end{array}$ & $\begin{array}{c}\text { Proportion of } \\
\text { illiterate } \\
\text { population }\end{array}$ & $\begin{array}{l}\text { Poverty } \\
\text { ratio }\end{array}$ & & \\
\hline $1992-93$ & -.07560 & -.03075 & .02741 & 0.24 & 7.9 \\
\hline to $2005-06$ & $(0.060)$ & $(0.116)$ & $(0.222)$ & & \\
\hline $1992-93$ to & -.06652 & .00085 & .03483 & 0.08 & 6.9 \\
\hline 1998-99 & $(0.282)$ & $(0.982)$ & $(0.341)$ & & \\
\hline $1998-99$ to & -.15835 & .03097 & .06004 & 0.25 & 17.2 \\
\hline $2005-06$ & $(0.026)$ & $(0.424)$ & $(0.100)$ & & \\
\hline
\end{tabular}

Note: 1. States: $\mathrm{n}=24$, df 23; 2 . () P value

Table 7.3c. Conditional $\beta$-convergence for neonatal mortality rate across the major states, India, 1981-2009

\begin{tabular}{|c|c|c|c|c|c|}
\hline \multirow[t]{2}{*}{ Period } & \multicolumn{3}{|c|}{$\beta$ coefficient } & \multirow{2}{*}{$\begin{array}{c}\text { Adjusted } \\
\mathrm{R}^{2}\end{array}$} & \multirow{2}{*}{$\begin{array}{l}\text { Speed of convergence } \\
\text { for infant mortality rate } \\
\text { (\% per annum })\end{array}$} \\
\hline & $\begin{array}{l}\text { Neonatal } \\
\text { mortality } \\
\text { rate }\end{array}$ & $\begin{array}{l}\text { Proportion } \\
\text { of illiterate } \\
\text { population }\end{array}$ & $\begin{array}{l}\text { Poverty } \\
\text { ratio }\end{array}$ & & \\
\hline 1981-2009 & -.00004 & .047557 & -.027748 & 0.40 & 16.3 \\
\hline 1981-1991 & -.04774 & .003983 & .080304 & 0.10 & 34.2 \\
\hline 1991-2001 & -.02930 & .053418 & -.035798 & 0.54 & 13.5 \\
\hline 2001-2009 & -.01630 & .105112 & -.093291 & 0.41 & \\
\hline
\end{tabular}

Note: 1. States: $\mathrm{n}=15$, df 14; 2 . () P value 


\subsubsection{Sigma convergence in averages of child health status}

In the early part of this study, we noted Quah (1996) and Young et al. (2008) observations that $\beta$-convergence is necessary but not a sufficient condition for Sigma convergence. In other words, a country experiencing $\beta$-convergence may not be experiencing a sigma convergence. Therefore, it is necessary to test for sigma convergence alongside the $\beta$ convergence. Figure 7.4 shows the results for Sigma convergence in averages of child health status. The sigma convergence results for child health indicators showed a mixed pattern: three out of two indicators indicated a convergence trend in child health status. The trends in standard deviation in children full immunisation showed evidence for divergence until 1998-99, but convergence thereafter. However, children underweight indicated a divergent trend that the dispersion in the percentage of underweight children gradually increased in recent periods (1992-93) compared to earlier periods (1998-99 and 2005-06). Results in case of NNMR also indicated a convergent trend over the period, the dispersion in NNMR declined from 16.2/1000 in 1981 to $10.4 / 1000$ live births in 2009 .

Figure 7.4. Sigma convergence in averages of child health indicators across the major states, India; 1981-2009

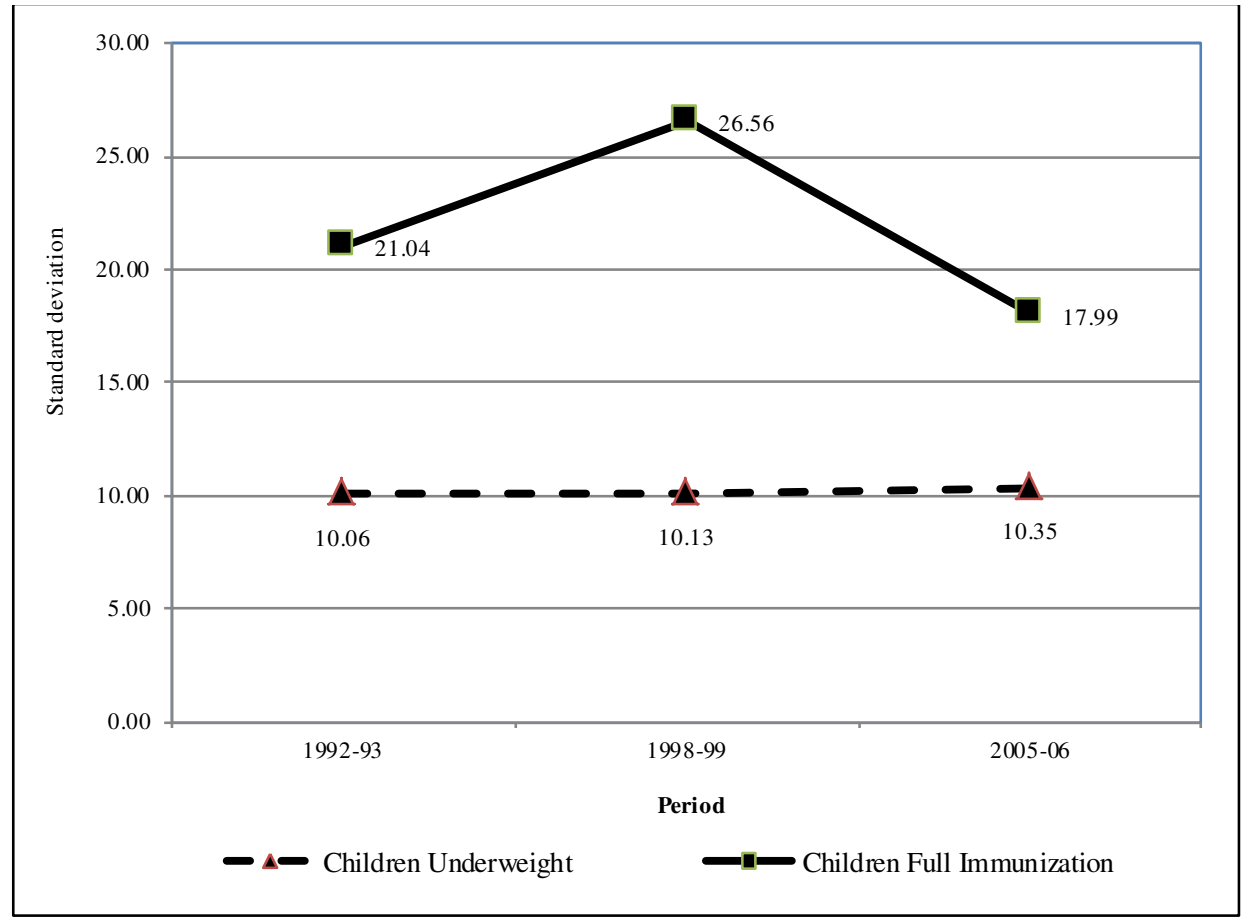




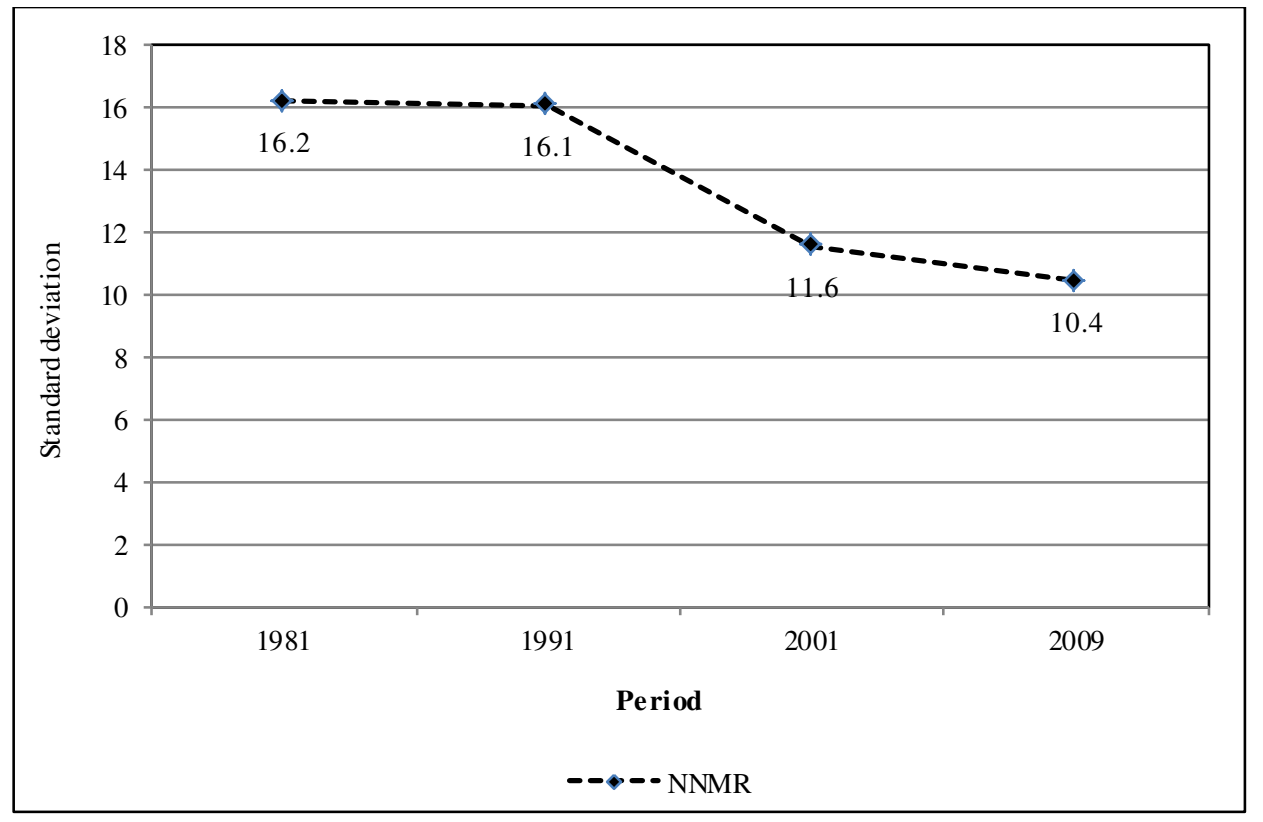

\subsubsection{Convergence in absolute and relative health inequalities}

To overcome the drawback of traditional convergence measures, this section assesses the convergence in inequalities in child health indicators such as children full immunisation, underweight and NNMR across the states. This section measured two types of inequality convergence: first, convergence in overall health inequalities and second, convergence in socioeconomic inequalities in child health indicators. As, this study pointed out earlier that, the overall health inequalities are classified into absolute inequalities measured in terms of AID and DMM and the relative inequalities measured in terms of Gini coefficient. Thus, convergence in absolute and relative health inequalities was measured based on a percentage reduction in DMM and Gini: a positive change in the percentage points indicate convergence and a negative value implies divergence. However, the convergence in socioeconomic inequality in child health was estimated using the sigma convergence measure in $\mathrm{CI}$ values of the states.

Figure $7.5 \mathrm{a}$ shows the trends of percentage of absolute convergence in children full immunisation, children underweight and NNMR among the major Indian states, 19812009. The results in case of children full immunisation revealed that the absolute inequalities indicate for divergence with an increase in inequalities by $34 \%$ during 199299, but later divergence was replaced with convergence with a decline in absolute inequalities by $25 \%$ during 1999-2006. However, in case of children underweight, the results showed divergence with an increase in AID by 6\% during 1992-1999 and 13\% in 
1999-2006. Overall, the results showed increasing divergence in children underweight across the Indian states. The percentage reduction in absolute inequality, measured by DMM, in NNMR was declined until 2001. During 2001-09, the earlier convergence was replaced with divergence. The volume of convergence in overall absolute inequality in NNMR during 1981-1991 was 15\%, which increased to 31\% during 1991-2001, but become negative during 2001-2009 (-8.3\%). However, in contrast to children full immunisation, the results of convergence in absolute inequality for NNMR revealed a trend of convergence replacing divergence for NNMR, a continued and increasing divergence trend for children underweight.

Figure 7.5a. Trends of percentage of absolute convergence in Children immunisation, Children underweight, NNMR among major Indian states, 1981-2009

Children full immunisation

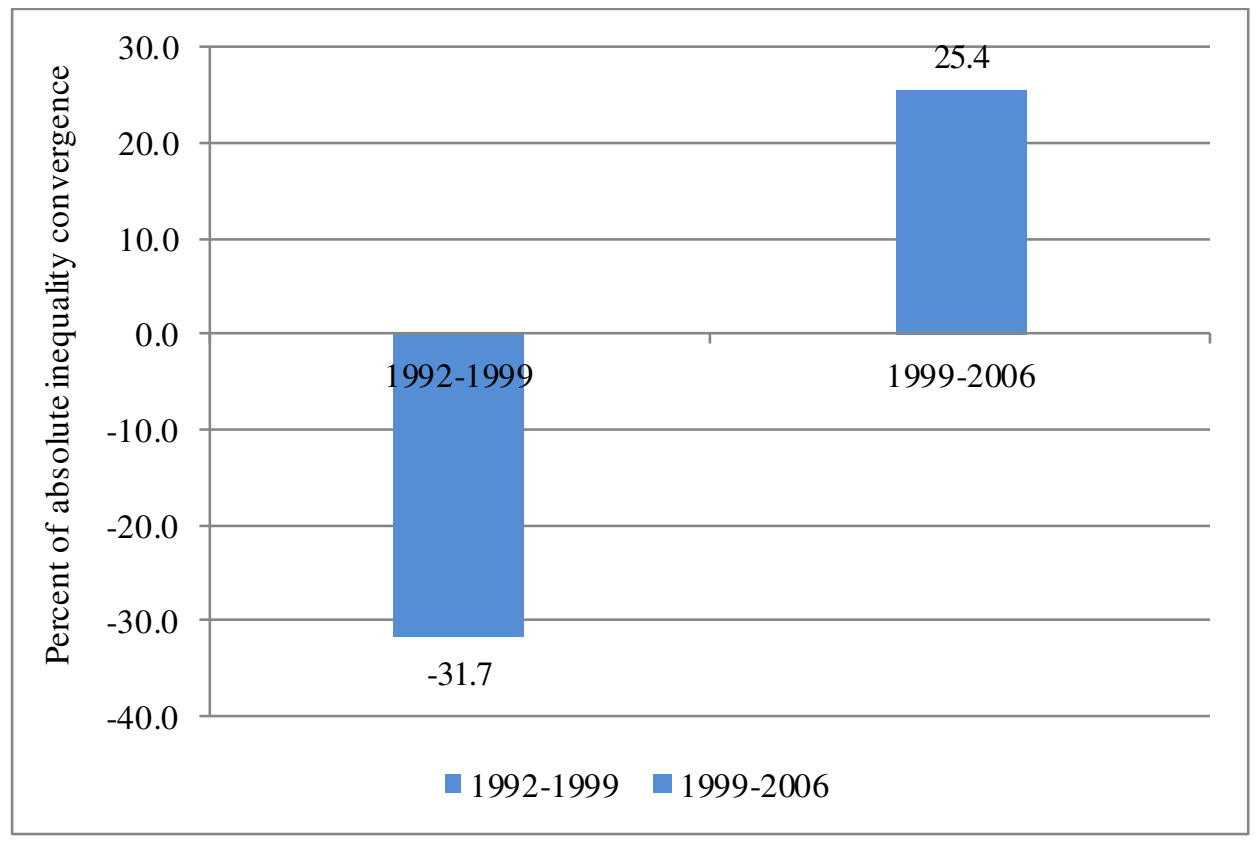


Children underweight

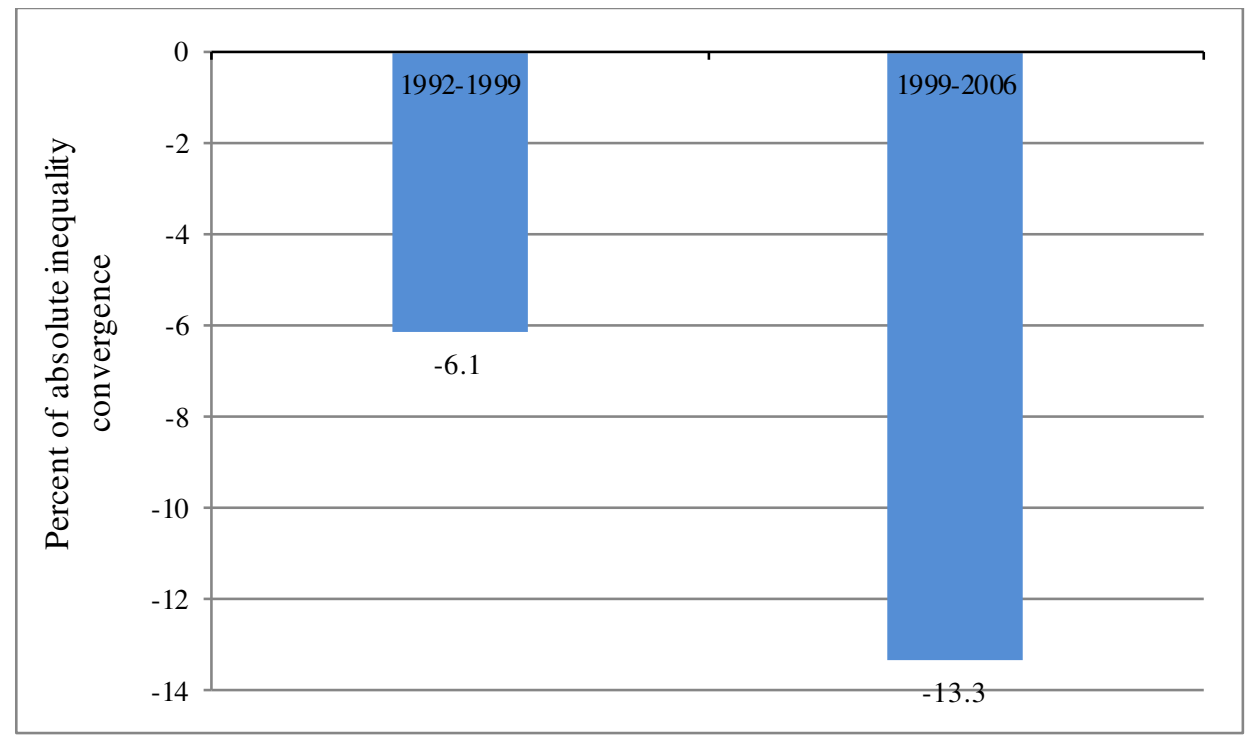

NNMR

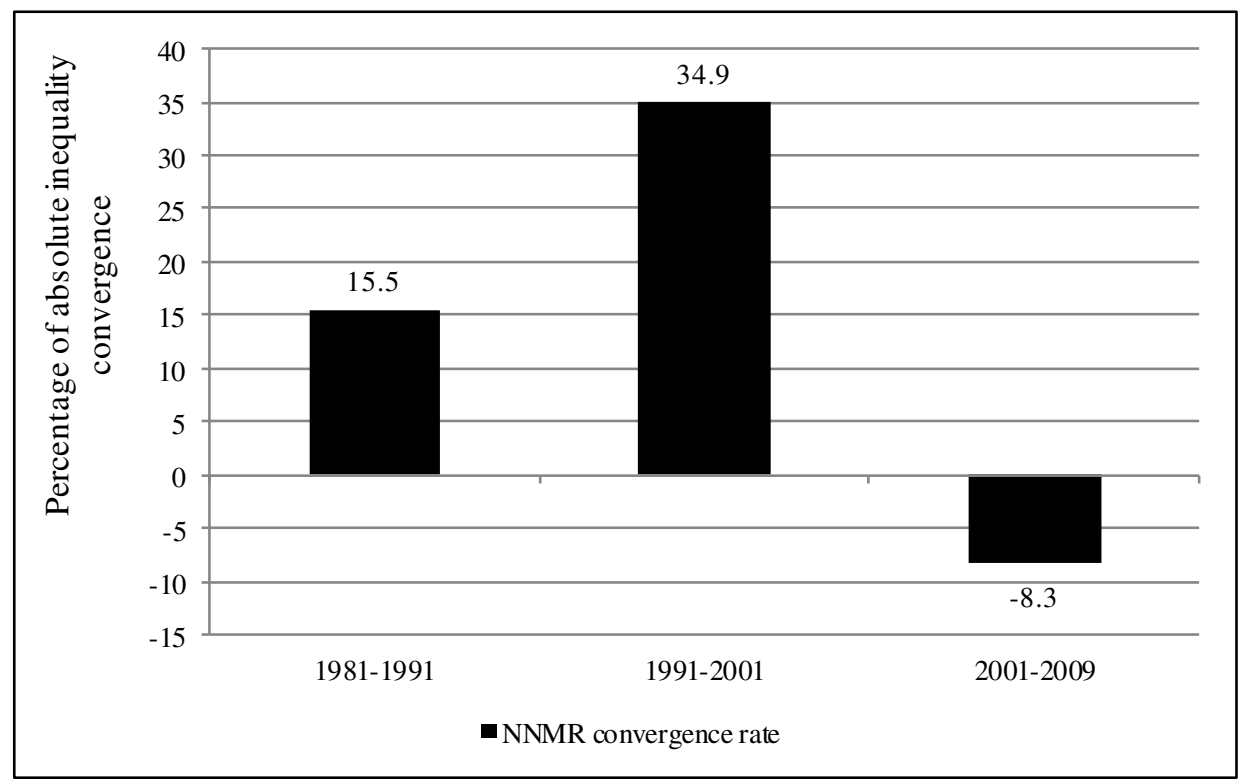

Convergence in relative overall inequality in child health was measured by Gini coefficient based on reduction in percentage of Gini for children full immunisation, children underweight and NNMR. The results in case of children full immunisation reveal divergence in relative inequalities in 1992-93, but the divergence was replaced with convergence in later period, 1999-2006. However, both children underweight and NNMR showed continued divergence in relative inequalities for the entire period, 1981-2009 that the rate of percentage reduction in Gini coefficient was negative for all three periods. The estimates of the relative convergence showed greater divergence in children underweight 
than in NNMR. However, there was a steady decline (from $-5 \%$ to $-2.5 \%$ ) in the volume of divergence for NNMR, but increasing (from -20 to $-29 \%$ ) for children underweight. In sum, the relative convergence in overall health inequality showed evidence of divergence than convergence, in two out three indicators (Figure 7.5b).

Figure.7.5b. Trends in convergence in relative inequalities based on the Gini estimates among the major states of India, 1981-2009

Children full immunisation

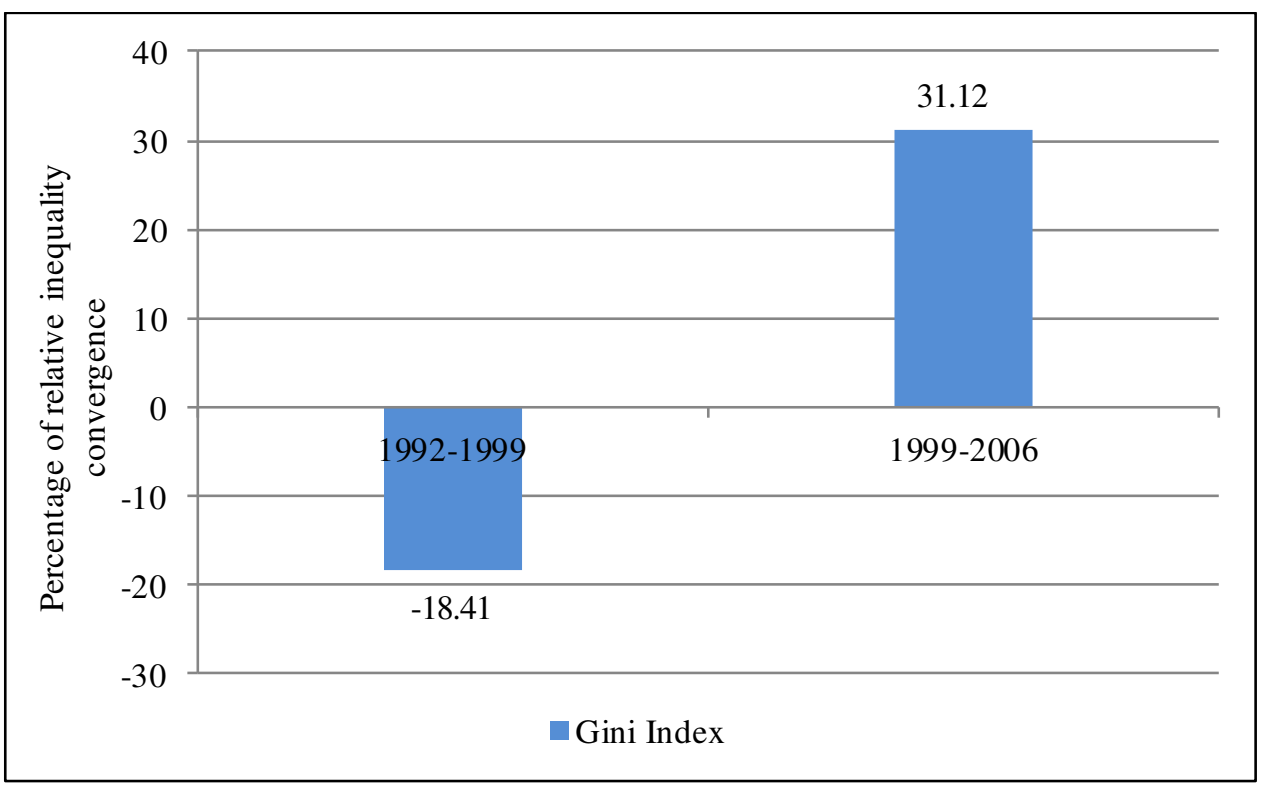

Children underweight

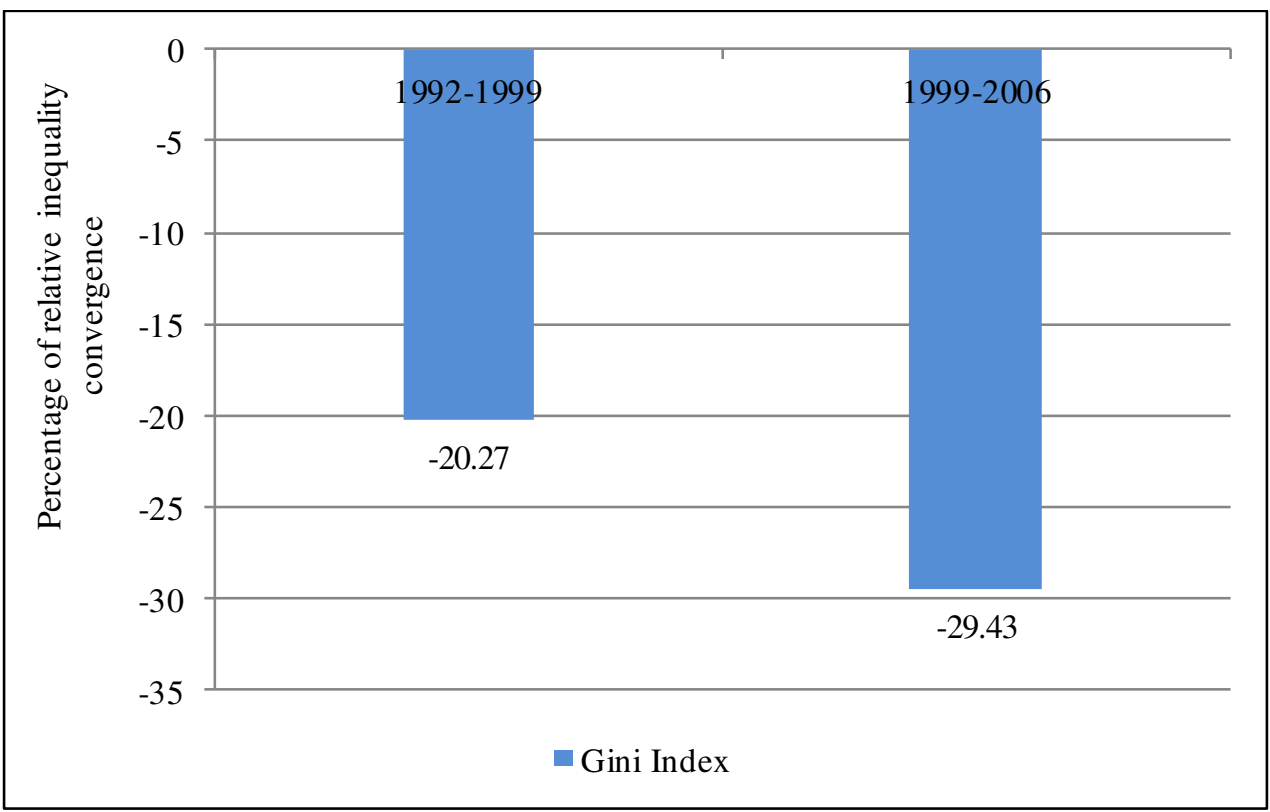


NNMR

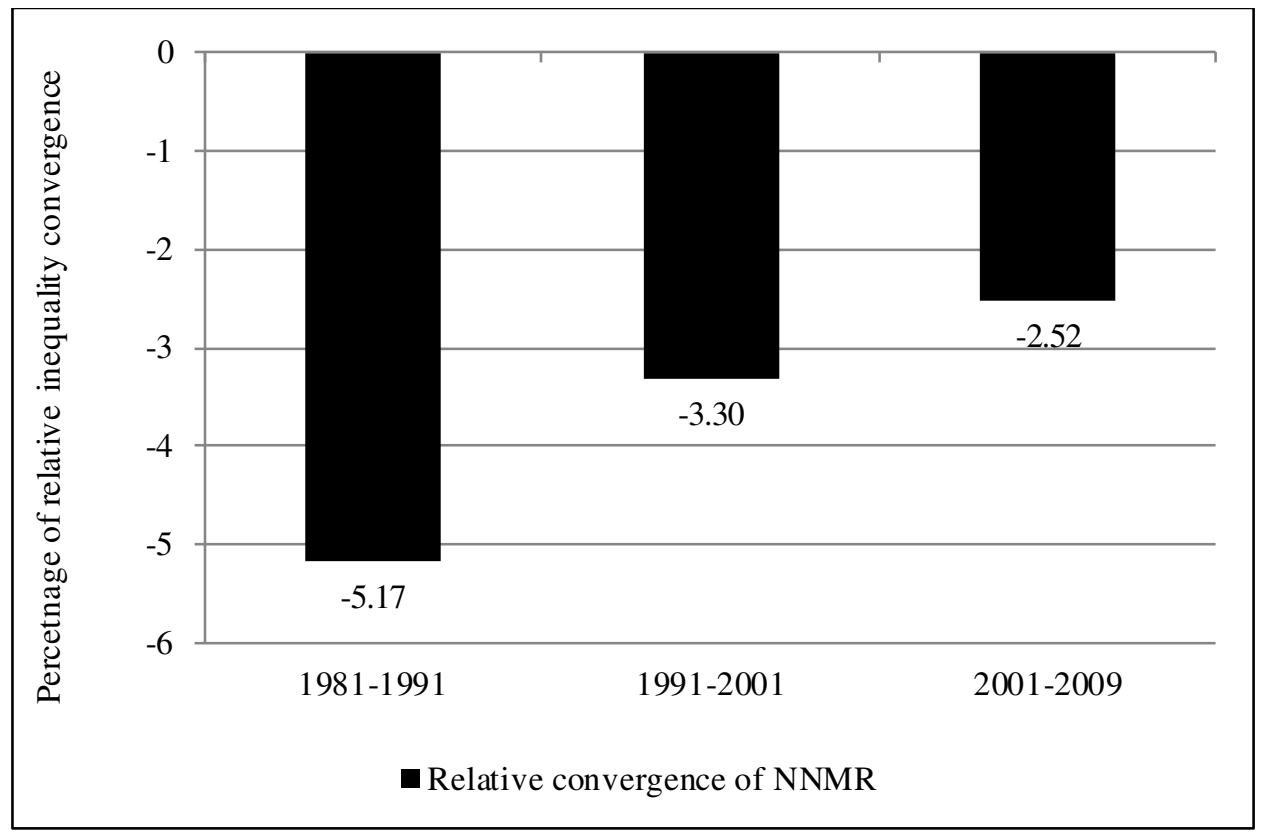

Figure 7.5c. Trends in percentage of absolute inequality convergence in Children full immunisation, Children underweight, NNMR among major Indian states by Rural-Urban, 1981-2009

Children full immunisation, Rural

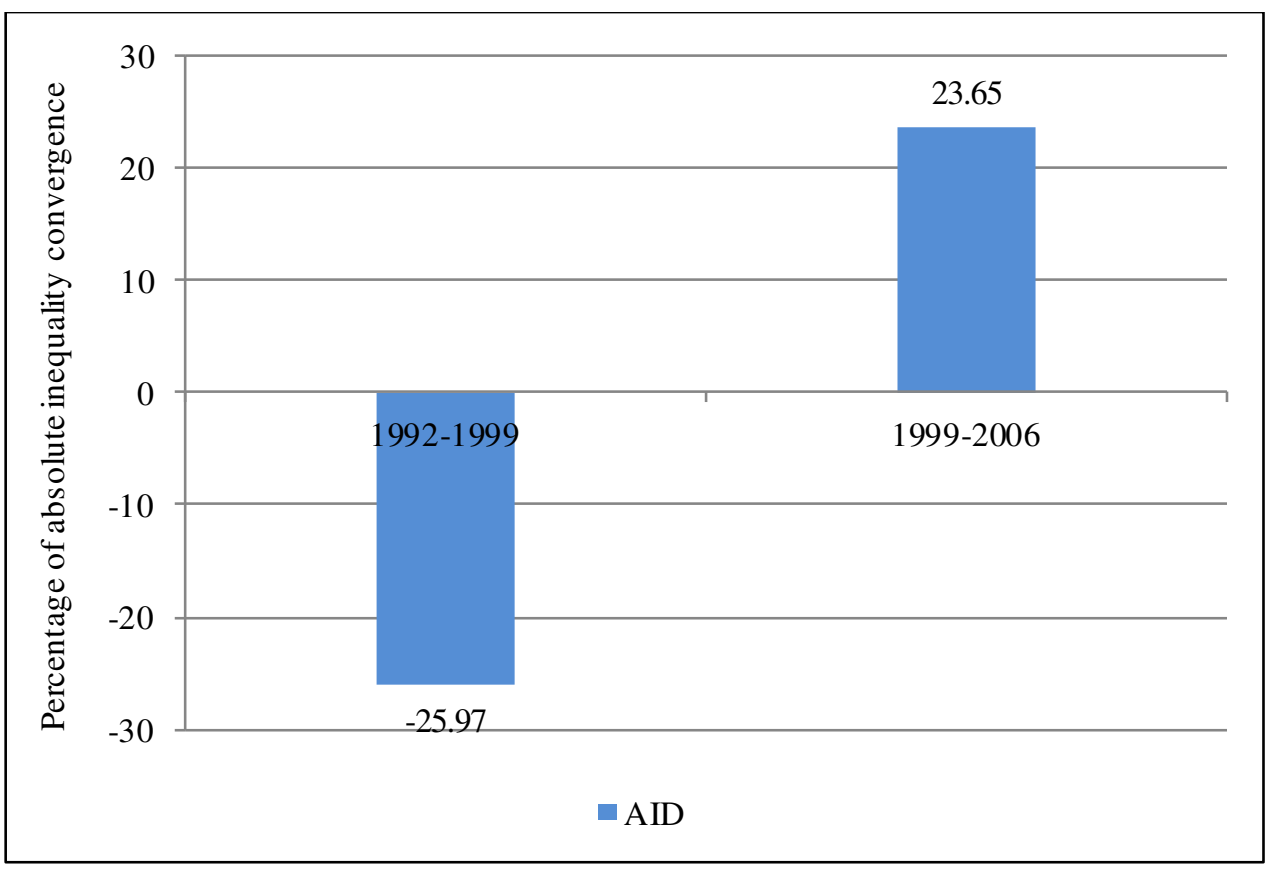


Children full immunisation, Urban

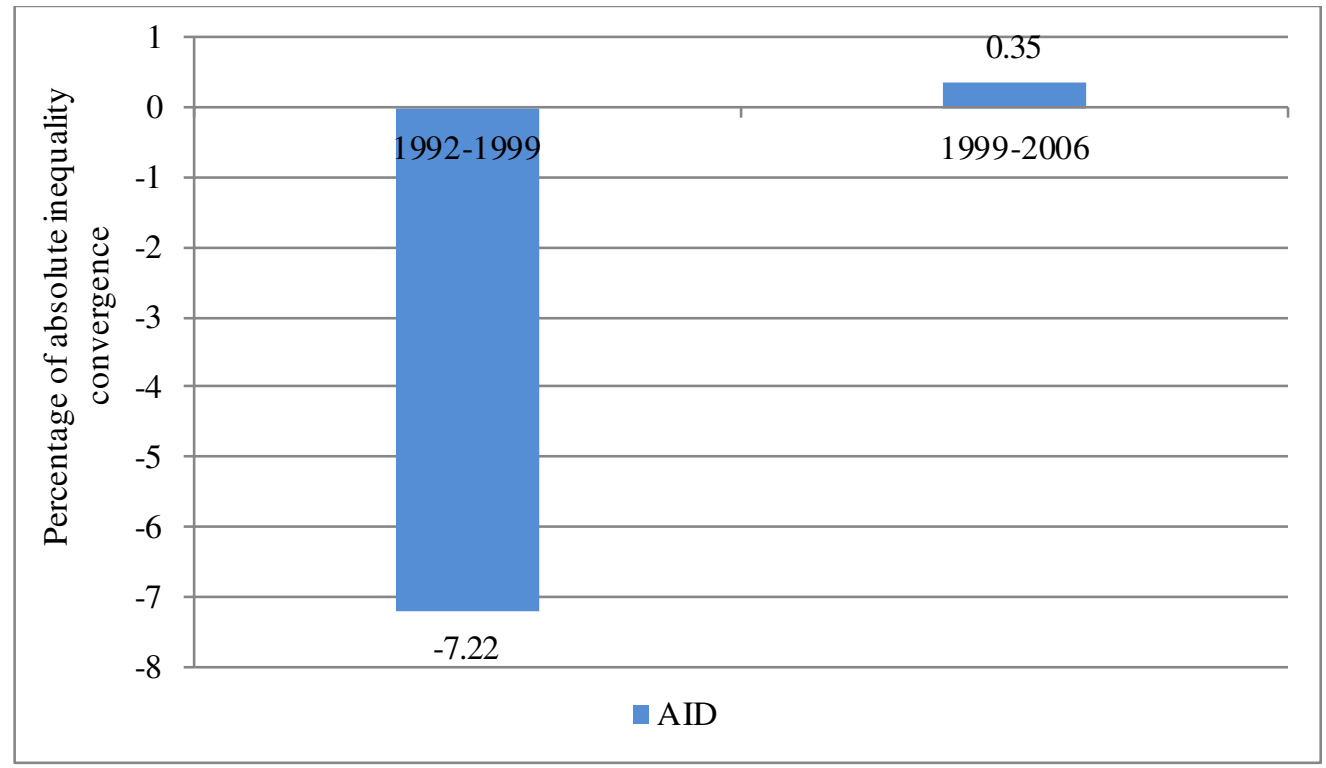

Children underweight, Rural

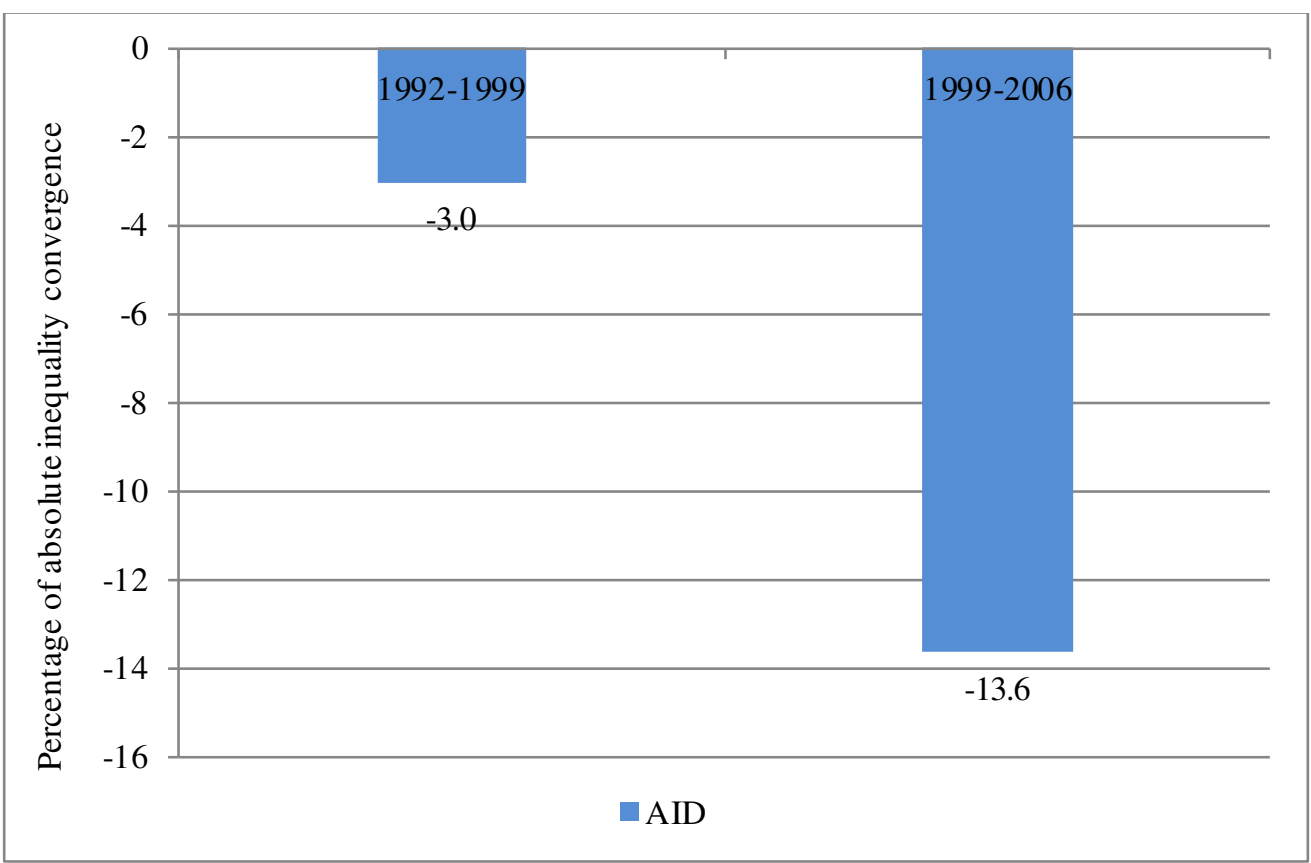


Children underweight, Urban

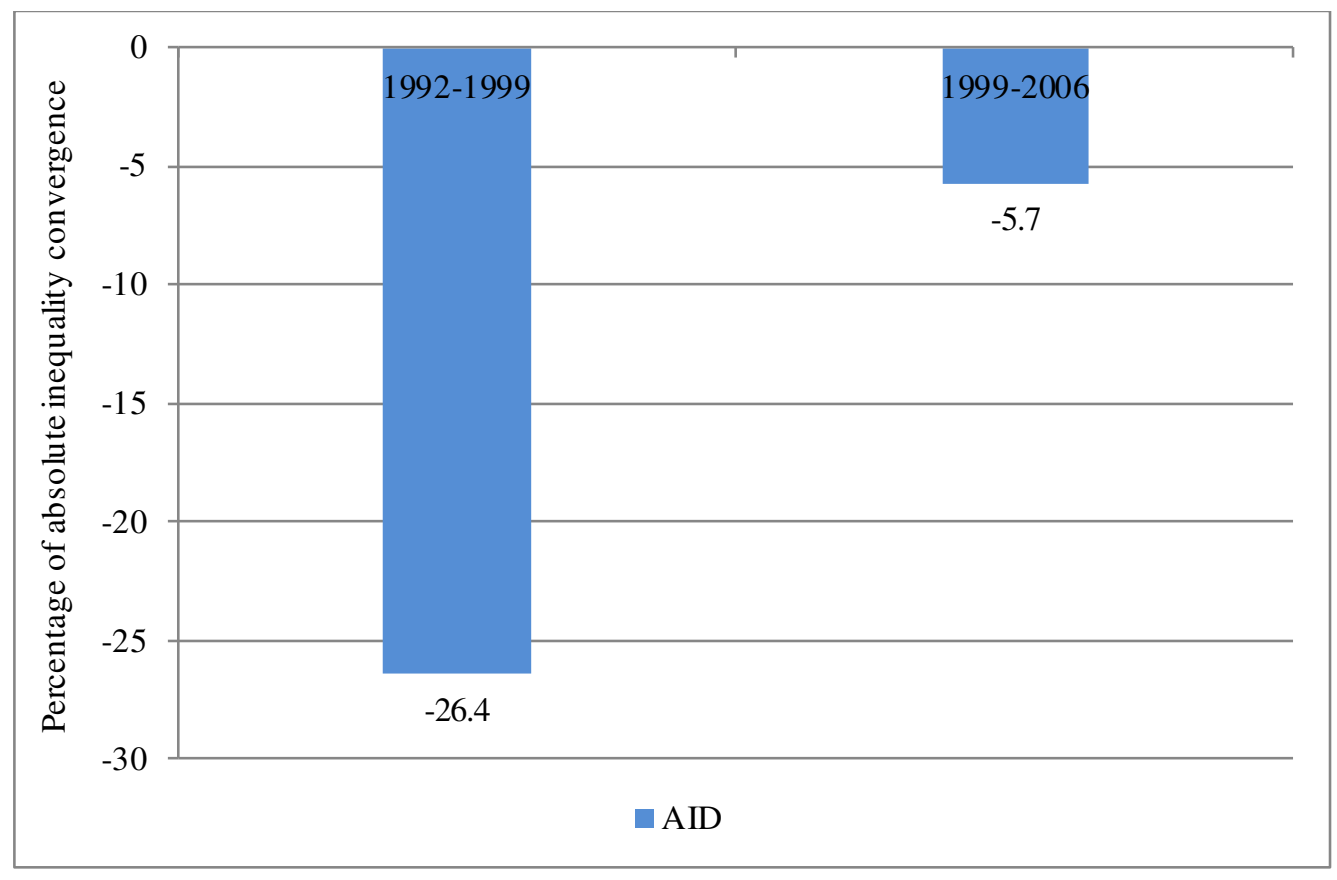

NNMR, Rural

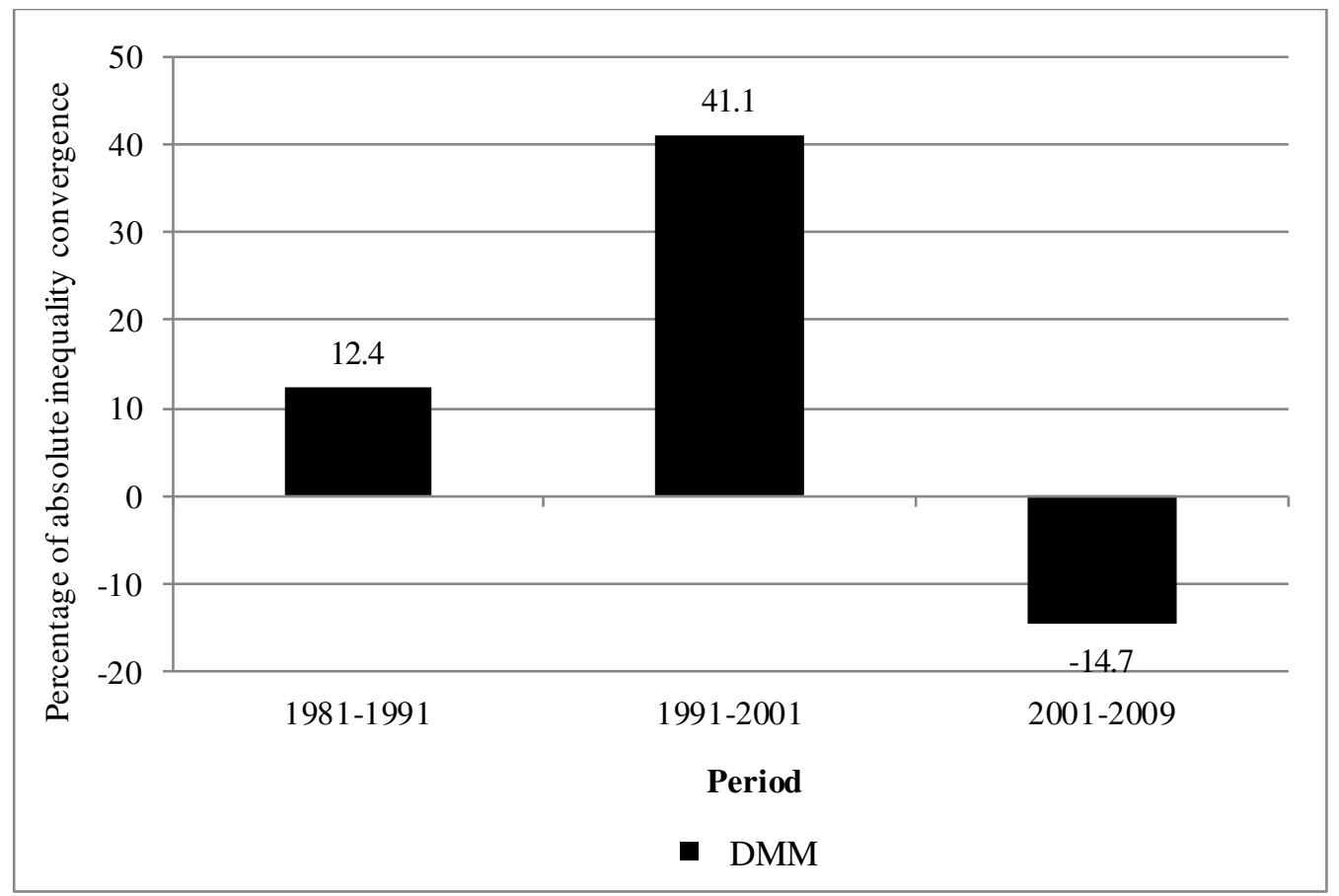


NNMR, Urban

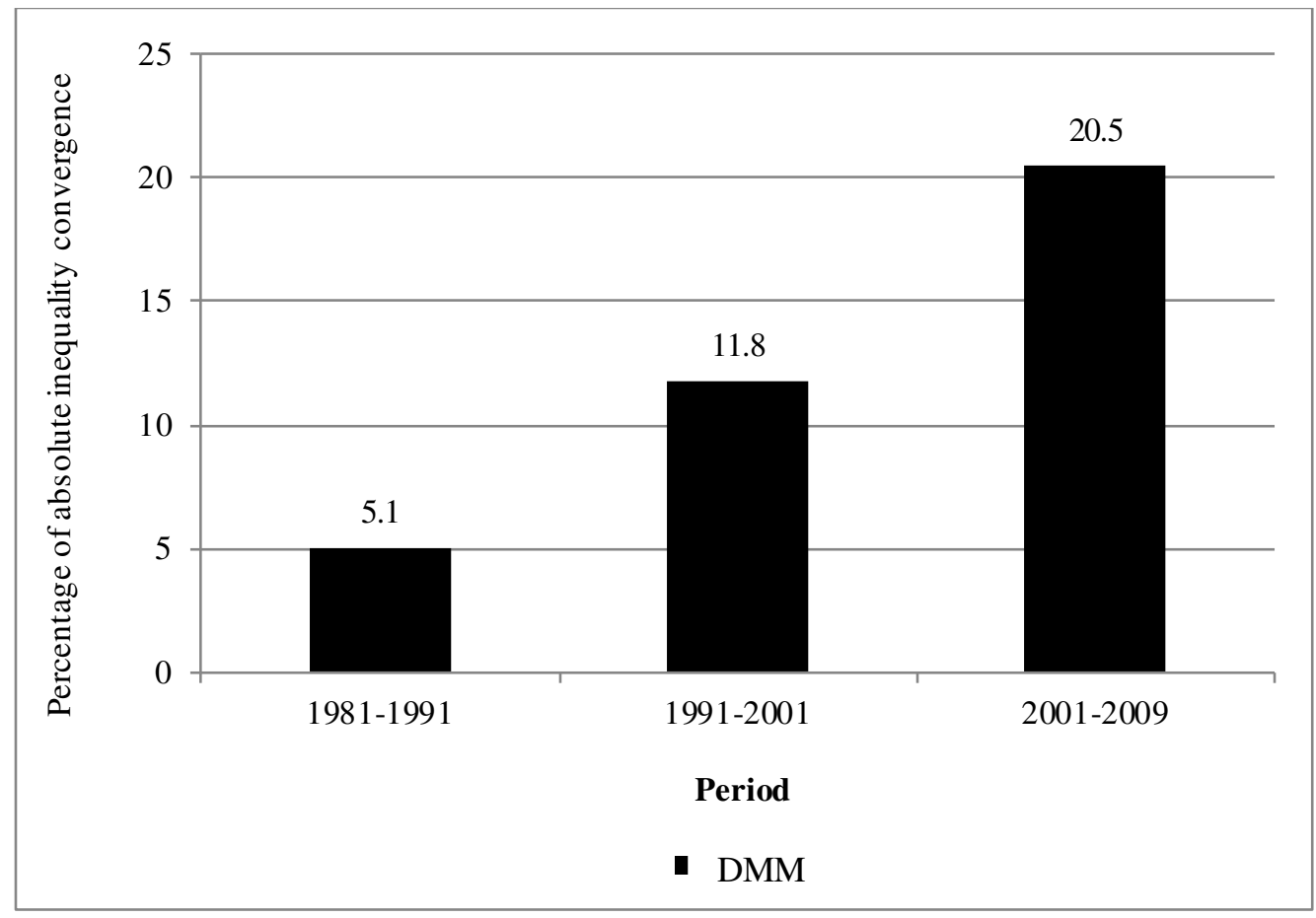

Figure $7.5 \mathrm{c}$ shows the trends of percentage of absolute convergence in children full immunisation, children underweight and NNMR among the major Indian states by ruralurban, 1981-2009. In case of children full immunisation in rural areas, the results reveal that inequalities in children full immunisation have experienced divergence by $-26 \%$ in 1992-99 which was replaced with convergence (24\%) in the recent period, 1999-2006. Similar results were also evident in urban areas, but both rate of divergence (-5\%) in 199299 and convergence $(0.35 \%)$ was very less in comparison with rural areas. In case of children underweight in rural areas, the results evident for the divergence and it was increased from -3 percent in 1992-1999 to -14 percent in 1999-2006. In urban areas, though results evident for divergence in both periods, but the rate of divergence was decreased from $-26 \%$ in 1992-93 to $-5.7 \%$ in 1999-2006. In case of NNMR in rural areas, the results suggested convergence in the first two periods, 1981-91 (12\%) and 1991-2001 (41\%), but earlier convergence was replaced with divergence in 2001-09 (-15\%). However, in urban areas, the results showed convergence for the entire period, 1981-2009 and volume of convergence was increased in the recent periods, 2001-2009. 
Figure 7.5d. Trends of percentage of relative inequalities convergence in Children full immunisation, Children underweight and NNMR among major Indian states by RuralUrban, 1981-2009

\section{Children full immunisation, Rural}

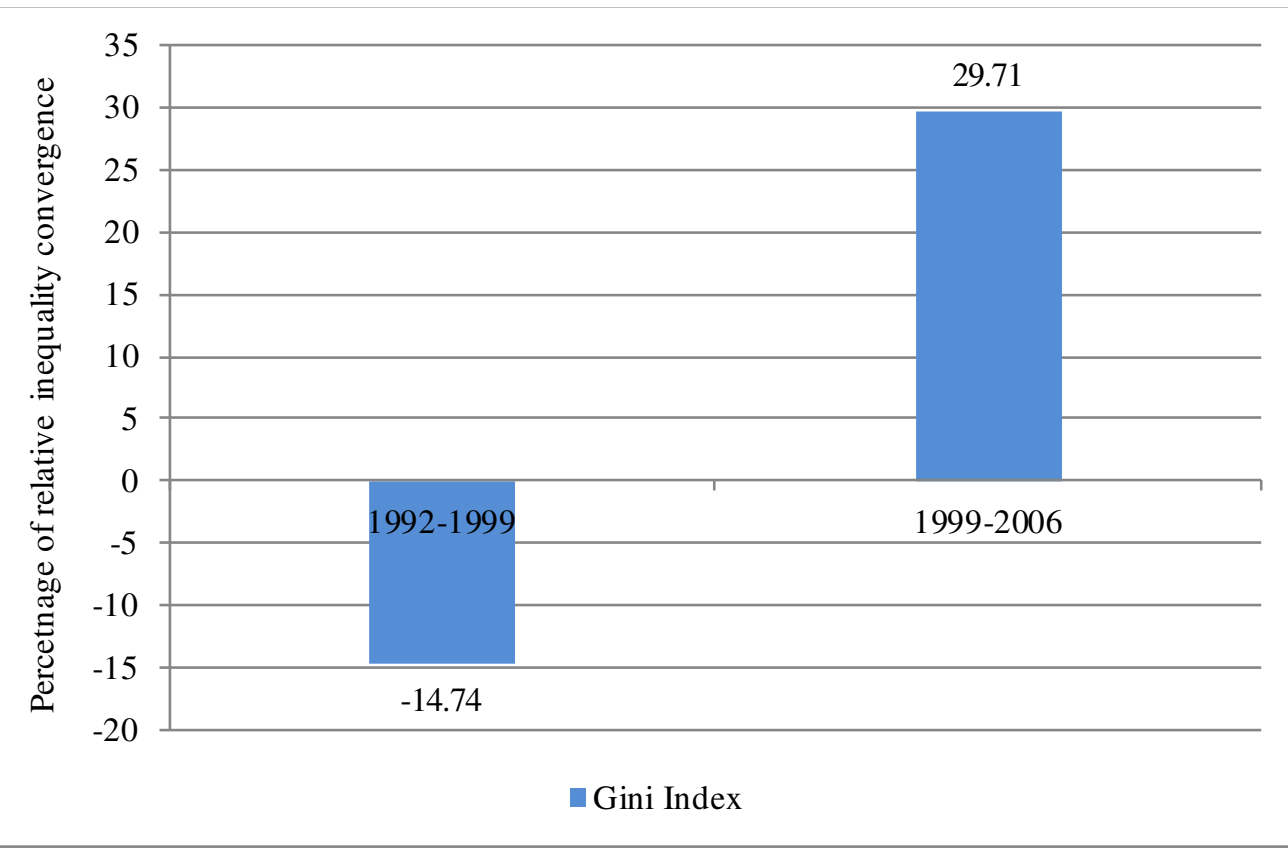

Children full immunisation, Urban

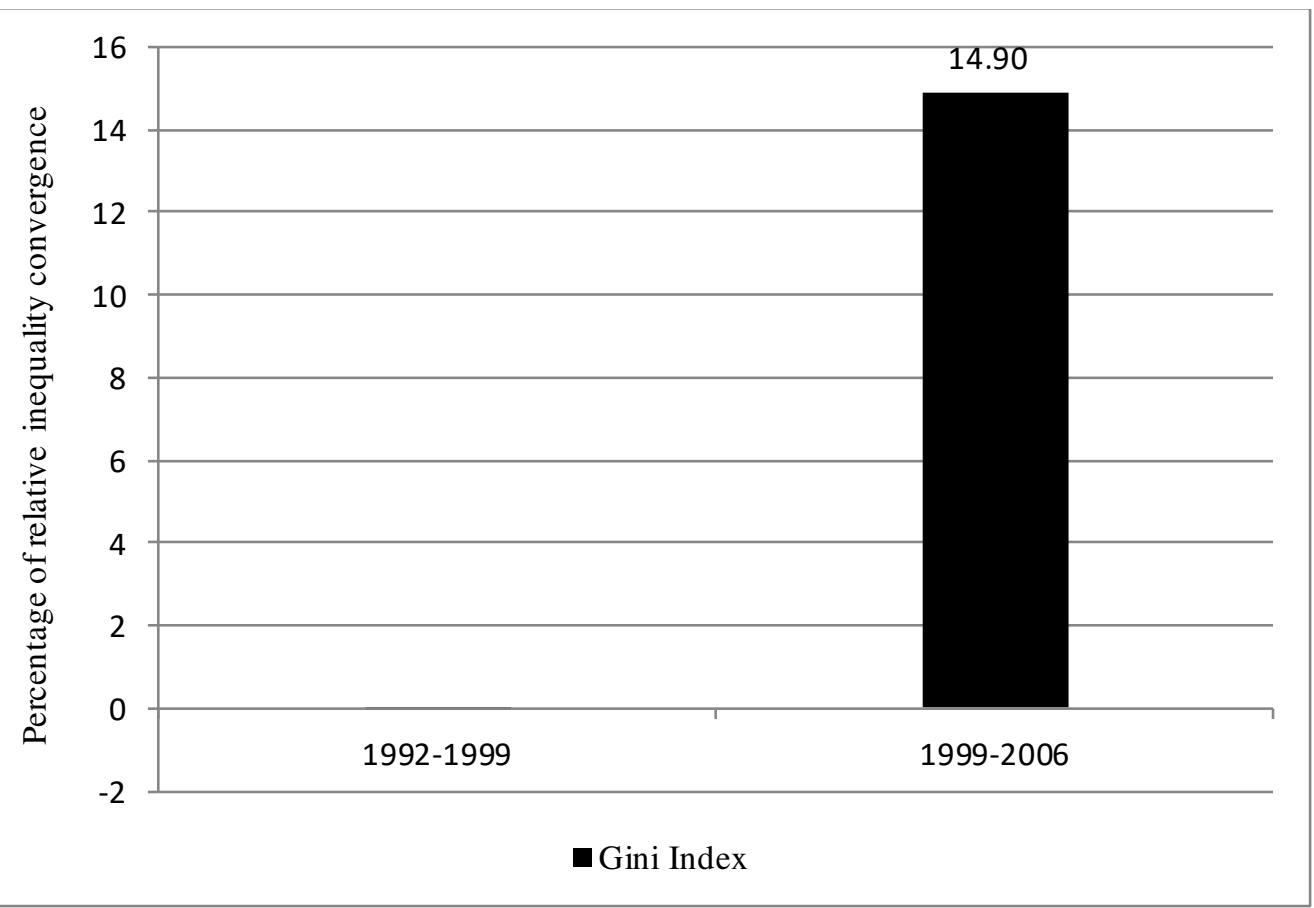


Children underweight, Rural

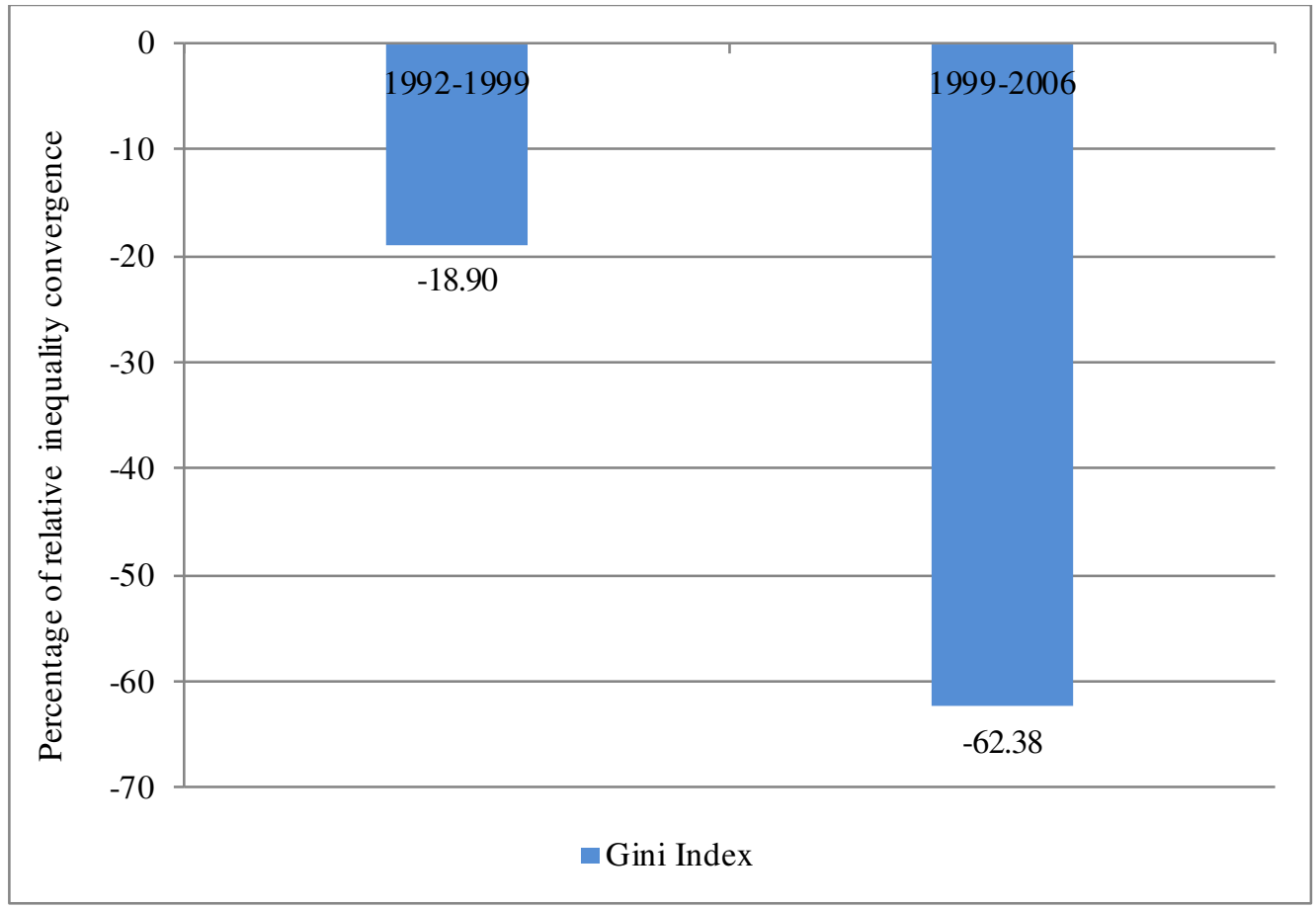

Children underweight, Urban

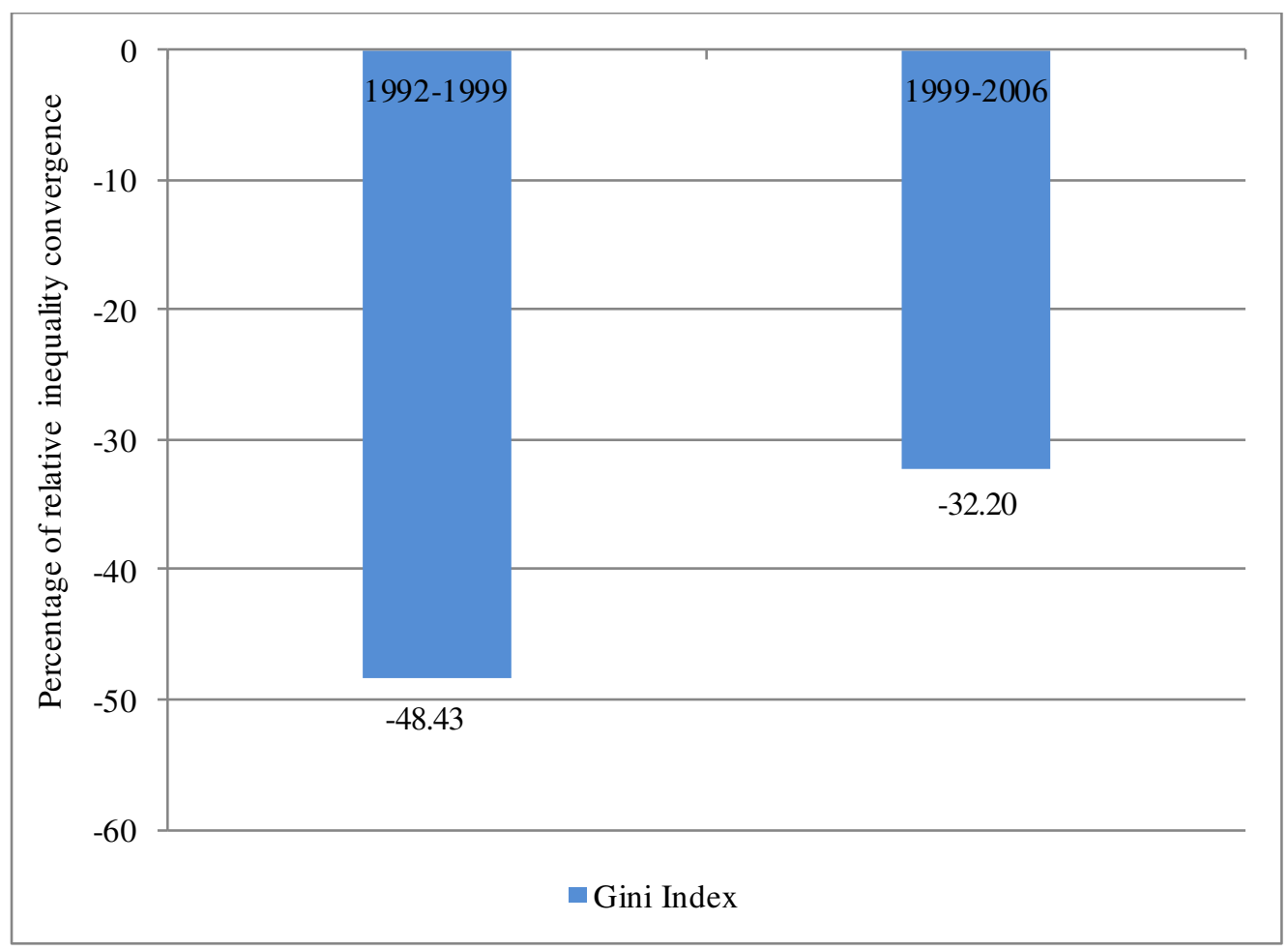


NNMR, Rural

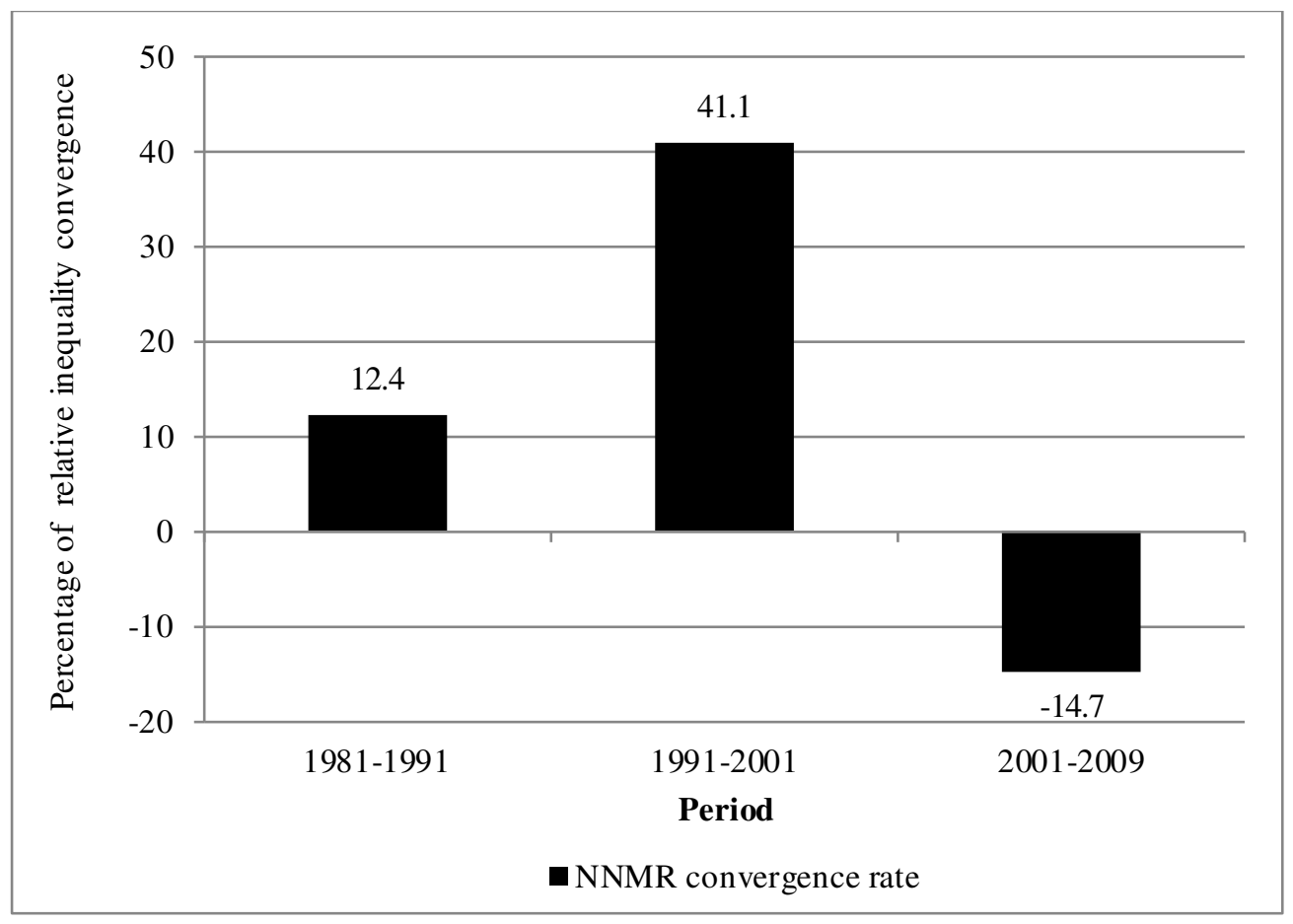

NNMR, Urban

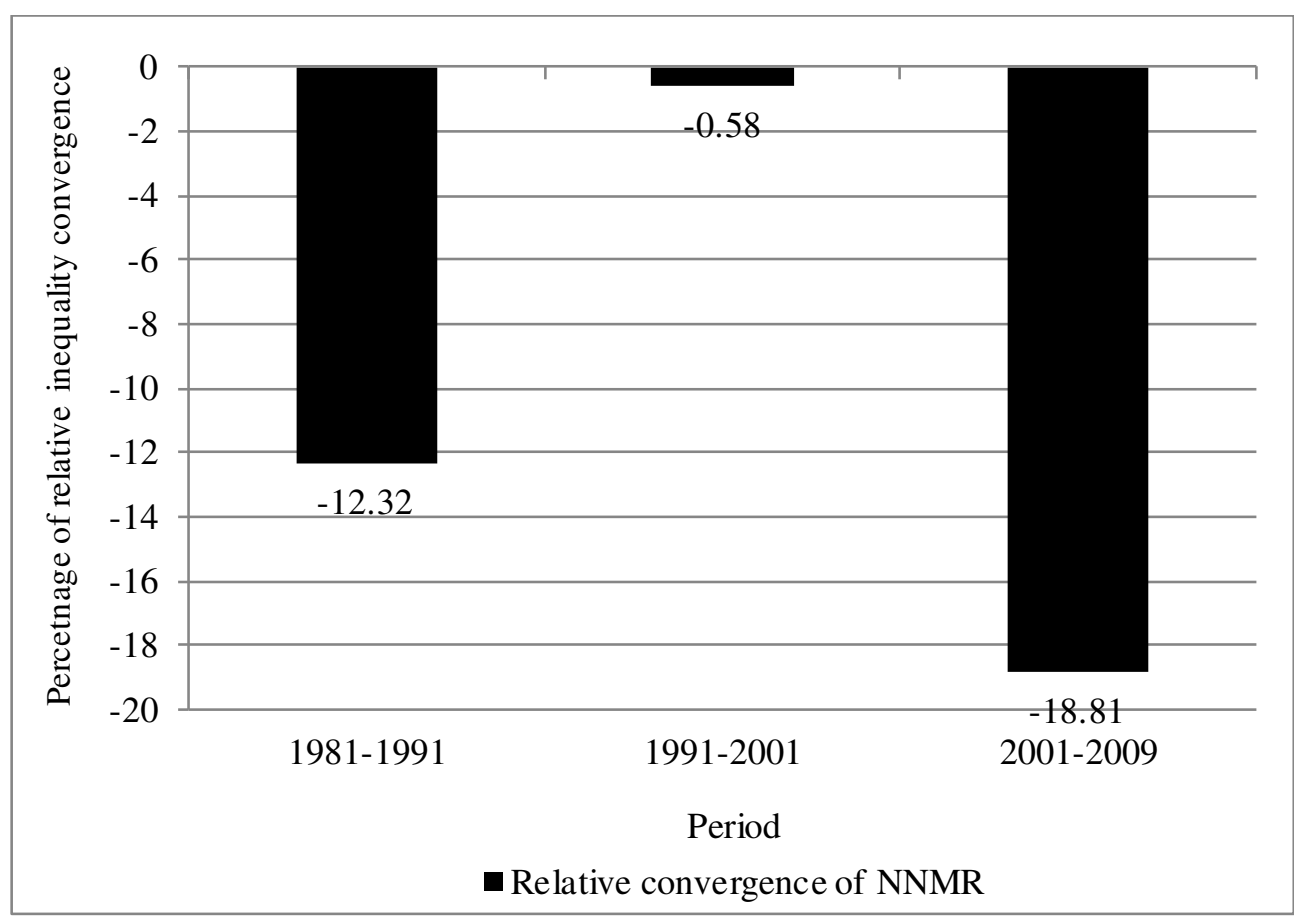


Figure $7.5 \mathrm{~d}$ show the trends of percentage of convergence in relative inequalities of children full immunisation, children underweight and NNMR among the major Indian states by Rural-Urban, 1981-2009. The results in case of children full immunisation in rural areas show divergence $(-14 \%)$ in 1992-1999, but replaced with convergence $(24 \%)$ during 1999-2006. However, results of relative inequality trends in children full immunisation in urban area showed insignificant volume of divergence in the periods, 1992-99, but later in 1999-2006, it was replaced with convergence (30\%). The relative inequality trends in case of children underweight showed overall divergence, but with a contrasting picture for rural and urban areas. In rural areas, the rate of divergence is increasing in the period, 1999-2006 (62\%) compared to earlier period, 1992-1999 (19\%). However, in case of urban areas the rate of divergence was decreased in the period 19992006 (48\%) compared to earlier periods, 1992-1999 (32\%). The results of relative convergence in case of NNMR in rural areas showed convergence until 2001, but earlier convergence was replaced with divergence in post-2001. However, in urban areas, relative convergence in NNMR showed continued divergence and such divergence was increased for the recent decade, 2001-2009.

\subsubsection{Sigma convergence in absolute and relative health inequalities}

The sigma convergence model was used to estimate the convergence in socioeconomic inequality (CIs) for child health indicators. Results indicated convergence trends for all the three indicators during 1992-99, thereafter, except for NNMR, estimates for child immunisation rates and child underweight showed evidence of divergence. However, the divergence was higher for children full immunisation rates (SD rose by 0.04248 points) compared with children underweight (SD rose by 0.00284 points). This indicated that the recent shift to divergence in socioeconomic inequality was more evident in case of child full immunisation rate than for children underweight. Overall, the evidences suggest diverging trend in socioeconomic inequality in child health for recent periods, pointing to dissimilar progress across the states. Therefore, the pattern of evidence suggests that the greater convergence in average health status was not guaranteed with the convergence in socioeconomic inequalities as it was observed in children full immunisation coverage and underweight (Figure 7.6a and b). 
Figure 7.6a. Sigma convergence in socioeconomic inequality (concentration indices) in child health indicators across the major states in India, 1992-2006

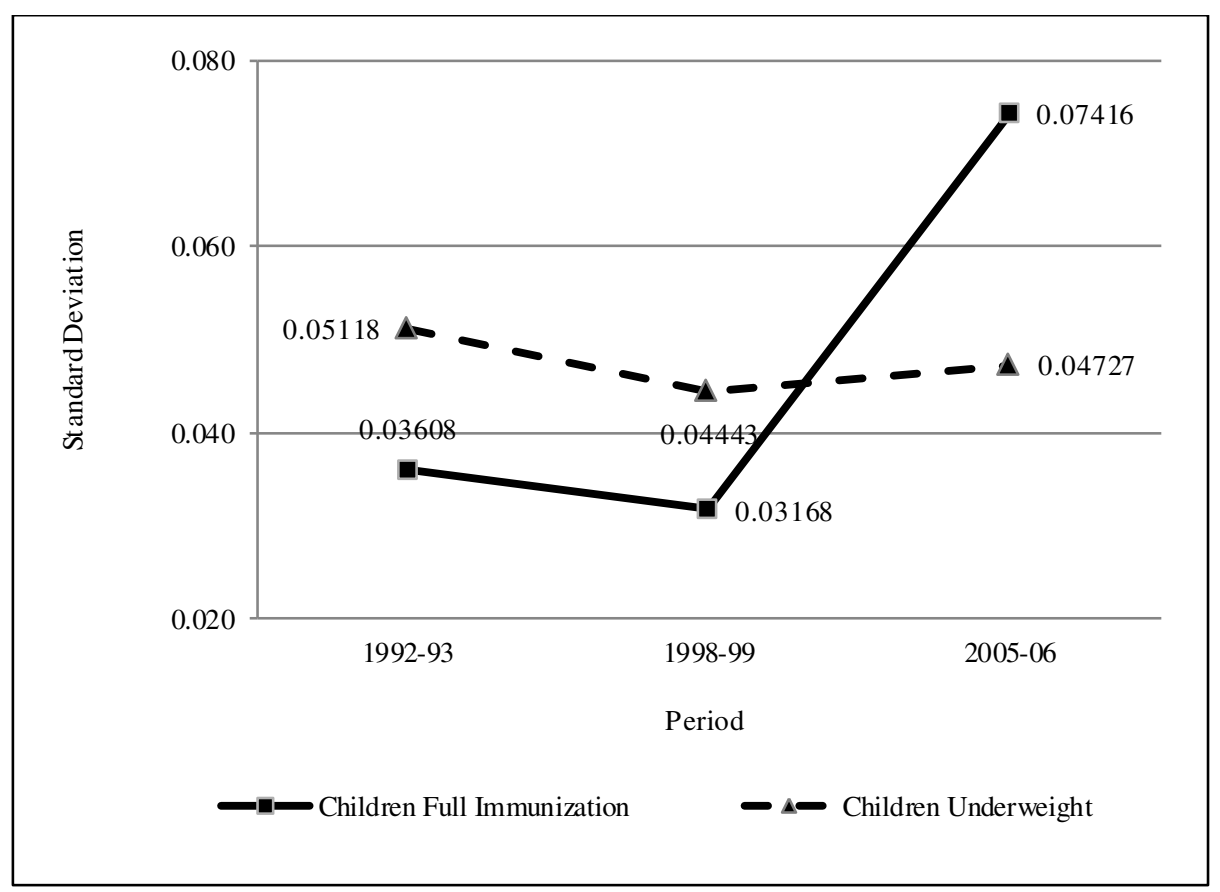

Figure 7.6b. Sigma convergence in socioeconomic inequality (concentration indices) in NNMR indicators across the major states in India,1992-2006

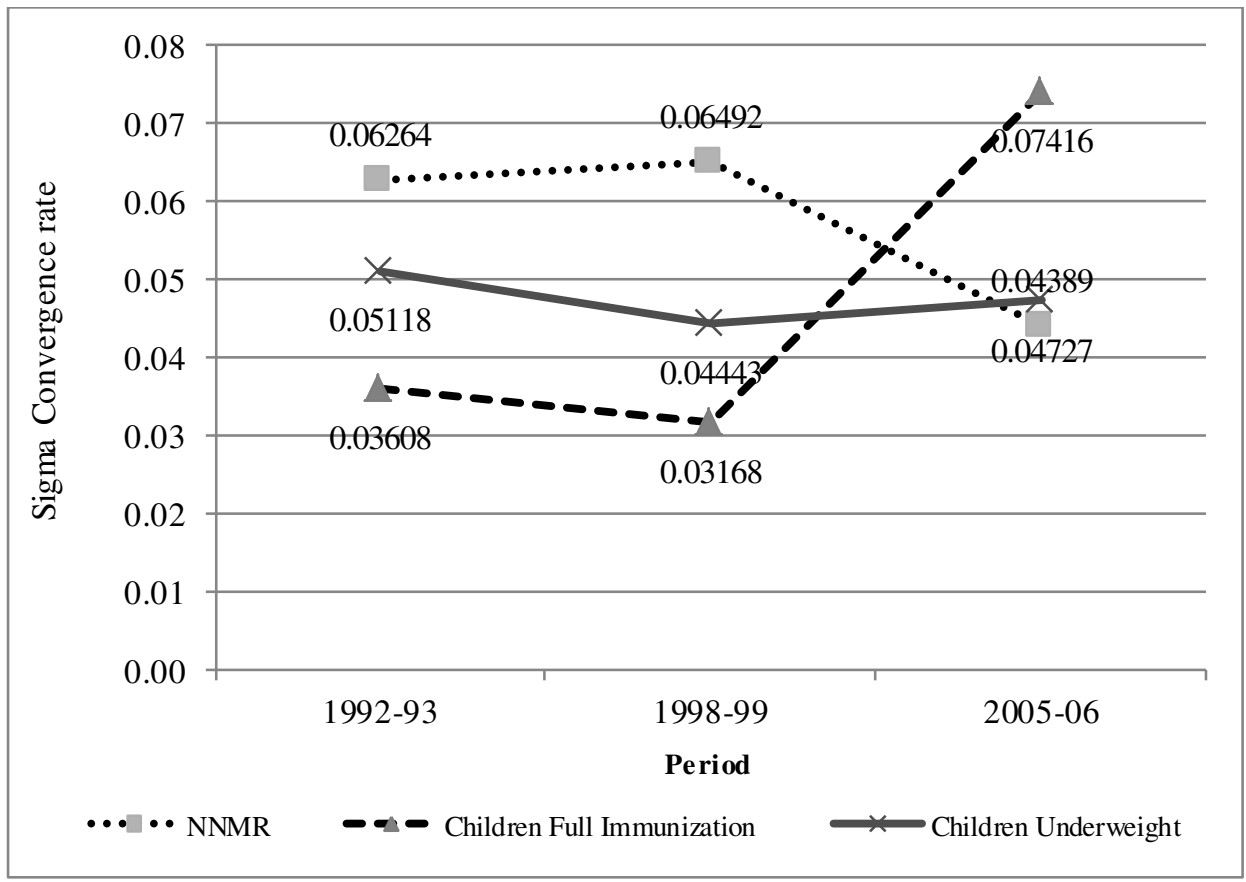




\subsubsection{Convergence clubs in child health utilisation and outcome indicators}

Figure 7.7a presents the changing patterns of children full immunisation clubs among the major states of India, 1992-2006. The results showed that during 1992-93, majority of the states fall under the clubs of children full immunisation levels between 21-40 percent and 41-60 percent. However, there are no states under the club of children full immunisation levels 80 percent and above. The states of Assam, Bihar, Meghalaya, Nagaland and Uttar Pradesh are in club 1 with lowest children full immunisation levels that, less than 20 percent. These states continue to occupy the same group until 1999. However, for the latest period, there are no states in the category of less than 20 percent of children full immunisation. The states of Goa, Himachal Pradesh, Kerala, and Tamil Nadu entered into the club of 80 percent and above of children full immunisation. However, out of these four states only Tamil Nadu survives to be in the same club while negative progress in the other three states made them to lose their place in this club for the period, 2005-06.

Figure 7.7a. Changing patterns of children immunisation clubs among the major states of India, 1992-2006

\begin{tabular}{|c|c|c|c|c|}
\hline Period & $\begin{array}{l}\text { Child } \\
\text { Immunisation } \\
\text { range }\end{array}$ & $1992-93$ & 1998-99 & 2005-06 \\
\hline 官 & Less than 20 & $\begin{array}{l}\text { Assam, Bihar, Meghalaya, } \\
\text { Nagaland, Uttar Pradesh }\end{array}$ & $\begin{array}{c}\text { Assam, Bihar, } \\
\text { Meghalaya, Nagaland, } \\
\text { Rajasthan }\end{array}$ & - \\
\hline 音 & $21-40$ & $\begin{array}{c}\text { Arunanchal Pradesh, } \\
\text { Madhya Pradesh, Manipur, } \\
\text { Orissa, Rajasthan, West } \\
\text { Bengal }\end{array}$ & $\begin{array}{l}\text { Arunanchal Pradesh, } \\
\text { Madhya Pradesh, Uttar } \\
\text { Pradesh }\end{array}$ & $\begin{array}{c}\text { Arunanchal Pradesh, Assam, } \\
\text { Bihar, Madhya Pradesh, } \\
\text { Meghalaya, Nagaland, } \\
\text { Rajasthan, Uttar Pradesh }\end{array}$ \\
\hline 罡 & $41-60$ & $\begin{array}{c}\text { Andhra Pradesh, Delhi, } \\
\text { Gujarat, Haryana, } \\
\text { Karnataka, Kerala, Mizoram }\end{array}$ & $\begin{array}{c}\text { Andhra Pradesh, Gujarat, } \\
\text { Jammu and Kashmir, } \\
\text { Manipur, Mizoram, } \\
\text { Orissa, West Bengal }\end{array}$ & $\begin{array}{l}\text { Andhra Pradesh, Gujarat, } \\
\text { Karnataka, Maharashtra, } \\
\text { Manipur, Mizoram, Orissa, } \\
\text { Punjab }\end{array}$ \\
\hline$\frac{Z}{E}$ & $61-80$ & $\begin{array}{c}\text { Goa, Himachal Pradesh, } \\
\text { Jammu and Kashmir, } \\
\text { Maharashtra, Punjab, Tamil } \\
\text { Nadu }\end{array}$ & $\begin{array}{c}\text { Delhi, Haryana, } \\
\text { Karnataka, Maharashtra, } \\
\text { Punjab }\end{array}$ & $\begin{array}{c}\text { Delhi, Goa, Haryana, } \\
\text { Himachal Pradesh, Jammu } \\
\text { and Kashmir, Kerala, West } \\
\text { Bengal }\end{array}$ \\
\hline$\frac{1}{\text { 主 }}$ & More than 80 & - & $\begin{array}{c}\text { Goa, Himachal Pradesh, } \\
\text { Kerala, Tamil Nadu }\end{array}$ & Tamil Nadu \\
\hline
\end{tabular}


Figure 7.7b Changing patterns of Children underweight clubs among the major states of India, 1992-2006

\begin{tabular}{|c|c|c|c|c|}
\hline Period & $\begin{array}{c}\text { Underweight } \\
\text { range }\end{array}$ & 1992-93 & 1998-99 & $2005-06$ \\
\hline$\frac{\text { ’̇ }}{\bar{z}}$ & $\begin{array}{l}\text { More than } \\
50 \%\end{array}$ & $\begin{array}{l}\text { Bihar, Karnataka, Madhya } \\
\text { Pradesh, Maharashtra, Orissa, } \\
\text { Uttar Pradesh, West Bengal }\end{array}$ & $\begin{array}{c}\text { Bihar, Madhya Pradesh, } \\
\text { Orissa, Rajasthan, Uttar } \\
\text { Pradesh }\end{array}$ & Bihar, Madhya Pradesh \\
\hline$\frac{\text { 三丨 }}{\bar{\Xi}}$ & $41-50$ & $\begin{array}{l}\text { Andhra Pradesh, Assam, Delhi, } \\
\text { Gujarat, Himachal Pradesh, } \\
\text { Jammu and Kashmir, Meghalaya, } \\
\text { Punjab, Rajasthan, Tamil Nadu }\end{array}$ & $\begin{array}{l}\text { Gujarat, Himachal } \\
\text { Pradesh, Karnataka, } \\
\text { Maharashtra, West } \\
\text { Bengal }\end{array}$ & $\begin{array}{l}\text { Gujarat, Meghalaya, } \\
\text { Orissa, Uttar Pradesh }\end{array}$ \\
\hline 总 & $31-40$ & $\begin{array}{c}\text { Arunanchal Pradesh, Goa, } \\
\text { Haryana, }\end{array}$ & $\begin{array}{l}\text { Andhra Pradesh, Assam, } \\
\text { Delhi, Haryana, Jammu } \\
\text { and Kashmir, } \\
\text { Meghalaya, Tamil Nadu }\end{array}$ & $\begin{array}{l}\text { Andhra Pradesh, } \\
\text { Arunanchal Pradesh, } \\
\text { Assam, Haryana, } \\
\text { Himachal Pradesh, } \\
\text { Karnataka, } \\
\text { Maharashtra, } \\
\text { Rajasthan, West } \\
\text { Bengal }\end{array}$ \\
\hline$\frac{\sum^{\prime}}{\vdots}$ & $21-30$ & $\begin{array}{l}\text { Kerala, Manipur, Mizoram, } \\
\text { Nagaland }\end{array}$ & $\begin{array}{l}\text { Arunanchal Pradesh, } \\
\text { Goa, Kerala, Manipur, } \\
\text { Mizoram, Nagaland, } \\
\text { Punjab }\end{array}$ & $\begin{array}{c}\text { Delhi, Goa, Jammu and } \\
\text { Kashmir, Kerala, } \\
\text { Manipur, Nagaland, } \\
\text { Punjab, Tamil Nadu }\end{array}$ \\
\hline 产 & Less than 20 & - & - & Mizoram \\
\hline
\end{tabular}

Changing patterns of children underweight clubs among the major states of India, 19922006 are presented in figure 7.7b. The results showed that for the period, 1992-93, the majority of states falling under club one and two which ranges 41 percent and above of children underweight. However, there were no states in the club of less than 20 percent of children underweight. By 1998-99, the states are distributed heavily in three clubs: two, three and four which ranges children underweight between 21 to 50 percent and share of club one that was 50 percent and above of children underweight was reduced. However, even this year, there was no state with less than 20 percent children underweight. In 200506, the majority of the states were distributed only in two clubs: three and four which ranges between children underweight of 31 to 50 percent and the share of club one (50 and above percent of children underweight) was further reduced and Mizoram was entered into club of less than 20 percent of children underweight. 
Figure 7.7c Changing patterns of neonatal mortality clubs among major states of India, 1981-2009

\begin{tabular}{|c|c|c|c|c|c|}
\hline Period & $\begin{array}{l}\text { NNMIR } \\
\text { range }\end{array}$ & 1981 & 1991 & 2001 & 2009 \\
\hline 完 & $\begin{array}{c}\text { More } \\
\text { than } 80\end{array}$ & $\begin{array}{c}\text { Madhya Pradesh, } \\
\text { Uttar Pradesh }\end{array}$ & - & - & - \\
\hline 音 & $61-80$ & $\begin{array}{c}\text { Assam, Bihar, } \\
\text { Gujarat, Orissa, } \\
\text { Tamil Nadu, West } \\
\text { Bengal }\end{array}$ & $\begin{array}{c}\text { Madhya Pradesh, } \\
\text { Orissa, Uttar Pradesh, } \\
\text { West Bengal }\end{array}$ & - & - \\
\hline 賗 & $41-60$ & $\begin{array}{c}\text { Andhra Pradesh, } \\
\text { Haryana, Himachal } \\
\text { Pradesh, Karnataka, } \\
\text { Maharashtra, } \\
\text { Punjab, Rajasthan }\end{array}$ & $\begin{array}{c}\text { Andhra Pradesh, } \\
\text { Assam, Bihar, Gujarat, } \\
\text { Karnataka, Rajasthan, } \\
\text { Tamil Nadu }\end{array}$ & $\begin{array}{l}\text { Andhra Pradesh, } \\
\text { Assam, Karnataka, } \\
\text { Madhya Pradesh, } \\
\text { Orissa, Rajasthan, } \\
\text { Uttar Pradesh, } \\
\text { West Bengal }\end{array}$ & $\begin{array}{c}\text { Madhya Pradesh, } \\
\text { Orissa, Rajasthan, } \\
\text { Uttar Pradesh }\end{array}$ \\
\hline 亥 & $21-40$ & Kerala & $\begin{array}{c}\text { Haryana, Himachal } \\
\text { Pradesh, Maharashtra, } \\
\text { Punjab }\end{array}$ & $\begin{array}{c}\text { Bihar, Gujarat, } \\
\text { Haryana, Himachal } \\
\text { Pradesh, } \\
\text { Maharashtra, } \\
\text { Punjab, Tamil Nadu }\end{array}$ & $\begin{array}{c}\text { Andhra Pradesh, } \\
\text { Assam, Bihar, } \\
\text { Gujarat, Haryana, } \\
\text { Himachal Pradesh, } \\
\text { Karnataka, } \\
\text { Maharashtra, } \\
\text { Punjab, West } \\
\text { Bengal }\end{array}$ \\
\hline$\frac{\dot{e}}{\Xi}>$ & $\begin{array}{c}\text { Less } \\
\text { than } 20\end{array}$ & - & Kerala & Kerala & Kerala, Tamil Nadu \\
\hline
\end{tabular}

Figure $7.7 \mathrm{c}$ presents the changing patterns of neonatal mortality clubs among the major states of India, 1981-2009. The results revealed that during 1981, Madhya Pradesh, Uttar Pradesh were the only two states in the club-I with NNMR of 80 per 1000 live births and above. The more number of states were in the club -III with NNMR of 40-60 per 1000 live births. However, there were no states under NNMR of less than 20 per 1000 live births in 1981. During 1991 and 2001, Kerala was the only state with NNMR of less than 20. Since 1991, there was no state with NNMR of 80+ per 1000 live births and further, from 2001, even the states with NNMR of 60+ per 1000 live births were also moved out. In 2009, the majority of the states were with NNMR of 21-40 per 1000 live births. The number of states in the club- $\mathrm{V}$ was also increased to two, with the joining of Tamil Nadu along with Kerala. 
Figure 7.7d Changing patterns of Children Immunisation clubs among social groups in major states of India, 1992-2006.

\begin{tabular}{|c|c|c|c|c|}
\hline Period & $\begin{array}{c}\text { Child Immunisation } \\
\text { range }\end{array}$ & $1992-93$ & $1998-99$ & $2005-06$ \\
\hline 㝘 & More than 80 & - & - & $\begin{array}{l}\text { Kerala (Hindu, Others; Richest); } \\
\text { Madhya Pradesh (Richest); Tamil } \\
\text { Nadu (OBC; Others; Middle, Richer); } \\
\text { Bihar (Richest); Haryana (Richest) }\end{array}$ \\
\hline 咅 & $61-80$ & $\begin{array}{c}\text { Haryana (Others); Kerala } \\
\text { (Hindu, Others); Maharashtra } \\
\text { (SC, Others; Hindu, Others); } \\
\text { Punjab (Others; Hindu); } \\
\text { Tamil Nadu (Others; Hindu, } \\
\text { Muslim) }\end{array}$ & $\begin{array}{l}\text { Andhra Pradesh (SC, Other; } \\
\text { Muslim; Richer); Gujarat (Others; } \\
\text { Richer); Haryana (Others; Middle, } \\
\text { Richer); Karnataka (OBC, Others; } \\
\text { Hindu; Richer); Kerala (OBC, } \\
\text { Others; Hindu; Middle, Richer); } \\
\text { Maharashtra (SC, ST,OBC, Others; } \\
\text { Hindu, Muslim, Others; Poorer, } \\
\text { Middle, Richer); Orissa (Richer); } \\
\text { Punjab (OBC, Others; Hindu, } \\
\text { Others; Richer); Tamil Nadu (SC, } \\
\text { OBC; Hindu, Muslim, Others; } \\
\text { Poorer, Middle, Richer); West } \\
\text { Bengal (Richer) }\end{array}$ & $\begin{array}{c}\text { Andhra Pradesh (Richest); Bihar } \\
\text { (Richer); Gujarat (Richest); Haryana } \\
\text { (SC, Others; Hindu; Middle, Richer); } \\
\text { Karnataka (Others; Middle, Richest); } \\
\text { Kerala (OBC, Others; Richer); } \\
\text { Madhya Pradesh (Others; Richer); } \\
\text { Maharashtra (OBC, Others; Richer, } \\
\text { Richest); Orissa (Middle, Richest); } \\
\text { Punjab (OBC, Others; Richest); Tamil } \\
\text { Nadu (SC; Hindu; Poorer, Richest); } \\
\text { West Bengal (Others; Hindu; Poorer, } \\
\text { Middle, Richer, Richest) }\end{array}$ \\
\hline
\end{tabular}




\begin{tabular}{|c|c|c|c|c|}
\hline $\begin{array}{l}\text { 章 } \\
\text { 亲 }\end{array}$ & $41-60$ & $\begin{array}{l}\text { Andhra Pradesh (Other; } \\
\text { Hindu, Muslims); Gujarat } \\
\text { (Others; Hindu, Muslim); } \\
\text { Haryana (SC, Others; Hindu); } \\
\text { Karnataka (SC, Others; } \\
\text { Hindu, Muslim); Kerala } \\
\text { (Muslim); Madhya Pradesh } \\
\text { (Muslim); Maharashtra (ST; } \\
\text { Muslim); Punjab (SC, } \\
\text { Others); Tamil Nadu (SC; } \\
\text { Others) }\end{array}$ & $\begin{array}{l}\text { Andhra Pradesh (OBC; Hindu, } \\
\text { Others; Poorer, Middle); Gujarat } \\
\text { (SC, ST, OBC; Hindu, Muslim; } \\
\text { Middle); Haryana (SC, OBC); } \\
\text { Karnataka (SC; Muslim; Poorer, } \\
\text { Middle); Kerala (Muslim; Poorer); } \\
\text { Madhya Pradesh (Muslim; Richer); } \\
\text { Orissa (SC, OBC, Others; Middle); } \\
\text { Punjab (SC; Middle); Rajasthan } \\
\text { (Richer); West Bengal (SC; Hindu; } \\
\text { Poorer, Middle) }\end{array}$ & $\begin{array}{l}\text { Andhra Pradesh (SC, OBC, Others; } \\
\text { Hindu, Muslim; Poorest, Middle, } \\
\text { Richer); Assam(OBC; Middle, } \\
\text { Richer); Gujarat (SC, OBC, Others; } \\
\text { Hindu; Richer); Haryana (OBC; } \\
\text { Poorer); Karnataka (SC, OBC; Hindu, } \\
\text { Muslim; Richer); Kerala (Muslim); } \\
\text { Madhya Pradesh (SC, OBC; Muslim; } \\
\text { Middle); Maharashtra (SC; Hindu, } \\
\text { Muslim, Others; Middle); Orissa (SC, } \\
\text { OBC, Others; Poorer, Richer); Punjab } \\
\text { (SC; Hindu, Others; Richer); } \\
\text { Rajasthan (Richest); Uttar Pradesh } \\
\text { (Richest); West Bengal (SC; Muslim; } \\
\text { Poorest) }\end{array}$ \\
\hline 豙 & $21-40$ & $\begin{array}{c}\text { Andhra Pradesh (SC, ST); } \\
\text { Assam (Hindu); Gujarat } \\
\text { (SC); Madhya Pradesh (SC, } \\
\text { Others; Hindu); Orissa (SC, } \\
\text { ST, Others); Rajasthan } \\
\text { (Others; Hindu); Uttar } \\
\text { Pradesh (Others; Hindu); } \\
\text { West Bengal (SC, ST, Others; } \\
\text { Hindu, Muslim) }\end{array}$ & $\begin{array}{c}\text { Assam (Hindu; Richer); Bihar } \\
\text { (Richer); Gujarat (Poorer); } \\
\text { Haryana (Poorer); Karnataka (ST); } \\
\text { Madhya Pradesh (OBC, Others; } \\
\text { Middle); Orissa (ST, Poorer), } \\
\text { Rajasthan (Others); Uttar Pradesh } \\
\text { (SC, Others; Hindu; Middle, } \\
\text { Richer); West Bengal (Others; } \\
\text { Muslim) }\end{array}$ & $\begin{array}{l}\text { Andhra Pradesh (ST; Poorer); Assam } \\
\text { (SC, ST, Others; Hindu; Poorer); } \\
\text { Bihar (SC, OBC, Others; Hindu; } \\
\text { Poorer, Middle); Gujarat (ST; } \\
\text { Muslim; Poorer, Middle); Karnataka } \\
\text { (ST; Poorest, Poorer); Madhya } \\
\text { Pradesh (ST; Hindu; Poorest, Poorer); } \\
\text { Maharashtra (ST; Poorest, Poorer); } \\
\text { Orissa (ST; Poorest); Punjab } \\
\text { (Middle); Rajasthan (SC, OBC, } \\
\text { Others; Hindu; Middle, Richer); Uttar } \\
\text { Pradesh (OBC, Others; Hindu; } \\
\text { Middle, Richer) }\end{array}$ \\
\hline 訔 & Less than 20 & $\begin{array}{c}\text { Assam (ST, Others; Muslim); } \\
\text { Bihar (SC, ST, Others; } \\
\text { Hindu, Muslim); Madhya } \\
\text { Pradesh (ST); Rajasthan (SC, } \\
\text { ST; Muslim); Uttar Pradesh } \\
\text { (SC, ST; Muslim) }\end{array}$ & $\begin{array}{l}\text { Assam(SC, ST, Others; Poorer, } \\
\text { Middle); Bihar (SC, ST, OBC, } \\
\text { Others; Hindu, Muslim; Poorer, } \\
\text { Middle); Madhya Pradesh (SC, ST; } \\
\text { Hindu; Poorer); Rajasthan (SC, ST, } \\
\text { OBC; Hindu, Muslim; Poorer, } \\
\text { Middle); Uttar Pradesh (ST, OBC; } \\
\text { Muslim; Poorer) }\end{array}$ & $\begin{array}{l}\text { Assam (Muslim; Poorest); Bihar } \\
\text { (Muslim, Others; Poorest); Gujarat } \\
\text { (Poorest); Haryana (Muslim, Others; } \\
\text { Poorest); Karnataka (Others); } \\
\text { Rajasthan (ST; Muslim, Others; } \\
\text { Poorest, Poorer); Uttar Pradesh (SC; } \\
\text { Muslim; Poorest, Poorer) }\end{array}$ \\
\hline
\end{tabular}


Figure 7.7e Changing patterns of Children Underweight clubs among social groups in major states of India, 1992-2006.

\begin{tabular}{|c|c|c|c|c|}
\hline Period & $\begin{array}{l}\text { Underweight } \\
\text { range }\end{array}$ & 1992-93 & 1998-99 & $2005-06$ \\
\hline 它 & More than $50 \%$ & $\begin{array}{l}\text { Andhra Pradesh (SC, ST; Others); } \\
\text { Madhya Pradesh (SC, ST, Others; Hindu, } \\
\text { Muslim, Others); Tamil Nadu (SC); West } \\
\text { Bengal (SC, ST, Others; Hindu, Muslim) }\end{array}$ & - & $\begin{array}{c}\text { Andhra Pradesh (Poorest, Poorer); Bihar } \\
\text { (Poorest, Poorer, Middle); Gujarat (SC, ST, } \\
\text { OBC; Hindu; Poorest, Poorer, Middle, } \\
\text { Richer }\end{array}$ \\
\hline 咅 & $41-50$ & $\begin{array}{l}\text { Andhra Pradesh (Others; Hindu, Muslim); } \\
\text { Tamil Nadu (Others; Hindu, Muslim, } \\
\text { Others); West Bengal (Others) }\end{array}$ & - & $\begin{array}{l}\text { Andhra Pradesh (Middle); Bihar (Richer); } \\
\text { Gujarat (Muslim); Madhya Pradesh (ST) }\end{array}$ \\
\hline 百 & $31-40$ & - & $\begin{array}{c}\text { Bihar (ST); Maharashtra }(\mathrm{ST}) ; \\
\text { Orissa }(\mathrm{ST})\end{array}$ & $\begin{array}{c}\text { Andhra Pradesh (Richer); Bihar (SC); } \\
\text { Gujarat (Others; Richest); Haryana (Muslim; } \\
\text { Poorest); Madhya Pradesh (SC, OBC, } \\
\text { Others; Hindu, Muslim; Poorest, Poorer, } \\
\text { Middle), Tamil Nadu (Poorer); Uttar Pradesh } \\
\text { (ST) }\end{array}$ \\
\hline 亥 & $21-30$ & $\begin{array}{l}\text { Bihar (SC, ST, Others; Hindu, Muslim); } \\
\text { Gujarat (ST); Karnataka (ST); Kerala } \\
\text { (ST); Maharashtra (SC, ST; Hindu); } \\
\text { Orissa (SC, ST; Hindu, Others); Punjab } \\
\text { (SC; Others); Rajasthan (ST) }\end{array}$ & $\begin{array}{l}\text { Bihar (SC; Hindu, Muslim; Poorer); } \\
\text { Gujarat (ST; Poorer); Karnataka } \\
\text { (SC, ST, Others; Muslim; Poorer); } \\
\text { Madhya Pradesh (ST; Poorer, } \\
\text { Middle); Maharashtra (OBC, } \\
\text { Others; Hindu; Poorer); Oris sa (SC, } \\
\text { OBC; Hindu, Others; Poorer); Tamil } \\
\text { Nadu (SC; Muslim; Poorer) }\end{array}$ & $\begin{array}{l}\text { Andhra Pradesh (Richest); Bihar (OBC, } \\
\text { Others; Hindu, Muslim; Richest); Haryana } \\
\text { (SC, OBC; Poorer); Karnataka (Poorest); } \\
\text { Kerala (Middle); Madhya Pradesh (Richer, } \\
\text { Richest); Maharashtra (Poorer); Orissa (ST, } \\
\text { Others); Rajasthan (SC, ST, Others; Hindu, } \\
\text { Muslim; Poorest); Tamil Nadu (SC, OBC; } \\
\text { Hindu, Muslim; Poorest, Middle); West } \\
\text { Bengal (ST) }\end{array}$ \\
\hline
\end{tabular}



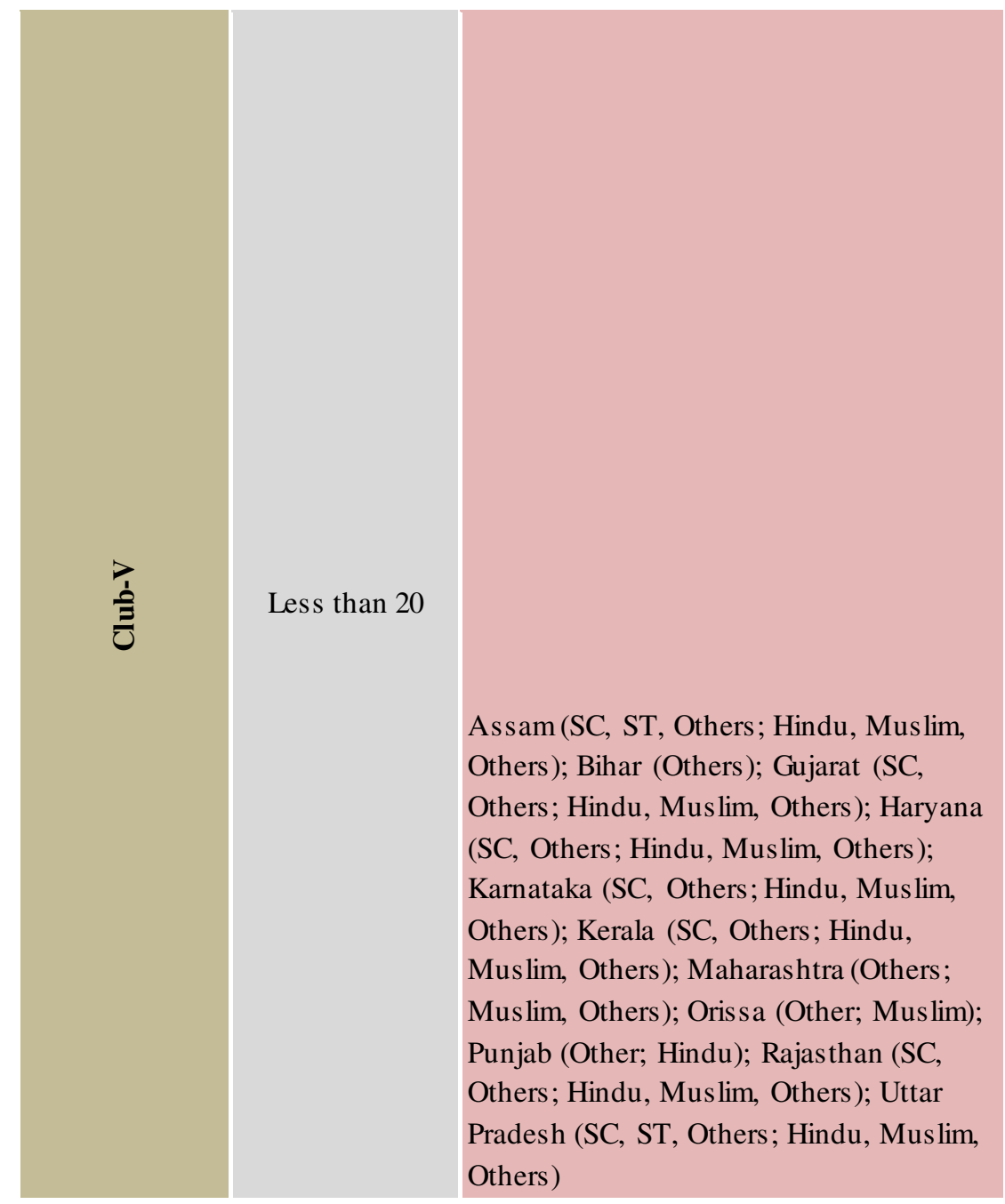

Andhra Pradesh (SC, ST, OBC, Others; Hindu, Muslims, Others Poorer, Middle, Richer); Assam (SC, ST, Others; Hindu, Muslims; Poorer, Middle, Richer); Bihar (OBC, Others; Middle, Richer); Gujarat (SC, OBC, Others; Hindu, Muslim; Middle, Richer); Haryana (SC, OBC, Others; Hindu, Muslim, Others; Poorer, Middle, Richer); Karnataka (OBC; Hindu; Middle, Richer); Kerala (SC, OBC, Others; Hindu, Muslim, Others; Poorer, Middle, Richer); Madhya Pradesh (SC,OBC, Others; Richer); Maharashtra (SC; Muslim, Others; Middle, Richer); Orissa (Others; Muslim; Middle, Richer); Punjab (SC, OBC, Others; Hindu, Muslim, Others; Poorer, Middle, Richer); Rajasthan (SC, ST, OBC, Others; Hindu, Muslim; Poorer, Middle, Richer); Tamil Nadu (OBC; Hindu, Others; Middle, Richer); Uttar

Pradesh (SC, ST, Others; Hindu, Muslim; Poorer, Middle, Richer); West Bengal (SC, ST, OBC, Others Hindu, Muslim; Poorer, Middle. Richer)

Andhra Pradesh (SC, ST, OBC, Others; Hindu, Muslims, Others); Assam (SC, ST, OBC, Others; Hindu, Muslims, Others; Poorer, Middle, Richer, Richest); Haryana (Others; Hindu, Others; Middle, Richer, Richest); Karnataka (SC, ST, OBC, Others; Hindu, Muslim, Others; Poorer, Middle, Richer, Richest); Kerala (SC, ST, OBC, Others; Hindu, Muslim, Others; Poorest, Poorer, Richer, Richest); Madhya Pradesh (Others); Maharashtra (SC, ST, OBC,

Others; Hindu, Muslim, Others; Poorest, Middle, Richer, Richest); Orissa (SC, OBC, Others; Hindu, Muslim; Poorer, Middle, Richer, Richest); Punjab (SC, OBC, Others; Hindu, Muslim, Others; Poorest, Poorer, Middle, Richer, Richest); Rajasthan (OBC; Others; Poorer, Middle, Richer, Richest); Tamil Nadu (Others; Others; Richest, Richer); Uttar Pradesh (SC, OBC, Others; Hindu, Muslim; Poorer, Middle, Richer, Richest); West Bengal (SC, OBC, Others; Hindu, Muslim; Poorer, Middle, Richer, Richest) 
Figure 7.7f Changing patterns of neonatal mortality clubs among social groups in major states of India, 1992-2006

\begin{tabular}{|c|c|c|c|c|}
\hline Period & $\begin{array}{c}\text { Neo natal } \\
\text { mortality } \\
\text { range }\end{array}$ & $1992-93$ & 1998-99 & 2005-06 \\
\hline 完 & More than 80 & Uttar Pradesh (ST); West Bengal (ST) & - & - \\
\hline 音 & $61-80$ & $\begin{array}{c}\text { Andhra Pradesh (SC); Assam } \\
\text { (Muslim); Bihar (SC, Muslim); Gujarat } \\
\text { (ST); Karnataka(SC, ST); Madhya } \\
\text { Pradesh (SC); Maharashtra (SC); } \\
\text { Orissa (SC, Other); Uttar Pradesh (SC, } \\
\text { Other; Hindu); West Bengal (SC) }\end{array}$ & $\begin{array}{l}\text { Andhra Pradesh (SC, Poorer); } \\
\text { Karnataka (ST, Poorer); Madhya } \\
\text { Pradesh (SC, ST, Hindu, Poorer, } \\
\text { Middle); Orissa (OBC); Rajasthan } \\
\text { (Poorer); Uttar Pradesh (SC, OBC, } \\
\text { Hindu, Poorer, Middle) }\end{array}$ & $\begin{array}{l}\text { Andhra Pradesh (SC, ST, Poorer, } \\
\text { Middle); Assam (SC, Poorest); } \\
\text { Gujarat (Poorest); Madhya Pradesh } \\
\text { (Poorer); Maharashtra (Poorest); } \\
\text { Rajasthan (SC); Uttar Pradesh } \\
\text { (Poorest) }\end{array}$ \\
\hline 䍗 & $41-60$ & $\begin{array}{l}\text { Andhra Pradesh (ST, Other; Hindu); } \\
\text { Assam (ST, Other; Hindu); Bihar (ST, } \\
\text { Others; Hindu); Gujarat (Others; } \\
\text { Hindu); Haryana (SC, Others; Hindu); } \\
\text { Karnataka (Others; Hindu, Muslim); } \\
\text { Madhya Pradesh (ST, Others; Hindu); } \\
\text { Orissa (ST); Rajasthan (SC, ST; } \\
\text { Hindu); Tamil Nadu (SC, Others); } \\
\text { Uttar Pradesh (Muslim); West Bengal } \\
\text { (Others; Hindu, Muslim) }\end{array}$ & $\begin{array}{l}\text { Andhra Pradesh (ST, OBC; Hindu); } \\
\text { Assam(Other); Bihar (SC, ST, } \\
\text { OBC; Hindu, Muslim; Poorer); } \\
\text { Gujarat (SC, OBC; Hindu; Poorer, } \\
\text { Middle); Haryana (Poorer); } \\
\text { Karnataka (SC, OBC; Hindu); } \\
\text { Madhya Pradesh (OBC, Others; } \\
\text { Muslim); Maharashtra (ST; Hindu, } \\
\text { Others; Poorer); Orissa (SC, ST, } \\
\text { Others; Poorer, Middle); Punjab } \\
\text { (SC; Middle); Rajasthan (SC, ST, } \\
\text { OBC, Others; Hindu, Muslim; } \\
\text { Middle), Tamil Nadu (Poorer); } \\
\text { Uttar Pradesh (ST, Others; Muslim; } \\
\text { Richer); West Bengal (ST) }\end{array}$ & $\begin{array}{l}\text { Andhra Pradesh (OBC, Others; Hindu, } \\
\text { Muslim; Poorest); Assam (ST, Others; } \\
\text { Hindu, Muslim; Poorer, Middle, } \\
\text { Richer); Bihar (SC, Others; Hindu, } \\
\text { Muslim; Poorest, Richest); Gujarat } \\
\text { (SC, ST, OBC; Hindu; Poorer, } \\
\text { Middle); Haryana (Poorer); Karnataka } \\
\text { (SC; Poorest, Poorer); Madhya } \\
\text { Pradesh (SC, ST, OBC; Hindu; } \\
\text { Poorest); Maharashtra (Others; } \\
\text { Middle); Orissa (SC, ST, OBC; } \\
\text { Poorest, Poorer, Richer); Rajasthan } \\
\text { (OBC, Others; Hindu; Poorest, } \\
\text { Middle, Richer); Uttar Pradesh (SC, } \\
\text { OBC; Hindu, Muslim; Poorer, Middle, } \\
\text { Richer); West Bengal (Others; } \\
\text { Muslim; Middle, Richer) }\end{array}$ \\
\hline
\end{tabular}




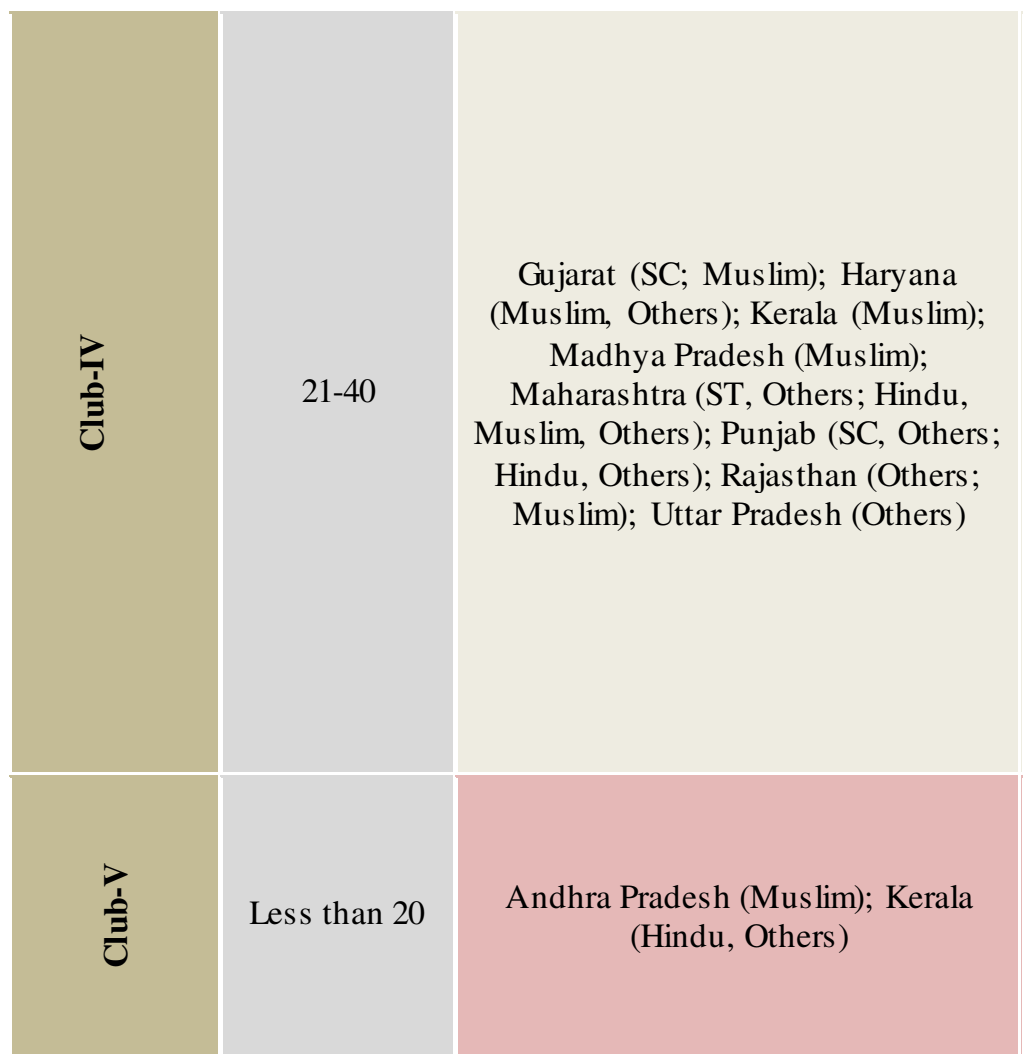

Andhra Pradesh (Others; Muslim; Middle, Richer); Assam (SC, ST, OBC; Hindu, Muslim; Poorer,

Middle, Richer); Bihar (Others; Middle, Richer); Gujarat (ST,

Others; Muslims; Richer); Haryana (SC, OBC, Other; Hindu; Middle,

Richer); Karnataka (Others;

Muslim; Middle, Richer); Kerala (Poorer); Madhya Pradesh (Richer); Maharashtra (SC, OBC, Others; Middle, Richer); Punjab (OBC

Others; Hindu, Others;

Rajasthan (Richer); Tamil Nadu (SC, OBC; Hindu, Muslim; Middle,

Richer); West Bengal (SC, Others;

Hindu, Muslim; Poorer, Middle)

Haryana (Muslim, Other); Kerala (OBC, Others; Hindu, Muslim,

Others; Middle, Richer);

Maharashtra (Muslim); Orissa

(Richer); West Bengal (Richer)
Andhra Pradesh (Richer, Richest); Assam (OBC); Bihar (OBC; Poorer, Middle, Richer); Gujarat (Others; Muslim; Richer, Richest); Haryana (SC, Others; Hindu, Muslim; Richer); Karnataka (ST, OBC, Others; Hindu, Muslim; Middle, Richer); Kerala (Middle); Madhya Pradesh (Others; Muslim; Middle, Richer, Richest); Maharashtra (SC, ST, OBC, Others; Hindu, Muslim; Poorer, Richer, Richest); Orissa (Others; Middle, Richest); Punjab (SC, Others; Hindu,

Others; Middle, Richer, Richest); Rajasthan (ST; Muslim, Poorer, Richest); Tamil Nadu (SC, OBC;

Hindu; Poorest, Poorer, Middle); Uttar Pradesh (Others; Richest); West Bengal (SC; Hindu; Poorest, Poorer) Assam (Richest); Haryana (OBC; Middle, Richest); Karnataka (Others; Richest); Kerala (OBC, Others; Hindu, Muslim, Others; Richer, Richest); Tamil Nadu (Others; Richer, Richest); West Bengal (Richest) 
Changing patterns of children immunisation clubs among social groups of the major states of India, 1992-2006 are presented in figure 7.7d. The results revealed that in 1992-93, no social group across the major states were in the club-I with 80+ percent of children full immunisation and the majority of the social groups were located in the club-III and IV with 20 to 60 percent children full immunisation levels. Also, during 1998-99, no states were in the club-I with $80+$ percent of children full immunisation and the majority of the social groups were located in the club-II and III with 20 to 60 percent children full immunisation levels. However, the number of social groups in the club-V showed an increase which indicates that there was a negative growth in children full immunisation in many social groups. In 2005-06, social groups such as Kerala (Hindu, Others; Richest); Madhya Pradesh (Richest); Tamil Nadu (OBC, Others, Middle, Richer); Bihar (Richest ${ }^{8}$ ); Haryana (Richest) has entered into $80+$ children full immunisation level. However, the number of social groups with children full immunisation level less than 20 percent was found to be more than that observed in 1992-93.

Figure 7.7e presents the changing pattern of children underweight clubs among social groups in major states of India, 1992-2006. The results in case of children underweight were not showed a clear pattern of change. In 1992-93, while the majority of the states were located in Club IV and V with children underweight of 21-30 percent and less than 20 percent respectively, but some states were also located in the clubs of children underweight 50 percent and more. In 1998-99, there were no states in the club I with children underweight 50 percent and more and a majority of the states during period were located in the Club V with children underweight less than 20 percent. In 2005-06, trends were reversed. Again, there were few states which re-entered into Club-I with children underweight more than 50 percent. However, the majority of the states were still located in the Club V with children underweight less than 20 percent.

Changing pattern of neonatal mortality clubs among the major states of India, 19812009 are presented in figure 7.7f. In 1992-93, the greater number of social groups was located in the club III with NNMR of 41-60 per 1000 live births. However, a few social groups were also located in the club-I with NNMR 80 per 1000 live births and above

8 Estimate based on 25-45 unweighted cases 
[Uttar Pradesh (ST); West Bengal (ST)] and NNMR less 20 [Andhra Pradesh (Muslim); Kerala (Hindu, Others)]. During 1998-99, there was no social group in the club of NNMR 80 and above. The greater number of social groups located in the Club-IV with NNMR of 21-40 and the number of social groups in Club-V was increased. In the latest period, 2005-06, there was no social group in the club of NNMR 80 and above. The number of social groups located in the Club-IV with NNMR of 21-40 and the number of social groups in Club-V was further increased. However, still it is noticed that a substantial number of social groups are located in the club-II and III with NNMR of more than 40-60.

Figure.7.8. Trends in Kernel density plots for children full immunisation (CIM), children underweight $(\mathrm{CU})$ and neonatal mortality rate (NNMR)

CIM_1992-93

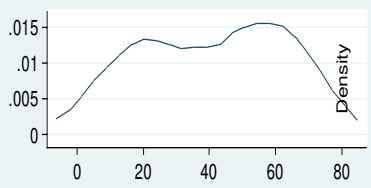

kernel $=$ epanechnikov, bandwidth $=9.95$

CU_1992-93
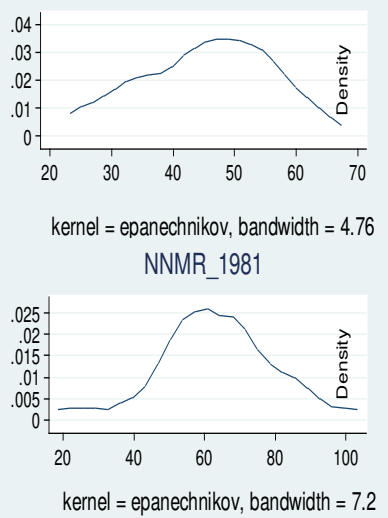

NNMR_2009

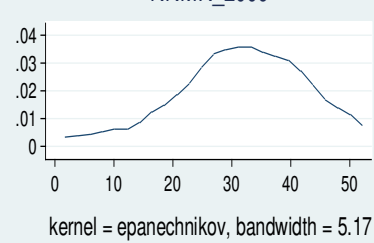

CIM_1998-99

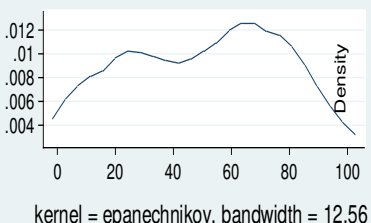

CU 1998-99
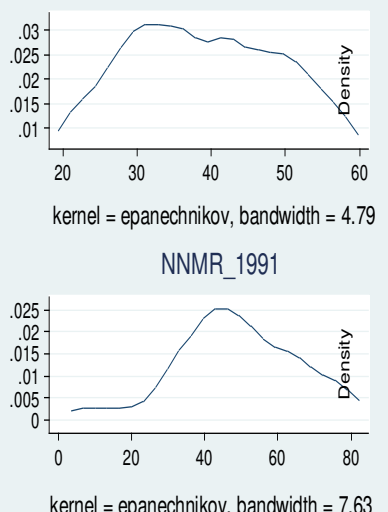

kernel $=$ epanechnikov, bandwidth $=7.63$
CIM_2005-06

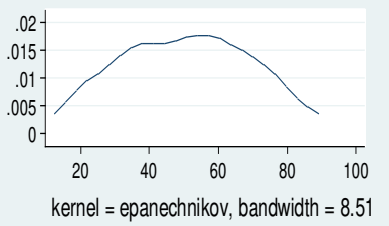

CU_2005-06

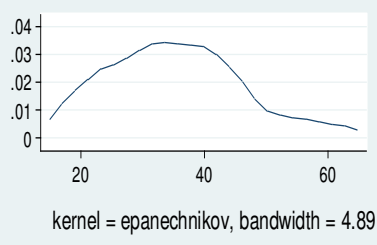

NNMR 2001

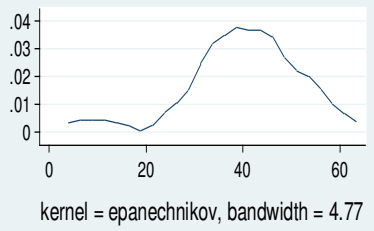

Another method used for determining the distribution of child health indicators across the states in terms of convergence clubs is shown in Fig.7.8. The figure shows the trends in Kernel density plots for children full immunisation (CIM), children underweight (CU) and neonatal mortality rate (NNMR). Trends in kernel density distribution plots for children full immunisation indicated a bi-modal distribution in 
1992-93 and multi-modal distribution in 1998-99, but a unimodal distribution in 200506 with a slight uneven surface. Thus, the results indicate evidence for convergence clubs in 1992-93 and 1998-99, but not absolute convergence, however, emerging absolute convergence in 2005-06. The results for children underweight show a reverse trend. In 1992-93, the kernel density distribution shows the unimodal distribution, but it was replaced with bi-modal distribution in 1998-99 and 2005-06. This evident that earlier convergence was replaced with convergence clubs in children underweight. The kernel density estimates for NNMR showed a unimodal distribution in 1981, which was replaced with multi-modal distribution in 1991 and bi-modal distribution 2001. However, an emerging unimodal distribution of NNMR was evident, though there was no evidence of absolute convergence of the distribution.

\subsection{Discussion}

Akin to fertility and mortality chapters, in this chapter also I have used both standard and inequality based convergence metrics to test the hypothesis of convergence in both averages and relative distribution of child health in India focusing on major states and socioeconomic groups for three key health indicators. The results of trend analyses for health averages and the inequalities of selected indicators suggested varying patterns. The gap across the states in average health status in terms of NNMR and full immunisation of children is closing. However, the gap remains the same for children underweight and showed a setback in convergence trend for children underweight in the recent period, 1998-2006. The trends in DMM and Gini coefficient suggested a decline in both absolute and relative dispersion initially, but indicated a reversal for the recent period, 2001-2011. The trends in socioeconomic inequalities in child health indicators suggested an increase in inequality in two out of three child health indicators.

A range of convergence metrics tested for child health averages and inequalities in this chapter revealed varying patterns of convergence by type of measure and time periods. The results indicate convergence in their averages, but with decreasing volume and speed of convergence for the recent period. The convergence in averages was principally driven by greater progress in laggard states and resultant catching-up process. However, the results indicated evidence of some recent setback in convergence in absolute and relative child health inequalities in children underweight, 
socioeconomic inequalities in children full immunisation and underweight, and relative inequalities in NNMR. From the late 1990s, results suggest that progress in converging trend in child health inequalities was being replaced by diverging trend. These anomalies may arise because of many reasons but here, I point out few important reasons. First, the divergence in children underweight for recent periods is more likely to be the result of unequal rate of progress in reduction of adult mortality across the states for the recent periods. Second, India has adopted globalization, liberalisation and privatisation policies since 1991, and different states of India benefited differently from these policies. Such differences may have led to increase in the relative income inequality across states and socioeconomic groups in the post 1990s and consequently widened the gaps in two out of three child health indicators. Third, explanation can also drawn from previous studies, for instance, Wagstaff (2002) provided alternative reasons for such situation: 'increasing per capita income and health averages also lead to increase in the relative dispersion of health across the different population of the developing countries' as India current experience suggests. Fourth, the difference in both absolute and relative convergence processes across the indicators can be attributed to the nature of the indicators and factors affecting them. For example, two process indicators: children full immunisation and children underweight are very much sensitive to fluctuation in state specific socioeconomic conditions, conundrums in health care policies and programmes and their implementation strategies. India is a country of huge geographic, socioeconomic and cultural diversity, therefore, state specific policies play a critical role in intra-state disparity between individual of various backgrounds. Hence, improving states average health status may not always result into convergence in relative health status. 


\section{CHAPTER 8 \\ Linkage of Demographic convergence and Child health inequalities}

\subsection{Introduction}

In the earlier chapters, this study made an attempt to quantify convergence for various demographic indicators and the results reveal that there is no evidence of absolute convergence in economic indicators, but there is an emerging convergence or decline in divergence in fertility indicators. Among mortality indicators, convergence during earlier phase is being replaced with divergence for recent decades (Chapter 3-6). Convergence estimates for child health indicators point to a divergence in their progress across the states and socioeconomic groups (Chapter 7). In general, as far as convergence estimates are concerned, there is evidence of decline in divergence of progress in fertility indicators and continued divergence in other demographic indicators. Further, it would be more interesting to see the implications of convergence/divergence of progress in socioeconomic and demographic indicators on child health inequalities. More specifically, the question needs to be investigated is whether, there is any noticeable linkage between socioeconomic, demographic progress and child health inequalities. Specially, how children's health is faring in the process of fertility decline and convergence across the states and socioeconomic groups.

Such an investigation is important because until recently, in both developed and developing countries, the literature on population and health subjects is focused on averages. Moreover, it becomes clear from the recent evidences that, the challenge for current health policy is to reduce inequality and not just to improve the average health or lengthen the life span. Child health inequalities have recently started to receive a good deal of attention in the developing world, where, inequalities are wide and increasing, thus, making more difficult to achieve the MDG-4 (Goland et al., 2012; Goli and Arokiasamy, 2014). A growing number of previous studies specifically focused on questions that, how large are the inequalities in health? And, how large are the health differences across the countries? What are the factors determining or contributing to it? (Wagstaff and Doorslaer, 1991; Kakwani et al., 1997; Gwatkin, 
2000; Wagstaff, 2002a, 2002b; Mormot, 2002; Mackenbach, 2003; van Doorslaer et al., 2004; Subramanian and Kawachi, 2004; Hosseinpoor et al., 2006; Pradhan and Arokiasamy, 2010; Marmot, 2005, 2010; Po and Subramanian, 2011; Goland et al., 2012; van Bon-Martern et al., 2012; Arokiasamy et al., 2012). Yet, a largely ignored part is that in many situations, efforts to achieve the health targets may increase inequalities with the better-off in a society benefitting disproportionately (Wagstaff et al., 2003; Goli and Arokiasamy, 2014). The understanding of the problem 'how the efforts to achieve the MDG-4 targets are resulting in an increase in inequalities in child health' is important. Such efforts direct us to understand three issues: 1 . What is the relationship between economic growth and child health inequalities? 2. What is the relationship between increases in social status and child health inequalities? 3 . What is the relationship between increases in child health averages and child health inequalities? These research questions have been discussed in detail below.

First question: What is the relationship between economic growth and child health inequalities? Economic growth has often been a powerful force for improved health status. Economic group differences in health are widely discussed subject in population and public health subjects. The previous empirical research has established a strong connection between 'international income inequality and inequality in health'. The studies have shown that common indicators of population health such as life expectancy and infant mortality are strongly correlated with average income levels, such that richer countries enjoy better health (Preston, 1975; Deaton, 2004; Subramanian et al., 2006, 2009; Harper and Lynch, 2007; Van de Poel et al., 2008; Subramanian, 2008; Speybroeck et al., 2009; Konda et al., 2009; Po and Subramanian, 2010). Also, the evidence from trends in health inequalities - in both the developing and developed world - supports the notion that health inequalities rise with rising per capita income. Moreover, many theories of development also predict a strong connection between international income inequality and inequality in health, reasoning that gains in population health often stem from the technological advances and improved nutrition associated with national income growth (e.g., Ram, 1982, 1992; Pritchett and Summers, 1996; Easterlin, 1996; Easterlin, 2004). The connection between health inequality and per capita income is probably due in part to technological change going hand-in-hand with economic development, paired with a propensity for the more well-off to take up new technology and innovation ahead of the poor. In other 
words, it is just that richer states absorb new health technology and purchasing power faster than poorer ones (Wagstaff, 2002).

Second question: What is the relationship between increase in social status and child health inequalities? Inequalities in health are also presented as the difference in health status between educational groups, geographic location, employment status, gender and ethnic groups (Marmot, 2010). Thus, the root interpretations of inequalities in health are the complex interaction between individual and household social, economic and environmental factors (Marmot and Wilkinson, 1999). Mackenbach (2002) points out that the most fundamental approach to study inequalities in health is to address directly inequalities in education and occupation which contributed maximum to health inequalities. The higher educated and people living in urban areas gets exposure to better health knowledge compared to their counterparts (e.g. Smith, 1999; GarciaGomez, van Kippersluis, O'Donnell, and van Doorslaer, 2013; Lleras-Muney, 2005; Conti, Heckman and Urzua, 2010; Van Kippersluis, O'Donnell, and van Doorslaer, 2011; Joe et al., 2009; Goli et al., 2013).

Studies have also focused on the linkage between 'Average Health Status (AHS) versus Health Inequalities (HI)' and many of them documented that health inequalities are increasing with increase in health averages (Contoyannis and Forster, 1999b; Victora and others, 2000, Wagstaff, 2002; Moser et al., 2005; Goli and Arokiasamy, 2013). However, very few studies attempted to explain the question: How does rising health averages pushing up health inequalities? Panayotov (2008) developed a framework showing the relationship between 'AHS and HI' (Figure 8.1). He said "improvement in average health status can mask widening of health inequalities. This is a situation where health gain and health equity are not interdependent as it complies with Kaldor-Hicks criterion for efficiency"9.

Figure 8.1 presents the association between AHS and $\mathrm{HI}$ in time as the outcomes of different combinations of distribution of the benefit among the population. The concrete curve for specific population is not something fixed or static. It is something

\footnotetext{
${ }^{9}$ Under Kaldor-Hicks efficiency, an outcome is considered more efficient if a Pareto-superior outcome can be reached by arranging sufficient compensation from those that are made better off to those that are made worse off so that all would end up no worse off than before.
} 
dynamic, which is constantly impacted by the distribution of the benefit from implementing policies, programs and interventions in the population. In other words, the concrete situation constantly changes depending on who-gets-what and how-much from implemented policies, programs and interventions in their interaction. Theoretically, there are eight possible combinations between AHS and HI and one when there is no change in both variables. Of these eight, four combinations ("major") have change in both variables - AHS and HI, and four ("minor") have change in any one variable while the other remains the same. Major combinations are represented in the following cases:

1) AHS increases and $\mathrm{HI}$ increase (red line)

2) AHS increases and HI decrease (green line)

3) AHS decreases and HI increase (black line, left and up)

4) AHS decreases and HI decrease (dashed black line, left and down)

Figure 8.1 Panayotov's framework showing the relationship between average health status and health inequalities

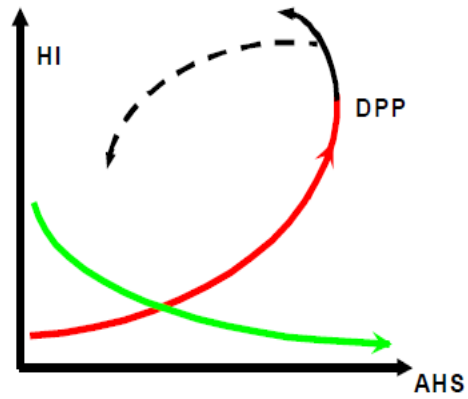

AHS - Average Health Status

$\mathrm{HI}$ - Health Inequalities

DPP - Dead Performance Point

The explanation of Panayotov's framework is also similar to that of the relationship between income growth and health inequalities. As said above, the rising inequalities will be more likely if new health technology is dispersed through the population unequally with the higher income groups adopting it ahead of the lower income groups. Many have argued this and discussed its implications for health inequalities. For instance, Contoyannis and Forster (1999b) do so in applying their aforementioned theoretical results to the issue of health inequalities in the United Kingdom. Victora and others (2000) emphasize the apparent faster adoption of new technology by the better-off. Wagstaff (2002) empirically established this relationship in the context of child health indicators for wider range of developing countries. 
On other hand, though, studies in health inequalities in India are plenty, but a significant body of research across multiple disciplines has been devoted to documenting and explaining the substantial disparities in health between socioeconomic groups. Many of these recent literatures have concentrated on estimating the predictors of health inequalities (e.g. Subramanian, 2006; Joe et al., 2009; Pradhan and Arokiasamy, 2010; Lauridsen and Pradhan, 2011; Po and Subramanian, 2011; Subramanyam and Subramanian, 2011; Arokiasamy et al., 2012; Jain et al., 2012; Goli et al., 2013; Goli and Arokiasmay, 2013). However, none of these studies on child health inequalities in India have focused on the linkage of demographic progress with child health inequalities.

Therefore, in this chapter, the study examined the questions: How children are faring under demographic transition and convergence? Do the fruits of decline in fertility distributing equally across the children of lower and upper socioeconomic groups or not? To our knowledge, this study for the first time creates a quantitative assessment of linkage between socioeconomic and demographic [fertility] transition, convergence and child health inequalities. In the period of continued demographic transition, the assessment of how different states and socioeconomic groups are moving and converging and its linkage with child health inequalities among children is important for realising the challenges of future health policy and determining priorities for convergence in health inequalities among children. Thus, the primary aim of this chapter is to quantify the association between demographic [fertility] progress, convergence and health inequalities among children in India.

\subsection{Data and Methods}

Data sources used in this chapter mainly come from SRS and three rounds of the NFHS. This study used scatter plots, simple and partial correlation plots and estimates, OLS and panel data regression models to establish the linkage between demographic [fertility] convergence and child health inequalities. A detailed discussion of these models is discussed in the chapter 2 . 


\subsection{Results}

\subsubsection{Time series association between trends in averages and inequalities in socioeconomic, demographic and health indicators}

Prior to the investigation of the actual research question of this study: do health inequalities are increasing with the progress in the averages of socioeconomic, demographic and health indicators, the association between trends in inequalities with progress in the averages of the same indicators are examined. The time series association between the trends in averages and inequalities in socioeconomic, demographic and health indicators were assessed to test the hypothesis that, whether the inequalities decrease with the progress in averages or not? The recent literature fosters that influence of progress in averages of socioeconomic and demographic indicators first evident on inequalities in same indicators further they have a cumulative effect on the child health inequalities (Wagstaff, 2002).

The results in case of NSDP revealed that increase in NSDP over the period among major states in India was associated with an increase in both absolute and relative inequalities. Figure 8.2 presents a scatter plot of the association between average NSDP and absolute inequalities was positive $(\mathrm{Cr}=0.9930)$. Similarly, the figure also showed that the association between average NSDP and relative inequalities was also positive $(\mathrm{Cr}=0.9850)$. In case of poverty ratios, the results indicated that decline in poverty ratios was negatively associated with inequalities, which demonstrate that the decline in the averages of poverty ratios were unable to contribute to the decrease in both absolute and relative inequalities in poverty ratios. However, the negative association was not very strong for absolute inequalities. While the negative association between the trends in averages of poverty ratios and absolute inequalities was very weak $(\mathrm{Cr}=-0.4154)$, but the negative association was very strong $(\mathrm{Cr}=-0.8844)$ between the trends in averages of poverty ratios and relative inequalities.

In contrast to the results of the NSDP and poverty ratios, the results in case of literacy rates revealed a different picture. The increase in averages of literacy rates contributed to decrease in both absolute and relative inequalities. The figure 8.2 showed that the association between trends in averages of literacy rates and absolute inequalities was negative $(\mathrm{Cr}=-0.8428)$. Similarly, the association between literacy rates and relative 
inequalities was also negative $(\mathrm{Cr}=-0.9902)$. Overall, the correlation results between the trends in averages and inequalities of socioeconomic indicators showed a positive association in economic indicators and a negative association in social indicators (Figure 8.2).

Figure 8.2. Times series association between growth in averages and inequalities in socioeconomic indicators, 1981-2010
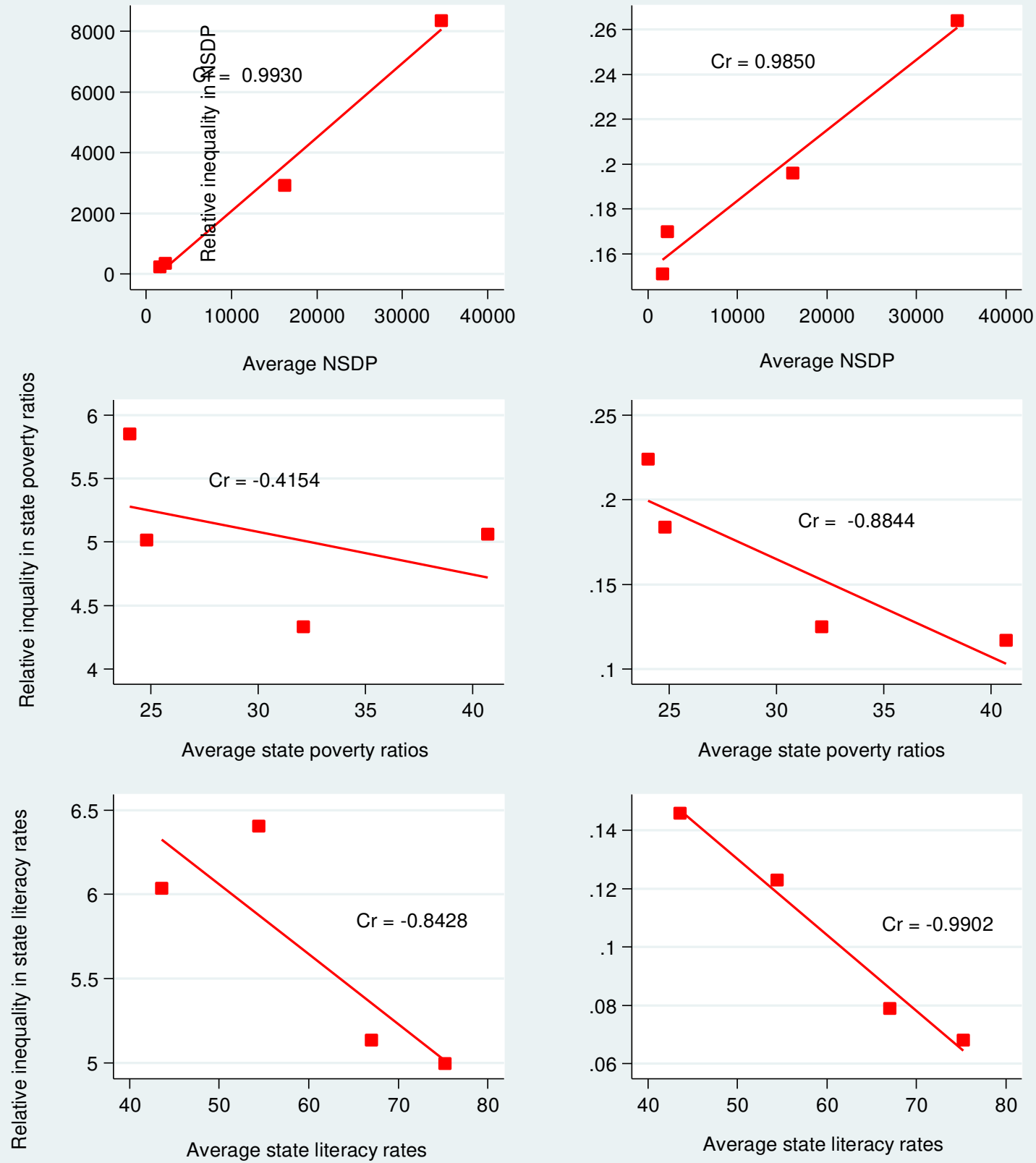
Figure 8.3 presents the association between averages and inequalities in TFR. The scatter plot showed the correlation between averages and absolute inequalities in TFR was positive $(\mathrm{Cr}=0.6198)$. Thus, indicate that the decline in TFR over the period among major states in India was associated with a decrease in absolute inequalities. However, the association between averages and relative inequalities in TFR was negative $(\mathrm{Cr}=-0.9546)$ that, the decline in TFR was associated with increase in relative inequalities. A similar pattern was also evident in case of TFR in urban areas. The results indicated that the decline in the averages of TFR was positively associated with decline in absolute inequalities in TFR $(\mathrm{Cr}=0.9923)$ which means that the decline in TFR was contributing to decline in absolute inequalities. The negative association between trends in averages of TFR and relative inequalities was strong $(\mathrm{Cr}=-0.9466)$ thus, indicating that the decline in TFR was associated with increase in relative inequalities in urban areas of India.

In rural areas, the results reveal that the decrease in averages of TFR also contributes to decrease in absolute inequalities in TFR. However, the positive association was not very strong $(\mathrm{Cr}=0.3103)$. The association between averages and relative inequalities in TFR has a strong negative correlation $(\mathrm{Cr}=-0.9185)$. Overall, the correlation results in case of trends in averages of TFR foster positive association with absolute inequalities and negative association with relative inequalities in it (Figure 8.3).

Figure 8.4 shows the time series association between averages and the inequalities of LEB. The results of scatter plot indicate that the association between trends in the averages and absolute inequalities of LEB was negative $(\mathrm{Cr}=-0.9989)$. On the contrary, the association between averages and relative inequalities of LEB was positive $(\mathrm{Cr}=0.9972)$. Overall, the results in case of LEB indicated that increase in averages over the period among major states in India was associated with a decrease in absolute inequalities and increase in relative inequalities. 
Figure 8.3. Times series association between growth in averages and inequalities in fertility indicators, 1981-2010
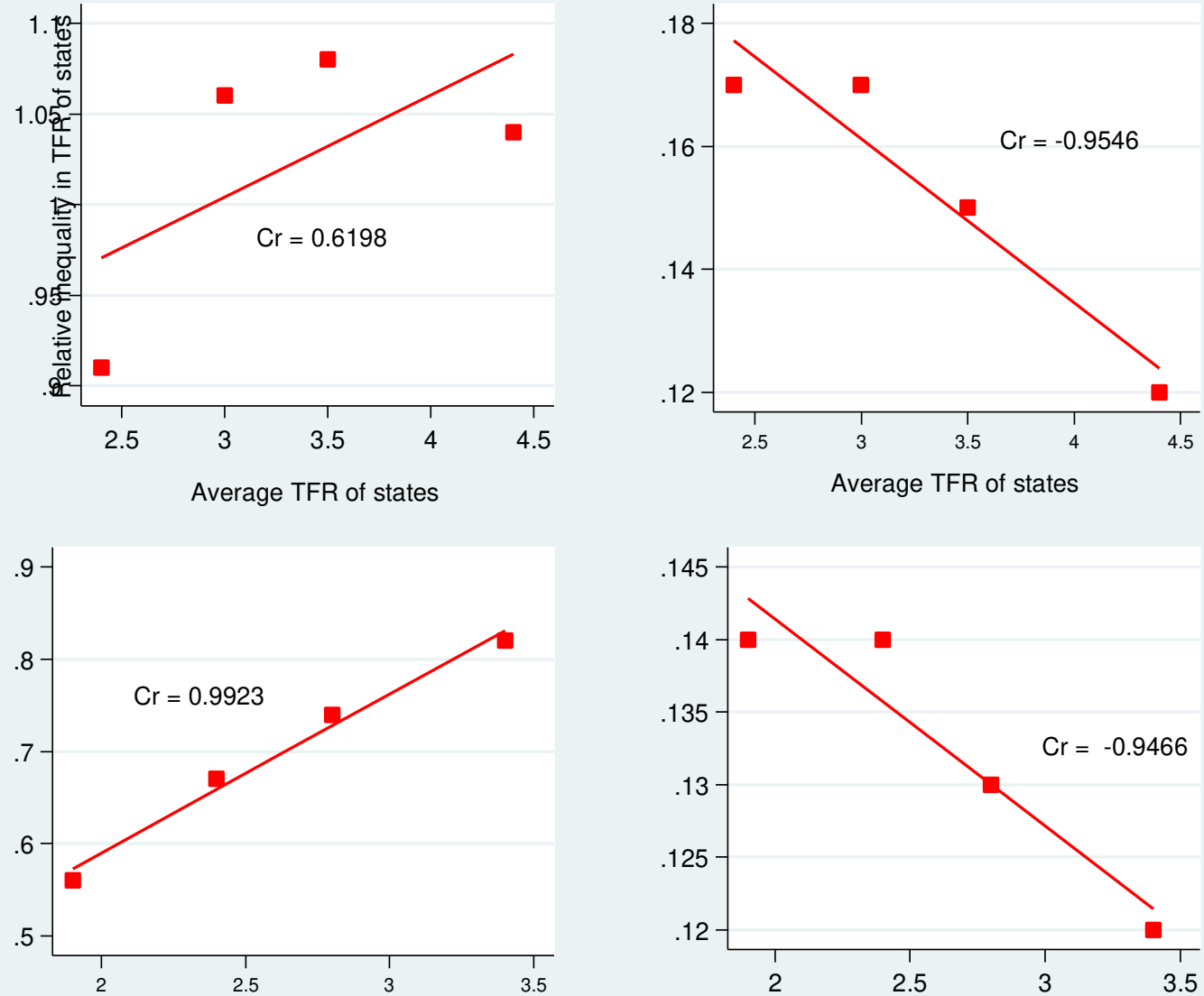

Average TFR of States in Urban areas

Average TFR of States in Urban areas

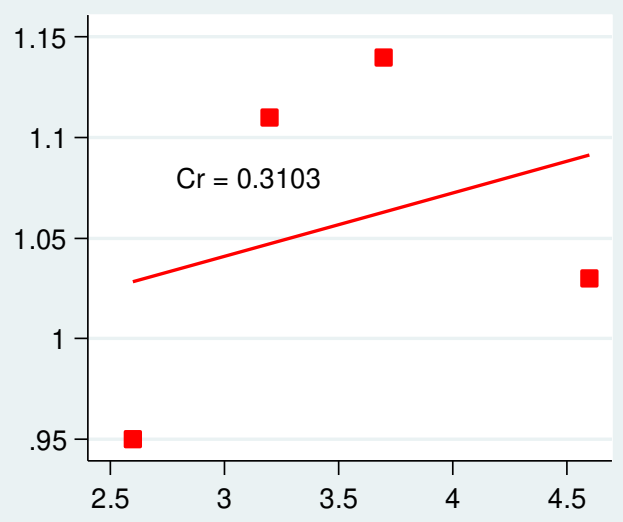

Average TFR of States in Rural areas

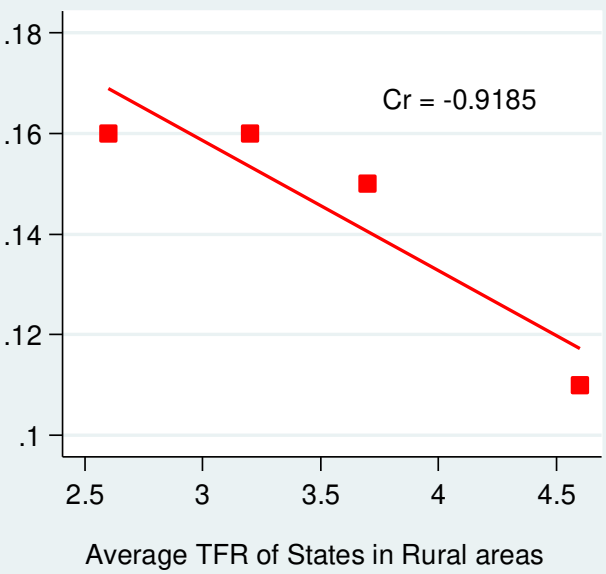


Figure 8.4. Times series association between growth in averages and inequalities in LEB, 1981-2006
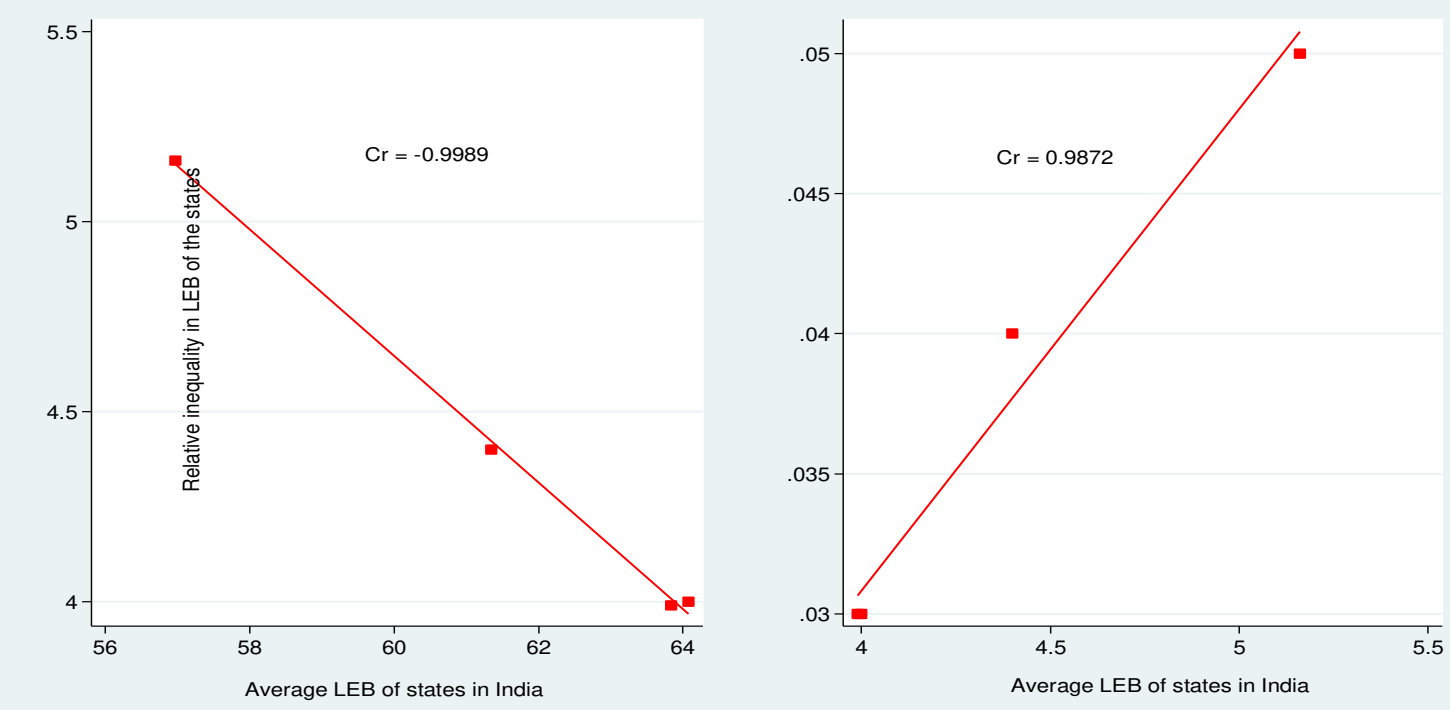

Figure 8.5 shows a scatter plot indicating the association between trends in decline in average and absolute inequalities of IMR was positive $(\mathrm{Cr}=0.9998)$, which indicate that the decrease in the averages of IMR was associated with a decrease in absolute inequalities across the major states in India. However, the association between averages of IMR and relative inequalities was negative $(\mathrm{Cr}=-0.9942)$. Thus, indicating that decline in averages of IMR is unable to contribute to decrease in inequalities in IMR. Akin to the trends in the overall IMR of the states, results in case of IMR in urban areas of the major state also indicated that decline in averages of IMR was positively associated with the decline in absolute inequalities in IMR $(\mathrm{Cr}=0.9824)$. This indicates that the decline in IMR was contributed to decline in absolute inequalities. Similarly, the trends in averages of IMR across the urban areas of the states was not showed a strong positive association with trends in relative inequalities because the correlation value was very low $(\mathrm{Cr}=0.0535)$.

In case of rural areas, the results of correlation plots for IMR revealed very strong positive association $(\mathrm{Cr}=0.9680)$ that, the decrease in averages of IMR was also contributing to the decrease in absolute inequalities in IMR. The correlation value between trends in the averages and relative inequalities of IMR was also positive, but the correlation value was showing very weak positive association $(\mathrm{Cr}=0.3683)$. Overall, the correlation results in case of trends in averages of IMR foster strong 
positive association with absolute inequalities and negative or weak positive association with relative inequalities (Figure 8.5).

Reflecting similar pattern in other demographic and health indicators, the results in case of NNMR also revealed that decline in NNMR over the period among major states in India was associated with a decline in absolute inequalities, but an increase in relative inequalities. Figure 8.5 presents a scatter plot which showed the association between trends in average of NNMR and absolute inequalities was positive $(\mathrm{Cr}=0.9323)$. However, the association between average of NNMR and relative inequalities was negative $(\mathrm{Cr}=-0.2994)$. In case of NNMR in urban areas, the results indicated that decline in averages of NNMR was positively associated with decline in absolute inequalities $(\mathrm{Cr}=0.7917)$ that, the decline in NNMR was contributed to decline in absolute inequalities. The negative association between trends in averages of NNMR and relative inequalities showed that, the decline in the averages of NNMR was associated with the increase in relative inequalities. However, the correlation was not very strong $(\mathrm{Cr}=-0.2897)$. The results in case of trends in NNMR in rural areas indicated high positive correlation $(\mathrm{Cr}=0.9186)$ that the decrease in the averages of NNMR was also contributing to decrease in absolute inequalities in NNMR. However, the association between NNMR and relative inequalities was negative $(\mathrm{Cr}=-0.9137)$. Taken as a whole, the correlation results in case of trends in averages of NNMR indicated positive association with absolute inequalities and negative association with relative inequalities in NNMR (Figure 8.6).

The scatter plots in case of children full immunisation are not showing any consistent pattern in terms of association between trends in averages of children full immunisation and inequalities in it (Figure 8.7). The results showed that increase in averages of children full immunisation over the period among major states in India was positively associated with absolute inequalities $(\mathrm{Cr}=0.1353)$, but negatively associated with relative inequalities $(\mathrm{Cr}=-0.3783)$. In case of urban areas, the results indicated that decline in children full immunisation was positively associated with decline in absolute inequalities meaning the decline in children full immunisation was contributed to decline in absolute inequalities. However, the positive association between trends in averages of children full immunisation and relative inequalities was not very strong $(\mathrm{Cr}$ 
$=0.0046)$. Therefore, it is difficult to say that with the decrease in averages of children full immunisation, the relative inequalities are declining.

Figure 8.5. Times series association between growth in averages and inequalities in IMR, 1981-2010

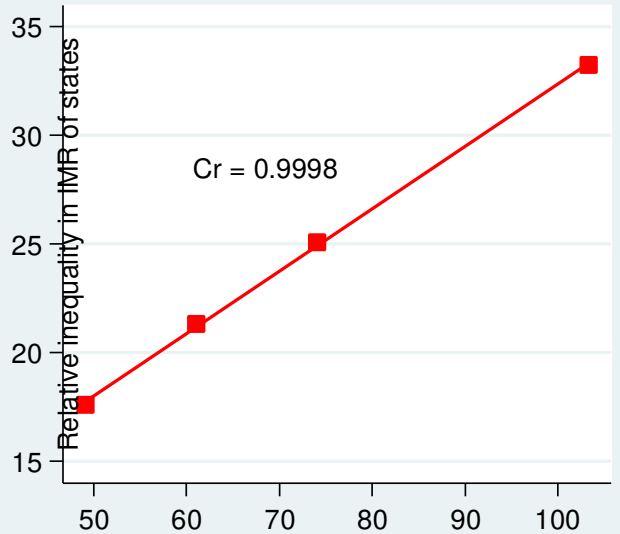

Average IMR of states

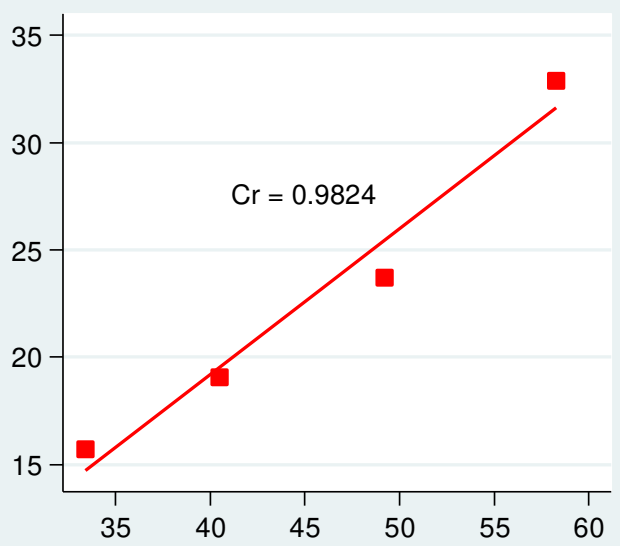

Average IMR of states in Urban areas

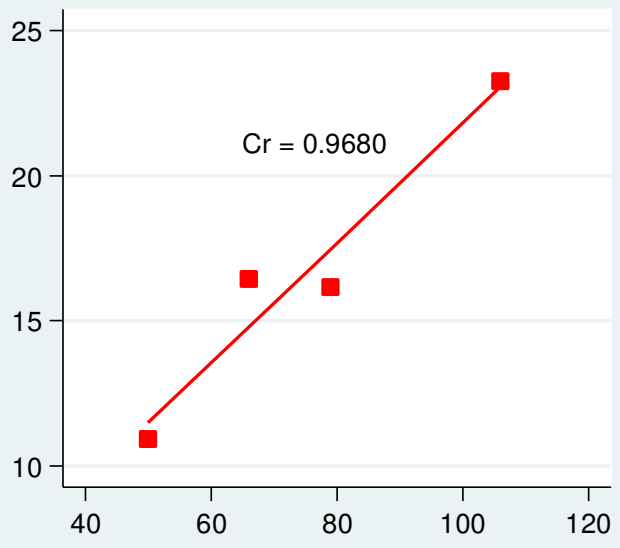

Average IMR of states in Rural areas

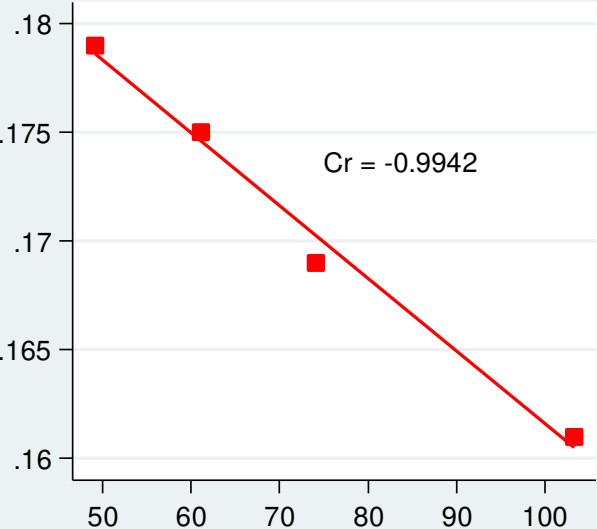

Average IMR of states

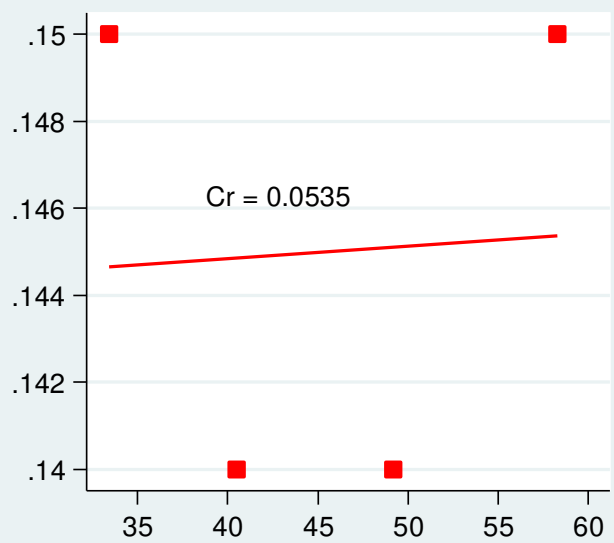

Average IMR of states in Urban areas

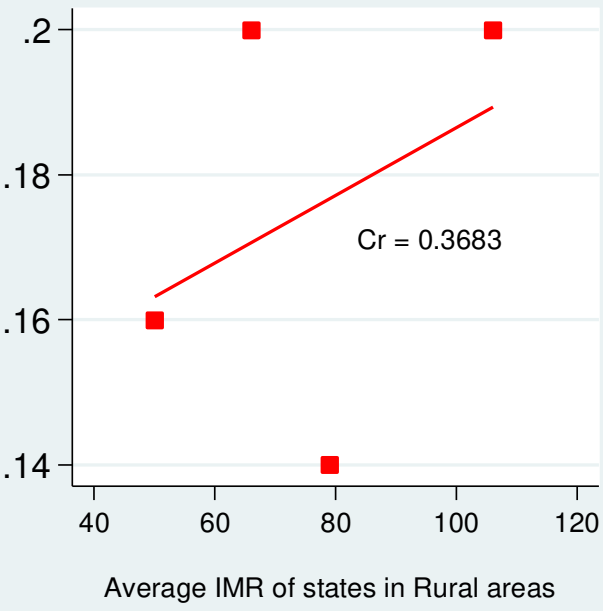


Figure 8.6. Times series association between trends in averages and inequalities in NNMR, 1981-2010
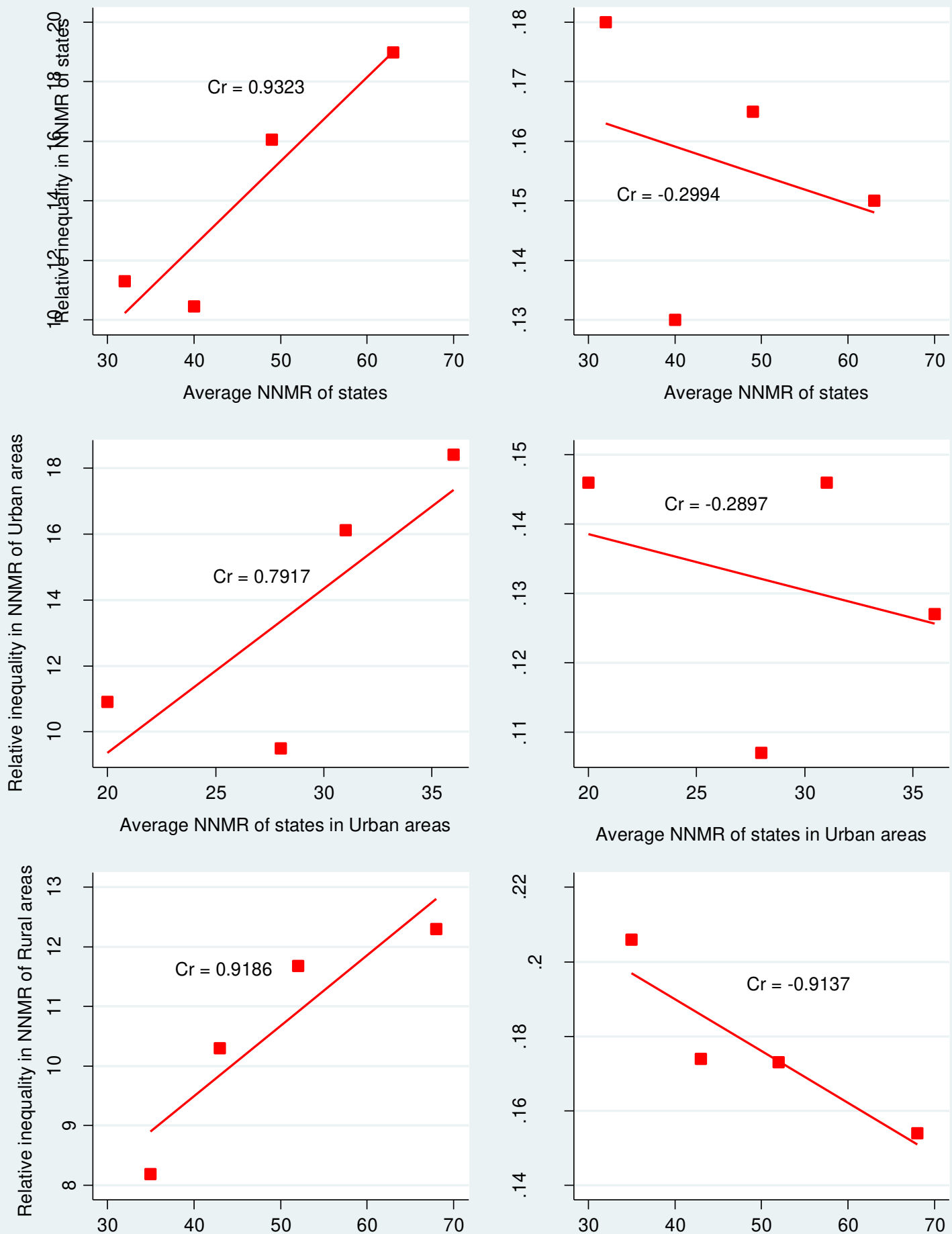

Average NNMR of states in Rural areas

Average NNMR of states in Rural areas 
In case of rural areas, the correlation results of trends in averages and inequalities in children full immunisation revealed that the decrease in averages of children full immunisation contributing to the decrease in absolute inequalities. However, the positive association was very weak $(\mathrm{Cr}=0.1016)$. The association between children full immunisation and relative inequalities was negative $(\mathrm{Cr}=-0.3474)$. Overall, the correlation results in case of trends in averages of children full immunisation foster positive association with absolute inequalities and negative association with relative inequalities. In general, the declining trends in children full immunisation were associated with increase in relative inequalities (Figure 8.7).

The time series correlation results for children underweight revealed that decline in children underweight over the period among major states in India was associated with an increase in both absolute and relative inequalities. For children underweight, the scatter plot in figure 8.8 indicated that the association between trends of decline in averages of children underweight and absolute inequalities were negative $(\mathrm{Cr}=$ 0.9516). Further, the association between averages of children underweight and relative inequalities were also negative $(\mathrm{Cr}=-0.9748)$. In case of urban areas, the results indicated that the decline in averages of children underweight was positively associated with the decline in absolute inequalities in children underweight. The decline in children underweight was contributed to decline in absolute inequalities. However, the negative association between trends in averages of children underweight and relative inequalities was very strong $(\mathrm{Cr}=-0.8915)$ which, indicate that the relative inequalities were increased with decrease in averages of children underweight.

The results in case of trends in averages and absolute inequalities in children underweight in rural areas revealed a strong negative association $(\mathrm{Cr}=-0.8436)$. This indicates that the decrease in averages of children underweight contributed to the decrease in absolute inequalities in children underweight. The association between trends of averages of children underweight and relative inequalities were also negative $(\mathrm{Cr}=-0.9001)$. Overall, the correlation results in case of trends in averages of children underweight foster negative association with both absolute and relative inequalities. By and large, the declining trends in children underweight were associated with increase in relative inequalities (Figure 8.8). 
Figure 8.7. Times series association between trends in averages and inequalities in children full immunisation, 1992-2006
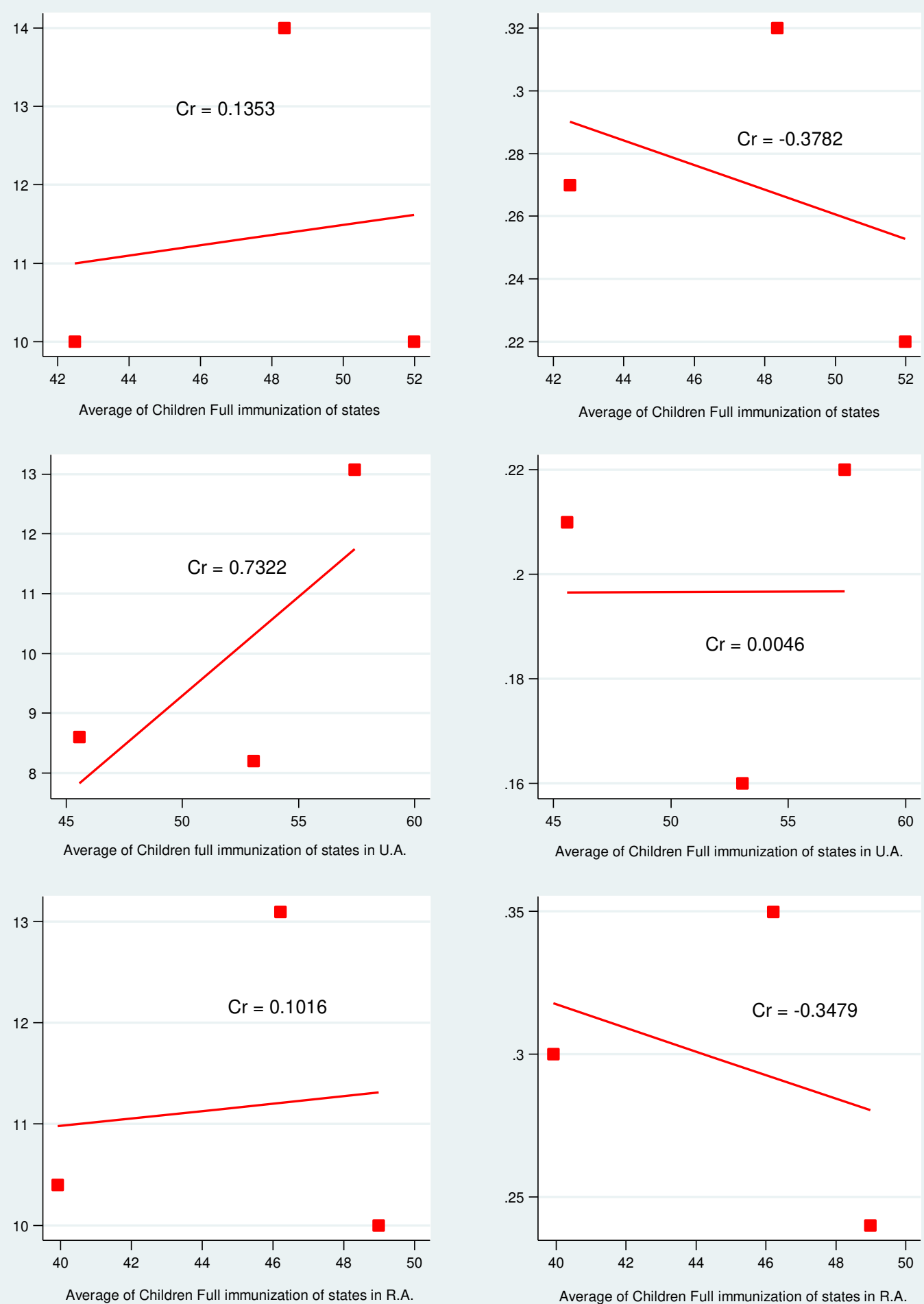

Note: UA: Urban Areas; RA: Rural Areas 
Figure 8.8. Times series association between trends in averages and inequalities in children underweight, 1992-2006
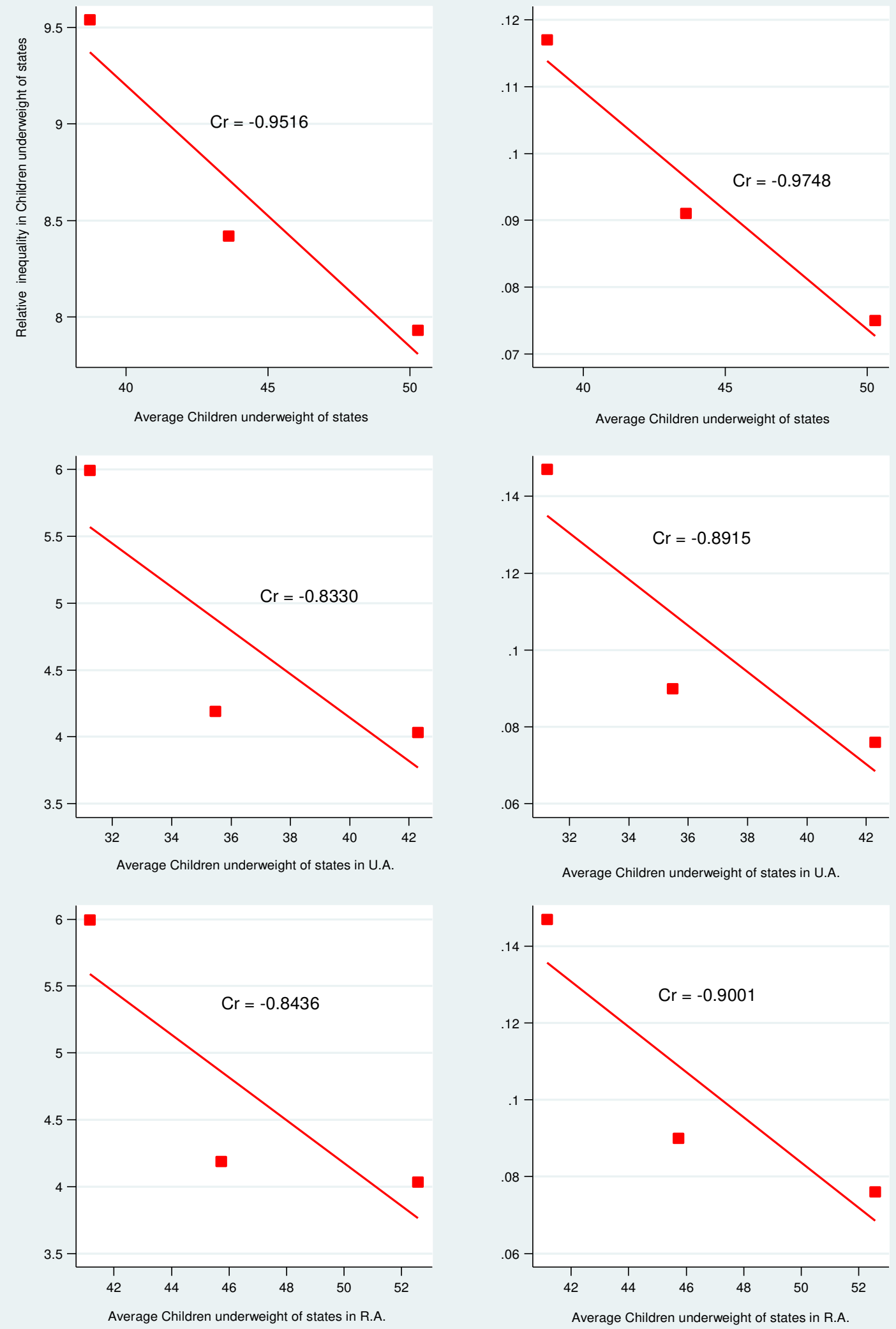

Note: UA: Urban Areas; RA: Rural Areas 


\subsubsection{Zero order correlation between change in averages of socioeconomic and demographic indicators and SES inequalities in child health}

Figure 8.9 shows the association between change in averages of socioeconomic, demographic, child health indicators and socioeconomic inequalities in child health during 1992-2006. The socioeconomic inequalities in child health indicators (children full immunisation, children underweight and NNMR) were measured in terms of Concentration Index (CI). The estimates of zero order correlation between NSDP and CI of three selected child health indicators evident that correlation values for all three child health indicators was positive. This reveals that the increase in NSDP was associated with more socioeconomic inequalities in all three children's health indicators during 1992-2006. However, this association was greater for children underweight $(\mathrm{r}=0$. 56) and smaller for NNMR ( $\mathrm{r}=0.14)$. Similarly, the association between changes in poverty ratios and socioeconomic inequalities in child health indicators showed a negative association which demonstrates that the decline in poverty ratios were also failed to contribute to the reduction in socioeconomic inequalities in all three child health indicators. The absolute negative association between the decline in poverty ratios and socioeconomic inequalities was greater in children underweight $(\mathrm{r}=-0.57)$ and lower in children full immunisation $(\mathrm{r}=-0.36)$. Compared to change in poverty ratios of the states, the change in per capita NSDP was more strongly associated with an increase in socioeconomic inequalities in child health indicators.

The estimates of zero order correlation between change in literacy rates and change in socioeconomic inequalities in children underweight, children full immunisation and NNMR foster the same relationship which evident in case of per capita NSDP and poverty ratios. The increase in averages of literacy rates was also associated with an increase in socioeconomic inequalities in all three child health indicators. While, the association between changes in literacy rate and children underweight was greater $(r=68)$, but it was lower in case of the association between change in averages of literacy rate and children full immunisation. The association between change in the TFR and children full immunisation also indicated that the decline in TFR couldn't help in reduction of socioeconomic inequalities in children full immunisation, children underweight and NNMR instead they have increased. In general, with a decline in TFR, the increase in socioeconomic inequalities were highest for children underweight, but 
other two indicators show more or less the same level of increase.

The association between change in averages of children full immunisation and socioeconomic inequalities in the same indicator showed a positive correlation $(r=0.39)$ which indicate that socioeconomic inequalities in children full immunisation increased with an increase in the averages of the same indicator. Similarly, in case of children underweight, the association between change in averages and socioeconomic inequalities showed a negative correlation $(\mathrm{r}=-0.38)$, which reveals that socioeconomic inequality increased with a decrease in the averages of the same indicator. The crosssectional association between change in averages of NNMR and socioeconomic inequalities in NNMR during 1992-2006 showed a positive association, but with a weak correlation $(\mathrm{r}=0$. 05). Therefore, it was not possible to say strongly that socioeconomic inequalities decreased with a decrease in averages of NNMR. Overall, the results foster that the progress in averages of socioeconomic and demographic indicators was associated with more unequal distribution of child health indicators. Furthermore, even the improvement in averages of child health indicators was unable to contribute to the reduction of socioeconomic inequalities in their respective indicators.

Figure 8.9. Zero order correlation (pooled cross-sectional association) between change in averages of per capita NSDP, Poverty ratios, Literacy rates, TFR and change in inequalities in child health indicators, 1992-2006

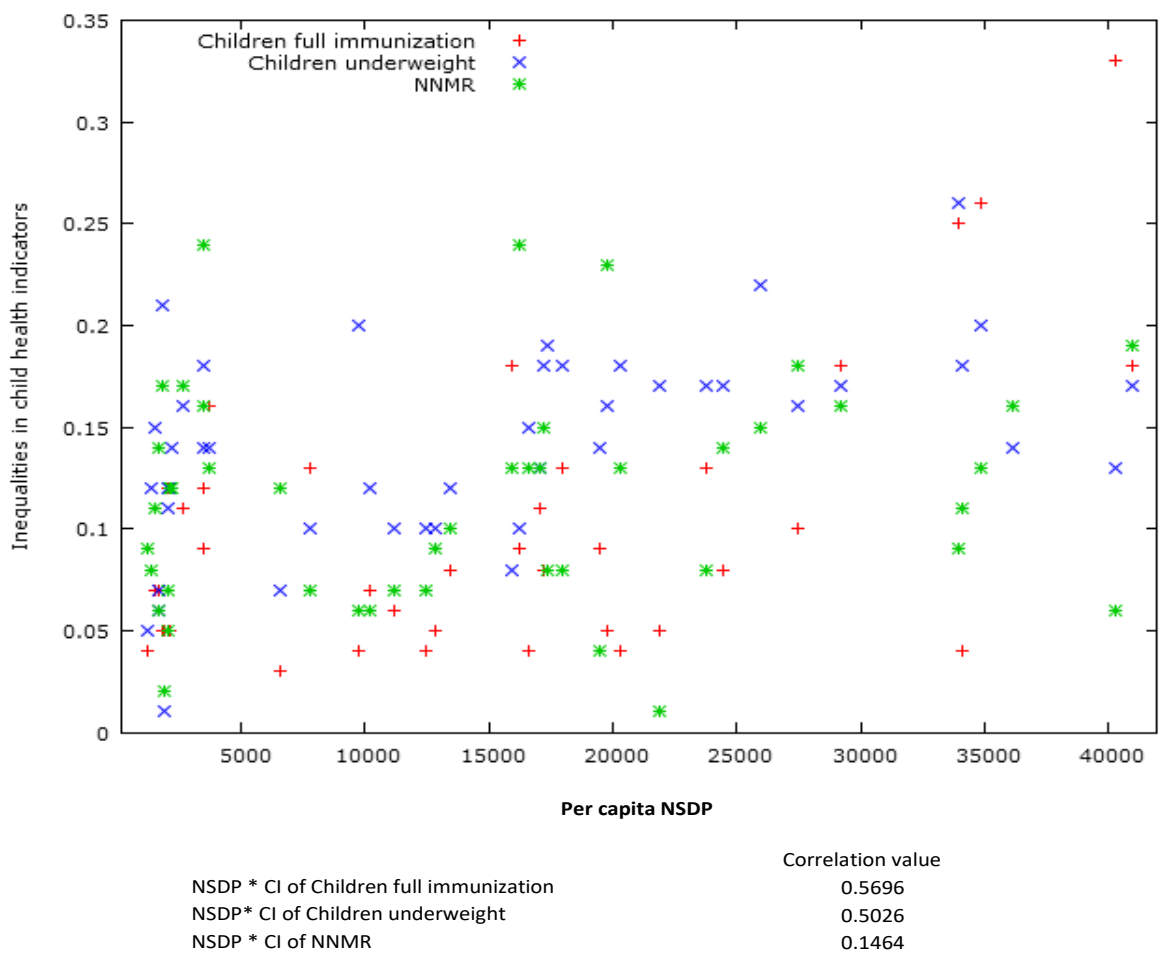



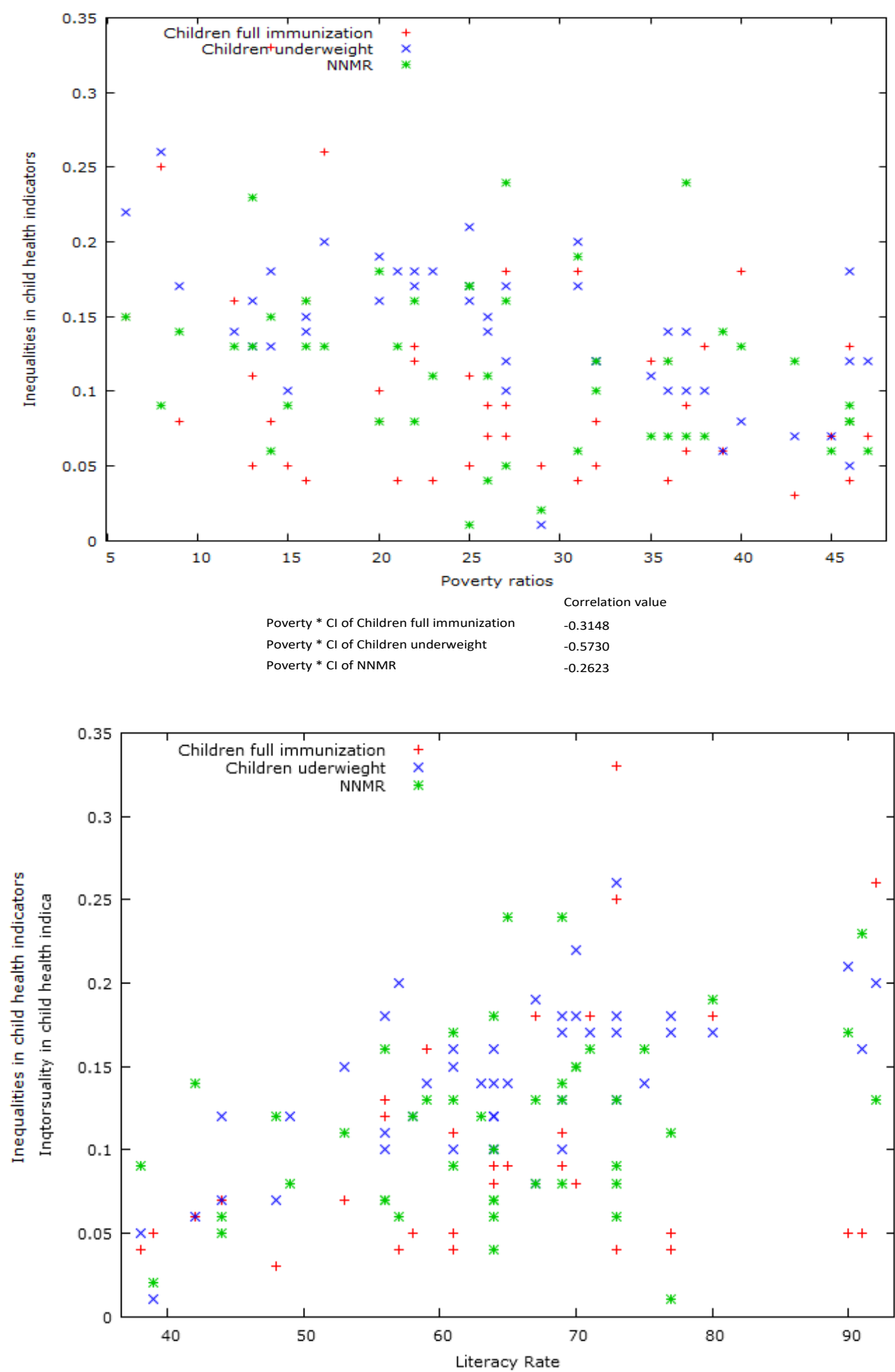

Correlation value

Literacy rate $* \mathrm{Cl}$ of Children full immunization

0.3682

Literacy rate ${ }^{*} \mathrm{Cl}$ of Children underweight $\quad 0.6817$ Literacy rate ${ }^{*} \mathrm{Cl}$ of NNMR 0.3884 

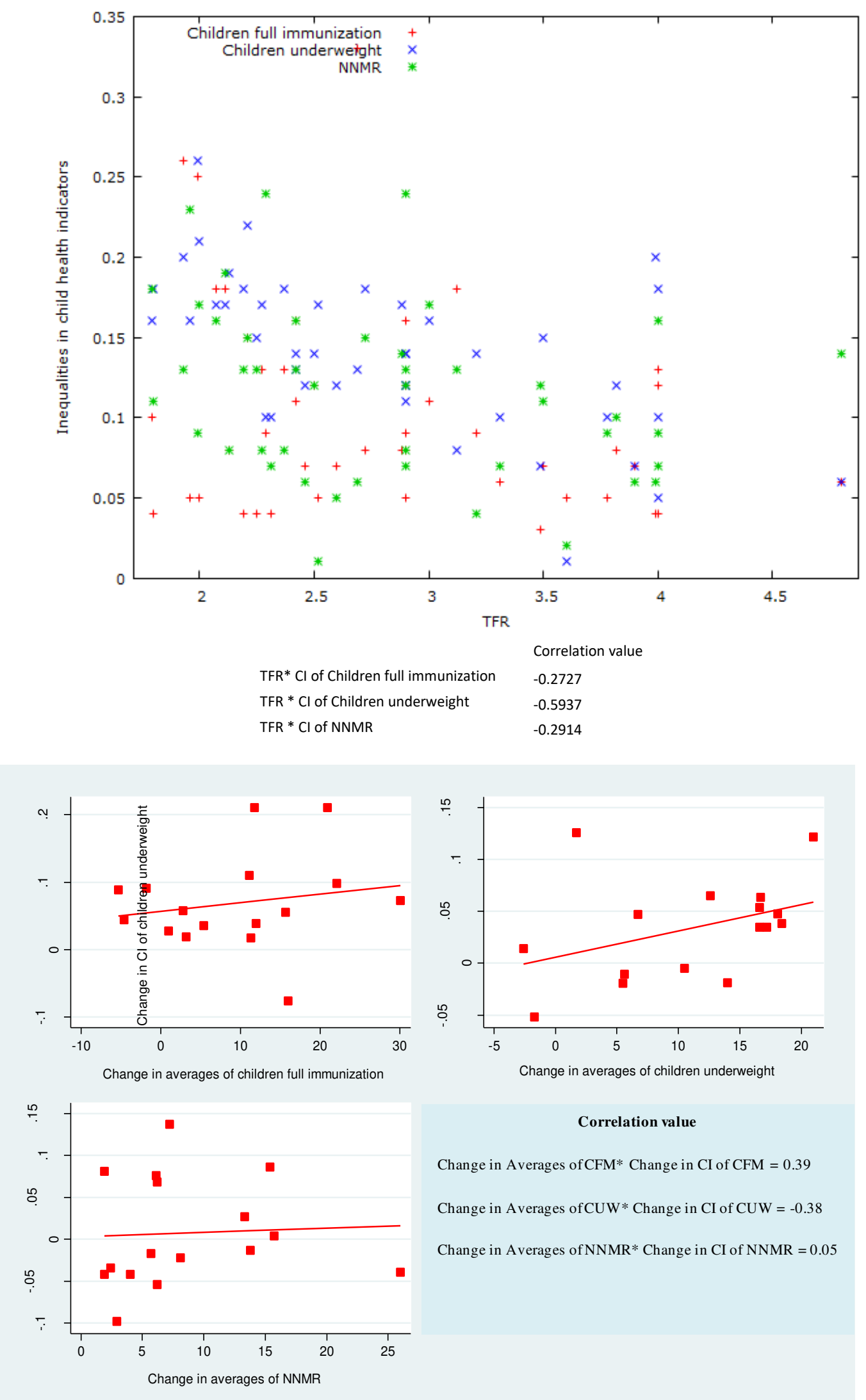
Figure 8.10. Partial correlation analyses (pooled cross-sections) showing three dimensional linkages of fertility with child health inequalities by adjusting to child health averages, 19922006

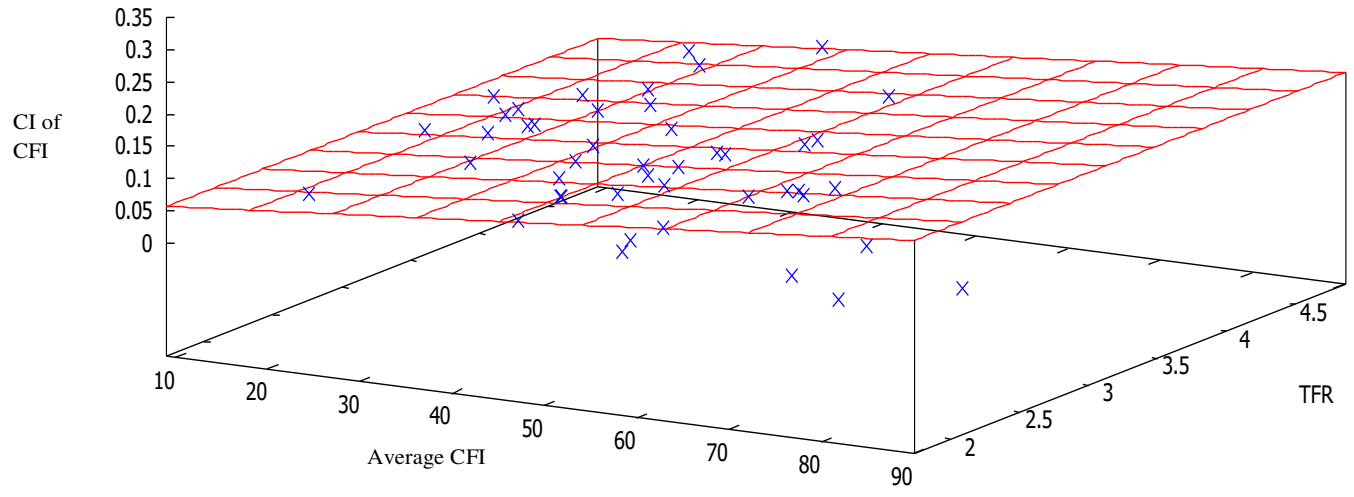

Adjusted correlation value $\mathrm{CI}$ of $\mathrm{CFM}^{*}$ Average $\mathrm{CFM}=0.388 * * *$

$$
\mathrm{CI} \text { of CFM*TFR } \quad=-0.273^{*}
$$

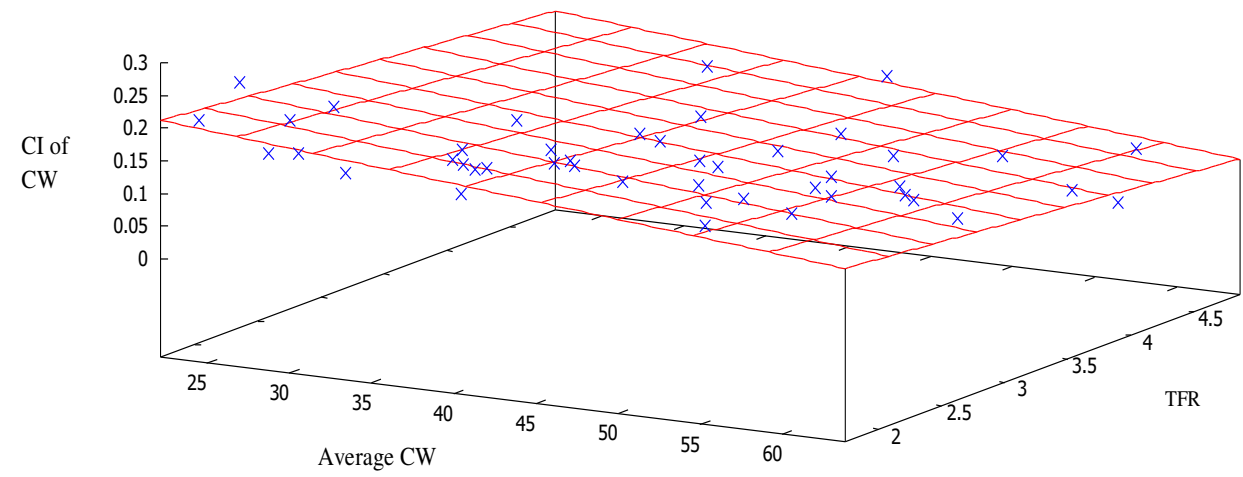

Adjusted correlation value $\mathrm{CI}$ of $\mathrm{CW} *$ Average $\mathrm{CW}=-0.672^{* * *}$

$$
\mathrm{CI} \text { of } \mathrm{CW} * \mathrm{TFR} \quad=-0.594 * * *
$$

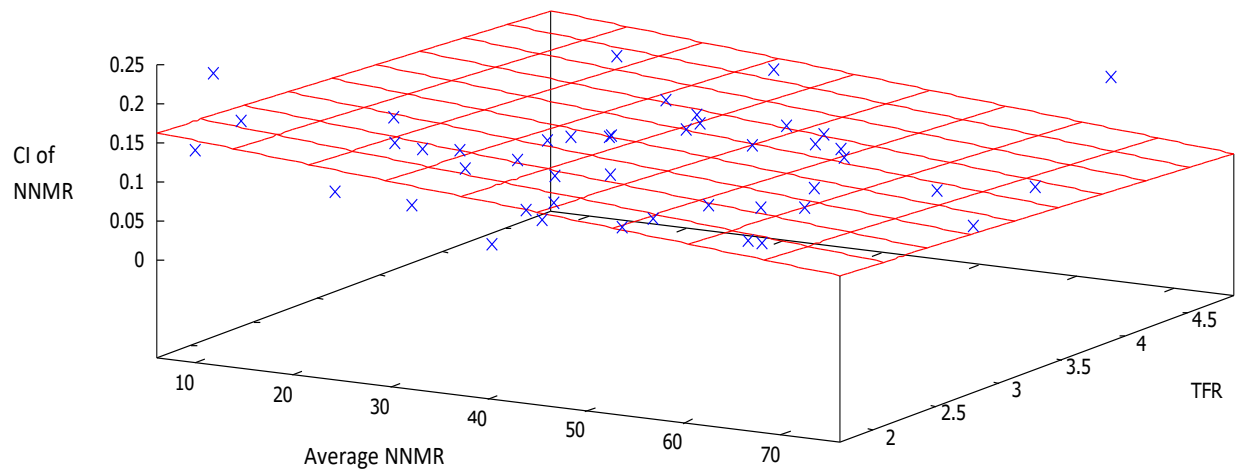

Adjusted correlation value CI of NNMR* Average NNMR $=-0.361 * * *$

CI of NNMR $*$ TFR $\quad=-0.291 * *$

Note: Significance levels: $* \mathrm{p}<0.05, * * \mathrm{p}<0.01, * * * \mathrm{p}<0.001$ 
Figure 8.10 presents the plots and estimates of partial correlation analyses showing three dimensional linkages of fertility with child health inequalities by adjusting to child health averages. The plot is showing the three dimensional linkage between CI of children full immunisation and TFR after adjusting to the averages of children full immunisation. The results showed that after adjusting to averages of children full immunisation, the association between inequalities in children full immunisation and TFR was negative with the correlation value of -0.273 which was also statistically significant $(p<0.05)$. The negative relationship indicates that the decline in TFR is associated with an increase in inequalities in children full immunisation. Similarly, in case of inequalities in children underweight and TFR linkage, the partial correlation plot and estimates showed that after adjusting averages of children underweight, the linkage between inequalities in children underweight and TFR was negative $(r=-0.59$, $\mathrm{p}<0.001$ ) that decline in TFR is not contributing to the reduction of inequalities in children underweight prevalence. The partial correlation plot and estimates of association between inequalities in NNMR and TFR after adjusting for averages of NNMR showed a negative relationship $(\mathrm{r}=-0.29, \mathrm{p}<0.01)$. This indicates that the decline in NNMR has not helped in the reduction of socioeconomic inequalities in NNMR.

\subsubsection{Pooled OLS regression estimates}

This section presents the estimates of adjusted effects of TFR based on pooled OLS regression. These estimates were based on three NFHS panels. As mentioned earlier in the methodology chapter that, the pooled OLS regression is the simplest regression model estimates of panel data, easy to interpret and understand causal relationships. The table 8.1 shows that the pooled OLS model estimates by using 45 observations included 3 cross-sectional units with a time-series length of 15 and the dependent variable is 'inequality in children full immunisation'. After controlling for important socioeconomic factors and averages of children full immunisation, the results showed that the TFR was associated positively with inequalities in children full immunisation. The positive association demonstrates that after adjusting for socioeconomic levels, the decline in TFR level was contributing to the decline in socioeconomic inequalities in children full immunisation. 
Table 8.1. Pooled OLS, using 45 observations; Included 3 cross-sectional units Time-series length $=15$

Dependent variable: Inequality in Children Full immunisation

\begin{tabular}{lcccc}
\hline Predictors & Coefficient & Std. Error & \multicolumn{2}{c}{ P-value } \\
\hline Total Fertility Rate & 0.0320 & 0.0175 & 0.0760 & $*$ \\
NDSP & 0.000004 & 0.0000012 & 0.0050 & $* * *$ \\
Poverty Ratio & 0.0003 & 0.00086 & 0.7252 & \\
Literacy Rate & 0.0003 & 0.0011 & 0.8020 & \\
Averages of Children & 0.0011 & 0.00060 & 0.0853 & $*$ \\
full immunisation & & & & \\
Year 1992_98 dummy & -0.0020 & 0.00191 & 0.3146 & \\
variable & & & & \\
Year 1998_06 dummy & -0.0037 & 0.0020 & 0.0763 & $*$ \\
variable & & & & \\
Constant & -0.09001 & 0.1117 & 0.4256 & \\
Adjusted R-squared & & 0.3669 & & \\
P-value(F) & & 0.0008 & & \\
\hline Signifincen & & & & \\
\end{tabular}

Significance level: $* \mathrm{p}<.10,{ }^{* *} \mathrm{p}<0.05, \mathrm{p}<0.01$

Table 8.2 presents the pooled OLS regression model estimates showing the effect of the decline in TFR on inequality in children underweight after controlling the other important socioeconomic factors. In contrast to the results of 'socioeconomic inequalities in children full immunisation', inequalities in children underweight showed a negative association $(\beta=-0.0117)$ with the decline in TFR. The negative association demonstrates that even after adjusting for socioeconomic levels, the decline in TFR level is contributing to the increase in socioeconomic inequalities in children underweight. However, this estimate was not statistically significant. In this model, only the literacy rate has emerged as a significant factor.

The pooled OLS regression estimates in case of NNMR are presented in table 8.3. After controlling for relevant socioeconomic factors, the association between socioeconomic inequalities in NNMR and decline in TFR was found positive $(\beta=0.0020)$. The positive association demonstrates that the decline in TFR was associated with a decline in socioeconomic inequalities in NNMR. However, the estimate was not statistically significant. Though, pooled OLS regression estimates presents the association between socioeconomic inequalities in child health indicators and fertility decline, but the panel data fixed effects model, unlike pooled OLS, explores the special features and provide more robust estimates. 
Table 8.2. Pooled OLS, using 45 observations; Included 3 cross-sectional units Time-series length $=15$

Dependent variable: Inequality in Children underweight

\begin{tabular}{lcccc}
\hline Predictors & Coefficient & Std. Error & p-value & \\
\hline Total Fertility Rate & -0.0117508 & 0.011155 & 0.29898 \\
NDSP & -0.00000095 & 0.00000083 & 0.26400 & \\
Poverty Ratio & -0.00107292 & 0.000661125 & 0.11311 & $*$ \\
Literacy Rate & 0.0012804 & 0.000737576 & 0.09089 & $*$ \\
Averages of Children & -0.00113229 & 0.000860954 & 0.19655 & \\
underweight & & & & \\
Year 1992_98 dummy & -0.000518886 & 0.00126194 & 0.68331 \\
variable & & & & \\
Year 1998_06 dummy & 0.00155733 & 0.00134422 & 0.25407 \\
variable & & & \\
Constant & 0.179072 & 0.0788123 & 0.02898 & $* *$ \\
Adjusted R-squared & & 0.510761 & \\
P-value(F) & & 0.000012 & & \\
\hline
\end{tabular}

Table 8.3. Pooled OLS, using 45 observations; Included 3 cross-sectional units

Time-series length $=15$

Dependent variable: Inequality in NNMR

\begin{tabular}{lccl}
\hline Predictors & Coefficient & Std. Error & p-value \\
\hline Total Fertility Rate & 0.0020808 & 0.0160131 & 0.89731 \\
NDSP & 0.000000116 & 0.0000012 & 0.92507 \\
Poverty Ratio & -0.000145738 & 0.000877845 & 0.86905 \\
Literacy Rate & 0.00203659 & 0.00128257 & 0.12082 \\
Averages of NNMR & -0.00053199 & 0.000883887 & 0.55093 \\
Year 1992_98 dummy & 0.00223986 & 0.00186319 & 0.23694 \\
variable & & & \\
Year 1998_06 dummy & -0.00342731 & 0.00199929 & 0.09485 \\
variable & & & \\
Constant & 0.00869381 & 0.131154 & 0.94751 \\
Adjusted R-squared & & 0.138105 & \\
P-value(F) & & 0.080355 & \\
\hline
\end{tabular}

\subsubsection{Panel data regression}

Results of panel data regression analysis with the lagged effects $(1992$, 1998) of TFR on inequality in Children full immunisation (2006) is presented in table 8.4. Prior to adjusting for relevant socioeconomic factors, fixed effect model estimates showed that TFR was negatively $(\beta=-0.0314, \mathrm{p}<0.05)$ associated with socioeconomic inequalities in children full immunisation. However, after adjusting for socioeconomic variables, the results showed a statistically significant positive association $(\beta=0.0152, \mathrm{p}<0.05)$ between TFR and socioeconomic inequalities in Children full immunisation. The 
positive association reveals a decline in TFR is associated with a decline in socioeconomic inequalities in children full immunisation. Table 8.5 presents the results of panel data regression analysis with the lagged effects (1992, 1998) of TFR on inequality in Children underweight (2006). Unlike, the results in case of socioeconomic inequalities in children full immunisation, the results of children underweight showed the negative association between socioeconomic inequalities in children underweight and TFR before and after adjusting to other socioeconomic factors. However, the size of effect declines when adjusting to other socioeconomic factors $(\beta=-0.0132, p<.10)$ compared to the unadjusted effect $(\beta=-0.0132, p<.01)$. Results of panel data regression analysis with the lagged effects $(1992,1998)$ of TFR on inequality in NNMR (2006) shown in Table 8.6. Similar to the results in case of socioeconomic inequalities in children full immunisation, the results of NNMR also showed a negative association $(\beta=-0.0257, \mathrm{p}<10)$ between socioeconomic inequalities and TFR before adjusting for relevant socioeconomic factors, but after adjusting for socioeconomic factors, the negative association is replaced with positive association $(\beta=0.0020)$. However, the positive association was not statistically significant. Change in the averages of the same indicators in all three selected child health indicators was inversely related to socioeconomic inequalities in the respective indicators which indicate that the rise in averages of children full immunisation, decline in children underweight and NNMR is associated with an increase in socioeconomic inequalities in the respective indicators. 
Table 8.4. Results of Panel data regression analysis with the lagged effects $(1992,1998)$ of TFR on inequality in Children full immunisation (2006)

\begin{tabular}{|c|c|c|c|}
\hline \multirow[t]{2}{*}{ Predictors } & \multicolumn{3}{|c|}{ Fixed effects } \\
\hline & Model I & Model II & Model III \\
\hline Total Fertility Rate & $-0.0314 * *$ & - & $0.0152 * * *$ \\
\hline NDSP & - & - & $0.000004 * * *$ \\
\hline Poverty Ratio & - & - & 0.0008 \\
\hline Literacy Rate & - & - & 0.0003 \\
\hline $\begin{array}{l}\text { Averages of Children full } \\
\text { immunis ation }\end{array}$ & - & $0.0016^{* * *}$ & $0.0013^{* *}$ \\
\hline Year 1992_98 dummy variable & $-0.0041 * *$ & $-0.0051 * * *$ & $-0.0021 * * *$ \\
\hline Year 1998_06 dummy variable & -0.0002 & -0.0006 & $-0.0039 * *$ \\
\hline Constant & $0.2126 * * *$ & $0.0567 * *$ & -0.0571 \\
\hline Adjusted R-squared & 0.2109 & 0.3512 & 0.4084 \\
\hline Wald $\mathrm{Chi}^{2}$ & $12.57 * * *$ & $18.39 * * *$ & $34.68 * * *$ \\
\hline P-value $(F)$ & 0.0129 & 0.0005 & 0.0007 \\
\hline No of observations & 45 & 45 & 45 \\
\hline \multicolumn{4}{|c|}{$\begin{array}{l}\text { Level of Significance: } * \mathrm{p}<0.10 ; * * \mathrm{p}<0.05 ; * * * \mathrm{p}<.01 \\
\text { Note: Fixed-effects, using } 45 \text { observations; included } 3 \text { cross -sectional units; Time-series length }=15 ; \\
\text { Dependent variable: Inequality in Children full immunisation }\end{array}$} \\
\hline \multicolumn{4}{|c|}{$\begin{array}{l}\text { Table 8.5. Results of Panel data regression analysis with the lagged affects }(1992,1998) \text { of TFR } \\
\text { on inequality in Children underweight (2006) }\end{array}$} \\
\hline \multirow[t]{2}{*}{ Predictors } & \multicolumn{3}{|c|}{ Fixed effect } \\
\hline & Model I & Model II & Model III \\
\hline Total Fertility Rate & $-0.0362 * * *$ & - & $-0.0132^{*}$ \\
\hline NDSP & - & - & -0.0000009 \\
\hline Poverty Ratio & - & - & -0.0011 \\
\hline Literacy Rate & - & - & $0.0012^{*}$ \\
\hline Averages of Children underweight & - & $-0.0027 * * *$ & -0.0008 \\
\hline Year 1992_98 dummy variable & $-0.0002 *$ & 0.0003 & -0.0005 \\
\hline Year 1998_06 dummy variable & 0.0022 & $0.0020 *$ & 0.0018 \\
\hline Constant & $0.2317 * * *$ & $0.2473 * * *$ & $0.1718^{* *}$ \\
\hline Adjusted R-squared & 0.3995 & 0.4387 & 0.5025 \\
\hline Wald $\mathrm{Chi}^{2}$ & $21.69 * * *$ & $31.54 * * *$ & $46.98 * * *$ \\
\hline P-value(F) & 0.0001 & 0.00003 & 0.00005 \\
\hline No of observations & 45 & 45 & 45 \\
\hline
\end{tabular}

Level of Significance: $* \mathrm{p}<0.10 ; * * \mathrm{p}<0.05 ; * * * \mathrm{p}<.01$

Note: Fixed-effects, using 45 observations; included 3 cross -sectional units; Time-series length $=15$;

Dependent variable: Inequality in Children full immunisation 
Table 8.6. Results of Panel data regression analysis with the lagged affects $(1992,1998)$ of TFR on inequality in NNMR (2006)

\begin{tabular}{lccc}
\hline Predictors & \multicolumn{3}{c}{ Fixed effect } \\
\cline { 2 - 4 } & Model I & Model II & Model III \\
\hline Total Fertility Rate & $-0.0257^{*}$ & - & 0020808 \\
NDSP & - & - & 0.0000002 \\
Poverty Ratio & - & - & -0.0003 \\
Literacy Rate & - & - & 0.0021 \\
Averages of NNMR & - & $-0.0017^{* * *}$ & -0.0005 \\
Year 1992_98 dummy variable & 0.0014 & 0.0023 & 0.0022 \\
Year 1998_06 dummy variable & -0.0015 & -0.0016 & $-0.0034^{*}$ \\
Constant & $0.1878^{* * *}$ & $0.1809^{* * *}$ & -0.0005 \\
Adjusted R-squared & 0.0143 & 0.1107 & 0.0909 \\
Wald Chi & $5.81^{*}$ & $10.08^{* *}$ & $14.05^{* *}$ \\
P-value(F) & 0.0621 & 0.0865 & 0.0904 \\
No of observations & 45 & 45 & 45 \\
\hline
\end{tabular}

Level of Significance: $* \mathrm{p}<0.10 ; * * \mathrm{p}<0.05 ; * * * \mathrm{p}<.01$

Note: Fixed-effects, using 45 observations; included 3 cross-sectional units; Time-series length $=15$;

Dependent variable: Inequality in Children full immunisation

\subsubsection{Demographic [Fertility] convergence and its linkages with Health inequalities}

This section assessed the association between demographic [Fertility] convergence and its linkage with child health inequalities. Demographic convergence was considered only in terms of fertility convergence. Though, in the conceptual framework I have also shown a linkage between mortality convergence and child health inequalities, but its direct association would be much lesser because child health and mortality indicators like IMR and LEB are closely associated. Therefore, the analyses have been restricted to only fertility convergence and child health inequalities.

The results in case of association between fertility convergence and inequalities in children full immunisation reveals that both absolute and relative inequalities in children full immunisation were low when there was a greater divergence $(\beta=.0706$, $\mathrm{p}<0.020$ ) in progress of fertility decline during 1981-91. The progress in fertility decline during 1991-2001 in India showed evidence of convergence (-0.012). In spite of convergence in progress of fertility decline, both absolute and relative inequalities in children full immunisation were higher in 1998-99 (AID=14; Gini index $=0.32$ ) 
compared to 1992-93 ( $\mathrm{AID}=10$; Gini index $=0.27$ ). Nevertheless, when the volume of convergence in progress of fertility decline have strengthened $(\beta=-0.046)$, both the absolute and relative inequalities in children full immunisation coverage have declined by 4 percentage points in AID and ten units in Gini index.

However, both absolute and relative inequalities in case of children underweight increased by 3 percentage points in AID and 4 units in the Gini index during 1992-2006 in spite of convergence of progress in fertility decline during 1991-2001 and 2001-09. The results in case of NNMR showed a different pattern compared to that of children full immunisation and children underweight. The absolute and relative inequalities in NNMR were high when there was divergence and declined with convergence until 1998-99. However, it increased when the volume of convergence was high for the period, 1999-2006. Though, the increase was not much in absolute inequalities which showed only a 1 percent rise, but relative inequalities, increased by 5 units. Overall, for the three child health indicators, the decline in absolute and relative inequalities was not consistently associated with the convergence in fertility decline. Two out of three indicators were increased with the increase in the volume of convergence over the progress of fertility decline (Figure 8.11 ).

Figure 8.11. Demographic Convergence and its linkages with child health inequalities

Fertility convergence and inequalities in children full immunisation, 1992-2006

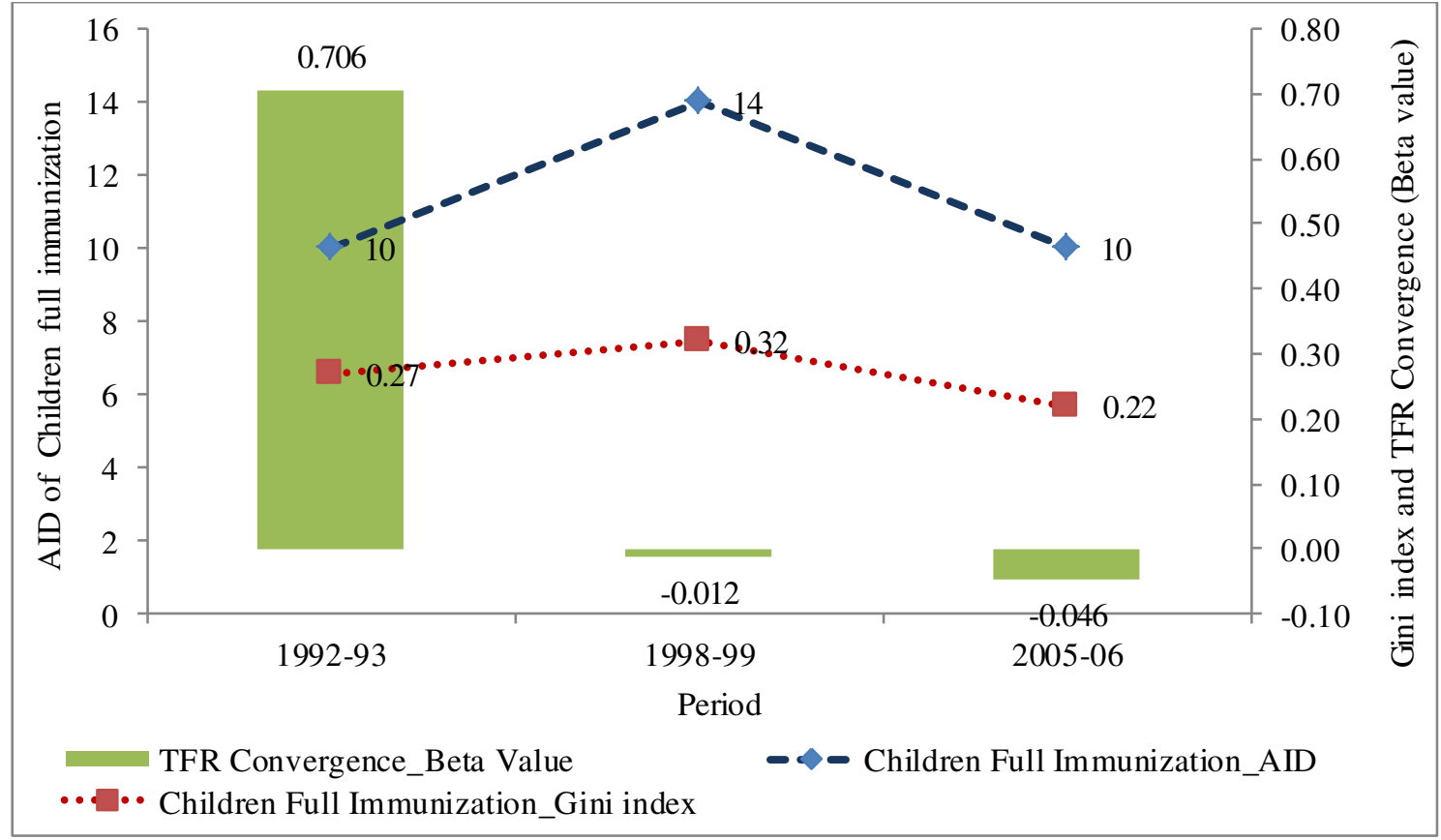


Fertility convergence and inequalities in children underweight

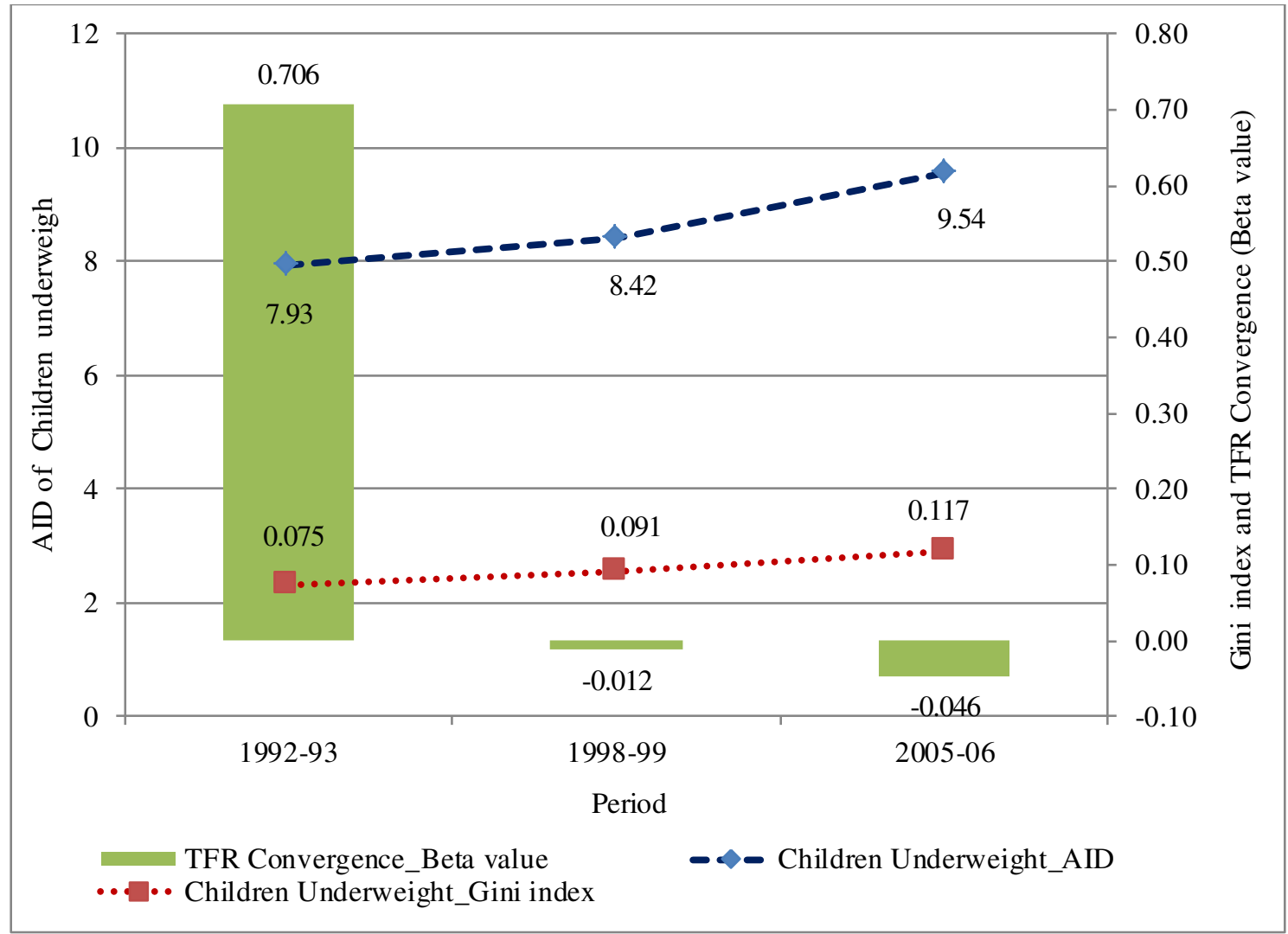

Fertility convergence and inequalities in neonatal mortality rate

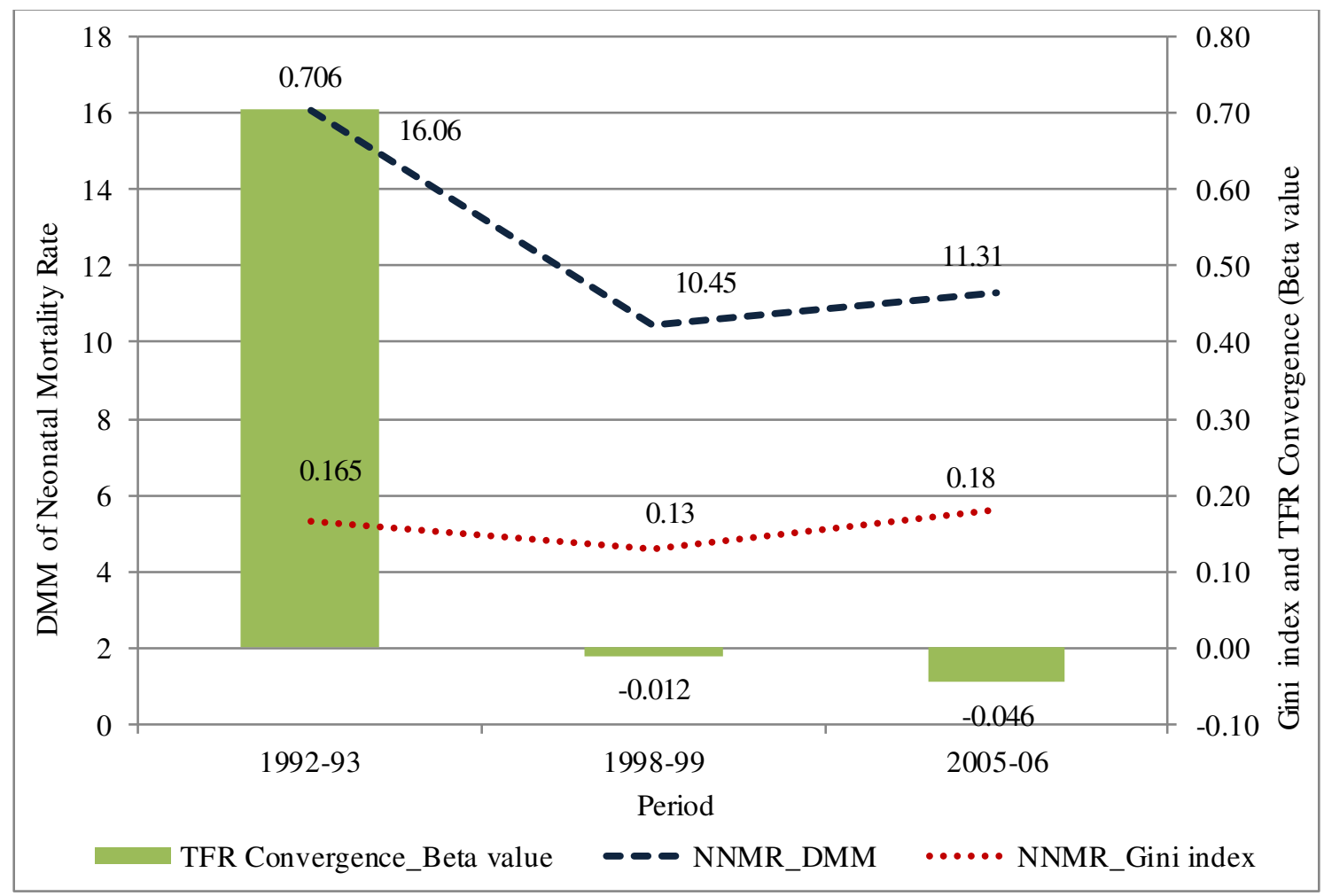




\subsection{Discussion}

The assessment of the association between change in averages of socioeconomic and demographic factors and inequalities in children full immunisation, children underweight and neonatal mortality rate during 1992-2006 revealed a number of intriguing findings. The association between change in averages, absolute and relative inequalities in children full immunisation, children underweight and neonatal mortality rate foster a decrease in absolute inequalities and an increase in relative with an increase in averages in the same indicators. The association between change in averages of children full immunisation, children underweight and neonatal mortality rate with socioeconomic inequalities in the same indicators suggested that socioeconomic inequalities in children full immunisation, children underweight and neonatal mortality rate increased with the increase in averages of children full immunisation and decreased in children underweight and neonatal mortality rate.

Second, the results indicated that the increase in NSDP is associated with an increase in relative inequalities in children full immunisation coverage, children underweight and neonatal mortality rate during 1992-2006. Similarly, the decline in poverty ratios is also contributing to the increase in relative inequalities in children's health status. The association between changes in literacy rate and children full immunisation, children underweight and neonatal mortality rate also foster the negative relationship that increase in literacy rate could help to reduce absolute inequalities, but not relative and socioeconomic inequalities in children underweight. The association between change in the TFR and children full immunisation, children underweight and neonatal mortality rate also indicate that the decline in TFR is associated with an increase in socioeconomic inequalities in children's health status.

The results of pooled OLS regression and fixed effects model estimates from Panel data regression revealed that before adjusting for other socioeconomic indicators like averages of NSDP, poverty ratios, literacy rates and the progress in demographic indicators like TFR was associated with an increase in inequalities in children's health status. However, after controlling for socioeconomic indicators (that steady condition of the states in term of socioeconomic status), the progress in TFR was associated with a decrease in inequalities in children's health status. Thus, it indicated that the benefits 
or fruits of demographic transition and convergence showed a more equal distribution among the societies with 'lesser pre-existing socioeconomic inequalities'. The plot of association between convergence in trends in the TFR and inequalities in children full immunisation showed that in spite of convergence in TFR during 1992-1998, both absolute and relative inequalities have increased. However, when fertility convergence progressed during the later period, 1998-2006, both the absolute and relative inequalities in children full immunisation has also declined. However, in spite of fertility convergence, SES inequalities in children underweight and neonatal mortality rate continued to rise. 


\section{CHAPTER 9}

\section{Distance to Line of equity and Line of highest achievement: How much demographic differences are avoidable in India}

\subsection{Background and rationale}

The earlier chapters foster that in spite of progress in averages of key demographic indicators in India, the differences across the states and key social groups continue to persist. Though, different measures of convergence in the averages and absolute differences in fertility, mortality and child health indicators are leading to different conclusions based on the type of measure and indicator, but relative and non-parametric measures of convergence continue to show the evidence of divergence in majority of the indicators (Chapters 4-7). Thus, for the majority of the indicators, convergence is yet to happen in terms of relative progress. Such conditions provide rationale to set priorities for the future prospects of demographic and health convergence across the states and social groups in India. However, it needs identification of laggard states and social groups from line of equity. Further, the quantification of avoidable differences and achievable progress in terms of demographic and health indicators is required for priority setting in the policies. Avoidable differences are potentially useful performance measure because their center of attention is on the objective of reducing the gap, namely, convergence. The identification and quantification of gaps will not only evaluate the progress under various intervention programs undergoing in India to avoid socioeconomic inequalities in demographic and health status, but they will be useful to set priorities towards a common goal of achieving the right to the highest attainable standards of health for all. Further, strengthen the existing policies and develop new strategies for future prospective of convergence.

However, till now, in the subject of demography the word 'avoidable' is widely used for only in mortality studies to estimate the 'cause-specific avoidable mortality' levels based on the 'multiple decrement life table approach' (Bauer et al., 1986; Car-Hill et al., 1987; Blumenthal, 1996; Anderson et al., 1978; Hisnanick et al., 1995; Baker et al., 1995; Gorey et al., 1997; Treurniet et al., 1999). However, in this chapter, I have not focused only on the avoidable mortality, but also for the first time extending this concept for other demographic indicators. The estimation of 'cause-specific avoidable 
mortality' is entirely a different procedure and not suitable for other demographic indicators like fertility and child health indicators. Moreover, the interest of this study is to focus on inequality and convergence, therefore, I have used the measures of difference, dispersion and inequality based graphical tools to show the scatterings and also quantify the avoidable size of differences in case of key demographic indicators. Thus, the key objective of this chapter is to plot the dispersion of states and quantify the avoidable size of differences in key demographic indicators.

\subsection{Methods and materials}

\subsubsection{Data}

The data source for fertility and mortality indicators in this chapter is the latest SRS, but for the data on child health indicators, the recent NFHS survey has been used (for more details see chapter II).

\subsubsection{Methods}

To make need assessment of future prospects of health equity across the states and socioeconomic groups, this chapter has used three methods: 1) Funnel plots, 2) Distance to line of equity plots and 3) Distance to line of highest achievement plot. Details of these methods have been discussed in chapter 2 (Methodology part).

\section{3. Results}

\subsubsection{Funnel plots}

Plotting of funnel charts need two components that 1) the indicator estimates in terms of percentage or in any other unit, and 2) the exposed population for which the indicator is measured. However, the SRS data won't provide the information of the exposed population for any of the indicators it measures. Therefore, these plots were measured only for the child health indicators which were estimated based on National Family Health Survey-3 data. Results of funnel plots in case of children full immunisation for the years 2005-06, revealed that out of 28 states, only 7 states were located between confidence limits of the national average. However, the 12 states were located above the upper confidence limits and 9 states were located below the lower confidence limits. There was a $60 \%$ gap between Tamil Nadu located above the upper confidence limits and Nagaland was well below the lower confidence limits. In case of 
children underweight, the results revealed that only seven states were above the upper confidence limits, but the majority of the states (12 states) were below the lower confidence limits. The only 10 states were located in between the confidence limits. Most of the states located above the upper confidence limits were socioeconomically poorer states. However, surprisingly economically developed states like Gujarat, was located above the upper confidence limit. The observation of the scattering of states showed that there was near about 30\% gap between the Madhya Pradesh, located above the upper confidence limit and Mizoram, located below the confidence limits (Figure 9.1).

Figure 9.1. Funnel charts: Measuring of dispersal of States from line of the national average in terms of child health care and outcome indicators

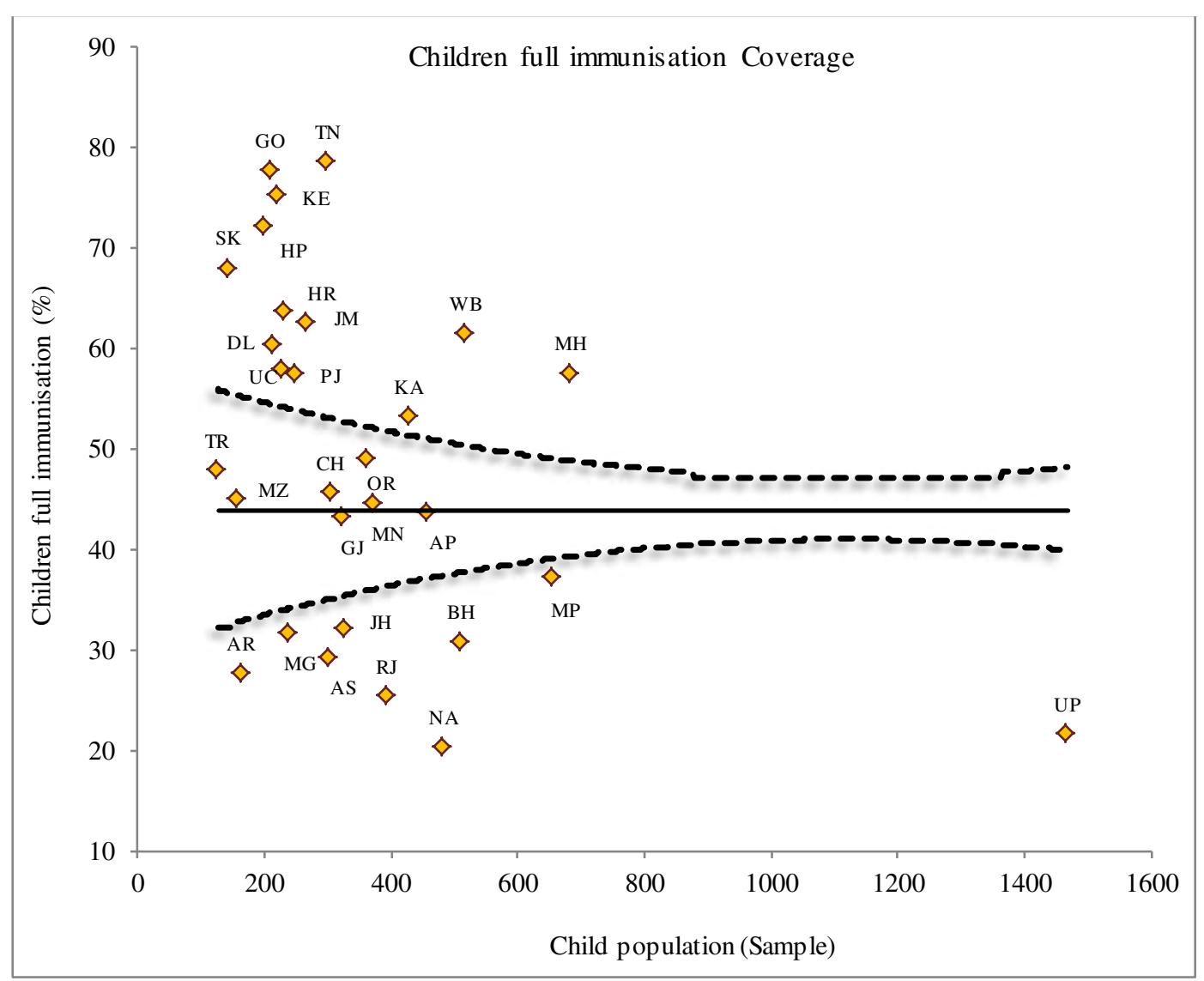



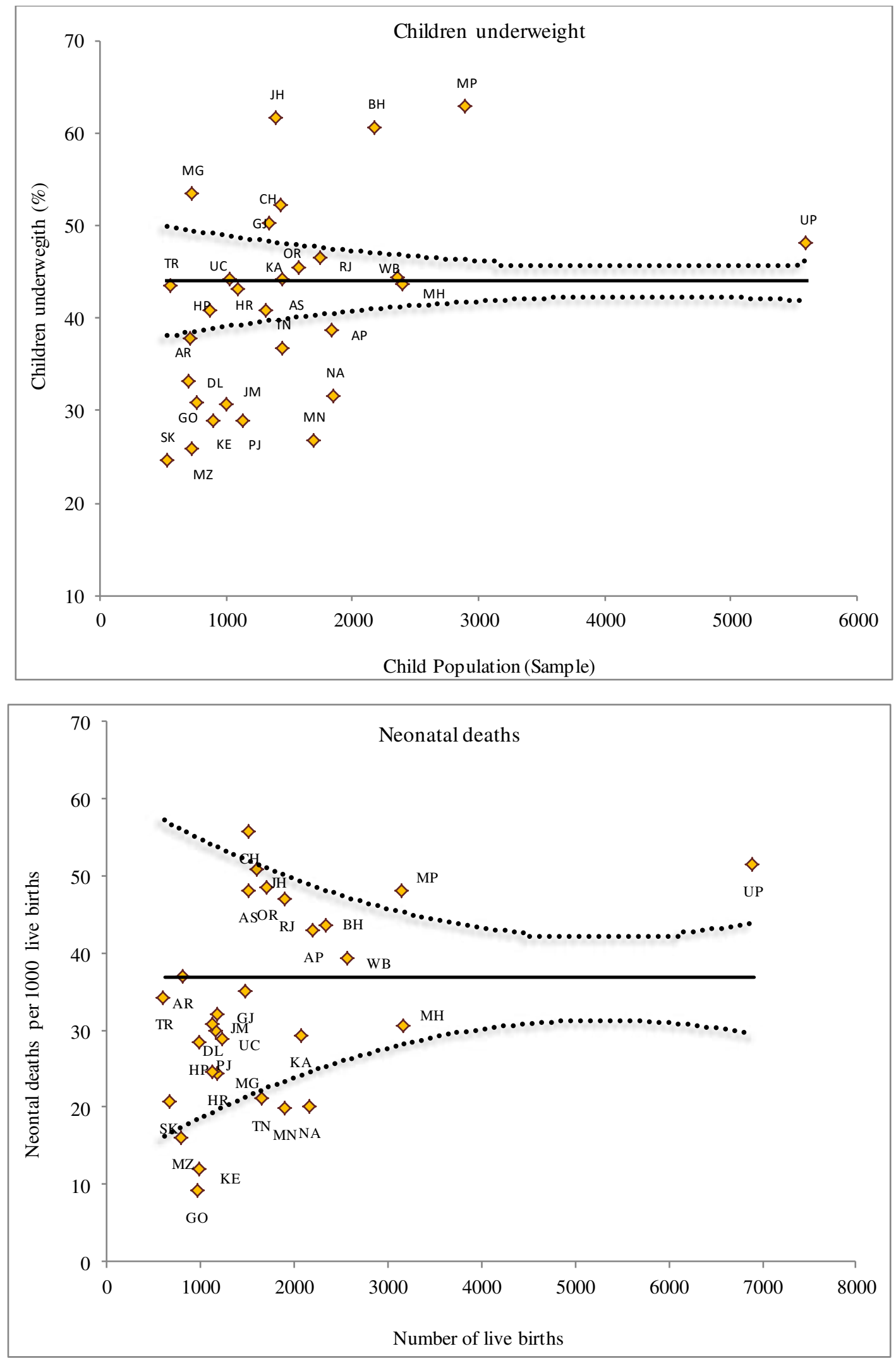


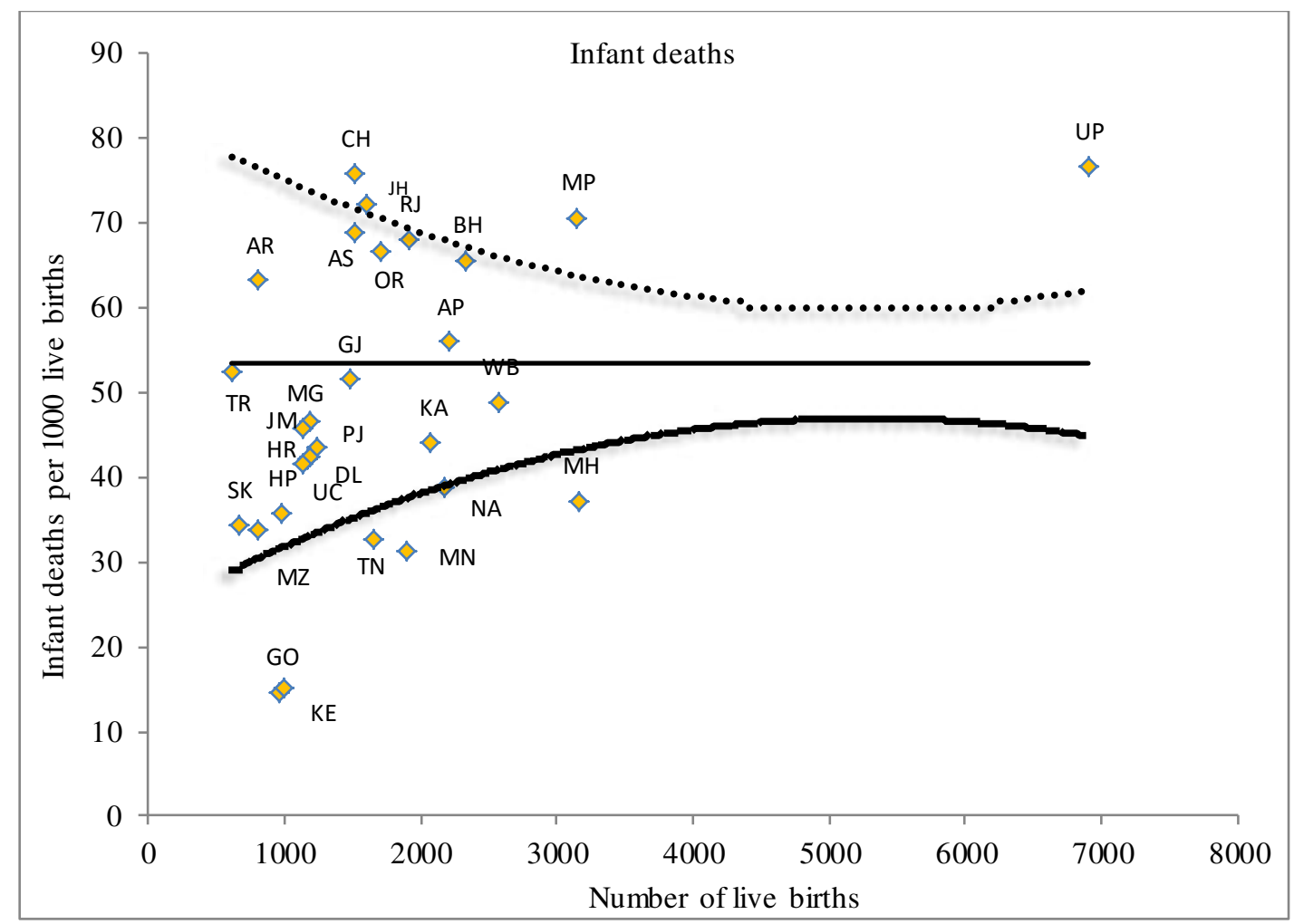

Note: doted lines are confidence limits. The middle straight line is the line showing the national average of the selected indicator.

Compared to children full immunisation and underweight, funnel plots for neonatal mortality across the states revealed that the majority of the states (19 states) were falling within the confidence limits. Only three states were located above the upper confidence limits and six states were located below the confidence limits. However, the interesting point need to be noted in case of neonatal mortality rate was the length of the confidence interval. It was large, ranging from 15-55 neonatal deaths per 1000 live births. This could be one reason why many of the states fall within the confidence limits. However, within the confidence limits scattering of the states was not around the national average, but skewed towards lines of upper and lower limits. Within the confidence limits, 12 states were located below the national average line and 7 states were located above the national average line. This indicates that the majority of the states fall below the national average lines which were located at a greater distance from the national average line.

A similar kind of situation was also evident in case of infant mortality rate that majority of the states were located within the confidence limits. Out of total 28, 18 were states located within the confidence limits and within this, 12 were below the national average 
and six states were placed above the line of the national average. Among remaining states, four states were located above the upper confidence limits and six states were located below the lower confidence limits. Akin to neonatal mortality, in case of infant mortality also the results showed that though scattering of infant deaths fall within the confidence limits, the confidence interval was much greater and scattering of the states within the confidence limits were skewed towards the lines of confidence limits. Probably, this could be the one reason why in spite of evidence of catching-up process in neonatal and infant mortality rates, the results in previous chapters showed that there was no evidence of clear convergence process in these indicators rather there was an evidence of convergence clubs. The funnel plots also strengthen the argument of

convergence clubs rather than absolute convergence. For achieving convergence, India need to reduce 75 infant deaths per 1000 live births between upper most located state of U.P and lower most located state of Kerala (Figure 9.1).

\subsubsection{Line of equity plots: TFR}

Figure 9.2 shows the line of equity plots in terms of TFR by place of residence and socioeconomic status. The scattering of states around line of equity in between rural and urban areas revealed that concentration of most of the states were highly skewed towards the rural areas, thus, indicating higher total fertility rate in rural areas in comparison with urban areas. The results also indicated that states like Rajasthan, Madhya Pradesh, Gujarat and Assam were located at a greater distance from the line of equity whereas states like Kerala, Tamil Nadu, Punjab and Andhra Pradesh were much nearer to the line of equity. The line of equity plot of TFR by literacy rate revealed that TFR in most of the states was highly skewed towards the illiterate women. The plot showed that Bihar, Uttar Pradesh, Assam and Madhya Pradesh were located very far from the line of equity whereas Karnataka, Andhra Pradesh, Maharashtra was located near the line of equity. Interestingly, the total fertility rate in south Indian states like Kerala and Tamil Nadu were more skewed towards the higher educated women.

The line of equity plot in terms of TFR by castes revealed that most of the states were more skewed towards the SC/ST caste. The states like Bihar, Uttar Pradesh, Rajasthan and M.P. were located far from line of equity whereas states like Andhra Pradesh and Maharashtra were located near the line of equity. Also, the results showed that TFR in 
few states such as West Bengal, Assam, Haryana and Kerala were more skewed towards the general castes. The results of line of equity plots of TFR by economic status showed that, except in Kerala, among all other states, the total fertility rate was highly skewed towards the poorest quintile women. The result also showed that states such as Uttar Pradesh, Rajasthan, Assam and Madhya Pradesh were located away from the line of equity, but southern Indian states like Kerala, Tamil Nadu, Andhra Pradesh and Karnataka were located very close to the line of equity. Overall, the results of line of equity plots in case of TFR by place of residence and socioeconomic factors showed that there is a bigger gap to be reduced in order to bring all the Indian states to the line of equity. Unless such distance is reduced, the future prospects of absolute convergence in TFR across the states and socioeconomic groups will not possible. Though, there was an evidence of catching of process by the demographically disadvantageous states such as Bihar, U.P., Rajasthan, M.P., Orissa and Assam, but still lot of gap need to be reduced between these states and south Indian states which were located much near to the line of equity.

Figure 9.2. Line of equity plots: Measuring the dispersion of states from the line of equity in terms of TFR by place of residence and socioeconomic status

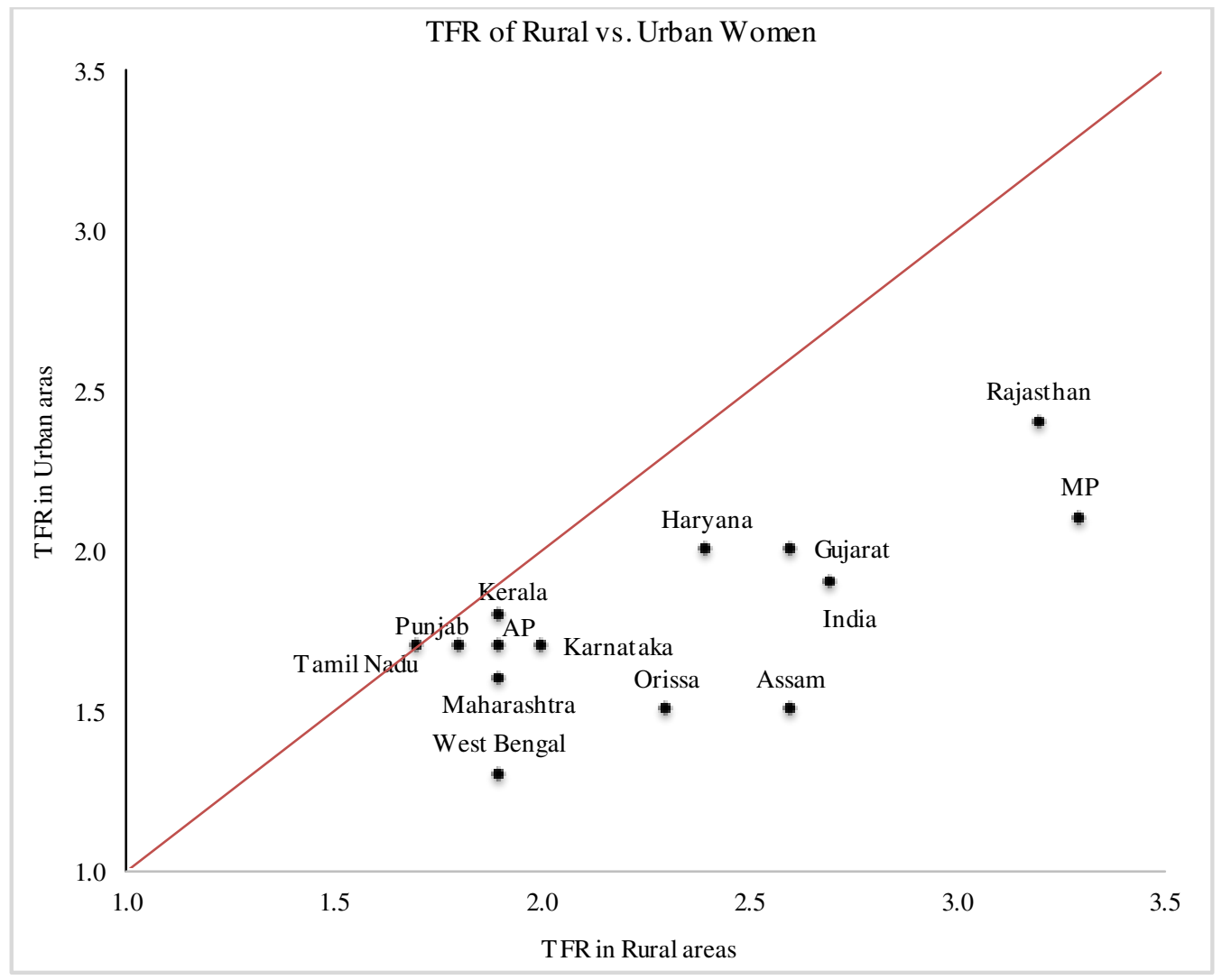



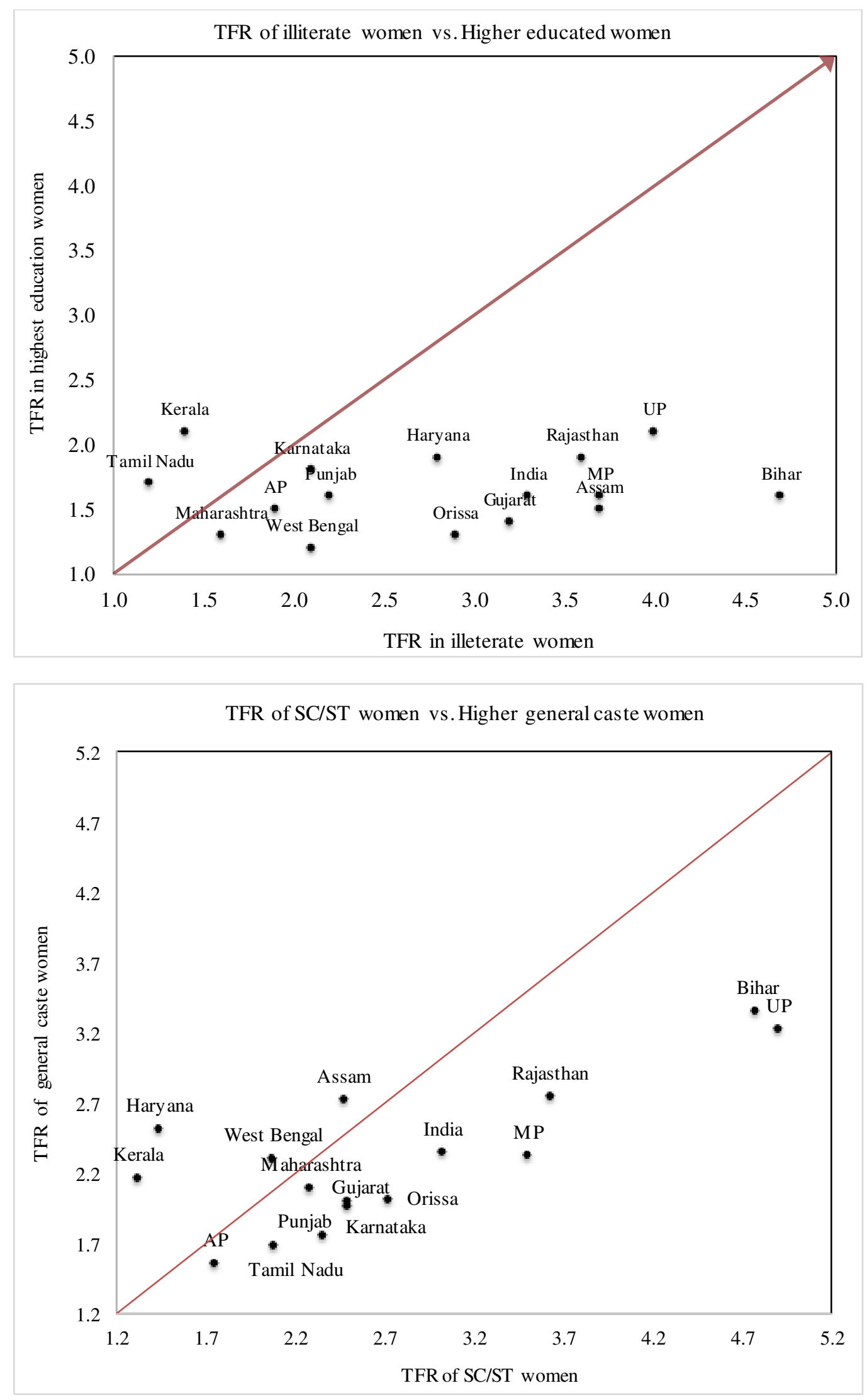


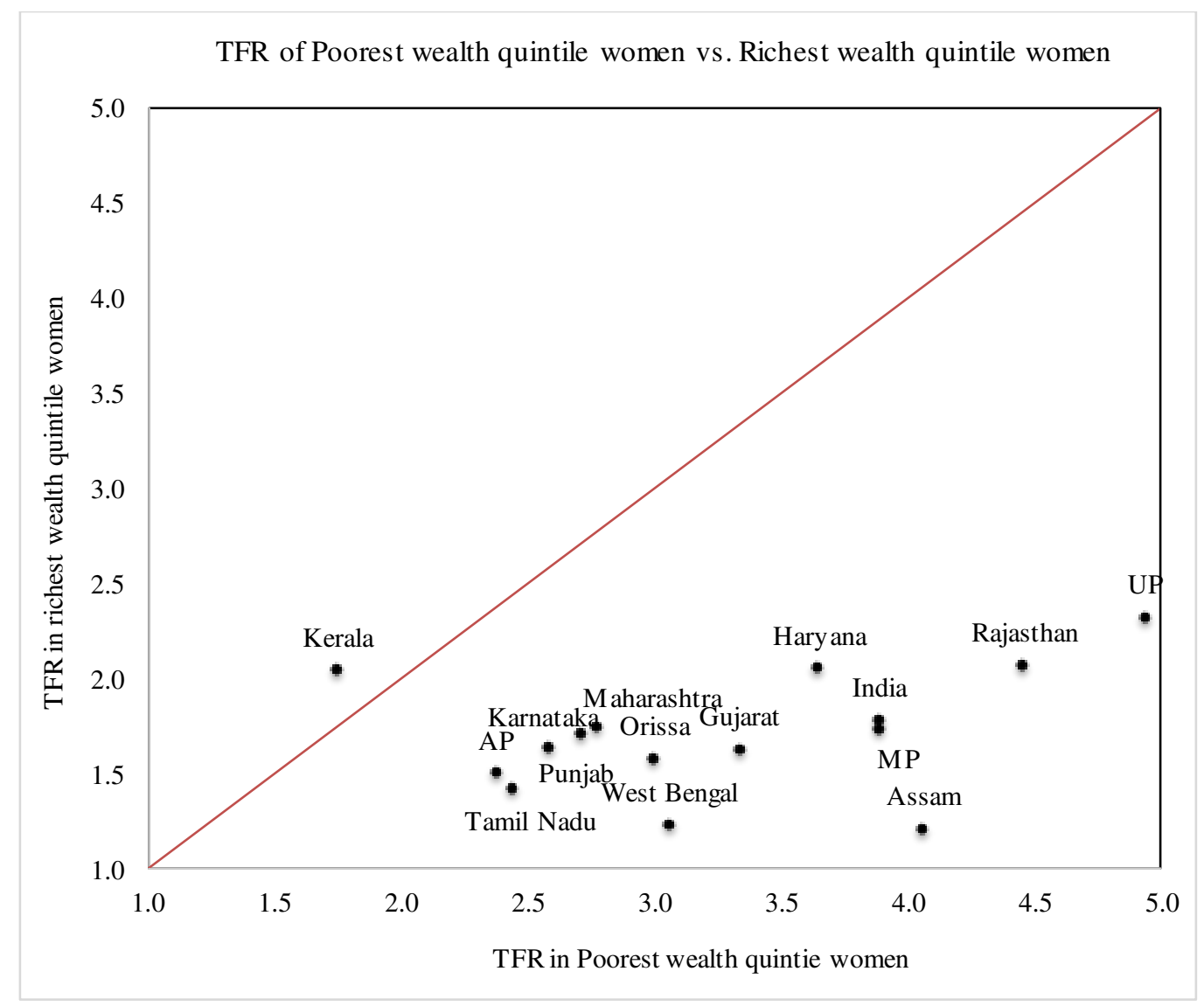

Mortality indicators

The results of the deviation of states from line of equity in terms of mortality indicators by place of residence are presented in figure 9.3. The results in case of LEB demonstrated that all the states were highly skewed towards the urban areas. The state like Kerala was located in close proximity to the line of equity. However, on the other hand, states like Bihar and Rajasthan are considered as the demographically disadvantageous states were located in greater distance from the line of equity. In general, the situation of other states was also not good, except Kerala, all other states were standing away from the line of equity. Figure 9.3 shows the results of the plot of line of equity in terms of infant mortality rate by place of residence. The results indicated that infant mortality rate among all the states were more skewed towards the rural areas. The result also revealed that most of the EAG states like Madhya Pradesh, Uttar Pradesh and Rajasthan were located away from the line of equity whereas Tamil Nadu, Maharashtra, Punjab and West Bengal were located close to the line of equity. 
In terms of infant mortality rate by wealth quintile, the result also showed that except Bihar, all the states were skewed towards the poorest wealth quintile. Bihar was located on the line of equity. The results also showed that few states such as Punjab and Kerala were closely concentrated near the line of equity whereas states like Gujarat and Maharashtra were located far from the line of equity. However, the location of Bihar on the line of equity needs to be understood carefully because this is a state where the prevalence of infant mortality is high. There could be one reason that the prevalence of infant mortality in Bihar is high among both rich and poor economic status households. For absolute convergence to happen in IMR across the states by socioeconomic groups there is a lot of gap which needs to be reduced between EAG and south Indian states.

Figure 9.3. Line of equity plots: Measuring the dispersion of states from the line of equity in terms of Mortality indicators by Place of Residence and Socioeconomic Status

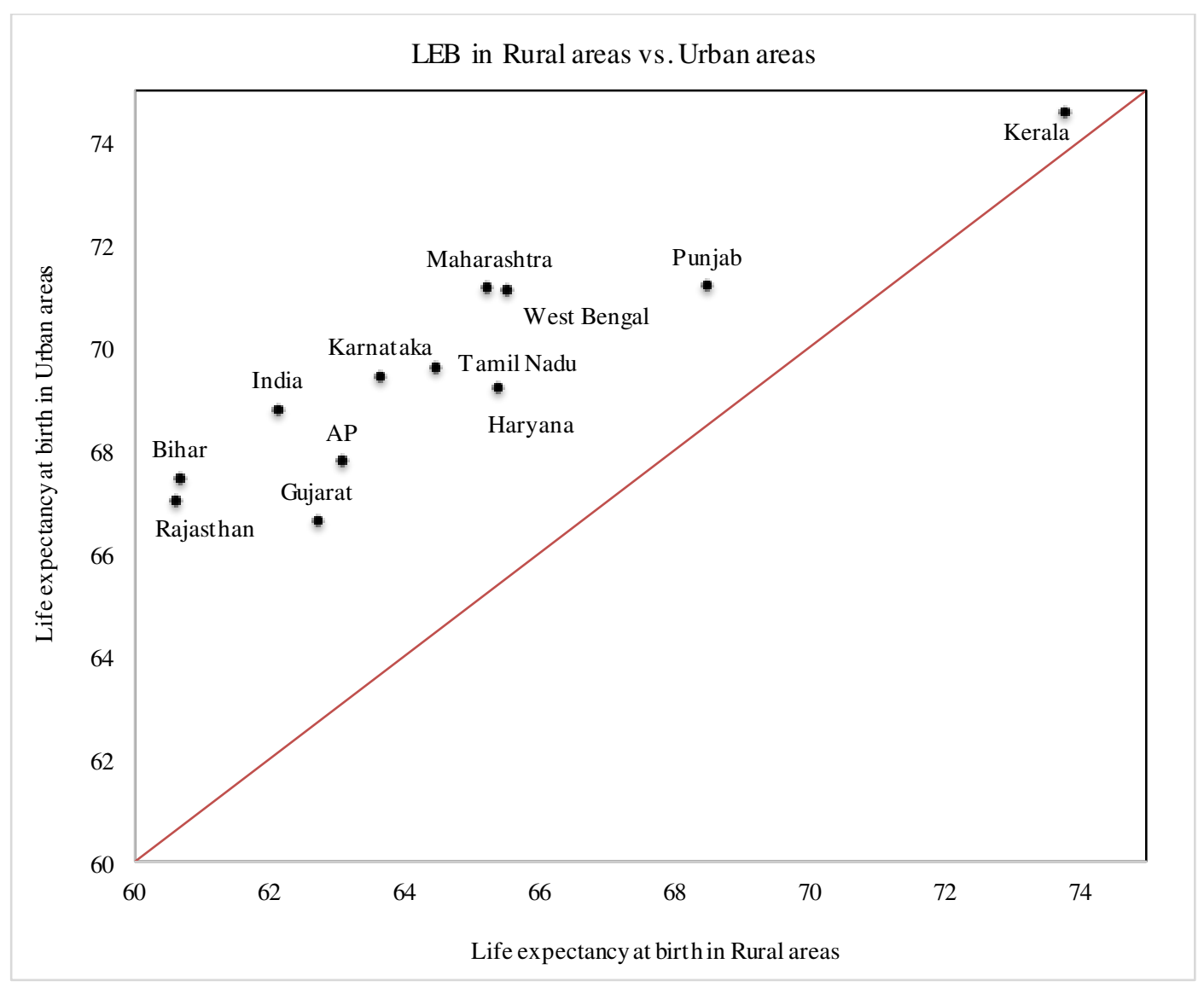



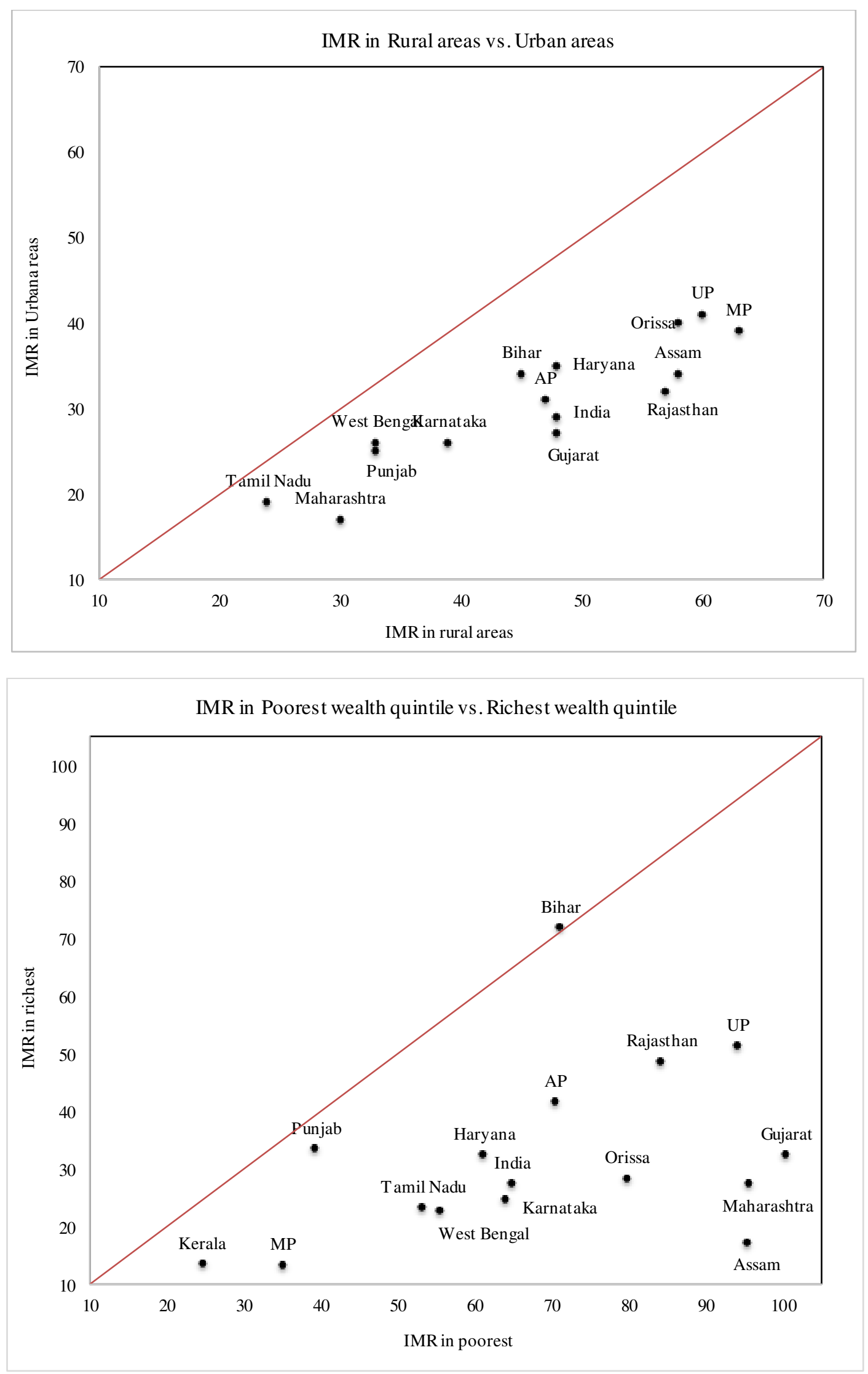
Child health care and outcome indicators

Figure 9.4 displays the plots of line of equity in terms of child health care and outcome indicators by place of residence and socioeconomic status. In case of full immunisation of children by place of residence, the result showed that most of the states were skewed towards the urban areas and located near the line of equity. Interestingly, the results also indicated that full immunisation coverage among children in three south Indian states and Maharashtra were skewed towards the rural areas. Further, Kerala and Tamil Nadu which are considered to be demographically advanced states with higher children full immunisation levels were located away from the line of equity whereas Assam was located close to the line of equity. The contrasting phenomena of rural advantage in these states need to be understood with separate study on these particular states. However, my wild guess is that the better rural health care system in these states led better health outcomes. The plot of line of equity in terms of full immunisation by education showed that among all the states, full immunisation coverage was highly skewed towards higher educated women. While Tamil Nadu was located nearer to the line of equity followed by Kerala, West Bengal and Maharashtra, the socioeconomically disadvantageous states such as Bihar, Uttar Pradesh and Rajasthan were located away from the line of equity. A similar situation was also evident in the plots of line of equity in case of children full immunisation coverage by the economic status of mothers. The results showed that coverage of children full immunisation was highly skewed towards the women with richest economic status compared to poorest economic status. Such inequality was very high in the economically developed states like Haryana and also economically weaker states like Bihar and M.P. However, two south Indian states, such as Tamil Nadu and Kerala were standing close to the line of equity.

The plots of line of equity in case of children underweight by place of residence revealed that the prevalence of children underweight was highly skewed towards the rural areas. Except, all India average, none of the states were located very near to the line of equity. There was no clear pattern emerged from the results. Among the states nearer to the line of equity includes both advantageous and disadvantageous such as Punjab, Tamil Nadu, Maharashtra, Uttar Pradesh and Gujarat including India. Similarly, those states which were located away from the line of equity also included 
the states like West Bengal, Kerala and Rajasthan. The results also showed that the concentration of children with underweight was highly skewed towards the illiterate women. A comparison of children underweight among illiterate and literate mothers indicated that almost all the states were located at a greater distance from the line of equity. Kerala was located somewhat nearer to the line of equity. On the other hand, state like Madhya Pradesh was located away from the line of equity. The plots of line of equity in children underweight by mother's economic status present a greater dispersal of states in terms of underweight among children of the poorest versus richest women. The results showed that the children underweight across the states was vastly skewed towards the poorest mothers compared to their counterparts. The Kerala was the only state located close to the line of equity, whereas, all the other states were positioned away from the line of equity. Moreover, Madhya Pradesh and Bihar were located at a greater distance from the line of equity. In case of NNMR by place of residence, the plot of line of equity presents a greater dispersal of states. Results indicated that the prevalence of NNMR was skewed towards the rural areas among all the states. However, the states like Kerala and Punjab were somewhat closely located to the line of equity in comparison with other states. The Empowered Action Group (EAG) states like Madhya Pradesh, Uttar Pradesh, Rajasthan and Orissa were located away from the line of equity. 
Figure 9.4. Line of equity plots: Measuring the dispersion of states from the line of equity in terms of child care and outcome indicators by place of residence and socioeconomic status
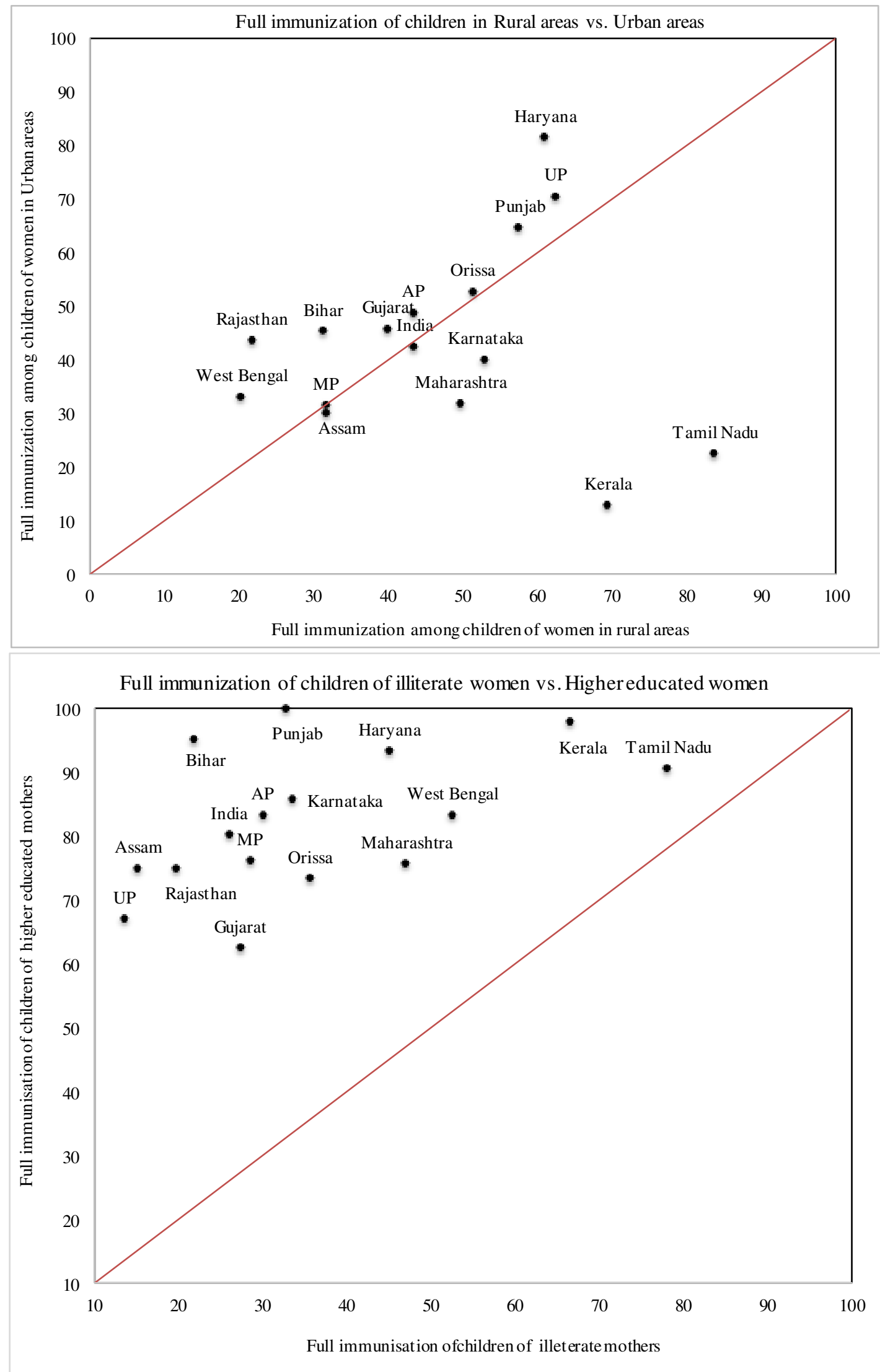

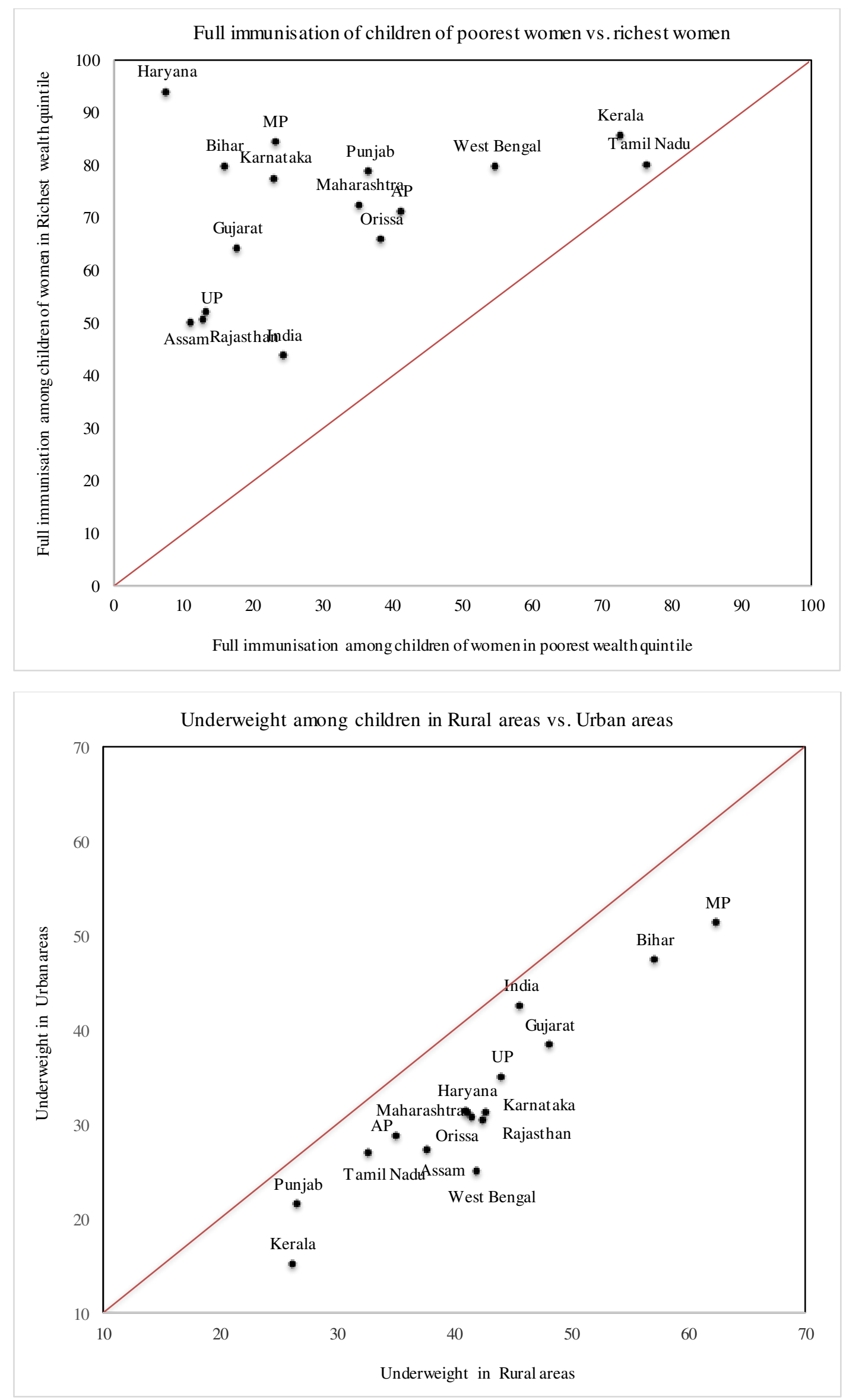

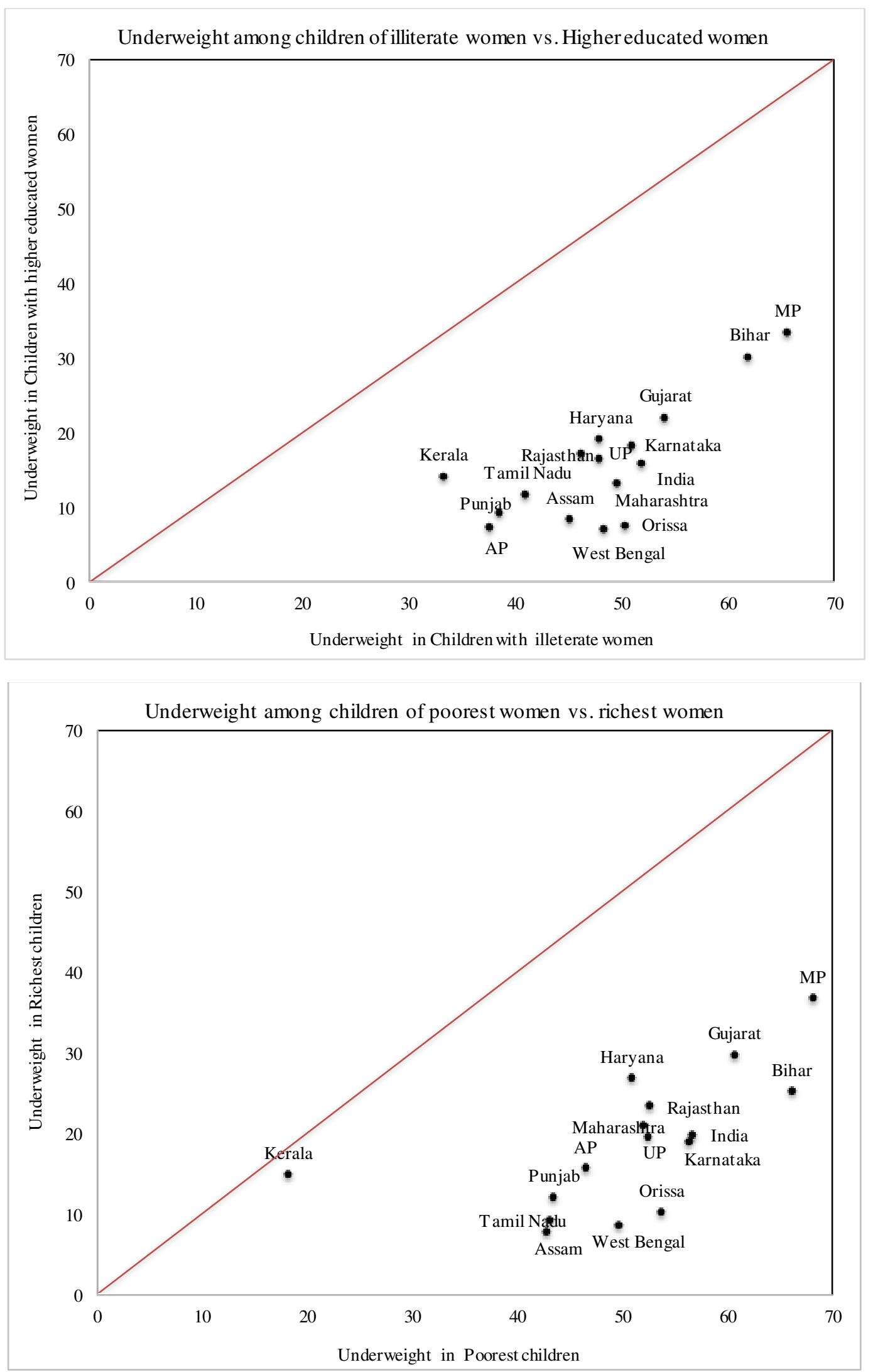


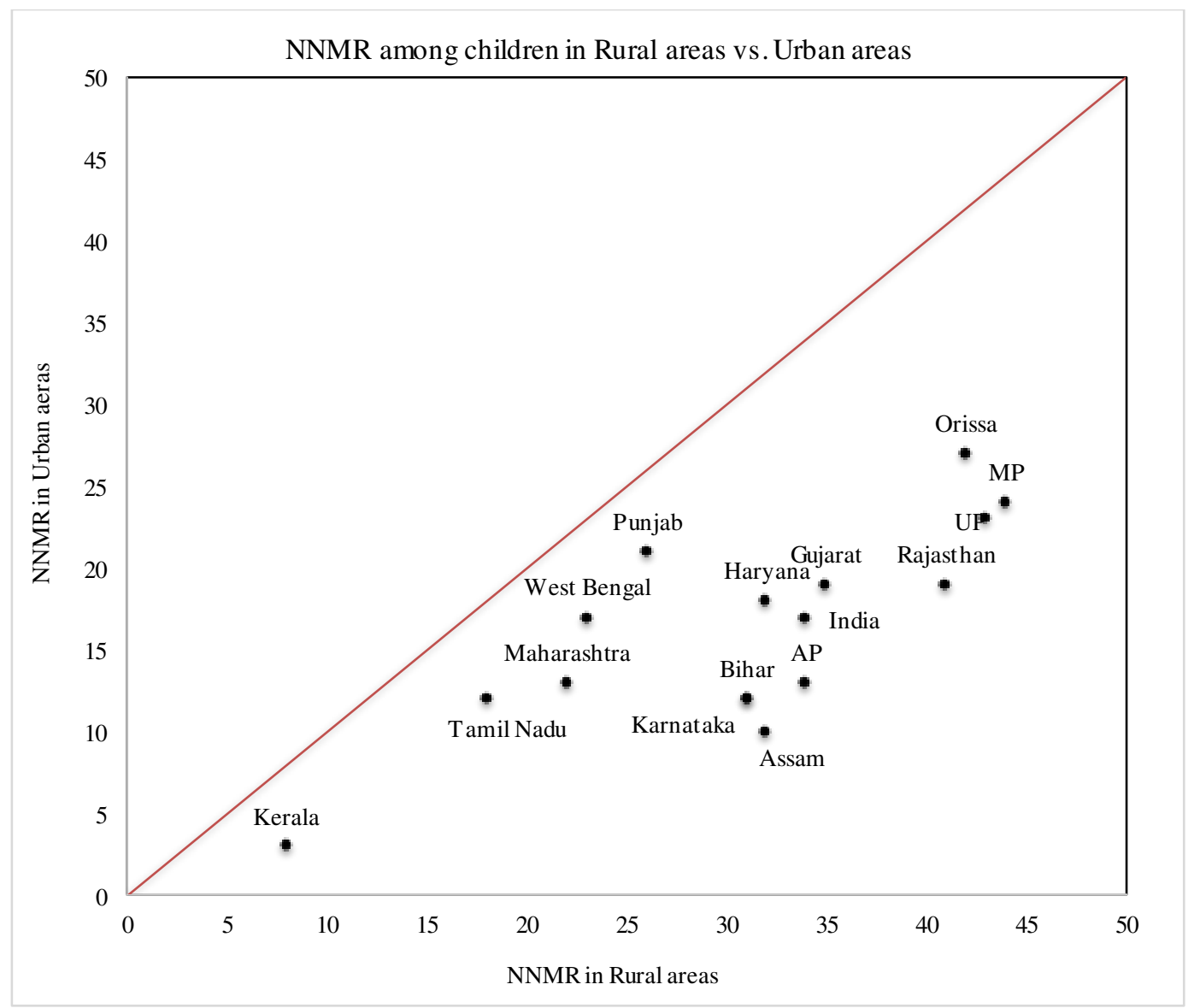

\subsubsection{Line of highest achievement: Avoidable demographic differences}

Figure 9.5 shows the measure of deviation of states from the line of highest achievement in terms of TFR by socioeconomic groups. The results showed that compared with reference line TFR of 2.1 , there was no avoidable fertility in seven states which comprises all the south Indian states and states like Maharashtra, Punjab and West Bengal. The level of avoidable TFR was greater in most of the north Indian states such as Bihar (1.5), Uttar Pradesh (1.3) and Madhya Pradesh (1). Similar results were evident in rural areas of the major states. There was no avoidable fertility in rural areas of the seven states, whereas, rural areas of Bihar (1.6), Uttar Pradesh (1.5) and Madhya Pradesh (1.2) have the highest avoidable fertility. However, in urban areas, only three states have avoidable TFR, but the urban areas of other states have already achieved below replacement level of TFR. The urban areas of Uttar Pradesh (1.4) and Bihar (1.4) have the highest avoidable TFR followed by Rajasthan (1.2). In case of illiterate women, the results revealed that there is no avoidable fertility among the south Indian states, Maharashtra, West Bengal and Punjab whereas states such as Bihar (3.5), Uttar Pradesh (2.8) and Madhya Pradesh (2.5) showed the highest avoidable fertility. 
Figure 9.5 Plots line of highest achievement: Measuring the dispersion of states from the line of highest achievement in terms of TFR by socioeconomic groups, 2011

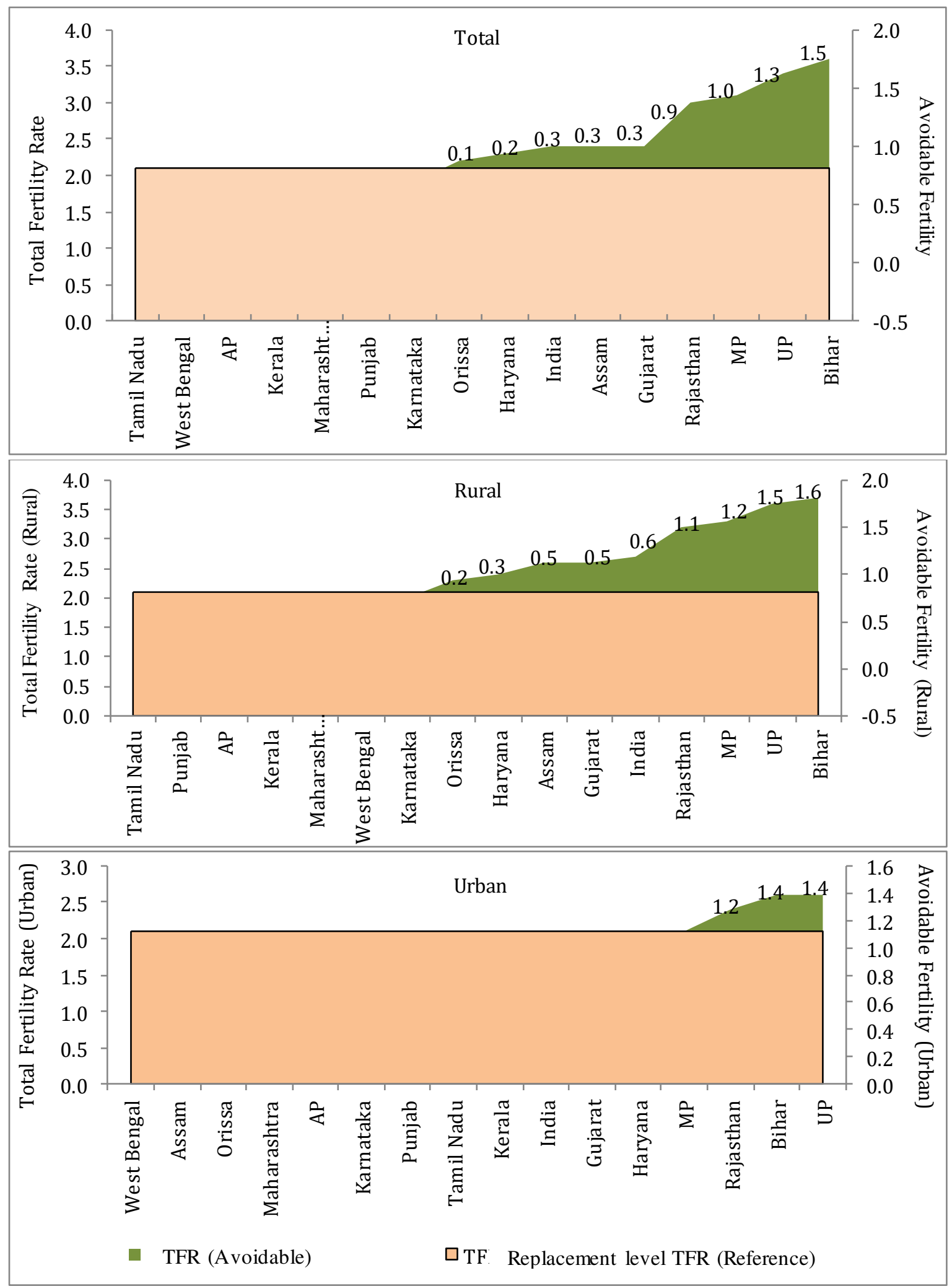



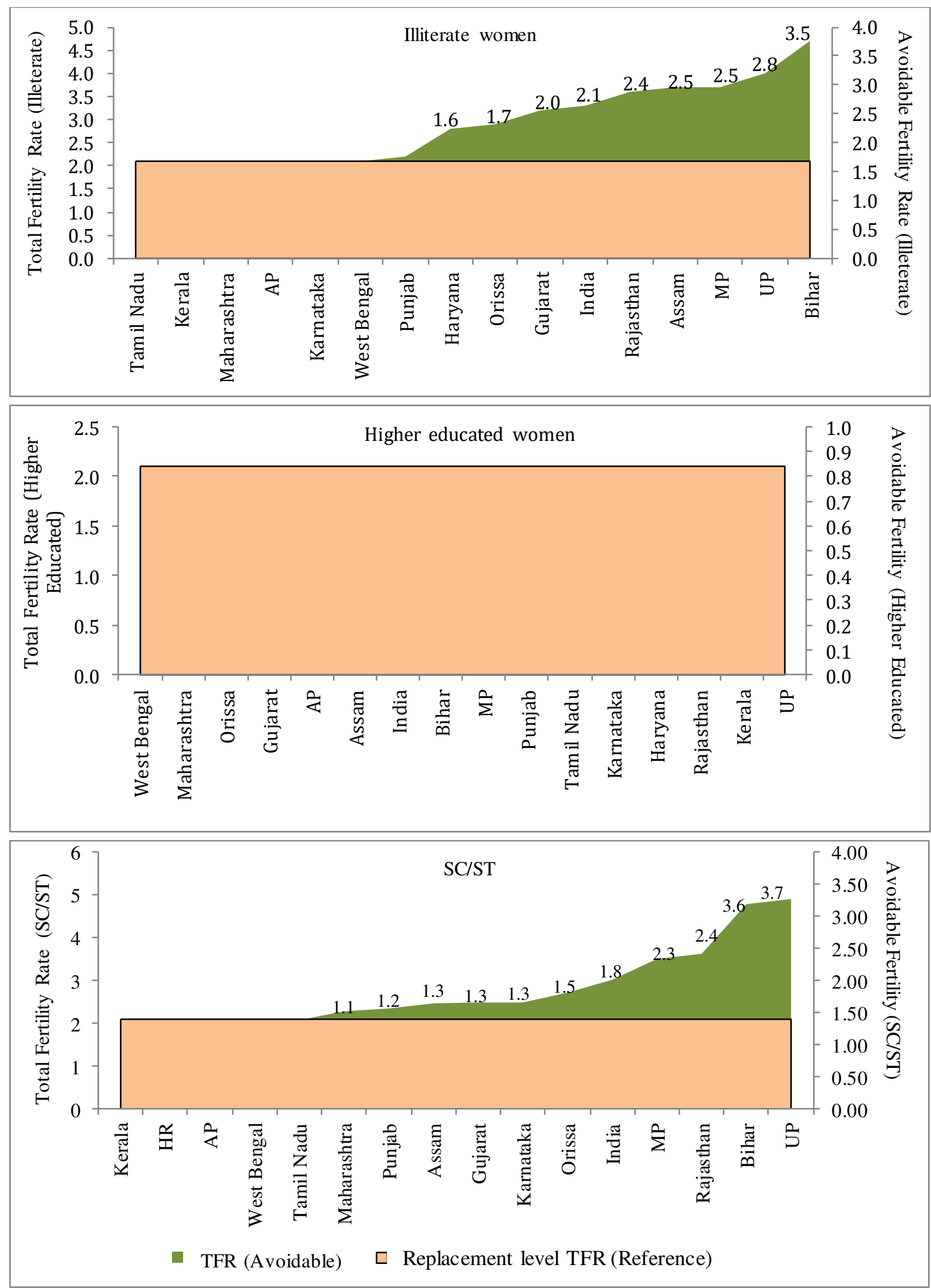

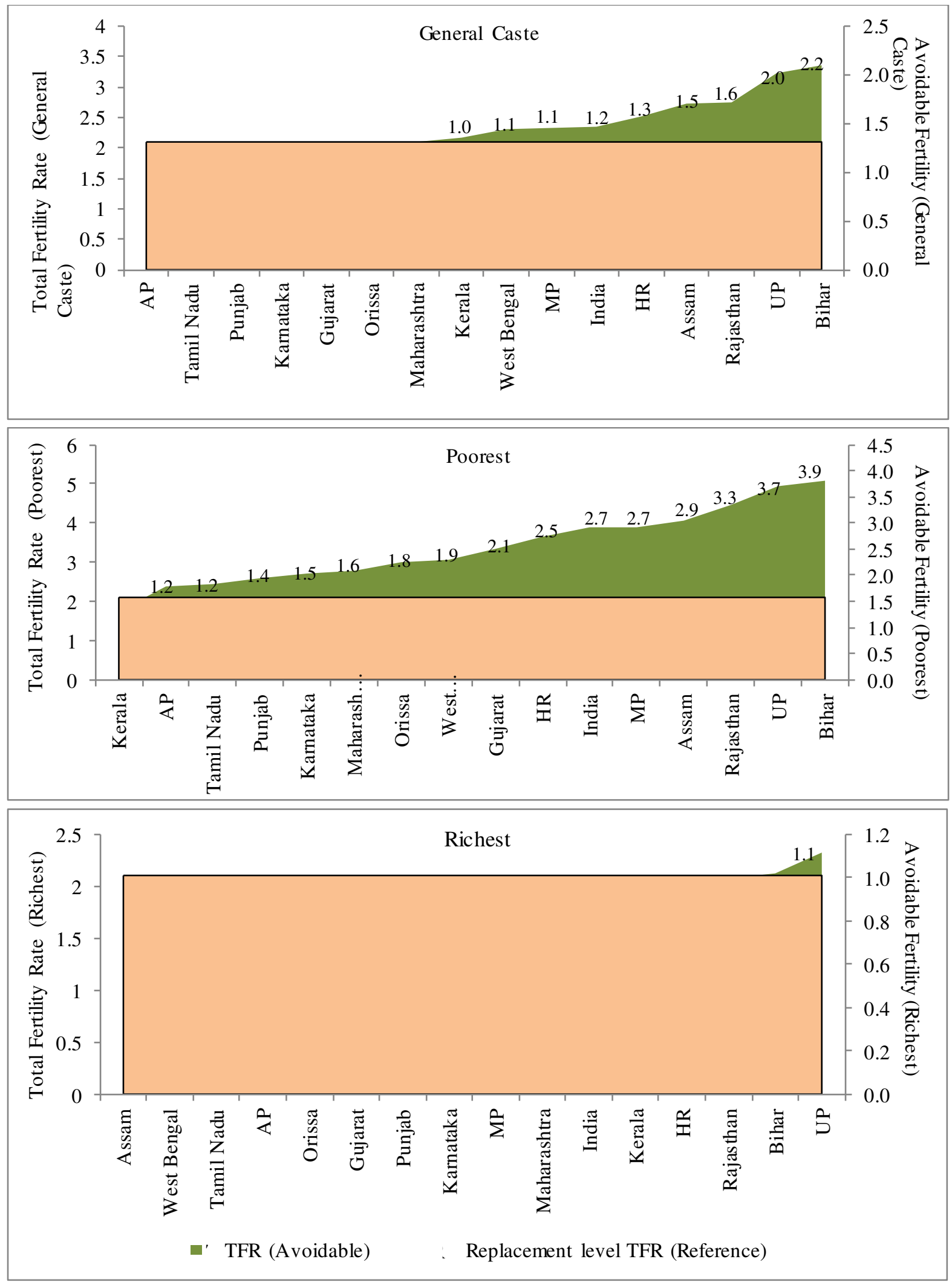
The results showed that there was no avoidable TFR among the highest educated women across all the states. In case of SC/ST caste women, the results showed that except five states, all the other major states have avoidable TFR. The highest avoidable TFR for SC/ST women was found in Uttar Pradesh (3.7), Bihar (3.6) and Madhya Pradesh (2.4). Among General caste women, seven states do not have avoidable TFR whereas, states like Bihar (2.2), Uttar Pradesh (2.0) and Rajasthan (1.6) have a highest avoidable TFR. Interestingly, the General caste of Kerala (1.0) also has some avoidable TFR. The results in case of women of poorest wealth quintile group showed that except Kerala, all the other states have avoidable TFR. However, avoidable difference was greater for the EAG states such as Bihar (3.9), Uttar Pradesh (3.7) and Rajasthan (3.3). The result showed that demographically the most advanced states like Kerala have the lowest avoidable TFR. However, among the richest women, except for Uttar Pradesh (1.1), there was no avoidable TFR among other states.

\section{Mortality indicators}

The results of the deviation of states from the line of highest achievement in terms of LEB among the rural areas showed that the achievable LEB was highest in all the major EAG states such as Madhya Pradesh, Assam, Orissa, Uttar Pradesh, Rajasthan and Bihar. In contrast, it was lowest in the rural areas of Kerala followed by Punjab and West Bengal. In case of urban areas, the results showed that Kerala has the lowest achievable life expectancy at birth, whereas, the highest achievable life expectancy at birth was found in Uttar Pradesh, followed by Madhya Pradesh. The results in terms of IMR showed that again, Kerala has the lowest avoidable infant mortality rate, whereas, Madhya Pradesh has the highest avoidable infant mortality rate. In case of both rural and urban areas, the results revealed that Kerala has achieved the lowest avoidable infant mortality rate followed by Tamil Nadu, whereas, Madhya Pradesh and Uttar Pradesh have the highest avoidable infant mortality rate. There are no avoidable infant deaths in urban areas of Kerala. Avoidable mortality estimates among poorest wealth quintile families revealed that Kerala has the lowest avoidable infant mortality rate followed by Punjab and Tamil Nadu, whereas, Gujarat has the highest avoidable infant mortality rate followed by Maharashtra and Assam. Among the richest wealth quintile families, Madhya Pradesh has the lowest avoidable infant mortality rate followed by 
Kerala and in same group Bihar showed the highest avoidable infant mortality rate (Figure 9.6).

Figure 9.6 Plots line of highest achievement: Measuring the dispersion of states from the line of highest achievement in terms of mortality indicators by socioeconomic groups
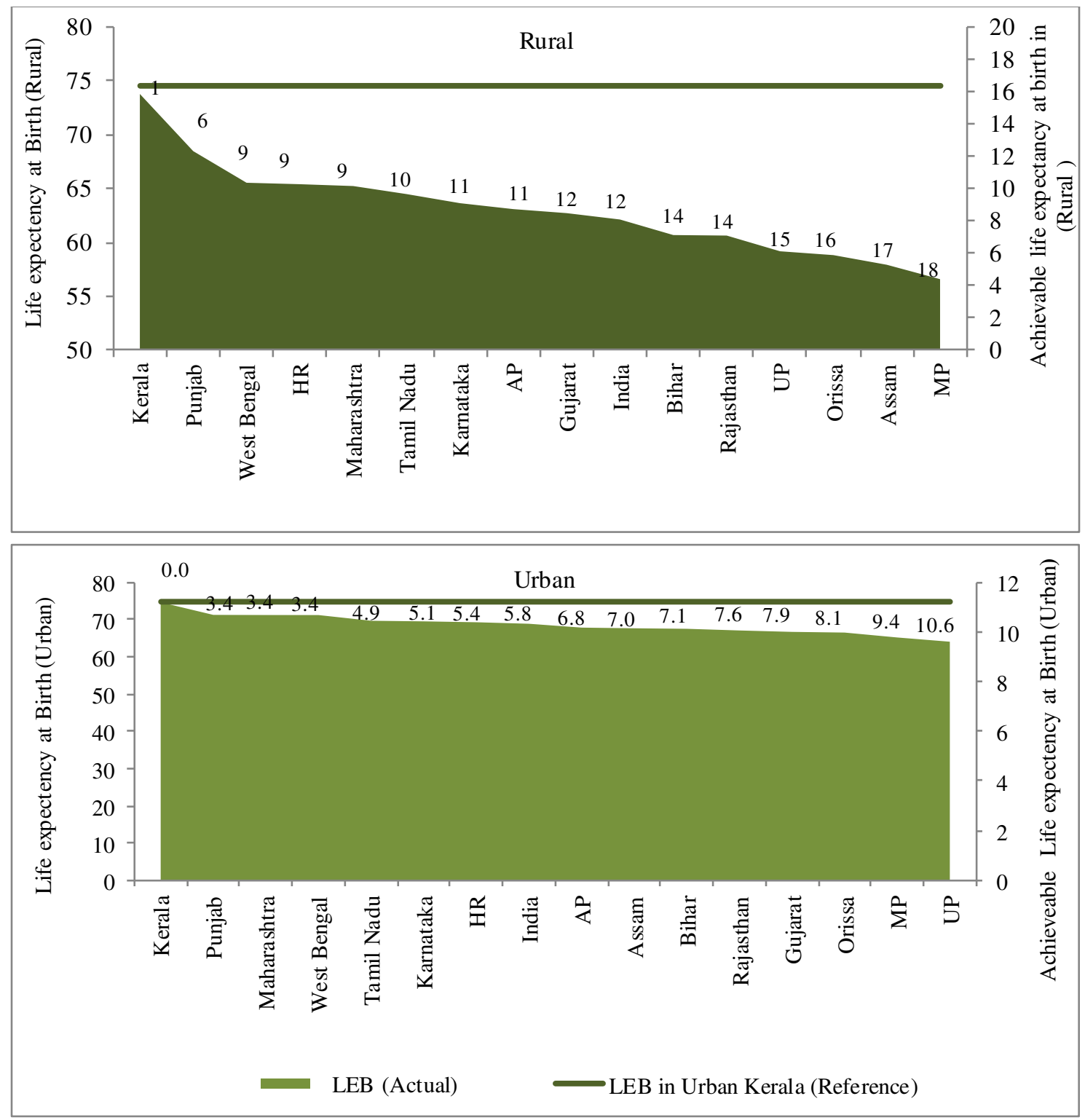

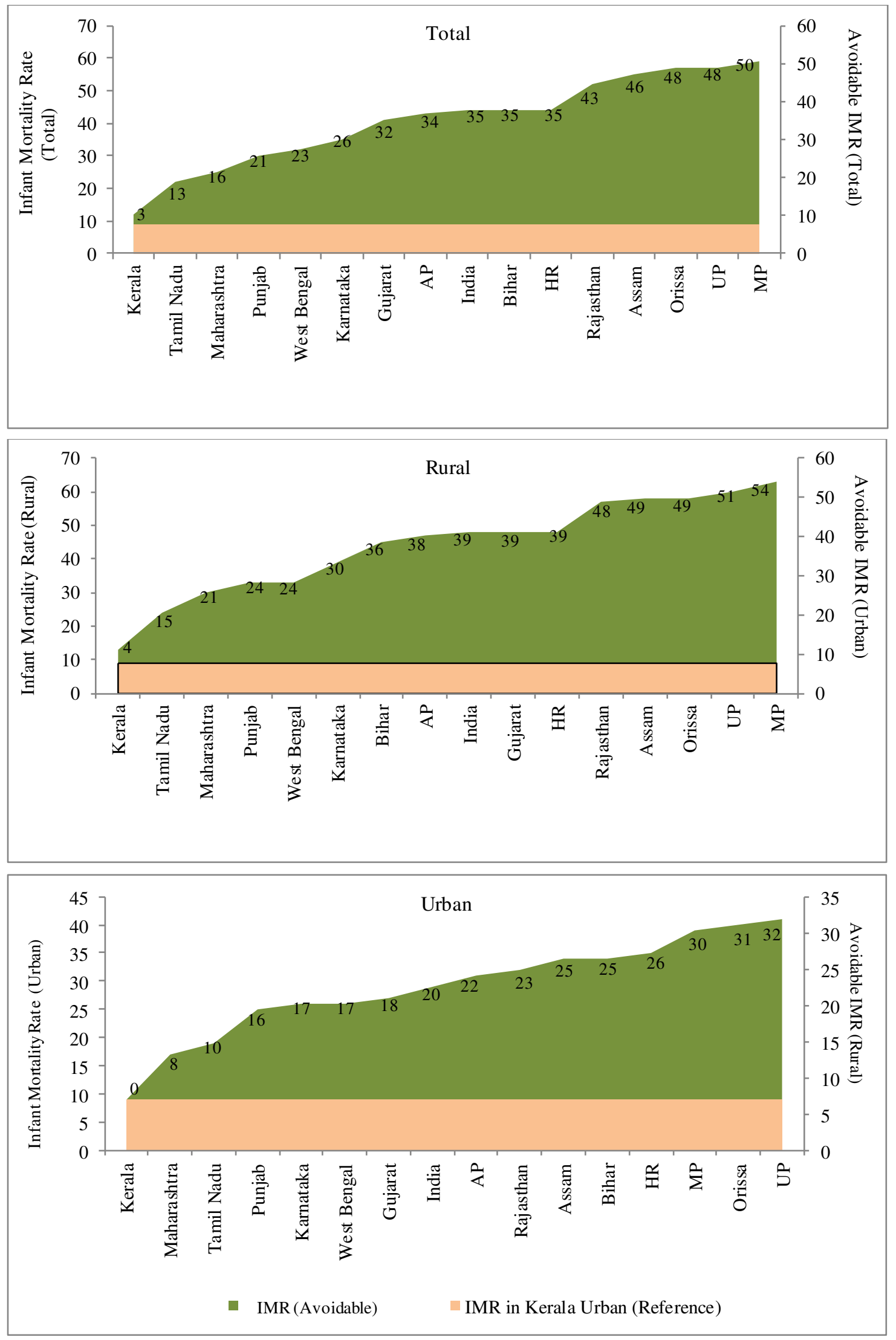

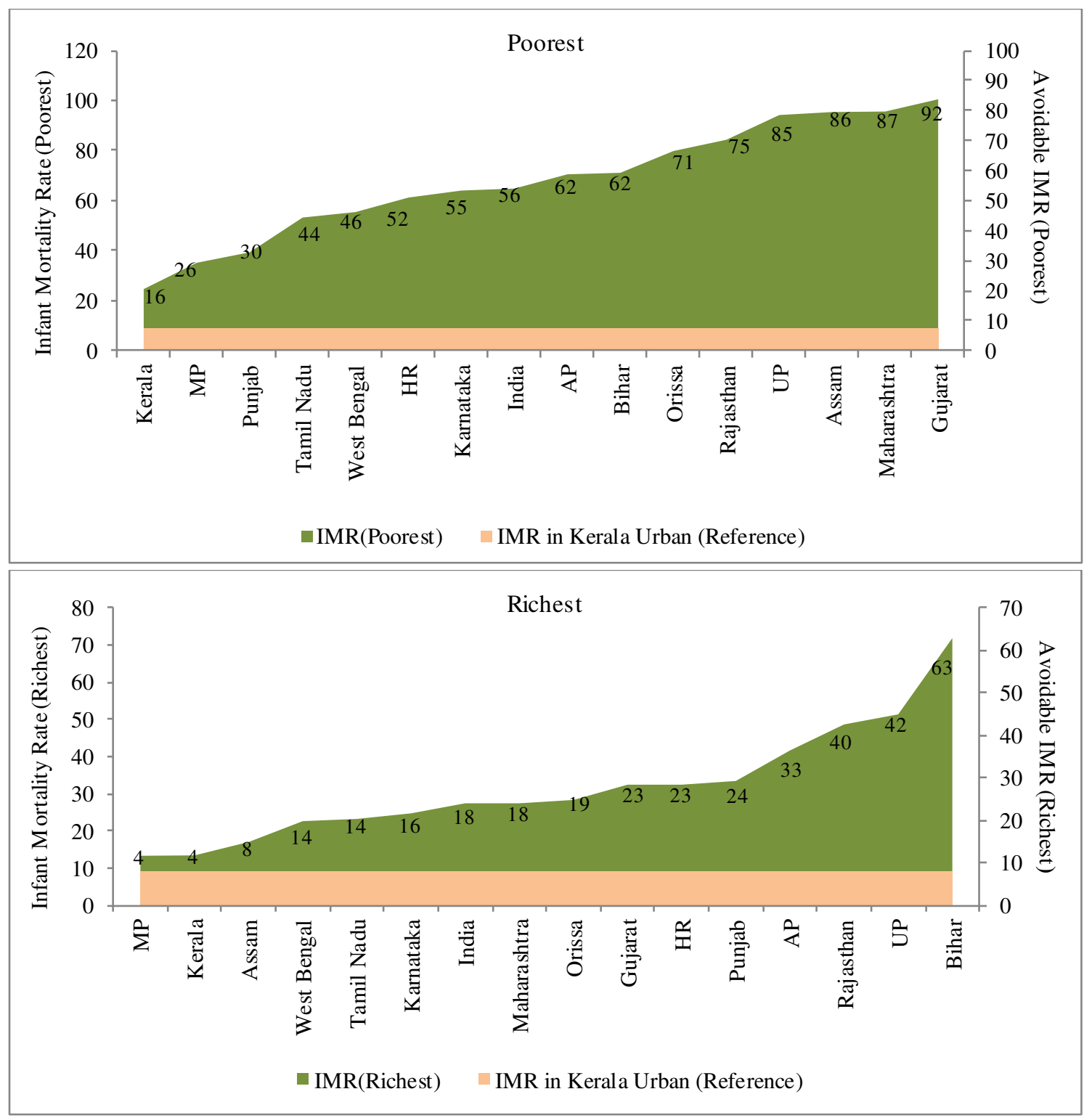

Children full immunisation

Figure 9.7 depicts the line of highest achievement plots showing the deviation of states from the line of highest achievement in terms of full immunisation among children across the social groups. In rural areas, the states standing nearest to the line of highest achievement was Tamil Nadu, whereas, the West Bengal was standing away from the line of highest achievement. As a result of this, the achievable full immunisation of children varies from $80 \%$ in rural West Bengal to only $16 \%$ in rural Tamil Nadu. In case of urban areas, the results revealed that Haryana has the highest $(87 \%)$ and Kerala has the lowest (16\%) amount of achievable children full immunisation. Similar kinds of results were also evident in case of children with 
illiterate mothers. Except, three states (Kerala, Tamil Nadu and West Bengal), all other states have more than $50 \%$ of achievable children full immunisation.

Figure 9.7. Plots line of highest achievement: Measuring the dispersion of states from the line of highest achievement in terms of full immunisation among children by socioeconomic groups

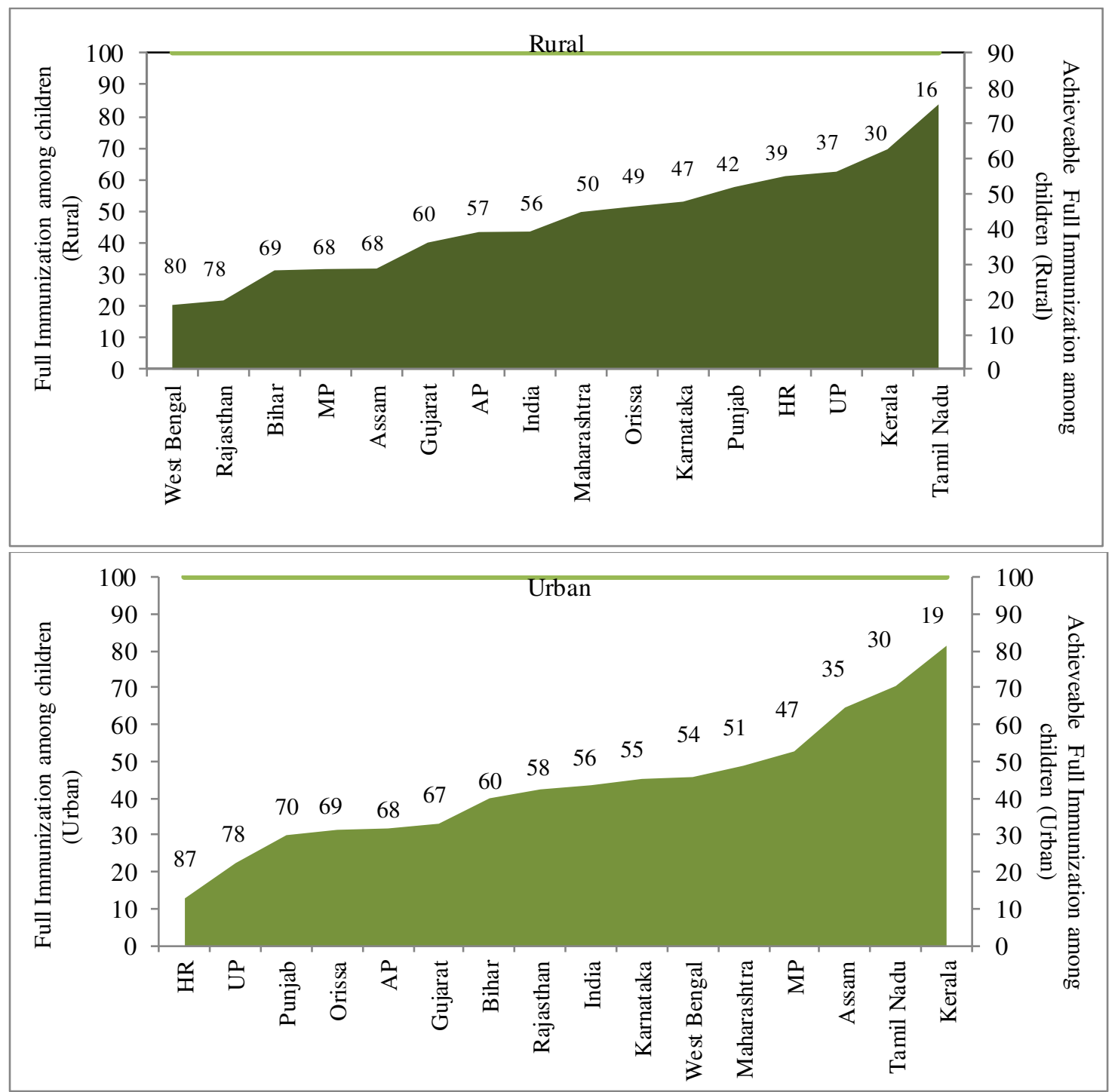



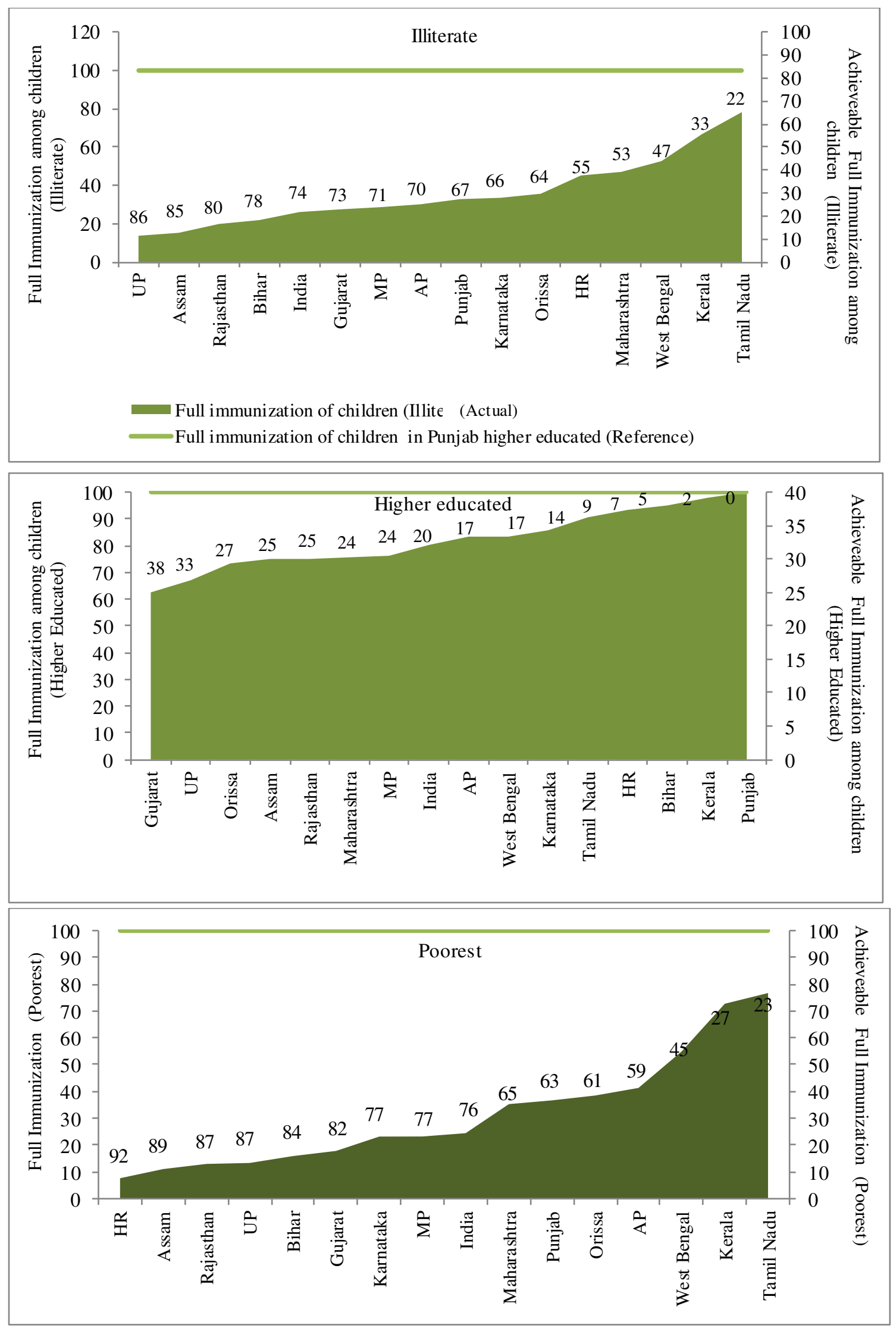


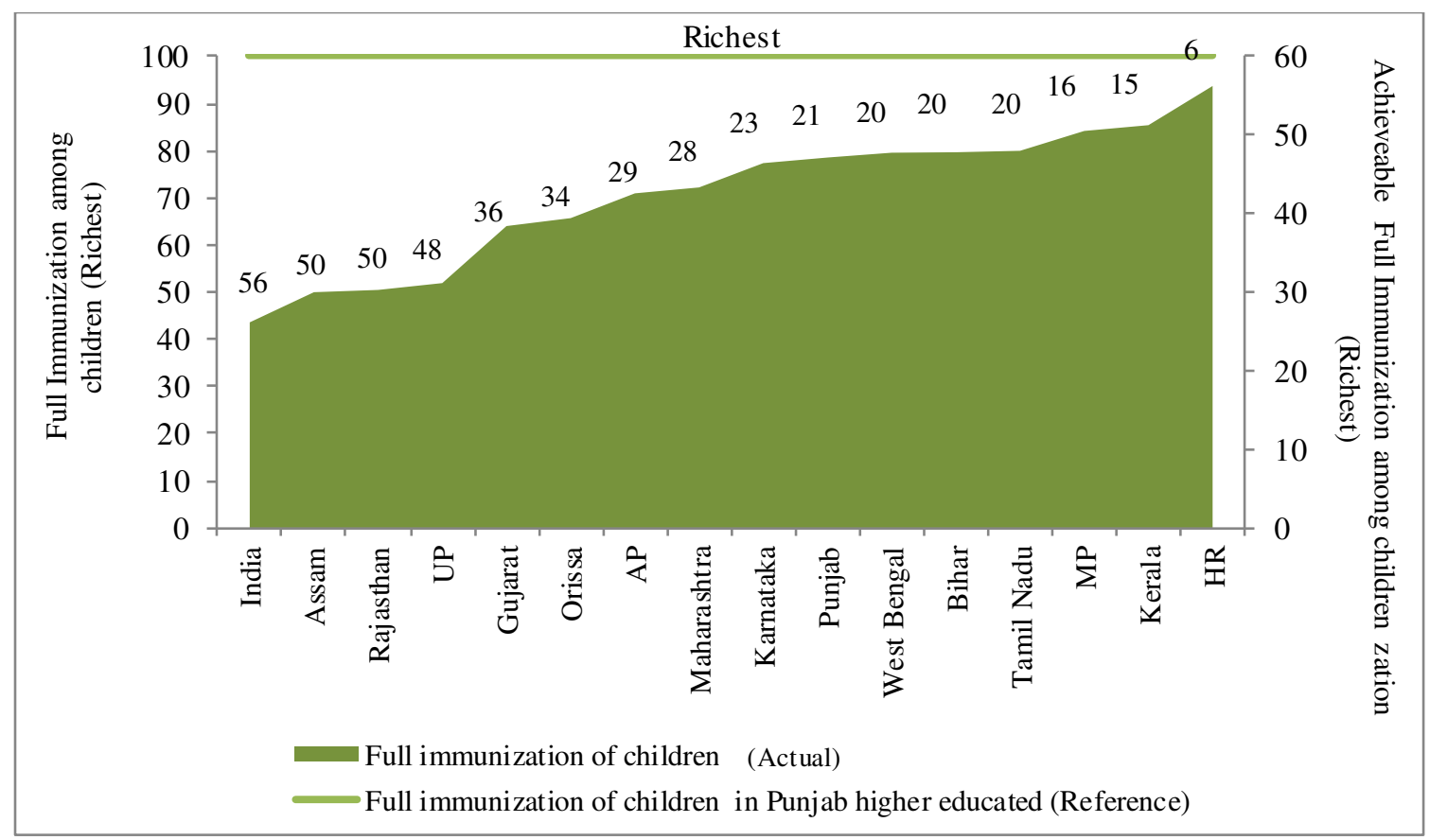

The results also showed that the achievable full immunisation is lowest among the children with higher educated mothers of few south Indian states. Surprisingly, the economically advanced state like Gujarat (38\%) has the highest achievable children full immunisation. On the other hand, there is no achievable full immunisation in Punjab and lowest in Kerala (2\%). In case of poorest wealth quintile households, the results reveal that the lowest achievable full immunisation was recorded among the children living in Tamil Nadu (23\%) and Kerala (27\%), whereas, the highest achievable full immunisation was recorded among the children living in Haryana (92\%) followed by Assam (89\%) which stands away from the line of highest achievement. Among the richest wealth quintile households, the plot of dispersal of states from the line of highest achievement showed that states such as Haryana was very close to the line of highest achievement with very low achievable children full immunisation, whereas, Assam (50\%), Rajasthan (50\%) and Uttar Pradesh (48\%) have highest achievable children full immunisation and stands away from the line of universal coverage.

\section{Children underweight}

Figure 9.8 presents the results of avoidable children underweight among states with reference to the line of highest achievement. In case of rural areas, the results show that Kerala has the lowest avoidable children underweight, whereas, Madhya 
Pradesh has the highest avoidable children underweight. Among urban areas, the lowest avoidable children underweight can be seen in most of the demographically advanced states such as Kerala (8\%) and Punjab (14\%). In contrast, EAG states like Madhya Pradesh (44\%) and Bihar (40\%) have the highest avoidable children underweight in urban areas. Among children of illiterate mothers, while Kerala (26\%) represents for the lowest avoidable children underweight, Madhya Pradesh (59\%) indicated for the highest avoidable children underweight followed by Bihar (55\%) and Gujarat (47\%). In case of children with higher educated mothers, the West Bengal showed evidence for the lowest avoidable children underweight, followed by Andhra Pradesh. In the same category, Madhya Pradesh (26\%) has the highest avoidable children underweight, followed by Bihar (23\%) and Gujarat (15\%). Kerala (11\%) has the lowest avoidable children underweight among the children whose mother belongs to poorest wealth quintile. In the same category, the highest avoidable underweight was recorded among the children living in the poorest households of Madhya Pradesh (30\%). Among the children of richest wealth quintile, Assam showed the lowest avoidable children underweight $(0.7 \%)$ followed by West Bengal (1.6\%), whereas, Madhya Pradesh has the highest avoidable children underweight $(29.6 \%)$.

Figure 9.8. Plots line of highest achievement: Measuring the dispersion of states from the line of highest achievement in terms of underweight among children by socioeconomic groups

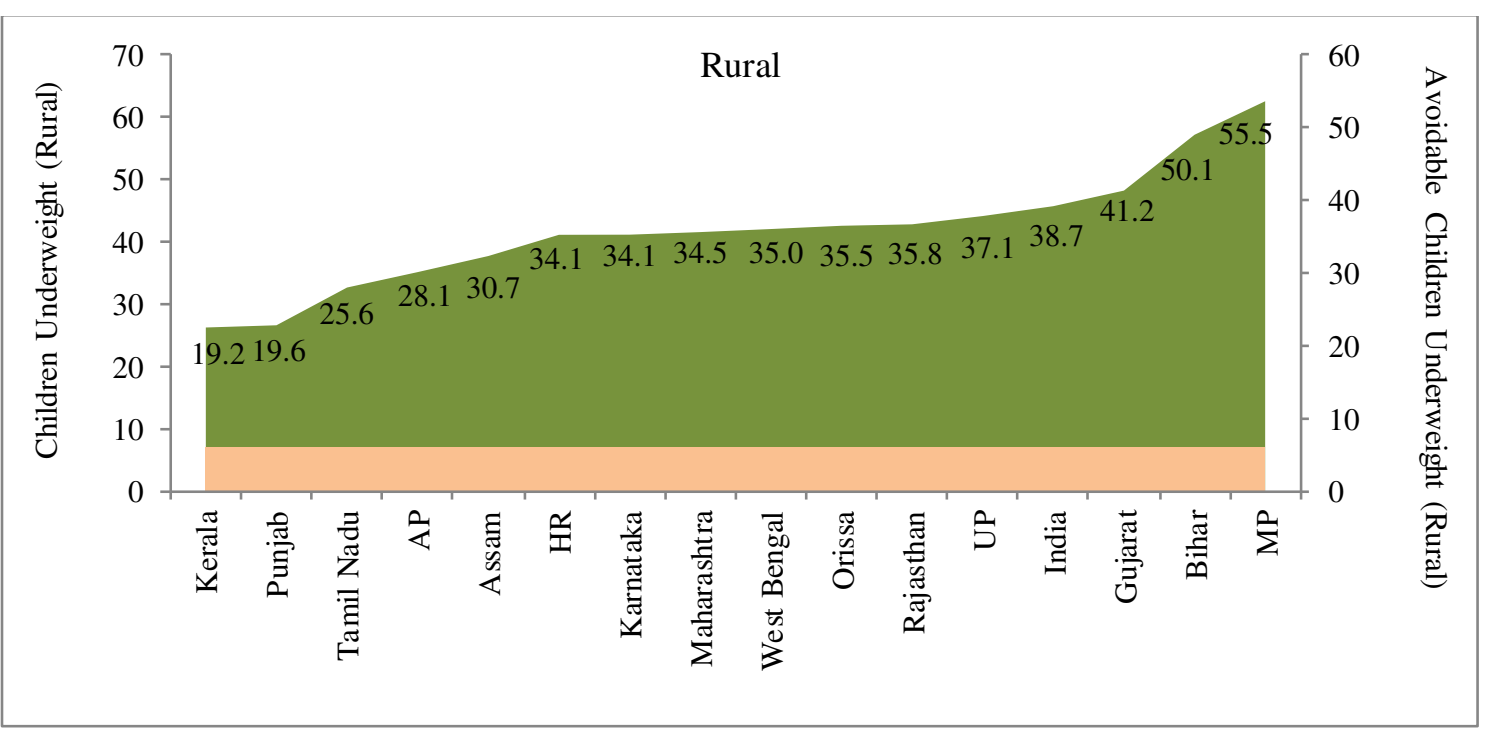



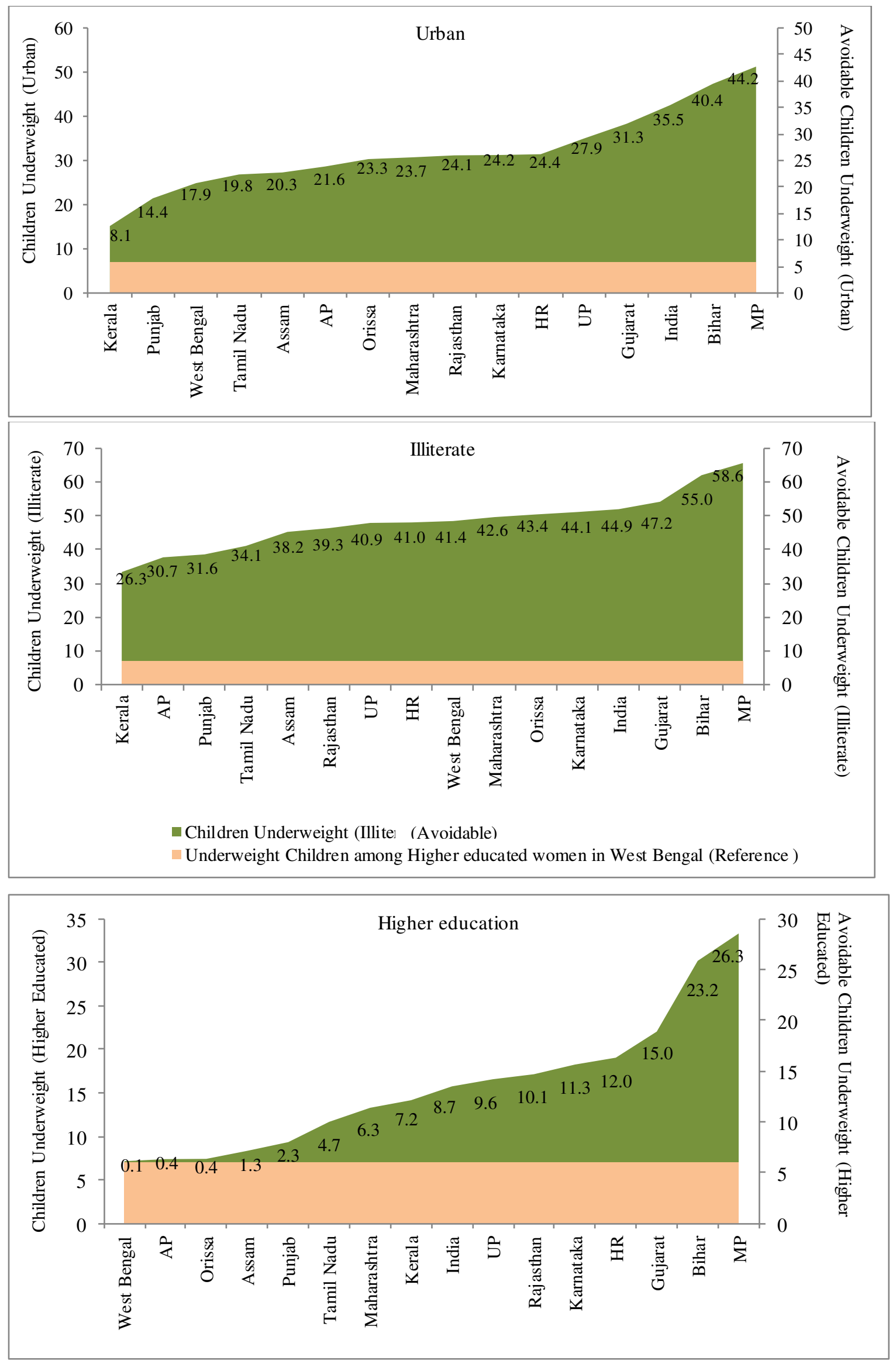

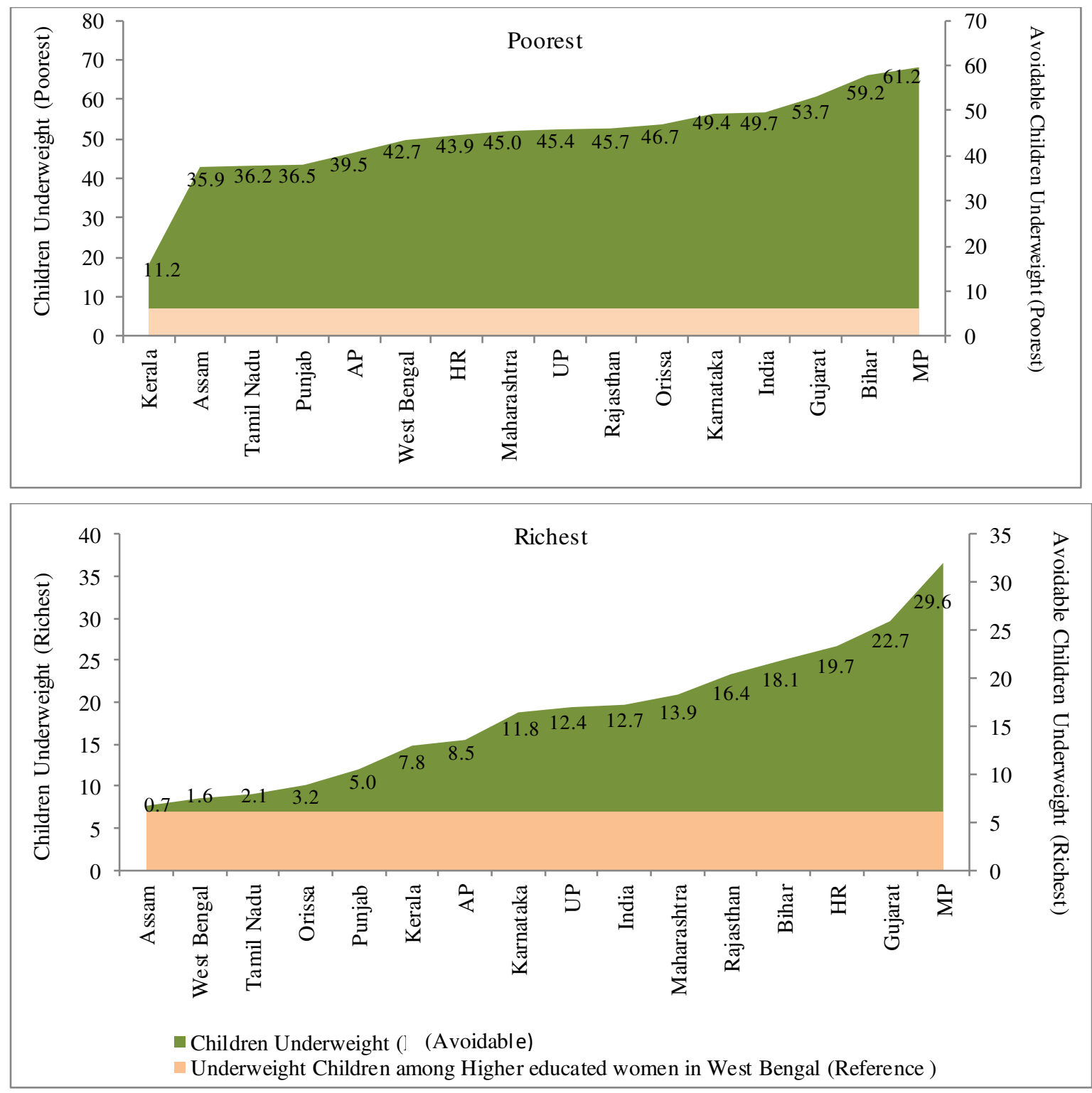

NNMR

Plots of line of highest achievement in terms of NNMR by socioeconomic groups present the avoidable neonatal mortality rate with reference to highest achievement in the decline of neonatal mortality (Figure 9.9). The results revealed that Kerala has the lowest avoidable neonatal mortality rate (4 deaths per 1000 live births), whereas, Madhya Pradesh has the highest avoidable NNMR (38 deaths per 1000 live births). In case of rural areas, again Kerala has the lowest avoidable NNMR (5 deaths per 1000 live births), whereas, Madhya Pradesh has the highest avoidable neonatal mortality (41 deaths per 1000 live births). In urban areas, there is no avoidable neonatal mortality in Kerala and lowest in Assam (7 deaths per 1000 live births). However, it is highest in urban areas of Orissa (24 deaths per 1000 live births), followed, by Madhya Pradesh (21 deaths per 1000 live births). 
Figure 9.9. Plots line of highest achievement: Measuring the dispersion of states from the line of highest achievement in terms of NNMR by socioeconomic groups
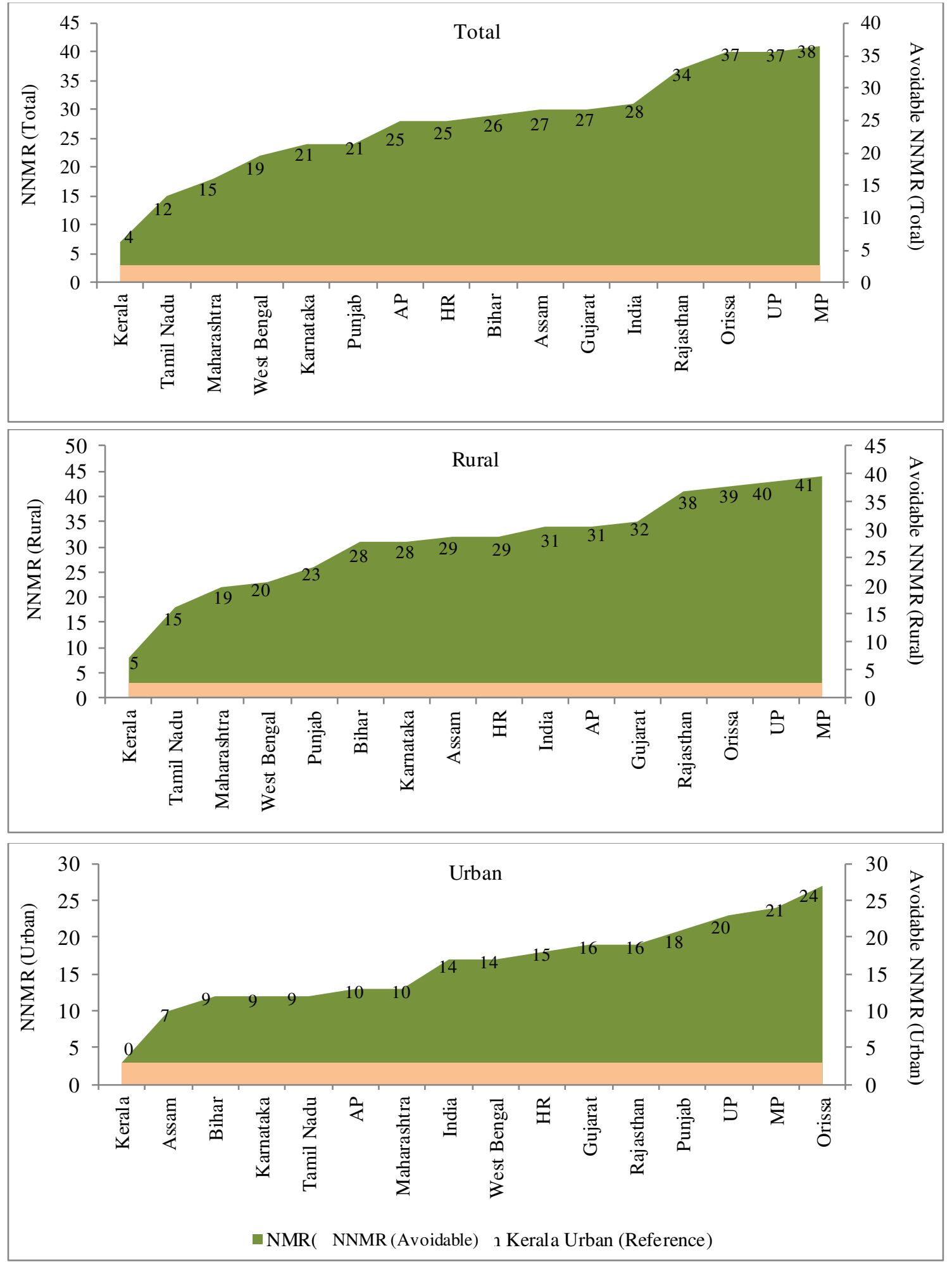


\subsection{Discussion}

In this chapter, this study used three types of measures 1) funnel charts 2) distance to line of hypothetical equity and 3) distance to the line of highest achievement. First, funnel chart measures the distance to a national average that how the states in terms of child health indicators are scattered from the line of national average under $95 \%$ confidence intervals. In case of children full immunisation, the results suggested that a greater number of states placed out of the $95 \% \mathrm{CI}$ line from the line of the national average. Only 7 states positioned within the 95\% confidence interval line and also close to the line of the national average. While 12 states were placed above the upper limit of $95 \%$ CI and 9 states were placed below the lower limit of 95\% CI. This indicates a huge gap among the states in terms of distribution of immunisation services among children across the states of India. In case of children underweight, only seven states placed out of the 95\% CI and 12 states placed below the $95 \% \mathrm{CI}$ of the national average of children underweight. This indicates that the scatterings of children underweight from national average reference are high. However, very few states positioned out of $95 \% \mathrm{CI}$ in case of neonatal mortality rate and infant mortality rate. This suggests that comparatively, in child health outcome indicators deviation from the national average is less.

The plots, which showed the distance to the line of equity for two opposite sides of the social groups, revealed that concentrations of TFR and IMR were highly skewed to demographically disadvantaged states and rural areas which were standing away from the line of equity. On the other hand, LEB concentration was more skewed towards demographically advanced states such as Kerala, Tamil Nadu, Maharashtra, Punjab and urban areas. The children with fully immunisation were highly skewed towards the urban area, higher educated mother, households with richest wealth quintile, thus, the distance to the line of equity between them and their counterparts was huge. In case of children underweight, the concentration of children with underweight was highly skewed towards lower socioeconomic groups and these groups were located at a greater distance from the line of equity.

The other important analyses of this chapter were the estimates and plots of avoidable differences in demographic and child health indicators. In case of children 
full immunisation, the highest achievement was $100 \%$ among children with higher educated mothers in Punjab and it is the reference line to estimate the achievable children full immunisation in other social groups and states. Across all the social groups and states, the highest achievable full immunisation was 92\% among the children living in the poorest households of Haryana. The highest achievement in terms of reduction of prevalence of children underweight was recorded in children of higher educated women in West Bengal $(0.1 \%$ children underweight). With reference to this, the highest avoidable underweight was recorded among children living in the poorest households of Madhya Pradesh. Similarly, in case of NNMR, the highest achievement was 3 per 1000 live births which was recorded in Kerala urban population. With reference to this, the avoidable neonatal mortality rate was 40 per 1000 live births in rural population of Madhya Pradesh. Overall, the results of this chapter fosters that the distance from the line of equity in all three child health indicators was greater across the states by socioeconomic groups. Similarly, the distance from the line of highest achievement was also very high. The average gap which needs to be filled to reach the line of equity in children full immunisation is $57 \%$. The average gap which needs to be filled to reach the line of equity in case of children underweight and NNMR is $37 \%$ and 28 deaths per 1000 live births respectively. Overall, the results in this chapter foster that there is a lot of gap that need to be reduced across the states and social groups to find absolute convergence in the progress of demographic and child health indicators in India. 


\section{CHAPTER 10}

\section{Summary and Conclusion}

There is a growing interest in the progression of worldwide convergence across diverse demographic indicators over the last one decade (Wilson, 2001, 2011, Neumayer 2003; Kenny 2005; McMichael et al. 2004; Moser et al., 2005; Dorius, 2008, 2010; Goli and Arokiasamy, 2014). Deaton (2013) viewed "the world is a better place than it used to be. People are wealthier and healthier, and live longer lives. Yet the escapes from destitution by so many have left inequalities between people and between nations". Also, the other literatures consistently show the diverse opinion in terms of convergence/divergence in key demographic indicators (mortality, fertility, health). With noted exceptions, the tendency towards declining between-country inequalities in demographic indicators have continued largely debated through the most recent available information. Analyses based on the recent data have serious limitations in terms of understanding the true trajectories of between and within country inequalities. Dorius (2010) argued that "the recent trend towards convergence mask a much more complex history with regard to between-country inequality. With recent data, it is difficult to predict the decisive turning points in the 'life-cycle of inequality'. The empirical record and the mechanics of correctly measuring 'between-country' convergence in non-pecuniary variables ${ }^{10}$ led him to suggest a generalised framework for studying inter-country or global inequality that he referred to as the 'inequality lifecycle hypothesis"". He further said "by explicitly linking patterns of inequality to the pattern of diffusion within a framework that includes a wider temporal span, a relatively predictable pattern of rising and then falling inter-country inequality has occurred in a diverse set of domains". The 'inequality life-cycle hypothesis' has an implied homeostatic component similar to the one put forward by Crenshaw (2000), where societies move from the conditions of low inequality or minimal disequilibrium over time. This transition starts from 'equilibrium' to 'disequilibrium' and back to 'equilibrium' is accompanied by often significant and relatively permanent changes in the averages of the variables (e.g. transition from high mortality to low mortality across all the states).

${ }^{10}$ By saying non pecuniary variables Dorius refers to demographic indicators 
Dorius (2008) and Wilson (2011) observations of predictable stages of demographic transition suggest that the "life-cycle of between-country inequality in three very different domains such as mortality, fertility and health tends to follow the same inverted U-shape" as the one first observed by Kuznets (1955) based on specific stages in the diffusion process. The inequality trends in demography (Fertility, Mortality and Health) sufficiently suggest the similar patterns of 'inequality life-cycle hypothesis' and subject to some degree of predictability. Yet, differences in onset, magnitude and duration of inequality, including noted reversals in inequality trends caution against deterministic explanations (Neumayer, 2004; Dorius, 2010). In a recent study Deaton (2013) takes an in-depth look at the historical and ongoing patterns behind the health and wealth of nations, and he addresses what needs to be done to help those left behind.

Although, most of the global studies looked at the between country inequalities, however, there is no empirical evidence of 'within-country' inequalities. There are many questions which went unaddressed in these global studies. Where to next from here? At least one next step in this regard is to take a closer look at the specific patterns of within country inequality in demographic variables. Whether, one can test convergence models in a developing country like India, both at sub-national level and across social groups? How thoroughly, the 'life cycle inequality hypothesis' can be tested in the Indian context, given the limitations of the availability of historical data on various population and health indicators? Do the convergence models help in priority setting for population and health policy in India?

Thus, the fundamental aim of this study is to provide cutting-edge summary measures in the form of convergence tools to assess and monitor the progress in population health status of India using available data. The approach of this study was mainly multidisciplinary, but the scope remains within the traditional boundaries of demography. This study attempted to develop a comprehensive framework to study the demographic transition, convergence and its linkage with health inequalities in India. It innovate a mechanism of bridging the gap between demographic convergence and health inequalities by taking the theories and methods found in economics, demography, sociology, geography and development. The combination of methods, theories and literature from sociology, economics, geography and development, facilitate greater cross-disciplinary approaches to the study of demographic and health 
transition, demographic and health convergence and health inequalities. It advances the empirical examination of demographic convergence assessment by assessing convergence not only in the averages, but also in the absolute and relative inequalities in population and health indicators.

Furthermore, this study makes four major contributions. First, it offers a methodological toolkit for the empirical analyses of demographic and health transition, and convergence, with particular attention to inequalities in progress of population and health indicators. It provides the first estimates of the critical change-points in socioeconomic, demographic and health transition. Second, it focuses on both absolute and relative inequalities in population and health indicators. Further, it provides the first estimates of convergence in population and health indicators for India. Third, it sheds new lights on 'health inequality analyses' by linking it to demographic progress and convergence. Fourth, for the first time, it estimates the gap between distance to the line of equity and the line of highest achievement. Thus, this study is a first time effort in terms of assessment of demographic convergence phenomena and its linkages with health inequalities in India. It revisits the old theories such a classic socioeconomic, demographic and health transition theories more clearly identifying the historical linkages between socioeconomic, demographic and health transition, and health inequalities.

\subsection{Summary of Findings}

Based on its rigorous conceptual exploration and statistical exercise, this study brought out number of intriguing findings and conclusions. I have presented a summary of findings in the sequence of analytical chapters below.

The demographic transition in India: evolutionary interpretation of long-term trends using change-point analysis

This chapter advanced the 'change-point analyses' as a new statistical tool for interpreting the progress and changes in population and health indicators for the greater understanding of the true trajectories of the population and health transition in India. The findings of this chapter suggest that the course of long-term demographic trends in India in key demographic indicators transiting from several major shifts. The shifts in IMR and TFR trends were closely connected with policy shifts in the family welfare 
program in India. The critical change-points recognized in pre-independence period in case of Population Size and LEB was mainly correlated with fluctuations in these indicators which emerged out of famines, several communicable diseases and epidemics. It has been observed that sustainable and the continued demographic transition began during the mid - 1960s. The findings of this chapter have not just helped in reassessing the historical past and the current demographic transition paths, but also advocating a new statistical tool for evaluating the demographic trends that are necessary for robust monitoring of the progress in population and health status in India.

Summary of convergence model results by indicator and type of convergence model

\begin{tabular}{|c|c|c|c|c|c|}
\hline \multirow{2}{*}{\multicolumn{2}{|c|}{ Selected indicators }} & \multicolumn{4}{|c|}{ Type of Convergence Model } \\
\hline & & \multirow{2}{*}{$\begin{array}{l}\text { Graphical } \\
\text { Plots } \\
\text { Catching-up } \\
\text { process }\end{array}$} & \multicolumn{2}{|c|}{ Parametric models } & \multirow{2}{*}{$\begin{array}{c}\text { Non-parametric } \\
\text { models } \\
\text { Absolute } \\
\text { Convergence }\end{array}$} \\
\hline & & & $\begin{array}{l}\text { Absolute } \beta \text { - } \\
\text { Convergence }\end{array}$ & $\begin{array}{c}\text { Conditional } \beta \text { - } \\
\text { Convergence }\end{array}$ & \\
\hline \multirow{4}{*}{$\begin{array}{l}\text { Per capita } \\
\text { NSDP } \\
\text { Literacy Rate }\end{array}$} & $\mathrm{EP}$ & No & No & Yes & No \\
\hline & RP & No & No & - & No \\
\hline & EP & Yes & Yes & Yes & No \\
\hline & RP & Yes & Yes & - & No \\
\hline \multirow{4}{*}{$\begin{array}{l}\text { Poverty } \\
\text { Ratios } \\
\text { HDI }\end{array}$} & EP & No & No & No & No \\
\hline & RP & No & No & - & No \\
\hline & EP & Yes & Yes & Yes & No \\
\hline & RP & Yes & Yes & - & No \\
\hline \multirow[t]{2}{*}{ LEB } & EP & No & Yes & Yes & No \\
\hline & RP & No & Yes & Yes & No \\
\hline \multirow[t]{2}{*}{ MMR } & $\mathrm{EP}$ & No & Yes & No & No \\
\hline & RP & Yes & - & - & No \\
\hline \multirow[t]{2}{*}{ IMR } & EP & No & Yes & Yes & No \\
\hline & $\mathrm{RP}$ & Yes & Yes & Yes & No \\
\hline \multirow[t]{2}{*}{ TFR } & EP & No & No & No & No \\
\hline & RP & Yes & Yes & No & No \\
\hline \multirow{6}{*}{$\begin{array}{l}\text { Children full } \\
\text { immunisation } \\
\text { Children } \\
\text { underweight } \\
\text { NNMR }\end{array}$} & EP & No & Yes & Yes & No \\
\hline & RP & Yes & Yes & Yes & No \\
\hline & EP & No & Yes & Yes & No \\
\hline & RP & No & Yes & Yes & No \\
\hline & EP & No & No & Yes & No \\
\hline & RP & Yes & No & Yes & No \\
\hline
\end{tabular}

Note: EP- Entire Period; RP- Recent Period

Socioeconomic convergence

The purpose of this chapter was to examine the progress in socioeconomic conditions across the major states of India by using convergence hypothesis. This made a more comprehensive assessment of socioeconomic convergence in terms of economic 
inequality, poverty ratios, literacy rate and Human Development Index (HDI) along with per capita NSDP. The findings suggested that in the period between 1981 and 2011, statistically significant absolute and conditional $\beta$-convergence in literacy rates and HDI was observed, but only conditional $\beta$-convergence was evident in case of per capita NSDP and poverty ratios. $\beta$-convergence estimates for the recent period (post2001) showed a divergence in per capita NSDP and poverty ratios, but convergence in literacy rates and HDI. Kernel density plots for socioeconomic indicators showed the existence of convergence clubs, but don't show the absolute convergence among all the indicators. Compared to other studies which suggest only parametric convergence measures to measure the socioeconomic convergence in India, this chapter suggests that use of the non-parametric convergence measures are crucial to gain more clear insights on the pathways of socioeconomic progress and to identify the short-term divergent paths.

\section{Fertility convergence}

This chapter investigated fertility convergence hypotheses in India across the states, rural-urban and socioeconomic spectrum. Both standard and innovative metrics of convergence were used for measuring the convergence. From a theoretical context, this chapter makes a critical contribution in advancing the knowledge on patterns of the progress in fertility transition. The assessment of India and the state level fertility transition demonstrates the evidence of transformation from progressive transition disequilibrium to progressive transition equilibrium phase. The finding also suggested that the earlier phase of divergence in fertility rates across the 15 major states of India was being replaced by emerging convergence in fertility rates for the recent period. The current trend pattern suggests a strong prospect of continued fertility convergence among the Indian states. On the whole, this chapter has advanced considerable knowledge in measuring the progress of fertility transition and convergence using a range of convergence models, both in terms of efficacy and equity across the states and socioeconomic spectrum.

\section{Mortality convergence}

The convergence metrics modelled in this chapter in case of averages and inequalities in mortality indicators also suggested varying patterns of convergence by type of 
measures and time periods. In general, the findings supported for the absolute convergence in the averages of selected mortality indicators, but with decreasing volume and speed of convergence for the recent period. Conditional $\beta$-convergence showed convergence in LEB and IMR, but the divergence in MMR. The convergence in averages was principally driven by greater progress in laggard states and resultant catching-up process. However, the results indicated evidence of some recent setback in convergence in absolute and relative health inequalities in LEB, MMR, socioeconomic and relative inequalities in IMR. For all the three mortality indicators, inequality measures of convergence showed a recent set back in convergence and Kernel density plots suggested divergence.

Convergence in child health care utilisation and outcomes

Akin to other chapters, this chapter also tested both average and inequality based convergence metrics to test the hypothesis of convergence in both average and relative distributions of child health status in India focusing on 15 major states and socioeconomic groups for four key child health indicators. The findings of trend analyses for child health averages and the inequalities of selected indicators suggested the trembling patterns. The findings of $\beta$-convergence measure suggested convergence in their averages, but with decreasing volume and speed of convergence for the recent period. Similarly, inequality based convergence tests suggested evidence of some recent setback in convergence in both absolute and relative health inequalities in children underweight, socioeconomic inequalities in children full immunisation and underweight and relative health inequalities in NNMR. From the late 1990s, results suggested that converging trends in child health inequalities were being replaced by diverging trend. However, non-parametric models which generally consider being more robust tools of convergence analyses (Bloom and Canning, 2007) showed evidence of convergence clubs, but not absolute convergence in child health indicators.

Linkage of demographic convergence with health inequalities in India

The assessment of the correlation between change in averages of socioeconomic and demographic factors and inequalities in children full immunisation, children underweight and neonatal mortality rate during 1992-2006 revealed a number of intriguing findings. The association between change in averages with absolute and 
relative inequality of children full immunisation, children underweight and neonatal mortality rate suggested that a decrease in absolute inequalities and increase in relative inequalities with an increase in averages in the same indicators. The association between change in averages of children full immunisation, children underweight, neonatal mortality rate and socioeconomic inequalities revealed that socioeconomic inequalities in children full immunisation, children underweight and neonatal mortality rate, increase with the increase in averages of children full immunisation, and decrease in children underweight and NNMR. The association between change in the TFR and children full immunisation, children underweight and neonatal mortality rate also indicated that decline in TFR lead to increase in socioeconomic inequalities in children's health status. The association between convergence in trends in the TFR and children full immunisation showed that in spite of convergence in the TFR during 1992-1998, both absolute and relative inequalities have increased. However, when the fertility convergence becomes stronger in the later period, 1998-2006, both the absolute and relative inequalities have also started declining. In spite of fertility convergence, inequalities in case of children underweight and neonatal mortality rate continued to rise. In general, after adjusting for the steady condition of the states in term of socioeconomic status, progress in TFR was associated with a decrease in inequalities in children's health status. Thus, it indicates that the benefits or fruits of demographic transition and convergence showed more equal distribution among the societies with lesser pre-existing socioeconomic inequalities.

Distance to line of equity and line of highest achievement: how much demographic differences can be avoided in India

This chapter plot and estimate the distance to line of national average and distance to line of equity, avoidable demographic differences in selected population and health indicators by using the latest available data for respective indicators. All three measures used in this chapter showed that there is a huge deviation of states and social groups from the line of national average, line of equity and line of high achievement. All the south Indian states like Kerala and Tamil Nadu, Himachal Pradesh in the northern India standing close to the line of equity and line of highest achievement. On the other hand, EAG states are standing away from the line of equity and line of highest achievement. The findings of measured avoidable demographic differences suggested that, there is a 
huge gap which needs to be reduced across the states and social groups to bring all the states and social groups in the line of equity and further to find the absolute convergence. Overall, this chapter fosters that India needs to aim for efficiency with equity to achieve the absolute convergence in population and health indicators in India.

\subsection{Conclusions}

This dissertation is an effort in terms of developing a comprehensive framework for the study of demographic transition, convergence and its linkage with child health inequalities in India. This study advances both theoretical and empirical examination of demographic convergence by assessing convergence not only in the averages, but also in the absolute and relative inequalities in population and health indicators. Taken as a whole, this study makes five critical research contributions:

First, this study interprets socioeconomic growth theory, demographic and health transition theory, convergence theory in terms of 'inequality life cycle hypothesis'. In Indian context, the study presented the first estimates of critical change-points of demographic and health transition based on 'change-point analyses'. Therefore, it introduces fresh research methods for the empirical analyses of demographic and health transition and more clearly identifying the historical linkages between 'critical changepoints' in demographic, health transition and population policy shifts. Second, the study systematically assessed the inequalities in the progress of population and health indicators across the states, rural-urban and socioeconomic groups. It focused on both absolute and relative inequalities in population and health indicators. Furthermore, it widens theoretical discussions on the origin and growth patterns of health inequalities in terms of 'inequality life cycle hypothesis'. Third, the study provides the estimates of convergence in population and health indicators in case of India, for the first time. Fourth, a fresh perspective is injected where the study links children's health inequalities with fertility progress and convergence. Fifth, the study estimates the distance to line of equity and line of highest achievement for the first time. In general, this study identifies the dynamic patterns of demographic transition and convergence in India and how these processes have implications on children's health inequalities. It evidently understood that inequalities in demographic indicators like fertility can diminish faster by "spill over" or "diffusion" effects compared to inequalities in health 
indicators. Moreover, inequalities are swimming against the progress in averages of both demographic and health indicators. This study identifies an avoidable demographic and health disparities, and the highest achievable targets of demographic and health status across the Indian states and socioeconomic groups.

Taken as a whole, the study fosters the importance of using effective demographic and health monitoring tools such as convergence models in countries such as India with huge socioeconomic and geographical disparities in demographic and health progress. According to the findings of this study, many of the demographic and health indicators are not showing evidence of absolute convergence in progress. The underlying convergence theory argues that as states develops demographically. They tend to converge towards the same policy agenda. The objective of achieving demographic convergence across the states and social group requires not only uniform population policies, but also uniformity in implementation and plan of action.

However, according to all classical convergence hypotheses (both the Liberal and Marxist), the convergence is inevitable, probably the final demographic regime (Wilson, 2013). To increase the pace of convergence, both engines ('spill-over' and 'intervention') are important. Though, the Spill-over model is inevitable in it and does not need a human interface to a large extent, but intervention is something which is required and pragmatically sounding. Now, it is confirmed from this study that a mere reduction in the number of children or fertility convergence in averages is not helping in distributing the fruits of demographic progress equally among the children of different states and socioeconomic groups. How children and their development (particularly in terms of health) are faring during the progress of demographic transition is an important concern for population policy in India. In principle, inequalities are avoidable as disparities in health stem out from identifiable policy options exercised by governments. Thus, they are amenable to policy interventions.

\subsection{Policy implications}

The findings of this study assumes importance, particularly, when there is a worldwide debate that the health policies is to focus on improvement of average health or distributional dimensions of health such as absolute and relative distributions and 
efficiency with the equity in health (Whitehead et al., 2001; Wagstaff, 2002; Victoria et al., 2003; Katherine et al., 2009; Blas and Kurup, 2010; Doris, 2010). In his recent landmark study, Deaton (2013) viewed "Inequality is often a consequence of progress. Not everyone gets rich at the same time, and not everyone gets immediate access to the latest life-saving measures". Further, growth, inequality, and catch up are the both side of the same coin. The dark side is what happens when the process is hijacked, so that catch-up never comes. Powerful and wealthy elites have choked off growth before, and they can do so again if they are allowed to undermine the institutions on which broadbased growth depends (Dreze and Sen, 2013; Deaton, 2013).

The outcome of the present study and other largely acceptable viewpoints are, for a fair society and healthy lives, there is a need to measure the health distribution beyond average health differentials (Marmot, 2010). Therefore, in terms of policy perspective, to add to equity and efficiency dimensions, this study suggests that the process of convergence in health and health inequalities can be used as a critical assessment tool for monitoring the health progress in existing health policies and programmes. Convergence in health and health inequalities is not only reflect a sense of equity across the states, regions and socioeconomic groups, but can also be an effective summary measure of overall health (absolute, relative and socioeconomic distribution of health) progress. In general, this study fosters the importance of using effective health monitoring tools such as convergence models in countries such as India, which has huge socioeconomic and geographical disparities in the progress of demographic and health inequalities. The policy analyst can use convergence measures as tools for health policy evaluations in India and states. In an effort to continuously track regional progress in population and health indicators, it is important to test the convergence hypothesis for every five years.

The future trends of both averages and inequalities in demographic and public health indicators across the states and socioeconomic groups depend on the population and health policies and strategic approaches of the federal and state governments of India. Therefore, setting health priorities towards convergence across the states is necessary. The argument of the underlying convergence theory is that as states develops economically, they tend to converge towards the same policy mix. The objectives and activities of national health systems become more alike. At present in India, though 
there is a convergence in health policies at conceptual level, the policy content and the preferred policy instruments for implementing such policy which continues to vary widely across the states.

Inequalities are avoidable to the extent that they stem from identifiable policy options exercised by governments, such as tax policy, regulation of business and labour, welfare benefits and social welfare funding (Dreze and Sen, 2013). Thus, health inequalities are, in principle, amenable to policy interventions. A government that cares about improving the health of the population needs effort towards alternative options in its policy setting process. There is a need to search for a new pathway to close demographic and health gap between states and social groups. Convergence should be a future sustainable population and development goal. The recent Lancet Commission report (2013) on "Grand Convergence: a future sustainable development goal has also identified this.

\subsection{Practical Implication}

Convergence hypotheses tested on India's demographic and health data in this work have been tested previously only for demographic and health data of the world or western countries. The methodology adopted in this study will assist in promoting the modeling methods for demographic and health progress assessment in India and its states. The unifying framework introduces here to understand inequality transition and convergence is a tool for bridging the crack between the disciplinary theories of such diverse subjects as political economy, human ecology, sociology and geography. By bridging these gaps, my framework promotes greater transparency between disciplines and allows for a crosscutting of theory and methods from studies of inequality which have previously relied on discipline-specific frameworks and theories for predicting and explaining inequality trends in various quantities of human welfare. It is my expectation that this framework will stimulate further comparative research and theory on long-term, within-country patterns of inequality and "inequality life cycle hypothesis".

Another practical implication of this study is that in the preparation of sub-national population projections, most demographers utilise a cohort-component model which 
requires assumptions concerning the future time paths and levels of migration, death rate and fertility rates (Connell, 1981). Now and then, target fertility levels for single countries are based which are established on historical fertility trends pertinent to their own country. However, assumption of convergence rates is often used in state population projections. The convergence models also help to test whether the underlying assumptions in state population projections are justified by estimating the national birth expectation data. In general, convergence is an appropriate statistical tool for assessing the interstate and inter-group progress in demographic and health transition.

\subsection{Limitations of the Study}

This study has limitation in terms of availability of long term reliable data for convergence analyses because the time frame in which these phases of divergence and convergence occur is much greater than a few decades, studies that focus only on a few decades often fail to capture the significant state level trends that take a century or more to unfold. Therefore, I failed to analyse and give evidence for complete 'life cycle hypotheses of transition and inequality' in demographic and population indicators in India. At the same time, with the current phase of the demographic transition in India, I failed to produce the evidence for "cycle of convergence, divergence and reconvergence". 


\section{References}

ABREU, M., DEGROOT, H. L., FLORAX, R. J, (2005), "Space and growth: a survey of empirical evidence and methods", Région et Développement, 21, 13-44.

AHLUWALIA, M. (2002), "State level performance under economic reforms in India", in KRUEGER, A. (ed) "Economic policy reforms and the Indian economy", University Of Chicago Press, Chicago, 91-128.

AIYAR, S. (2001), "Growth theory and convergence across Indian states: a panel study", in CALLEN, T., REYNOLDS, P., TOWE, C. (eds) "India at the crossroads: sustaining growth and reducing poverty”, International Monetary Fund, Washington DC, 143-169.

ALAGARAJAN, M. (2003), "An analysis of fertility differentials by religion in Kerala state: a test of the interaction hypothesis", Population Policy and Research Review, 22, 557-74.

ALAGARAJAN, M., KULKARNI P.M. (1998), "Fertility differentials by religion in Kerala - a period parity progression ratio analysis", Demography India, 27 (1), 21328.

ALAGARAJAN, M., KULKARNI P.M. (2008), "Religious differentials in fertility in India: is there a convergence'? Economic and Political Weekly, 43 (48), 44-53.

ANDERSON, B.A., HÄRM, E. (1979), Human Fertility in Russia since the Nineteenth Century, Princeton, NJ: Princeton University Press.

ANGELES, L. (2010), "Demographic transitions: analyzing the effects of mortality on fertility”, Journal of Population Economics, 23 (1), 99-120.

AROKIASAMY, P., GOLI S. (2012). Fertility convergence in the Indian states: an assessment of changes in averages and inequalities in fertility. Genus: A Journal of Population Studies, 68 (1), 65-88.

AROKIASAMY, P., SHAHNAWAZ M., SRINIVAS G. (2010), "Reverse causation effect of fertility on infant mortality reduction: exploring evidences from India", Paper presented in First Asian Population Association Conference, New Delhi, November 16-20.

AROKIASAMY, P. (2009), "Fertility decline in India: contributions by uneducated women using contraception", Economic and Political Weekly, 44, (30). 
BALABDAOUI, F., BOCQUET-APPEL, J.P., LAJAUNIE, C., IRUDAYA RAJAN, S. (2001), "Space-time evolution of the fertility transition in India, 1961-1991, International Journal of Population Geography, 7, 129-148.

BANDOPADHYAY, S., 2004. Twin peaks - distribution dynamics of economic growth across Indian states. In: A. Shorrocks and R. van der Hoeven, eds. Growth, inequality and poverty: prospects for pro-poor growth. Oxford: Oxford University Press.

BARRO, R.J., SALA-I-MARTIN X. (1991), "Convergence across states and regions", Brookings Papers on Economic Activity, 1, 107-158.

BARRO, R.J., SALA-I-MARTIN X. (1992), “Convergence”, The Journal of Political Economy, 100, 223-251.

BARRO, R.P., FERREIRA F.H.G., VEGA J.R.M., CHANDUI, J.S., GIUGALE J.M. (2009), Measuring inequality of opportunity in Latin America and Caribbean, World Bank, Washington DC.

BARRO, R.J. (1991), "Economic growth in a cross-section of countries", The Quarterly Journal of Economics, 106, 407-44.

BARRO, R.J., SALA-I-MARTIN X. (1995), "Economic Growth", Mcgraw-Hill, New York.

- 1992. “Convergence”, Journal of Political Economy, 100, 223-251.

BARRO, ROBERT J \& SALA-I-MARTIN, X. (1997), “Technological Diffusion, Convergence, and Growth," Journal of Economic Growth, 2 (1), 1-26.

BAUMOL, W.J. (1986), "Productivity growth, convergence and welfare: what the long-run data show", American Economic Review, 76, 1072-85.

BAUMONT C., ERTUR C., GALLO J. L. (2002). "The European Regional Convergence Process, 1980-1995: Do Spatial Regimes and Spatial Dependence Matter?" Econometrics 0207002, EconWPA.

BECKER, G.S., PHILIPSON J.T., SOARES R.R. (2005), "The quantity and quality of life and the evolution of world inequality", The American Economic Review, 95 (1), 277-291. 
BHAT, P.N.M. (1989), "Mortality and fertility transition in India, 1881-1961: a reassessment", in DYSON T. (eds) "India's historical demography: studies in famine diseases and society", Curzon press, London, 73-118.

BHAT, P.N.M., PRESTON S.H., DYSON T. (1984), "Vital rates in India, 1961-1981", Committee On Population And Demography, Report no.24. National Academy Press, Washington D.C.

BHAT, P.N.M., RAJAN S.I. (1997), "Demographic transition since independence", in ZACHARIAH K.C., RAJAN S.I. (eds) "Kerala's demographic transition: determinants and consequences, Sage Publications: New Delhi, India, 33-78.

BHATTACHARYA, B.B., SAKTHIVEL S. (2004), "Regional growth and disparity in India: comparison of pre and post-reform decades", Economic and Political Weekly, 39, 1071-77.

BLAS, E., KURUP S.A. (2010), "Synergy for equity", in BLAS E., KURUP S.A. (eds) "Equity, social determinants and public health programmes", World Health Organization, 261-284.

BLOOM, D.E., WILLIAMSON J.G. (1998), "Demographic transition and economic miracles in emerging Asia", World Bank Economic Review, 12, 419-55.

BLOOM, D.E., CANNING D. (2007), "Mortality traps and the dynamics of health transitions", Proceedings of the National Academy of Sciences, 104 (41), 1604416049.

BONGAARTS, J. (2003), "Completing the fertility transition in the developing world: the role of educational differences and fertility preferences", Working Paper, Policy Research Division, New York: Population Council.

BONGAARTS, J. (2008) Fertility Transitions in Developing Countries: Progress or Stagnation? Working Paper No. 7. Population Council, New York.

BOURGUIGNON, F. MORRISON C. (2002), "Inequality among world citizens: 1820 1992,"American Economic Review, 92 (4), 727-744.

BOX, G., LUCEÑO A. (1997), "Statistical control by monitoring and feedback adjustment", Wiley, New York.

BROWN, R.L., PRUS G.S. (2004), "Social transfers and income inequality in old age: a multinational perspective", North American Actuarial Journal, 8 (4). 
CALDWELL, JOHN C. (1976), "Toward a restatement of demographic transition theory", Population and Development Review, 2 (3/4), 321-66.

CALDWELL, JOHN C., SANTOW, G. (1989), Introduction. Pp 48-109 in CALDWELL, JOHN C and SANTOW, G. (eds) "Selected Readings in the Cultural, Social and Behavioural Determinants of Health", Canberra: Australian National University.

CALDWELL, K.B., CALDWELL, P., MCDONALD, F.P., SCHINDLMAYR, T. (2006), Demographic Transition Theory. Dordrecht, the Netherlands.

CLELAND, J.C. 1990. The idea of the health transition. Pp. 48-109 in CLELAND, J.C. et al. (ed.) What We Know about Health Transition, Canberra: Australian National University.

CALDWELL, J.C. (2004), "Demographic theory: a long view", Population and Development Review, 30(2), 297-316.

CASELli, F., GERARDO E., FERNANDO, L. (1996), " Reopening the Convergence Debate: A New Look at Cross-Country Growth Empirics," Journal of Economic Growth, 1(3), 363-89.

CASHIN, P., SAHAY, R. (1996), "Regional economic growth and convergence in India", Finance and Development, 33, 49-52.

CASTERLINE, J.B. (2001), "The pace of fertility transition: national patterns in the second half of the twentieth century", Population and Development Review, 27, 1752.

CASTERLINE, J.B. (2011), 'Review of Tim Dyson's population and development: the demographic transition. Population and Development Review, 37 (2), 395-397.

CASTERLINE J.B. (2001), "The pace of fertility transition: National patterns in the second half of the twentieth century," in BULATAO R.A., CASTERLINE J.B. (eds) "Global Fertility Transition", Population and Development Review, Supplement to Vol. 27, New York: Population Council, pp. 17-52.

CENSUS OF INDIA. (1871-2001), "Final population totals", Office of the Registrar General of India: New Delhi.

CENSUS OF INDIA. (2011), "Provisional population totals", Office of the Registrar General of India: New Delhi. 
CENSUS OF INDIA. (2011), "Drop-in-article: Census of India 2011", Office of the Registrar General of India: New Delhi.

CHANDRASEKHAR, S. (1972), "Infant mortality, population growth and family planning in India", Routledge, London.

CHAURASIA A.R. (2009) Mortality Transition in India 1970-2005, paper presented in IUSSP Conference, Morocco, September and October.

CHOW, GREGORY C. (1960), "Tests of Equality Between Sets of Coefficients in Two Linear Regressions". Econometrica 28 (3): 591-605.

CLARK, R. (2011), "World health inequality: convergence, divergence, and development", Social Science and Medicine, 72, 617- 624.

COALE, A.J. (1973), "The demographic transition," IUSSP Liege International Population Conference. Liege: IUSSP, 1, 53-72.

COALE, ed. (1987), The Decline of Fertility in Europe, Princeton, NJ: Princeton University Press.

COLE, M.A., NEUMAYER E. (2003), "The pitfalls of convergence analysis: is the income gap really widening?" Applied Economics Letters, 10, 355-357.

COLEMAN, D.A. (2002), "Populations of the industrial world - a convergent demographic community?" International Journal of Population Geography, 8, 319344.

COLEMAN, D. (2004), "Why we don't have to believe without doubting in the "Second Demographic Transition" - some agnostic comments". Vienna Yearbook for Population Research, 11-24.

CUTLER D., A. DEATON, A. LLERAS-MUNEY (2006), The Determinants of Mortality Journal of Economic Perspectives, 20 (3): 97-120.

DARWENT, D. (1969), "Growth poles and growth centers in regional planning- a review", Environment and Planning, 1, 5-32.

DAS, K. (1993), "Planning and regional differentiation in India: strategies and practices", Journal of Indian School of Political Economy, 5, 603-632.

DASGUPTA, D., et al., (2001), "Growth and interstate disparities in India", Economic and Political Weekly, 1. July, 2413-2422. 
DASGUPTA, M. (2005), "Public health in India: dangerous neglect, reviewed work(s)", Economic and Political Weekly, 40(49), 5159-5165.

DATTA, G., RAVALLION, M., (2002), "Is India's economic growth leaving the poor behind'? Journal of Economic Perspectives, 16, 89-108.

DAVIS, K. (1945), "The World Demographic Transition", Annals of the American Academy of Political and Social Science, 237, 1-11.

DAVIS, K. (1951), The population of India and Pakistan, Princeton University Press, Princeton.

DAVIS, K. (1963), "The theory of change and response in modern demographic history", Population Index, 29, 345-366.

DEATON, A. CHRISTINA P. (2001), "Mortality, education, income and inequality among American cohorts," in David A. Wise, ed., Topics in Aging Volume, Chicago University press.

DEATON, A., (2003), "Health, Inequality, and Economic Development," Journal of Economic Literature, American Economic Association, 41 (1), 113-158.

DEATON A. (2001). Inequalities in Income and Inequalities in Health Chapter 10 in Finis Welch (ed.), The causes and consequences of increasing inequality, pp. 285313, Chicago: Chicago University Press (2001)

DEATON A., DREZE J. (2002) Poverty and Inequality in India: a Re-examination, Economic and Political Weekly, 37 (36): 3729-3748.

DEATON A. (2013). What's wrong with inequality? The Lancet, 38: 363.

DEATON, A. S., PAXSON, C. (2004), "Mortality, Income, and Income Inequality over Time in Britain and the United States,"NBER Chapters, in: Perspectives on the Economics of Aging, pages 247-286 National Bureau of Economic Research, Inc.

DEATON, A., and DREZE, J. (2009), Nutrition in India: facts and interpretation. Economic and Political Weekly, 45 (7), 42e65.

DEATON, A. (2011), "What does the empirical evidence tell us about the injustice of health inequalities?," Working Papers 1284, Princeton University, Woodrow Wilson School of Public and International Affairs, Research Program in Development Studies.

DEATON, A. (2013). The Great Escape: Health, Wealth, and the Origins of Inequality, Princeton University Press: Princeton 
DE BARROS, R.P., FRANCISCO H.G., FERREIRA J.R., VEGA M., CHANDUVI J.S. (2009), "Measuring inequality of opportunities in Latin America and the Caribbean", Conference edition, The World Bank.

DEBORAH, L. XIE Y. (2009), "Socioeconomic status and health differentials in China convergence or divergence at older ages?" Population Studies Research Center Report 09-690. University of Michigan, USA.

DHOLAKIA, R.H. (1994), "Spatial dimensions of accelerations of economic growth in India”, Economic and Political Weekly, 29, 2303-2309.

- (2003), "Regional disparity in economic and human development in India", Economic and Political Weekly, 27, 4166-4172.

- (1985), Regional disparity in economic growth in India. Himalaya, New Delhi.

DIPRETE, T.A., GREGORY M.E. (2006), "Cumulative advantage as a mechanism for inequality: a review of theoretical and empirical developments", Annual Review of Sociology, 32, 71-297.

DOEPKE, M. (2005), "Child mortality and fertility decline: does the barro-becker model fit the facts?" Journal of Population Economics, 18, 337-366.

DORIUS, S.F. (2008), "Global demographic convergence? a reconsideration of changing inter-country inequality in fertility", Population and Development Review, 34 (3), 519-539.

DORIUS, S. F. (2010), "Demystifying inter-country inequality: data, measures, and long-run trends in the inter-country and global distribution of human well-being”. An unpublished Dissertation in Sociology and Demography, the Pennsylvania State University. https:/etda.libraries.psu.edu/paper/11051/4963

DORIUS, S.F., FIREBAUGH G. (2010), "Trends in global gender inequality", Social Forces, 88 (5), 1941-1968.

DRÈZE, J., KHERA R. (2012), "Regional patterns of human and child deprivation in India”, Economic and Political Weekly, 47, 42-49.

DRÈZE, J., SEN, A.K. (2013). An Uncertain Glory, India and Its Contradictions. New Delhi: Penguin

DUPRE-MATTHEW, E. (2008), "Educational differences in health risks and illness over the life course: a test of cumulative disadvantage theory", Social Science Research, 37, 1253-1266. 
DATTA, G., RAVALLION, M., (2002), “Is India's economic growth leaving the poor behind'? Journal of Economic Perspectives, 16, 89-108.

DYSON, T. (1989), "The population history of Berar since 1881 and its potential wider significance", Indian Economic and Social History Review, 26 (2), 167-201.

DYSON, T. (2004), "India's population - the future”, in DYSON T., ROBERT C., VISARIA L., (eds) "21st Century India: Population, Environment and Human Development”, Oxford, Oxford University Press.

DYSON, T. (2010), "Population and development: the demographic transition”, Zed Book publication, London and New York.

EASTERLIN-RICHARD, A. (2000), "The worldwide standard of living since 1800", The Journal of Economic Perspectives, 14, 7-26.

EFRON, B., TIBSHIRANI R. (1993), "An introduction to the bootstrap", Chapman and Hall, New York.

EGGER, P., PFAFFERMAYR M. (2003), “On testing conditional sigma-convergence”, Working Paper, Department of Economics, University of Innsbruck, Austria.

EVANS, M.D.R. (1986), "American fertility patterns: a comparison of white and nonwhite cohorts born 1903-56”, Population and Development Review, 12 (2), 267-293.

EVANS, G.W., ELYSE K.E. (2002),. Socioeconomic status and health: the potential role of environmental risk exposure, Annual Review of Public Health, 23, 303-331.

FINGLETON, B., LÓPEZ-BAZO E., (2006), "Empirical growth models with spatial effects," Papers in Regional Science, 85(2), 177-198.

FINGLETON, B. (2005), Testing the 'new economic geography': a comparative analysis based on EU regional data, Urban/Regional 0504003, EconWPA.

FRANKLIN, R.S. (2002), "Fertility convergence across Italy's regions, 1952- 1995", Paper presented at the 41st Annual Meeting, Western Regional Science Association, Monterey, CA.

FRANKLIN, R.S. (2003), "Italian Fertility, 1864 to 1961: An analysis of regional trends", Paper prepared for the 43rd European Congress of the Regional Science Association, August 27-30, 2003, Jyväskylä, Finland.

FRIEDMAN, M. (1992), "Do old fallacies ever die"? Journal of Economic Literature, 30, 2129-2132. 
FRIEDMANN, J. (1956), 'Locational aspects of economic development", Land Economics, 32, 213-227.

- (1964), "Regional development in post-industrial society", Journal of the American Institute of Planners, 32 (3), 84-90.

FUJISAKI, I., PEARLSTINE V.E., MILLER M. (2008), "Detecting population decline of birds using long-term monitoring data", Population Ecology, 50, 275-284.

FUJITA, M., KRUGMAN P., VENABLES A., 1999. The spatial economy: cities, regions, and international trade, MIT Press, Cambridge, MA.

GÄCHTER, M., ENGELBERT T. (2011), "Health status convergence at the local level: empirical evidence from Austria”, International Journal for Equity in Health, $10,34$.

GALOR, O. (1996), "Convergence? Inferences from Theoretical Models," CEPR Discussion Papers 1350, C.E.P.R. Discussion Papers.

GAKIDOU, E.E., MURRAY C.J.L., FRENK J. (2001), A framework for measuring health inequality, WHO working paper, Geneva, Switzerland.

GHOSH, A. (1956), "Demographic trends in India during 1901-50", Population Studies, 9(3), 217-36.

GHOSH, B., MARJIT S., NEOGI C. (1998), "Economic growth and regional divergence in India 1960 to 1995", Economic and Political Weekly, 33, 1623-1630.

GHOSH, M. (2006), "Economic growth and human development in Indian states", Economic and Political Weekly, 41, 3321-3329.

- (2011), "Regional Disparities in education, health and human development in India", Indian Journal of Human Development 5, 5-28.

GOESLING, B., FIREBAUGH G. (2004), "The trend in international health inequality", Population and Development Review, 30, 131-146.

GOLDBERG M., MELCHIOR M., LECLERC A., LERT F. (2003), "Épidémiologie et déterminants sociaux des inégalités de santé", Revue d'épidémiologie et de santé publique; 51, 381-401.

GOLI S., AROKIASAMY, P., (2014). Maternal and Child Mortality Indicators across 187 countries in the World: Converging or Diverging. Global Public Health: An 
International Journal for Research, Policy and Practice, DOI: $10.1080 / 17441692.2014 .890237$

GOLI, S., REDDY, B., AROKIASAMY, P., (2014). Socioeconomic Progress across the major Indian states: Converging and Diverging. MPRA Paper No. 48978 and forthcoming in Social Science Research

GOLI, S., AROKIASAMY, P., (2013). Demographic transition in India: an evolutionary interpretation of population and health trends using 'change-point analysis’ PLoS ONE, 8(10), e76404. doi:10.1371/journal.pone.0076404.

GOLI, S., AROKIASAMY, P., (2013). Trends in health and health inequalities among major states of India: assessing progress through convergence models. Health Economics, Policy and Law. DOI: 10.1017/S1744133113000042.

GOLI, S., ABDUL, J., 2013. What is the cause of the decline in maternal mortality in India? Evidences from time series and cross-sectional analyses. J Biosoc. Sci. 22, $1-15$.

GOLI, S., 2012. Rural-Urban Divide in Health Status. Geography and You, September - October Issue.

GOLI, S., RIDDHI, D., AND AROKIASAMY, P. (2013). Pathways of economic inequalities in maternal and child health in urban India: A Decomposition analysis. PLOS One Medical Journal. DOI 10.1371/journal.pone.0058573.

GOVERNMENT OF INDIA (1971-2007), "Sample Registration System Year Books", Office of Registrar General of India, New Delhi.

GOVERNMENT OF INDIA (1973-2006), "Poverty Estimates Reports", Planning Commission, Government of India, New Delhi.

GRAHAM H. (2004a), "Social determinants and their unequal distribution: clarifying policy understandings", The Milbank Quarterly, 82 (1), 101-124.

GRAHAM H. (2004b), "Tackling health inequalities in England: remedying health disadvantages, narrowing gaps or reducing health gradients?" Journal of Social Policy, 33 (1), 115-131.

GROSSMAN G.M., HELPMAN, E. (1994), "endogenous innovation in the theory of growth," Journal of Economic Perspectives, American Economic Association, 8(1), 23-44. 
GUILMOTO, C.Z., RAJAN S.I. (2001), "Spatial pattern of fertility transition in Indian districts", Population and Development Review, 24 (4), 713-738.

GUJARATI, D. (2007). Basic Econometrics. New Delhi: Tata McGraw-Hill. pp. 278284.

GWATKIN, D.R. (2002), "Reducing health inequalities in developing countries", in Detels R., McEwen J., Beaglehoel R., Tanaka H. (eds) Oxford Textbook of Public Health, Oxford University Press, 1791-809.

GWATKIN, D.R. (2000), "Health inequalities and the health of the poor: what do we know? What can we do"? Bulletin of the World Health Organization, 78(1), 3-17.

ISLAM, N. (2003). "What have We Learnt from the Convergence Debate?" Journal of Economic Surveys, 17(3), 309-362.

ISLAM, N. (1998). "Growth Empirics: A Panel Data Approach- A Reply," The Quarterly Journal of Economics, 113(1), 325-329.

HAINES M.R. (2002), "Ethnic differences in demographic behaviour in the United States: has there been convergence"? Working Paper 9042, National Bureau of Economic Research, Cambridge (MA).

HALL, P. (1992), “Regional planning”, Routledge, London, New York.

Harper, S., Lynch, J. (2007), "Trends in socioeconomic inequalities in adult health behaviours among U.S. states, 1990-2004”, Public Health Reproductive, 122, 177 89.

HAVEMAN R, WOLFF E, (2004). "The concept and measurement of asset poverty: Levels, trends and composition for the U.S., 1983-2001"Journal of Economic Inequality, 2(2), 145-169.

HAVEMAN R, WOLFF E, (1995). "The Determinants of Children's Attainments: A Review of Methods and Findings," Journal of Economic Literature, American Economic Association, 33(4), 1829-1878.

HERBERTSSON, T.T., ORSZAG J.M., ORSZAG P.R. (2000), Population dynamics and convergence in fertility rates, Accessed at www.econ.bbk.ac.uk/wp/ewp/ewp9921.pdf

HINKLEY, D., SCHECHTMAN E. (1987), "Conditional bootstrap methods in the mean-shift model', Biometrika, 74(1), 85-93. 
HINKLEY, D.V. (1971), "Inference about the change-point from cumulative sum tests", Biometrika, 58(3), 509-523.

HIRSCHMAN, A.O. (1958), The strategy of economic development, Yale University Press, New Haven.

HIRSCHMAN, C. (1994), "Why fertility changes", Annual Review of Sociology 20, 203-233.

HOOVER, E.M. (1971), An introduction to regional economics, Alfred A. Knopf, New York.

HOSSEINI H., HAGHSHENAS N.M. (2009), "Dynamics of development and fertility convergence in Muslim countries", paper presented at the $26^{\text {th }}$ IUSSP International population conference, Morocco, 27 September-2 October.

HOSSEINPOOR A.R., DOORSLAER E.V., SPEYBROECK N., M. NAGHAVI, K. MOHAMMAD, R. MAJDZADEH, ET AL. (2006), "Decomposing socioeconomic inequality in the infant mortality in Iran", International Journal of Epidemiology, 35, 1211-19.

http://planningcommission.nic.in/data/datatable/index.php?data=datatab http://www.equityhealthj.com/content/10/1/34.

IIPS, MACRO INTERNATIONALS (1992-2006), "National Family Health Survey (13) data", Ministry of Health and Family Welfare, Government of India. http://www.nfhsindia.org/.

INSTITUTE OF APPLIED MANPOWER RESEARCH. (2011), “India Human Development Report 2011", Oxford University Press, New Delhi.

ISLAM, N. (2003), "What have We Learnt from the Convergence Debate?" Journal of Economic Surveys, 17(3), 309-362.

JAMES, K.S. (1995), "Demographic transition and education in Kerala", Economic and Political Weekly, 30(51), 3274-76.

JAMES, K.S., NAIR S.B. (2005), "Accelerated decline in fertility in India since the 1980s: trends among Hindus and Muslims", Economic and Political Weekly, 45(5), 375-84.

JAIN K. GOLI S., AROKIASAMY P., (2012). Are Self-reported Morbidities Deceptive in Measuring Socioeconomic Inequalities in India: A Case of Obstetric Morbidity in EAG States. Indian Journal of Medical Research, 136, 35-42. 
JHA, R. (2004), Reducing poverty and inequality in India: has the liberalization helped? in CORNIA, G.A. (ed) Inequality, Growth and Poverty in an Era of Liberalization and Globalization, Oxford University Press, New York, 297-327.

JOHNSON, P.A. (2000), "A non-parametric analysis of income convergence across the US”, Economic Letters, 69, 219-223.

KALEDIENE, R., PETRAUSKIENE, J. (2004), "Socioeconomic transition, inequality, and mortality in Lithuania", Economics and Human Biology, 2 (1), 87-95.

KANGASHARJU, A. (1998), " $\beta$-convergence in Finland: regional differences in speed of convergence", Applied Economics, 30, 679-687.

KAR, S., SAKTHIVEL S. (2007), "Reforms and regional inequality in India", Economic and Political Weekly, 42(47), 69-77.

KARKAL, M. (1985), "Maternal and infant mortality: reviewed Work(s)", Economic and Political Weekly, 20(43), 1835-1837.

KATHERINE, E.S., DAVID J.H., BLACKMAN T ET AL. (2009), "Divergence or convergence? health inequalities and policy in a developed Britain", Critical Social Policy, 29, 216-242.

KAKWANI, NC, WAGSTAFF A, VAN DOORSLAER E. (1997), Socioeconomic inequalities in health: measurement, computation and statistical inference. Journal of Econometrics, 77, 87-104.

KAWACHI, I, SUBRAMANIAN S, et al. (2002), A glossary for health inequality. Journal of Epidemiology and Community Health, 56, 647-52.

KENNY, C. (2005a), "There's more to life than money: examining the link between income, health and education, mimeo, World Bank.

KIRK, D.. (1996), "Demographic Transition Theory." Population Studies 50:361.

KITAGAWA, E.M., HAUSER, M.P. (1973), Differential mortality in the United States: a study in socioeconomic epidemiology. Cambridge, MA: Harvard University Press.

KOTZAMANIS, B., DUQUENNE M.N. (2006), "Les disparité démographiques en Grèce, convergence OR divergence'? Balkan Demographic Paper no. 7. Demobalk Network. 
KONDA, N, SUBRAMANIAN S, et al. (2009) Income inequality, mortality and self rated health: Meta analysis of multilevel studies. British Medical Journal, 339 b4471.

KRUGMAN, P. (1979), "Increasing returns, monopolistic competition and international trade", Journal of International Economics, 9, 469-479.

- (1991a), "Increasing returns and economic geography", Journal of Political Economy, 99, 483-499.

- (1991b), Geography and Trade. MIT Press, Cambridge.

KUMAR, A., MOHANTY S.K. (2011), Socioeconomic differentials in childhood immunisation in India, 1992-2006, Pop Research, 28, 301-324 DOI 10.1007/s12546-011- 9069 .

KULKARNI, P.M. (2011), "Towards an explanation of India's fertility transition", Paper presented at the George Simmons Memorial Lecture, 33rd Annual Conference of the IASP, Lucknow, November 11-13, 2011.

KULKARNI, P.M., ALAGARAJAN M. (2005), "Population growth, fertility and religion in India”, Economic and Political Weekly, 45(5), 403-11.

KUMAR, S., RUSSELL, R.R. (2002), "Technological change, technological catch-up, and capital deepening: relative contributions to growth and convergence", American Economic Review, 92, 527-548.

KURIAN, N.J. (2000), "Widening regional disparities in India - some indicators", Economic and Political Weekly, 35, 538-550.

- (2007), "Widening economic and social disparities: implications for India", Indian Journal of Medical Research, 126, 374-380.

KULKARNI, P.M. (2011), "Towards an explanation of India's fertility transition", George Simmons Memorial Lecture, 33rd Annual Conference of the IASP, Lucknow, November 11-13, 2011.

KUZNETS, S. (1957), "Quantitative aspects of economic growth of nations-II", Economic Development and Cultural Change, 5, 3-111.

LANZIERI, G. (2010), "Is there a fertility convergence across member states of the European union'? Working Paper 12. United Nations Statistical Commission and Economic Commission for Europe. 
LEE, R. (2003), The Demographic Transition: Three Centuries, University of Pennsylvania.

LEE, R.D., REHER D.S. (eds) (2011), "Demographic transition and its consequences", Supplement to Population and Development Review, 37.

LEGRAND, T. K., PHILLIPS. J. F., (1996). "The Effect of Fertility Reductions on Infant and Child Mortality: Evidence from Matlab in rural Bangladesh." Population Studies, 50, 51-68.

LESTHAEGE, R., CAMILLE V. (2001): "Ready, willing and able: a conceptualization of transitions to new behavioural norms" in CASTERLINE B.J. (ed) "Diffusion processes and fertility transition: selected perspectives", National Academy Press, Washington DC, 240-64.

LESTHAEGHE, R., (1983), "A Century of Demographic and Cultural Change in Western Europe: An Exploration of Underlying Dimensions." Population and Development Review 9, 411-435.

LIPTON, M. (1977), Why poor people stay poor: urban bias in world development, Harvard University Press, Cambridge, MA.

LOWRY, D., XIE Y. (2009), "Socioeconomic status and Health differentials in China: Convergence or Divergence at older ages"? Population Studies Research Center Report 09-690, University of Michigan.

LUCAS, R.E. (1988), "On the Mechanics of Economic Development", Journal of Monetary Economics, 22 (1), 3-42.

LUCAS, R.E. (2000), "Some macroeconomics for the 21st Century", Journal of Economic Perspectives, 14 (1), 159-168.

MACKENBACH, J.P., BOS V., ANDERSEN O., ET AL. (2003), "Widening socioeconomic inequalities in mortality in six western European countries", Int $J$ Epideomiol, 32, 830-37.

MACKENBACH, J.P., STRONK K. (2002), "A strategy for reducing health inequalities in the Netherland", British Medical Journal, 325, 1029-32.

MACLANAHAN. (2004), "Diverging destinies: How children are faring under the second demographic transition", Demography, 41(4), 607-627. 
MAHALANOBIS, P.C., BHATTACHARYA D. (1976), "Growth of population in India and Pakistan-1801-1961”, Artha Vijnana, 18, 1-10.

MANKIW, N.G., WEIL D.N., ROMER D. (1992), "A contribution to the empirics of economic growth", The Quarterly Journal of Economics, 107, 407-437.

MARJIT, S., MITRA, S., (1996), "Convergence in regional growth rates: Indian research agenda”, Economic and Political Weekly, 17 August, 2239-2242.

MARMOT, M. (2002), The influence of income on health: view of an epidemiologist. Health Affairs, 21 31- 46.

MARMOT, M. (2005), Social determinants of health inequalities. The Lancet, 365, 1099-104.

MARMOT, M. (2010), "Fair society healthy lives [The marmot review]", ULC institute for health equity, London.

MARMOT, M. (2010) Fair society healthy lives, a marmot review: Strategic Review of Health Inequality in England Post -2010, 2010.

MACKENBACH, JP, STRONK K. (2002), A strategy for reducing health inequalities in the Netherland. British Medical Journal, 325, 1029-32.

MCKEOWN T (1976), The Modern Rise of Population. London, Edward Arnold.

MCCOMBIE J. S. L. (1981), What Still Remains of Kaldor's Laws? The Economic Journal, 91 (361), 206-216.

MAYER-FOULKES, D. (2003), "Convergence clubs in cross-country life expectancy dynamics", in ROLPH V. D. H., SHORROCS A. F. (eds) "Prospective on poverty and growth”, United Nations University Press, Tokyo, 144-171.

McMICHAEL, A.J. (2001), Human frontiers, environmental and disease: past patterns, uncertain, Cambridge University Press, Cambridge.

MCMICHAEL, A.J., MCKEE M., SHKOLNIKOV V., VALKONEN T. (2004), "Mortality trends and setbacks: global convergence or divergence?" Lancet, 363, 1155-59.

MCNAY, K., AROKIASAMY P., CASSEN H.R. (2004), "Female education and fertility decline: recent development in the relationship", Population Studies, 41(1), 5-30. 
MERRICK, T. (2001), Population and poverty: a review of reviews, in BIRDSALL N., KELLEY A.C., SINDING S. (eds) "Population matters: demographic change, economic growth, and poverty in the developing world", Oxford University Press, New York, 201-212.

MISRA, R.P. (ed) (1969), Regional planning: concepts, techniques and policies. The University of Mysore, Mysore.

MITRA, K.C. (2005), Encyclopaedia of population studies and demography: critical issues in development dynamics, Dominant Publishers, New Delhi.

MONTERO-GRANADOS, R., JIMENEZ D.J., MARTIN J. (2007), 'Decentralisation and convergence in health among the provinces of Spain (1980-2001)", Social Science and Medicine, 64(6), 1253-1264.

MONTGOMERY, M. R., CASTERLINE J. B. (1996), "Social influence, social learning and new models of fertility", Population and Development Review, 22,151-165.

MONTGOMERY, K. (1999), The Demographic Transition, Geography, UWC.

MOSER, K., SHKOLNIKOV V., LEON D. (2005), "World mortality 1950-2000: divergence replaces convergence from the late 1980s", Bulletin of the World Health Organization, 83(3), 202-209.

MUKHERJEE, M (1969), National income of India: trends and structure, Statistical Publishing Society, Calcutta.

MYRDAL, G. (1957), "Economic theory and under-developed region", Gerald Duckworks, London.

NAGARAJ, R., VAROUDAKIS A., VEGANZONES M. (2000), 'Long-run growth trends and convergence across Indian states", Journal of International Development, 12, 45-70.

NAMBOODIRI, K (1996) A Primer on Population Dynamics. New York, SpringerVerlag.

NAVANEETHAM, K, DHARMALINGAM, A (2002). Utilisation of maternal health care services in Southern India. Social Science and Medicine, 55, 1849-1869.

NAZRUL, I. (2003), "What have we learnt from the convergence debate"? Journal of Economic Surveys, 17, 311-362. 
NEUMAYER, E. (2004), "HIV/Aids and cross-national convergence in life expectancy", Population and Development Review, 30(4), 727-742.

NEUMAYER, E. (2004), "Recessions lower (some) mortality rates: evidence from Germany", Social Science and Medicine, 58(6), 1037-1047.

NOTESTIEN, F.W. (1945), "Population: the long view", in SCHULTZ (ed) "Food for the world", University of Chicago Press, Chicago.

O'CONNELL, M. (1981), 'Regional fertility patterns in the United States: convergence or divergence"? International Regional Science Review, 6, 1-14.

O' Donnell et al. (2008), Analysis health equity using household survey data: a guide to techniques and their implementation. World Bank Institute. Washington DC.

OEPPEN J. (1999), "The health and wealth of nations since 1820", Paper presented at the Annual Meeting of the Social Science History Association, Fort Worth, TX.

OFFICE OF REGISTRAR GENERAL OF INDIA (1971-2007), "Compendium of Sample Registration System", Registrar General of India, Ministry of Home Affairs, Government of India.

OFFICE OF REGISTRAR GENERAL OF INDIA (2009), Sample Registration System Year Book, Government of India, New Delhi.

http://censusindia.gov.in/Vital_Statistics/SRS/Sample_Registration_System.aspx.

OFFICE OF REGISTRAR GENERAL OF INDIA AND CENSUS COMMISSIONER (1981-2011), “Census 1981-2011”, Ministry of Home Affairs, Government of India, New Delhi. http://censusindia.gov.in/.

OFFICE OF REGISTRAR GENERAL OF INDIA (1981-2007), Compendium of Sample Registration System Year Books, Government of India, New Delhi. http://censusindia.gov.in/Vital_Statistics/SRS/Sample_Registration_System.aspx.

OMRAN A.R. (1971), "The epidemiological transition: theory of the epidemiology of population change", Milbank memorial fund quarterly, 49, 509-538.

ORTIZ I., CUMMINS M., (2013), "The Age of Austerity - A Review of Public Expenditures and Adjustment Measures in 181 Countries." 2013. New York and Geneva: IPD and South Centre 
OTTAVIANO, G.I.P., THISSE J. (2004), "Agglomeration and economic geography", in HENDERSON V., THISSE JACQUES F. (eds) "Handbook of regional and urban economics. North-Holland press: Amsterdam, 2563-2608.

OTtAVIANO G., PUGA, D., (1997). Agglomeration in a global Economy: A Survey, CEP Discussion Papers dp0356, Centre for Economic Performance, LSE.

PAGE E.S. (1957), "On problems in which a change in parameter occurs at an unknown point”, Biometrika, 44, 248-252.

PAL, P., GHOSH J. (2007), "Inequality in India: a survey of recent trends", DESA Working Paper No. 45, ST/ESA/2007/DWP/45. United Nations, New York.

PALlONI, A. (1990), The meaning of the health transition. Pp. xvi-xvii in Caldwell John et al. (ed.) What We Know about Health Transition, Canberra: Australian National University.

PANAYOTOV J. (2008). Public Health and Average Health Status: Do Health Inequalities Matter, ICARE, Melbourne, Australia

https://www.academia.edu/209927/Public_Health_and_Average_Health_Status_Do_H ealth_Inequalities_Matter_Panayotov_Matrix_

PARR, J.B. (1999), "Growth-pole strategies in regional economic planning: a retrospective view”, Urban Studies, 36, 1195- 1215.

PERLMAN, R.L. (2008), "Socioeconomic inequalities in ageing and health", The Lancet, 372, Supplement 1, S34-S39.

PERROUX, F. (1950), "Economic space: theory and applications", Quarterly Journal of Economics, 64, 89-104.

PETTITT, A.N. (1980), "A simple cumulative sum type statistic for the change-point problem with zero-one observations", Biometrika, 671, 79-84.

PFAFFERMAYR, M. (2007), "Conditional Beta- and Sigma-Convergence in Space: A Maximum Likelihood Approach,"Working Papers 2007-17, Faculty of Economics and Statistics, University of Innsbruck.

PFAFFERMAYR, M. (2009), "Conditional [beta] and [sigma]-convergence in space: A maximum likelihood approach," Regional Science and Urban Economics, 39(1), 6378. 
PO JYT., SUBRAMANIAN SV (2010) Mortality burden and socioeconomic status in India. PLOS ONE, 6 e16844.

PRADHAN, M., SAHN E.D., YOUNGER, D.S. (2003), "Decomposing world health inequality", Journal of Health Economics, 22, 271-293.

PRASAD, P.H. (1988), "Roots of uneven regional growth in India", Economic and Political Weekly, 23, 1689-1692.

PRADHAN J and AROKIASAMY P, (2010) Socioeconomic inequality in child survival in India: a decomposition analysis. Health Policy, 2, 114-20.

PATHAK, K.P., SINGH, A. (2011), "Trends in malnutrition among children in India: growing inequalities across different economic groups", Social Science and Medicine, 73, 576-585

PRESTON, S. H. (1976), Mortality pattern in national populations, Academic press, New York.

PRESTON, S.H. (1980), Causes and consequences of mortality declines in less developed countries during the twentieth century, in EASTERLIN R.A. ed. Population and economic change in developing countries. The University of Chicago Press, Chicago and London, 289-360.

PRESSAT R., (1985), Contribution des écarts de mortalité par âge à la différence des vies moyennes, Population, 40 (4-5) 766-770.

PRITCHETT. (1996), "Forget convergence: divergence past, present and future", Finance and Development, 33, 40-43.

PUGA, D. (1996), The Rise and Fall of Regional Inequalities, CEP Discussion Papers dp0314, Centre for Economic Performance, LSE.

QUAH, D.T. (1993), "Empirical cross-section dynamics in economic growth", European Economic Review, 37, 426-434.

- (1996a), "Empirics for economic growth and convergence", European Economic Review, 40, 1353-1375.

- (1996b), "Twin peaks: growth and convergence in models of distribution dynamics", The Economic Journal, 106, 1045-1055. 
RAILEANU S.M. (2011), "Exploring the economic convergence in the European Union's new member states by using non- parametric models", Romanian Journal of Economic Forecasting, 14, 20-40.

RAM, R. (1998), "Forty years of the life span revolution: an exploration of the roles of convergence, income, and policy", Economic Development and Cultural Change, 46(4), 849-857.

RAM U, JHA P, RAM F, et al. (2013), Neonatal, 1-59 month, and under-5 mortality in 597 Indian districts, 2001 to 2012: estimates from national demographic and mortality surveys. Lancet Glob Health; 1, e219-26.

RAO H. (1984), Regional Disparities and Development in India, Ashish Publishing House, New Delhi.

RAO M.G., SHAND R., KALIRAJAN K.P. (1999), "Convergence of incomes across Indian states: a divergent view”, Economic and Political Weekly, 34, 769-778.

RAVALLION (2003), 'Measuring Aggregate Welfare in Developing Countries: How Well do National Accounts and Surveys Agree?' Review of Economics and Statistics, 85, 645-652.

RBI (1955-2011), Handbook of statistics. New Delhi: Reverse Bank of India, Government of India.

REGISTRAR GENERAL OF INDIA (1971-2007), "Compendium of Sample Registration System Year Books". Government of India, New Delhi. http://censusindia.gov.in/Vital_Statistics/SRS/Sample_Registration_System.aspx

REGISTRAR GENERAL OF INDIA (2009), Sample Registration System Year Book. Government of India, New Delhi.

REGISTRAR GENERAL OF INDIA, CENSUS COMMISSIONER (1971-2011), “Census 1971-2011". Ministry of Home Affairs, Government of India, New Delhi. Available at http://censusindia.gov.in/

REHER, D.S. (2004), "The demographic transition revisited as a global process", Population Space and Place, 10, 19-41.

RELE, J.R. (1987), "Fertility levels and trends in India, 1951-81", Population and Development Review, 13(3), 513-530. 
RELE, J.R. (1982), "Trends and differentials in fertility in population of India", Country Monograph Series No.10, ST/ESCAP/220. New York: ESCAP, U.N.:91108.

REY, S., MONTOURI, D.B. (1999), "US regional income convergence: a spatial econometric perspective", Regional Studies, 33, 143-156.

ROMER, P.M. (1986), "Increasing returns and long-run growth", Journal of Political Economy, 94, 1002-1037.

- (1990), "Endogenous technological change", Journal of Political Economy, 98, 71102.

ROSS, C. E., CHIA-LING W.U. (1995), "The Link between Education and Health", American Sociological Review, 60, 719-745.

ROSS, C. E., CHIA-LING W.U. (1996), "Education, Age, and the Cumulative Advantage in Health", Journal of Health and Social Behaviour, 37,104-20.

ROWE, M L, THAPA BK et al. (2005), How does schooling influence maternal health practices? Evidence from Nepal. In: David EB (Eds) Special Issue on education and public health, 512-533.

ROSS, J., EDWARD A., KATHERINE A. (2004), "Plateaus during the rise of contraceptive prevalence," International Family Planning Perspectives, 30(1), $39-44$.

ROY, C., DATTA U. (1992), 'Inter-state and intra-state variations in economic development and standard of living", Economic and Political Weekly, 27, 26512655 .

- (1993), "Inter-state and intra-state variations in economic development and standard of living”, Journal of Indian School of Political Economy, 1, 47-68.

SAIKIA, N., JASILIONIS D., RAM F., SHKOLNIKOV V. (2010), “Estimates of mortality under age 60 in India and its states, 1970-2004", MPIDR Technical Report 2006-2010 August .

SALA-I-MARTIN, X. (1996a), "Regional cohesion: evidence and theories of regional growth and convergence", European Economic Review, 40, 1325-52.

- (1996b), "The classical approach to convergence analysis", The Economic Journal, 106, 1019-1036. 
SALA-I-MARTIN, XAVIER X. (1996), "The classical approach to convergence analysis", The Economic Journal, 106, 1019-1036.

SARKER, P.C. (1994), "Regional imbalances in Indian economy over plan periods", Economic and Political Weekly, 27(11), 621-633.

- (1995), "Regional disparities in India: issues and measurement", PhD Dissertation, University of Bombay, Bombay.

SCIUBBA, J. (2006), "The Implications of Demographic Convergence", Paper presented at the annual meeting of the International Studies Association, San Diego, California, USA, Mar 22, 2006.

SCOTT, D.W. (1978), "Choosing the window width when estimating a density", Biometrika, 65, 1-11.

- (1979), “On optimal and data based histograms”, Biometrika, 66, 605-610.

SEGALL, M., G. TIPPING H. LUCAS, DUNG T.V, TAM N.T, VINH D.X., HUONG D.L. (2000), "Health Care Seeking by the Poor in Transitional Economies: the Case of Vietnam", Institute of Development Studies, Research Report No. 43, Brighton, Sussex.

SEN, A. (1973), “On Economic Inequality”, New York, Norton. (Expanded edition with a substantial annexe by James E. Foster and A. Sen, 1997).

SEN, A. (1983), 'Development: which way now", The Economic Journal, 93, 745-762.

- (1998), 'Mortality as an indicator of economic success and failure", The Economic Journal 108, 1-25.

SHIBUYA, K., BOERMA J.T. (2005), "Measuring progress towards reducing health inequalities", Bulletin of the World Health Organization, 83(3), 161-240.

SHKOLINKOV, V.M. ET AL. (2011), "Increasing absolute mortality disparities by education in Finland, Norway and Sweden, 1971-2000", Journal of Epidemiology and Community Health, 66, 372-378.

SHKOLNIKOV, V.M., ANDREEV E.M., BEGUN A.Z. (2003), "Gini coefficient as a life table function: computation from discrete data, decomposition of differentials and empirical examples", Demographic Research, 8(11), 305-358. 
SHABNAM, J, GIFFORD M and DALAL K. (2011), Socioeconomic inequalities in the use of delivery care services in Bangladesh: a comparative study between 2004 and 2007. Health, 3, 762-771.

SHIFFMAN, J, (2000) Can poor countries surmount high maternal mortality? Study in Family Planning 31 274-289.

SIBANDA, T., SIBANDA N. (2007), "The CUSUM chart method as a tool for continuous monitoring of clinical outcomes using routinely collected data", BMC Medical Research Methodology, 7(46), 1-7.

SINDING, S.W. (2001), Foreword. In Bulatao R.A., Caterline J.B., eds. Global Fertility Transition, Population and Development Review, A supplement to Vol. 27, 9-10.

SINGH, A, PATHAK PK, PAN W, (2011) Infant and Child Mortality in India in the Last Two Decades: A Geospatial Analysis. PLoS ONE, 6(11), e26856. Doi: 10.1371/ journal. Pone .0026856

SIRIOPPULOS, C., ASTERIOU D. (1997), "Testing the convergence hypothesis for Greece", Managerial and Decision Economics, 18, 383-389.

SIRIOPOULOS, S.C., ASTERIOU D. (1998), "Testing for convergence across the Greek regions", Regional Studies, 32, 537-546.

SOLOW, R.M. (1956), "A contribution to theory of economic growth", The Quarterly Journal of Economics, 70, 65-94.

SPEYBROECK, N, HOSSEINPOOR AR, et al, (2010) Decomposition socioeconomic health inequality. International Journal of Public Health, 55, 347-351.

SPOORENBERG T., DOMMARAJU P. (2012) Regional Fertility Transition in India: An Analysis Using Synthetic Parity Progression Ratios, International Journal of Population Research, Volume 2012, Article ID 358409, 20 pages.

SRINIVASAN, K., CHANDER SHEKHAR, AROKIASAMY P. (2007), "Reviewing reproductive and child health programme in India", Economic and Political Weekly, 42(27): 2931-39.

SUBRAMNAIAN, S.V., KAWACHI I. (2004), Income Inequality and Health: what have you learn so far? Epidemiological Review, 26, 78- 91.

SUBRAMNAIAN, S, et al. (2006), "The mortality divide in India: the different contribution of gender, caste and standard of living across the life course", American Journal of Public Health, 5, 818-825. 
SUBRAMANIAN, S. (2008), "Health inequalities in India: the axes of stratification", The Brown Journal of World Affairs, 6, 127-38.

SUBRAMANYAM M.A., KAWACHI I., et al. (2010), "Socioeconomic inequalities in childhood under nutrition in India: analyzing trends between 1992 and 2005", PLoS One, 5 e11392.

SUBRAMANYAM, M.A., SUBRAMANYAM S.V. (2011), Research on social inequalities in health in India. Indian Journal of Medical Research, 133, 461-463.

SUTCLIFFE, B. (2005), "A converging or diverging world: SESA", Working Paper No. 2 ST/ESA/SWP/2.

SUBRAMANIAN, S. V., and Smith, G. D. (2006), Patterns, distribution, and determinants of under- and overnutrition: a population-based study of women in India. American Journal of Clinical Nutrition, 84(3), 633e640.

SUBRAMANIAN, S. V., ERTEL, K. (2009), Self-rated health may be adequate for broad assessments of social inequalities in health. International Journal of Epidemiology, 38, 319-320.

SUTCLIFFE, B. (2005), “A Converging or Diverging World?" DESA Working Paper No. 2 ST/ESA/2005/DWP/2.

SUZUKI, E., KASHIMA, S., KAWACHI, I., SUBRAMANIAN, S.V. (2005), Social and geographic inequalities in premature adult mortality in Japan: an observational study from 1970 to 2005. British Medical Journal, 2, e000425.

SUVEDI BK, et al. (2009), Nepal maternal mortality and morbidity study: summary of preliminary findings. Family Health division, Department of Health Services, Ministry of Health, Government of Nepal.

TAYLOR, W. (2000a), Change-Point Analyzer 2.0 Shareware Programme, Taylor Enterprises, Libertyville, Illinois. Available online at: http:/www.variation.com/cpa.

TAYLOR, W. (2011), "Change-point analysis: a powerful new tool for detecting changes", Taylor Enterprises, Libertyville, Illinois. Available online at: http://www. variation.com/cpa.

TAYLOR, S. (2009), "Wealth, health and equity: convergence to divergence in late 20th century globalization", British Medical Bulletin, 91, 29-48.

TEWARI, R.T. (1985), "Inter-regional disparities in levels of development", in. Mishra P.G. (ed) "Regional structure of development and growth in India", Ashish Publishing House, New Delhi, 102-23. 
THEIL, H. (1967). Economics and Information Theory. Chicago: Rand McNally and Company.

THE LANCET (2013), The Grand Convergence: Future Sustainable Goal', the Lance Commission Sustainable Goal. The Lancet, 383 (9913), 187.

THOMPSON, W.S. (2003), “Encyclopedia of Population 2”, 939-40.

TONOYAN, T., LUSINE, M. (2012), "Health inequalities in Armenia - analysis of survey results", International Journal for Equity in Health, 11:32.

TRIVEDI, K. (2002), "Regional convergence and catch-up in India between 1960 and 1992”, Working Paper No. 2003-W01. Nuffield College, University of Oxford, Oxford.

UNICEF (2011), "Global Inequality: Beyond the Bottom Billion - A Review of Income Distribution in 141 Countries." UNICEF.

http://www.uniceforg/socialpolicy/index_58230.html

UNITED NATIONS DEVELOPMENT PROGRAMME. (2011), "Human Development Index Report-2011”, India, New York, USA.

UNITED NATIONS. (1999), "World Population Prospects: The Revision 1999", Population database, Department of Economic And Social Affairs, Population Division, New York.

UNITED NATIONS. (2002), "Fertility levels and trends in countries with intermediate levels of fertility," in Completing the Fertility Transition, Report of the Expert Group Meeting on Completing the Fertility Transition, Department of Economic and Social Affairs, Population Division.ESA/P/WP.172/Rev.1.NewYork:UnitedNations,

(http//www.un.org/esa/population/publications/completingfertility/completingfertilit y.htm).

UNITED NATIONS (2003), Levels and Trends of Contraceptive Use as Assessed in 2002. Department of Economic and Social Affairs New York: United Nations.ST/ESA/SER.A/190.

VALLIN, J., MESLÉ, F. (2004), "Convergences and Divergences in Mortality: A New Approach of Health Transition

VAN DE POEL ET AL. (2008), "Socioeconomic inequality in malnutrition in developing countries", Bulletin of the World Health Organization, 86, 282-91. 
VAN DE WALLE E., KNODEL J. (1980), "Europe's fertility transition: new evidences and lessons for today's developing world", Population Bulletin, 34(6), 3-44.

VAN DE WALLE E. (1990), How do we define the health transition? Pp. xiv-xv in Caldwell J. et al. (ed.) What We Know about Health Transition, Canberra: Australian National University.

VARBLANE, U., VAHTER, P. (2005), "An Analysis of the Economic Convergence Process in the Transition Countries. University of Tartu", Economics and Business Working Paper No. 37.

VICTORA, C.G., WAGSTAFF A., SCHELLENBERG J.A., GWATKIN D., CLAESON M., HABICHT P.J. (2003), "Applying an equity lens to child health and mortality: more of the same is not enough", Lancet, 362(9379): 233-41.

VAUPEL J.W. (1986), How changes in age-specific mortality affects life expectancy. Population Studies, 40,147-157.

VAUPAL J.W, ROMO C. (2002), Decomposing change in the life expectancy: A bouquet of formulas in honour of Nathan Keyfitz's 90th birthday, Demography 40, 201-16.

VINING D.R. (1986), Social versus reproductive success: The central theoretical problem of human socio-biology. Behavioural and Brain Sciences, 9, 167-187.

VISARIA, L. (2004b), "The continuing fertility transition", in Dyson T., Cassen R., Visaria L. (eds) "Twenty-First Century India-Population, Economy, Human Development, and the Environment", New Delhi: Oxford University Press, 57-73.

VISARIA, L. (2011), "Demographic transition in South India", Special Series Paper, Population Foundation of India, New Delhi.

VISARIA, P., VISARIA L. (1994), "Demographic transition: accelerating fertility decline in 1980s", Economic and Political Weekly, 29(51/52), 3281-3292.

VISARIA, P., VISARIA L. (2003), "Long-Term Population Projections for Major States, 1991-2001”, Economic and Political Weekly, 38(45): 4763-4775.

VISARIA, L. (2004a), "Mortality trends and the health transition", in DYSON T., CASSEN R. VISARIA L. (eds), "Twenty-first century India-population, economy, human development, and the environment", Oxford University Press, New Delhi, $32-56$. 
VNA BON-MARTENS ET AL. (2012), "Health inequality in the Netherland: a cross sectional study of a role of Type D (distressed) personality", British Medical Journal Public Health, 12, 46.

WAGSTAFF, A. (2000), "Socioeconomic inequalities in child mortality: a comparison across nine developing countries", Bulletin World Health Organization, 78, 19-29.

WAGSTAFF, A. (2002), "Inequalities in health in developing countries: swimming against the Tide"? Working Paper, World Bank, Washington DC, USA.

WAGSTAFF, A., PACT P., DOORSLAER E.V. (1991), "On the measurement of inequalities in health", Social Science Medicine, 33(5), 545-557.

WAGSTAFF, A., WATANABE, N. (2000), Socioeconomic inequalities in child malnutrition developing world. In Policy research working paper, 2434. Washington D.C: World Bank.

WAGSTAFF, A, VAN DOORSLAER E and WANTANBE N. (2003) On decomposition the cause of health sector inequality, with as an application to malnutrition inequality in Vietnam. Journal of Econometrics, 112, 219-27.

WAGSTAFF, A., and DOORSLAER, V. E. (2004), Overall versus socioeconomic health inequality: a measurement framework and two empirical illustrations. Health Economics, 13(3), 297e301.

WAGSTAFF, A. (2002a). Caring more about the poor: Inequality aversion, health inequalities and health achievements. Washington D.C: World Bank.

WAGSTAFF, A. (2002b), Poverty and health sector inequalities. Bulletin of World Health Organization, 80(2), 97e105.

WATKINS, S.C. (1990), "From provinces into nations: demographic integration in Western Europe, 1870-1960", Princeton University Press, Princeton.

WEEKS, J.R., GETIS A., HILL A.G., GADALLA S., RAHED T. (2004), "The fertility transition in Egypt: intra-urban pattern in Cairo", Annals of the Association of American Geographers, 94(1), 74-93.

WHITEHEAD, M., DAHGREN G., EVANS T. (2001), "Equity and health sector reforms: can low-income countries escape the medical poverty trap"? Lancet, 358, 833-36.

WHITEHEAD, M. (1992), "Perspectives in health inequity", International Journal of Health Services, 22, 429-445.

WHITEHEAD, M., PETTICREW, M., GRAHAM, H., MACINTYRE, S., BAMBRA, C., EGAN, M. (2004), "Evidence for public health policy on inequalities: 2: 
Assembling the evidence jigsaw", Journal of Epidemiology and Community Health. 58, 817-821.

WHITEHEAD, M., DAHLGREN, G. (2006), Levelling up (part 1): A discussion paper on concepts and principles for tackling social inequities in health. Copenhagen: WHO Regional Office for Europe.

WILLIAMSON, J.G. (1965), "Regional inequality and the process of national development: a description of the patterns", Economic Development and Cultural Change, 13, 3-45.

WILKINSON, R., MARMOT, M. (2003), Social determinants of health: the solid facts. World Health Organization, Europe.

WILLSON, A.E., SHUEY K.M., ELDER G.H., JR (2007), “Cumulative advantage processes as mechanisms of inequality in life course health", American Journal of Sociology, 112, 1886-1924.

WILSON, C. (2001), "On the scale of global demographic convergence 1950-2000", Population Development Review, 27, 155-71.

WILSON, C. (2011), “Understanding global demographic convergence since 1950", Population Development Review, 37(2), 375-88.

WILSON, C., AIREY P. (1999), "How can a homeostatic perspective enhance demographic transition theory", Population Studies, 53(2), 117-128.

WOOLDRIDGE, J. (2002), "Econometric Analysis of Cross Section and Panel Data", Cambridge: MIT Press.

WORKIE, M. (2003), "Absolute convergence across time and space: new empirical evidence for an old debate", Journal of Economics, 51, 1270 -1290.

WORLD, HEALTH ORGANIZATION (2001), "On the measuring of health inequalities", Report on WHO technical consultation Geneva, Switzerland, November 7-8.

WORLD HEALTH ORGANIZATION (2002), "World report on violence and health. 9-11”, World Health Organization, Geneva.

WORLD HEALTH ORGANIZATION. (2007), "The world health on health inequalities, inequality, and social determinants of health", Population and Development Review, 33(4), 567-585. 
WORLD HEALTH ORGANIZATION. (2008), World health report on primary health care: now more than ever, World Health Organization, Geneva.

WORLD HEALTH ORGANIZATION. (2012). WHO Glossary of Public Health Terms, World Health Organisation, Geneva.

YOUNG, A.T., HIGGINS M.J., LEVY D. (2008), " 2 -convergence versus $\beta$ convergence: evidence form U.S. county-level data", Journal of Money, Credit and Banking, 40, 1083-1093. 


\section{Annexure}

Comments on the dissertation entitled "Demographic Convergence and its Linkages with Health Inequalities in India" submitted by Mr. Srinivas Goli for the Ph.D degree to the International Institute for Population Sciences, Mumbai.

The dissertation entitled "Demographic Convergence and its Linkages with Health Inequalities in India" by Mr. Srinivas Goli addresses an important and the growingly significant topic of "convergence" to the demographic trends in India using states as units and also selected socioeconomic factors as literacy and below poverty line groups. The term "convergence" has different connotations in different disciplines. In mathematics it signifies the narrowing of the averages in income or output per capita between different groups. Inequalities in income and various development parameters, between the rich and the poor within a country, between countries and between ethnic groups are hotly debated and emotionally charged topics. The whole works of Marx and Engles are devoted to these topics and recommend systemic methods to reduce inequalities. The concept of Human Development Index and various other indices based on which countries are being compared, regularly by the United Nations and ranked are basically methods to address this issue of inter country inequalities and suggest methods of reducing the gaps. There is a growing feeling throughout the world that gross inequalities between the rich and poor endanger the peace and stability of the human race. Hence, the emphasis on convergence.

Application of the principles and techniques of study of convergence specifically to demographic and health parameters using various measures of inequality, seem to be relatively new, probably starting with the works of Dorius (2010) and Wilson (2001, 2011). The later author has elaborated on these in a number of articles but all based on inter country comparisons of demographic parameters in developed countries. Hence, this topic in demography is hardly fifteen years old and it is a creditable thing for Mr. Goli to have taken up this problem for analysis of convergence of parameters in India on fertility, mortality, child health care utilization and outcomes and linkages of demographic convergence and child health inequalities. He has done a commendable job of developing appropriate tool kits for measuring convergence (mostly expanding on those developed earlier for developed countries) and using them on them on Indian data and coming to very interesting conclusions. There are ten chapters in his thesis and each one of them is substantially rich and analytically forceful, but there are many editorial mistakes which the candidate could have corrected by re-reading the draft. For example the second line of chapter I "Introduction" reads "........negligible population growth characterized by characterized by high birth and low death rates...." It should be high death rates. Similarly, many sentences are incomplete and confusing. 
The candidate uses the "inequality life-cycle hypothesis" developed by Dorius which states that during demographic and health transition inequalities between groups and countries first increase and then decrease i.e. Equilibrium disequilibrium- equilibrium seems to the mantra that the candidate is sold to and proves its validity across the states in India. There is a major difference between the convergence of demographic and socioeconomic parameters in the developed countries of the west and what is taking place in India and other developing countries. In the later the convergence is forced by the government of the day by specific policies and programs. In China all couples have one child families and there is a sharp convergence of fertility across all groups and geographic areas, but it is all forced by the government. The equilibriumdisequilibrium-equilibrium hypothesis or change-point-analyses' may not hold here. There is only one change point, the one child policy implemented in 1979. In India, in 1977 the CBR dropped by 4 points in one year because of overall 8 million vasectomies done during 1975-76. But these two years are not coming-out as change-points in the Goli's analyses why?

The summary of result in the application of various convergence models on different indicators (page 270) given in the last chapter is very interesting. He has used the method of so- called "catching-up process", Absolute $\beta$ - convergence, conditional $\beta$ convergence and non- parametric models (Absolute convergence) on different socioeconomic, demographic and child health parameters and comes to the conclusion that in none of the parameters all the four methods lead to the same conclusion that none of the parameters all the four methods lead to the same conclusion either for the entire period, 1971- 2001 or for any sub-periods. For example on IMR, Mr Goli finds from his analysis the following conclusion. Significance of convergence: Yes; nonsignificance of convergence: No.

\begin{tabular}{|l|c|c|}
\hline \multicolumn{1}{|c|}{ Method } & \multicolumn{2}{|c|}{ Period } \\
\hline $\begin{array}{c}\text { Entire Period (1981- } \\
\text { 2009) }\end{array}$ & $\begin{array}{r}\text { Recent Period (1991- } \\
\text { 2009) }\end{array}$ \\
\hline $\begin{array}{l}\text { Graphical Plots } \\
\text { (Catching up process }\end{array}$ & No & Yes \\
\hline Absolute $\beta$ - convergence & Yes & Yes \\
\hline $\begin{array}{l}\text { Conditional } \beta- \\
\text { convergence }\end{array}$ & Yes & Yes \\
\hline $\begin{array}{l}\text { Non- parametric models } \\
\text { (Absolute convergence) }\end{array}$ & No & No \\
\hline
\end{tabular}

If we use many indexes this type of conclusion is likely to occur and it is hard to reconcile the differences. In economics usually only $\beta$ - convergence, which is the regression coefficient of the rate of growth on the level is used to study convergence (where the $\beta$ - convergence is negative since people with higher levels of income tend to 
grow slower than the poorer, thus reducing the gap between the rich and poor), has been widely used. More indicators and tools are used; the more divergent are the result! The explanations given for the differences in the results are not convincing. This requires elaboration in the thesis under each chapter.

Finally, I wonder why the candidate did not use the HDI index, so advocated by the United Nations to study convergence. The whole of the United Nations Development Program is working on it and we have in India state level data on HDI for a number of years.

On the whole the thesis is an outcome of a brilliant piece of research by the candidate and deserves to be published after some shift editing. I recommend the award of $\mathrm{PhD}$ degree to the candidate.

Dated: 10-6-2014 


\section{Examiner's Report}

Title of Ph D thesis "Demographic Convergence and its Linkages with Health Inequalities in India"

\section{Student's name: Mr. Srinivas Goli}

With the changing demographic scenario and most demographic indicators progressing towards the final stages of transition, the major concern in India has been on heterogeneity across region and socioeconomic groups. While the demographic heterogeneity in India is well known, how far the differences are converging or diverging has been a matter of great interest. However, there were no systematic studies to understand the convergence issue in India until now. This thesis is an excellent exposition of the theory of convergence and its linkages with health inequality in India. The study has several merits. (1) This is a very comprehensive study considering the convergence of various economic, social and demographic indicators in addition to considering linkages between convergence and inequality. (2) The thesis is also has used appropriate statistical techniques for the analysis. Some innovative measures are also used that separates this work from the usual multivariate analysis. (3) There is a well thought out chapter plan and flow for the dissertation, which makes an easy reading. (4) The survey of literature is also extensive and covered major work in the area of work.

The student needs to be congratulated for this excellent work. Some of my comments which may be useful for further research and while publishing the thesis are as follows:

1. Chapter 3 of the thesis looks into the long-term trend in demographic transition using an innovative statistical tool. However, when analyzing the long-term trend and looks at the socioeconomic explanations for such trend the policy impacts often get neglected. The policy shifts and other, calamities can make a sudden structural shift in the indicator as compared to socioeconomic changes which are normally smooth. But The Economists normally use Chow test to understand such breaks which provide useful information. If the breaks are identified, the data need to be smoothened using any methods so that the longterm trends are clear.

2. The core chapter of the thesis deals with the issue of convergence. The chapter 4, 5, 6 and 7 analyze the convergence in various indicators. While chapter four considers convergence in socioeconomic indicators, chapter five looks at the fertility rate convergence. The analyses found that while economic variables are diverging, the fertility variables are converging in recent years. Perhaps, it reaffirms the fact that fertility transition is not critically related to economic factors in India. The mortality convergence has some setbacks in recent years. The child health care utilization has shown converging although at a lesser magnitude. To some extent, those indicators having an upper and lower bound 
appear to be converging as against economic variables like income which does not have an upper bound. Beta convergence and sigma convergence methodology are, perhaps, not a very powerful tool when the indicators have an upper and lower bound like in the cases of percentages. Perhaps, it would be also useful for analyses such indicator using other inequality measures as well. Moreover, it will be interesting to further examine whether there is any convergence pattern depending upon the levels of the indicators. It is possible that at least for indicators with upper bound like in the cases of percentages, the indicators diverge in the initial levels, while it converges at the later stages when the levels themselves become high.

3. The convergence of indicator has also close connection with the arguments on quantity-quality trade-off. At the lower levels of progress, the attempt would be to ensure increased converge or progress. But when the progress become high in terms of its level, the attempt also would turn more towards ensuring quality of the services of such indicator. Therefore, it would be also important to consider some indicators of quality when the overall level of an indicator itself is high will provide useful information on the convergent pattern from low to high levels.

4. Chapter 8 deals with two critical questions on the impact of economic and social indicators on health inequality. In addition to the plotting of data, pooled regression analysis is also used to understand the relationship. Any pooled regression analysis on demographic indicators in India needs to incorporate state effect as the major variation both in level and trends in demographic indicators are due to state effect. Perhaps, state effect can be due to policy and governance factor within the state in addition to the state specific effects. It is possible to strengthen the analysis by adding state variables also into the picture.

5. Chapter 9 is innovative in quantifying avoidable size of difference in demographic indicators across the states. The statistical tools used for the analyses are also innovative and the findings have lot of significance in terms of understanding the magnitude of difference and for policy formulation.

Overall, the thesis has provided excellent analyses of demographic convergence and inequalities in India. I have no hesitation in recommending this thesis for the award of $\mathrm{PhD}$ degree. I also strongly recommend publication of the thesis as a Book or in the form of different articles in reputed journals. I wish the student all the best. 


\section{Point by Point Reply to Reviewers Comments}

Acknowledgement: I would like to express thankfulness to two examiners for their thoughtful and constructive comments. I am highly grateful to both examiners for appreciating the work.

\section{Examiner I}

Comment 1. There are ten chapters in his thesis and each one of them is substantially rich and analytically forceful but there are many editorial mistakes which the candidate could have corrected by re reading the draft.

Reply: The suggestion on editorial mistakes is well taken. The thesis thoroughly edited.

Comment 2. There is a major difference between the convergence of demographic and socio-economic parameters in the developed countries of the west and what is taking place in India and other developing countries. In the later the convergence are forced by the government of the day by specific policies and programmes. In China all couples have one child families and there is a sharp convergence of fertility across all groups and geographic areas, but it is all forced by the government. The equilibrium-disequilibrium-equilibrium hypothesis or change-point-analyses' may not hold here. There is only one change point, the one child policy implemented in 1979. In India, in 1977 the CBR dropped by 4 points in one year because of overall 8 million vasectomies done during 1975-76. But these two years are not coming-out as change-points in the Goli's analyses why?

Reply: Yes, we agree with examiner's observation that the demographic transition in developing countries is much faster than expected. For example, Caldwell (2004: 297) stated 'Demographic transition theory has been largely transformed over the last half-century from grand theory to short-term theory, often endowed with such immediacy as to so limit our vision of the future that even population policy makers are made difficult." Demographic theorists lost their nerve as the globalisation of decline in mortality and fertility proceeded much more rapidly than they anticipated". However, this is hardly affecting the 'Change-Point analyses'. I clearly stated in Chapter 3 [page 52, 53] that the change-points marked in the graphical plots are the critical change-points which are Cumulative Sum (CUSUM) of changes in an indicator over a period. Here, the prediction of a critical change-points hardly affected by short-term fluctuations in an indicator. Moreover, we have not categorically said anywhere that the reasons for changes are only attributable to any one of the development, urbanization, economic growth, medical and contraceptive technology or government policies; rather, we have correlated our predicted change-points with 
corresponding multiple factors such as policy shifts, other developmental factors, unexpected natural hazards, epidemic and other relevant changes. Our analyses clearly showed that 1976 is the critical change-point in the transition of TFR [Chapter 3, page 62]. A decline of about one child per woman during the decade of 1971-81, or to refer to the midpoints of this period, approximately 1975-76, the year 1976 represents the first critical change-point of fertility trend in India [Chapter 3, page 62].

Comment 3: If we use many indices, it is difficult to make a single conclusion and it is hard to reconcile the differences. In economic usually only Beta convergence has been widely used, otherwise, if we use more tools, more divergent are the results. The explanations given for the differences in the results are not convincing. This requires elaborations in the thesis under each chapter.

Reply: The suggestion is well taken and more detailed explanations are incorporated in the revised thesis. However, we have given adequate explanations in the methods section where, we have discussed each technique and purpose of its application. Moreover, within the field of economics, from where most of these methods came, there is no agreement among the scholars about a single and standard measure of convergence. For example, Barro and Sala-I-Martin (1994, 1995) used $\beta$ convergence. Romer (1994) preferred sigma convergence over $\beta$ convergence, Wilson $(2001,2011)$ preferred only simple graphical plots of cumulative population distribution by initial level of the indicator, Dorius (2008, 2010) used both classical econometric tools like $\beta$ and sigma convergence. Bloom and Canning (2003) and Vollmer (2013) recommended non-parametric tools. Given, this diverse opinion on the application of convergence measures, we have decided to experiment many of the tools which have different purpose for its application. Agreed to the fact that this is the first time effort on this issue in the Indian context, theoretically there was no clear preceding evidence about which measure suits more in Indian context and its nature of transition and geographic diversity. It is a matter of great interest to test different models of convergence of Indian demographic data. However, we clearly stated in the conclusions of the each chapter and in the overall conclusion that, the Non-parametric measures are more robust tools for measuring convergence in socioeconomic, demographic and health indicators. According to the results of this particular technique in our study, we have not found absolute convergence yet, in any of the indicators selected in this study.

Comment 4: Why the candidate did not use the HDI Index, so advocated by the United Nations to study convergence. The whole of United Nations Development Programme is working on it and we have in India state level data on HDI for a number of years. 
Reply: We would like to point out that this important indicator was already considered in the analysis. The convergence analyses for $\underline{\mathrm{HDI}}$ were presented in Chapter 4 on Socioeconomic Convergence.

\section{$\underline{\text { Examiner II }}$}

Comment 1. The authors used Change-Point analyses for predicting critical changes, but The Economists normally use Chow test to understand ChangePoint analyses. If the breaks are identified, the data needs to be smoothened using any methods so that the long-terms trends clear.

Reply: There are several approaches to perform a trend analyses and 'changepoint analyses' (Page, 1955; Chow, 1960; Page, 1957; Hinkley, 1971; Hinkley and Schechtman, 1987; Pettitt, 1980; Box and Luceño, 1997; Sibanda and Sibanda, 2007; Fujisaki et al. 2008; Taylor, 2000a; Taylor, 2011). The Chow test is a statistical and econometric test of whether the coefficients in two linear regressions on different data sets are equal or The Chow test, commonly used in econometrics to test for structural breaks. For a linear model with one known single break in the main, the Chow test is often used. If the single break in mean is unknown, then Hartley's test may be appropriate. Other challenges are where there is: a known number of unknown breaks in the mean; an unknown number of (unknown) breaks in mean; breaks in variance. In these cases, the Chow test is not applicable (Gujarati, 2007). However, the purpose of using 'Change-Point analyser over other tools is, 'The procedure of performing a 'Change-Point analyses' is very flexible, as it can be carried out on all the types of time series data as well as attribute data, that data from non-normal distributions, ill-behaved and complaint data, and data with outliers' (Taylor, 2011). Moreover, the smoothing mechanism is an inbuilt process within the procedure of change-point analyses. The outliers in any population data create additional distinction in the data, making it more complicated to detect a change. The 'change-point analyses' technique is more robust to such outliers. Moreover, other tools will not give change-wise error rate.

Comment 2. It will be interesting to examine whether there is any convergence pattern depending upon the levels of indicators. It is possible that at least for indicators with upper bound like in the case of percentages, the indicators diverge in the initial levels while it converges at the later stages when the level themselves become high.

Reply: The hypotheses of this thesis are based on the same argument which reviewer is pointing to. These hypotheses have covered in many ways in the analysis. For example, I have carried out convergence analyses by sub-periods and the estimates for recent period, which are taking care of examiner's argument. A Non-parametric model like Kernel density plots at different time- 
points also gives some hints in the direction of progress in average levels and convergence process. Moreover, the convergence club charts drawn based on level of indicators at different time points also takes into account the examiner arguments.

Comment 3. The convergence of indicator has also close connection with the arguments on quantity-quality trade-off. At the lower levels of progress, the attempt would be to ensure increased converge or progress. But when the progress become high in terms of its level, the attempt also would turn more towards ensuring quality of the services of such indicator. Therefore, it would be also important to consider some indicators of quality when the overall level of an indicator itself is high will provide useful information on the convergent pattern from low to high levels.

Reply: We agree with reviewer's observation and the suggestion is well taken. However, at present the prime focus of current population and health policies is towards the convergence in basic indicators and once they are achieved then we can also consider convergence in quality of life indicators such as Healthy Life Expectancy (HALE). I will consider this suggestion in my future endeavours.

Comment 4. Chapter 8 deals with two critical questions on the impact of economic and social indicators on health inequality. In addition to the plotting of data, pooled regression analysis is also used to understand the relationship. Any pooled regression analysis on demographic indicators in India needs to incorporate state effect as the major variation both in level and trends in demographic indicators are due to state effect. Perhaps, state effect can be due to policy and governance factor within the state in addition to the state specific effects. It is possible to strengthen the analysis by adding state variables also into the picture.

Reply: The suggestion is well taken and I will try to consider this suggestion in my future endeavours. However, the unit of analyses for this model is states, so partially their effects were already controlled in the model. Nevertheless, I am accepting that the addition of state dummies will give more details. 


\begin{abstract}
This thesis comes out at a time when the debate on between-state and social group demographic and health inequalities continues to be largely debated based on most recent available information. However, analyses based on the recent demographic and health data have serious limitations in terms of understanding the true trajectories of between-state and social group inequalities. With the changing demographic scenario and most demographic indicators progressing towards the final stages of transition, the major concern in India has been heterogeneity across region and socioeconomic groups. While the demographic heterogeneity in India is well known, how far the differences are converging or diverging has been a matter of great interest. This study attempted to develop a comprehensive framework to study the demographic transition, convergence and its linkage with health inequalities in India. It innovate a mechanism of bridging the gap between demographic convergence and health inequalities by taking the theories and methods found in other social science disciplines. It revisits the old theories such as classic socioeconomic, demographic and health transition theories more clearly identifying the historical linkages between socioeconomic, demographic, health transition and health inequalities. It advances the empirical examination of demographic convergence assessment by assessing convergence not only in the averages, but also in the absolute and relative inequalities in population and health indicators. The core chapters of the thesis deal with the issue of convergence. Chapter 4, 5, 6 and 7 analyse the convergence using various indicators. While chapter four considers convergence in socioeconomic indicators, chapter 5 and 6 looks at the mortality and fertility convergence. Chapter 8 links fertility decline with child health inequalities and chapter 9 identifies the avoidable demographic difference. Chapter 10 presents summary and conclusion. The analyses foster that while economic variables are diverging, fertility variables are converging in recent years. Perhaps, it re-affirms the fact that fertility transition is not critically related to economic factors in India. The mortality convergence has some setbacks in recent years. The child health care utilization has shown converging although at a lesser magnitude. Moreover, fertility decline is associated with increasing in child health inequalities in a context where there are pre-existing socioeconomic inequalities. Inequality is often a consequence of progress. Not everyone gets rich at the same time, and not everyone gets immediate access to the latest life-saving measures. Further, growth, inequality, and catch up are the both sides of the same coin. The dark side is what happens when the process is hijacked, so that catch-up never comes. Powerful and wealthy elites have choked off demographic and health progress before, and they can do so again if they are allowed to undermine the institutions on which broad-based demographic and health progress depends. Now, it is confirmed from this study that a mere reduction in the number of children or fertility convergence in averages are not helping in distributing the fruits of demographic progress equally among the children of different states and socioeconomic groups. How far children and their development (particularly in terms of health) are faring during the progress of demographic transition is an important concern for population policy in India. In principle, inequalities are avoidable as disparities in health stem out from identifiable policy options exercised by governments. Thus, they are amenable to policy interventions. In general, this study promotes the importance of using effective health monitoring tools such as convergence models in countries such as India, which has huge socioeconomic and geographical disparities in the progress of demographic and health inequalities. The policy analyst can use convergence measures as tools for health policy evaluations in India and states. In an effort to continuously track regional progress in population and health indicators, it is important to test the convergence hypothesis for every five years.
\end{abstract}

